Algoritmos e técnicas de validação em agrupamento de dados multi-representados, agrupamento possibilístico e bi-agrupamento 



\title{
Algoritmos e técnicas de validação em agrupamento de dados multi-representados, agrupamento possibilístico e bi-agrupamento
}

\author{
Danilo Horta \\ Orientador: Prof. Dr. Ricardo José Gabrielli Barreto Campello \\ Tese apresentada ao Instituto de Ciências Matemáticas \\ e de Computação - ICMC-USP, como parte dos \\ requisitos para obtenção do título de Doutor em \\ Ciências - Ciências de Computação e Matemática \\ Computacional. VERSÃO REVISADA.
}


Ficha catalográfica elaborada pela Biblioteca Prof. Achille Bassi e Seção Técnica de Informática, ICMC/USP, com os dados fornecidos pelo(a) autor(a)

\begin{tabular}{|c|c|}
\hline \multirow[t]{3}{*}{$\begin{array}{l}\mathrm{HH} 881 \mathrm{a} \\
\mathrm{a}\end{array}$} & $\begin{array}{l}\text { Horta, Danilo } \\
\text { Algoritmos e técnicas de validação em agrupamento } \\
\text { de dados multi-representados agrupamento } \\
\text { possibilistico e bi-agrupamento / Danilo Horta; } \\
\text { orientador Ricardo José Gabrielli Barreto Campello. } \\
\text {-- São Carlos, } 2013 \text {. } \\
\quad 202 \text { p. }\end{array}$ \\
\hline & $\begin{array}{l}\text { Tese (Doutorado - Programa de Pós-Graduação em } \\
\text { Ciências de Computação e Matemática Computacional) -- } \\
\text { Instituto de Ciências Matemáticas e de Computação, } \\
\text { Universidade de São Paulo, } 2013 \text {. }\end{array}$ \\
\hline & $\begin{array}{l}\text { 1. Agrupamento de dados. I. Campello, Ricardo } \\
\text { José Gabrielli Barreto, orient. II. Título. }\end{array}$ \\
\hline
\end{tabular}


Aos meus pais pelo apoio incansável ao longo do período de elaboração deste trabalho. 



\section{Agradecimentos}

Ao prof. Ricardo Campello pelo comprometimento e pela orientação acadêmica. Aos colegas e amigos de laboratório pelas conversas e pelo incentivo.

À Solange Rezende pelo apoio e sabedoria.

Agradecimentos à Fundação de Amparo à Pesquisa do Estado de São Paulo (FAPESP) e ao Conselho Nacional de Desenvolvimento Científico e Tecnológico (CNPq) pelo contínuo apoio por meio de auxílios científico-financeiros. 



\section{Resumo}

Existem bases para as quais os dados são naturalmente representados por mais de uma visão. Por exemplo, imagens podem ser descritas por atributos de cores, textura e forma. Proteínas podem ser caracterizadas pela sequência de aminoácidos e pela representação tridimensional. A unificação das diferentes visões de uma base de dados pode ser problemática porque elas podem não ser comparáveis entre si ou podem apresentar diferentes graus de importância. Esses graus de importância podem, inclusive, se manifestar de maneira local, de acordo com a subestrutura dos dados em questão. Isso motivou o surgimento de algoritmos de agrupamento de dados capazes de lidar com bases multi-representadas (i.e., que possuem mais de uma visão dos dados), como o algoritmo $\mathrm{SCAD}$. Esse algoritmo se mostrou promissor em experimentos relatados na literatura, mas possui problemas críticos identificados neste trabalho que o impedem de funcionar em determinados cenários. Tais problemas foram solucionados por meio da proposição de uma nova versão do algoritmo, denominada ASCAD, fundamentada em provas formais sobre a sua convergência. Foram desenvolvidas versões relacionais do algoritmo ASCAD, capazes de lidar com bases descritas apenas por relações de proximidade entre os objetos. Foi desenvolvido também um índice de validação interna e relativa de agrupamento voltado para dados multi-representados.

A avaliação de agrupamento possibilístico e de bi-agrupamento por meio da comparação entre solução encontrada e solução de referência (validação externa) também foi explorada. Algoritmos de bi-agrupamento têm ganhado um interesse crescente da comunidade de análise de expressão gênica. No entanto, pouco se conhece do comportamento e das propriedades das medidas voltadas para validação externa de bi-agrupamento, o que motivou uma análise teórica e empírica dessas medidas. Essa análise mostrou que a maioria das medidas de biagrupamento possui problemas críticos e destacou duas delas como sendo as mais promissoras. Foram inclusas nessa análise três medidas de agrupamento particional não exclusivo, cujo uso na comparação de bi-agrupamentos é possível por meio de uma nova abordagem de avaliação de bi-agrupamento proposta nesta tese.

Agrupamento particional não exclusivo faz parte de um domínio mais geral de soluções, i.e., o domínio dos agrupamentos possibilísticos. Observou-se algumas falhas conceituais importantes das medidas de agrupamento possibilístico, o que motivou o desenvolvimento de novas medidas e de uma análise empírica e conceitual envolvendo 34 medidas. Uma das medidas propostas se destacou como sendo a única que apresentou avaliações imparciais com relação ao número de grupos, o valor máximo de similaridade ao comparar a solução ideal encontrada com a solução de referência e avaliações sensíveis às diferenças das soluções em todos os cenários considerados. 


\section{Abstract}

There are data sets for which the instances are naturally represented by more than one view. For example, images can be described by attributes of color, texture, and shape. Proteins can be characterized by the amino acid sequence and by their three-dimensional description. The unification of different views of a data set can be problematic because they may not be comparable or may have different degrees of importance. These degrees of importance may even manifest itself locally, according to the data substructures. This prompted the emergence of clustering algorithms capable of handling multi-represented data sets (i.e., data sets having more than one view) as the SCAD algorithm. This algorithm has shown promising results in experiments reported in the literature, but it has critical problems identified in this work that hinder its application in certain scenarios. These problems were solved here by proposing a new version of the algorithm, called ASCAD, based on formal proofs about its correctness. We developed relational versions for ASCAD, capable of handling data sets described only by the proximities between the instances. We also developed an index for internal and relative validation of multi-represented data clusterings.

The evaluation of possibilistic clustering and bi-clustering by comparing the found and reference solutions (external validation) was also explored. Bi-clustering algorithms have gained increasing interest from the community of gene expression analysis. However, little is known of the behavior and properties of the measures aimed at external validation of bi-clustering, which motivated a theoretical and empirical analysis of these measures in this work. This analysis showed that most bi-clustering measures has critical issues and highlighted two of the measures as being the most promising. We included in this analysis three measures of non-exclusive partitional clustering, whose use in comparing bi-clusterings is possible through a new approach proposed in this thesis.

Non-exclusive partitional clustering belong to a more general domain of solutions, i.e., the domain of possibilistic clusterings. There are some important conceptual flaws in the measures of possibilistic clustering, which motivated us to develop new measures and to conceptually and empirically analyse 34 measures. One of the proposed measures stood out as being the one who presented unbiased evaluations regarding the number of clusters, the maximum similarity when comparing the optimal solution with the reference one, and evaluations sensitive to solution differences in all scenarios considered. 



\section{CONTEÚDO}

ÍNDICE DE NOTAÇÃO

LISTA DE ABREVIAÇÕES $\quad$ Xv

\begin{tabular}{|lll}
\hline & Introdução & 1
\end{tabular}

1.1 Resumo das contribuições $\ldots \ldots \ldots \ldots$. . . . . . . . . . 4

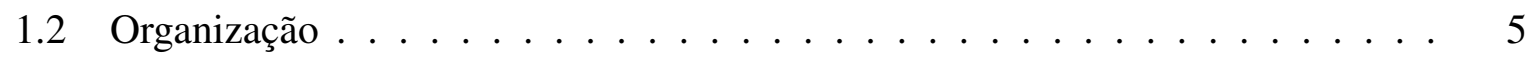

2 AGRUPAMENTO DE DADOS 9

2.1 Fundamentação . . . . . . . . . . . . . . . . . . . . 9 9

2.2 Tipos de agrupamento . . . . . . . . . . . . . . . . . . 11

2.3 Agrupamento em subespaços $\ldots \ldots \ldots$. . . . . . . . . . . . . . 13

$2.3 .1 \quad$ Subespaços alinhados com os eixos . . . . . . . . . . . . . . 14

2.3 .2 Subespaços arbitrariamente orientados . . . . . . . . . . . . . . . . . . . . . . . . . . . . .

2.3 .3 Bi-agrupamento . . . . . . . . . . . . . . . . . 19

2.4 Algoritmos para dados multi-representados $\ldots \ldots \ldots \ldots . \ldots . \ldots 22$

2.4 .1 Algoritmo SCAD . . . . . . . . . . . . . . . . 23

2.4 .2 Algoritmo CARD . . . . . . . . . . . . . . . . 27

2.5 Validação de agrupamento . . . . . . . . . . . . . . . . . . . . . 29

2.5 .1 Índice da Silhueta . . . . . . . . . . . . . . . . . . . . 31

2.5 .2 Silhueta Simplificada . . . . . . . . . . . . . . . 32

2.5 .3 Silhueta Simplificada Nebulosa . . . . . . . . . . . . . . 33

2.6 Considerações . . . . . . . . . . . . . . . . . . . . . 33

3 ALGORITMOS E TÉCNICAS EM DADOS MULTI-REPRESENTADOS 35

$3.1 \quad$ SCAD ajustado $\ldots \ldots \ldots \ldots \ldots \ldots$

3.1 .1 Versão rígida . . . . . . . . . . . . . . . . . . . 38

3.1 .2 Complexidade computacional . . . . . . . . . . . . . 39

3.1.3 Análise da função $\mathcal{Q}_{m, q}$ e do expoente de ponderação . . . . . . . . . . 41

3.2 Versão relacional do SCAD ajustado . . . . . . . . . . . . . . . . . . . 41 
3.3 Versão não euclidiana do SCAD ajustado relacional . . . . . . . . . . . . . . . 43

3.4 Provas de convergência . . . . . . . . . . . . . . . . . . 45

3.5 Silhueta para dados multi-representados . . . . . . . . . . . . . . . 49

3.6 Experimentos . . . . . . . . . . . . . . . . . . . 50

3.6 .1 SCAD contra SCAD ajustado . . . . . . . . . . . . . . . . . 51

3.6.2 Robustez à configuração do expoente de ponderação . . . . . . . . . . 52

3.6 .3 Robustez a atributos ruidosos . . . . . . . . . . . . . 55

3.6 .4 Ponderação de representações . . . . . . . . . . . . . . . . 60

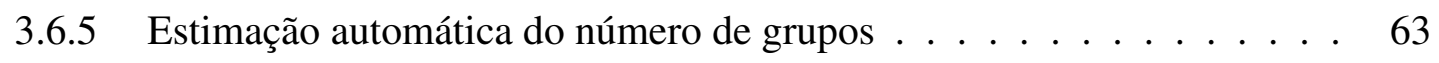

3.7 Considerações . . . . . . . . . . . . . . . . . . . . . . 70

4 MEDIDAS DE COMPARAÇÃO DE AGRUPAMENTOS

4.1 Fundamentação . . . . . . . . . . . . . . . . . . . . . . 72

4.2 Medidas para agrupamento possibilístico . . . . . . . . . . . . . . 74

4.2 .1 Revisão da literatura . . . . . . . . . . . . . . . . . 74

4.2 .2 Comentários sobre as medidas . . . . . . . . . . . . . . . . 78

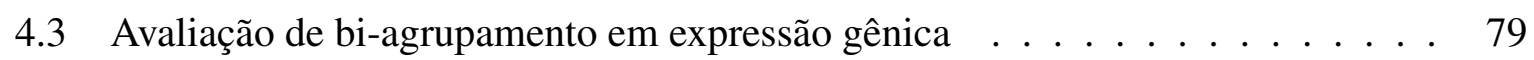

4.3 .1 Revisão da literatura . . . . . . . . . . . . . . . . . . . . . . . . . . . . . . 80

4.3 .2 Comentários sobre as medidas . . . . . . . . . . . . . . . . . 84

4.4 Considerações . . . . . . . . . . . . . . . . . . . . . . . . . . . . . . . . . 84

5 NOVAS MEDIDAS PARA AGRUPAMENTO POSSIBILÍSTICO 87

5.1 Medida 13FRI . . . . . . . . . . . . . . . . . . . . . . . 87

$5.1 .1 \quad$ Ajuste para aleatoriedade . . . . . . . . . . . . . . . . . . 90

5.1 .2 Considerações . . . . . . . . . . . . . . . . . 92

5.2 Medida 13GRI $\ldots \ldots \ldots \ldots \ldots$

5.3 Complexidade computacional e implementação . . . . . . . . . . . . . . . . 94

5.4 Experimentos . . . . . . . . . . . . . . . . . . . . 95

5.4 .1 Similaridade entre agrupamentos . . . . . . . . . . . . . . 96

5.4 .2 Agrupamentos gerados aleatoriamente . . . . . . . . . . . . . . 104

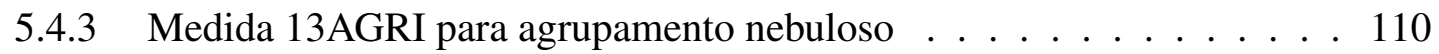

5.4 .4 Medida 13AGRI como estatística de estabilidade . . . . . . . . . . . . 113

5.5 Provas teóricas . . . . . . . . . . . . . . . . . . . . . . . . . . . . . . 114

5.6 Considerações . . . . . . . . . . . . . . . . . . . . . . . . . . 119

6 DESENVOLVIMENTO DE MEDIDAS PARA BI-AGRUPAMENTO 121

6.1 Trabalhos relacionados . . . . . . . . . . . . . . . . . . . 122

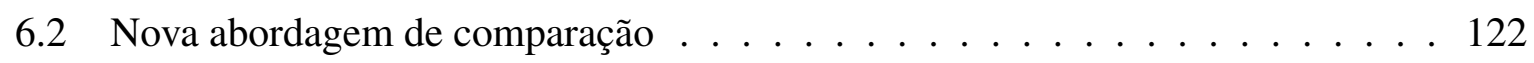

6.3 Análise teórica das medidas . . . . . . . . . . . . . . . . . . . . . . . . . . 124

6.3 .1 Considerações . . . . . . . . . . . . . . . . . . 130

6.4 Experimentos . . . . . . . . . . . . . . . . . . . . . . 131 
$6.4 .1 \quad$ Experimento $1 \ldots \ldots \ldots \ldots \ldots$

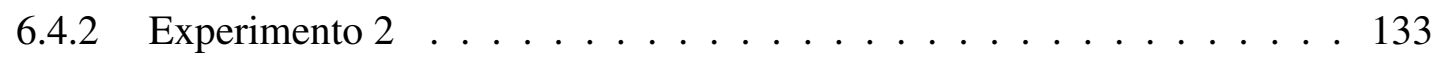

6.4 .3 Experimento $3 \ldots \ldots \ldots \ldots \ldots$

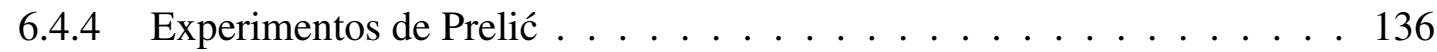

6.4 .5 Experimentos de Eren . . . . . . . . . . . . . . . . . . 137

6.5 Provas teóricas . . . . . . . . . . . . . . . . . . . . . . 138

6.6 Considerações . . . . . . . . . . . . . . . . . . . . . . . 153

7 Conclusões 155

7.1 Trabalhos futuros . . . . . . . . . . . . . . . . . . . . . . . . . . . 157

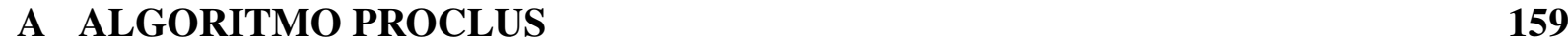

B MEDIDAS 09EBC E 10CSI EM TERMOS DE CONJUNTOS

C ALGORITMOS BASEADOS EM MEDÓIDES PARA DADOS MULTI$\begin{array}{ll}\text { REPRESENTADOS } & 165\end{array}$

C.1 Fundamentação . . . . . . . . . . . . . . . . . . . . . . . . 166

C.2 Algoritmos . . . . . . . . . . . . . . . . . . . . . . 167

C.3 Inicialização . . . . . . . . . . . . . . . . . . . . . . . . . . . 168

C.4 Atributos como visões distintas da base $\ldots \ldots \ldots \ldots$. . . . . . . . . . . . 169

C.5 Considerações . . . . . . . . . . . . . . . . . . . . . . . . . . 173

D Medida-F para bi-agrupamento de Turner et al.

E COMENTÁRIOS SOBRE TESTE DE HIPÓTESE

E.1 Hipótese estatística . . . . . . . . . . . . . . . . . . . . . . . . . . 177

E.2 Wilcoxon rank-sum test . . . . . . . . . . . . . . . . . . . . . . . . . . . 179

F COMMENTS ON "REL. GEN. CLU. VAL. IND."

F.1 Introduction . . . . . . . . . . . . . . . . . . . . . . . 183

F.2 Distances between Centroids - Relational Case. . . . . . . . . . . . . . . 184

F.3 Conclusions . . . . . . . . . . . . . . . . . . . . . . . . . . . 188 



\section{ÍNDICE DE NOTAÇÃO}

$\tilde{x} \quad$ letras minúsculas e itálicas com til denotam objetos

$\tilde{x}_{i} \quad i$-ésimo objeto

$x \quad$ letras minúsculas e itálicas denotam escalares

$x_{i} \quad i$-ésimo escalar

x letras minúsculas denotam vetores (quando usado em uma operação matricial, $\mathrm{x}$ é interpretado como um vetor coluna)

$\mathrm{x}^{i} \quad i$-ésimo vetor

$\mathrm{x}_{i} \quad i$-ésimo atributo do vetor $\mathrm{x}$

$\mathrm{X} \quad$ letras maiúsculas denotam matrizes

$\mathrm{X}^{i} \quad i$-ésima matriz

$\mathrm{X}_{i,:} \quad i$-ésima linha da matriz $\mathrm{X}$

$\mathrm{X}_{:, i} \quad i$-ésima coluna da matriz $\mathrm{X}$

$\mathrm{X}_{i, j} \quad$ elemento da $i$-ésima linha e $j$-ésima coluna da matriz $\mathrm{X}$

$X \quad$ letras maiúsculas e itálicas denotam conjuntos

$X_{i} \quad i$-ésimo conjunto

$\mathbb{N}_{i, j} \quad$ conjunto de inteiros $\{i, i+1, \ldots, j\}$

$\vec{x} \quad$ letras minúsculas e itálicas com seta denotam eixos 



\section{LISTA DE ABREVIAÇÕES}

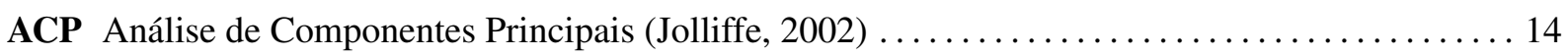

ALOI Amsterdam Library of Object Images (Geusebroek et al., 2005) ..................... 35

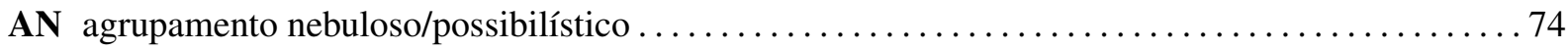

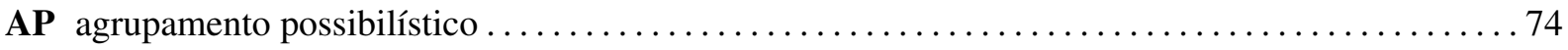

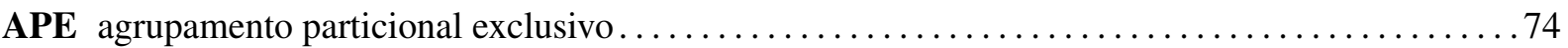

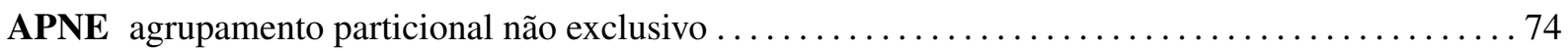

ARI Adjusted Rand Index (Hubert e Arabie, 1985) .................................. 31

ASCAD Adjusted Simultaneous Clustering and Attribute Discrimination ....................4

BC BCubed (Amigó et al. 2009) ................................................ 73

CARD Clustering and Aggregating Relational Data (Frigui et al., 2007) ....................2 2

CCA Cheng and Church's Algorithm (Cheng e Church, 2000)........................21

COSA Clustering Objects on Subsets of Attributes (Friedman e Meulman, 2004)................ 16

DBSCAN Density-Based Spatial Clustering of Applications with Noise (Ester et al., 1996) ........22

EM Expectation Maximization (Dempster et al. 1977) ................................ 12

EMGM Expectation Maximization for Gaussian Mixture (Dempster et al., 1977) ............... 96

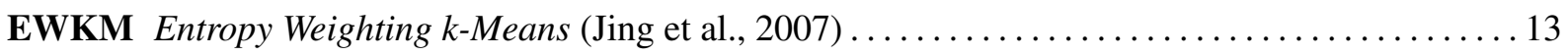

FCM Fuzzy c-Means $(\overline{\text { Dunn, }}, 1973 ;$ Bezdek, 1981) ............................... 12

FCMdd Fuzzy c-Medoids (Krishnapuram et al., 2001) ............................... 166

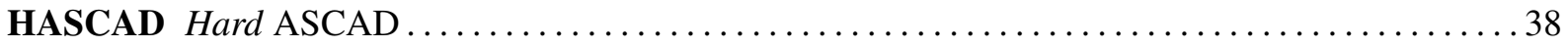


IPCM2 Improved Possibilistic c-Means 2 (Zhang e Leung, 2004) ......................... 97

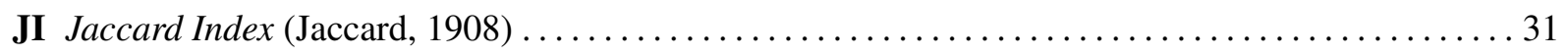

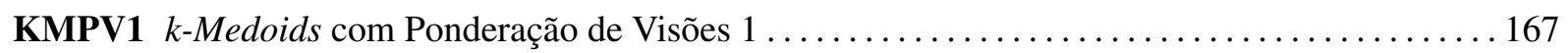

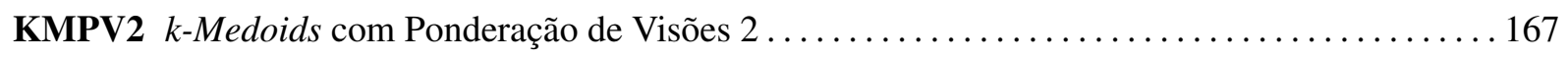

LAC Locally Adaptive Clustering (Domeniconi et al., 2004) ........................... 16

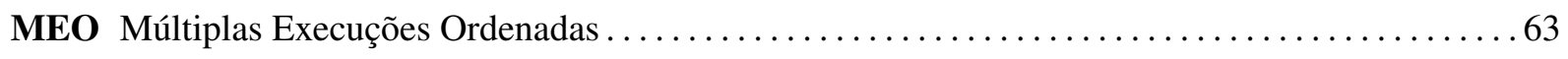

NERFCM Non-Euclidean Relational Fuzzy c-Means (Hathaway e Bezdek, 1994)...............227

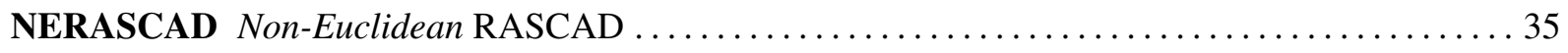

PAM Partitioning Around Medoids (Kaufman e Rousseeuw, 1990) ..........................15

PROCLUS Projected Clustering Aggarwal et al., 1999) ............................... 15

RASCAD Relational Adjusted Simultaneous Clustering and Aspect Discrimination ............. 35

RFCM Relational Fuzzy c-Means (Hathaway et al., 1989) ............................... 10

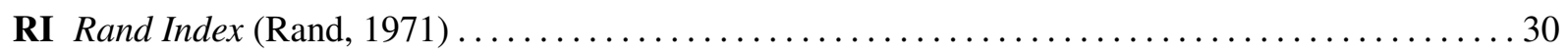

SCAD Simultaneous Clustering and Attribute Discrimination (Frigui e Nasraoui, 2004) ...........2

SS Silhueta Simplificada (Hruschka et al., 2004, 2006, Vendramin et al., 2010) ................. 33

SSN Silhueta Simplificada Nebulosa (Campello e Hruschka, 2006) ........................ 33

SSNPR Silhueta Simplificada Nebulosa para Ponderação de Representações . . . . . . . . . . . . . 50

SUBCLU Density-Connected Subspace Clustering (Kailing et al., 2004a) ....................96

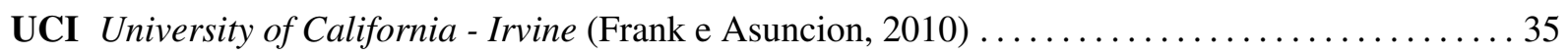

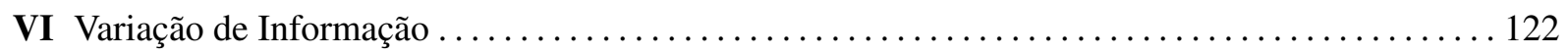




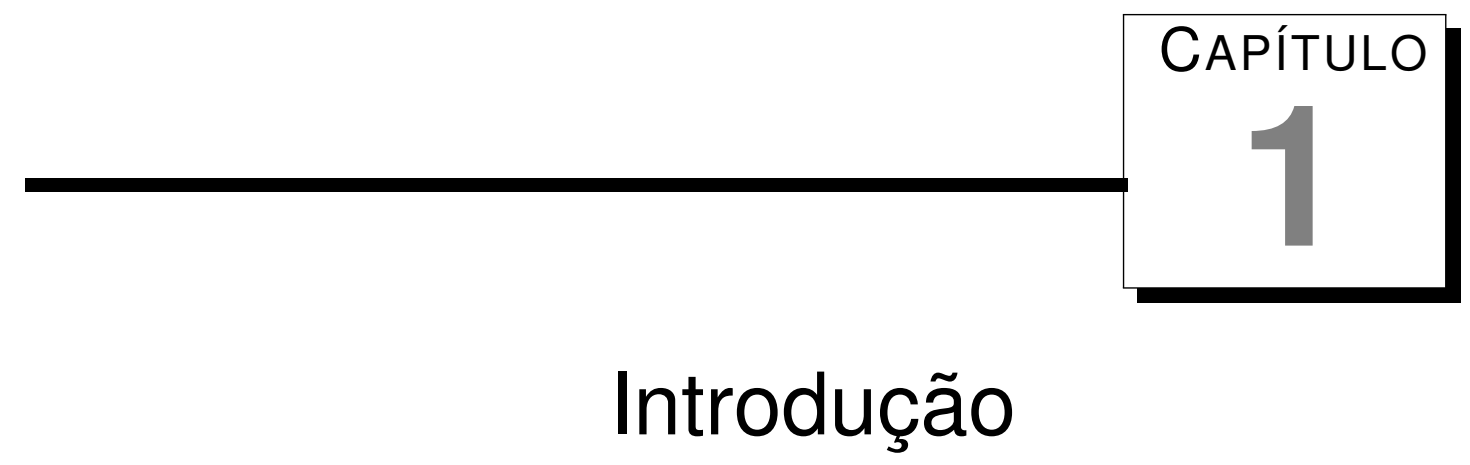

Agrupamento de dados é um problema conceitual fundamental na mineração de dados, o qual objetiva determinar um conjunto finito de categorias que descrevam os dados de acordo com as similaridades entre os seus objetos (Kaufman e Rousseeuw, 1990; Everitt et al., 2001). A solução para esse problema frequentemente é o objetivo final do procedimento de mineração, possuindo um amplo espectro de aplicações que inclui desde segmentação de imagens e mercado até categorização de documentos e de funções biológicas em dados de expressão gênica (Jain et al., 1999; Xu e Wunsch, 2005). A solução de um problema de agrupamento também pode ajudar a resolver outros problemas relacionados, como classificação de padrões e extração de regras (Wang e Fu, 2005).

É comum representar os objetos de uma base de dados por vetores de atributos. Dessa forma, cada objeto pode ser visto como um ponto no respectivo espaço de atributos, o qual frequentemente possui uma distância associada como medida de dissimilaridade entre os objetos. Atributos com pouca ou nenhuma relevância são muitas vezes usados na descrição de bases de dados, os quais podem atrapalhar o processo de agrupamento (Almuallim e Dietterich, 1991; Kira e Rendell, 1992b a; Wettschereck et al., 1997). O uso de atributos indesejáveis acontece porque dificilmente se sabe ao certo quais atributos devem ser utilizados na descrição de uma dada base de dados (Kira e Rendell, 1992b). Existe uma literatura extensa de seleção e ponderação de atributos para combater esse problema (Kira e Rendell, 1992b; Blum e Langley, 1997; Mitra et al., 2002; Liu e Yu, 2005). No entanto, essas técnicas assumem que a importância de cada atributo é uniforme para as diferentes subestruturas dos dados, o que nem sempre é o caso. Por esses motivos, foram desenvolvidos algoritmos de agrupamento que ponderam os atributos a medida que revelam subestruturas da base de dados (Frigui e Nasraoui, 2000; Frigui e Salema, 2003; Frigui e Nasraoui, 2004; Frigui et al., 2007). Esses algoritmos atribuem graus potencialmente diferentes de relevância aos atributos de acordo com a subestrutura do grupo em questão. Tais métodos são denominados de algoritmos de projeção suave na literatura de agrupamento 
de dados em subespaços (Kriegel et al., 2009), a qual consiste em algoritmos que buscam por subestruturas escondidas nos subespaços de atributos.

O algoritmo Simultaneous Clustering and Attribute Discrimination (Frigui e Nasraoui, 2004) (SCAD) é capaz de ponderar, da forma descrita acima, não apenas atributos, mas também subconjuntos de atributos, dependendo da configuração escolhida pelo usuário. Tais subconjuntos podem ser entendidos como diferentes representações ou visões dos dados. De fato, existe uma variedade de aplicações nas quais os dados são multi-representados (Kailing et al., 2004b; Bickel e Scheffer, 2004; Achtert et al., 2006b), tais como agrupamento de páginas da web (e.g., representação extrínseca, composta por textos de âncoras que apontam para a página em questão, e representação intrínseca, composta pelos textos da própria página), agrupamento de proteínas (caracterizadas pela sequência de aminoácidos, pela estrutura secundária e pela representação tridimensional) e agrupamento de imagens (descritas por atributos de cores, textura e forma). As diferentes representações dos dados são comumente consolidadas em uma única representação de maneira que os algoritmos de agrupamento tradicionais possam atuar. No entanto, essa unificação pode ser problemática quando as representações não são comparáveis. Isto é, como seria possível unificar os atributos de uma sequência de aminoácidos com a representação tridimensional da molécula? Além disso, diferentes representações podem apresentar diferentes graus de importância para diferentes subestruturas dos dados.

O algoritmo SCAD oferece uma maneira de contornar os problemas citados acima ao agregar ponderadamente as diferentes representações dos mesmos dados. A importância do algoritmo SCAD para o cenário de dados multi-representados e de agrupamento em subespaços motivou a definição da

Hipótese 1: Algoritmos de agrupamento de dados com ponderação automática de representações auxilia no processo de agrupamento em bases com representações que possuem graus de importância distintos.

No entanto, o algoritmo SCAD, bem como a sua versão relacional chamada de Clustering and Aggregating Relational Data (Frigui et al., 2007) (CARD), possuem problemas que podem impedi-los de funcionar em determinadas aplicações. Por exemplo, as suas equações não consideram todos os pontos críticos na otimização das variáveis, tornando-os vulneráveis a situações de falha. Esses problemas foram identificados ao decorrer deste trabalho e motivaram o

Objetivo 1: Analisar e solucionar os problemas dos algoritmos SCAD e CARD que o impedem de funcionar em certos cenários. Avaliar experimentalmente os algoritmos resultantes, incluindo o cenário no qual a base de dados possui diferentes representações com graus distintos de relevância.

São apresentadas provas teóricas que comprovam a convergência dos novos algoritmos nos cenários outrora problemáticos, além de experimentos corroborando com a eficácia dos algoritmos ajustados em cenários de multi-representação dos dados. Outras contribuições foram derivadas ao longo do processo que culminou no cumprimento do Objetivo 1, como a proposição de uma versão do algoritmo SCAD ajustado para agrupamento particional exclusivo e uma análise da complexidade computacional dos algoritmos, todas detalhadas nesta tese. 
Os algoritmos de agrupamento que ponderam representações dos dados produzem soluções que são compostas não somente pelos agrupamentos encontrados, mas também pelos pesos de relevância. Como qualquer solução encontrada por algoritmos de agrupamento, essas também devem ser avaliadas por métodos de validação de agrupamento. No entanto, não foram encontrados índices de validação de agrupamento que consideram os pesos na avaliação; em especial, índices de validação relativa, capazes de discriminar qual solução, dentre um conjunto de soluções candidatas, é a melhor com base somente nas soluções em si e nos dados (i.e., sem informação externa). Ao ignorar os pesos encontrados pelo algoritmo, parte da solução não está sendo avaliada, o que motivou a

Hipótese 2: Validação relativa de agrupamento que considera a ponderação de representações pode desempenhar-se melhor que a validação relativa tradicional na qual a ponderação é desconsiderada.

O índice da silhueta simplificada (Hruschka et al., 2004, 2006), usado em validação relativa de agrupamento, tem apresentado um desempenho destacado em estudos comparativos recentes (Vendramin et al., 2008, 2009, 2010), o que, em conjunto com a Hipótese 2, motivou o

Objetivo 2: Desenvolver uma versão da silhueta simplificada que considere a ponderação de representações associada ao agrupamento. Compará-la com a versão sem ponderação.

Dado que a ponderação de atributos é um caso particular da ponderação de representações (cada representação pode consistir de apenas um atributo), a silhueta simplificada desenvolvida também pode ser usada para agrupamento que tem associado a si uma ponderação dos atributos. Portanto, esse índice relativo é também aplicável a soluções geradas por algoritmos de agrupamento em subespaços baseados em projeção suave. $\mathrm{O}$ índice desenvolvido foi comparado experimentalmente com a versão que não considera a ponderação das representações da base de dados, mostrando um desempenho superior aferido por dois índices de validação externa de agrupamento de dados.

Uma abordagem que tem atraído interesse da comunidade de análise de expressão gênica (Cheng e Church, 2000; Madeira e Oliveira, 2004; Prelić et al., 2006; Busygin et al., 2008; De França et al., 2013), e que também lida com o problema de atributos pouco relevantes, é a abordagem de bi-agrupamento de dados (Mirkin, 1996). Novos algoritmos dessa abordagem, chamados de algoritmos de bi-agrupamento de dados, têm sido propostos todos os anos, o que demanda estudos comparativos e o uso de índices para a validação externa. No entanto, os índices clássicos de agrupamento de dados não são diretamente aplicáveis em bi-agrupamentos, i.e., em soluções produzidas por algoritmos de bi-agrupamento. Alguns índices de validação externa de bi-agrupamento foram recentemente propostos (Liu e Wang, 2007; Santamaría et al. 2007; Lee et al., 2009; Hochreiter et al., 2010), invariavelmente em trabalhos nos quais a principal contribuição foi a proposição de um novo algoritmo de bi-agrupamento ou a comparação experimental de algoritmos de bi-agrupamento. Como consequência, propriedades importantes que um índice de validação externa deve possuir foram negligenciadas, acarretando em problemas críticos identificados durante este trabalho. Observou-se também que um bi-agrupamento poderia ser representado de outra forma, a saber, como um agrupamento particional não exclu- 
sivo, possibilitando o uso de índices de validação externa aplicáveis a tais agrupamentos. Esse tipo de agrupamento é um caso particular de um outro que engloba quase todas as soluções geráveis por algoritmos de agrupamento não hierárquicos, denominado de agrupamento possibilístico. Como a área de índices para a validação externa de agrupamento possibilístico não está consolidada, estabeleceu-se a

Hipótese 3: É possível desenvolver um índice de validação externa para agrupamento possibilístico que apresenta avaliações mais justas que os atuais.

A hipótese acima motivou o

Objetivo 3: Desenvolver um índice de validação externa para agrupamento possibilístico que apresenta avaliações mais justas que os atuais.

O comportamento do índice proposto foi avaliado por meio de experimentos com soluções de algoritmos de agrupamento de diferentes naturezas e em cenários cujos resultados destacariam avaliações desejáveis das avaliações indesejáveis. Ademais, o índice proposto foi também usado como uma estatística que mede a estabilidade de um conjunto de agrupamentos, apresentando bons resultados em bases de dados reais.

A conclusão do Objetivo 3 forneceu um índice que, por meio de uma nova metodologia de avaliação, é aplicável a bi-agrupamentos, motivando a

Hipótese 4: É possível validar bi-agrupamentos por meio de um índice de validação externa de agrupamento particional não exclusivo de tal modo que a validação seja superior, em termos de propriedades conceituais, que a de índices propostos especificamente para bi-agrupamento.

Essa hipótese implicou no

Objetivo 4: Propor uma metodologia que permita o uso de índices de agrupamento particional não exclusivo para validar externamente bi-agrupamentos. Analisar teoricamente os diversos índices.

A qualidade dos índices na validação externa de bi-agrupamento foi aferida teoricamente, por meio de oito propriedades conceituais propostas no presente trabalho, e empiricamente, envolvendo modelos de bi-grupos e algoritmos de bi-agrupamento propostos em trabalhos importantes da literatura de expressão gênica. Essa análise salientou a considerável deficiência que a maioria dos índices externos para bi-agrupamento possui e destacou dois índices como sendo os mais confiáveis.

\subsection{Resumo das contribuições}

Seguem as principais contribuições desta tese.

1. Aprimoramento do algoritmo SCAD para agrupamento de dados multi-representados, originando o algoritmo Adjusted Simultaneous Clustering and Attribute Discrimination (ASCAD.

2. Desenvolvimento da versão relacional do algoritmo ASCADe prova da equivalência entre as duas versões. 
3. Proposição de um índice de validação interna e relativa que considera a ponderação das diferentes representações dos dados.

4. Revisão de 28 medidas de similaridade entre agrupamentos mais gerais que os agrupamentos particionais exclusivos.

5. Revisão de 14 medidas de similaridade entre bi-agrupamentos.

6. Proposição das medidas 13FRI, 13AFRI, 13GRI e 13AGRI, sendo esta última a mais geral, voltada para agrupamentos possibilísticos e ajustada para aleatoriedade.

7. Comparação empírica das medidas propostas com 32 outras em conjuntos de agrupamentos gerados por algoritmos de diferentes naturezas.

8. Proposição de uma nova abordagem para a comparação de bi-agrupamentos.

9. Definição formal de oito propriedades para medidas de similaridade entre biagrupamentos

10. Análises teórica e empírica de 16 medidas de bi-agrupamento com base nas propriedades propostas.

\subsection{Organização}

No Capítulo 2 são apresentados conceitos gerais da área de agrupamento de dados. São definidos os seguintes tipos de agrupamento de dados: particional exclusivo, particional não exclusivo, nebuloso e possibilístico. Esses quatro tipos de agrupamento se relacionam entre si de forma hierárquica, de forma que o algoritmo possibilístico engloba todos os outros. A área de agrupamento em subespaços é introduzida como uma forma de lidar com problemas advindos de bases de dados com muitos atributos ou com atributos apresentando diferentes graus de relevância para diferentes subestruturas dos dados. Em especial, foi definido o bi-agrupamento de dados como uma abordagem de agrupamento que tem ganhado espaço na literatura de análise de expressão gênica. A área de agrupamento de dados multi-representados é definida como aquela na qual as bases se encontram representadas por mais de uma visão dos dados. Dois algoritmos de agrupamento de dados multi-representados chamados SCAD e CARD são definidos e alguns de seus problemas são discutidos. São apresentadas três abordagens empregadas na validação de agrupamento: validação externa, validação interna e validação relativa. O índice da Silhueta (Rousseeuw, 1987; Falkenauer, 1998; Everitt et al., 2001) bem como duas variantes são definidas como medidas utilizadas nas validações interna e relativa.

No Capítulo 3 os algoritmos SCAD e CARD são discutidos mais a fundo, trazendo a tona outros problemas conceituais que eles apresentam. São propostas modificações para esses dois algoritmos sustentadas por provas matemáticas a fim de sanar os problemas encontrados. É provado que os passos dos novos algoritmos de fato minimizam as suas funções-custo. Também é provado que os novos algoritmos, embora sejam capazes de lidar com bases de dados 
de naturezas distintas (relacionais e não relacionais, vide Seção 2.1), são equivalentes, sob uma suposição razoável. Uma das versões do índice da Silhueta é generalizada para incluir a ponderação das representações dos dados que os algoritmos propostos geram na avaliação da solução de agrupamento. Os experimentos indicam que os algoritmos em questão são robustos a atributos ruidosos, são melhores que as suas contrapartidas que não ponderam representações e que o índice proposto é superior à versão da qual ele deriva. É também demonstrado experimentalmente que o índice proposto pode ser usado em conjunto com uma abordagem repetitiva para a estimação do melhor número de grupos.

No Capítulo 4 é feita uma revisão de medidas de similaridade para agrupamento de dados mais gerais que o tradicional agrupamento particional exclusivo e para bi-agrupamento de dados. É argumentado que falta fundamentação para as medidas que comparam agrupamentos mais gerais que o particional exclusivo, no sentido de que as equações utilizadas foram deduzidas para o tipo de agrupamento particional exclusivo e o seu uso em contextos mais gerais deveria ser formalmente justificado. Mostra-se um exemplo no qual a aplicação de tais medidas resulta em valores inesperados e contra-intuitivos. Outros problemas das medidas revisadas são também discutidos, os quais são tratados mais a fundo no Capítulo5. São apresentadas quatro abordagens comumente empregadas na literatura de expressão gênica para avaliar a eficácia de algoritmos de bi-agrupamento, sendo que uma delas consiste no uso de medidas de similaridade para comparar soluções encontradas com as de referência. Como não existe uma análise teórica das medidas de similaridade para bi-agrupamentos, é esperado que várias delas apresentem problemas conceituais. Um desses problemas consiste na associação entre os bi-grupos da solução encontrada e os da solução de referência, o que resulta na insensibilidade quanto à homogeneidade das soluções. Essa e outras questões são tratadas mais a fundo no Capítulo 6 .

No Capítulo 5 é desenvolvida a medida 13FRI voltada para agrupamentos particionais exclusivo, particionais não exclusivo e nebulosos. Por meio de um modelo nulo para a geração de agrupamentos proposto nesta tese, a medida 13FRI é ajustada para aleatoriedade de forma a atribuir valores próximos de zero na comparação de soluções geradas de forma independente, originando a medida 13AFRI. A medida 13AFRI é generalizada para lidar também com agrupamentos possibilísticos, dando origem à medida 13AGRI, a qual também é ajustada para aleatoriedade. Cada componente das medidas é justificado e as afirmações não triviais são formalmente provadas. É apresentada uma implementação eficiente das medidas, sob a qual uma análise da complexidade computacional para o pior caso é feita. As medidas propostas são comparadas experimentalmente com 32 outras em conjuntos de agrupamentos gerados por algoritmos de diferentes naturezas, em dezenas de bases reais e artificiais.

No Capítulo 6 são apresentadas oito propriedades relevantes para a comparação de biagrupamentos. Propõe-se uma nova abordagem para a comparação de bi-agrupamentos por meio da transformação de soluções de bi-agrupamento em agrupamentos particionais não exclusivos. Essa abordagem permite o uso de medidas desenvolvidas para agrupamentos particionais não exclusivos na comparação de bi-agrupamentos. As medidas de bi-agrupamento revisadas no Capítulo 4 em conjunto com duas medidas de agrupamento particional não exclusivo utiliza- 
das por meio da abordagem proposta são analisadas de acordo com as propriedades propostas. Prova-se formalmente quais propriedades cada uma das medidas apresenta. A relevância das propriedades é verificada por meio de experimentos envolvendo modelos de bi-grupos e algoritmos de bi-agrupamento selecionados de estudos importantes da literatura de expressão gênica.

No Capítulo 7 são fornecidas as conclusões desta tese e sugeridos trabalhos futuros.

No Apêndice $\mathbf{A}$ é revisado um algoritmo de agrupamento em subespaços, por motivos ilustrativos da área. No Apêndice $\mathbf{B}$ duas medidas aplicáveis em agrupamentos particionais não exclusivos são redefinidas por meio de conjuntos, de forma a facilitar a análise de medidas relatada no Capítulo 6. No Apêndice $\mathbf{C}$ são propostos algoritmos baseados em medóides para dados multi-representados e apresentados alguns experimentos analisando certas características deles. Esses algoritmos foram fundamentados de maneira semelhante aos algoritmos propostos no Capítulo 3. No Apêndice $\mathbf{E}$ são feitos comentários gerais sobre teste de hipótese, em especial sobre o teste estatístico proposto por Wilcoxon. No Apêndice Fé apresentado um artigo submetido ao jornal científico "IEEE Transactions on Fuzzy Systems", mas que foi rejeitado devido a uma publicação sobre o mesmo assunto que ocorreu no mesmo jornal enquanto o artigo em questão estava sendo analisado pelos revisores. 



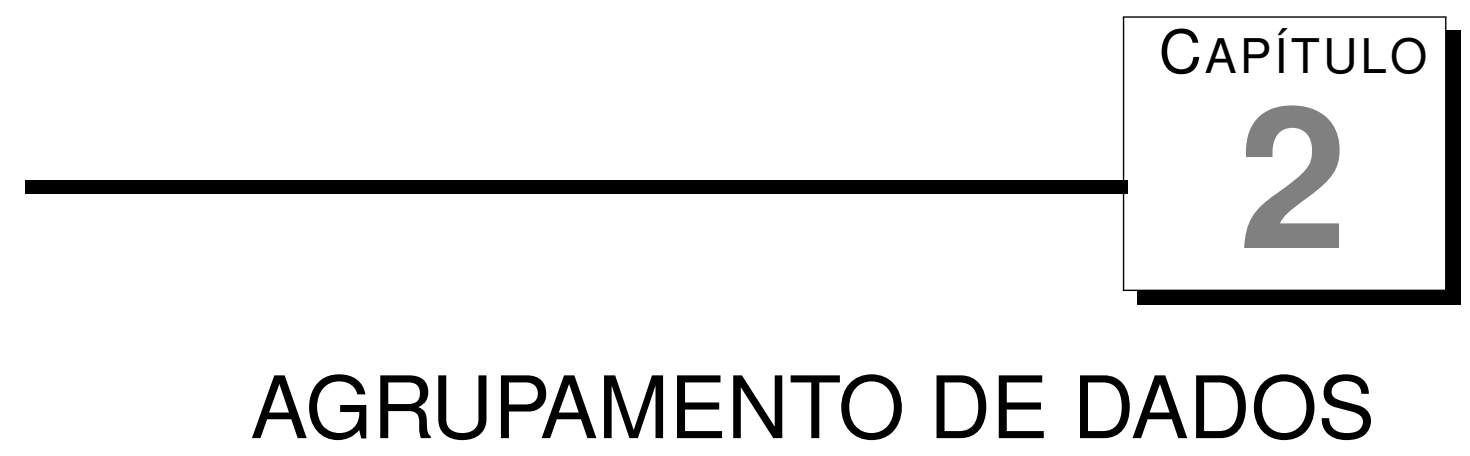

Neste capítulo são definidos conceitos fundamentais sobre agrupamento de dados que permeiam toda a tese. Existem diversos tipos de soluções de agrupamento. Quatro deles são definidos na Seção 2.2, sendo utilizados nas técnicas e análises desenvolvidas durante o presente trabalho. Uma visão geral da área de agrupamento em subespaços é fornecida na Seção 2.3, na qual são apresentadas as duas principais motivações para o surgimento do campo de pesquisa. Uma delas motivou o desenvolvimento dos algoritmos SCAD e CARD, definidos na Seção 2.4 sob a categorização de algoritmos que lidam com dados multi-representados. A validação de agrupamento é introduzida na Seção 2.5. como uma etapa importante do agrupamento de dados. Considerações são dadas na Seção 2.6 .

\subsection{Fundamentação}

Agrupamento de dados consiste na organização de uma coleção de objetos $O \triangleq$ $\left\{\tilde{o}_{1}, \tilde{o}_{2}, \ldots, \tilde{o}_{n}\right\}$ em grupos com base em similaridades entre esses objetos (Jain et al., 1999; Everitt et al., 2001; Theodoridis e Koutroumbas, 2006) ou com base em algum tipo de coerência entre os objetos (Madeira e Oliveira, 2004). Por meio dessa organização, espera-se que objetos de um mesmo grupo apresentem uma forte semelhança ou coerência entre si. Concomitantemente, espera-se que objetos de grupos distintos apresentem uma fraca semelhança ou coerência entre si. A esse tipo de organização se dá o nome de agrupamento de dados, o que pode ser obtido por meio da aplicação de um algoritmo de agrupamento de dados.

Existem pelo menos três maneiras comumente utilizadas para a representação de uma base de dados para agrupamento. Na representação vetorial, cada objeto é denotado por um vetor de atributos. Suponha que a base de dados $O$ seja representada em um espaço $p$-dimensional (i.e., de $p$ atributos) e possua $n$ objetos. O objeto $\tilde{o}_{j}$ é, então, denotado pelo vetor $\mathrm{x}^{j}$, no qual $\mathrm{x}_{s}^{j}$ é 
o valor correspondente ao $s$-ésimo atributo. A coleção de vetores $X \triangleq\left\{\mathrm{x}^{1}, \mathrm{x}^{2}, \ldots, \mathrm{x}^{n}\right\} \subset \mathbb{R}^{n}$ representa a base de dados $O$.

Uma segunda forma de representação consiste no uso da chamada matriz de dados. Considerando, novamente, que a base de dados possua $n$ objetos e que o seu espaço de atributos seja $p$-dimensional, uma matriz de dados $\mathrm{X} \in \mathbb{R}^{n \cdot p}$, na qual a $j$-ésima linha transposta denota o vetor de atributos do $j$-ésimo objeto, $\tilde{o}_{j}$, é utilizada na representação matricial para descrever a base $O$. Pode-se dizer que $\left(\mathrm{X}_{j,:}\right)^{\mathrm{T}}$ é o vetor coluna que representa o objeto $\tilde{o}_{j}$ no espaço de atributos $\mathbb{R}^{p}$.

A escolha de representar a base de dados por meio de uma matriz de dados $\mathrm{X}$ ou por um conjunto de vetores $X$ ocorre geralmente por conveniência, dado que ambas são equivalentes. Por exemplo, no contexto de bi-agrupamento de dados, costuma-se descrever os bi-grupos como submatrizes da matriz de dados, o que torna o uso da representação matricial conveniente. Ambas as representações são utilizadas nesta tese.

A terceira forma de representação da base de dados consiste no uso de uma matriz $\mathrm{R} \in \mathbb{R}^{n \cdot n}$ que fornece a relação de proximidade entre cada par de objetos, de forma que $\mathrm{R}_{j_{1}, j_{2}}$ fornece a proximidade entre os objetos $\tilde{o}_{j_{1}}$ e $\tilde{o}_{j_{2}}$. A matriz R é comumente chamada de matriz relacional dos dados. Os valores de proximidade contidos em $\mathrm{R}$ podem ser ou de similaridade ou de dissimilaridade, mas não de ambos ao mesmo tempo, e devem respeitar algumas propriedades. Por motivos de adequação ao presente trabalho, é adotada a definição de matriz relacional fornecida por Hathaway e Bezdek (1994), na qual R é uma matriz de dissimilaridades que obedece as seguintes restrições:

$$
\begin{array}{ll}
\mathrm{R}_{j_{1}, j_{2}} \geq 0 & \forall j_{1}, j_{2} \in \mathbb{N}_{1, n}, \\
\mathrm{R}_{j, j}=0 & \forall j \in \mathbb{N}_{1, n} \mathrm{e} \\
\mathrm{R}_{j_{1}, j_{2}}=\mathrm{R}_{j_{2}, j_{1}} & \forall j_{1}, j_{2} \in \mathbb{N}_{1, n} .
\end{array}
$$

Observe que não há atributos na representação relacional dos dados, significando que vários algoritmos de agrupamento (e.g., k-Means (MacQueen, 1967)) não podem ser diretamente aplicados porque precisam dos atributos, enquanto existem outros (e.g., algoritmo hierárquico de ligação única (Everitt et al., 2001) e Relational Fuzzy c-Means (Hathaway et al., 1989) ( $($ RFCM) que foram projetados especificamente para lidar com a representação relacional. Esses últimos são chamados de algoritmos de agrupamento de dados relacionais (ou simplesmente por algoritmos relacionais), enquanto os outros são chamados de algoritmos de agrupamento de dados não relacionais.

Os algoritmos relacionais são importantes por vários motivos. Primeiro, visto que toda base de dados representada por $\mathrm{X}$ ou $X$ também pode ser representada por uma matriz relacional $\mathrm{R}$ por meio do cálculo das distâncias entre as suas linhas (no caso de X) ou entre os seus vetores (no caso de $X$ ), algoritmos relacionais são mais gerais que algoritmos não relacionais. Segundo, e o mais importante, existem áreas nas quais relações de proximidade são tudo que o usuário tem acesso para representar os dados. Esse pode ser o caso de aplicações que preservam a privacidade, nas quais os atributos não podem ser revelados (e.g., informações bancárias). Além disso, existem várias áreas do conhecimento, tais como taxonomia numérica, engenharia 
sísmica, projeto de sistemas inteligentes, recuperação de documentos, gerenciamento, engenharia industrial e ciências sociais, nas quais os dados relacionais são comumente encontrados (Hathaway et al., 1989; Dave e Sen, 2002).

\subsection{Tipos de agrupamento}

São apresentados aqui quatro tipos de agrupamento de dados e diferentes formas de representá-los. Esses tipos relacionam-se hierarquicamente, conforme ilustra a Figura 2.1 .

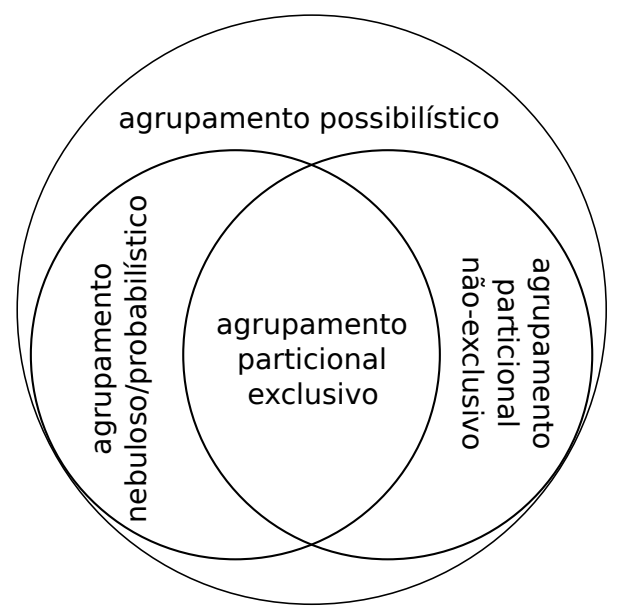

Figura 2.1: Diagrama de Venn para o agrupamento possibilístico e as suas especializações.

No agrupamento particional exclusivo ${ }^{1}$, cada objeto pertence a um, e somente um, grupo. Esse tipo de agrupamento pode ser representado por uma coleção $C \triangleq\left\{C_{1}, C_{2}, \ldots, C_{k}\right\}$ de $k$ conjuntos não vazios e disjuntos tais que $C_{1} \cup C_{2} \cup \cdots \cup C_{k}=O$. Desse modo, $C_{i}$ representa o $i$-ésimo grupo e contém os objetos relativos (e exclusivos) a este. Esse é o tipo de agrupamento gerado, por exemplo, pelo k-Means.

Em determinados cenários, é esperado que um objeto possa participar de mais de uma categoria. Nesse caso, faz-se útil o agrupamento particional não exclusivo ${ }^{2}$, no qual é permitida a interseção entre os conjuntos de $C$. Note que um agrupamento particional exclusivo é também um caso particular de agrupamento particional não exclusivo e, portanto, o segundo é uma generalização do primeiro, como ilustrado na Figura 2.1 .

Outros modelos de agrupamento de dados bastante conhecidos na literatura são aqueles que permitem que os objetos pertençam a todos os grupos com um grau de pertinência. Geralmente é assumido que esse grau de pertinência varia dentro do intervalo real [0,1]. No entanto, não é possível estabelecer uma representação de tais agrupamentos por meio da teoria tradicional dos conjuntos, como foi feito com os agrupamentos particionais exclusivos e não exclusivos. Uma representação para agrupamentos dessa natureza se dá por meio de uma matriz de agrupamento $\mathrm{U} \triangleq\left[\mathrm{U}_{i, j}\right] \in \mathbb{R}^{k \cdot n}$. O elemento $\mathrm{U}_{i, j}$ denota o grau de pertinência do objeto $\tilde{o}_{j}$ ao $i$-ésimo grupo.

\footnotetext{
${ }^{1}$ Frequentemente denotado apenas por agrupamento particional ou agrupamento. Em inglês, é referido por partition, hard clustering, crisp clustering ou simplesmente clustering.

${ }^{2}$ Referido também como agrupamento com sobreposição, ou overlapping clustering em inglês.
} 
De acordo com as restrições impostas à matriz de agrupamento $\mathrm{U}$, tem-se diferentes tipos de agrupamento. Considere, primeiramente, matrizes U que seguem as restrições

$$
\begin{array}{lr}
0 \leq \mathrm{U}_{i, j} \leq 1 & \forall i \in \mathbb{N}_{1, k} \mathrm{e} \forall j \in \mathbb{N}_{1, n}, \\
0<\sum_{j=1}^{n} \mathrm{U}_{i, j} & \forall i \in \mathbb{N}_{1, k} \mathrm{e} \\
0<\sum_{i=1}^{k} \mathrm{U}_{i, j} & \forall j \in \mathbb{N}_{1, n},
\end{array}
$$

em que $n$ é o número de objetos e $k$ é o número de grupos. A Propriedade (2.2a) garante que a menor pertinência de um objeto a um grupo é 0 e que a maior pertinência é 1; a Propriedade 2.2b) garante que não existe grupo vazio (i.e., cuja soma das pertinências dos objetos a ele seja 0 ); a Propriedade (2.2c) garante que todo objeto possui algum grau de pertinência. Uma matriz com essas propriedades pode ser chamada de matriz de agrupamento possibilístico ${ }^{3}$ Anderson et al. 2010) e é gerada por vários algoritmos de agrupamento de dados (Krishnapuram e Keller, 1993, Davenport e Hathaway, 1995, Pal et al., 2005). Para fins didáticos, define-se o conjunto de todas as matrizes possibilísticas de $n$ objetos e $k$ grupos como

$$
M_{\mathrm{p}}(k) \triangleq\left\{\mathrm{U} \in \mathbb{R}^{k \cdot n} \mid \text { Propriedades (2.2a), 2.2b) e (2.2c) sejam satisfeitas }\right\}
$$

ou simplesmente $M_{\mathrm{p}}$.

Ao adicionar a propriedade

$$
\sum_{i=1}^{k} \mathrm{U}_{i, j}=1 \quad \forall j \in \mathbb{N}_{1, n}
$$

ao conjunto de Propriedades 2.2a), 2.2b) e 2.2c), tem-se o que convencionou-se denominar de matriz de agrupamento probabilístico ou agrupamento nebuloso 4 . Nesse caso, a soma das pertinências de um objeto a todos os grupos precisa ser igual a 1. Essa propriedade, na interpretação nebulosa da matriz, transmite a ideia de que o total de pertinência de um objeto (ou seja, 1) é distribuído entre os grupos do agrupamento. Diz-se que o objeto $\tilde{o}_{j}$ pertence ao $i$-ésimo grupo com um grau de pertinência $\mathrm{U}_{i, j}$. Já na interpretação probabilística, o significado é outro. Diz-se que o objeto $\tilde{o}_{j}$ tem uma probabilidade $\mathrm{U}_{i, j}$ de pertencer inequivocamente ao $i$-ésimo grupo. Ou seja, nessa última interpretação, o problema de agrupamento continua sendo de natureza particional, mas o agrupamento representado por U carrega em si a dúvida quanto ao agrupamento particional não exclusivo correto. Novamente por motivos didáticos, define-se

$$
M_{\mathrm{n}}(k) \triangleq\left\{\mathrm{U} \in M_{\mathrm{p}}(k) \mid \text { Propriedade (2.4) seja satisfeita }\right\} ;
$$

ou simplesmente $M_{\mathrm{n}}$. Por motivos de simplicidade, daqui em diante os agrupamentos probabi-

\footnotetext{
${ }^{3}$ Do inglês possibilistic clustering.

${ }^{4} \mathrm{O}$ uso dos termos "nebuloso" (fuzzy) ou "probabilístico" (probabilistic) depende da interpretação da pertinência de $\mathrm{U}_{i, j}$. Por exemplo, os algoritmos Fuzzy c-Means (Dunn, 1973, Bezdek, 1981) (FCM) e Expectation Maximization (Dempster et al., 1977) (EM) fornecem, respectivamente, as interpretações nebulosa e probabilística.
} 
lísticos e nebulosos serão conjuntamente referidos por agrupamentos nebulosos.

Adicionando a propriedade

$$
\mathrm{U}_{i, j} \in\{0,1\} \quad \forall i \in \mathbb{N}_{1, k} \text { e } \forall j \in \mathbb{N}_{1, n}
$$

ao conjunto de Propriedades (2.2a), 2.2b) e (2.2c), tem-se matrizes que representam agrupamentos particionais não exclusivos, as quais pertencem ao conjunto

$$
M_{\mathrm{ne}}(k) \triangleq\left\{\mathrm{U} \in M_{\mathrm{p}}(k) \mid \text { Propriedade (2.6) seja satisfeita }\right\}
$$

ou simplesmente $M_{\text {ne }}$. Finalmente, os agrupamentos particionais exclusivos podem também ser representados por matrizes U que satisfazem as Propriedades (2.2a), 2.2b), 2.2c), 2.4) e (2.6). Esse tipo de matriz é sumarizado por meio do conjunto

$$
M_{\mathrm{e}}(k) \triangleq\left\{\mathrm{U} \in M_{\mathrm{ne}}(k) \mid \text { Propriedade (2.4) seja satisfeita }\right\}
$$

ou simplesmente $M_{\mathrm{e}}$.

Existem algoritmos que associam ao agrupamento gerado outras informações potencialmente úteis ao usuário. Por exemplo, algoritmos de agrupamento em subespaços (Agrawal et al., 1998; Kriegel et al., 2009) costumam associar a cada grupo o subconjunto de atributos cujo subespaço contribuiu para a detecção do grupo. Esse é o caso do algoritmo Entropy Weighting k-Means (Jing et al., 2007) (EWKM) que associa a cada grupo um vetor de pesos indicando a importância de cada atributo para a descoberta do grupo. Outra forma especial de agrupamento é o bi-agrupamento (Madeira e Oliveira, 2004). Esse tipo de solução consiste em um conjunto de bi-grupos, cada qual representando uma submatriz da matriz de dados (assumindo que $\mathrm{X}$ esteja representando a base). Os algoritmos de bi-agrupamento podem ser classificados como um subtipo de algoritmo de agrupamento em subespaços (Madeira e Oliveira, 2004), cuja área é discutida na Seção 2.3. Maiores detalhes sobre bi-agrupamento de dados são fornecidos na Seção 2.3.3.

\subsection{Agrupamento em subespaços}

Os algoritmos de agrupamento em subespaços surgiram como uma nova estratégia para lidar com problemas advindos de bases de dados que possuem muitos atributos ou cujos atributos podem apresentar diferentes graus de relevância para diferentes subestruturas dos dados. Sabe-se que bases de dados de alta dimensionalidade apresentam características complicadoras como a perda progressiva de contraste das distâncias entre os pontos que representam os objetos e a diminuição exponencial da densidade de pontos, que impõem dificuldades aos algoritmos clássicos de agrupamento de dados (Aggarwal et al., 2001; Steinbach et al., 2003). Além disso, é comum que vários dos atributos de uma base de alta dimensionalidade sejam redundantes ou ruidosos. Filtros de seleção de atributos (Mitra et al., 2002; Liu e Yu, 2005) 
ou outras técnicas de redução de dimensionalidade, como Análise de Componentes Principais (Jolliffe, 2002) ( $\mathrm{ACP}$, podem ser empregadas para combater a alta dimensionalidade. No entanto, quando a alta dimensionalidade está acompanhada de atributos que possuem relevâncias distintas para diferentes subestruturas dos dados, tais métodos tendem a falhar por assumirem graus globais de relevância de atributo.

Note que a utilidade de um algoritmo de agrupamento em subespaços não se limita às bases de alta dimensionalidade. Qualquer base de dados com mais de um atributo pode apresentar o fenômeno da relevância de atributos diferenciada pela subestrutura dos dados considerada. Esse é o caso de muitas bases de expressão gênica, nas quais milhares de genes são comumente representados por poucas dezenas de atributos (condições experimentais). É sabido que raramente os genes de um grupo de interesse serão semelhantes entre si ou coesos sob todas as condições experimentais investigadas (Cheng e Church, 2000; Kluger et al., 2003; Madeira e Oliveira, 2004; Divina e Aguilar-Ruiz, 2006; Prelić et al., 2006; Busygin et al., 2008; Yang et al., 2011). Esse é o principal motivo do interesse dos pesquisadores da área de expressão gênica nos algoritmos de bi-agrupamento.

Baseando-se na categorização proposta por Kriegel et al. (2009), pode-se classificar as técnicas de agrupamento em subespaços em algoritmos que: procuram por grupos em subespaços alinhados com os eixos; procuram por grupos em subespaços arbitrariamente orientados; e procuram por grupos baseados em padrão da matriz de dados (bi-agrupamento). Essas três categorias são detalhadas nas seções seguintes.

\subsubsection{Subespaços alinhados com os eixos}

Kriegel et al. (2009) definem subespaços alinhados com os eixos como sendo subespaços formados pela seleção de um subconjunto dos atributos do espaço original de atributos ou pela ponderação dos atributos. No primeiro caso, os vetores (assumindo uma representação vetorial da base) no novo subespaço possuem apenas os atributos do subconjunto selecionado, cujos valores permanecem inalterados. No segundo caso, os vetores no novo subespaço possuem todos os atributos do espaço original, porém os seus valores são alterados de acordo com a ponderação do subespaço em questão.

Os algoritmos de subespaços alinhados com os eixos podem ser subdivididos nas seguintes categorias:

- Algoritmos baseados em projeção. Geralmente os algoritmos de agrupamento dessa categoria procuram por projeções nas quais os pontos (que representam os objetos no espaço dos atributos) possam ser considerados próximos. Cada grupo de objetos tem associado a si um subconjunto dos atributos da base. De modo geral, as técnicas desta categoria são baseadas em algoritmos tradicionais nos quais a função de distância é especializada para lidar com os subespaços.

- Algoritmos baseados em projeção suave. Cada grupo tem associado a si uma ponderação dos atributos da base por meio da qual os pontos podem ser considerados próximos. Isto 
é, cada atributo da base contribui para a formação de um dado grupo, mas de forma ponderada.

- Algoritmos baseado na enumeração dos subespaços. Consiste em algoritmos que buscam grupos em todos os subespaços geráveis pela escolha de subconjuntos de atributos. O espaço é subdividido por meio de uma grade (auto-adaptável ou não) e a construção dos grupos acontece na união ou divisão das suas células.

Cada uma dessas subcategorias é detalhada nas seções seguintes.

\subsubsection{Algoritmos baseados em projeção}

A abordagem geral desses algoritmos consiste na integração de distâncias especializadas em algoritmos de agrupamento tradicionais (i.e., que não discriminam atributos) (Kriegel et al. 2009) ou em algoritmos baseados nos tradicionais. Por exemplo, o algoritmo Projected Clustering (Aggarwal et al., 1999) (PROCLUS), descrito no Apêndice A, é baseado no algoritmo Partitioning Around Medoids (Kaufman e Rousseeuw, 1990) (PAM). O algoritmo PROCLUS faz uso da distância de Manhattan resultante ao ignorar completamente certos atributos, referida por distância de Manhattan segmental (Aggarwal et al. 1999). Seja $X \triangleq\left\{\mathrm{x}^{1}, \ldots, \mathrm{x}^{n}\right\} \subset \mathbb{R}^{p}$ um conjunto de vetores $p$-dimensionais que representa uma base de dados de $n$ objetos. Sejam $D_{i} \subset \mathbb{N}_{1, p}$ o conjunto de atributos (referidos por índices) relevantes ao $i$-ésimo grupo de um agrupamento hipotético e $\mathrm{v}^{i} \in \mathbb{R}^{p}$ o protótipo que representa o $i$-ésimo grupo. A distância de Manhattan segmental do vetor $\mathrm{x}^{j}$ ao vetor $\mathrm{v}^{i}$ é definida como

$$
\sum_{s \in D_{i}}\left|\mathrm{x}_{s}^{j}-\mathrm{v}_{s}^{i}\right|
$$

em que $|\cdot|$ fornece o valor absoluto. Observe que a distância tradicional de Manhattan foi reformulada para considerar apenas um determinado subespaço por grupo.

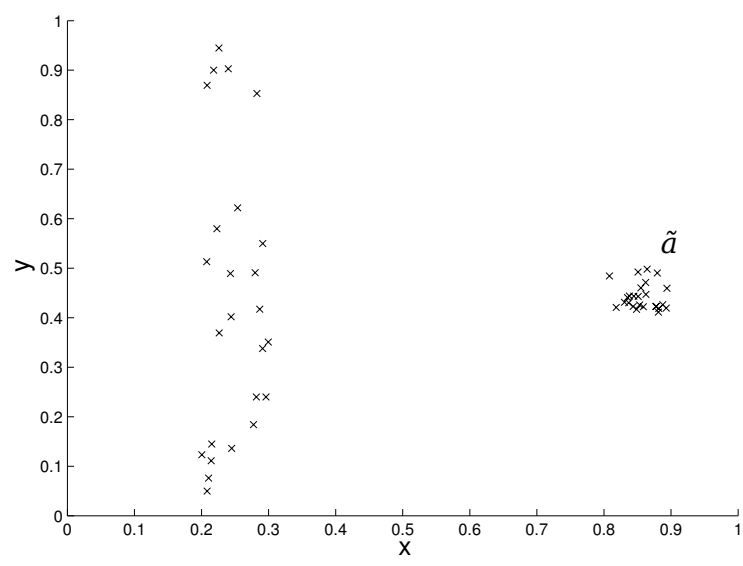

(a) Projeção no plano $\vec{x}-\vec{y}$.

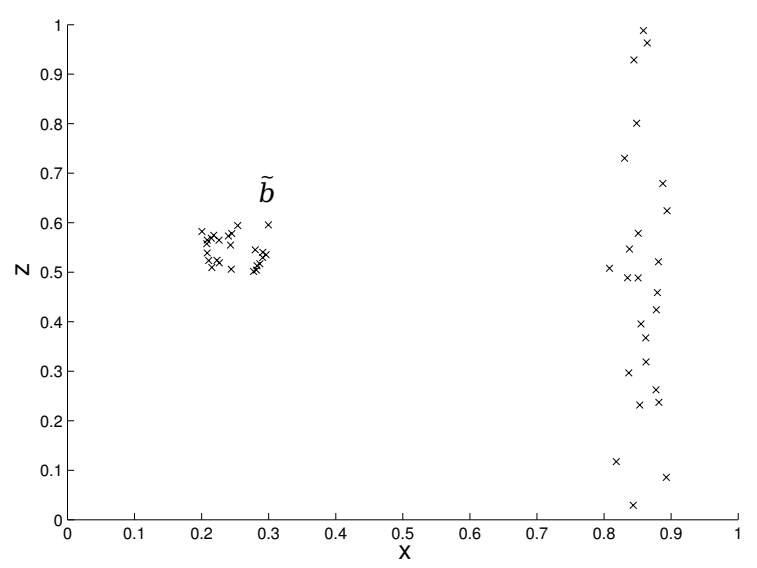

(b) Projeção no plano $\vec{x}-\vec{z}$.

Figura 2.2: Dificuldade associada à pré-seleção de atributos, como exemplificada por Aggarwal et al. (1999). 
$\mathrm{O}$ algoritmo PROCLUS foi criado devido à necessidade de selecionar atributos por grupo, tarefa que não é possível de ser realizada por uma pré-seleção de atributos. Na Figura 2.2. encontram-se ilustradas duas projeções de uma base de dados tridimensional. As projeções ortogonais nos planos $\vec{x}-\vec{y}$ e $\vec{x}-\vec{z}$ são ilustradas, respectivamente, pelas Figs. 2.2(a) e 2.2(b), Na Figura 2.2(a), o conjunto de objetos denotado por $\tilde{a}$ tem seus elementos próximos entre si, o que não acontece quando esses mesmos objetos estão no espaço tridimensional original ou quando são projetados no plano $\vec{x}-\vec{z}$. Pode-se dizer algo análogo sobre o conjunto de objetos denotado por $\tilde{b}$ na Figura 2.2(b). Em outras palavras, não existe um subconjunto de atributos que, ao mesmo tempo, faça com que cada um dos conjuntos de objetos $\tilde{a}$ e $\tilde{b}$ tenham seus elementos próximos entre si, formando dois grupos de maneira evidente. Portanto, uma seleção prévia de atributos não conseguiria evidenciar os grupos $\tilde{a}$ e $\tilde{b}$ de forma tão clara quanto às projeções por grupo que um algoritmo baseado em projeção faria.

\subsubsection{Algoritmos baseados em projeção suave}

Os algoritmos dessa categoria procuram lidar com o problema da relevância dos atributos por meio de pesos distribuídos entre os atributos. Um peso de relevância é associado, por grupo, a cada um dos atributos. Sejam $p$ o número de atributos da base de dados e $k$ o número de grupos. Esses pesos podem ser representados por uma matriz $\mathrm{Y} \triangleq\left[\mathrm{Y}_{i, s}\right] \in \mathbb{R}^{k \cdot p}$, chamada de matriz de ponderação, tal que

$$
\begin{aligned}
\mathrm{Y}_{i, s} \in[0,1] & \forall i \in \mathbb{N}_{1, k} \text { e } \forall s \in \mathbb{N}_{1, p}, \\
\sum_{s=1}^{p} \mathrm{Y}_{i, s}=1 & \forall i \in \mathbb{N}_{1, k} .
\end{aligned}
$$

Desse modo, $\mathrm{Y}_{i, s}$ denota a relevância do $s$-ésimo atributo ao $i$-ésimo grupo. Isto é, quanto maior o valor de $\mathrm{Y}_{i, s}$, maior a relevância do atributo. Por motivos didáticos, define-se o conjunto de todas as matrizes de ponderação como

$$
M_{\mathrm{y}}(k) \triangleq\left\{\mathrm{Y} \in \mathbb{R}^{k \cdot p} \mid \mathrm{Y} \text { satisfaça as Propriedades 2.10a e 2.10b }\right\} \text {. }
$$

Quando o número de grupos correspondente é evidente, será usado apenas $M_{\mathrm{y}}$.

Pode-se citar os métodos Locally Adaptive Clustering (Domeniconi et al., 2004) (LAC), Clustering Objects on Subsets of Attributes (Friedman e Meulman, 2004) (COSA) e os métodos derivados do algoritmo $\mathrm{SCAD}$ como exemplos da classe de algoritmos em questão. O algoritmo LAC é baseado nas iterações do k-Means com a adição da etapa de cálculo dos pesos de atributos. A distância euclidiana ponderada é usada para o cálculo das distâncias entre os objetos e os protótipos dos grupos. Por exemplo, a distância do $j$-ésimo objeto ao $i$-ésimo grupo é dada por

$$
\sqrt{\sum_{s=1}^{p} \mathrm{Y}_{i, s} \cdot\left(\mathrm{x}_{s}^{j}-\mathrm{v}_{s}^{i}\right)^{2}}
$$

em que $p$ é o número de atributos da base de dados, $\mathrm{x}_{s}^{j}$ é o valor do $s$-ésimo atributo do vetor 
$\mathrm{x}^{j}$ que representa o $j$-ésimo objeto e $\mathrm{v}_{s}^{i}$ é o valor do $s$-ésimo atributo do vetor $\mathrm{v}^{i}$ que representa o $i$-ésimo grupo. Desse modo, quanto menor a relevância do atributo, menor é o impacto do respectivo atributo no cálculo da distância.

Já o algoritmo COSA funciona como um invólucro no qual um algoritmo de agrupamento relacional de dados qualquer pode ser usado. A partir das soluções encontradas pelo algoritmo de agrupamento embutido, o método COSA encontra, por grupo, pesos de relevância para os atributos. $\mathrm{O}$ seu esquema geral pode ser descrito da seguinte forma:

1. Seja $R \in \mathbb{R}^{n \cdot n}$ a matriz relacional que representa a base de dados.

2. Inicializa a matriz de ponderação $\mathrm{Y} \in M_{\mathrm{y}}$.

3. Calcula a nova matriz relacional $\mathrm{R}$ de acordo com $\mathrm{Y}$.

4. Aplica o algoritmo de agrupamento embutido fornecendo a ele a matriz relacional $\mathrm{R}$.

5. Calcula a nova matriz de ponderação Y a partir do agrupamento gerado no passo anterior.

6. Se a matriz Y se estabilizou, para. Caso contrário, volta ao Passo3

Dos algoritmos da categoria em questão, os mais importantes para o presente trabalho são o SCAD e CARD, descritos na Seção 2.4.

\subsubsection{Algoritmos baseados na enumeração dos subespaços}

De acordo com Kriegel et al. (2009), algoritmos baseados na enumeração dos subespaços são aqueles que procuram por todos os grupos em todos os subespaços formados pela escolha de subconjuntos dos atributos da base. Naturalmente, uma busca exaustiva por todos os subespaços possíveis é computacionalmente inviável. Por exemplo, em uma base de dados com 30 atributos, existem

$$
\sum_{s=1}^{30} \frac{30 !}{(30-s) ! s !}=1.073 .741 .823
$$

subespaços possíveis. Uma das maneiras mais comuns para lidar com essa explosão combinatorial consiste no uso de ideias e conceitos do clássico algoritmo Apriori (Agrawal e Srikant, 1994) de aprendizado de regras de associação. Essa abordagem foi empregada no primeiro algoritmo baseado em subespaço, denominado CLIQUE (Agrawal et al., 1998). O seu primeiro passo é particionar o espaço dos dados em uma grade de células alinhadas com os eixos. As bordas dessas células possuem um comprimento fixo $\xi$, fornecido pelo usuário. Uma célula é considerada densa quando possui um número mínimo $\tau$, definido pelo usuário, de pontos e um grupo é formado por um conjunto máximo de células densas adjacentes. Observe que a projeção de uma célula $s$-dimensional contendo $t$ pontos em qualquer subespaço $(s-1)$-dimensional (e, por indução, qualquer espaço de menor dimensionalidade) contém pelo menos $t$ pontos (Agrawal et al., 1998); a Figura 2.3 ajuda a ilustrar o problema. Isso sugere uma estratégia bottom-up na procura por células densas em subespaços de menor para maior dimensionalidade. Começando com as células unidimensionais, células densas $s$-dimensionais são encontradas a 
partir das células densas $(s-1)$-dimensionais de maneira semelhante ao que o algoritmo Apriori faz com transações (Kriegel et al., 2009). Uma heurística é também utilizada para ignorar um número maior de subespaços na procura por subespaços e conjuntos máximos de células densas (grupos).

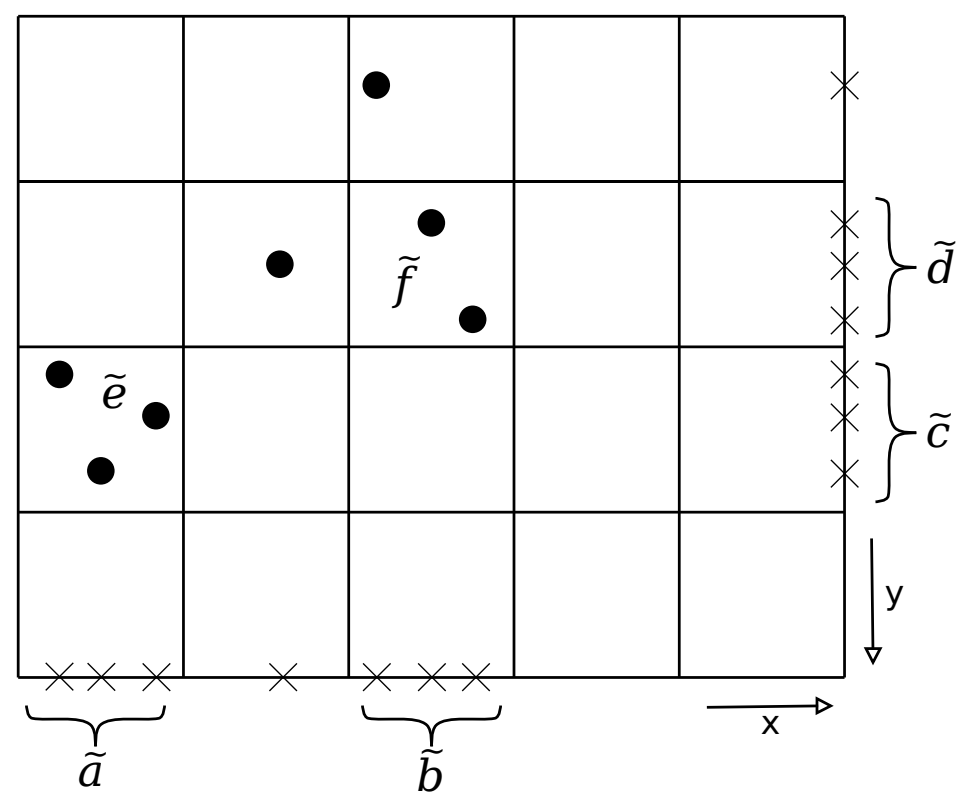

Figura 2.3: Base de dados bidimensional de sete objetos. Seja $\tau=3$ o limiar de densidade. Nesse caso, tem-se as células densas $\tilde{a}$ e $\tilde{b}$ no subespaço da coordenada $\vec{x}$, as células densas $\tilde{c}$ e $\tilde{d}$ no subespaço da coordenada $\vec{y}$ e a célula densa $\tilde{e}$ no espaço original dos dados. Observe que a célula $\tilde{f}$ não é densa, porém as suas duas projeções a são.

Note que o número de células da grade aumenta exponencialmente com o número de dimensões. Além disso, o usuário deve fornecer o tamanho das células e o limiar do número de pontos, parâmetros que influenciam diretamente a solução e que são difíceis de serem configurados. Para lidar com esses problemas, foi proposto o método MAFIA (Nagesh et al., 2001), uma variante do algoritmo CLIQUE, cujas dimensões das células da grade se adaptam à distribuição dos dados. Outras variantes do algoritmo CLIQUE são os métodos ENCLUS (Cheng et al., 1999) e $n$ Cluster (Liu et al., 2007).

\subsubsection{Subespaços arbitrariamente orientados}

Algoritmos desta categoria procuram por grupos em subespaços nos quais as projeções dos objetos se encontram densas, próximas umas às outras. Esses subespaços podem ser de dimensão arbitrária e de qualquer orientação. Formalmente, seja $\mathbb{R}^{p}$ o espaço de atributos da base de dados. Algoritmos de agrupamento do tipo em questão buscam por grupos em subespaços afins $S+$ a (subespaço $S \subset \mathbb{R}^{p}$ de afinidade a) nos quais subconjuntos de pontos se encontram densamente projetados. A Figura 2.4 apresenta um exemplo. Os pontos estão espalhados e próximos da reta $\tilde{a}$, sendo esta ortogonal ao plano $\tilde{b}$. Portanto, os pontos em questão apresentariam uma alta variância caso fossem projetados sobre a reta $\tilde{a}$. Contrariamente, os pontos apresentariam uma baixa variância caso fossem projetados sobre o plano $\tilde{b}$. Para um algoritmo de agrupamento 
de dados, a segunda situação favorece o agrupamento dos respectivos objetos.

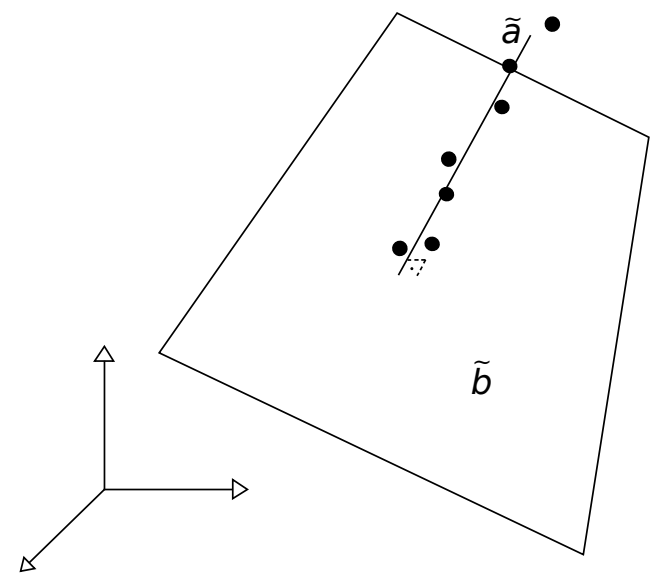

Figura 2.4: Pontos em um espaço tridimensional. Observe que os pontos estariam próximos entre si se fossem projetados no plano $\tilde{b}$.

Uma abordagem comumente empregada em algoritmos de agrupamento baseados em subespaços arbitrariamente orientados consiste no uso da técnica $\mathrm{ACP}$ em subconjuntos dos pontos da base de dados. A aplicação da técnica $\mathrm{ACP}$ em uma seleção de pontos resulta em uma base ortonormal na qual os seus vetores podem ser ordenados de acordo com a variância da projeção dos pontos. Considere uma ordenação decrescente de acordo com os valores de variância. $\mathrm{O}$ primeiro vetor dessa ordenação representa a direção de maior variância da seleção de pontos. O segundo vetor denota a direção de maior variância e que é ao mesmo tempo perpendicular ao primeiro vetor. $\mathrm{O}$ terceiro vetor denota a direção de maior variância e que é ao mesmo tempo perpendicular ao primeiro e ao segundo vetor, e assim por diante. Caso essa seleção de pontos corresponda a um grupo escondido em um subespaço, a projeção dos pontos no subespaço gerado pelos últimos vetores dessa ordenação tende a ser densa, devido a uma menor variância.

Diversos algoritmos de agrupamento da subárea em questão baseiam-se na ferramenta ACP ou em técnicas similares para encontrar subespaços interessantes, cada qual adotando uma abordagem diferente para a seleção de subespaços, uma medida de distância ou uma maneira particular de escolher os subconjuntos de objetos (Aggarwal e Yu, 2000; Böhm et al., 2004; Achtert et al., 2006a, 2007). Kriegel et al. (2009) fornecem também uma visão geral de outras abordagens (e.g., transformada de Hough, dimensão fractal e amostragem aleatória) para encontrar subespaços interessantes.

\subsubsection{Bi-agrupamento}

Os algoritmos de bi-agrupamento procuram por submatrizes da matriz de dados que consistem em padrões preestabelecidos. Cada submatriz encontrada é descrita por um bi-grupo, o que nada mais é que uma tupla de um conjunto de linhas e um conjunto de colunas da matriz de dados. Formalmente, sejam $\mathrm{X} \in \mathbb{R}^{n \cdot p}$ uma matriz de dados, $R \triangleq\{1,2, \ldots, n\}$ o conjunto de índices denotando suas linhas e $C \triangleq\{1,2, \ldots, p\}$ o conjunto de índices denotando suas colunas. Um bi-grupo $B_{i} \triangleq\left(B_{i}^{\mathrm{r}}, B_{i}^{\mathrm{c}}\right)$ é uma tupla de um conjunto não vazio $B_{i}^{\mathrm{r}} \subset \mathbb{N}_{1, n}$ de índices representando linhas e um conjunto não vazio $B_{i}^{c} \subset \mathbb{N}_{1, p}$ de índices representando colunas da matriz 
de dados X. A coleção $B \triangleq\left\{B_{1}, B_{2}, \ldots, B_{k}\right\}$ de bi-grupos forma um bi-agrupamento dos dados representados por X. Considere o bi-agrupamento representado pela Figura 2.5. Usando a notação estabelecida, tem-se os dois bi-grupos $B_{1}=(\{1,2,3\},\{1,2\})$ e $B_{2}=(\{2\},\{2,3\})$. O conjunto $B=\left\{B_{1}, B_{2}\right\}$ representa o bi-agrupamento correspondente $\mathrm{e}^{5}$

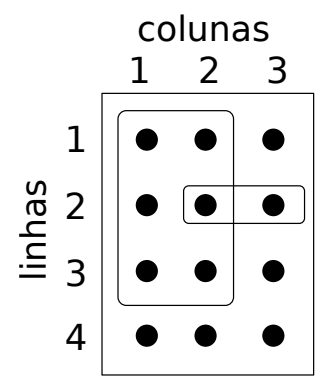

Figura 2.5: Matriz $\mathrm{X} \in \mathbb{R}^{4 \cdot 3}$ de dados bi-agrupados em dois bi-grupos.

Existem definições de bi-agrupamento que impõem algumas outras restrições, como não permitir sobreposição entre bi-grupos $\left(B_{i}^{\mathrm{r}} \cap B_{l}^{\mathrm{r}}=B_{i}^{\mathrm{c}} \cap B_{l}^{\mathrm{c}}=\emptyset\right.$ para todo $\left.i \neq l\right)$ ou exigir que $\cup_{i=1}^{k} B_{i}^{\mathrm{r}}=R$ e $\cup_{i=1}^{k} B_{i}^{\mathrm{c}}=C$ (Madeira e Oliveira, 2004). Entretanto, essas pressuposições são muito restritivas em certos contextos. Considere, por exemplo, dados de expressão gênica, os quais contêm tipicamente centenas ou milhares de genes, algumas vezes o genoma inteiro de um organismo. É esperado que alguns genes não participem de nenhum processo biológico sob as condições monitoradas. Além disso, em um conjunto de dados transcriptômicos múltiplas vias genéticas (genetic pathways) podem estar ativos em uma condição e um gene pode participar de diferentes caminhos genéticos sob condições distintas. Para detectar essas interações entre genes, os bi-grupos precisam se sobrepor (Hochreiter et al., 2010). O mesmo pode ser dito sobre documentos textuais descritos por conjuntos de palavras (bag of words), nos quais um documento pode pertencer a categorias diferentes dependendo das palavras consideradas. Por essas razões, é adotado aqui a já mencionada e a forma mais geral de definição de bi-agrupamento.

Acredita-se que Hartigan (1972) tenha apresentado os primeiros algoritmos capazes de agrupar simultaneamente as linhas e colunas de uma matriz de dados X. Mais tarde em (Mirkin, 1996) esse novo tipo de agrupamento de dados foi definido como bi-agrupamento (também chamado de co-agrupamento ou agrupamento de duas vias/dois modos), mas somente depois da publicação de Cheng e Church (2000) que essa classe de algoritmos de agrupamento despertou a atenção da comunidade acadêmica. O paradigma de bi-agrupamento de dados ganhou espaço em determinados campos de pesquisa. Por exemplo, no campo de expressão gênica, dado que é improvável que um conjunto de genes (linhas da matriz de dados) tenha seus elementos similares uns aos outros sob todas as condições investigadas (colunas) e vice-versa (Cheng e Church, 2000; Madeira e Oliveira, 2004; Prelić et al., 2006; Busygin et al., 2008). A abordagem de bi-agrupamento ganhou atenção também nos campos de análise de texto, de log de páginas da internet e de cesta de compras (market basket em inglês) (Dhillon et al., 2003). Por exemplo, na análise de textos, alguém pode estar interessado em encontrar grupos de documentos (linhas

\footnotetext{
${ }^{5}$ Note que as linhas e/ou colunas de um bi-grupo não precisam ser contíguas. Em ilustrações os bi-grupos costumam ser representados de maneira contígua apenas por motivos didáticos.
} 
da matriz de dados) similares e o seu relacionamento com as palavras (colunas da matriz de dados). A Figura 2.6 sugere uma tendência crescente no interesse por bi-agrupamento de dados na literatura científica.

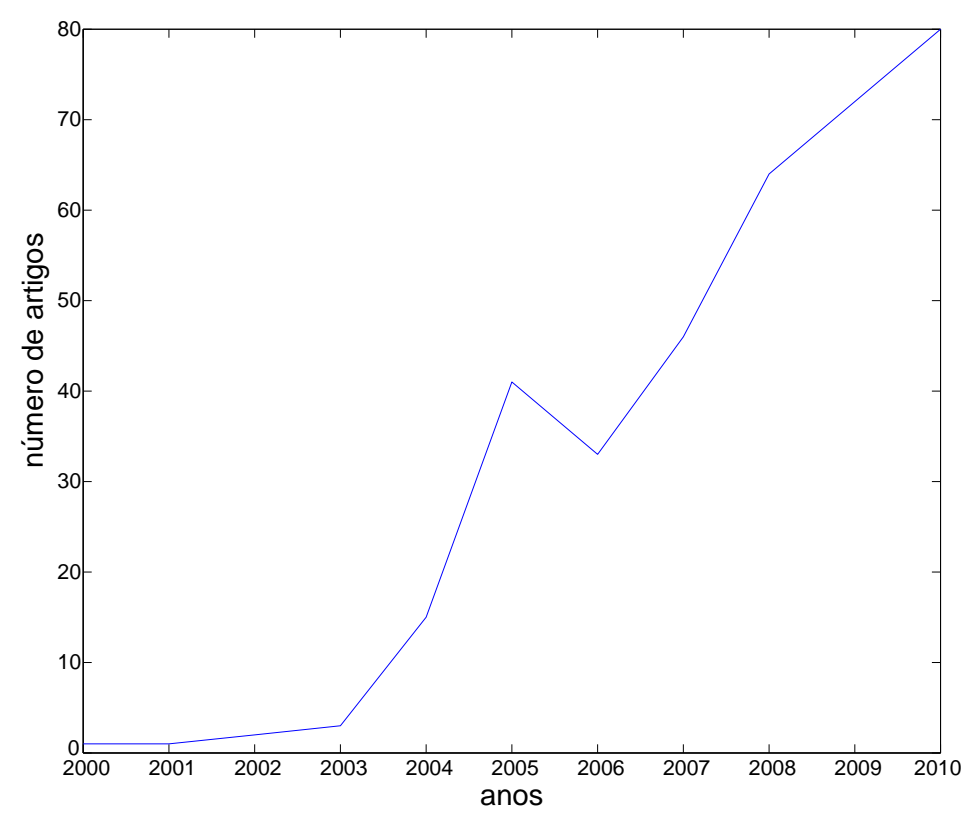

Figura 2.6: Tendência do número de artigos contendo a palavra-chave biclustering pela base de dados da SciVerse Scopus.

Considere o Cheng and Church's Algorithm (Cheng e Church, 2000) (CCA). Sua funçãocusto avalia bi-grupos individualmente. Portanto, o custo de um bi-agrupamento pode ser dado pela média dos custos de cada bi-grupo. Considere um grupo qualquer $B_{i}$. Sejam

$$
\begin{aligned}
& \mathrm{X}_{B_{i}^{\mathrm{r}}, s} \triangleq \sum_{t_{1} \in B_{i}^{\mathrm{r}}} \mathrm{X}_{t_{1}, s} /\left|B_{i}^{\mathrm{r}}\right|, \\
& \mathrm{X}_{j, B_{i}^{\mathrm{c}}} \triangleq \sum_{t_{2} \in B_{i}^{\mathrm{c}}} \mathrm{X}_{j, t_{2}} /\left|B_{i}^{\mathrm{c}}\right| \mathrm{e} \\
& \mathrm{X}_{B_{i}^{\mathrm{r}}, B_{i}^{\mathrm{c}}} \triangleq \sum_{\substack{t_{1} \in B_{i}^{\mathrm{r}} \\
t_{2} \in B_{i}^{\mathrm{c}}}} \mathrm{X}_{t_{1}, t_{2}} /\left|B_{i}^{\mathrm{r}}\right|\left|B_{i}^{\mathrm{c}}\right|
\end{aligned}
$$

as médias da $s$-ésima coluna de acordo com $B_{i}$, da $j$-ésima linha de acordo com $B_{i}$ e da submatriz correspondente ao bi-grupo $B_{i}$, respectivamente. O custo do bi-grupo $B_{i}$, chamado de média do resíduo quadrático, é dado por

$$
\frac{1}{\left|B_{i}^{\mathrm{r}}\right|\left|B_{i}^{\mathrm{c}}\right|} \sum_{\substack{t_{1} \in B_{i}^{\mathrm{r}} \\ t_{2} \in B_{i}^{\mathrm{c}}}}\left(\mathrm{X}_{t_{1}, t_{2}}-\mathrm{X}_{B_{i}^{\mathrm{r}}, t_{2}}-\mathrm{X}_{t_{1}, B_{i}^{\mathrm{c}}}+\mathrm{X}_{B_{i}^{\mathrm{r}}, B_{i}^{\mathrm{c}}}\right)^{2}
$$

Repare que a Fórmula 2.12 não faz uso de distâncias entre os objetos, o que geralmente ocorre com os outros tipos de algoritmos de agrupamento de dados. De fato, o que a funçãocusto do algoritmo CCA avalia é o que Kriegel et al. (2009) chamam de coerência de valores. Nesse modelo, os elementos de uma submatriz são perfeitamente coerentes quando podem ser descritos pela equação

$$
X_{j, s}=\mu+r_{j}+c_{s}
$$


Isto é, o valor de $X_{j, s}$ é dado pela soma de uma constante $\mu$ com os deslocamentos $r_{j}$ e $c_{s}$ correspondentes à $j$-ésima linha e à $s$-ésima coluna. Essa propriedade é satisfeita pela Funçãocusto 2.12, dado que o seu menor valor (zero) é alcançado quando

$$
\mathrm{X}_{j, s}=-\mathrm{X}_{B_{i}^{\mathrm{r}}, B_{i}^{\mathrm{c}}}+\mathrm{X}_{B_{i}^{\mathrm{r}, s}}+\mathrm{X}_{j, B_{i}^{\mathrm{c}}}
$$

para todo $j \in B_{i}^{\mathrm{r}}$ e $s \in B_{i}^{\mathrm{c}}$. Além disso, o valor dado pela Fórmula (2.12) pode ser entendido como o resíduo que representa o quão próximo do Modelo (2.13) o bi-grupo $B_{i}$ está. Portanto, pode-se dizer que o algoritmo CCA busca por bi-grupos que seguem o Modelo (2.13), que nada mais é que um padrão para os valores da submatriz correspondente.

O modelo apresentado acima é bem ilustrativo da área de algoritmos de bi-agrupamento, pois diversos modelos adotados na respectiva literatura podem ser entendidos como casos especiais do modelo de coerência de valores (Kriegel et al., 2009). Por exemplo, os algoritmos publicados em (Getz et al., 2000; Califano et al., 2000; Sheng et al., 2003) buscam por bi-grupos que seguem o Modelo 2.13) quando $r_{j}=0$ ou $c_{s}=0$.

\subsection{Algoritmos para dados multi-representados}

Dados multi-representados são aqueles cujas representações são fornecidas por mais de uma visão dos mesmos (Kailing et al., 2004b; Xia et al., 2010). A principal premissa para o desenvolvimento de técnicas que consideram a multi-representação dos dados é a de que a construção de um espaço de atributos comum para bases multi-representadas, de forma que os algoritmos clássicos de agrupamento possam atuar, nem sempre é possível ou não implica em uma boa descrição, como exemplificado na Seção 1 . O algoritmo baseado no Density-Based Spatial Clustering of Applications with Noise (Ester et al., 1996) (DBSCAN) proposto por Kailing et al. (2004b) para lidar com multi-representação possibilita o uso de distâncias distintas para as diferentes representações, o que permitiu um bom desempenho nos seus experimentos em agrupamento de imagens. Como outro exemplo, Bickel e Scheffer (2004) propõem versões dos algoritmos kMeans e EM para lidar com bases de páginas da web descritas por duas visões (texto da própria página e textos de hyperlinks entrantes), baseando-se na ideia de co-treinamento da literatura de aprendizado supervisionado (Blum e Mitchell, 1998).

Frigui e Nasraoui (2004) propuseram o algoritmo SCAD que, além de não restringir os dados a um único espaço de atributos, é capaz de ponderar as diferentes representações dos dados de acordo com a subestrutura em questão. Por exemplo, no problema de segmentação de imagem em regiões homogêneas, um pixel pode ser descrito pela sua posição (coordenadas horizontal e vertical), cor e por atributos de textura. Considere o problema de segmentação da Figura 2.7. Frigui e Nasraoui (2004) aplicaram o algoritmo FCM no conjunto de pixeis sem e com as coordenadas de posição, resultando nas segmentações ilustradas pela Figura 2.8(a) e Figura 2.8(b), respectivamente.

Visivelmente o uso de informações de posição ajudou o algoritmo FCM na segmentação da imagem. Sem o uso das posições, o algoritmo FCM fragmentou a imagem ao categorizar 


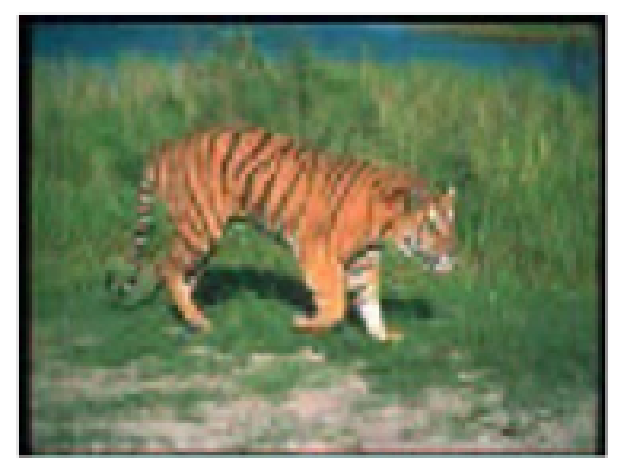

Figura 2.7: Imagem de um tigre publicada por Frigui e Nasraoui (2004).

pixeis espalhados como sendo da mesma região. Com o uso das posições, a região do tigre foi encontrada com boa precisão, o que não aconteceu com o plano de fundo. O plano de fundo foi subdivido em três grupos que não representam categorias aparentes na imagem. A explicação para essa divisão artificial do plano de fundo está nas grandes distâncias entre os pontos que pertencem a ele. Com o uso do algoritmo SCAD, capaz de atribuir relevância aos atributos de maneira específica para cada grupo, uma melhor solução de segmentação foi encontrada (Figura 2.9). Ao atribuir menor relevância aos atributos de posição, o plano de fundo da imagem foi segmentado em grupos relacionados ao gramado, à área descampada e à área afetada pela sombra do animal. Embora não seja um agrupamento ideal, certamente representa um avanço na segmentação.

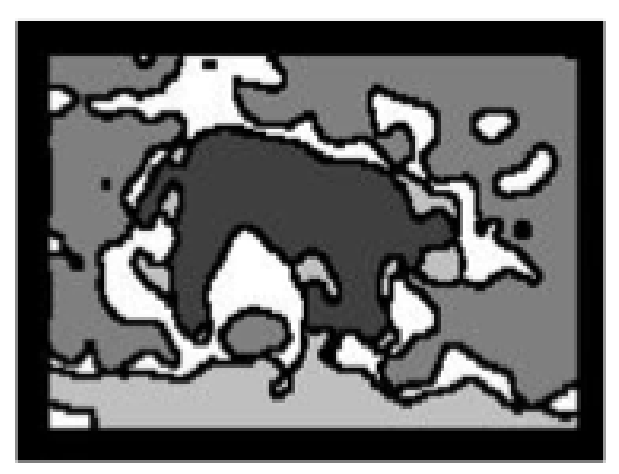

(a) FCM $]$ sem as posições dos pixeis.

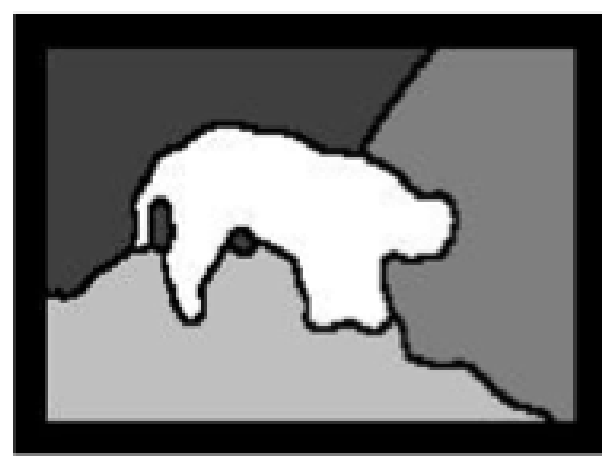

(b) FCM

Figura 2.8: Resultados de segmentação obtidos pelo algoritmo FCM, publicada por Frigui e Nasraoui (2004).

Os algoritmos SCAD e CARD são detalhados nas Seções 2.4.1 e 2.4.2 devido à sua importância para este trabalho.

\subsubsection{Algoritmo SCAD}

Frigui e Nasraoui (2000) propuseram a primeira versão do algoritmo SCAD, na qual os atributos eram ponderados individualmente (o que explica a parte "Attribute Discrimination" 


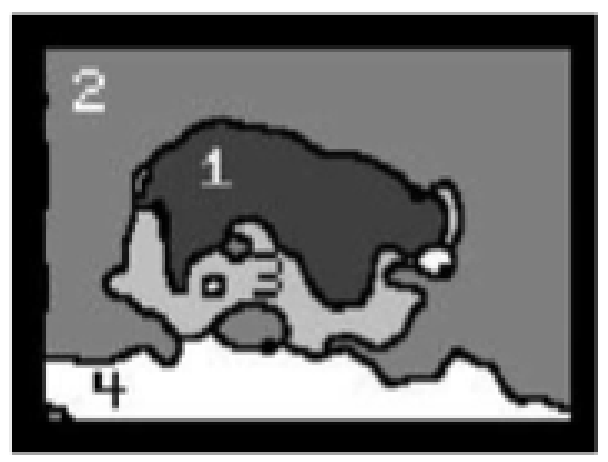

Figura 2.9: SCAD com as posições dos pixeis, publicada por Frigui e Nasraoui (2004).

do seu nome). A função-custo desse algoritmo é definida como

$$
\sum_{i=1}^{k} \sum_{j=1}^{n}\left(\mathrm{U}_{i, j}\right)^{m} \sum_{s=1}^{p} \mathrm{Y}_{i, s} \cdot\left(\mathrm{x}_{s}^{j}-\mathrm{v}_{s}^{i}\right)^{2}+\sum_{i=1}^{k} \delta_{i} \sum_{s=1}^{p}\left(\mathrm{v}_{s}^{i}\right)^{2}
$$

em que $\delta_{i}$ é uma variável de ponderação que deve ser escolhida de maneira que os dois termos da função-custo tenham a mesma ordem de magnitude (Frigui e Nasraoui, 2000) e $m \in[1, \infty$ ) é o expoente de "nebulosidade" (do inglês fuzzifying exponent), o mesmo do clássico algoritmo Fuzzy c-Means (Dunn, 1973; Bezdek, 1981) (FCM). O segundo termo da Expressão (2.14) serve para impedir que as equações de atualização atribuam, para todo grupo, importância máxima a apenas um atributo e nenhuma importância aos outros. A atualização das variáveis $\delta_{i}$ ocorre a cada iteração por meio de uma heurística. No entanto, não foi provado que essa estratégia de atualização leva à convergência do algoritmo e, logo, não há garantias teóricas de estabilidade da primeira versão do algoritmo $\mathrm{SCAD}$.

Frigui e Salema (2003) propuseram uma nova versão do algoritmo SCAD, agora com a capacidade de ponderar subconjuntos de atributos (representações da base de dados), ao invés de apenas atributos individualmente. Em (Frigui e Nasraoui, 2004) surge a terceira versão do algoritmo SCAD, dessa vez com uma nova função-custo na qual o segundo termo da Expressão 2.14) não está presente . Essa versão serviu de base para a criação de outros algoritmos, como um algoritmo de agrupamento semi-supervisionado (Frigui e Mahdi, 2007) e um algoritmo de agrupamento relacional (Frigui et al., 2007). Daqui em diante o termo SCAD irá se referir à essa última versão, i.e., à versão proposta em (Frigui e Nasraoui, 2004), definida adiante nesta mesma seção. O algoritmo SCAD é analisado e melhorado na Seção 3.1 .

As funções-custo de vários algoritmos de agrupamento, incluindo as dos algoritmos SCAD e CARD (Seção 2.4.2) e de vários outros algoritmos de agrupamento, são instâncias de uma mesma classe de funções-custo. Considere o conjunto

$$
M_{\mathrm{d}} \triangleq\left\{\mathrm{D} \in \mathbb{R}^{k \cdot n} \mid \mathrm{D}_{i, j} \geq 0 \quad \forall i \in \mathbb{N}_{1, k}, \forall j \in \mathbb{N}_{1, n}\right\}
$$


A classe de funções-custo em questão é dada por $\mathcal{G}_{m}: M_{\mathrm{p}} \times M_{\mathrm{d}} \mapsto \mathbb{R}$ e definida por

$$
\mathcal{G}_{m}(\mathrm{U}, \mathrm{D}) \triangleq \sum_{i=1}^{k} \sum_{j=1}^{n}\left(\mathrm{U}_{i, j}\right)^{m} \cdot \mathrm{D}_{i, j}
$$

em que $\mathrm{U} \in M_{\mathrm{p}}$. A distância do objeto $\tilde{o}_{j}$ ao $i$-ésimo grupo é dada por $\mathrm{D}_{i, j}$. As diferentes formas de calcular essa distância, o valor de $m$ e quais pontos do domínio de $\mathcal{G}_{m}$ são usados, dão origem às instâncias dessa classe de funções.

Seja

$$
M_{\mathrm{v}}(a) \triangleq\left\{\left\{\mathrm{v}^{1}, \mathrm{v}^{2}, \ldots, \mathrm{v}^{k}\right\} \subset \mathbb{R}^{a}\right\}
$$

o conjunto de todos os possíveis conjuntos de $k$ protótipos contidos no espaço $\mathbb{R}^{a}$ e considere uma base de dados representada por $X \triangleq\left\{\mathrm{x}^{1}, \ldots, \mathrm{x}^{n}\right\} \subset \mathbb{R}^{a}$. Dado um conjunto de protótipos $V \in M_{\mathrm{v}}(a)$ e uma matriz de agrupamento $\mathrm{U} \in M_{\mathrm{p}}$, grande parte dos algoritmos que buscam minimizar a Função (2.16) calculam as distâncias representadas em $\mathrm{D} \triangleq\left[\mathrm{D}_{i, j}\right] \in M_{\mathrm{d}}$ por

$$
\mathrm{D}_{i, j} \triangleq\left\|\mathrm{x}^{j}-\mathrm{v}^{i}\right\|^{2} \quad \forall i \in \mathbb{N}_{1, k}, \forall j \in \mathbb{N}_{1, n}
$$

em que $\|\cdot\|$ é qualquer norma induzida por produto interno no espaço $\mathbb{R}^{a}$. Pode-se citar as funções-custo dos algoritmos FCM e k-Means como instâncias da Função $\mathcal{G}_{m}$ e de adoção do cálculo das distâncias por meio da Def. (2.18).

Frigui e Nasraoui (2004) estenderam o termo $\mathrm{D}_{i, j}$ da Função (2.16) para agregar diferentes representações de uma base de dados. Seja $D \triangleq\left\{\mathrm{D}^{1}, \ldots, \mathrm{D}^{p}\right\} \subset \mathbb{R}^{k \cdot n}$ um conjunto de $p$ matrizes contido em

$$
M_{\mathrm{dp}} \triangleq\left\{\left\{\mathrm{D}^{1}, \ldots, \mathrm{D}^{p}\right\} \subset \mathbb{R}^{k \cdot n} \mid \mathrm{D}^{s} \in M_{\mathrm{d}} \quad \forall s \in \mathbb{N}_{1, p}\right\}
$$

A função-custo em questão é dada por $\mathcal{G}_{m, q}: M_{\mathrm{n}} \times M_{\mathrm{dp}} \times M_{\mathrm{y}} \mapsto \mathbb{R} \mathrm{e}$

$$
\mathcal{G}_{m, q}(\mathrm{U}, D, \mathrm{Y}) \triangleq \sum_{i=1}^{k} \sum_{j=1}^{n}\left(\mathrm{U}_{i, j}\right)^{m} \cdot \sum_{s=1}^{p}\left(\mathrm{Y}_{i, s}\right)^{q} \cdot \mathrm{D}_{i, j}^{s}
$$

em que $q \in[1, \infty)$ é o expoente de ponderação e $m \in[1, \infty)$ é o expoente de nebulosidade. A diferença essencial entre as Funções 2.16) e (2.20) consiste no cômputo da dissimilaridade entre objetos e grupos. A última agrega, por meio de um conjunto de pesos de representações, $p$ distâncias para compor a distância final entre um objeto e um grupo. O expoente $q$ é responsável pelo quão nebuloso os elementos da matriz Y são. Isto é, quando $q$ se aproxima de $1, \mathrm{Y}_{i, s}$ tende a assumir valores binários (isto é, próximos de zero ou próximos de um); quando $q$ tende ao infinito, $\mathrm{Y}_{i, s}$ tende a assumir o valor $1 / p$ (Frigui e Nasraoui, 2004).

$\mathrm{O}$ algoritmo $\mathrm{SCAD}$ otimiza um caso particular de $\mathcal{G}_{m, q}$, no qual protótipos são usados para representar os grupos. Seja $\dot{X} \triangleq\left\{X_{1}, X_{2}, \ldots, X_{p}\right\}$ o conjunto de $p$ representações, cada qual descrita por um conjunto $X_{s} \triangleq\left\{\mathrm{x}^{1, s}, \mathrm{x}^{2, s}, \ldots, \mathrm{x}^{n, s}\right\} \subset \mathbb{R}^{a_{s}}$ de $n$ vetores de atributos contidos no espaço de atributos $\mathbb{R}^{a_{s}}$ correspondente à $s$-ésima representação. Portanto, $\mathrm{X}^{j, s}$ é o vetor 
que representa o objeto $\tilde{o}_{j}$ sob a $s$-ésima representação. $\mathrm{O} i$-ésimo grupo é representado por $p$ protótipos $\mathrm{v}^{i, s} \in \mathbb{R}^{a_{s}}$, cada qual correspondendo a uma diferente visão dos dados. É definido $V_{s} \triangleq\left\{\mathrm{v}^{1, s}, \mathrm{v}^{2, s}, \ldots, \mathrm{v}^{k, s}\right\}$ como sendo o conjunto de $k$ protótipos correspondentes à $s$-ésima representação. O conjunto desses conjuntos é denotado por $\dot{V} \triangleq\left\{V_{1}, V_{2}, \ldots, V_{p}\right\}$ e está contido no conjunto

$$
M_{\mathrm{vp}} \triangleq\left\{\left\{V_{1}, V_{2}, \ldots, V_{p}\right\} \mid V_{s} \in M_{\mathrm{v}}\left(a_{s}\right) \quad \forall s \in \mathbb{N}_{1, p}\right\} .
$$

Seja a função $\mathcal{D}_{\text {ve }}: M_{\mathrm{vp}} \mapsto M_{\mathrm{dp}}$, definida como $\mathcal{D}_{\text {ve }}(\dot{V} ; \dot{X}) \triangleq\left\{\mathrm{D}^{s}\right\}_{s=1}^{p} \in M_{\mathrm{dp}}, \mathrm{D}^{s} \triangleq\left[\mathrm{D}_{i, j}^{s}\right]$ $\left(\forall s \in \mathbb{N}_{1, p}\right)$ e

$$
\mathrm{D}_{i, j}^{s} \triangleq\left\|\mathrm{x}^{j, s}-\mathrm{v}^{i, s}\right\|_{\mathrm{e}}^{2} \quad \forall i, j, s,
$$

em que $\|\cdot\|_{\text {e }}$ é a norma euclidiana para o espaço correspondente. A função $\mathcal{D}_{\text {ve }}$ é usada para definir a distância dos objetos aos grupos, considerando as diferentes representações dos dados. $\mathrm{O}$ algoritmo SCAD minimiza a função $\mathcal{G}_{m, q}^{\mathrm{ve}}: M_{\mathrm{n}} \times M_{\mathrm{vp}} \times M_{\mathrm{y}} \mapsto \mathbb{R}$, definida por

$$
\begin{aligned}
\mathcal{G}_{m, q}^{\mathrm{ve}}(\mathrm{U}, \dot{V}, \mathrm{Y} ; \dot{X}) & \triangleq \mathcal{G}_{m, q}\left(\mathrm{U}, \mathcal{D}_{\mathrm{ve}}(\dot{V} ; \dot{X}), \mathrm{Y}\right) \\
& =\sum_{i=1}^{k} \sum_{j=1}^{n}\left(\mathrm{U}_{i, j}\right)^{m} \cdot \sum_{s=1}^{p}\left(\mathrm{Y}_{i, s}\right)^{q} \cdot\left\|\mathrm{x}^{j, s}-\mathrm{v}^{i, s}\right\|_{\mathrm{e}}^{2}
\end{aligned}
$$

para $q \in(1, \infty)$ e $m \in(1, \infty)$. O Algoritmo 2.1 fornece uma visão geral do algoritmo SCAD.

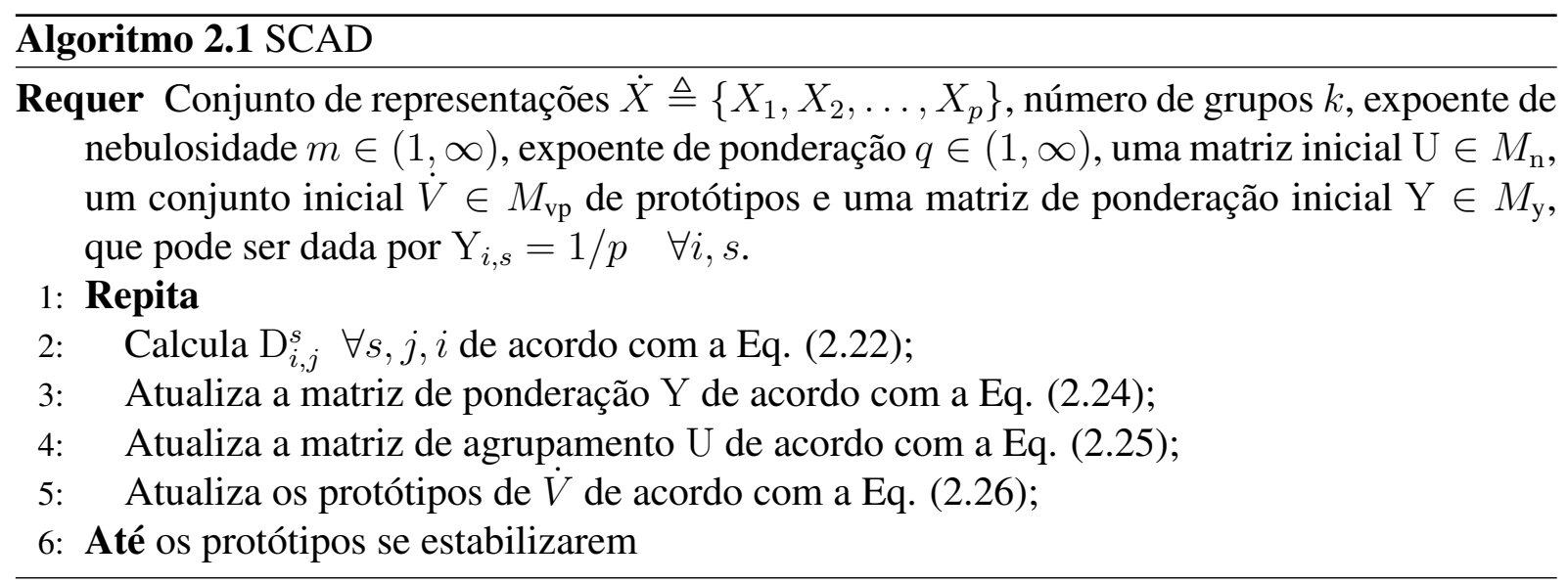

Em relação ao Passo 3, foi concluído em (Frigui e Nasraoui, 2004) que

$$
\mathrm{Y}_{i, s} \triangleq\left(\sum_{l=1}^{p}\left(\frac{\sum_{j=1}^{n}\left(\mathrm{U}_{i, j}\right)^{m} \cdot \mathrm{D}_{i, j}^{s}}{\sum_{j=1}^{n}\left(\mathrm{U}_{i, j}\right)^{m} \cdot \mathrm{D}_{i, j}^{l}}\right)^{\frac{1}{q-1}}\right)^{-1}
$$

minimiza a Função 2.23) quando as variáveis U e $\dot{V}$ são mantidas constantes. Analogamente, em (Frigui e Nasraoui, 2004) afirma-se que a equação

$$
\mathrm{U}_{i, j} \triangleq\left(\sum_{l=1}^{k}\left(\frac{\sum_{s=1}^{p}\left(\mathrm{Y}_{i, s}\right)^{q} \cdot \mathrm{D}_{i, j}^{s}}{\sum_{s=1}^{p}\left(\mathrm{Y}_{l, s}\right)^{q} \cdot \mathrm{D}_{l, j}^{s}}\right)^{\frac{1}{m-1}}\right)^{-1}
$$


minimiza a Função 2.23 quando as variáveis $\dot{V}$ e Y são mantidas constantes. Entretanto, os autores não provaram que as duas equações acima realmente minimizam a função-custo, mas apenas que alguns dos pontos críticos são dados pelas mesmas. Em determinadas situações, o algoritmo SCAD pode, inclusive, falhar, ao ignorar determinados pontos críticos na otimização das variáveis U e Y. Essa questão e outras são discutidas em maior profundidade na Seção 3.1 .

A atualização que ocorre no Passo 5 é dada por

$$
\mathrm{v}^{i, s} \triangleq \sum_{j=1}^{n}\left(\mathrm{U}_{i, j}\right)^{m} \cdot \mathrm{x}^{j, s} / \sum_{j=1}^{n}\left(\mathrm{U}_{i, j}\right)^{m}
$$

a qual minimiza a Função 2.23 quando as variáveis U e Y são mantidas constantes. O vetor $\mathrm{v}^{s, i}$ resultante da Eq. 2.26) é denominado de centróide; especificamente, centróide do $i$-ésimo grupo sob o $s$-ésimo aspecto. Frigui e Nasraoui (2004) mostraram que os pontos críticos da Função (2.23), ao manter U e Y constantes, podem ser encontrados pela Eq. 2.26). Horta e Campello (2012) provaram que tais pontos críticos são, também, mínimos globais e que não há necessidade de se exigir que a distância usada na Def. (2.22) seja euclidiana.

A seção seguinte apresenta uma versão relacional do algoritmo SCAD.

\subsubsection{Algoritmo CARD}

No contexto relacional, não há atributos para representar os objetos, mas apenas relações de proximidade entre eles (Seção 2.1). Nesse caso, uma base $O$ de $n$ objetos pode ser representada por uma coleção $R \triangleq\left\{\mathrm{R}^{1}, \mathrm{R}^{2}, \ldots, \mathrm{R}^{p}\right\} \subset \mathbb{R}^{n \cdot n}$ de $p$ matrizes relacionais. Os elementos dessas matrizes são identificados pela notação $\mathrm{R}_{j, l}^{s}$, a qual indica o valor do elemento da matriz $\mathrm{R}^{s}$ que reside na $j$-ésima linha e na $l$-ésima coluna. A função-custo do algoritmo CARD pode ser definida como $\mathcal{K}_{m, q}: M_{\mathrm{n}} \times M_{\mathrm{y}} \mapsto \mathbb{R}$, na qual

$$
\mathcal{K}_{m, q}(\mathrm{U}, \mathrm{Y} ; R) \triangleq \sum_{i=1}^{k} \frac{\sum_{j=1}^{n} \sum_{l=1}^{n}\left(\mathrm{U}_{i, j}\right)^{m} \cdot\left(\mathrm{U}_{i, l}\right)^{m} \cdot \sum_{s=1}^{p}\left(\mathrm{Y}_{i, s}\right)^{q} \cdot \mathrm{R}_{j, l}^{s}}{2 \sum_{j=1}^{n}\left(\mathrm{U}_{i, j}\right)^{m}}
$$

em que $q \in(1, \infty)$ é o expoente de ponderação e $m \in(1, \infty)$ é o expoente de nebulosidade. Embora a função $\mathcal{K}_{m, q}$ seja pouco intuitiva, ela possui uma forte relação com a Função (2.23). Essa relação justifica o seu uso e é descrita na Seção 3.2 .

Assim como o algoritmo SCAD foi baseado no FCM o algoritmo CARD foi baseado no Non-Euclidean Relational Fuzzy c-Means (Hathaway e Bezdek, 1994) (NERFCM), a versão relacional do FCM $\mathrm{O}$ Algoritmo 2.2 fornece uma visão geral do método CARD.

Como no contexto relacional não existem atributos, não é possível adotar o conceito usual de centróide (Eq. (2.26)). Ao invés disso, os protótipos dos grupos são centróides relacionais, definidos por

$$
\hat{\mathrm{v}}^{i} \triangleq\left[\begin{array}{llll}
\left(\mathrm{U}_{i, 1}\right)^{m} & \left(\mathrm{U}_{i, 2}\right)^{m} & \ldots & \left(\mathrm{U}_{i, n}\right)^{m}
\end{array}\right]^{\mathrm{T}} \frac{1}{\sum_{j=1}^{n}\left(\mathrm{U}_{i, j}\right)^{m}} \quad \forall i \in \mathbb{N}_{1, k} .
$$




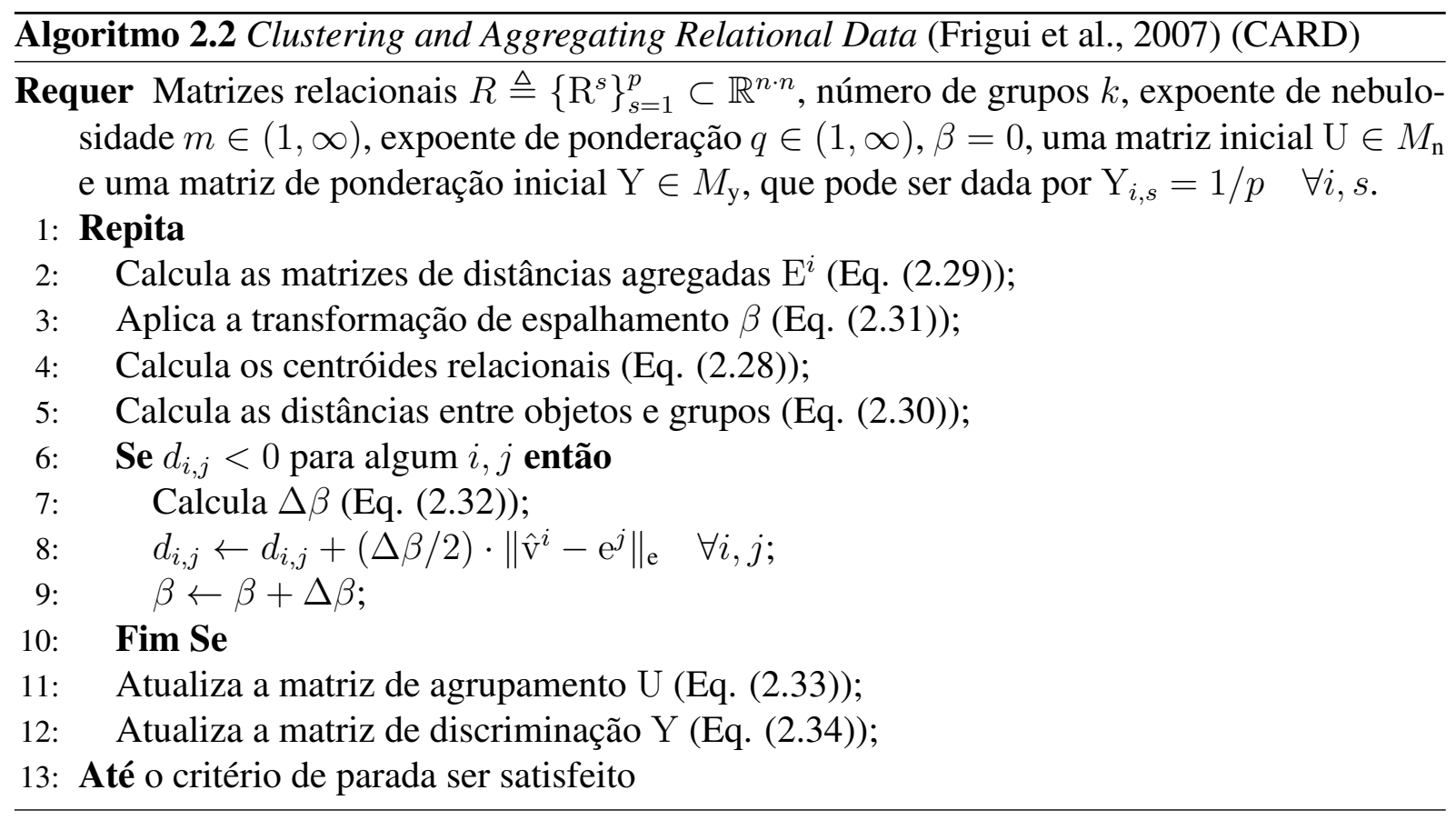

Esse tipo de centróide foi primeiramente introduzido por Hathaway et al. (1989) e não possui uma interpretação intuitiva, sendo apenas um artefato de um teorema contido no mesmo artigo. O seu significado é melhor discutido na Seção 3.2 .

A partir das matrizes relacionais e da matriz de ponderação $\mathrm{Y}$, o algoritmo CARD constrói uma matriz de distâncias $\mathrm{E}^{i}$ para cada grupo e em cada iteração. Essas matrizes são definidas como

$$
\mathrm{E}_{j, l}^{i} \triangleq \sum_{s=1}^{p}\left(\mathrm{Y}_{i, s}\right)^{q} \cdot \mathrm{R}_{j, l}^{s} \quad \forall i \in \mathbb{N}_{1, k}, \forall j, l \in \mathbb{N}_{1, n}
$$

ao agregar as matrizes relacionais por meio de uma soma ponderada. Uma vez feito esses cálculos e usando os centróides relacionais, essas matrizes de agregação são usadas na equação

$$
d_{i, j} \triangleq\left(\mathrm{E}^{i} \cdot \hat{\mathrm{v}}^{i}\right)_{j}-\left(\hat{\mathrm{v}}^{i}\right)^{\mathrm{T}} \cdot \mathrm{E}^{i} \cdot \hat{\mathrm{v}}^{i} / 2
$$

para calcular as distâncias totais (i.e., considerando todas as representações da base) dos objetos aos grupos. Portanto, $d_{i, j}$ representa a distância total do $j$-ésimo objeto ao $i$-ésimo grupo. A Eq. 2.30) é a mesma usada em (Hathaway et al., 1989), mas com matrizes de distância diferentes.

Quando uma ou mais das matrizes $E^{i}$ não é euclidiana, é possível que a Eq. (2.30) forneça distâncias negativas (Hathaway e Bezdek, 1994). Nesse caso, Frigui et al. (2007) sugeriram aplicar a transformação de espalhamento $\beta$, desenvolvida em (Hathaway e Bezdek, 1994), para aproximar as matrizes não euclidianas de suas versões euclidianas ${ }^{6}$. A transformação de espalhamento $\beta$ é definida por

$$
\mathrm{E}_{\beta}^{i} \triangleq \mathrm{E}^{i}+\beta \cdot(\mathbb{1}-\mathrm{I})
$$

${ }^{6}$ É provado em (Hathaway e Bezdek, 1994) que toda matriz relacional não euclidiana se torna euclidiana ao aplicar a transformação de espalhamento $\beta$ para um valor suficientemente grande de $\beta$. 
em que $\mathbb{1}$ é uma matriz $n$-por- $n$ de uns e I é a matriz identidade $n$-por- $n$. O valor de $\beta$ é escolhido de forma que o grau de distorção da matriz correspondente seja o menor possível, mas que faça todas as distâncias $d_{i, j}$ serem não negativas. Formalmente, o valor de $\beta$ é deslocado a cada iteração por

$$
\Delta \beta \triangleq \max _{i, j}\left\{-2 \cdot d_{i, j} /\left\|\hat{\mathrm{v}}^{i}-\mathrm{e}^{j}\right\|_{\mathrm{e}}^{2}\right\}
$$

em que $\mathrm{e}^{j}$ denota a $j$-ésima coluna da matriz identidade $n$-por- $n$. Note que as matrizes $\mathrm{E}^{i}$ se alteram a cada iteração, devido aos novos valores dos elementos da matriz Y. No entanto, o valor $\beta$ da iteração passada, calculado a partir da necessidade de distorcer as matrizes $\mathrm{E}^{i}$ daquela iteração, é reutilizado na iteração atual nas novas matrizes $\mathrm{E}^{i}$. Isso não está em acordo com as deduções matemáticas de Hathaway e Bezdek (1994) nas quais Frigui et al. (2007) se basearam, em que é pressuposto que as matrizes a serem distorcidas não se alteram, a não ser pela transformação de espalhamento, no decorrer das iterações. Essa questão é melhor trabalhada na Seção 3.3 para um novo algoritmo relacional.

As matrizes de agrupamento e de ponderação são atualizadas da seguinte forma. De modo análogo à Eq. (2.25), a atualização de U para o algoritmo CARDé definida por

$$
\mathrm{U}_{i, j} \triangleq\left(\sum_{l=1}^{k}\left(\frac{d_{i, j}}{d_{l, j}}\right)^{\frac{1}{m-1}}\right)^{-1} .
$$

A atualização da matriz $\mathrm{Y}$ é dada por

$$
\mathrm{Y}_{i, s} \triangleq\left(\sum_{l=1}^{p}\left(\frac{\hat{D}_{i, s}}{\hat{D}_{i, l}}\right)^{\frac{1}{q-1}}\right)^{-1}
$$

na qual

$$
\hat{D}_{i, s} \triangleq \sum_{j=1}^{n} \sum_{t=1}^{n}\left(\mathrm{U}_{i, j}\right)^{m} \cdot\left(\mathrm{U}_{i, t}\right)^{m} \cdot \mathrm{R}_{j, t}^{s} .
$$

Frigui et al. (2007) não provaram que as equações de atualização de $\mathrm{Y}$ e U fornecem, pelo menos, um mínimo local para a Função 2.27). Além disso, pode ocorrer divisão por zero nessas equações em cenários encontrados na prática (Seção 3.6.1). A Seção 3.2 detalha melhor essas questões e propõe um algoritmo melhor fundamentado e que possui uma relação direta com o algoritmo SCAD, Será provado que os passos de otimização desse novo algoritmo realmente minimizam a Função 2.27). Também será provado que a nova versão (ajustada) do algoritmo SCAD desenvolvida nesta tese é equivalente a esse novo algoritmo relacional, dada pressuposições razoáveis.

\subsection{Validação de agrupamento}

Quase todos os algoritmos de agrupamento produzem uma solução mesmo quando são aplicados em bases de dados que não apresentam qualquer subestrutura (Tan et al., 2005); por 
exemplo, quando os pontos que representam os objetos formam uma nuvem uniforme no espaço de atributos. Os mesmos algoritmos podem ser aplicados várias vezes na mesma base de dados para gerar várias soluções a fim de evitar mínimos locais das funções-objetivo que tentam otimizar. No entanto, as soluções geradas são boas ou foram obtidas meramente ao acaso? Qual das soluções geradas é a melhor? Para responder esse tipo de questão existe a validação de agrupamento, a qual consiste no processo de avaliar os resultados gerados por algoritmos de agrupamento (Halkidi et al., 2002a).

Existem três abordagens empregadas na validação de agrupamento (Halkidi et al., 2002a): validação externa, validação interna e validação relativa. Todas elas utilizam índices que aferem características da solução ou medem a similaridade entre duas soluções. Na validação externa, pode-se usar uma estratégia que mede o grau com o qual os dados produzidos pelo algoritmo de agrupamento confirmam conhecimento prévio a respeito da base de dados (Jain e Dubes, 1988). Porém, o mais comum na validação externa é a medição da similaridade entre o agrupamento gerado pelo algoritmo e um agrupamento de referência, o que é feito por meio da aplicação de índices 7. Esse agrupamento de referência é uma estrutura pré-definida que impomos à base de dados e que reflete a nossa intuição sobre a estrutura da base. Essa pré-estrutura é, em geral, uma solução que acreditamos ser a ideal e essa linha de validação é bastante usada em trabalhos que avaliam experimentalmente algoritmos de agrupamento.

Pode-se citar o Rand Index (Rand, 1971) (RI) como um índice muito conhecido na área de validação externa de agrupamentos particionais exclusivos, definido da seguinte forma. Sejam $\mathrm{U} \in M_{\mathrm{e}} \mathrm{e} \mathrm{V} \in M_{\mathrm{e}}$ duas matrizes de agrupamento particional exclusivo, no qual um deles seria um agrupamento gerado e o outro a solução ideal. Considere as variáveis de pareamento definidas como se segue:

- $a$ : número de pares de objetos contidos nos mesmos grupos tanto em U como em V;

- $b$ : número de pares de objetos contidos em grupos diferentes em U mas nos mesmos grupos em $\mathrm{V}$;

- $c$ : número de pares de objetos contidos nos mesmos grupos em U mas em grupos diferentes em $\mathrm{V}$;

- $d$ : número de pares de objetos contidos em grupos diferentes tanto em $\mathrm{U}$ como em $\mathrm{V}$.

O índice RI]é dado por

$$
\mathrm{RI}(\mathrm{U}, \mathrm{V}) \triangleq \frac{a+d}{a+b+c+d}
$$

Existem críticas contundentes e algumas amplamente conhecidas ao índice RI (Hubert e Arabie, 1985; Jain e Dubes, 1988; Saporta e Youness, 2002; Vendramin et al., 2010). Uma das principais críticas está no fato do índice $[\mathrm{RI}$ atribuir a mesma importância aos termos $a$ e $d$. Logo, o índice RI não faz qualquer distinção entre pares unidos e pares separados tanto na solução de referência como na solução sendo avaliada. Essa política é discutível, particularmente

\footnotetext{
${ }^{7}$ Observe que validação é uma estratégia para avaliar, enquanto um índice é uma medida objetiva, podendo fazer parte da estratégia de validação.
} 
quando o agrupamento particional exclusivo é interpretado como sendo um conjunto de grupos de elementos unidos, enquanto as separações são simplesmente consequências do procedimento de união. Além disso, sabe-se que o termo $d$ pode dominar os outros três termos, fazendo com que o índice se torne incapaz de distinguir apropriadamente soluções boas das ruins (Sharan e Shamir, 2000; Jiang et al., 2004). Essa situação se torna particularmente crítica quando o número de grupos aumenta, visto que o valor da variável $d$ tende a aumentar também.

Felizmente há uma coleção de outros índices capazes de produzir resultados mais acurados, como é o caso do Jaccard Index (Jaccard, 1908) (JI) e da medida Adjusted Rand Index (Hubert e Arabie, 1985) (ARI), esta última definida como

$$
\operatorname{ARI}(\mathrm{U}, \mathrm{V}) \triangleq \frac{a-\frac{(a+c)(a+b)}{a+b+c+d}}{\frac{a+c+a+b}{2}-\frac{(a+c)(a+b)}{a+b+c+d}}
$$

$\mathrm{O}$ índice $\mathrm{ARI}$ surge quando o índice $\mathrm{RI}$ é normalizado de forma que a esperança seja zero quando os dois agrupamentos são gerados aleatoriamente. Como consequência, o índice ARI é um dos índices externos mais utilizados na literatura de agrupamento de dados. Nas Seções 4.2.1 são definidos índices de validação externa também para os tipos mais gerais de agrupamento, por motivos de análise e comparação com índices propostos nesta tese.

$\mathrm{Na}$ validação interna, os resultados encontrados são avaliados somente com relação aos próprios dados da base, sem a ajuda de informações sobre a solução ideal, objetivando responder a pergunta "Esta solução que encontrei é uma boa solução?". Já na validação relativa, o objetivo consiste em apontar a melhor solução de um conjunto de agrupamentos. Por exemplo, quando se gera diversas soluções ao aplicar um mesmo algoritmo mas com parâmetros de entrada distintos (Halkidi et al., 2002a). Os índices usados nas validações relativas e internas são, em geral, os mesmos (Jain e Dubes, 1988).

Existem vários índices para validação relativa ou interna voltados para agrupamentos particionais exclusivos (Milligan e Cooper, 1985; Halkidi et al., 2001). Alguns dos mais populares são o índice de Davies-Bouldin (Davies e Bouldin, 1979; Jain e Dubes, 1988), o Critério da Razão de Variância (também chamado de índice de Calinski-Harabasz) (Calinski e Harabasz, 1974; Everitt et al. 2001), o índice de Dunn (Dunn, 1974; Halkidi et al., 2002b) e o índice da Silhueta.

Em estudos comparativos recentes (Vendramin et al., 2009, 2010), o índice da Silhueta se desempenhou melhor que dezenas de outros índices relativos e internos projetados para agrupamentos particionais exclusivos. O índice da Silhueta é descrito na seção seguinte. Também são descritas duas variantes do índice da Silhueta nas Seções 2.5.2 e 2.5.3 devido à importância dessas para o presente trabalho.

\subsection{1 Índice da Silhueta}

O índice da Silhueta é baseado em considerações geométricas sobre a compactação e a separação dos grupos e foi concebido para lidar com agrupamento particional exclusivo, $U \in$ $M_{\mathrm{e}}$. Para defini-lo, considere que o objeto $\tilde{o}_{j}$ pertence ao $p_{j}$-ésimo grupo, $\mathrm{U}_{p_{j}, j}=1$. Então, 
seja a distância média desse objeto a todos os outros objetos do $p_{j}$-ésimo grupo denotada por

$$
a_{p_{j}, j} \triangleq \sum_{l=1}^{n} \mathrm{U}_{p_{j}, l} \cdot d\left(\tilde{o}_{j}, \tilde{o}_{l}\right) /\left(\sum_{l=1}^{n} \mathrm{U}_{p_{j}, l}-1\right),
$$

em que $d\left(\tilde{o}_{j}, \tilde{o}_{l}\right)$ representa a distância entre os objetos $\tilde{o}_{j}$ e $\tilde{o}_{l}$. Note que a média é em relação às distâncias do objeto $\tilde{o}_{j}$ aos outros do mesmo grupo, por isso a subtração no denominador. Seja

$$
b_{t, j} \triangleq \sum_{l=1}^{n} \mathrm{U}_{t, l} \cdot d\left(\tilde{o}_{j}, \tilde{o}_{l}\right) / \sum_{l=1}^{n} \mathrm{U}_{t, l}
$$

a distância média de $\tilde{o}_{j}$ a todos os objetos do $t$-ésimo grupo, $t \neq p_{j}$. Finalmente, seja $b_{q_{j}, j}$ o mínimo de $b_{t, j}$ computado para $t=1,2, \ldots, k, t \neq p_{j}$, representando a dissimilaridade média do objeto $\tilde{o}_{j}$ ao seu grupo vizinho mais próximo. Então, a silhueta do objeto $\tilde{o}_{j}$ é definida como

$$
s_{j} \triangleq\left(b_{q_{j}, j}-a_{p_{j}, j}\right) / \max \left\{a_{p_{j}, j}, b_{q_{j}, j}\right\},
$$

em que o denominador é apenas um termo de normalização. Note que $s_{j} \in[-1,1]$. Quanto maior o valor de $s_{j}$, melhor é a atribuição do objeto $\tilde{o}_{j}$ ao $p_{j}$-ésimo grupo. No caso de grupo unitário, i.e., se ele consiste apenas de um objeto $\tilde{o}_{j}$, então é assumido por convenção que $s_{j}=0$ (Kaufman e Rousseeuw, 1990). Isso previne que o índice da Silhueta, definido como a média de $s_{j}$ para $j=1,2, \ldots, n$, i.e.,

$$
\mathbb{S}_{\mathrm{sil}}(\mathrm{U}) \triangleq(1 / n) \sum_{j=1}^{n} s_{j}
$$

de avaliar a solução trivial $k=n$ (em que cada objeto da base de dados forma o seu próprio grupo) como sendo a melhor. O melhor agrupamento é esperado ocorrer quando o índice da Silhueta é maximizado, o que implica em minimizar a distância intragrupo $\left(a_{p_{j}, j}\right)$ e maximizar a distância intergrupo $\left(b_{q_{j}, j}\right)$.

\subsubsection{Silhueta Simplificada}

O índice da Silhueta depende do cálculo de todas as distâncias entre todos os objetos, o que é bastante custoso computacionalmente. Para contornar esse problema, Hruschka et al. (2004, 2006) propuseram a substituição das distâncias entre objetos pelas distâncias entre objetos e grupos. Nesse caso, o termo $a_{p_{j}, j}$ da Eq. 2.37) é redefinido como a dissimilaridade do objeto $\tilde{o}_{j}$ ao seu grupo (e.g., dissimilaridade entre $\tilde{o}_{j}$ e o protótipo do seu grupo), o $p_{j}$-ésimo grupo,

$$
a_{p_{j}, j} \triangleq \mathrm{D}_{p_{j}, j}
$$

em que $\mathrm{D}_{p_{j}, j}$ possui a interpretação do mesmo termo da Função 2.16. Similarmente, o termo $b_{t, j}$ é calculado como a dissimilaridade do objeto $\tilde{o}_{j}$ ao $t$-ésimo grupo, e $b_{q_{j}, j}$ se torna a dissimi- 
laridade do objeto $\tilde{o}_{j}$ ao grupo mais próximo:

$$
b_{q_{j}, j} \triangleq \mathrm{D}_{q_{j}, j}
$$

A ideia do índice da Silhueta Simplificada (Hruschka et al., 2004, 2006; Vendramin et al. 2010) (SS) é substituir a média das distâncias entre objetos pelas distâncias entre objetos e grupos. Essa modificação reduz a complexidade computacional do índice da Silhueta, $\mathcal{O}\left(n^{2}\right)$, para $\mathcal{O}(n k)$, em que $n$ é o número de objetos da base e $k$ é o número de grupos. A versão simplificada apresentou resultados similares aos da versão original em extensivos estudos comparativos (Vendramin et al., 2009, 2010).

\subsubsection{Silhueta Simplificada Nebulosa}

O índice $\mathbf{S S}$ não faria uso explícito da matriz de um agrupamento nebuloso em seus cálculos caso fosse aplicado para avaliar tal tipo de agrupamento. Nesses casos, tal matriz de agrupamento $\mathrm{U} \in M_{\mathrm{n}}$ seria usada apenas para impor à base de dados um agrupamento particional exclusivo $\hat{\mathrm{U}} \in M_{\mathrm{e}}$ no qual o índice $\underline{\mathrm{SS}}$ poderia ser aplicado, de acordo com

$$
\hat{\mathrm{U}}_{i, j} \triangleq \begin{cases}1 & \mathrm{U}_{i, j}=\max \left\{\mathrm{U}_{1, j}, \mathrm{U}_{2, j}, \ldots, \mathrm{U}_{k, j}\right\} \\ 0 & \text { caso contrário }\end{cases}
$$

Consequentemente, o índice SS pode não ser capaz de discriminar grupos de dados sobrepostos, mesmo que esses grupos tenham suas regiões distintas com maior densidade de dados, porque ela negligencia informações contidas na matriz U sobre os graus com que os grupos se sobrepõem. Essa informação pode ser usada para revelar as regiões com alta densidade de dados ao salientar a importância dos objetos concentrados na vizinhança dos protótipos enquanto reduzse a importância dos objetos das áreas de sobreposição. Para tanto, uma versão generalizada do índice SS, referida por Silhueta Simplificada Nebulosa (Campello e Hruschka, 2006) (SSN), foi definida como

$$
\mathbb{S}_{\mathrm{ssn}}(\mathrm{U}) \triangleq \sum_{j=1}^{n}\left(\mathrm{U}_{p_{j}, j}-\mathrm{U}_{q_{j}, j}\right) \cdot s_{j} / \sum_{j=1}^{n}\left(\mathrm{U}_{p_{j}, j}-\mathrm{U}_{q_{j}, j}\right),
$$

em que $s_{j}$ é a silhueta simplificada do objeto $\tilde{o}_{j}, \mathrm{U}_{p_{j}, j}$ e $\mathrm{U}_{q_{j}, j}$ são o primeiro e o segundo maiores elementos da $j$-ésima coluna da matriz de agrupamento, respectivamente.

\subsection{Considerações}

Neste capítulo foram apresentados alguns conceitos da área de agrupamento de dados que são particularmente importantes no contexto desta tese. Os quatros tipos de solução de agrupamento não hierárquico mais presentes na literatura foram formalmente definidos. Foi apresentada uma visão geral da área de agrupamento em subespaços, fomentada a partir da necessidade 
de lidar, em especial, com duas características de certas bases de dados: alta dimensionalidade e importância diferenciada dos atributos para as diferentes subestruturas dos dados. Ambas as características apresentam novos desafios para a área de agrupamento de dados, visto que os algoritmos clássicos tendem a falhar em tais cenários.

O algoritmo $\mathrm{SCAD}$ foi descrito como um algoritmo capaz de ponderar atributos individualmente, tornando-o membro da subcategoria dos algoritmos baseados em projeção suave e apto a lidar com o problema da importância diferenciada dos atributos, bem como de ponderar e agregar diferentes representações dos dados. A sua versão relacional denominada CARD foi descrita e alguns dos problemas teóricos dos algoritmos SCAD e CARD foram apresentados, os quais serão, em conjunto com outros problemas, solucionados no Capítulo 3 .

Algoritmos de bi-agrupamento foram introduzidos como técnicas para lidar com a busca de padrões na matriz de dados. Essa abordagem permite a discriminação local de atributos, de acordo com as subestruturas dos dados. Tais algoritmos têm despertado um interessente crescente da comunidade de análise de expressão gênica, a qual têm produzido várias técnicas de bi-agrupamento nos últimos anos (Madeira e Oliveira, 2004; Tanay et al., 2005; Busygin et al. 2008; Eren et al., 2012).

A validação de agrupamento foi apresentada como uma tarefa importante na análise de resultados, com o objetivo de aferir a legitimidade e comparar soluções de agrupamento. Existem três tipos de validação de agrupamento: validação externa, validação interna e validação relativa. A primeira emprega índices que comparam os agrupamentos gerados com informações preconcebidas sobre os dados. A segunda tem como objetivo destacar agrupamentos que representam de fato uma estrutura inerente aos dados. A terceira emprega índices para comparar as soluções de um conjunto de soluções, com o objetivo de destacar a melhor dentre elas. Alguns índices de validação externa e interna/relativa de particular interesse para este trabalho, como o ARI e a Silhueta, foram descritos. 
CAPÍTULO

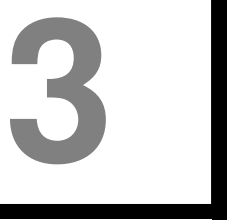

\section{NOVOS ALGORITMOS E TÉCNICA DE VALIDAÇÃO EM AGRUPAMENTO DE DADOS MULTI-REPRESENTADOS}

Neste capítulo são discutidos alguns dos problemas dos algoritmos SCAD e CARD e são apresentados novos algoritmos de agrupamento para dados multi-representados que não possuem os problemas apontados. Na Seção 3.1 são feitas críticas ao algoritmo SCAD e uma versão ajustada, denominada ASCAD, é proposta. Uma versão particional exclusivo do algoritmo ASCAD é definida na Seção 3.1.1 apenas para ilustrar que versões não nebulosas dos algoritmos em questão podem ser facilmente desenvolvidas a partir da base teórica aqui apresentada. No contexto relacional, os algoritmos Relational Adjusted Simultaneous Clustering and Aspect Discrimination (RASCAD) e Non-Euclidean RASCAD (NERASCAD) são propostos nas Seções 3.2 e 3.3 . Provas da convergência dos algoritmos propostos são apresentadas na Seção 3.4 .

A Seção 3.6 tem como objetivos principais avaliar a capacidade dos algoritmos propostos em lidar com bases de dados ruidosas (ruído nos atributos) e com bases de dados descritas por múltiplas representações. No primeiro caso, as bases foram geradas artificialmente com graus crescente de ruído. No segundo, foram usadas bases reais formadas a partir de imagens da coleção Amsterdam Library of Object Images (Geusebroek et al., 2005) (ALOI). Na Seção 3.6.1, os algoritmos SCAD e ASCAD são comparados em uma base da University of California - Irvine (Frank e Asuncion, 2010) ( $\overline{\mathrm{UCI}}$ ), na qual o algoritmo SCAD falha mas o algoritmo ASCAD não. É avaliada também a robustez do algoritmo ASCAD quanto à escolha do parâmetro $q$ (Seção 3.6.2) em bases reais da UCI Por fim, a Seção 3.7 apresenta as considerações sobre o capítulo. 


\subsection{SCAD ajustado}

Existem alguns ajustes importantes que podem ser feitos na formulação original do algoritmo SCAD (Algoritmo 2.1). Primeiro, SCAD requer como entrada a matriz de ponderação Y e os protótipos (contidos em $\dot{V}$ ) para cada grupo e representação da base, entre outros parâmetros. Não é desejável requerer que o usuário forneça essas duas variáveis, as quais podem influenciar a solução final. Esse pré-requisito pode ser facilmente eliminado ao alterar a ordem dos passos do algoritmo. Observe que a Eq. (2.26) precisa apenas da matriz de agrupamento U e que ela produz os centróides correspondentes. Assim, a Eq. 2.24 pode ser usada depois de inicializar U e aplicar a Eq. (2.26). Isso justifica a ordem dos passos do algoritmo ASCAD definido pelo Algoritmo 3.1. Consequentemente, o algoritmo ASCAD requer apenas que o usuário inicialize a matriz de agrupamento nebuloso $\mathrm{U} \in M_{\mathrm{n}}$, além das definições de $m \in(1, \infty)$ e $q \in(1, \infty)$. Uma estratégia útil de inicialização para esse passo é dada na Seção 3.6, a qual foi adotada em todos os experimentos relatados na mesma seção. Além disso, uma sugestão para o valor do expoente $q$ é também dada na Seção 3.6 .

Um outro problema com o algoritmo $\underline{\mathrm{SCAD}}$ advém dos passos de otimização das variáveis U e Y. Considere uma representação $X_{s} \in X$ do conjunto de dados no qual os atributos assumem valores de um conjunto pequeno de números ordinais. Por exemplo, um atributo que pode assumir apenas os valores contidos em $\{-1,0,1\}$, os quais denotam rejeição, neutralidade e aceitação. Pode ser que exista um grupo $i \in \mathbb{N}_{1, k}$ tal que $\mathrm{x}^{j, s}=\mathrm{v}^{i, s}$ para todo $j \in \mathbb{N}_{1, n}$ sujeito a $\mathrm{U}_{i, j}>0$. Nesse caso, tem-se

$$
\sum_{j=1}^{n}\left(\mathrm{U}_{i, j}\right)^{m} \cdot \mathrm{D}_{i, j}^{s}=\sum_{j=1}^{n}\left(\mathrm{U}_{i, j}\right)^{m} \cdot\left\|\mathrm{x}^{j, s}-\mathrm{v}^{i, s}\right\|_{\mathrm{e}}^{2}=0
$$

e o passo de otimização dado pela Eq. 2.24) simplesmente falharia devido à divisão por zero. Uma observação similar pode ser feita para o passo de otimização de U. Sempre quando a fórmula $\sum_{s=1}^{p}\left(\mathrm{Y}_{i, s}\right)^{q} \cdot \mathrm{D}_{i, j}^{s}$ assume o valor zero, o passo de otimização dado pela Eq. 2.25) falha. A modificação proposta para o algoritmo SCAD não sofre de tais problemas porque as fórmulas dos seus passos foram rigorosamente deduzidas. O Algoritmo 3.1 fornece uma visão geral do algoritmo ASCAD e seus passos são detalhados a seguir.

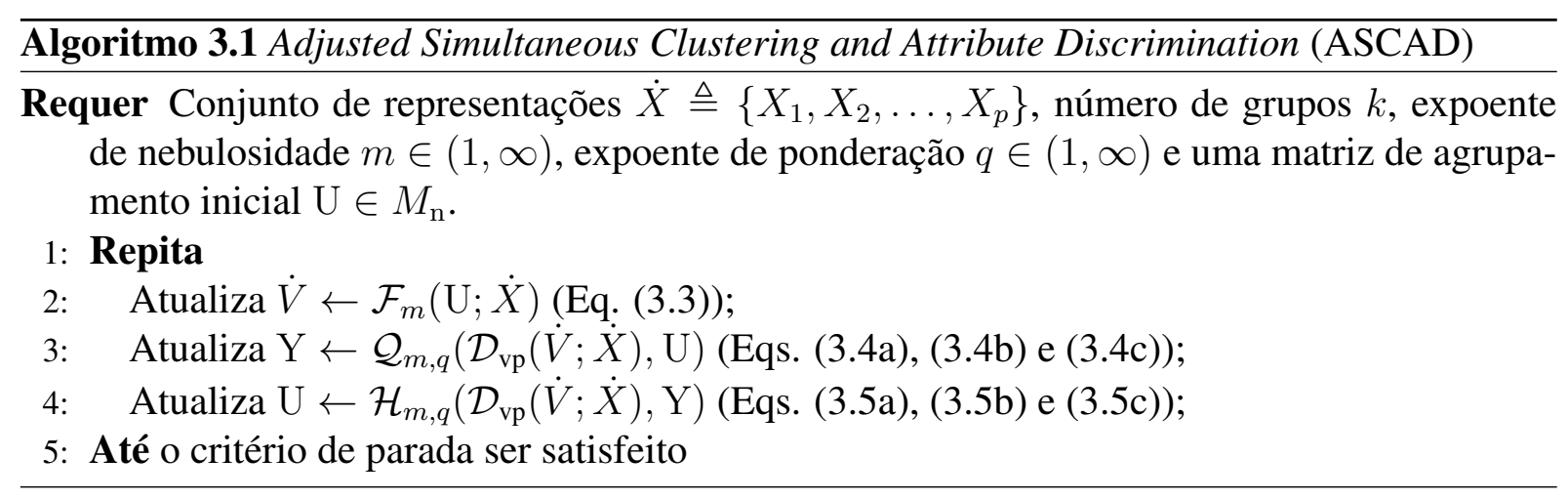

O Passo 2 do Algoritmo 3.1 é idêntico ao Passo 5 do Algoritmo 2.1. Entretanto, é provado 
no Teorema 3.3 que os valores de $\mathrm{D}_{i, j}^{s}$ (Eq. (2.22) ) não precisam ser gerados a partir da distância euclidiana quadrática. $\mathrm{O}$ algoritmo ASCAD minimiza uma função-custo na qual as distâncias são calculadas pela função $\mathcal{D}_{\mathrm{vp}}: M_{\mathrm{vp}} \mapsto M_{\mathrm{dp}}$, definida como $\mathcal{D}_{\mathrm{vp}}(\dot{V} ; \dot{X}) \triangleq\left\{\mathrm{D}^{s}\right\}_{s=1}^{p} \in M_{\mathrm{dp}}$, $\mathrm{D}^{s} \triangleq\left[\mathrm{D}_{i, j}^{s}\right]\left(\forall s \in \mathbb{N}_{1, p}\right) \mathrm{e}$

$$
\mathrm{D}_{i, j}^{s} \triangleq\left\|\mathrm{x}^{j, s}-\mathrm{v}^{i, s}\right\|_{s}^{2} \quad \forall i, j, s,
$$

em que $\|\cdot\|_{s}$ é uma norma qualquer induzida por produto interno no espaço $\mathbb{R}^{a_{s}}$. Compare $\mathcal{D}_{\text {vp }}$ com a função $\mathcal{D}_{\text {ve }}$ definida pela Eq. (2.22). A função-custo $\mathcal{G}_{m, q}^{\mathrm{vp}}: M_{\mathrm{n}} \times M_{\mathrm{vp}} \times M_{\mathrm{y}} \mapsto \mathbb{R}$ é definida por

$$
\begin{aligned}
\mathcal{G}_{m, q}^{\mathrm{vp}}(\mathrm{U}, \dot{V}, \mathrm{Y} ; \dot{X}) & \triangleq \mathcal{G}_{m, q}\left(\mathrm{U}, \mathcal{D}_{\mathrm{vp}}(\dot{V} ; \dot{X}), \mathrm{Y}\right) \\
& =\sum_{i=1}^{k} \sum_{j=1}^{n}\left(\mathrm{U}_{i, j}\right)^{m} \cdot \sum_{s=1}^{p}\left(\mathrm{Y}_{i, s}\right)^{q} \cdot\left\|\mathrm{x}^{j, s}-\mathrm{v}^{i, s}\right\|_{s}^{2},
\end{aligned}
$$

para $q \in(1, \infty)$ e $m \in[1, \infty)$. Compare $\mathcal{G}_{m, q}^{\mathrm{vp}}$ com a função $\mathcal{G}_{m, q}^{\mathrm{ve}}$ definida pela Eq. (2.23). Repare que as normas $\|\cdot\|_{s}$ podem ser únicas para cada representação $s \in \mathbb{N}_{1, p}$ da base de dados, resultando em diferentes distâncias. Mantendo as variáveis U e Y constantes, o mínimo global da função $\mathcal{G}_{m, q}^{\mathrm{vp}}$ é dado pela função $\mathcal{F}_{m}: M_{\mathrm{p}} \mapsto M_{\mathrm{vp}}$, definida por $\mathcal{F}_{m}(\mathrm{U} ; \dot{X}) \triangleq \dot{V}^{*} \triangleq$ $\left\{V_{1}^{*}, V_{2}^{*}, \ldots, V_{k}^{*}\right\}$ em que $V_{i}^{*}=\left\{\mathrm{v}^{i, 1 *}, \mathrm{v}^{i, 2 *}, \ldots, \mathrm{v}^{i, p *}\right\} \mathrm{e}$

$$
\mathrm{v}^{i, s *} \triangleq \sum_{j=1}^{n}\left(\mathrm{U}_{i, j}\right)^{m} \cdot \mathrm{x}^{j, s} / \sum_{j=1}^{n}\left(\mathrm{U}_{i, j}\right)^{m} \quad \forall i \in \mathbb{N}_{1, k}, \forall s \in \mathbb{N}_{1, p} \text {. }
$$

No Passo 3 , as variáveis U e $\dot{V}$ permanecem constantes e a matriz de ponderação Y é atualizada por meio da função $\mathcal{Q}_{m, q}: M_{\mathrm{dp}} \times M_{\mathrm{p}} \mapsto M_{\mathrm{y}}$, a qual é definida como $\mathcal{Q}_{m, q}(D, \mathrm{U}) \triangleq$ $\mathrm{Y}^{*} \triangleq\left[\mathrm{Y}_{i, s}^{*}\right] \mathrm{e}$

$$
\mathrm{Y}_{i, s}^{*} \triangleq\left(\sum_{l=1}^{p}\left(\frac{\sum_{j=1}^{n}\left(\mathrm{U}_{i, j}\right)^{m} \cdot \mathrm{D}_{i, j}^{s}}{\sum_{j=1}^{n}\left(\mathrm{U}_{i, j}\right)^{m} \cdot \mathrm{D}_{i, j}^{l}}\right)^{\frac{1}{q-1}}\right)^{-1}
$$

para todo par $(i, s) \in \mathbb{N}_{1, k} \times \mathbb{N}_{1, p}$ tal que $\sum_{j=1}^{n}\left(\mathrm{U}_{i, j}\right)^{m} \cdot \mathrm{D}_{i, j}^{l}>0 \quad \forall l \in \mathbb{N}_{1, p}$. Quando o par $(i, s)$ não possui essa propriedade, a Eq. (3.4a) não é bem definida para os termos $\mathrm{Y}_{i, 1}^{*}, \mathrm{Y}_{i, 2}^{*}, \ldots, \mathrm{Y}_{i, p}^{*}$. Esses termos são definidos da seguinte forma:

$$
\begin{array}{ll}
\mathrm{Y}_{i, s}^{*} \triangleq 0 & \text { para } \sum_{j=1}^{n}\left(\mathrm{U}_{i, j}\right)^{m} \cdot \mathrm{D}_{i, j}^{s}>0, \\
\mathrm{Y}_{i, s}^{*} \triangleq 1 / z_{i} & \text { para } \sum_{j=1}^{n}\left(\mathrm{U}_{i, j}\right)^{m} \cdot \mathrm{D}_{i, j}^{s}=0,
\end{array}
$$

em que $z_{i}$ é o número de representações que faz a fórmula $\sum_{j=1}^{n}\left(\mathrm{U}_{i, j}\right)^{m} \cdot \mathrm{D}_{i, j}^{l}$ igualar-se a zero. Formalmente,

$$
z_{i} \triangleq\left|\left\{l \in \mathbb{N}_{1, p} \mid \sum_{j=1}^{n}\left(\mathrm{U}_{i, j}\right)^{m} \cdot \mathrm{D}_{i, j}^{l}=0\right\}\right|,
$$

na qual $|\cdot|$ é a cardinalidade de conjunto. A atualização de Y provida pela função $\mathcal{Q}_{m, q}$ fornece 
um mínimo global para a função-custo $\mathcal{G}_{m, q}($ Função 2.20$)$ e, assim, para a função-custo $\mathcal{G}_{m, q}^{\mathrm{vp}}$, mantendo as variáveis $\mathrm{U}$ e $\dot{V}$ constantes e assumindo $D=\mathcal{D}_{\mathrm{vp}}(\dot{V} ; \dot{X})$ (Teorema 3.1).

Por fim, o Passo 4 do algoritmo ASCAD consiste na minimização de $\mathcal{G}_{m, q}$ e, portanto, de $\mathcal{G}_{m, q}^{\text {vp }}$ com respeito à matriz de agrupamento $\mathrm{U}$, enquanto $\mathrm{Y}$ e $\dot{V}$ são mantidas constantes e assumindo $D=\mathcal{D}_{\mathrm{vp}}(\dot{V} ; \dot{X})$ (Teorema 3.2). Considere a função $\mathcal{H}_{m, q}: M_{\mathrm{dp}} \times M_{\mathrm{y}} \mapsto M_{\mathrm{n}}$, definida como $\mathcal{H}_{m, q}(D, \mathrm{Y}) \triangleq \mathrm{U}^{*} \triangleq\left[\mathrm{U}_{i, j}^{*}\right] \mathrm{e}$

$$
\mathrm{U}_{i, j}^{*} \triangleq\left(\sum_{l=1}^{k}\left(\frac{\sum_{s=1}^{p}\left(\mathrm{Y}_{i, s}\right)^{q} \cdot \mathrm{D}_{i, j}^{s}}{\sum_{s=1}^{p}\left(\mathrm{Y}_{l, s}\right)^{q} \cdot \mathrm{D}_{l, j}^{s}}\right)^{\frac{1}{m-1}}\right)^{-1}
$$

para todo par $(i, j) \in \mathbb{N}_{1, k} \times \mathbb{N}_{1, n}$ tal que $\sum_{s=1}^{p}\left(\mathrm{Y}_{l, j}\right)^{q} \cdot \mathrm{D}_{l, j}^{s}>0 \quad \forall l \in \mathbb{N}_{1, k}$. Quando o par $(i, j)$ não satisfaz essa propriedade, a Eq. 3.5a não é bem definida para os termos $\mathrm{U}_{1, j}^{*}, \mathrm{U}_{2, j}^{*}, \ldots, \mathrm{U}_{k, j}^{*}$. Esses termos são definidos como se segue:

$$
\begin{array}{ll}
\mathrm{U}_{i, j}^{*} \triangleq 0 & \text { para } \sum_{s=1}^{p}\left(\mathrm{Y}_{i, s}\right)^{q} \cdot \mathrm{D}_{i, j}^{s}>0 \\
\mathrm{U}_{i, j}^{*} \triangleq 1 / z_{j} & \text { para } \sum_{s=1}^{p}\left(\mathrm{Y}_{i, s}\right)^{q} \cdot \mathrm{D}_{i, j}^{s}=0
\end{array}
$$

em que $z_{j}$ é o número de grupos que faz a fórmula $\sum_{s=1}^{p}\left(\mathrm{Y}_{l, s}\right)^{q} \cdot \mathrm{D}_{l, j}^{s}$ igualar-se a zero. Formalmente,

$$
z_{j} \triangleq\left|\left\{l \in \mathbb{N}_{1, k} \mid \sum_{s=1}^{p}\left(\mathrm{Y}_{l, s}\right)^{q} \cdot \mathrm{D}_{l, j}^{s}=0\right\}\right|
$$

Na próxima seção propõe-se uma versão do algoritmo ASCAD para agrupamento particional exclusivo.

\subsubsection{Versão rígida}

Uma versão particional exclusiva do algoritmo ASCAD é proposta, referida por Hard ASCAD ( $($ HASCAD $)$. Nesse contexto, um objeto $\tilde{o}_{j}$ pertence ou não pertence ao $i$-ésimo grupo e as matrizes de agrupamento pertencem ao conjunto $M_{\mathrm{e}}$. A função-custo $\mathcal{G}_{1, q}^{\mathrm{vph}}: M_{\mathrm{e}} \times M_{\mathrm{vp}} \times M_{\mathrm{y}} \mapsto$ $\mathbb{R}$ do algoritmo HASCADé definida por

$$
\begin{aligned}
\mathcal{G}_{q}^{\mathrm{vph}}(\mathrm{U}, \dot{V}, \mathrm{Y} ; \dot{X}) & \triangleq \mathcal{G}_{1, q}^{\mathrm{vp}}(\mathrm{U}, \dot{V}, \mathrm{Y} ; \dot{X}) \\
& =\mathcal{G}_{1, q}\left(\mathrm{U}, \mathcal{D}_{\mathrm{vp}}(\dot{V} ; \dot{X}), \mathrm{Y}\right) \\
& =\sum_{i=1}^{k} \sum_{j=1}^{n} \mathrm{U}_{i, j} \cdot \sum_{s=1}^{p}\left(\mathrm{Y}_{i, s}\right)^{q} \cdot\left\|\mathrm{x}^{j, s}-\mathrm{v}^{i, s}\right\|_{s}^{2},
\end{aligned}
$$

para $q \in(1, \infty)$. Observe que a Função 3.6 pode ser considerada uma instância da Função (3.2), na qual o expoente $m$ é definido como 1. Essa função-custo é muito semelhante à do algoritmo k-Means. Elas se diferem, essencialmente, na maneira como o custo correspondente à associação de um objeto $\tilde{o}_{j}$ a um grupo (digamos, ao $i$-ésimo grupo) é definido; enquanto 
o algoritmo k-Means define esse custo como $\left\|\mathrm{x}^{j}-\mathrm{v}^{i}\right\|^{2}$, o algoritmo HASCAD define como $\sum_{s=1}^{p}\left(\mathrm{Y}_{i, s}\right)^{q} \cdot\left\|\mathrm{x}^{j, s}-\mathrm{v}^{i, s}\right\|_{s}^{2}$.

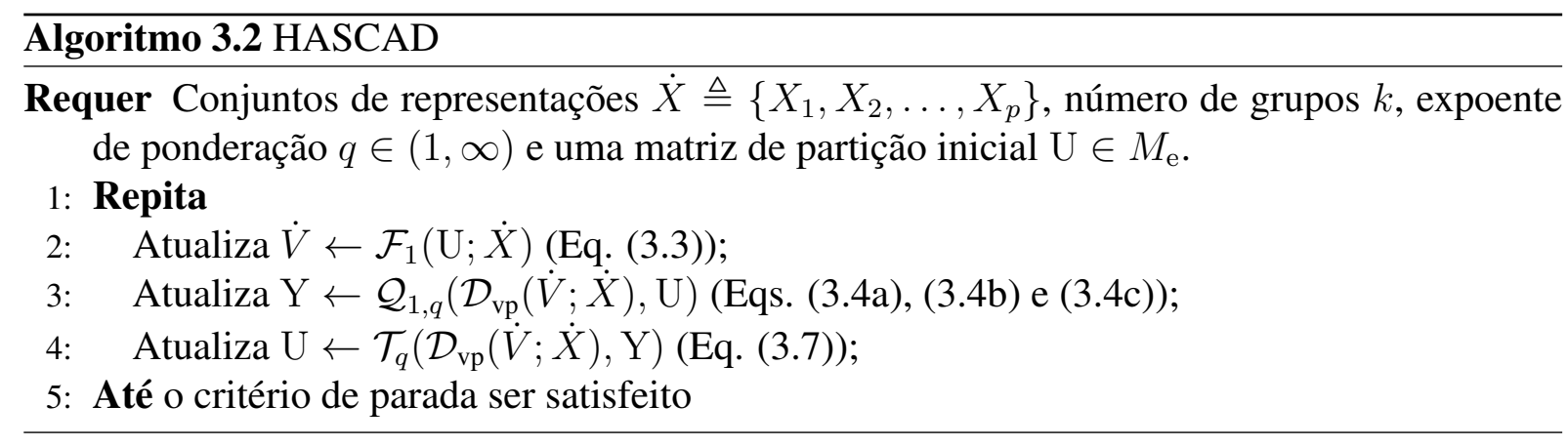

O algoritmo HASCAD também minimiza a sua função-custo ao otimizar alternadamente as variáveis. O Algoritmo 3.2 apresenta em linhas gerais o algoritmo HASCAD. Os Passos $2 \mathrm{e} 3 \mathrm{do}$ Algoritmo 3.1 são idênticos aos Passos 2 e 3 do Algoritmo 3.2, exceto pela configuração $m=$ 1. O Passo 4, por outro lado, difere substancialmente do passo correspondente do algoritmo ASCAD, Seja $\mathcal{T}_{q}: D_{\mathrm{dp}} \times M_{\mathrm{y}} \mapsto M_{\mathrm{e}}$ uma função definida por $\mathcal{T}_{q}(D, \mathrm{Y}) \triangleq \mathrm{U}^{*} \triangleq\left[\mathrm{U}_{i, j}^{*}\right] \mathrm{e}$

$$
\mathrm{U}_{i, j}^{*} \triangleq\left\{\begin{array}{cc}
1 & \text { se } \sum_{s=1}^{p}\left(\mathrm{Y}_{i, s}\right)^{q} \cdot \mathrm{D}_{i, j}^{s}=\min _{l \in \mathbb{N}_{1, k}}\left\{\sum_{s=1}^{p}\left(\mathrm{Y}_{l, s}\right)^{q} \cdot \mathrm{D}_{l, j}^{s}\right\} \\
0 & \text { se } \sum_{s=1}^{p}\left(\mathrm{Y}_{i, s}\right)^{q} \cdot \mathrm{D}_{i, j}^{s}>\min _{l \in \mathbb{N}_{1, k}}\left\{\sum_{s=1}^{p}\left(\mathrm{Y}_{l, s}\right)^{q} \cdot \mathrm{D}_{l, j}^{s}\right\}
\end{array}\right.
$$

Em palavras, para minimizar $\mathcal{G}_{q}^{\mathrm{vph}}$ com relação à matriz $\mathrm{U}$, o algoritmo simplesmente associa os objetos de $O$ aos grupos mais próximos, de acordo com $\sum_{s=1}^{p}\left(\mathrm{Y}_{i, s}\right)^{q} \cdot \mathrm{D}_{i, j}^{s}$. Se um empate ocorre (i.e., se um objeto possui a mesma distância para mais de um grupo e essa distância é a menor possível), o algoritmo simplesmente associa aleatoriamente o objeto responsável a um dos grupos mais próximos. Decompondo o lado direito da Eq. (3.6) em $n$ termos

$$
\sum_{i=1}^{k} \mathrm{U}_{i, j} \cdot \sum_{s=1}^{p}\left(\mathrm{Y}_{i, s}\right)^{q} \cdot\left\|\mathrm{x}^{j, s}-\mathrm{v}^{i, s}\right\|_{s}^{2}=\sum_{i=1}^{k} \mathrm{U}_{i, j} \cdot \sum_{s=1}^{p}\left(\mathrm{Y}_{i, s}\right)^{q} \cdot \mathrm{D}_{i, j}^{s} \quad j=1,2, \ldots, n,
$$

é simples verificar que $\mathcal{T}_{q}$ realmente provê um mínimo global para $\mathcal{G}_{q}^{\mathrm{vph}}$, mantendo Y e $\dot{V}$ constantes!

\subsubsection{Complexidade computacional}

A análise da complexidade do algoritmo ASCAD (Algoritmo 3.1) pode ser decomposta em três partes: uma para cada passo. O Passo 2 calcula os centróides por meio da Eq. (3.3), a qual pode ser computada da seguinte forma. Calcule $\sum_{j=1}^{n}\left(\mathrm{U}_{i, j}\right)^{m}$ para todo $i \in \mathbb{N}_{1, k}$ em $\mathcal{O}(k n)$, no qual $k$ é o número de grupos e $n$ é o número de objetos. Usando esses $k$ valores,

\footnotetext{
${ }^{1}$ Existe a possibilidade de se obter um ou mais grupos vazios. Pela Condição $2.2 \mathrm{~b}$, tem-se uma matriz de agrupamento degenerada. Nesse caso, há indícios de que a base de dados possui menos grupos do que o configurado pelo usuário. Portanto, uma estratégia simples para lidar com essa situação é a remoção dos grupos vazios, permitindo que o algoritmo prossiga com os grupos remanescentes e uma matriz de agrupamento não degenerada.
} 
calcule o centróide $\mathrm{v}^{i, s *}$ para todo $i \in \mathbb{N}_{1, k}$ e $s \in \mathbb{N}_{1, p}$ em $\mathcal{O}(k n p)$, no qual $p$ é o número de representações. Portanto, o Passo 2 possui complexidade computacional de $\mathcal{O}(k n+k n p)=$ $\mathcal{O}(k n p)$.

O Passo 3 consiste no cômputo de $\mathrm{Y}_{i, s}^{*}$ por meio das Eqs. 3.4a), 3.4b) e 3.4c). Para o pior caso, o Passo 3 precisa calcular a Eq. (3.4a) para todo $i \in \mathbb{N}_{1, k}$ e $s \in \mathbb{N}_{1, p}$. A Eq. (3.4a) pode ser escrita de forma equivalente como

$$
\mathrm{Y}_{i, s}^{*}=\left(\left(\sum_{j=1}^{n}\left(\mathrm{U}_{i, j}\right)^{m} \cdot \mathrm{D}_{i, j}^{s}\right)^{\frac{1}{q-1}} \cdot \sum_{l=1}^{p}\left(\sum_{j=1}^{n}\left(\mathrm{U}_{i, j}\right)^{m} \cdot \mathrm{D}_{i, j}^{l}\right)^{\frac{1}{1-q}}\right)^{-1}
$$

Pode-se calcular a equação acima como se segue. Seja $a_{s}$ o número de atributos da $s$-ésima representação e assuma que o cômputo de $\mathrm{D}_{i, j}^{s}$ demanda $\mathcal{O}\left(a_{s}\right)$ de tempo computacional (a mesma complexidade computacional para o cálculo da distância euclidiana). Calcule

$$
\sum_{l=1}^{p}\left(\sum_{j=1}^{n}\left(\mathrm{U}_{i, j}\right)^{m} \cdot \mathrm{D}_{i, j}^{l}\right)^{\frac{1}{1-q}} \quad \forall i \in \mathbb{N}_{1, k}
$$

em $\mathcal{O}\left(k n \sum_{s=1}^{p} a_{s}\right)$. Use esses valores para calcular $\mathrm{Y}_{i, s}^{*}$ em $\mathcal{O}\left(n a_{s}\right)$, para cada $i \in \mathbb{N}_{1, k}$ e $s \in \mathbb{N}_{1, p}$. Logo, o cálculo do Passo 3 demanda $\mathcal{O}\left(k n \sum_{s=1}^{p} a_{s}\right)$ de tempo computacional.

O Passo 4 usa as Eqs. 3.5a), 3.5b) e (3.5c) para calcular a matriz de agrupamento U*. Para o pior caso, o Passo 4 precisa computar a Eq. (3.5a) para cada $i \in \mathbb{N}_{1, k}$ e $j \in \mathbb{N}_{1, n}$. Similarmente à Eq. 3.4a), a Eq. 3.5a pode ser equivalentemente descrita como

$$
\mathrm{U}_{i, j}^{*}=\left(\left(\sum_{s=1}^{p}\left(\mathrm{Y}_{i, s}\right)^{q} \cdot \mathrm{D}_{i, j}^{s}\right)^{\frac{1}{m-1}} \cdot \sum_{l=1}^{k}\left(\sum_{s=1}^{p}\left(\mathrm{Y}_{l, s}\right)^{q} \cdot \mathrm{D}_{l, j}^{s}\right)^{\frac{1}{1-m}}\right)^{-1} .
$$

Seja novamente $a_{s}$ o número de atributos da $s$-ésima representação e assuma que o cálculo de $\mathrm{D}_{i, j}^{s}$ demanda $\mathcal{O}\left(a_{s}\right)$ (como, por exemplo, a distância Euclidiana) de tempo computacional. Calcule

$$
\sum_{l=1}^{k}\left(\sum_{s=1}^{p}\left(\mathrm{Y}_{l, s}\right)^{q} \cdot \mathrm{D}_{l, j}^{s}\right)^{\frac{1}{1-m}} \quad \forall j \in \mathbb{N}_{1, n}
$$

em $\mathcal{O}\left(k n \sum_{s=1}^{p} a_{s}\right)$. Use esses valores para calcular $\mathrm{U}_{i, j}^{*}$ em $\mathcal{O}\left(\sum_{s=1}^{p} a_{s}\right)$, para cada $i \in \mathbb{N}_{1, k} \mathrm{e}$ $j \in \mathbb{N}_{1, n}$. Logo, o cômputo do Passo 4 demanda $\mathcal{O}\left(k n \sum_{s=1}^{p} a_{s}\right)$ de tempo computacional.

Seja $t$ o número de iterações. Conclui-se que o algoritmo ASCAD demanda $\mathcal{O}(t(k n p+$ $\left.\left.k n \sum_{s=1}^{p} a_{s}+k n \sum_{s=1}^{p} a_{s}\right)\right)=\mathcal{O}\left(t k n \sum_{s=1}^{p} a_{s}\right)$ de tempo computacional. O algoritmo FCM possui complexidade assintótica de $\mathcal{O}(t k n a)$ (Kolen e Hutcheson, 2002), na qual $a$ é o número de atributos da base de dados. No caso do conjunto de atributos descrevendo a base de dados provida ao FCM ser apenas a união dos conjuntos disjuntos de atributos descrevendo as representações da base de dados provida ao algoritmo $\operatorname{ASCAD~(i.e.,~} a=\sum_{s=1}^{p} a_{s}$ ), pode-se dizer 
que ambos os algoritmos tem a mesma complexidade assintótica.

\subsubsection{Análise da função $\mathcal{Q}_{m, q}$ e do expoente de ponderação}

Seja $\hat{d}_{i, l} \triangleq \sum_{j=1}^{n}\left(\mathrm{U}_{i, j}\right)^{m} \cdot \mathrm{D}_{i, j}^{l}$ a soma ponderada das distâncias dos objetos ao $i$-ésimo grupo com relação à $l$-ésima representação. A Eq. 3.4a, então, pode ser reescrita como

$$
\mathrm{Y}_{i, s}^{*}=1 /\left(\left(\frac{\hat{d}_{i, s}}{\hat{d}_{i, 1}}\right)^{\frac{1}{q-1}}+\cdots+\left(\frac{\hat{d}_{i, s}}{\hat{d}_{i, s-1}}\right)^{\frac{1}{q-1}}+1+\left(\frac{\hat{d}_{i, s}}{\hat{d}_{i, s+1}}\right)^{\frac{1}{q-1}}+\cdots+\left(\frac{\hat{d}_{i, s}}{\hat{d}_{i, p}}\right)^{\frac{1}{q-1}}\right) .
$$

Considere a atualização dos pesos de ponderação para o $i$-ésimo grupo. Na maioria dos casos, espera-se ter $\hat{d}_{i, l}>0 \quad \forall l \in \mathbb{N}_{1, p}$. Nesses casos, os elementos da $i$-ésima linha de $\mathrm{Y}^{*}$ são computados exclusivamente por meio da Eq. 3.8. Portanto, quanto menor o valor $\hat{d}_{i, s}$ da soma ponderada das distâncias dos objetos ao $i$-ésimo grupo, com relação à $s$-ésima representação, menores são as frações $\left(\hat{d}_{i, s} / \hat{d}_{i, l}\right)^{1 /(q-1)} \quad \forall l \neq s$ da Eq. 3.8 e, portanto, mais próximo de 1 será o valor de $\mathrm{Y}_{i, s}^{*}$. Contrariamente, quanto maior o valor de $\hat{d}_{i, s}$, mais próximo de 0 será o valor de $\mathrm{Y}_{i, s}^{*}$. Conclui-se que a etapa de otimização de $\mathrm{Y}$, para um dado grupo, atribui maior importância às representações que avaliam os objetos como estando próximos do grupo correspondente. O mesmo ocorre para o caso em que existe $l$ tal que $\hat{d}_{i, l}=0$. Nesse contexto, a otimização fornecida pela função $\mathcal{Q}_{m, q}$ distribui igualmente os pesos de relevância às representações que anulam a soma das distâncias dos objetos ao $i$-ésimo grupo (Eq. (3.4c)), enquanto atribui importância nula às outras representações (Eq. (3.4b)).

Quanto à escolha do valor do expoente $q$, considere, novamente, a atualização dos pesos de ponderação para o $i$-ésimo grupo e o caso no qual $\hat{d}_{i, l}>0 \quad \forall l \in \mathbb{N}_{1, p}$. Sejam $d \triangleq \min _{l \in\{1, \ldots, p\}}\left\{\hat{d}_{i, l}\right\}$ e $n_{\text {min }}$ a quantidade de $l \in \mathbb{N}_{1, p}$ tais que $\hat{d}_{i, l}=d$. Quando $q$ tende a 1 pela direita, os termos $\left(\hat{d}_{i, s} / \hat{d}_{i, l}\right)^{1 /(q-1)}$ da Eq. 3.8 cujas frações $\hat{d}_{i, s} / \hat{d}_{i, l}$ são menores que 1 tendem a zero, enquanto os termos $\left(\hat{d}_{i, s} / \hat{d}_{i, l}\right)^{1 /(q-1)}$ cujas frações $\hat{d}_{i, s} / \hat{d}_{i, l}$ são maiores que 1 tendem ao infinito. Portanto, ter-se-á $\mathrm{Y}_{i, s}^{*} \approx 0$ para $\hat{d}_{i, s}>d$ e $\mathrm{Y}_{i, s}^{*} \approx 1 / n_{\min }$ para $\hat{d}_{i, s}=d$. Contrariamente, quando $q$ tende ao infinito, os termos $\left(\hat{d}_{i, s} / \hat{d}_{i, l}\right)^{1 /(q-1)}$ da Eq. 3.8 tendem a 1. Portanto, ter-se-á $\mathrm{Y}_{i, s}^{*} \approx 1 / p$ para todo $s \in \mathbb{N}_{1, p}$. Logo, a finalidade do expoente $q$ é a de regular o grau de ponderação das representações. Quanto menor o valor de $q$, mais drástica é a ponderação das representações efetuada pela função $\mathcal{Q}_{m, q}$. Contrariamente, quanto maior o valor de $q$, mais branda é a ponderação das representações.

\subsection{Versão relacional do SCAD ajustado}

Considere uma base de dados $O$ de $n$ objetos e sejam $R \triangleq\left\{\mathrm{R}^{s}\right\}_{s=1}^{p}, \mathrm{R}^{s} \triangleq\left[\mathrm{R}_{j, l}^{s}\right] \in \mathbb{R}^{n \cdot n}$ para todo $s \in \mathbb{N}_{1, p}, \dot{X} \triangleq\left\{X^{s}\right\}_{s=1}^{p}$ e $X^{s} \triangleq\left\{\mathrm{x}^{j, s}\right\}_{j=1}^{n} \subset \mathbb{R}^{a_{s}}$ para todo $s \in \mathbb{N}_{1, p}$. Diz-se que $\dot{X}$ é uma realização de $\mathrm{R}$ se, e somente se, $\mathrm{R}_{j, l}^{s} \triangleq\left\|\mathrm{x}^{j, s}-\mathrm{x}^{l, s}\right\|_{s}^{2}$ para todo $s, j, l$, em que $\|\cdot\|_{s}$ pode ser qualquer norma induzida por produto interno do espaço $\mathbb{R}^{a_{s}}$. Será apresentada agora a relação entre a função-custo do algoritmo CARD (Eq. 2.27) e a função-custo do algoritmo 
ASCAD (Eq. (3.2)). Suponha que $\dot{X}$ seja uma realização de $R$. Sejam $\dot{V} \triangleq\left\{V_{1}, V_{2}, \ldots, V_{p}\right\} \in$ $M_{\mathrm{vp}}$ (Eq. (2.21) o conjunto contendo conjuntos de protótipos tais que $V_{s} \triangleq\left\{\mathrm{v}^{i, s}\right\}_{i=1}^{k}$ para todo $s \in \mathbb{N}_{1, p}$ e $\mathrm{U} \in M_{\mathrm{n}}$ uma matriz de agrupamento nebuloso. Quando os protótipos em $\dot{V}$ são centróides (Eq. (3.3) ) dos seus respectivos grupos e representações dos dados, tem-se $\mathcal{K}_{m, q}(\mathrm{U}, \mathrm{Y} ; R)=\mathcal{G}_{m, q}^{\mathrm{vp}}(\mathrm{U}, \dot{V}, \mathrm{Y} ; \dot{X})$ (Teorema 3.4). Consequentemente, não existe diferença (a não ser por motivos práticos) entre minimizar $\mathcal{K}_{m, q}(\mathrm{U}, \mathrm{Y} ; R)$ ou $\mathcal{G}_{m, q}^{\mathrm{vp}}\left(\mathrm{U}, \mathcal{F}_{m}(\mathrm{U} ; \dot{X}), \mathrm{Y} ; \dot{X}\right)$, em que $\mathcal{F}_{m}$ é a função que calcula os centróides (Eq. (3.3)).

Como já mencionado na Seção 3.1, a função $\mathcal{F}_{m}$ fornece um mínimo global de $\mathcal{G}_{m, q}^{\mathrm{vp}}$ quando as variáveis U e Y são mantidas constantes (Teorema 3.3). Logo, por meio da estratégia de otimizar as variáveis alternadamente, pode-se usar a função $\mathcal{K}_{m, q}$ para minimizar a função $\mathcal{G}_{m, q}^{\mathrm{vp}}$ e vice-versa. Frigui et al. (2007) assumiram (sem provar) que essa relação entre as equações existia e, a partir dela, desenvolveram o algoritmo CARD, os autores ora partiam da função $\mathcal{K}_{m, q}$ para deduzir as equações de otimização, ora partiam da função $\mathcal{G}_{m, q}^{\mathrm{vp}}$. Contrariamente, o algoritmo RASCAD proposto nesta seção foi deduzido a partir das funções $\mathcal{G}_{m, q}^{\mathrm{vp}}$ e $\mathcal{G}_{m, q}$.

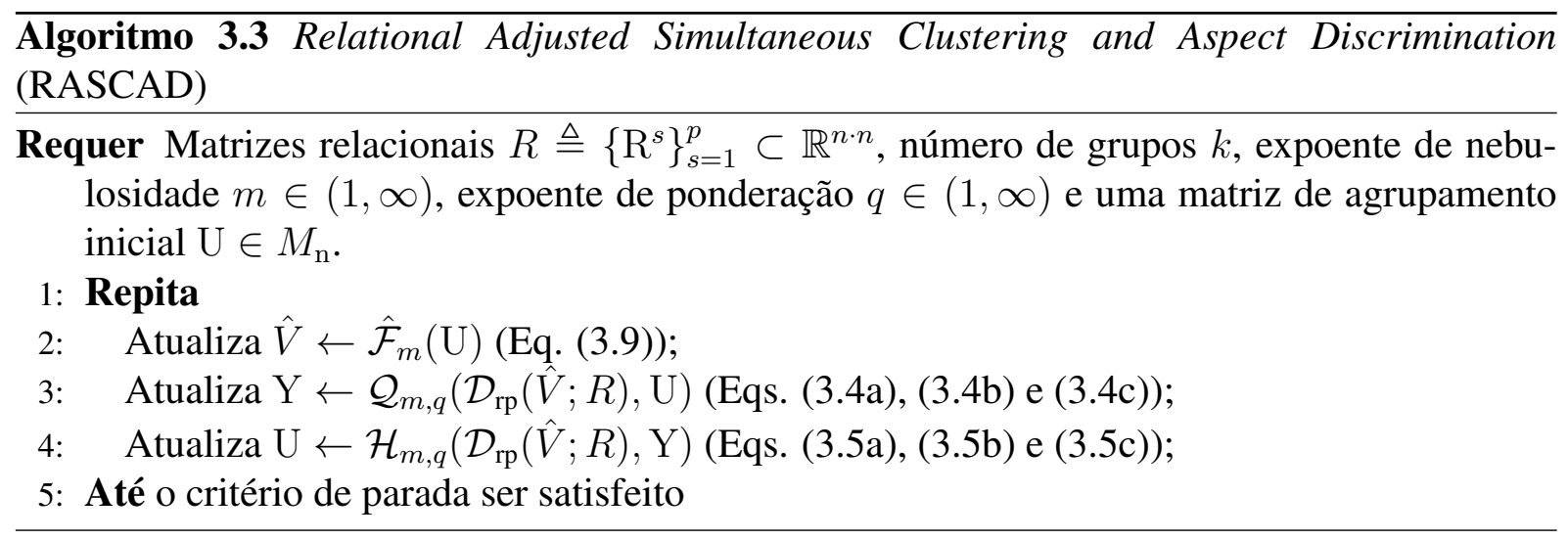

O Algoritmo 3.3 apresenta uma visão geral do algoritmo RASCAD O Passo 2, presente também no algoritmo CARD, faz uso da função $\hat{\mathcal{F}}_{m}(\mathrm{U}): M_{\mathrm{n}} \mapsto M_{\mathrm{v}}(n)$ (Eq. (2.28)), em que $\hat{\mathcal{F}}_{m}(\mathrm{U}) \triangleq \hat{V}^{*} \triangleq\left\{\hat{\mathrm{V}}^{i *}\right\}_{i=1}^{k} \subset \mathbb{R}^{n} \mathrm{e}$

$$
\hat{\mathrm{v}}^{i *} \triangleq\left[\begin{array}{llll}
\left(\mathrm{U}_{i, 1}\right)^{m} & \left(\mathrm{U}_{i, 2}\right)^{m} & \ldots & \left(\mathrm{U}_{i, n}\right)^{m}
\end{array}\right]^{\mathrm{T}} \frac{1}{\sum_{j=1}^{n}\left(\mathrm{U}_{i, j}\right)^{m}} \quad \forall i \in \mathbb{N}_{1, k},
$$

para calcular as distâncias $\mathrm{D}_{i, j}^{s}$ usadas pelos Passos 3 e 4 (explicação mais adiante). Os pontos definidos pela Eq. (3.9) são chamados de centróides relacionais (Hathaway e Bezdek, 1994). Seja $\dot{X}$ uma realização de $R$ e $\mathrm{U} \in M_{\mathrm{n}}$ uma matriz de agrupamento da base de dados em questão. É verdade que

$$
\left\|\mathrm{x}^{j, s}-\mathrm{v}^{i, s *}\right\|_{s}^{2}=\left(\mathrm{R}^{s} \cdot \hat{\mathrm{v}}^{i *}\right)_{j}-\left(\hat{\mathrm{v}}^{i *}\right)^{\mathrm{T}} \cdot \mathrm{R}^{s} \cdot \hat{\mathrm{v}}^{i *} / 2
$$

na qual os centróides $\mathrm{v}^{i, s *}$ e $\hat{\mathrm{v}}^{i *}$ são dados pelas Eqs. (3.3) e 3.9), respectivamente (Hathaway e Bezdek, 1994). Em palavras, as distâncias $\left\|\mathrm{x}^{j, s}-\mathrm{v}^{i, s *}\right\|_{s}^{2}$ podem ser calculadas por meio da matriz relacional correspondente e sem o uso dos vetores de atributos.

Seja a função $\mathcal{D}_{\text {rp }}: M_{\mathrm{v}}(n) \mapsto M_{\mathrm{dp}}$ definida como $\mathcal{D}_{\mathrm{rp}}(\hat{V} ; R) \triangleq\left\{\mathrm{D}^{s}\right\}_{s=1}^{p} \in M_{\mathrm{dp}}, \mathrm{D}^{s} \triangleq\left[\mathrm{D}_{i, j}^{s}\right]$ 
$\left(\forall s \in \mathbb{N}_{1, p}\right) \mathrm{e}$

$$
\mathrm{D}_{i, j}^{s} \triangleq\left(\mathrm{R}^{s} \cdot \hat{\mathrm{v}}^{i}\right)_{j}-\left(\hat{\mathrm{v}}^{i}\right)^{\mathrm{T}} \cdot \mathrm{R}^{s} \cdot \hat{\mathrm{v}}^{i} / 2 \quad \forall i, j, s .
$$

A função $\mathcal{D}_{\text {rp }}$ é usada nos Passos 3 e 4 para calcular as distâncias entre os objetos e os grupos, fundamentada no fato expresso pela Eq. (3.10). Nesses dois passos, a função $\mathcal{G}_{m, q}$ e, portanto, a função $\mathcal{G}_{m, q}^{\mathrm{vp}}$ são minimizadas de acordo com as variáveis $\mathrm{Y}$ e $\mathrm{U}$ da mesma forma que ocorre com o Algoritmo 3.1 (Teoremas 3.1 $\mathrm{e} 3.2$ ).

Note que o lado direito das Eqs. 2.30) e (3.11) diferem apenas na matriz relacional usada. Enquanto o uso da matriz relacional $\mathrm{R}^{s}$ na Eq. (3.11) garante o importante fato expresso pela Eq. (3.10), o mesmo não pode ser afirmado sobre a Eq. (2.30). Essa diferença permite a prova da equivalência dos algoritmos ASCAD e RASCAD, Observe que $\mathcal{D}_{\text {rp }}\left(\hat{\mathcal{F}}_{m}(\mathrm{U}) ; R\right)=$ $\mathcal{D}_{\text {vp }}\left(\mathcal{F}_{m}(\mathrm{U} ; \dot{X}) ; \dot{X}\right)$, devido à Eq. (3.10) e assumindo que $\dot{X}$ é uma realização de $R$. Portanto, os Passos 3 e 4 do Algoritmo 3.3 e os Passos 3 e 4 do Algoritmo 3.1 produzem as mesmas matrizes $\mathrm{Y}$ e U, dado que a mesma matriz de partição U foi fornecida ao Passo 2 do Algoritmo 3.3 e ao Passo 2 do Algoritmo 3.1 .

Uma matriz relacional $\mathrm{R}^{s} \in \mathbb{R}^{n \cdot n}$ é euclidiana se, e somente se, existem $a_{s} \in \mathbb{N}_{1, \infty}$ e um conjunto $\left\{\mathrm{x}^{1, s}, \mathrm{x}^{2, s}, \ldots, \mathrm{x}^{n, s}\right\} \subset \mathbb{R}^{a_{s}}$ de pontos tais que $\mathrm{R}_{j, l}^{s}=\left\|\mathrm{x}^{j, s}-\mathrm{x}^{l, s}\right\|_{s}^{2} \forall j, l \in \mathbb{N}_{1, n}$, na qual $\|\cdot\|_{s}$ é qualquer norma induzida por produto interno em $\mathbb{R}^{a_{s}}$ (Mardia et al., 1979). Logo, existe uma realização $\dot{X}$ de $R=\left\{\mathrm{R}^{s}\right\}_{s=1}^{p}$ se, e somente se, as matrizes relacionais de $R$ são euclidianas. Portanto, pode-se dizer que os algoritmos RASCAD e ASCAD são equivalentes quando existe uma realização $\dot{X}$ de $R$, no sentido de que ambos os algoritmos produzem o mesmo resultado quando lhe são fornecidas respectivamente as representações $\dot{X}$ e $R$ e os mesmos parâmetros de entrada.

Os casos particulares das minimizações realizadas pelas funções $\mathcal{Q}_{m, q}$ e $\mathcal{H}_{m, q}$, tratados pelas Eqs. (3.4b), 3.4c), 3.5b) e 3.5c), não foram considerados no desenvolvimento do algoritmo CARD. Como discutido na Seção 2.4.1, são situações importantes e que impedem que o algoritmo CARD seja aplicado em certos cenários.

\subsection{Versão não euclidiana do SCAD ajustado relacional}

Como observado em (Hathaway e Bezdek, 1994) para o algoritmo RFCM, a distância definida pela Eq. (3.11) pode se tornar negativa se a matriz relacional $\mathrm{R}^{s}$ não for euclidiana, i.e., se não existe $X_{s}$ que seja uma realização de $\mathrm{R}^{s}$. Um simples exemplo ilustrando esse comportamento foi dado em (Hathaway e Bezdek, 1994). Para superar esse problema e fazer com que o algoritmo RFCM funcionasse com qualquer matriz relacional $\mathrm{R}^{s}$, Hathaway e Bezdek (1994) propuseram aplicar a transformação de espalhamento $\beta$ na matriz relacional toda vez que uma distância negativa fosse obtida. A implementação desse método no esquema do algoritmo RFCM deu origem ao algoritmo NERFCM. Essa ideia foi usada no desenvolvimento do algoritmo CARD e é adotada aqui também, dando origem ao algoritmo NERASCAD.

Seja $\mathbb{1} \in \mathbb{R}^{n \cdot n}$ uma matriz de apenas uns e $\mathrm{I} \in \mathbb{R}^{n \cdot n}$ a matriz identidade $n$-por- $n$. A 


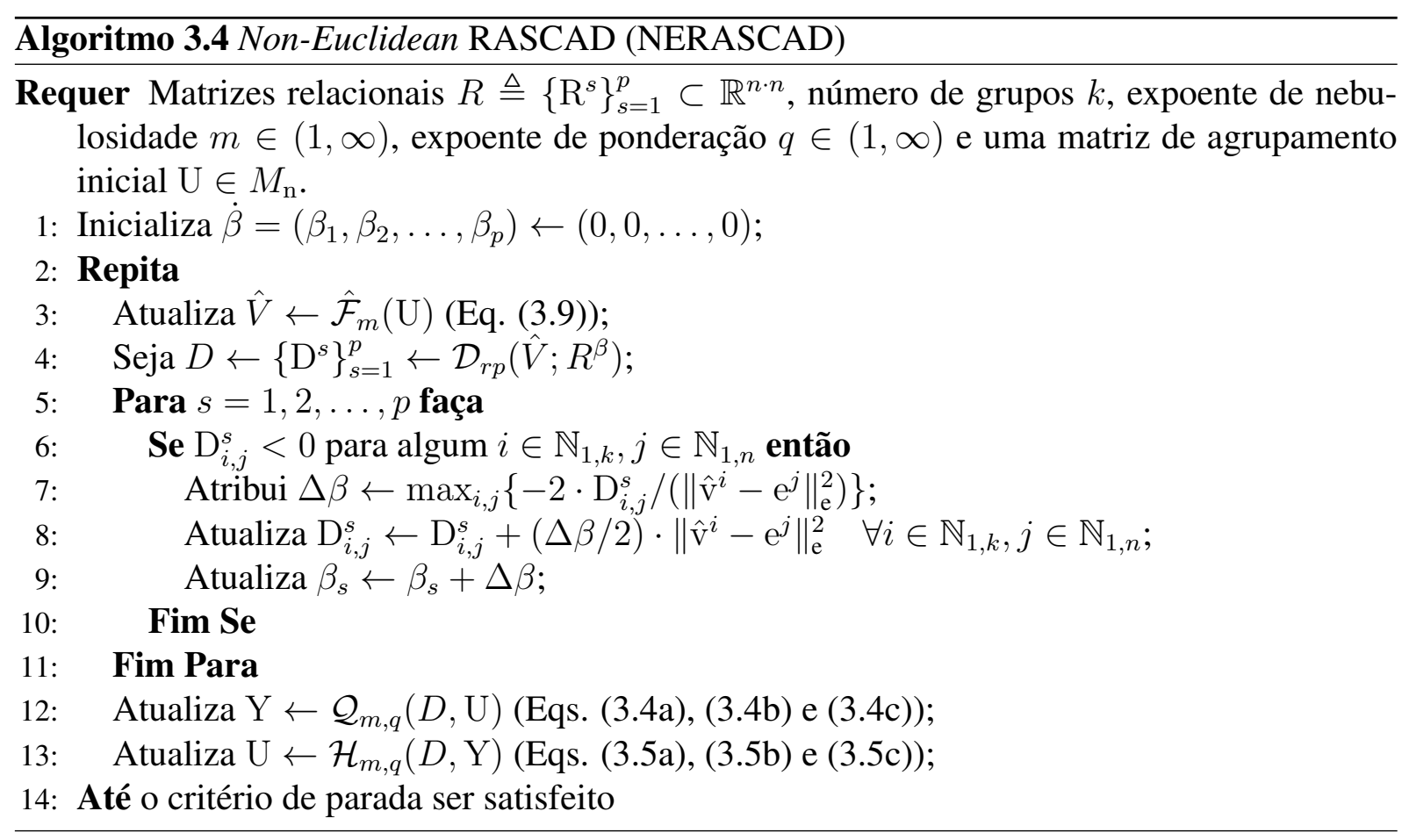

transformação de espalhamento $\beta$ de uma matriz $\mathrm{R}$ é definida como $\mathrm{R}^{\beta} \triangleq \mathrm{R}+\beta \cdot(\mathbb{1}-\mathrm{I})$, o que faz com que $\mathrm{R}$ se aproxime de ou se torne uma matriz relacional euclidiana ${ }^{2}$ A notação $\mathrm{R}^{s, \beta_{s}}$ será usada para denotar a transformação de espalhamento $\beta_{s}$ da matriz relacional $\mathrm{R}^{s}$, de modo que $\mathrm{R}^{s, \beta_{s}} \triangleq \mathrm{R}^{s}+\beta_{s} \cdot(\mathbb{1}-\mathrm{I})$. Portanto, $\mathrm{R}^{s}=\mathrm{R}^{s, 0}\left(\beta_{s}=0\right)$. Será adotado uma $p$ upla $\dot{\beta} \triangleq\left(\beta_{1}, \beta_{2}, \ldots, \beta_{p}\right)$ para se referir às $p$ transformações $\beta_{s}$ das matrizes relacionais de $R=\left\{\mathrm{R}^{s}\right\}_{s=1}^{p}$, de modo que $R^{\dot{\beta}} \triangleq\left\{\mathrm{R}^{s, \beta_{s}}\right\}_{s=1}^{p}$.

O Algoritmo 3.4 fornece uma visão geral do algoritmo NERASCAD Inicialmente, as matrizes relacionais são utilizadas sem transformação alguma. Por isso o algoritmo começa inicializando $\beta_{s}=0$ para todo $s \in \mathbb{N}_{1, p}$. Os Passos 7 e 8 fazem uso de vetores $n$-dimensionais $\mathrm{e}^{j}$

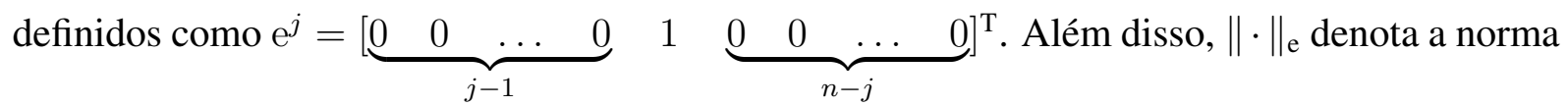
euclidiana para o espaço vetorial $\mathbb{R}^{n}$.

Suponha que a condição do Passo 6 seja satisfeita para algum $s \in \mathbb{N}_{1, p}$. Isto é, suponha que existam valores negativos em $\mathrm{D}^{s}$. Os Passos $7 \mathrm{e} 8$ garantem que todo elemento da matriz $\mathrm{D}^{s}$ será não negativo e que $\mathrm{D}^{s}$ será idêntica à matriz que seria gerada pela Eq. (3.11) se $\mathrm{R}^{s, \beta_{s}}$, com o novo valor $\beta_{s}$ (Passo 9), fosse usada. Esses fatos são provados em (Hathaway e Bezdek, 1994).

Se as matrizes relacionais de $R=\left\{\mathrm{R}^{s}\right\}_{s=1}^{p}$ são euclidianas, a condição do Passo 6 do Algoritmo 3.4 nunca será satisfeita (Hathaway e Bezdek, 1994). Isto é, a Eq. (3.11) de fato fornecerá distâncias entre pontos em algum espaço vetorial. Nesse caso, RASCAD e NERASCAD produziriam a mesma solução se aplicados para o mesmo conjunto de matrizes relacionais.

Observe que ambos os algoritmos RASCAD e NERASCAD não requerem a inicialização da matriz de ponderação Y, contrariamente ao algoritmo CARD (Frigui et al., 2007). Este

\footnotetext{
${ }_{2}$ Hathaway e Bezdek $(1994)$ provaram que toda matriz relacional não euclidiana se torna euclidiana para um valor suficientemente alto de $\beta$.
} 
último requer o parâmetro $\mathrm{Y}$ devido à ordem dos seus passos e ao uso dos elementos de $\mathrm{Y}$ para calcular as distâncias agregadas (Eq. (2.29)).

\subsection{Provas de convergência}

Teorema 3.1. Considere o seguinte como dado: $n \in \mathbb{N}_{1, \infty}, k \in \mathbb{N}_{1, \infty}, m \in[1, \infty), q \in(1, \infty)$, $\mathrm{U} \in M_{\mathrm{p}}, D \triangleq\left\{\mathrm{D}^{s}\right\}_{s=1}^{p} \in M_{\mathrm{dp}} e \mathrm{D}^{s} \triangleq\left[\mathrm{D}_{i, j}^{s}\right] \in \mathbb{R}^{k \cdot n}$ para todo $s \in \mathbb{N}_{1, p}$. Sejam $\Psi: M_{\mathrm{y}} \mapsto \mathbb{R}$ definido por $\Psi(\mathrm{Y}) \triangleq \mathcal{G}_{m, q}(\mathrm{U}, D, \mathrm{Y})$ e a função $\mathcal{Q}_{m, q}$ definida pelas Eqs. (3.4a), (3.4b) e (3.4c). É verdade que

$$
\Psi\left(\mathcal{Q}_{m, q}(D, \mathrm{U})\right)=\min _{\mathrm{Y} \in M_{\mathrm{y}}}\{\Psi(\mathrm{Y})\}
$$

Prova. Ao reescrever a Eq. (2.20) como

$$
\mathcal{G}_{m, q}(\mathrm{U}, D, \mathrm{Y})=\sum_{i=1}^{k} \sum_{s=1}^{p}\left(\mathrm{Y}_{i, s}\right)^{q} \cdot d_{i, s}
$$

em que

$$
d_{i, s} \triangleq \sum_{j=1}^{n}\left(\mathrm{U}_{i, j}\right)^{m} \cdot \mathrm{D}_{i, j}^{s},
$$

torna-se fácil concluir que a solução $\mathrm{Y}^{*}$ do problema $\min _{\mathrm{Y} \in M_{\mathrm{y}}}\{\Psi(\mathrm{Y})\}$, quando $d_{i_{0}, s_{0}}=0$, necessariamente satisfaz

$$
\mathrm{Y}_{i_{0}, s}^{*}=0 \quad \forall s \in \mathbb{N}_{1, p} \mid d_{i_{0}, s}>0 .
$$

Por outro lado, os termos

$$
\mathrm{Y}_{i_{0}, s}^{*} \quad \forall s \in \mathbb{N}_{1, p} \mid d_{i_{0}, s}=0
$$

podem assumir quaisquer valores, desde que $\mathrm{Y}^{*} \in M_{\mathrm{y}}$. Para ser justo, esses valores são definidos como na Eq. (3.4c). Observe que essa atualização para $\left.Y_{i_{0}, s}^{*}\right|_{s=1} ^{p}$ satisfaz as Propriedades (2.10), visto que

$$
\sum_{s=1}^{p} \mathrm{Y}_{i_{0}, s}^{*}=\sum_{\forall s \in \mathbb{N}_{1, p} \mid d_{i_{0}, s}=0} \mathrm{Y}_{i_{0}, s}^{*}=1
$$

e $\mathrm{Y}_{i_{0}, s}^{*} \geq 0 \quad \forall s \in \mathbb{N}_{1, p}$. Dadas essas considerações e assumindo, sem perda de generalidade, que os $k_{1}$ grupos tais que $d_{i, s}>0$ para todo $s \in \mathbb{N}_{1, p}$ são os primeiros, i.e.,

$$
\begin{array}{r}
\forall i \in \mathbb{N}_{1, k_{1}} \nexists s \in \mathbb{N}_{1, p} \text { tal que } d_{i, s}=0 \\
\forall i \in \mathbb{N}_{k_{1}+1, k} \exists s \in \mathbb{N}_{1, p} \text { tal que } d_{i, s}=0
\end{array}
$$

pode ser concluído que as Eqs. (3.4b) e (3.4c) determinam as últimas $k-k_{1}$ linhas de uma eventual solução $\mathrm{Y}^{*}$ para o problema $\min _{Y \in M_{y}}\{\Psi(Y)\}$.

Segue de $\mathrm{Y}^{*} \triangleq \mathcal{Q}_{m, q}(D, \mathrm{U})$ que 


$$
\begin{aligned}
\Psi\left(\mathrm{Y}^{*}\right) & =\sum_{i=1}^{k_{1}} \sum_{s=1}^{p}\left(\mathrm{Y}_{i, s}^{*}\right)^{q} \cdot d_{i, s}+\sum_{i=k_{1}+1}^{k} \sum_{s=1}^{p}\left(\mathrm{Y}_{i, s}^{*}\right)^{q} \cdot d_{i, s} \\
& =\sum_{i=1}^{k_{1}} \sum_{s=1}^{p}\left(\mathrm{Y}_{i, s}^{*}\right)^{q} \cdot d_{i, s} .
\end{aligned}
$$

Assim, considere a função $\Phi: M_{\mathrm{y}}\left(k_{1}\right) \mapsto \mathbb{R}$ definida por

$$
\Phi(\dot{\mathrm{Y}}) \triangleq \sum_{i=1}^{k_{1}} \sum_{s=1}^{p}\left(\dot{\mathrm{Y}}_{i, s}\right)^{q} \cdot d_{i, s}
$$

Para finalizar a prova, é necessário encontrar a solução

$$
\dot{\mathrm{Y}}^{*} \triangleq \underset{\dot{\mathrm{Y}} \in M_{\mathrm{y}}\left(k_{1}\right)}{\arg \min }\{\Phi(\dot{\mathrm{Y}})\}
$$

que corresponde à submatriz $\mathrm{Y}_{1: k_{1},:}^{*}$ (i.e., às primeiras $k_{1}$ linhas de $\mathrm{Y}^{*}$ ).

Para satisfazer a Propriedade 2.10b), considere o Lagrangiano

$$
\Phi_{\lambda}(\dot{\mathrm{Y}}, \Lambda) \triangleq \sum_{i=1}^{k_{1}} \sum_{s=1}^{p}\left(\dot{\mathrm{Y}}_{i, s}\right)^{q} \cdot d_{i, s}-\sum_{i=1}^{k_{1}} \lambda_{i}\left(\sum_{s=1}^{p} \dot{\mathrm{Y}}_{i, s}-1\right)
$$

em que $\dot{\mathrm{Y}} \in M_{\mathrm{y}}\left(k_{1}\right)$ e $\Lambda \triangleq\left(\lambda_{1}, \ldots, \lambda_{k_{1}}\right) \in \mathbb{R}^{k_{1}}$. Precisamos encontrar o ponto $\left(\dot{\mathrm{Y}}^{*}, \Lambda^{*}\right)$ no qual as derivadas parciais igualam-se a zero (ao final da dedução, pode-se notar que a Propriedade 2.10a é satisfeita automaticamente). Considere

$$
\begin{array}{lrl}
\frac{\partial \Phi_{\lambda}}{\partial \dot{\mathrm{Y}}_{i, s}}(\dot{\mathrm{Y}}, \Lambda) & =q\left(\dot{\mathrm{Y}}_{i, s}\right)^{q-1} \cdot d_{i, s}-\lambda_{i} & \forall i \in \mathbb{N}_{1, k_{1}}, \forall s \in \mathbb{N}_{1, p} \\
\frac{\partial \Phi_{\lambda}}{\partial \lambda_{i}}(\dot{\mathrm{Y}}, \Lambda)=-\sum_{s=1}^{p} \dot{\mathrm{Y}}_{i, s}+1 & \forall i \in \mathbb{N}_{1, k_{1}}
\end{array}
$$

Igualando as derivadas parciais no ponto $\left(\dot{\mathrm{Y}}^{*}, \Lambda^{*}\right)$ a zero, fornece

$$
\begin{aligned}
\dot{\mathrm{Y}}_{i, s}^{*} & =\left(\lambda_{i}^{*} / q d_{i, s}\right)^{1 /(q-1)} & \forall i \in \mathbb{N}_{1, k_{1}}, \forall s \in \mathbb{N}_{1, p}, \\
\sum_{s=1}^{p} \dot{\mathrm{Y}}_{i, s}^{*} & =1 & \forall i \in \mathbb{N}_{1, k_{1}} .
\end{aligned}
$$


Substituindo (3.13) em (3.14) fornece

$$
\begin{aligned}
\sum_{s=1}^{p}\left(\lambda_{i}^{*} / q d_{i, s}\right)^{1 /(q-1)}=1 \quad \forall i \in \mathbb{N}_{1, k_{1}} \quad \Rightarrow \\
\left(\lambda_{i}^{*}\right)^{1 /(q-1)}=\left(\sum_{s=1}^{p} 1 /\left(q d_{i, s}\right)^{1 /(q-1)}\right)^{-1} \quad \forall i \in \mathbb{N}_{1, k_{1}} .
\end{aligned}
$$

Usando (3.15) em (3.13) fornece a Eq. 3.4a). Consequentemente, $\dot{\mathrm{Y}}_{i, s}^{*}>0 \quad \forall i \in \mathbb{N}_{1, k_{1}}, \forall s \in$ $\mathbb{N}_{1, p}$ e, portanto, $\dot{\mathrm{Y}}^{*} \in M_{\mathrm{y}}\left(k_{1}\right)$. Assim, $\dot{\mathrm{Y}}^{*}$ é um ponto crítico de $\Phi$.

Claramente, se $\dot{\mathrm{Y}}^{*}$ é um mínimo global de $\Phi$, então $\mathrm{Y}^{*}$ é um mínimo global de $\Psi$. Então, considere a matriz Hessiana $\mathrm{H}_{\Phi_{\lambda}}$ de $\Phi_{\lambda}$, com relação à matriz $\dot{Y}$. As suas derivadas parciais de segunda ordem são (tenha em mente a Eq. (3.12)

$$
\begin{array}{ll}
\frac{\partial^{2} \Phi_{\lambda}}{\partial \dot{\mathrm{Y}}_{i, s} \partial \dot{\mathrm{Y}}_{i, s}}\left(\dot{\mathrm{Y}}^{*}, \Lambda^{*}\right)=q(q-1)\left(\dot{\mathrm{Y}}_{i, s}^{*}\right)^{q-2} \cdot & d_{i, s}>0 \\
& \forall i \in \mathbb{N}_{1, k_{1}}, \forall s \in \mathbb{N}_{1, p} \\
\frac{\partial^{2} \Phi_{\lambda}}{\partial \dot{\mathrm{Y}}_{i, s} \partial \dot{\mathrm{Y}}_{t, l}}\left(\dot{\mathrm{Y}}^{*}, \Lambda^{*}\right)=0 & \\
& \forall(i, s) \neq(t, l) .
\end{array}
$$

Logo, $\mathrm{H}_{\Phi_{\lambda}}$ é uma matriz diagonal com elementos não nulos dados pela Eq. 3.16). Devido à positividade dos determinantes dos menores principais, a matriz $\mathrm{H}_{\Phi_{\lambda}}$ é definida positiva. Assim, $\left(\dot{\mathrm{Y}}^{*}, \Lambda^{*}\right)$ é um mínimo global de $\Phi_{\lambda}$ e, consequentemente, $\dot{\mathrm{Y}}^{*}$ é um mínimo global de $\Phi$. Portanto, $\mathrm{Y}^{*}=\mathcal{Q}_{m, q}(D, \mathrm{U})$ é um mínimo global de $\Psi$, na qual as primeiras $k_{1}$ linhas são dadas pela Eq. (3.4a) e as últimas $k-k_{1}$ linhas são dadas pelas Eqs. (3.4b) e (3.4c).

Teorema 3.2. Considere o seguinte como dado: $n \in \mathbb{N}_{1, \infty}, k \in \mathbb{N}_{1, \infty}, m \in(1, \infty), q \in[1, \infty)$, $\mathrm{Y} \in M_{\mathrm{y}}, D \triangleq\left\{\mathrm{D}^{s}\right\}_{s=1}^{p} \in M_{\mathrm{dp}} e \mathrm{D}^{s} \triangleq\left[\mathrm{D}_{i, j}^{s}\right] \in \mathbb{R}^{k \cdot n}$ para todo $s \in \mathbb{N}_{1, p}$. Sejam $\Psi: M_{\mathrm{p}} \mapsto \mathbb{R}$ definido como $\Psi(\mathrm{U}) \triangleq \mathcal{G}_{m, q}(\mathrm{U}, D, \mathrm{Y})$ e a função $\mathcal{H}_{m, q}$ definida pelas Eqs. (3.5a), 3.5b) $e$ (3.5c). É verdade que

$$
\Psi\left(\mathcal{H}_{m, q}(D, \mathrm{Y})\right)=\min _{\mathrm{U} \in M_{\mathrm{p}}}\{\Psi(\mathrm{U})\}
$$

Prova. A prova é análoga à do Teorema 3.1 .

Teorema 3.3. Considere o seguinte como dado: $n \in \mathbb{N}_{1, \infty}, k \in \mathbb{N}_{1, \infty}, m \in[1, \infty), q \in[1, \infty)$, $\mathrm{U} \in M_{\mathrm{p}}, \mathrm{Y} \in M_{\mathrm{y}}, \dot{X} \triangleq\left\{X_{1}, X_{2}, \ldots, X_{p}\right\}$, em que $X_{s} \triangleq\left\{\mathrm{x}^{1, s}, \mathrm{x}^{2, s}, \ldots, \mathrm{x}^{n, s}\right\} \subset \mathbb{R}^{a_{s}}$ para todo $s \in \mathbb{N}_{1, p}$ e $\|\cdot\|_{s}$ é qualquer norma induzida por produto interno em $\mathbb{R}^{a_{s}}$, para todo $s \in \mathbb{N}_{1, p}$. Sejam $\Psi: M_{\mathrm{vp}} \mapsto \mathbb{R}$ definida como $\Psi(\dot{V}) \triangleq \mathcal{G}_{m, q}^{\mathrm{vp}}(\mathrm{U}, \dot{V}, \mathrm{Y} ; \dot{X})$, a função $\mathcal{G}_{m, q}^{\mathrm{vp}}$ definida pela Eq. (3.2) e a função $\mathcal{F}_{m}$ definida pela Eq. (3.3). É verdade que

$$
\Psi\left(\mathcal{F}_{m}(\mathrm{U} ; \dot{X})\right)=\min _{\dot{V} \in M_{\mathrm{vp}}}\{\Psi(\dot{V})\}
$$

Prova. Seja $\dot{V} \triangleq\left\{V_{1}, V_{2}, \ldots, V_{p}\right\}$, em que $V_{s} \triangleq\left\{\mathrm{v}^{i, s}\right\}_{i=1}^{k} \subset \mathbb{R}^{a_{s}}$ para todo $s \in \mathbb{N}_{1, p}$. É 
fácil ver que o problema de minimização pode ser resolvido ao resolver cada um dos seguintes problemas independentes:

$$
\begin{aligned}
& \min _{\mathrm{v}^{i, s} \in \mathbb{R}^{a s}}\left\{\Phi_{i, s}\left(\mathrm{v}^{i, s}\right)\right\} \triangleq\left\{\sum_{j=1}^{n}\left(\mathrm{U}_{i, j}\right)^{m} \cdot\left(\mathrm{Y}_{i, s}\right)^{q} \cdot\left\|\mathrm{x}^{j, s}-\mathrm{v}^{i, s}\right\|_{s}^{2}\right\} \\
& \forall i \in \mathbb{N}_{1, k}, \forall s \in \mathbb{N}_{1, p}
\end{aligned}
$$

Dado que não existe restrição para a minimização de $\Phi_{i, s}$ e dado que essa função possui derivadas direcionais contínuas em todos os pontos, todos os seus pontos críticos podem ser encontrados ao analisar os pontos cujas derivadas direcionais são zero. Sejam $\vec{d} \in \mathbb{R}^{a_{s}}$ um vetor direcional e $\mathrm{v}^{i, s *} \in \mathbb{R}^{a_{s}}$ um ponto. Além disso, seja

$$
h_{i, s}(t) \triangleq \Phi_{i, s}\left(\mathrm{v}^{i, s *}+t \overrightarrow{\mathrm{d}}\right)=\sum_{j=1}^{n}\left(\mathrm{U}_{i, j}\right)^{m} \cdot\left(\mathrm{Y}_{i, s}\right)^{q} \cdot\left\|\mathrm{x}^{j, s}-\mathrm{v}^{i, s *}-t \overrightarrow{\mathrm{d}}\right\|_{s}^{2},
$$

em que $t \in \mathbb{R}$. Ao tomar a sua derivada, tem-se

$$
\begin{aligned}
\frac{d h_{i, s}}{d t}(t) & =\left(\mathrm{Y}_{i, s}\right)^{q} \cdot d\left(\sum_{j=1}^{n}\left(\mathrm{U}_{i, j}\right)^{m} \cdot\left\langle\mathrm{x}^{j, s}-\left(\mathrm{v}^{i, s *}+t \overrightarrow{\mathrm{d}}\right), \mathrm{x}^{j, s}-\left(\mathrm{v}^{i, s *}+t \overrightarrow{\mathrm{d}}\right)\right\rangle_{s}\right) / d t \\
& =\left(\mathrm{Y}_{i, s}\right)^{q}\left(\sum_{j=1}^{n}\left(\mathrm{U}_{i, j}\right)^{m} \cdot\left\langle-\overrightarrow{\mathrm{d}}, \mathrm{x}^{j, s}-\left(\mathrm{v}^{i, s *}+t \overrightarrow{\mathrm{d}}\right)\right\rangle_{s}\right. \\
& \left.+\sum_{j=1}^{n}\left(\mathrm{U}_{i, j}\right)^{m} \cdot\left\langle\mathrm{x}^{j, s}-\left(\mathrm{v}^{i, s *}+t \overrightarrow{\mathrm{d}}\right),-\overrightarrow{\mathrm{d}}\right\rangle_{s}\right) \\
& =-2\left(\mathrm{Y}_{i, s}\right)^{q} \sum_{j=1}^{n}\left(\mathrm{U}_{i, j}\right)^{m} \cdot\left\langle\overrightarrow{\mathrm{d}}, \mathrm{x}^{j, s}-\left(\mathrm{v}^{i, s *}+t \overrightarrow{\mathrm{d}}\right)\right\rangle_{s}
\end{aligned}
$$

em que $\|\cdot\|_{s}^{2}=\langle\cdot, \cdot\rangle_{s}$. Ao configurar $t=0$, obtém-se uma derivada direcional de $\Phi_{i, s}$ no ponto $\mathrm{v}^{i, s *}$ :

$$
\begin{aligned}
\frac{d h_{i, s}}{d t}(0) & =-2\left(\mathrm{Y}_{i, s}\right)^{q} \sum_{j=1}^{n}\left(\mathrm{U}_{i, j}\right)^{m} \cdot\left\langle\overrightarrow{\mathrm{d}}, \mathrm{x}^{j, s}-\mathrm{v}^{i, s *}\right\rangle_{s} \\
& =-2\left(\mathrm{Y}_{i, s}\right)^{q}\left\langle\overrightarrow{\mathrm{d}}, \sum_{j=1}^{n}\left(\mathrm{U}_{i, j}\right)^{m} \cdot \mathrm{x}^{j, s}-\mathrm{v}^{i, s *} \cdot \sum_{j=1}^{n}\left(\mathrm{U}_{i, j}\right)^{m}\right\rangle_{s}
\end{aligned}
$$

Assumindo que $\mathrm{Y}_{i, s}>0$, é fácil ver que $\frac{d h_{i, s}}{d t}(0)=0$ para todo vetor direcional $\overrightarrow{\mathrm{d}}$ quando

$$
\mathrm{v}^{i, s *}=\sum_{j=1}^{n}\left(\mathrm{U}_{i, j}\right)^{m} \cdot \mathrm{x}^{j, s} / \sum_{j=1}^{n}\left(\mathrm{U}_{i, j}\right)^{m}
$$

Quando $\mathrm{Y}_{i, s}=0, \frac{d h_{i, s}}{d t}(0)$ será zero independentemente do valor de $\mathrm{v}^{i, s *}$. Consequentemente, 
a função $\mathcal{F}_{m}(\mathrm{U} ; \dot{X})$ fornece um ponto crítico para a função $\Psi$. Agora, resta mostrar que esse ponto é mínimo.

Encontrando a sua segunda derivada, tem-se

$$
\frac{d^{2} h_{i, s}}{d t^{2}}(t)=2\left(\mathrm{Y}_{i, s}\right)^{q} \sum_{j=1}^{n}\left(\mathrm{U}_{i, j}\right)^{m} \cdot\|\overrightarrow{\mathrm{d}}\|_{s}^{2} \geq 0
$$

Isso prova que $\mathrm{v}^{i, s *}$, como definido em (3.18), é um ponto mínimo de $\Phi_{i, s}$. O fato que (3.18) fornece o único ponto crítico de $\Phi_{i, s}$ quando $\mathrm{Y}_{i, s}>0$ e que $\Phi_{i, s}$ é sempre igual a zero quando $\mathrm{Y}_{i, s}=0$, prova que $\mathrm{v}^{i, s *}$ é também um ponto de mínimo global. Como resultado, $\mathcal{F}_{m}(\mathrm{U} ; \dot{X})$ fornece não apenas um ponto crítico para a função $\Psi$, mas também um mínimo global. Isso prova o teorema.

Teorema 3.4. Considere o seguinte como dado: $n \in \mathbb{N}_{1, \infty}, k \in \mathbb{N}_{1, \infty}, m \in[1, \infty), q \in(1, \infty)$, $\dot{X} \triangleq\left\{X_{1}, X_{2}, \ldots, X_{p}\right\}$, em que $X_{s} \triangleq\left\{\mathrm{x}^{1, s}, \mathrm{x}^{2, s}, \ldots, \mathrm{x}^{n, s}\right\} \subset \mathbb{R}^{a_{s}}$ para todo $s \in \mathbb{N}_{1, p}, R \triangleq$ $\left\{\mathrm{R}^{1}, \mathrm{R}^{2}, \ldots, \mathrm{R}^{p}\right\} \subset \mathbb{R}^{n \cdot n}$ de p matrizes relacionais, $\mathrm{R}_{j, l}^{s} \triangleq\left\|\mathrm{x}^{j, s}-\mathrm{x}^{l, s}\right\|_{s}^{2}$ para todo $j, l \in \mathbb{N}_{1, n}$ e $s \in \mathbb{N}_{1, p}$ e $\|\cdot\|_{s}$ é qualquer norma induzida por produto interno em $\mathbb{R}^{a_{s}}$, para todo $s \in \mathbb{N}_{1, p}$. É verdade que $\mathcal{K}_{m, q}(\mathrm{U}, \mathrm{Y} ; R)=\mathcal{G}_{m, q}^{\mathrm{vp}}\left(\mathrm{U}, \mathcal{F}_{m}(\mathrm{U} ; \dot{X}), \mathrm{Y} ; \dot{X}\right)$, cujas funções $\mathcal{K}_{m, q}, \mathcal{G}_{m, q}^{\mathrm{vp}}$ e $\mathcal{F}_{m}$ são definidas pelas Eqs. (2.27), (3.2) e (3.3), respectivamente.

Prova. Seja ${ }^{i, s *}$, para todo $i \in \mathbb{N}_{1, k}$ e $s \in \mathbb{N}_{1, p}$, definido pela Eq. 3.3. Para provar o teorema, basta demonstrar que

$\sum_{s=1}^{p}\left(\mathrm{Y}_{i, s}\right)^{q}\left(\sum_{j=1}^{n} \sum_{l=1}^{n}\left\|\mathrm{x}^{j, s}-\mathrm{x}^{l, s}\right\|^{2}\left(\mathrm{U}_{i, j}\right)^{m}\left(\mathrm{U}_{i, l}\right)^{m} / 2 \sum_{l=1}^{n}\left(\mathrm{U}_{i, l}\right)^{m}\right)=\sum_{s=1}^{p}\left(\mathrm{Y}_{i, s}\right)^{q}\left(\sum_{j=1}^{n}\left(\mathrm{U}_{i, j}\right)^{m}\left\|\mathrm{x}^{j, s}-\mathrm{v}^{i, s *}\right\|^{2}\right)$

para todo $i \in \mathbb{N}_{1, k}$, que, por sua vez, basta provar que

$$
\sum_{j=1}^{n} \sum_{l=1}^{n}\left\|\mathrm{x}^{j, s}-\mathrm{x}^{l, s}\right\|^{2}\left(\mathrm{U}_{i, j}\right)^{m}\left(\mathrm{U}_{i, l}\right)^{m} / 2 \sum_{l=1}^{n}\left(\mathrm{U}_{i, l}\right)^{m}=\sum_{j=1}^{n}\left(\mathrm{U}_{i, j}\right)^{m}\left\|\mathrm{x}^{j, s}-\mathrm{v}^{i, s *}\right\|_{s}^{2}
$$

para todo $i \in \mathbb{N}_{1, k}$ e $s \in \mathbb{N}_{1, p}$, o que é feito nas Eqs. (3)-(6) de (Tucker, 1987).

\subsection{Silhueta para dados multi-representados}

O índice da Silhueta definido na Seção 2.5.1 depende do cálculo de todas as distâncias entre todos os objetos. Esse cômputo pode ser substituído por uma versão simplificada baseada em distâncias entre objetos e grupos (protótipos). Essa versão simplificada, referida por $\underline{\text { SS, foi }}$ definida na Seção 2.5.2. Na Seção 2.5.3 foi definida uma versão do índice SS capaz de lidar com agrupamentos nebulosos, referida por SSN

Observe que os índices $[\mathrm{SS}$ e $[\mathrm{SSN}$ usam apenas distâncias entre objetos e grupos. Essa propriedade faz com que esses dois índices sejam candidatos a avaliar agrupamentos gerados pelos algoritmos ASCAD, HASCAD e NERASCAD, Sejam os termos dados pelas Eqs. (2.40) 
e 2.41) redefinidos como

$$
\begin{aligned}
& a_{p_{j}, j} \triangleq \sum_{s=1}^{p}\left(\mathrm{Y}_{p_{j}, s}\right)^{q} \cdot \mathrm{D}_{p_{j}, j}^{s} \\
& b_{q_{j}, j} \triangleq \sum_{s=1}^{p}\left(\mathrm{Y}_{q_{j}, s}\right)^{q} \cdot \mathrm{D}_{q_{j}, j}^{s}
\end{aligned}
$$

em que $q$ é o expoente de ponderação e os termos $\mathrm{D}_{p_{j}, j}^{s}$ e $\mathrm{D}_{q_{j}, j}^{s}$ possuem a mesma interpretação do termo correspondente da Função 2.20). O índice resultante devido aos termos acima é chamado de Silhueta Simplificada Nebulosa para Ponderação de Representações (SSNPR). Na Seção 3.6.5 o índice SSNPRé experimentalmente avaliado ao ser usado em uma abordagem de estimação automática do número de grupos.

\subsection{Experimentos}

Esta seção tem como objetivos principais avaliar a capacidade dos algoritmos propostos em lidar com bases de dados ruidosas (ruído nos atributos) e com bases de dados descritas por múltiplas representações. No primeiro caso, bases de dados geradas artificialmente com graus crescentes de ruído são usadas. No segundo caso, são usadas bases reais formadas a partir de imagens da coleção ALOI

Na Seção 3.6.1, os algoritmos SCAD e ASCAD são executados em uma base real bem conhecida da UCI na qual o algoritmo SCAD falha mas o algoritmo ASCAD não. Será avaliada a robustez do algoritmo ASCAD quanto à escolha do parâmetro $q$ (Seção 3.6.2) em bases da UCI

Nesta seção são apresentados vários experimentos envolvendo os algoritmos SCAD, ASCAD e FCM. A distância euclidiana quadrática foi usada pelos algoritmos em todos os experimentos, o que implica que NERASCAD RASCAD e ASCAD produziriam exatamente as mesmas soluções, assumindo a mesma configuração de parâmetros. Esse fato foi discutido nas Seções 3.2 e 3.3 e torna suficiente reportar os resultados de apenas um dos algoritmos nos experimentos desta seção. De maneira análoga, os algoritmos NERFCM/RFCM e FCM são equivalentes (Hathaway e Bezdek, 1994). Portanto, dos algoritmos citados acima, apenas ASCAD e FCM participaram dos experimentos que se seguem.

Os algoritmos SCAD, ASCAD ECM possuem o expoente de nebulosidade $m$. Uma regra comumente empregada é aquela na qual $m=2$. Essa regra foi adotada em todos os experimentos apresentados aqui. O expoente de ponderação $q$, por outro lado, é pouco explorado e ainda não possui um valor padrão. Na Seção 3.6.2 o algoritmo ASCAD mostrou bons resultados na avaliação da robustez quanto à escolha de $q$ para o conjunto de valores inteiros $\{2,3, \ldots, 15\}$ em duas bases reais bem conhecidas (Iris e Glass). No processo de desenvolvimento do algoritmo ASCAD, vários experimentos preliminares foram conduzidos, os quais sugeriram valores de $q$ por volta de 10 como sendo promissores. Em determinadas ocasiões, foi observado que valores de $q$ próximos de 1 ou 2 fizeram com que o algoritmo ASCAD exagerasse na ponderação das 
representações ao atribuir quase todo o peso de relevância a apenas uma delas, praticamente ignorando todas as outras. Em outras ocasiões, um valor muito alto para $q$ fez com que o algoritmo praticamente não realizasse a ponderação de representações (esse comportamento é discutido em (Frigui e Nasraoui, 2004)). Adicionalmente, valores muito altos para $q$ implicam em valores muito baixos de $\sum_{s=1}^{p}\left(\mathrm{Y}_{i, s}\right)^{q} \cdot \mathrm{D}_{i, j}^{s}$, o que pode causar erros críticos de arredondamento de ponto-flutuante no cálculo da Eq. 3.5a). Por esses motivos e para facilitar o entendimento dos resultados, foi adotado $q=10$ em todos os experimentos (excetuando os experimentos de robustez à seleção de $q$ apresentados na Seção 3.6.2). De qualquer modo, essa falta de orientação para a escolha de $q$ apenas favorece o algoritmo FCM em comparações com o algoritmo ASCAD.

Para inicializar a matriz de agrupamento U para os algoritmos ASCAD, SCAD e FCM, a seguinte estratégia foi adotada. Escolha aleatoriamente, sem reposição, $k$ objetos da base de dados. Cada um dos objetos escolhidos é uma semente que representa um grupo. Associe todos os objetos aos grupos correspondentes às sementes mais próximas e construa a matriz $\mathrm{U} \in$ $M_{\mathrm{e}}$ que represente essa solução de agrupamento. Essa estratégia estabelece a primeira matriz de agrupamento (particional exclusivo) para todas as execuções dos algoritmos mencionados acima.

Dois índices externos aplicáveis na comparação de agrupamentos nebulosos foram usados para avaliar as soluções encontradas. Os dois correspondem a generalizações do índice ARI definido na Seção 2.5. Eles são denotados aqui por 07CARI e 10AARI, foram propostos em (Campello, 2007) e (Anderson et al., 2010), respectivamente, e são descritos nesta tese no Capítulo 4 .

Por fim, é importante mencionar que os algoritmos SCAD e ASCAD não foram comparados em termos de acurácia, dado que os seus passos de otimização são iguais quando (por sorte) situações de falha do algoritmo SCAD não ocorrem.

\subsubsection{SCAD contra SCAD ajustado}

Como exemplo de um cenário no qual o algoritmo SCAD falha, procurou-se no repositório da UCI por uma base de dados possuindo atributos discretos (veja a discussão sobre o assunto na Seção 3.1). A primeira base de dados encontrada foi a Original Breast Cancer Wisconsin (BCancer), a qual possui nove atributos de inteiros e 699 objetos classificados nas categorias "maligno" e "benigno". Os nove atributos representam nove características citológicas atribuídas por um oncologista (Ferris e Mangasarian, 1995): espessura da moita, uniformidade do tamanho da célula, uniformidade do formato da célula, adesão marginal, tamanho da célula epitelial, núcleos nus, bland chromatin, nucléolos normais e mitoses $3^{3}$. O oncologista analisou cada amostra e atribuiu um valor inteiro de 1 a 10 a cada uma dessas características celulares.

Os objetos com valores faltantes foram descartados, restando 683 objetos da base BCancer. $\mathrm{O}$ algoritmo $\mathrm{SCAD}$ foi aplicado 100 vezes com a seguinte configuração: $k=2, m=2 \mathrm{e}$

\footnotetext{
${ }^{3}$ Do inglês clump thickness, uniformity of cell size, uniformity of cell shape, marginal adhesion, single epithelial cell size, bare nuclei, bland chromatin, normal nucleoli e mitoses, respectivamente.
} 
$q=10$. Além disso, cada atributo foi considerado como uma diferente representação da base de dados.

O algoritmo $\mathrm{SCAD}$ não finalizou em quatro das 100 execuções devido a divisões por zero. Por exemplo, em uma das execuções, a primeira matriz $U$ representava um agrupamento no qual o primeiro grupo $C_{1}$ (observe que a matriz inicial $\mathrm{U}$ é sempre uma matriz de agrupamento particional exclusivo) possuía 44 objetos. Todos esses objetos apresentavam o mesmo valor para o quinto atributo no primeiro cálculo de $\mathrm{Y}$, o que levou o algoritmo SCAD a uma situação, como predito na Seção 3.1, na qual ele falha.

Pode-se argumentar que a base BCancer possui vários objetos idênticos e que talvez isso tenha causado a falha do algoritmo SCAD, Então, removeu-se os pontos idênticos da base BCancer, sobrando apenas 448 objetos. Novamente, o algoritmo SCAD falhou várias vezes devido a divisões por zero. Contrariamente, o algoritmo ASCAD pôde finalizar todas as suas execuções, usando os mesmos parâmetros.

\subsubsection{Robustez à configuração do expoente de ponderação}

Vários experimentos foram executados com as bases Iris e Glass do repositório da UCI. A base Iris consiste em três classes de plantas descritas por quatro atributos numéricos. Duas dessas classes possuem uma forte sobreposição. A Figura 3.1 mostra a projeção da Iris nas suas primeiras duas dimensões, sugerindo que duas das classes são sobrepostas e que não há uma clara separabilidade entre elas (ao desconsiderar a classificação), a ponto de fazer sentido considerar essas duas classes como sendo apenas um grupo. Logo, é bastante comum adotar $k^{*}=2$ como o número de grupos ótimo e unir essas duas classes (Wu e Yang, 2005), o que foi feito aqui.

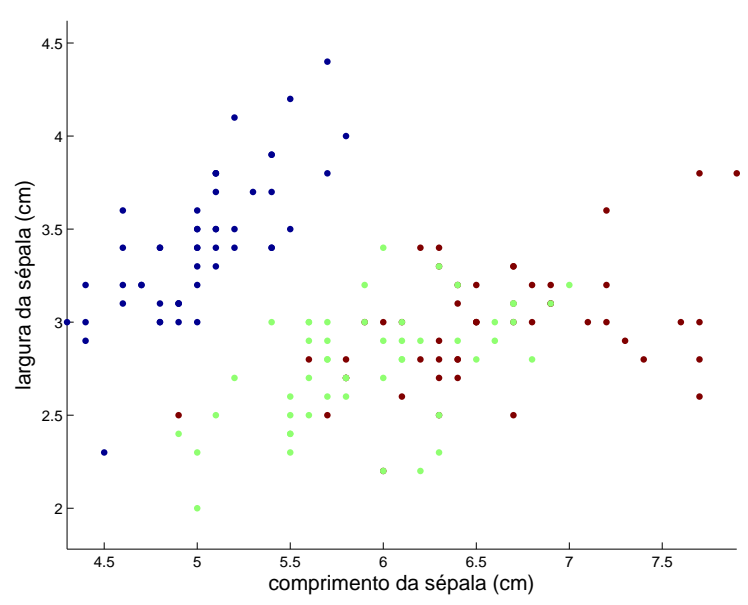

Figura 3.1: Iris projetada nas duas primeiras dimensões. As cores representam os rótulos.

A base Glass possui 214 objetos representados em um espaço de nove dimensões. Ela consiste em seis classes representando seis tipos de vidros. Foi aplicado o índice da Silhueta (Seção 2.5.1) na classificação da base Glass para verificar se essa rotulação pode ser considerada um agrupamento. Adotando a distância euclidiana para medir as dissimilaridades entre os objetos, obtivemos um valor negativo $(-0.09)$ para o índice da Silhueta, o que indica fortemente que as 
classes possuem um alto grau de sobreposição e que, portanto, a classificação dada não pode ser interpretada como um agrupamento dos dados. A Figura 3.2(a) ajuda a ilustrar a forte sobreposição que existe entre as classes da base Glass. As classes, então, foram unidas duas por vez de acordo com as distâncias euclidianas intergrupo medidas pelas distâncias entre os centróides correspondentes. Esse processo foi executado até sobrarem apenas duas classes. O índice da Silhueta elegeu a classificação em duas classes como sendo o melhor agrupamento, o qual obteve o valor 0.46 de avaliação. A Figura 3.2(b) ilustra o resultado. Portanto, a classificação em duas classes foi adotada como o agrupamento de referência para a base de dados Glass.

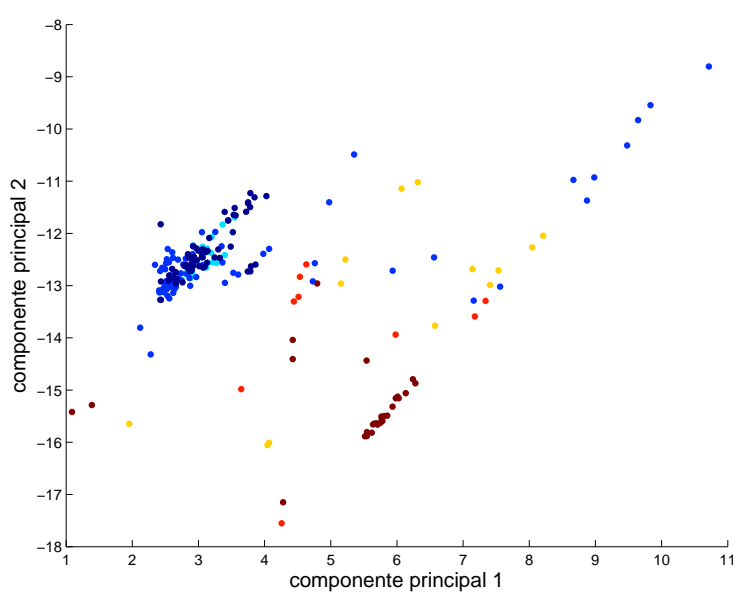

(a) Rótulos originais.

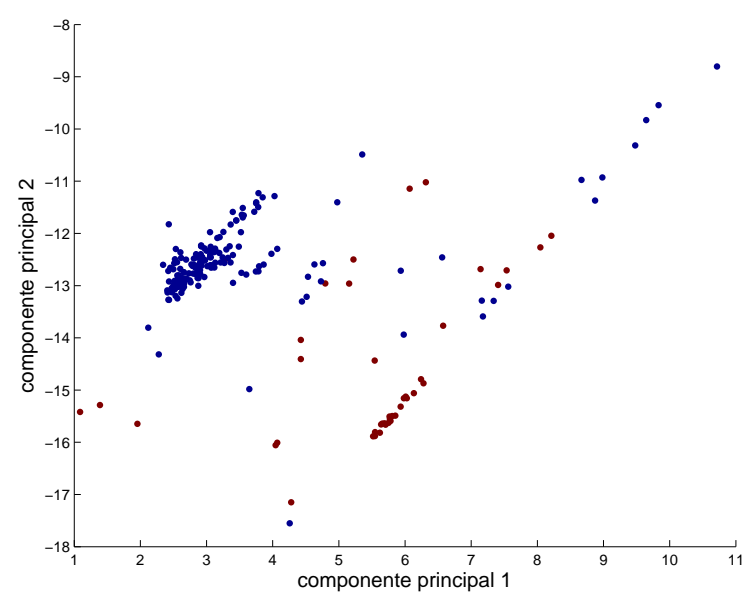

(b) Rótulos resultantes de uniões dos grupos mais próximos.

Figura 3.2: Base de dados Glass projetada nas duas primeiras componentes principais ( $\mathrm{ACP}$. As cores representam os rótulos.

Os algoritmos ASCAD e FCM foram aplicados 100 vezes para cada $q \in\{2,3, \ldots, 15\}$ (note que apenas o algoritmo ASCAD necessita do parâmetro $q$ ) com o número ótimo de grupos $k^{*}$ e o melhor agrupamento, de acordo com a respectiva função-custo, foi considerado como a solução encontrada. Cada atributo foi considerado como sendo uma única visão da base. A Tabela 3.1 apresenta as pontuações, de acordo com os índices 10AARI e 07CARI, alcançadas pelas soluções da base de dados Iris. As soluções do algoritmo ASCAD pontuaram melhor que as soluções do algoritmo FCM em quase todos os valores de $q$. As exceções ocorreram para os valores $q=13,14,15$, em diferenças insignificantes de avaliação pelo índice 07CARI.

Os algoritmos ASCAD e FCM também foram aplicados na base de dados Glass com a mesma configuração experimental. A Tabela 3.2 mostra os resultados. O algoritmo ASCAD exibiu a melhor pontuação para cada valor de $q$. Além disso, a qualidade das soluções geradas não sofreu alteração, até a terceira casa decimal, para diferentes valores de $q$. Novamente o algoritmo ASCAD desempenhou melhor que o FCM, agora em todas as configurações.

Os resultados desta seção indicam que o algoritmo ASCAD foi robusto à escolha do parâmetro q. O algoritmo ASCAD também superou o $\mathrm{FCM}$ em duas bases reais amplamente utilizadas na literatura. A próxima seção avalia a robustez do algoritmo ASCAD com relação a atributos ruidosos. 
Tabela 3.1: Médias dos valores fornecidos pelos índices ao variar o expoente de ponderação $q$ para a base Iris. As únicas três derrotas do algoritmo ASCAD estão destacadas.

\begin{tabular}{cccccc} 
& \multicolumn{3}{c}{ Índice 10AARI } & \multicolumn{2}{c}{ Índice 07CARI } \\
\hline \hline$q$ & ASCAD & FCM & ASCAD & FCM \\
\hline 2 & 0.819 & 0.768 & 0.784 & 0.734 \\
3 & 0.794 & 0.768 & 0.755 & 0.734 \\
4 & 0.785 & 0.768 & 0.745 & 0.734 \\
5 & 0.781 & 0.768 & 0.741 & 0.734 \\
6 & 0.778 & 0.768 & 0.739 & 0.734 \\
7 & 0.777 & 0.768 & 0.737 & 0.734 \\
8 & 0.776 & 0.768 & 0.736 & 0.734 \\
9 & 0.775 & 0.768 & 0.736 & 0.734 \\
10 & 0.775 & 0.768 & 0.735 & 0.734 \\
11 & 0.774 & 0.768 & 0.734 & 0.734 \\
12 & 0.774 & 0.768 & 0.734 & 0.734 \\
13 & 0.773 & 0.768 & $\mathbf{0 . 7 3 3}$ & 0.734 \\
14 & 0.773 & 0.768 & $\mathbf{0 . 7 3 3}$ & 0.734 \\
15 & 0.773 & 0.768 & $\mathbf{0 . 7 3 3}$ & 0.734
\end{tabular}

Tabela 3.2: Médias dos valores fornecidos pelos índices ao variar o expoente de ponderação $q$ para base Glass.

\begin{tabular}{ccccc} 
& \multicolumn{2}{c}{ Índice 10AARI } & \multicolumn{2}{c}{ Índice 07CARI } \\
\hline \hline$q$ & ASCAD & FCM & ASCAD & FCM \\
\hline $2,3, \ldots, 15$ & 0.529 & 0.409 & 0.529 & 0.372
\end{tabular}




\subsubsection{Robustez a atributos ruidosos}

Os experimentos relatados nesta seção foram conduzidos para avaliar a capacidade do algoritmo ASCAD em distinguir entre atributos relevantes e ruidosos. Para esse fim, as representações consideradas aqui são os próprios atributos da base de dados. Cem bases de dados para cada número de grupos $k^{*}=2,4,6,8$ e para cada número de atributos $a=3,10,50$ foram geradas pelo gerador de bases descrito em (Vendramin et al., 2009, 2010), o qual segue, em linhas gerais, o gerador descrito nos estudos clássicos (Milligan, 1981; Milligan e Cooper. 1985). Essas bases de dados serão referidas por bases MCL. Cada grupo de cada base de dados possui 20 objetos. Assim, por exemplo, as bases de dados com $k^{*}=4$ possuem 80 objetos cada. Sobreposição entre os grupos é permitida em todas exceto na primeira dimensão. A distribuição normal multivariada foi utilizada para gerar os objetos dentro de um mesmo grupo, de maneira que a estrutura resultante pudesse ser considerada como consistindo de grupos naturais que exibem as propriedades de isolamento externo e coesão interna. A Figura 3.3(a) apresenta uma das bases de dados com $k^{*}=8$ grupos e $a=3$ atributos, cujas cores representam os rótulos dos objetos. Essa base de dados apresenta uma tendência nítida de agrupamento. Os detalhes sobre os centros e as variâncias dessas distribuições normais são precisamente descritos em (Milligan. 1981).

Para cada um dessas 1200 bases de dados MCL descritas acima, novas foram criadas pela adição de ruído em seus atributos, da seguinte maneira. Dada uma base de dados, seus atributos são divididos aleatoriamente em três grupos (sejam $G_{a}, G_{b}$ e $G_{c}$ ). A primeira base de dados com ruído é gerada pela modificação aleatória de cada elemento da submatriz de dados correspondente aos atributos em $G_{c}$, o que dá origem a uma base de dados com atributos relevantes apenas em $G_{a}$ e $G_{b}$. A segunda base de dados é criada pela modificação aleatória de $50 \%$ dos elementos da submatriz correspondente aos atributos em $G_{b}$ e $100 \%$ dos elementos correspondentes aos atributos em $G_{c}$. Por fim, a terceira base de dados é gerada pela modificação aleatória de $100 \%$ dos elementos das submatrizes correspondentes aos atributos em $G_{b}$ e $G_{c}$. Esses quatro tipos de bases de dados são denotados por 0-0-0, 0-0-1, 0-0.5-1 e 0-1-1, em que cada valor corresponde ao ruído de $G_{a}, G_{b}$ e $G_{c}$, respectivamente. Por exemplo, 0-0.5-1 denota as bases de dados com $50 \%$ de ruído em $G_{b}$ e $100 \%$ de ruído em $G_{c}$.

A Figura 3.3(b) apresenta a base de dados da Figura 3.3(a) com a inserção de 50\% e 100\% de ruído nas dimensões $\vec{x}$ e $\vec{y}$. Essa certamente é uma base de dados mais complexa, visto que os grupos não mais estão bem separados e coesos. No entanto, ainda sim existe uma sutil tendência de agrupamento, como pode ser vista em suas projeção nas Figuras 3.3(c) e 3.3(d)

Os algoritmos FCM e ASCAD foram executados 30 vezes para cada base de dados com o seu respectivo parâmetro $k$ configurado igual ao número de grupos $k^{*}$ da base de dados. $\mathrm{O}$ agrupamento com o menor valor da função-custo gerada a partir dessas 30 execuções foi considerado como a solução final. Para avaliar os agrupamentos resultantes, ambos os índices 10AARI e 07CARI foram usados para mensurar a compatibilidade entre o agrupamento nebuloso encontrado e a partição de referência. Como os dois índices forneceram resultados bastante similares, apenas o índice 07CARI foi usado para criar os gráficos da Figura 3.4(a) à 


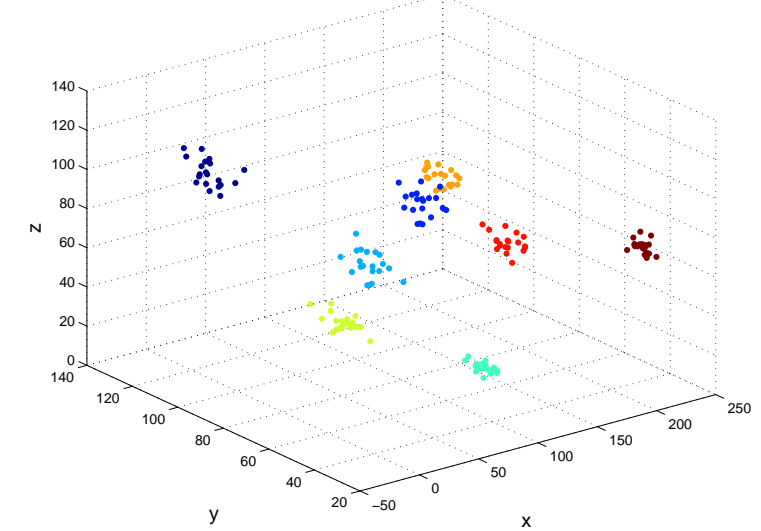

(a) MCL $\operatorname{com} k^{*}=8$ e $a=3$, sem ruído.

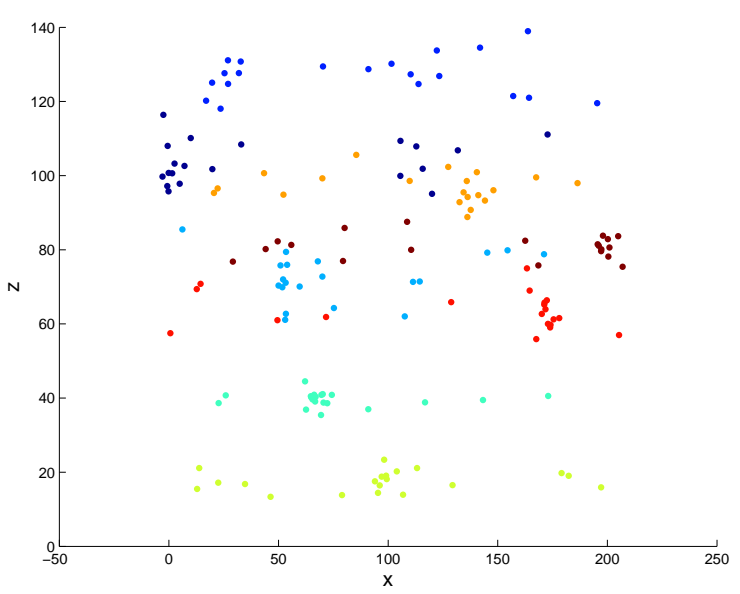

(c) Base da Figura 3.3(b) projetada no plano $\vec{x}-\vec{z}$.

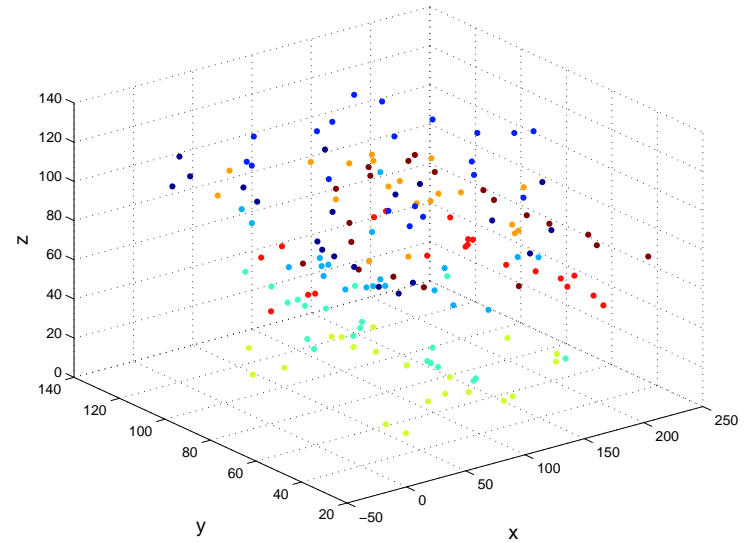

(b) MCL com $k^{*}=8$ e $a=3,50 \%$ e $100 \%$ de ruído em $\vec{x}$ e $\vec{y}$, respectivamente.

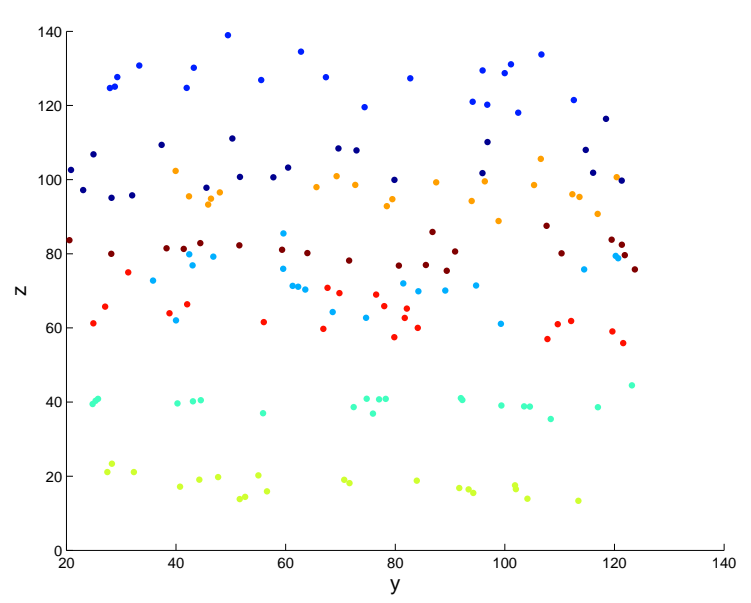

(d) Base da Figura $3.3(\mathrm{~b})$ projetada no plano $\vec{y}-\vec{z}$.

Figura 3.3: Exemplos de bases MCL geradas. 


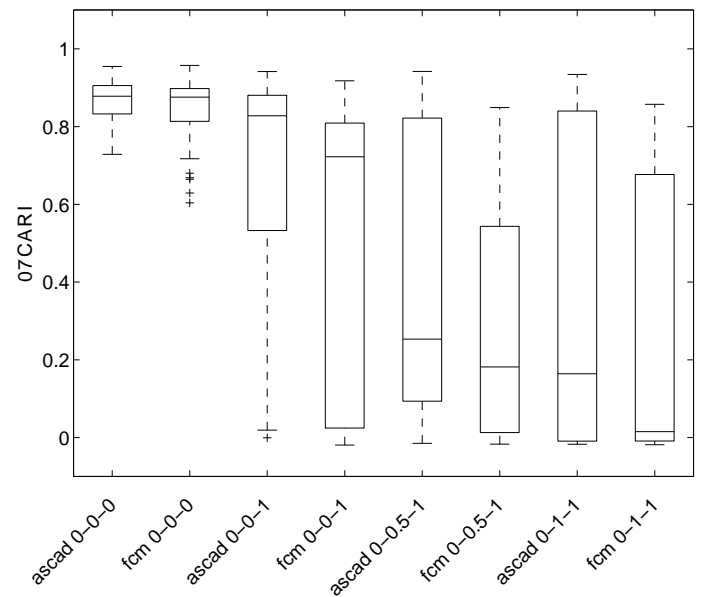

(a) MCL $\operatorname{com} k^{*}=2$ e $a=3$.

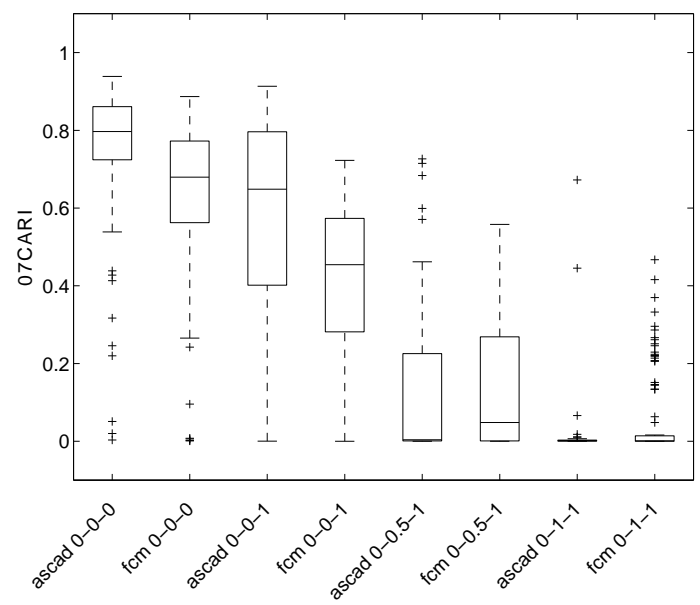

(c) MCL com $k^{*}=2$ e $a=50$.

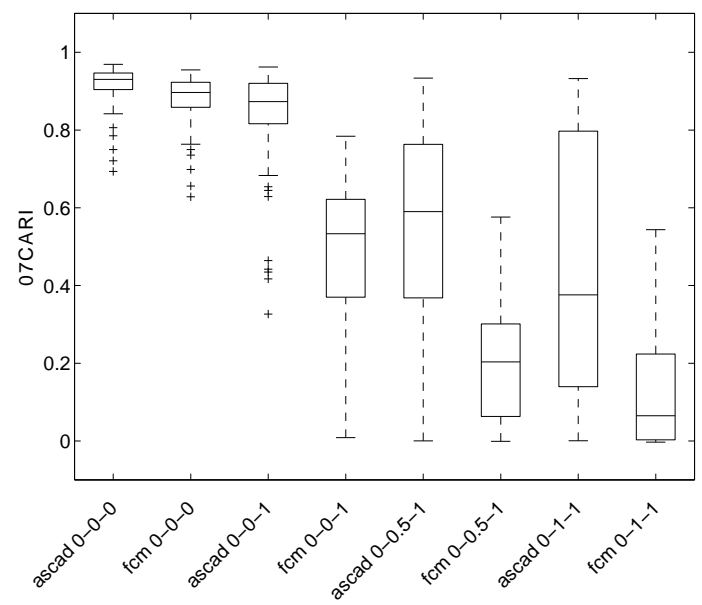

(e) MCL com $k^{*}=4$ e $a=10$.

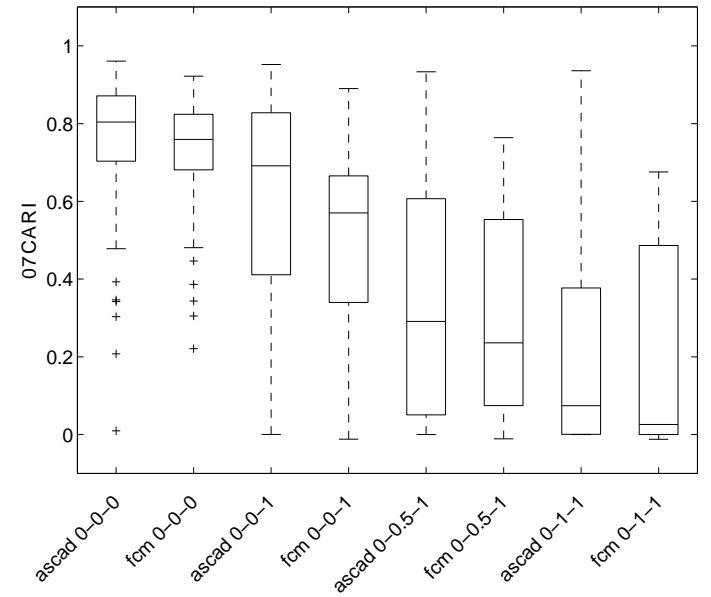

(b) MCL com $k^{*}=2$ e $a=10$.

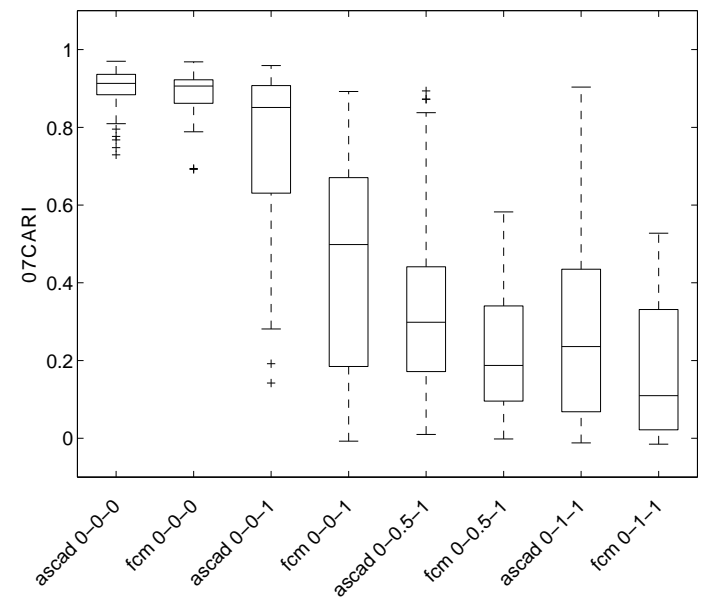

(d) MCL com $k^{*}=4$ e $a=3$.

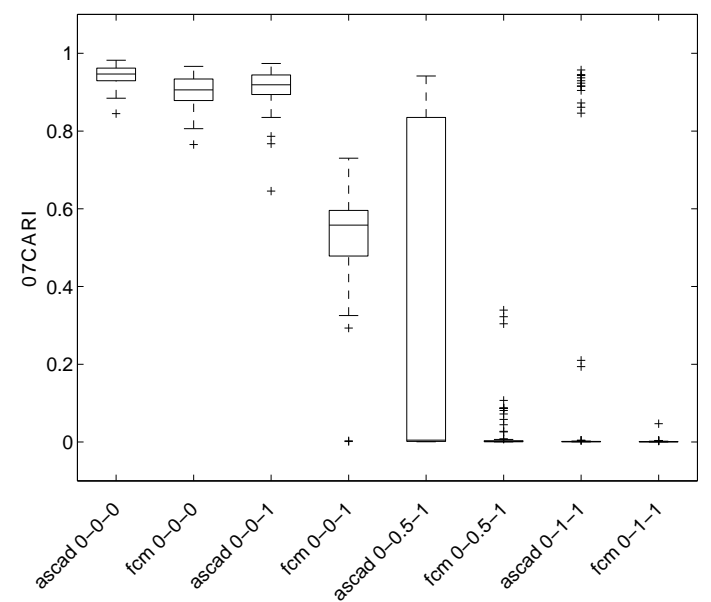

(f) MCL com $k^{*}=4$ e $a=50$.

Figura 3.4: Resultados para as bases MCL com $k^{*}=2,4$ e $a=3,10,50$ (ASCAD vs FCM. 


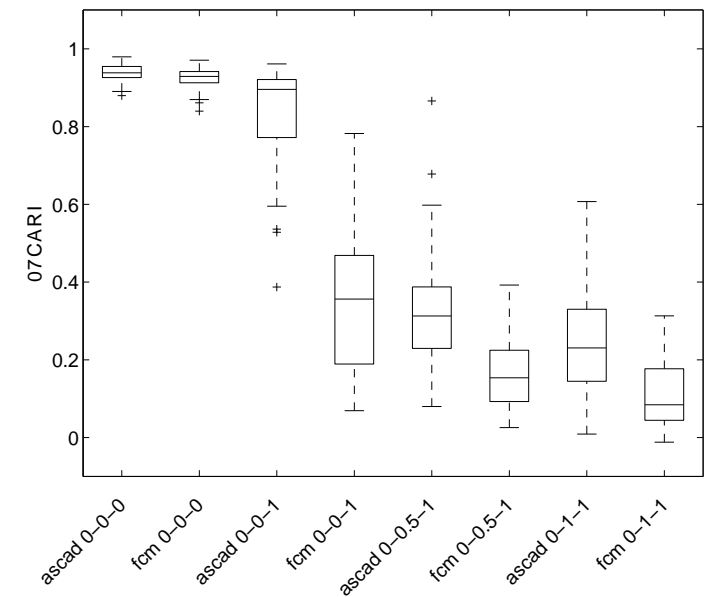

(a) MCL $\operatorname{com} k^{*}=6$ e $a=3$.

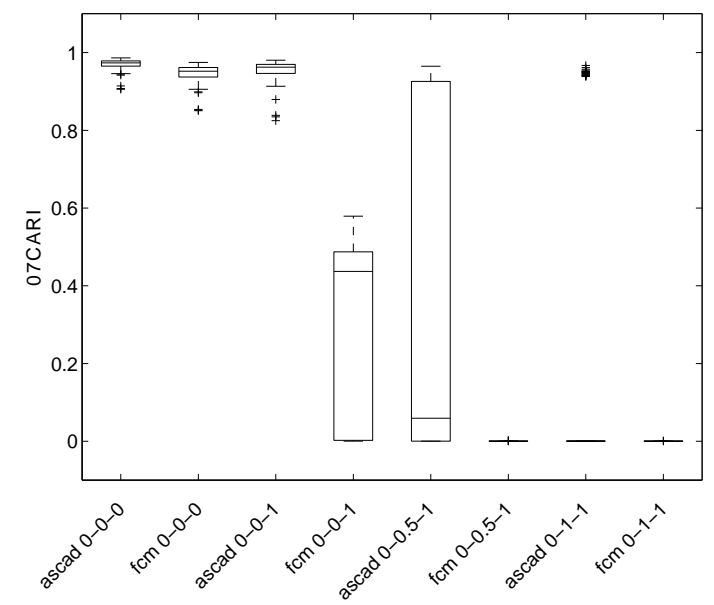

(c) MCL com $k^{*}=6$ e $a=50$.

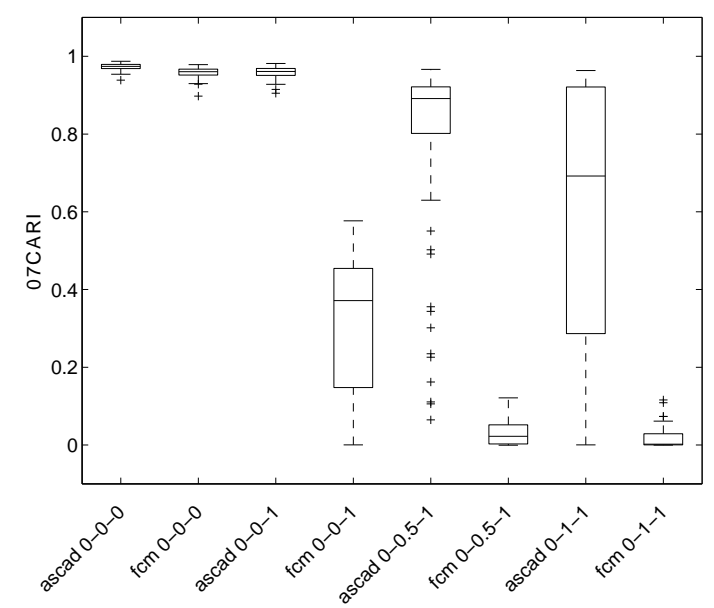

(e) MCL com $k^{*}=8$ e $a=10$.

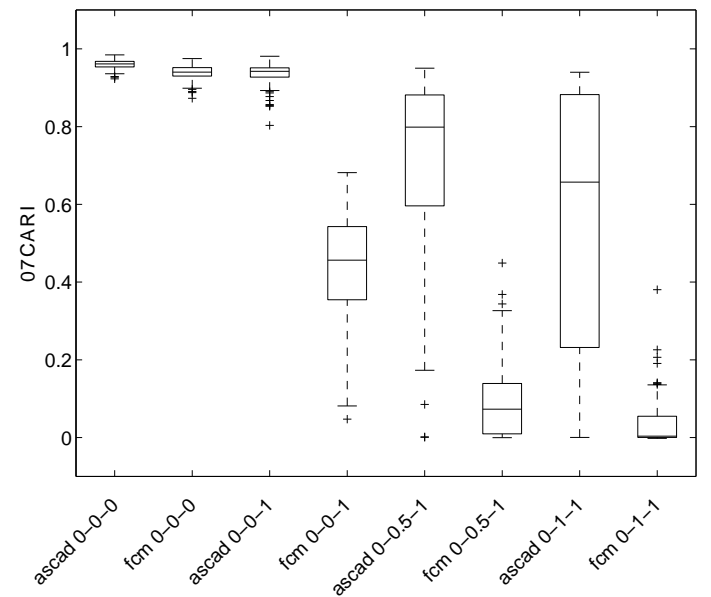

(b) MCL com $k^{*}=6$ e $a=10$.

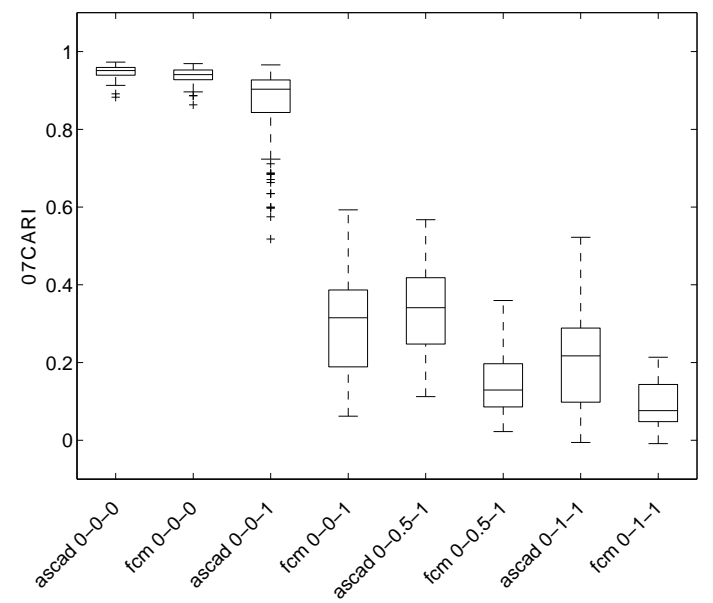

(d) MCL com $k^{*}=8$ e $a=3$.

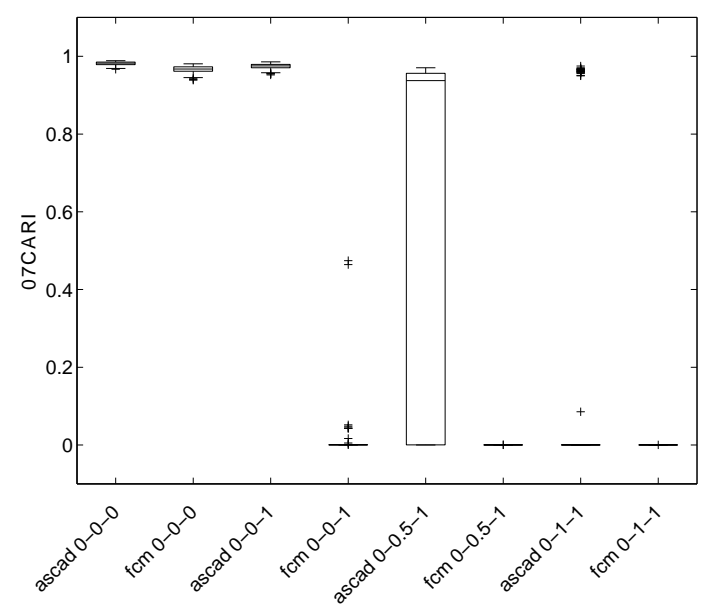

(f) MCL $\operatorname{com} k^{*}=8$ e $a=50$.

Figura 3.5: Resultados para as bases MCL com $k^{*}=6,8$ e $a=3,10,50$ (ASCAD vs FCM. 
Tabela 3.3: Percentual de vitórias, de acordo com o índice 07CARI, do algoritmo ASCAD sobre o algoritmo FCM para as bases MCL.

\begin{tabular}{lcccc}
\multicolumn{5}{c}{ vitórias do algoritmo } \\
\hline \hline BasC de dados & $0-0-0$ & $0-0-1$ & $0-0.5-1$ & $0-1-1$ \\
\hline MCL $k^{*}=2$ e $a=3$ & 54 & 87 & 78 & 71 \\
MCL $k^{*}=2$ e $a=10$ & 74 & 77 & 66 & 68 \\
MCL $k^{*}=2$ e $a=50$ & 99 & 89 & $\mathbf{4 8}$ & $\mathbf{4 9}$ \\
MCL $k^{*}=4$ e $a=3$ & 73 & 99 & 77 & 80 \\
MCL $k^{*}=4$ e $a=10$ & 99 & 100 & 91 & 83 \\
MCL $k^{*}=4$ e $a=50$ & 100 & 100 & 88 & 73 \\
MCL $k^{*}=6$ e $a=3$ & 90 & 100 & 85 & 71 \\
MCL $k^{*}=6$ e $a=10$ & 100 & 100 & 96 & 96 \\
MCL $k^{*}=6$ e $a=50$ & 100 & 100 & 90 & 76 \\
MCL $k^{*}=8$ e $a=3$ & 92 & 100 & 86 & 67 \\
MCL $k^{*}=8$ e $a=10$ & 100 & 100 & 100 & 98 \\
MCL $k^{*}=8$ e $a=50$ & 100 & 100 & 84 & 73
\end{tabular}

Figura 3.5(f),

As Figuras de 3.4(a) a 3.5(f) mostram que o algoritmo ASCAD foi superior ao FCM em quase todas as configurações de bases de dados. Alguns resultados (0-0-1 para $k^{*}=8$ e $a=3$, 0-0-1 para $k^{*}=6,8$ e $a=10$ e 0-0-1 para $k^{*}=8$ e $a=50$, por exemplo) apresentaram uma alta disparidade na qualidade das soluções. Embora poder-se-ia argumentar que isso é esperado dado que o espaço de busca do algoritmo ASCAD é maior e engloba o do algoritmo FCM, o primeiro possui um problema de otimização mais complexo a resolver.

Como as mesmas bases de dados foram usadas para ambos os algoritmos ASCAD e FCM. é possível fazer um pareamento entre os resultados e gerar os percentuais de vitórias do algoritmo ASCAD para os diferentes cenários (Tabela 3.3). Com o aumento da quantidade de ruído, espera-se que a tendência de agrupamento se enfraqueça (veja a Figura 3.3(b) para se ter uma ideia do enfraquecimento da tendência de agrupamento) e que ambos os algoritmos passem a não encontrar soluções tão boas. Esse comportamento é o que parece causar a menor discrepância no desempenho entre os algoritmos ASCAD e FCM apresentado nas colunas 0-0.5-1 e 0-1-1. De qualquer modo, fica evidente que o algoritmo ASCAD apresentou soluções com maior acurácia que o algoritmo FCM e foi capaz de lidar melhor com o ruído inserido do que esse último.

O teste de Wilcoxon pareado de dois lados (Apêndice E) foi aplicado nos valores de 07CARI e os resultados encontram-se na Tabela 3.4. Note que em apenas quatro casos, cujos valores estão em negrito, o teste estatístico não rejeitou a hipótese de que as distribuições de 07CARI possuem a mesma mediana (assumindo que as distribuições são simétricas) com o nível de significância $\alpha=0.01$. Portanto, há fortes indícios de que os resultados obtidos não se devem ao acasd 4

Esses resultados indicam que o algoritmo ASCAD pode ser útil para agrupar bases de dados

\footnotetext{
${ }^{4} \mathrm{O}$ Apêndice E Efornece uma visão geral do teste de Wilcoxon e de testes de hipótese de modo geral.
} 
Tabela 3.4: Resultados do teste de Wilcoxon pareado de dois lados para os valores de 07CARI dos algoritmos ASCAD E FCM para as bases MCL.

\begin{tabular}{|c|c|c|c|c|}
\hline Base de dados & $0-0-0$ & $0-0-1$ & $0-0.5-1$ & $0-1-1$ \\
\hline MCL $k^{*}=2$ e $a=3$ & 0.11 & 0.00 & 0.00 & 0.00 \\
\hline $\operatorname{MCL} k^{*}=2$ e $a=10$ & 0.00 & 0.00 & 0.04 & 0.04 \\
\hline $\operatorname{MCL} k^{*}=2$ e $a=50$ & 0.00 & 0.00 & 0.25 & 0.04 \\
\hline $\operatorname{MCL} k^{*}=4$ e $a=3$ & 0.00 & 0.00 & 0.00 & 0.00 \\
\hline $\operatorname{MCL} k^{*}=4$ e $a=10$ & 0.00 & 0.00 & 0.00 & 0.00 \\
\hline MCL $k^{*}=4$ e $a=50$ & 0.00 & 0.00 & 0.00 & 0.00 \\
\hline $\operatorname{MCL} k^{*}=6$ e $a=3$ & 0.00 & 0.00 & 0.00 & 0.00 \\
\hline $\operatorname{MCL} k^{*}=6$ e $a=10$ & 0.00 & 0.00 & 0.00 & 0.00 \\
\hline MCL $k^{*}=6$ e $a=50$ & 0.00 & 0.00 & 0.00 & 0.00 \\
\hline $\operatorname{MCL} k^{*}=8$ e $a=3$ & 0.00 & 0.00 & 0.00 & 0.00 \\
\hline $\operatorname{MCL} k^{*}=8$ e $a=10$ & 0.00 & 0.00 & 0.00 & 0.00 \\
\hline $\operatorname{MCL} k^{*}=8$ e $a=50$ & 0.00 & 0.00 & 0.00 & 0.00 \\
\hline
\end{tabular}

com atributos ruidosos por ser capaz de atribuir uma menor relevância a eles.

\subsubsection{Ponderação de representações}

São apresentados nesta seção vários experimentos envolvendo a coleção de imagens ALOI para avaliar a capacidade de ponderação de representações do algoritmo ASCAD e, portanto, dos algoritmos RASCAD e NERASCAD, A biblioteca ALOI é composta por 1000 categorias, cada qual contendo dezenas de fotografias de um objeto específico. Embora uma dada categoria tenha apenas imagens de um mesmo objeto, suas imagens são distintas devido a uma variação sistemática dos ângulos de visão e iluminação e da cor de iluminação. A Figura 3.6 apresenta três imagens de cada um dos grupos de xícara e de urso. Cada uma dessas duas categorias possui características particulares de cor, formato e textura. Portanto, é esperado que uma descrição razoável de tais objetos forme uma base de dados com tendência de agrupamento.

Pela seleção aleatória dessas categorias e as interpretando como grupos, 100 bases de dados foram criadas para cada $k^{*}=2,3,4,5$. As 400 bases de dados resultantes são denotadas por bases de dados ALOI. Cada grupo dessas 400 bases de dados é uma amostra sem reposição de tamanho 25 da categoria correspondente. Para representar essas fotografias, seis descritores de imagens foram extraídos: momentos de cor (144 atributos), estatísticas de textura extraídas da matriz de coocorrência de níveis de cinza (88 atributos), histograma de borda de Sobel (128 atributos), estatísticas de primeira ordem do histograma de níveis de cinza (6 atributos), propriedades da matriz de comprimento de corrida (run-length matrix, em inglês) de níveis de cinza (44 atributos) e o próprio histograma de níveis de cinza (256 atributos). Cada um desses descritores correspondem a uma visão diferente da base de dados e foi usado como tal pelo algoritmo ASCAD, Mais precisamente, seis matrizes de dados com 111 atributos em média representaram cada base de dados para o algoritmo ASCAD, enquanto para o algoritmo FCM cada base de dados foi representada por apenas uma matriz de dados formado pela concatenação dessas seis 


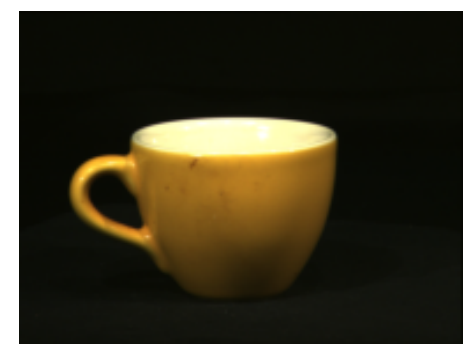

(a) Xícara na configuração 1 .

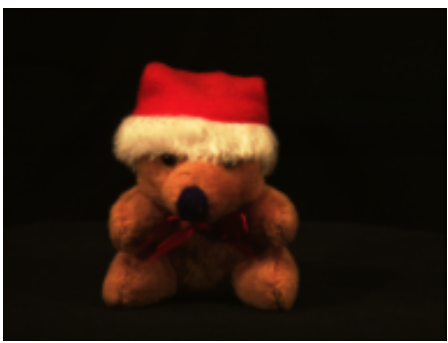

(d) Urso na configuração 1.

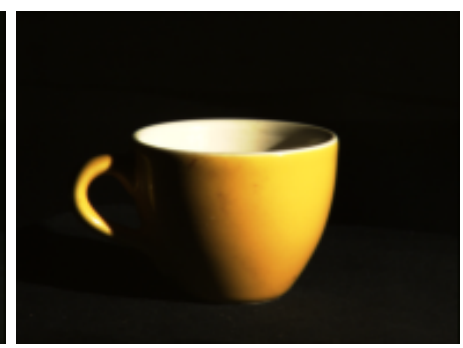

(b) Xícara na configuração 2.

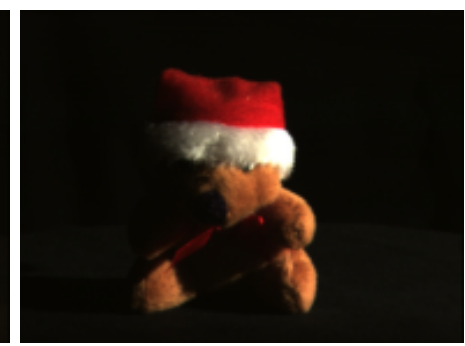

(e) Urso na configuração 2.

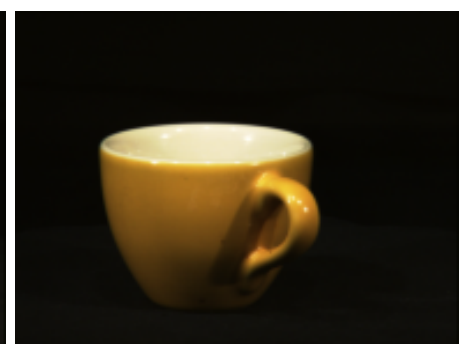

(c) Xícara na configuração 3.

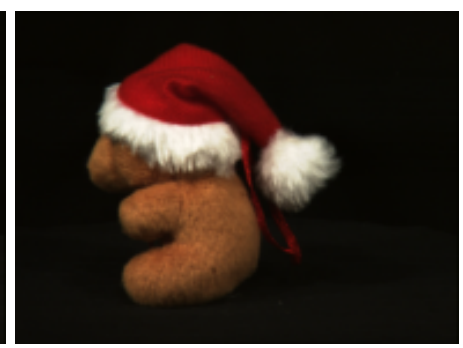

(f) Urso na configuração 3.

Figura 3.6: Amostras de duas categorias da coleção de imagens ALOI matrizes de dados.

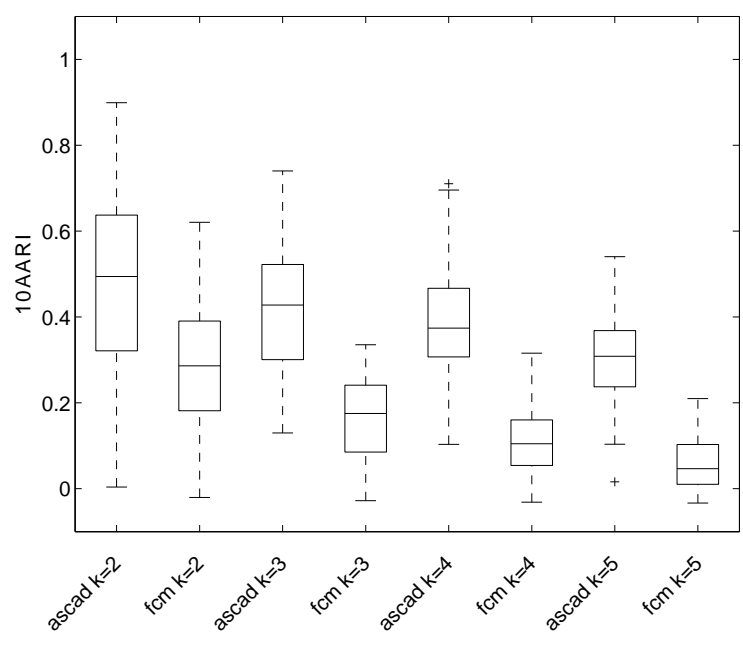

(a) Índice 10AARI.

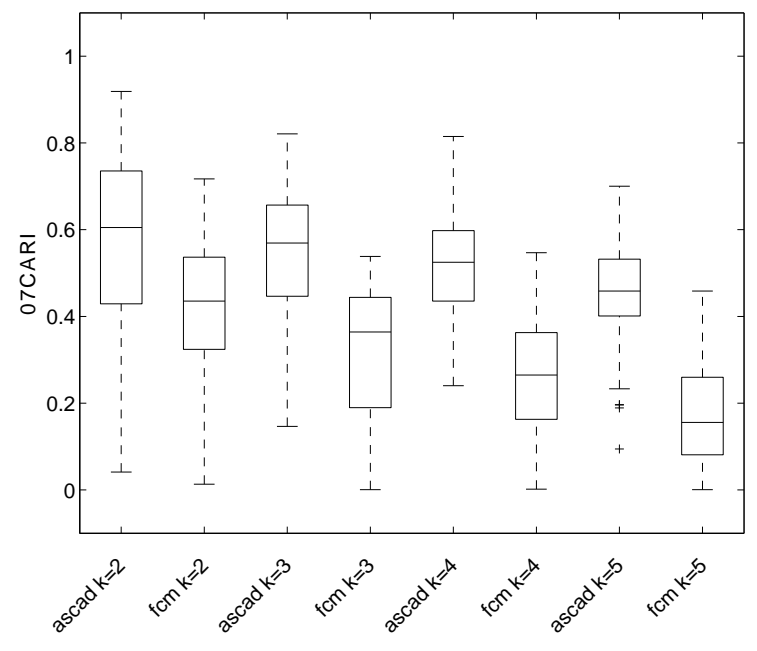

(b) Índice 07CARI.

Figura 3.7: Desempenho dos algoritmos ASCAD e FCM para as bases de dados ALOI sem redução na dimensionalidade (666 atributos).

Foram adotadas duas versões dessas bases. A versão original, contendo os 666 atributos, e uma versão de um atributo apenas por representação da base (i.e., seis atributos). Essa última versão foi obtida aplicando $\mathrm{ACP}$ em cada matriz de dados $\mathrm{X}^{s}$, separadamente. Note que embora cada representação dos dados nessa segunda versão seja composto por apenas uma atributo, ainda sim cada representação se refere a uma representação diferente da base de dados.

Os algoritmos FCM e ASCAD foram executados 30 vezes para cada base de dados com o número ideal de grupos $k^{*}$. A distância euclidiana quadrática foi adotada por ambos os algoritmos. O agrupamento nebuloso com o menor valor de função-custo desses 30 agrupamentos foi escolhido como a solução final. 


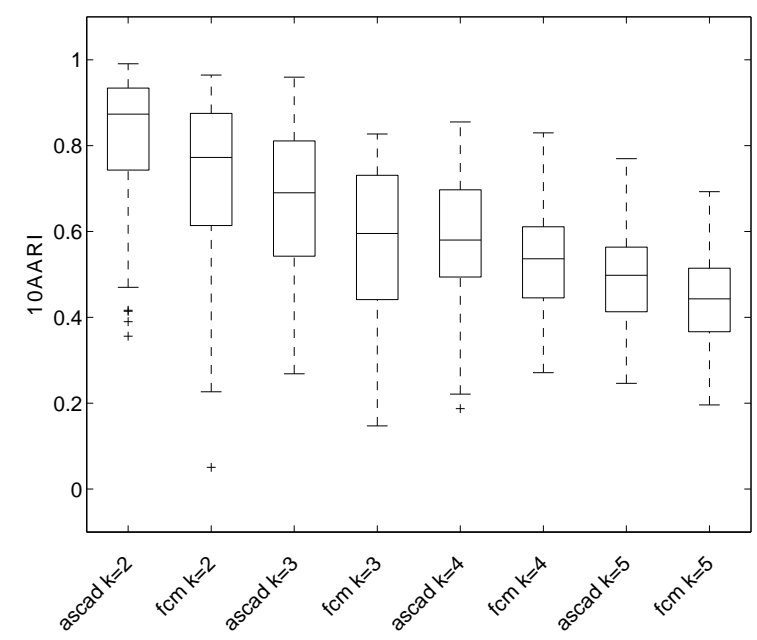

(a) Índice 10AARI.

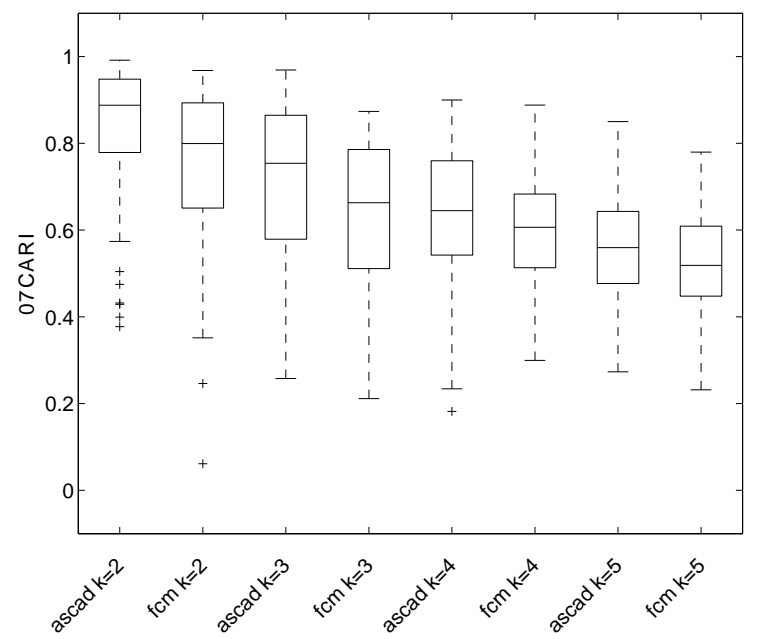

(b) Índice 07CARI.

Figura 3.8: Desempenho dos algoritmos ASCAD e FCM para as bases de dados ALOI com redução na dimensionalidade (seis atributos).

Tabela 3.5: Percentual de vitórias do algoritmo ASCAD sobre o algoritmo FCM para as bases ALOI sem redução na dimensionalidade (666 atributos).

\begin{tabular}{lcccc}
\multicolumn{5}{c}{ vitórias do algoritmo } \\
\hline \hline Índice & $k^{*}=2$ & $k^{*}=3$ & $k^{*}=4$ & $k^{*}=5$ \\
\hline 10AARI & 98 & 100 & 100 & 100 \\
07CARI & 94 & 99 & 100 & 100
\end{tabular}

As Figuras 3.7 e 3.8 apresentam os resultados de acordo com os índices 10AARI e 07CARI para as bases de dados sem e com redução de dimensionalidade, respectivamente. $\mathrm{O}$ algoritmo ASCAD foi superior ao FCM, em especial para as bases de dados sem redução de dimensionalidade. Nas bases de 666 atributos é de se esperar que vários dos atributos tenham pouca relevância ou atrapalhem o processo de agrupamento. É provável que essa maior discrepância nos desempenhos para as bases sem redução de dimensionalidade se deva à ponderação de representações que o algoritmo ASCADrealiza.

Tabela 3.6: Percentual de vitórias do algoritmo ASCAD sobre o algoritmo FCM para as bases ALOI com redução na dimensionalidade (6 atributos).

\begin{tabular}{lcccc}
\multicolumn{5}{c}{ vitórias do algoritmo } \\
\hline \hline Índice & $k^{*}=2$ & $k^{*}=3$ & $k^{*}=4$ & $k^{*}=5$ \\
\hline 10AARI & 96 & 88 & 89 & 87 \\
07CARI & 95 & 83 & 79 & 80
\end{tabular}

Como os resultados dos algoritmos são pareados, pôde-se calcular os percentuais de vitórias do algoritmo ASCAD frente ao algoritmo FCM, apresentados nas Tabelas 3.5 e 3.6. Esses resultados mostram que o algoritmo ASCAD quase não perdeu para o FCM nas bases ALOI sem redução de dimensionalidade e ganhou na maior parte dos cenários nas bases ALOI com redução de dimensionalidade. As Tabelas $3.7 \mathrm{e} 3.8$ corroboram com a validade das conclusões, 
as quais apresentam os valores-p do teste de Wilcoxon. Houve significância estatística em todos os cenários, tomando $\alpha=0.01$.

Tabela 3.7: Resultados do teste de Wilcoxon pareado de dois lados para os valores de 10AARI e 07CARI dos algoritmos ASCAD e FCM para as bases ALOI sem redução na dimensionalidade (666 atributos).

\begin{tabular}{|c|c|c|c|c|}
\hline \multicolumn{5}{|c|}{ valores-p ASCAD vs FCM } \\
\hline Índice & $k^{*}=2$ & $k^{*}=3$ & $k^{*}=4$ & $k^{*}=5$ \\
\hline 10AARI & 0.00 & 0.00 & 0.00 & 0.00 \\
\hline 07CARI & 0.00 & 0.00 & 0.00 & 0.00 \\
\hline
\end{tabular}

Tabela 3.8: Resultados do teste de Wilcoxon pareado de dois lados para os valores de 10AARI e 07CARI dos algoritmos ASCAD e FCM para as bases ALOI com redução na dimensionalidade (6 atributos).

\begin{tabular}{|c|c|c|c|c|}
\hline \multicolumn{5}{|c|}{ valores-p (ASCAD vs FCM } \\
\hline Índice & $k^{*}=2$ & $k^{*}=3$ & $k^{*}=4$ & $k^{*}=5$ \\
\hline 10AARI & 0.00 & 0.00 & 0.00 & 0.00 \\
\hline 07CARI & 0.00 & 0.00 & 0.00 & 0.00 \\
\hline
\end{tabular}

\subsubsection{Estimação automática do número de grupos}

Foi proposto na Seção 3.5 o índice SSNPR para incorporar a matriz de ponderação Y no índice SSN] Para determinar a sua efetividade na avaliação de agrupamentos com números de grupos distintos, o método Múltiplas Execuções Ordenadas ( $\mathrm{MEO}$, comumente adoptado na literatura e descrito a seguir, foi aplicado para estimar o número de grupos nas bases MCL descritas na Seção 3.6.3. Ou seja, contrariamente aos experimentos anteriores, o número de grupos não é fornecido. Para gerar agrupamentos e as matrizes de ponderação Y correspondentes por meio da abordagem MEO, o algoritmo ASCAD foi empregado. Por essa razão, a instância do método MEO é referida de MEO ASCAD, o qual é descrito agora em mais detalhes.

$\mathrm{O}$ algoritmo MEO ASCAD executa o algoritmo ASCAD $t=30$ vezes para um número crescente de grupos $k$. Para cada valor de $k$, os $t$ agrupamentos encontrados pelo ASCAD são avaliados por meio de um índice de validação relativa (SSNPR neste trabalho) para que o agrupamento de melhor qualidade seja armazenado para referência futura. Depois de executar o algoritmo ASCAD para cada valor de $k$ em uma determinada faixa, o melhor agrupamento (de acordo com o índice de validação relativa) é escolhido. Para este trabalho, o conjunto de inteiros $\{2,3, \cdots, \sqrt{n}\}$ foi adotado (o limite superior de $\sqrt{n}$ é comumente usado como uma "regra de ouro" na literatura (Pal e Bezdek, 1995; Pakhira et al., 2005)), no qual $n$ é o número de objetos da base de dados.

As Figuras 3.9 e 3.10 apresentam os resultados das avaliações de acordo com o índice 07CARI. Os resultados para o índice 10AARI foram bem semelhantes, portanto foram omitidos. Como pode ser visto, o algoritmo MEO ASCAD obteve bons resultados em comparação 


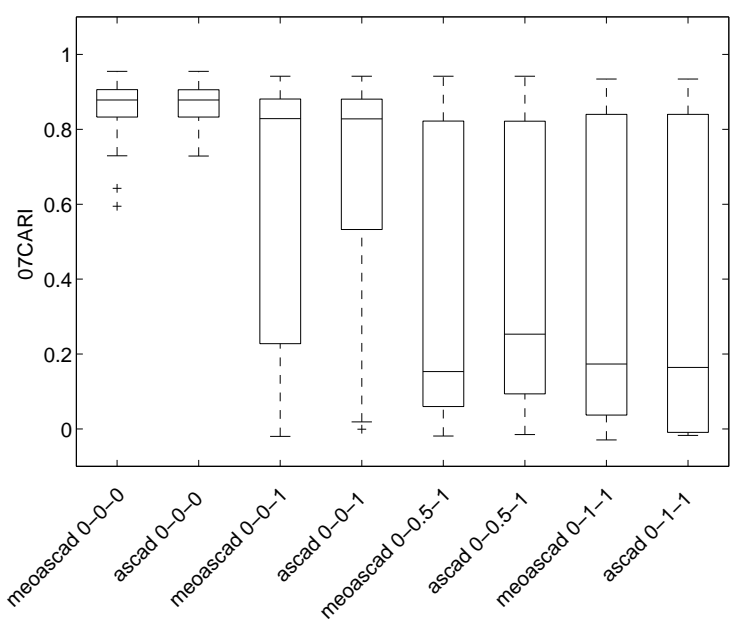

(a) MCL $\operatorname{com} k^{*}=2 \mathrm{e} a=3$.

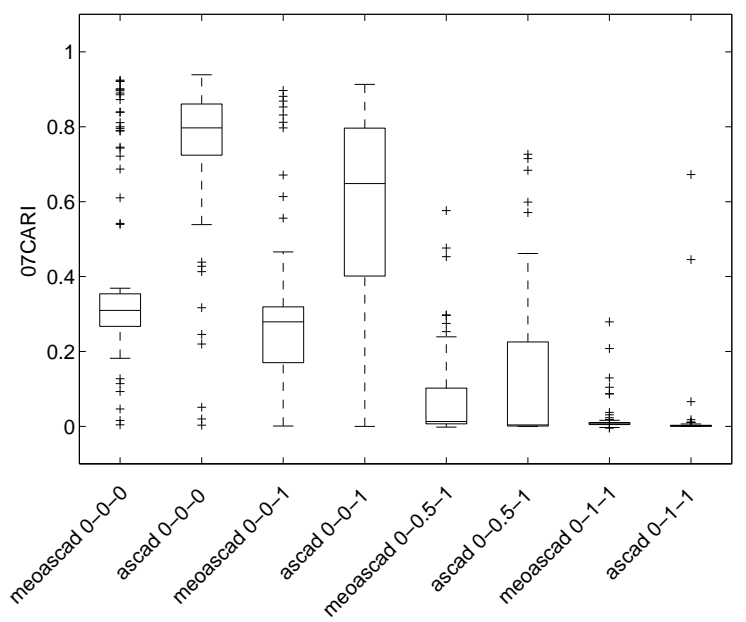

(c) MCL com $k^{*}=2$ e $a=50$.

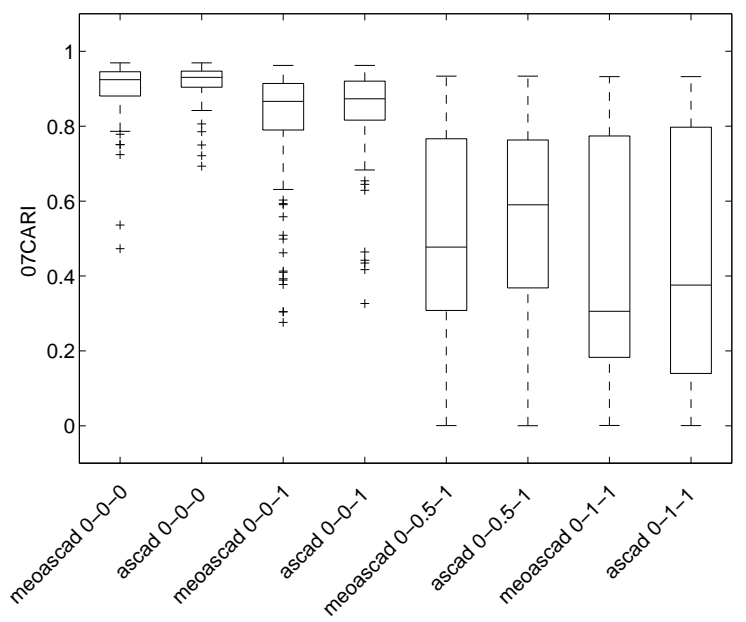

(e) MCL com $k^{*}=4$ e $a=10$.

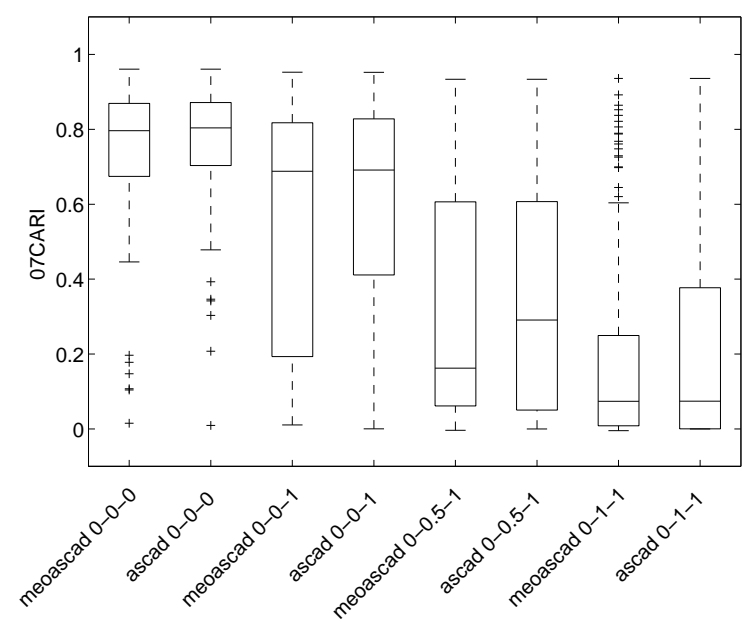

(b) MCL com $k^{*}=2$ e $a=10$.

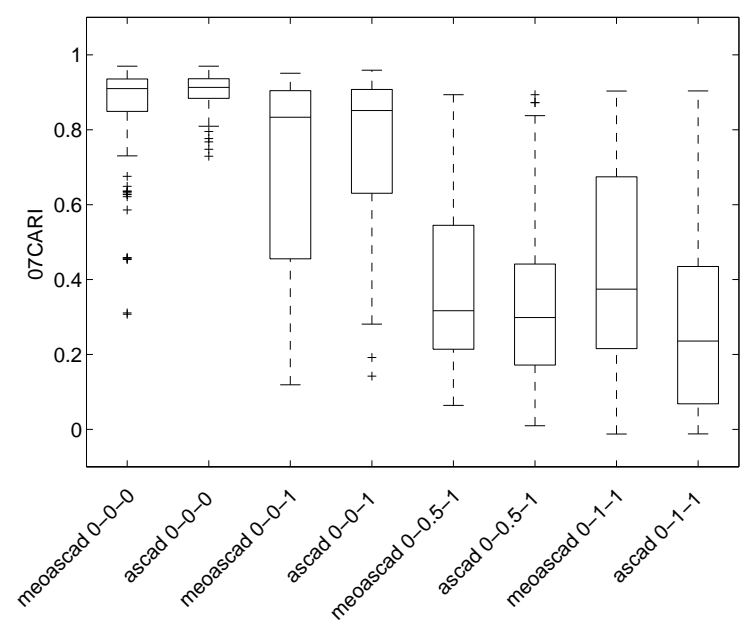

(d) MCL $\operatorname{com} k^{*}=4$ e $a=3$.

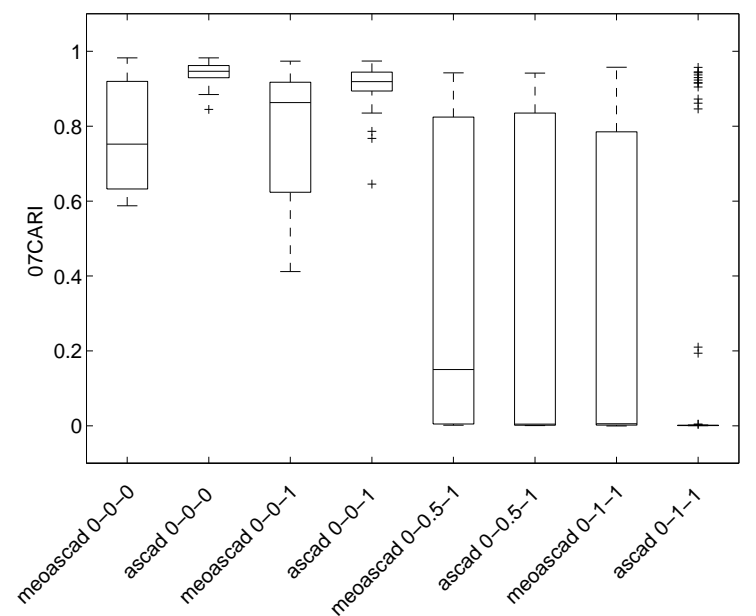

(f) $k^{*}=4$ e $a=50$.

Figura 3.9: Valores do índice 07CARI para o algoritmo MEOASCAD aplicado nas bases MCL de $k^{*}=2,4$ e $a=3,10,50$. 


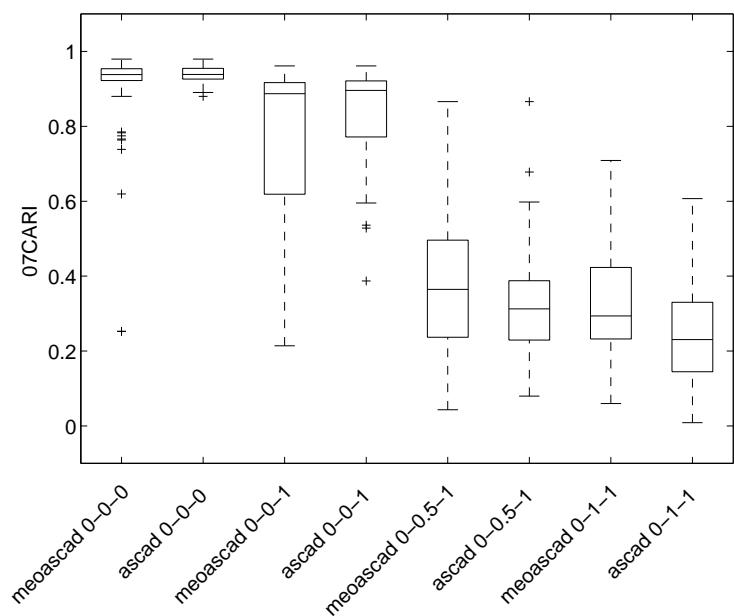

(a) MCL com $k^{*}=6$ e $a=3$.

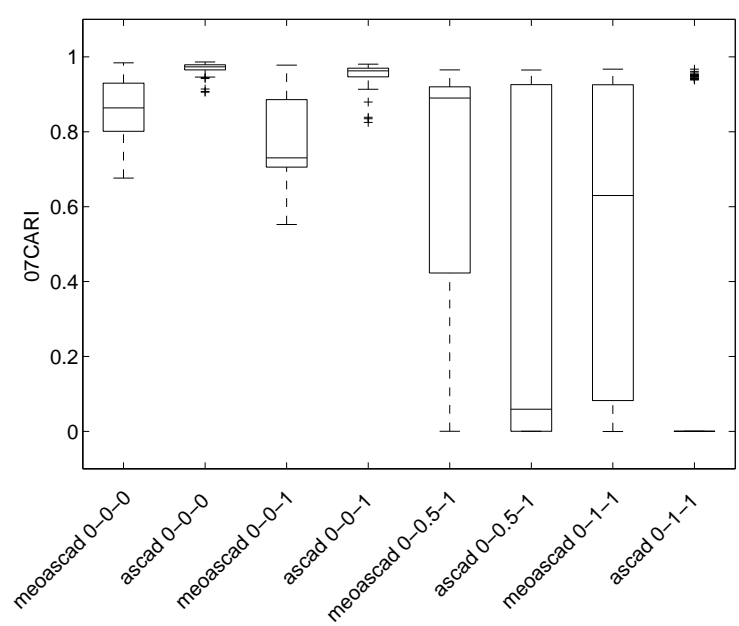

(c) MCL com $k^{*}=6$ e $a=50$.

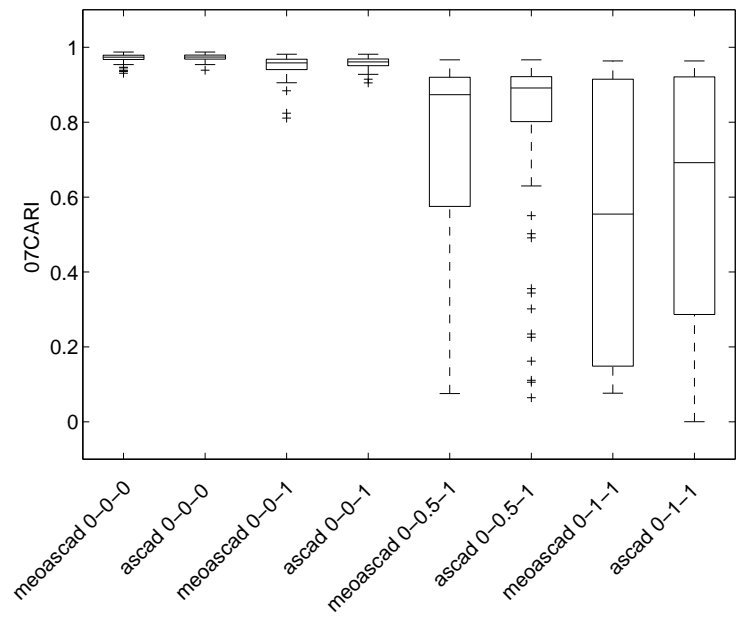

(e) MCL com $k^{*}=8$ e $a=10$.

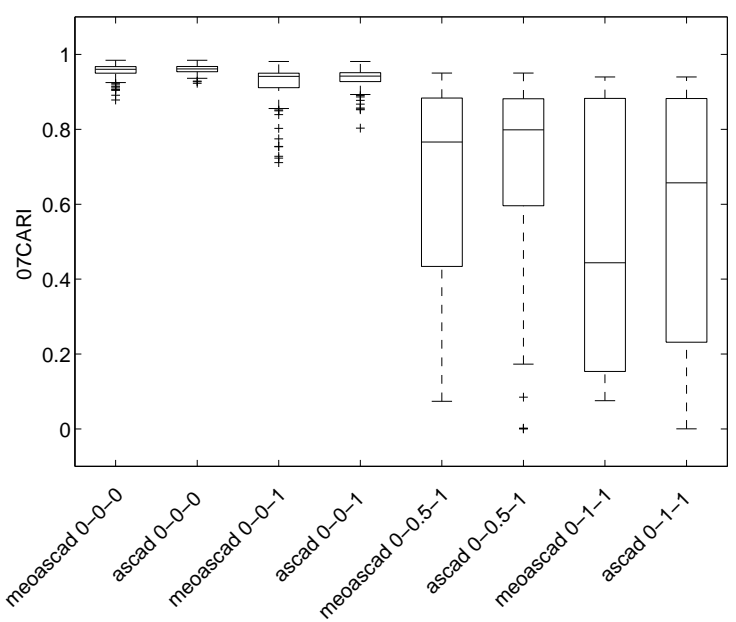

(b) MCL $\operatorname{com} k^{*}=6$ e $a=10$.

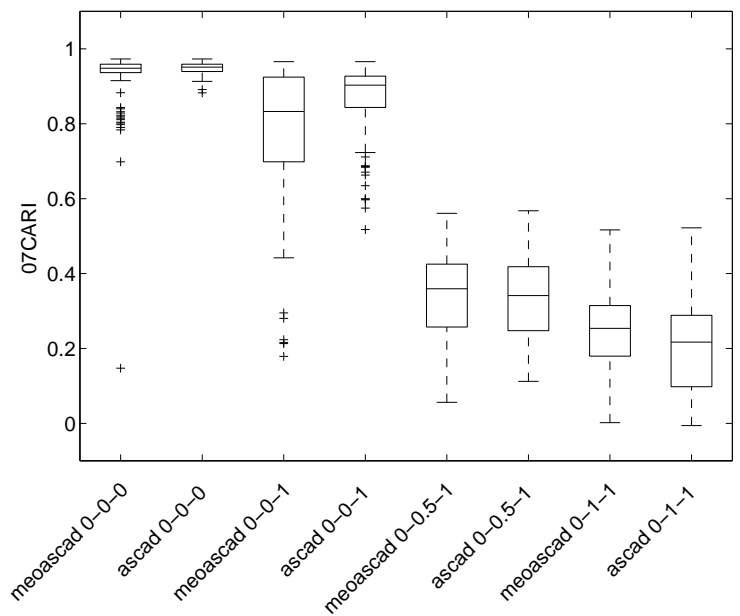

(d) MCL com $k^{*}=8$ e $a=3$.

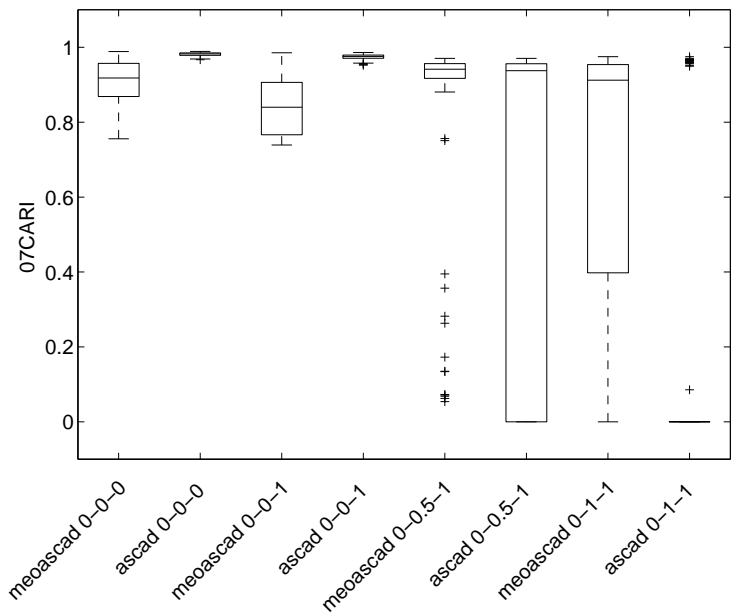

(f) $\mathrm{MCL} \operatorname{com} k^{*}=8$ e $a=50$.

Figura 3.10: Valores do índice 07CARI para o algoritmo MEOASCAD aplicado nas bases MCL de $k^{*}=6,8$ e $a=3,10,50$. 
com os resultados do algoritmo ASCAD, quando o número de grupos ideal é fornecido ao algoritmo. Há, portanto, fortes indícios de que o índice SSNPR pôde guiar corretamente o algoritmo MEO ASCAD na busca por agrupamentos.

O método MEO ASCAD foi também aplicado com o índice SSN para verificar se o uso da ponderação no índice SSNPR foi realmente um avanço na avaliação das soluções. As Figs. 3.11 e 3.12 mostram que a silhueta com ponderação de fato teve um desempenho consideravelmente superior ao da silhueta nebulosa original na maioria dos cenários para as bases MCL, mensurado pelo índice 07CARI. Os resultados para as bases reais ALOI apresentados pelas Figs. 3.13 apontam uma ligeira superioridade do índice SSNPR. As Tabelas 3.9 e 3.10 mostram a comparação pareada para as bases ALOI, o que comprova que o índice SSNPR de fato foi superior ao índice SSN

Tabela 3.9: Percentual de vitórias do algoritmo MEOASCAD com o índice SSNPR sobre o mesmo algoritmo com o índice SSN para as bases ALOI sem redução na dimensionalidade (666 atributos).

vitórias do índice SSNPR $(\%)$

\begin{tabular}{lcccc}
\hline \hline Índice & $k^{*}=2$ & $k^{*}=3$ & $k^{*}=4$ & $k^{*}=5$ \\
\hline 10AARI & 65 & 51 & 51 & 48 \\
07CARI & 65 & 57 & 64 & 51
\end{tabular}

Tabela 3.10: Percentual de vitórias do algoritmo MEOASCAD com o índice SSNPR sobre o mesmo algoritmo com o índice SSN para as bases ALOI com redução na dimensionalidade (6 atributos).

\begin{tabular}{lcccc}
\multicolumn{5}{c}{ vitórias do índice } \\
\hline \hline Índice & $k^{*}=2$ & $k^{*}=3$ & $k^{*}=4$ & $k^{*}=5$ \\
\hline 10AARI & 66 & 76 & 62 & 70 \\
07CARI & 61 & 72 & 58 & 63
\end{tabular}

\footnotetext{
${ }^{5}$ Os valores de $k$ nos rótulos das figuras representam as diferentes bases de dados. O número de grupos foi estimado pela própria abordagem empregada (i.e., MEOASCAD em conjunto com o respectivo índice interno).
} 


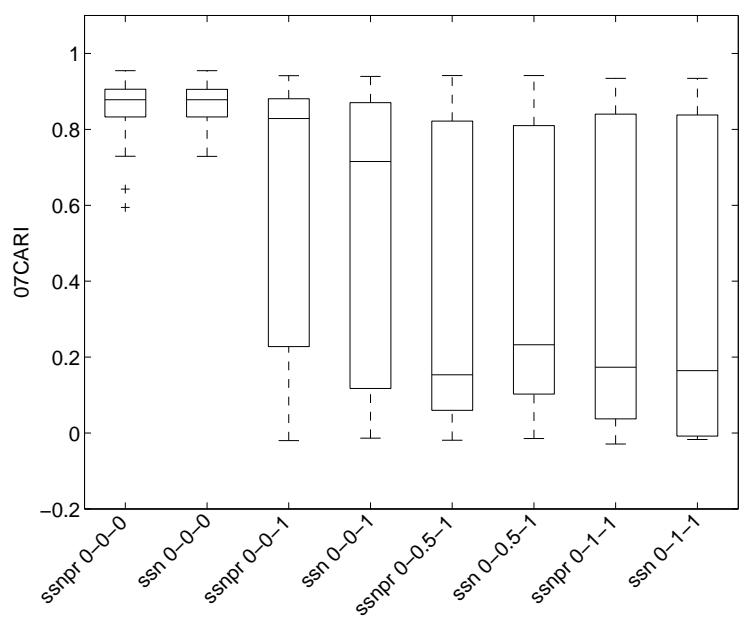

(a) MCL com $k^{*}=2$ e $a=3$.

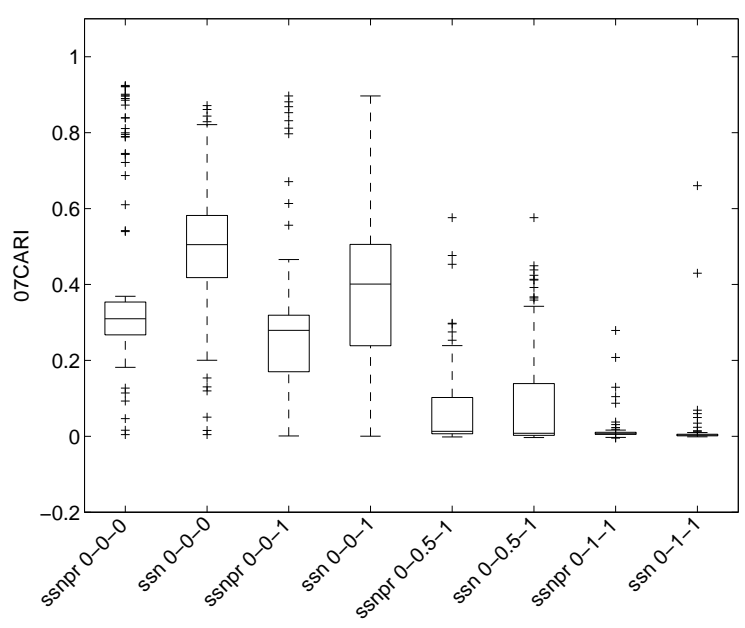

(c) MCL com $k^{*}=2$ e $a=50$.

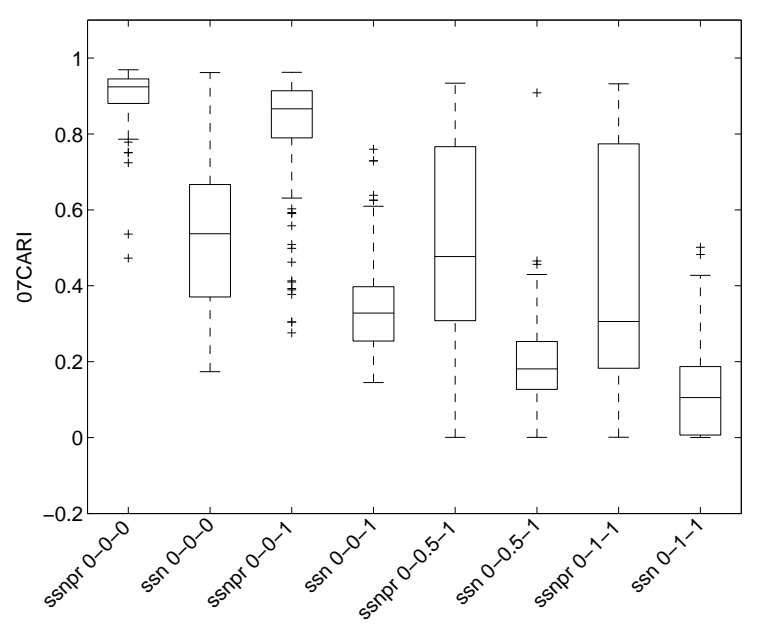

(e) MCL com $k^{*}=4$ e $a=10$.

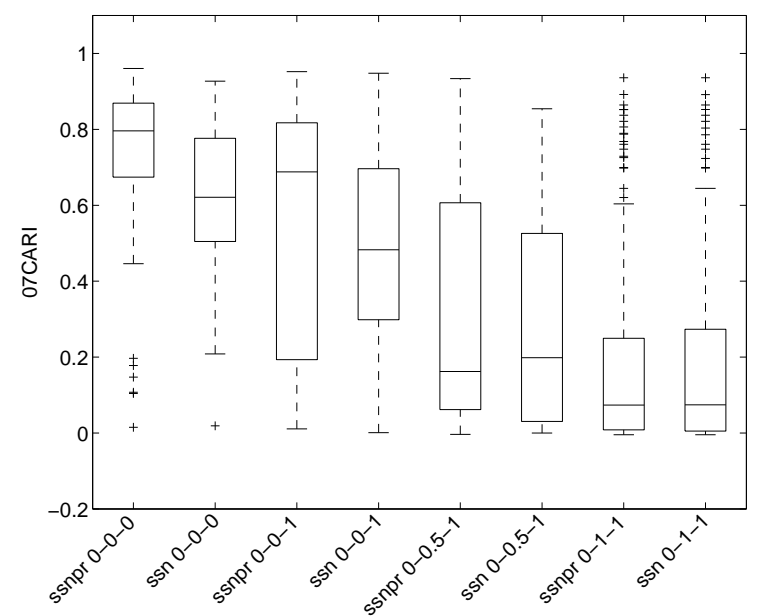

(b) MCL com $k^{*}=2$ e $a=10$.

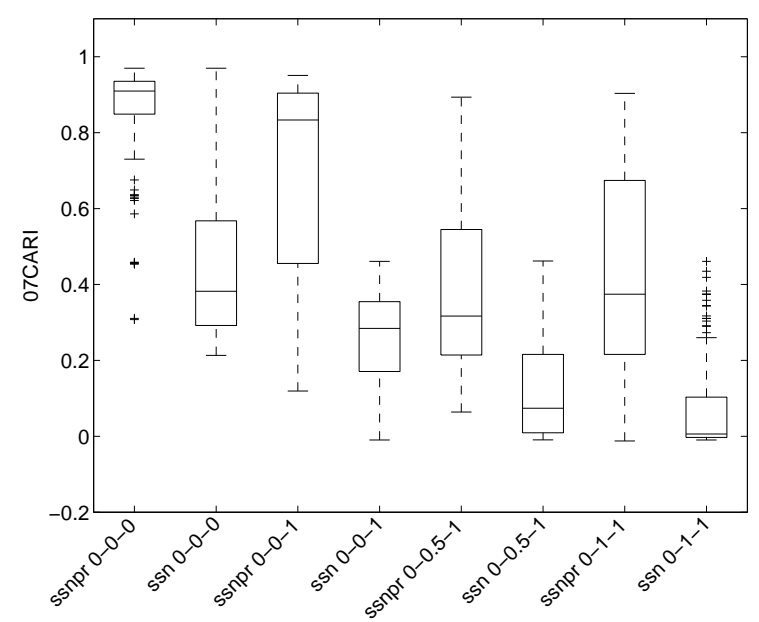

(d) MCL com $k^{*}=4$ e $a=3$.

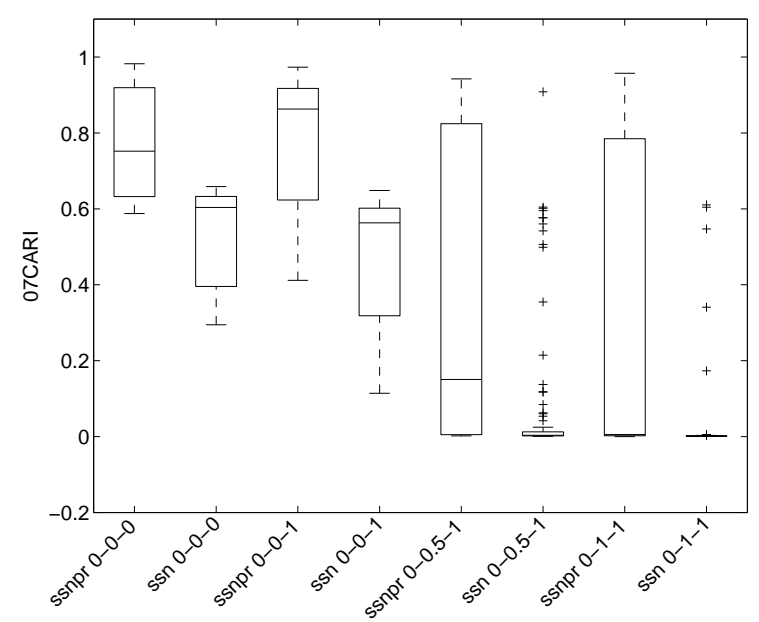

(f) $k^{*}=4$ e $a=50$.

Figura 3.11: Valores do índice 07CARI para o algoritmo MEO ASCAD com o índice SSN e com o índice SSNPR nas bases MCL de $k^{*}=2,4$ e $a=3,10,50$. 


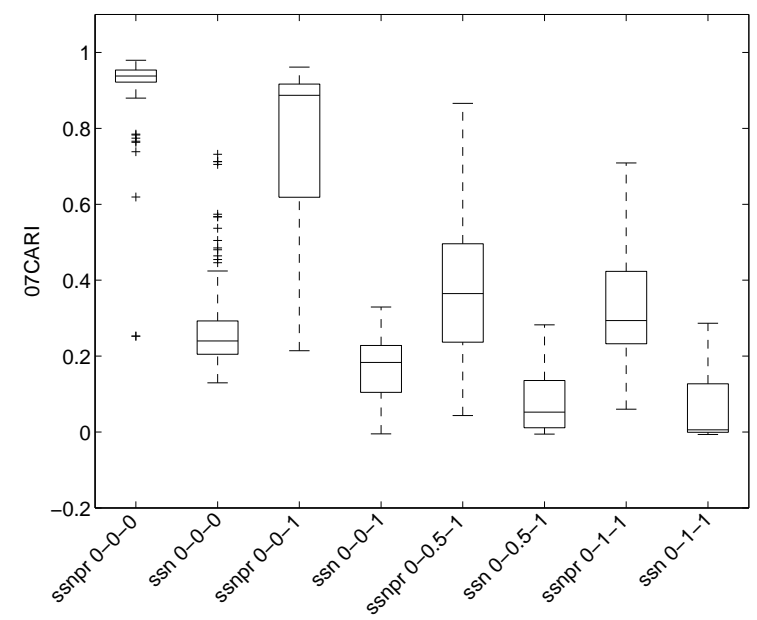

(a) MCL $\operatorname{com} k^{*}=6$ e $a=3$.

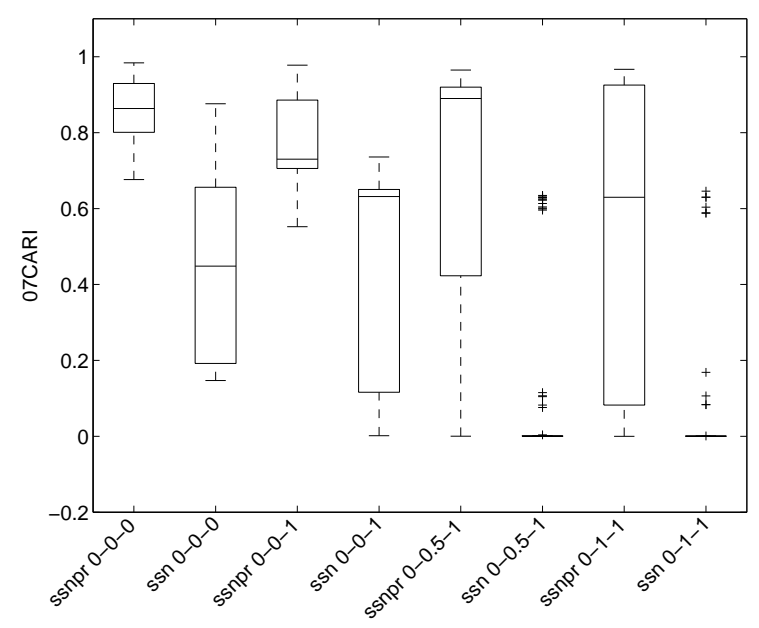

(c) MCL com $k^{*}=6$ e $a=50$.

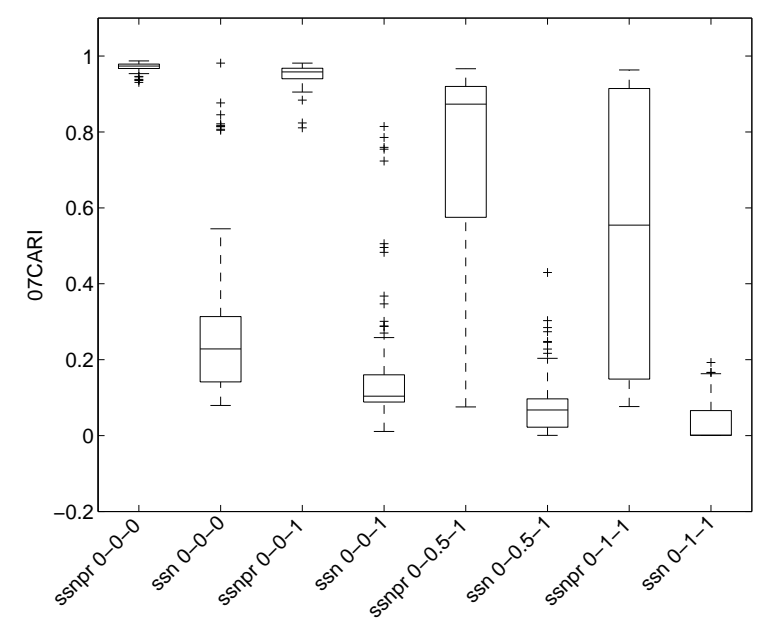

(e) MCL com $k^{*}=8$ e $a=10$.

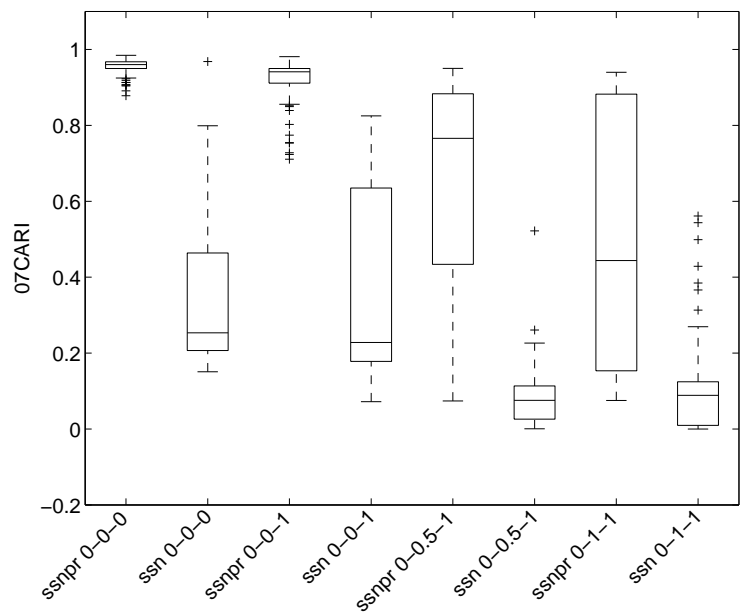

(b) MCL com $k^{*}=6$ e $a=10$.

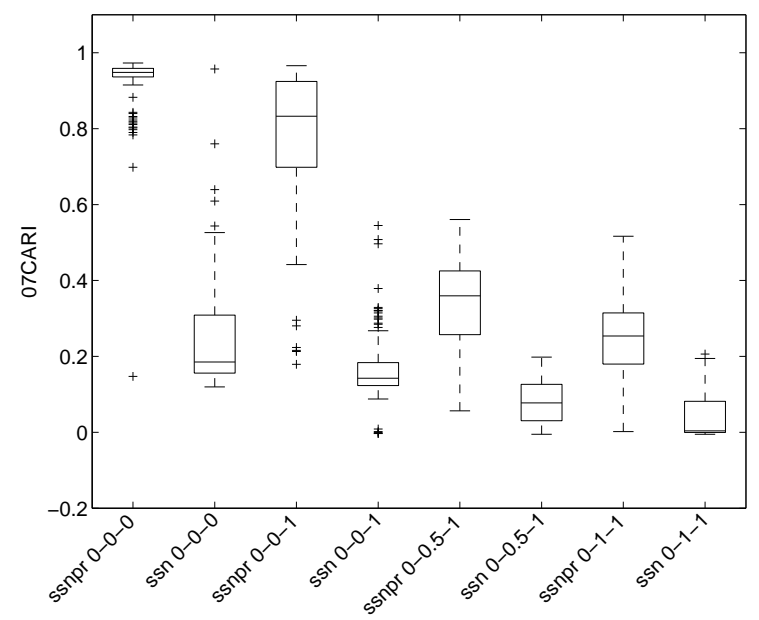

(d) MCL $\operatorname{com} k^{*}=8$ e $a=3$.

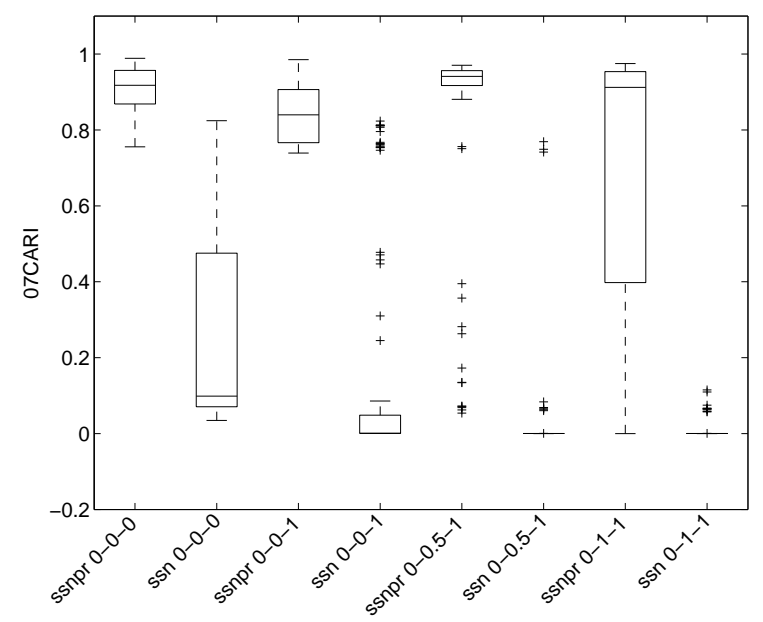

(f) MCL com $k^{*}=8$ e $a=50$.

Figura 3.12: Valores do índice 07CARI para o algoritmo MEOASCAD com o índice SSN e com o índice SSNPR nas bases MCL de $k^{*}=6,8$ e $a=3,10,50$. 


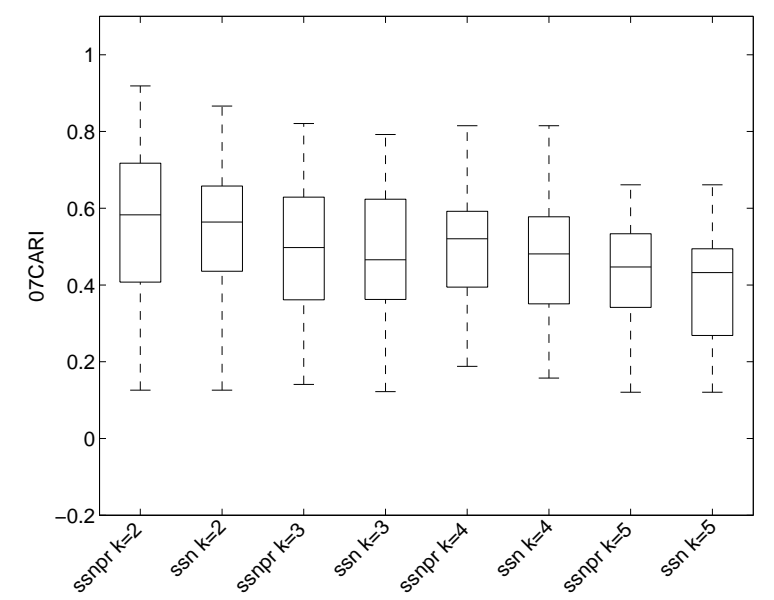

(a) ALOI sem redução na dimensionalidade (666 atributos).

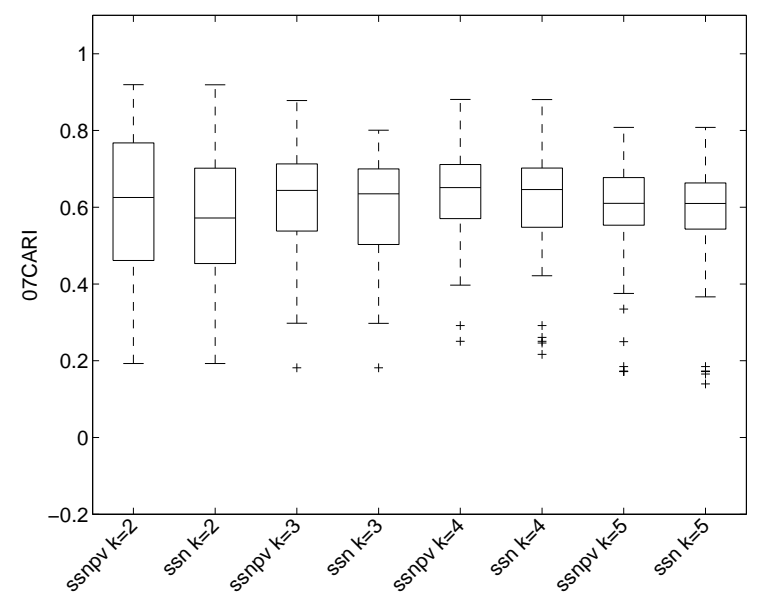

(b) ALOI com redução na dimensionalidade (seis atributos).

Figura 3.13: Valores do índice 07CARI para o algoritmo MEO ASCAD com o índice SSN e com o índice SSNPR nas bases ALOI. 


\subsection{Considerações}

Na Seção 3.1 foram discutidos alguns dos problemas do algoritmo SCAD dentre os quais incluem a possibilidade de falha em sua execução. Na mesma seção esses problemas foram sanados, dando origem ao algoritmo ASCAD. Por motivos ilustrativos, foi definido o algoritmo HASCAD, uma versão particional exclusiva do algoritmo ASCAD, na Seção 3.1.1. A análise relatada na Seção 3.1 .2 mostrou que a complexidade computacional assintótica do algoritmo ASCAD é linear em relação aos números de objetos e de grupos. De fato, ASCAD apresenta a mesma complexidade do algoritmo FCM.

O algoritmo CARD, versão relacional do SCAD, apresenta problemas conceituais e pode falhar em determinadas situações de maneira semelhante à sua versão não relacional. Essas questões foram discutidas nas Seções 3.2 e 3.3, complementando o que já foi apresentado na Seção 2.4.2.

Uma versão relacional do algoritmo ASCAD, denominada RASCAD, foi apresentada na Seção 3.2. Foi provado que eles são equivalentes, sob uma hipótese realista na prática. Esse fato é importante porque garante que o algoritmo RASCAD tem o mesmo desempenho, em termos de acurácia, que o algoritmo ASCAD mesmo sem a presença dos atributos da base, simplificando a análise experimental de ambos.

Uma generalização do algoritmo RASCAD, denominada NERASCAD, capaz de lidar também com matrizes relacionais não euclidianas foi desenvolvida. Essa versão é aplicável a qualquer tipo de base representada por matrizes relacionais.

Os teoremas desenvolvidos na Seção 3.4 provam que os algoritmos propostos de fato minimizam suas funções-custo, fornecendo uma garantia de convergência e uma base teórica para análises futuras.

Os experimentos relatados na Seção 3.6 mostram, entre outras coisas, que o algoritmo ASCAD é capaz de lidar com dados multi-representados e com dados ruidosos. A primeira característica foi investigada em um grande conjunto de imagens reais, representadas por seis representações distintas, nas quais experimentos mostraram que o algoritmo ASCAD se desempenhou melhor que o algoritmo FCM. Os resultados apontam que o algoritmo ASCAD foi superior, com significância estatística, tanto nos experimentos com dados multi-representados como nos experimentos com dados ruidosos.

Foi proposto o índice SSNPR, o qual avalia internamente agrupamentos nebulosos que possui associado a si uma ponderação das diferentes representações dos dados. Esse índice foi comparado com a sua versão anterior, [SSN, na qual a ponderação não é considerada, e mostrou resultados claramente superiores em quase todas as bases adotadas. O índice proposto também foi usado em uma abordagem que estima o número de grupos, apresentando resultados comparáveis com os resultados obtidos quando o número de grupos ideal foi fornecido. 


\section{$-4$ MEDIDAS DE COMPARAÇÃO DE AGRUPAMENTOS}

Como discutido na Seção 2.5, a abordagem mais comum na comparação de algoritmos de agrupamento de dados consiste na comparação das similaridades entre os agrupamentos gerados por tais algoritmos e um agrupamento de referência. Isto é, um algoritmo $\tilde{a}$ é comumente considerado melhor que $\tilde{b}$ para uma dada base $X$ se $\tilde{a}$ produz agrupamentos que são mais similares (de acordo com uma medida de agrupamento) a uma solução de referência de $X$ do que os agrupamentos gerados por $\tilde{b}$. Como vários algoritmos de agrupamento são publicados todos os anos, o desenvolvimento de medidas cada vez mais efetivas para comparar agrupamentos é indispensável (Vinh et al., 2009, 2010).

Medidas de similaridade entre agrupamentos também são usadas para agrupamento consensual (consensus clustering), avaliação da estabilidade de agrupamento e até para a quantificação da perda de informação (Strehl e Ghosh, 2003; Monti et al., 2003; Yu et al., 2007; Beringer e Hüllermeier, 2007; Vinh e Epps, 2009). Técnicas de agrupamento consensual objetivam encontrar soluções de alta qualidade combinando várias soluções obtidas por diferentes métodos, inicializações do algoritmo ou perturbações da mesma base de dados. Essa combinação é alcançada ao produzir uma agrupamento que compartilhe o máximo de informação, quantificada por uma medida de similaridade, com as soluções originais (Strehl e Ghosh, 2003).

No contexto de avaliação da estabilidade de agrupamento, o método que gerou um conjunto de soluções é considerado estável se o conjunto apresenta um baixo grau de variedade de soluções (Kuncheva e Vetrov, 2006). Pode-se aplicar um algoritmo de agrupamento várias vezes em subamostras do conjunto original de dados para diferentes números de grupos. O número de grupos do conjunto de soluções que apresentou a menor diversidade é considerado uma boa estimativa do número real de grupos (Borgelt e Kruse, 2006, Vinh e Epps, 2009).

Uma outra aplicação interessante para medidas de similaridade consiste na quantificação 
da perda de informação (Beringer e Hüllermeier, 2007). Para melhorar a efetividade de uma técnica de agrupamento (e.g., no contexto de agrupamento em fluxo de dados), pode-se mapear os dados em um espaço de menor dimensionalidade e só então realizar o agrupamento dos dados. Se a transformação ocorre com pouca perda de informação, aferida por uma medida de similaridade de agrupamento, espera-se que uma eventual estrutura presente nos dados tenha se preservada no espaço transformado.

As aplicações acima apresentadas, dentre outras não mencionadas, motivaram o desenvolvimento de medidas de similaridade para agrupamentos mais gerais que os particionais exclusivos e para bi-agrupamentos. Neste capítulo, portanto, é apresentada uma revisão crítica de medidas de similaridade para agrupamentos possibilísticos (Seção 4.2) e para bi-agrupamentos (Seção 4.3). A fim de situar melhor as medidas para bi-agrupamento, na Seção 4.3 são também discutidas as formas mais comuns de avaliação de bi-agrupamento em dados de expressão gênica. A seção seguinte apresenta conceitos fundamentais para o assunto do capítulo. Considerações finais são feitas na Seção 4.4 .

\subsection{Fundamentação}

As medidas mais populares para comparar agrupamentos particionais exclusivos são aquelas baseadas em contagem de pares, o que inclui os índices RI e ARI definidos na Seção 2.5. Uma abordagem comum para calcular tais medidas consiste na obtenção da matriz de contingência (Albatineh et al., 2006). Sejam $\mathrm{U} \in M_{\mathrm{e}}$ e $\mathrm{V} \in M_{\mathrm{e}}$ dois agrupamentos com $k_{\mathrm{U}}$ e $k_{\mathrm{V}}$ grupos, respectivamente, da mesma base de dados com $n$ objetos. A tabela de contingência é definida pela Tabela 4.1, em que $\mathrm{N}=\mathrm{UV}^{\mathrm{T}}$ é a matriz de contingência e $\mathrm{N}_{r, t}$ é o número de objetos que simultaneamente pertencem ao $r$-ésimo grupo de $\mathrm{U}$ e ao $t$-ésimo grupo de $\mathrm{V}$. As somas marginais $\mathrm{N}_{+, t} \triangleq \sum_{r=1}^{k_{\mathrm{U}}} \mathrm{N}_{r, t}$ e $\mathrm{N}_{r,+} \triangleq \sum_{t=1}^{k_{\mathrm{V}}} \mathrm{N}_{r, t}$ representam os tamanhos dos grupos e a soma total $\mathrm{N}_{+,+} \triangleq \sum_{r, t=1}^{k_{\mathrm{U}}, k_{\mathrm{V}}} \mathrm{N}_{r, t}=n$ representa o número de objetos da base. A matriz de contingência é então usada para calcular as variáveis de pareamento $a, b, c$ e $d$ definidas de maneira descritiva na Seção 2.5 (Jain e Dubes, 1988; Albatineh et al., 2006). Essas variáveis podem ser calculadas da seguinte forma:

$$
\begin{aligned}
& a=\sum_{r, t=1}^{k_{\mathrm{U}}, k_{\mathrm{V}}}\left(\begin{array}{c}
\mathrm{N}_{r, t} \\
2
\end{array}\right)=\frac{1}{2} \sum_{r, t=1}^{k_{\mathrm{U}}, k_{\mathrm{V}}} \mathrm{N}_{r, t}^{2}-\frac{\mathrm{N}_{+,+}}{2} \\
& b=\sum_{r=1}^{k_{\mathrm{U}}}\left(\begin{array}{c}
\mathrm{N}_{r,+} \\
2
\end{array}\right)-a=\frac{1}{2} \sum_{r=1}^{k_{\mathrm{U}}} \mathrm{N}_{r,+}^{2}-\frac{1}{2} \sum_{r, t=1}^{k_{\mathrm{U}}, k_{\mathrm{V}}} \mathrm{N}_{r, t}^{2} \\
& c=\sum_{t=1}^{k_{\mathrm{V}}}\left(\begin{array}{c}
\mathrm{N}_{+, t} \\
2
\end{array}\right)-a=\frac{1}{2} \sum_{t=1}^{k_{\mathrm{V}}} \mathrm{N}_{+, t}^{2}-\frac{1}{2} \sum_{r, t=1}^{k_{\mathrm{U}}, k_{\mathrm{V}}} \mathrm{N}_{r, t}^{2} \\
& d=\left(\begin{array}{c}
\mathrm{N}_{+,+} \\
2
\end{array}\right)-(a+b+c)=\frac{1}{2} \mathrm{~N}_{+,+}^{2}-\frac{1}{2}\left(\sum_{r=1}^{k_{\mathrm{U}}} \mathrm{N}_{r,+}^{2}+\sum_{t=1}^{k_{\mathrm{V}}} \mathrm{N}_{+, t}^{2}\right)+\frac{1}{2} \sum_{r, t=1}^{k_{\mathrm{U}}, k_{\mathrm{V}}} \mathrm{N}_{r, t}^{2}
\end{aligned}
$$


Tabela 4.1: Tabela de contingência.

\begin{tabular}{c|cccc|c}
$\mathrm{U} / \mathrm{V}$ & $\mathrm{V}_{1,:}$ & $\mathrm{V}_{2,:}$ & $\cdots$ & $\mathrm{V}_{k_{\mathrm{V}},:}$ & Somas \\
\hline $\mathrm{U}_{1,:}$ & $\mathrm{N}_{1,1}$ & $\mathrm{~N}_{1,2}$ & $\cdots$ & $\mathrm{N}_{1, k_{\mathrm{V}}}$ & $\mathrm{N}_{1,+}$ \\
$\mathrm{U}_{2,:}$ & $\mathrm{N}_{2,1}$ & $\mathrm{~N}_{2,2}$ & $\cdots$ & $\mathrm{N}_{2, k_{\mathrm{V}}}$ & $\mathrm{N}_{2,+}$ \\
$\vdots$ & $\vdots$ & $\vdots$ & $\ddots$ & $\vdots$ & $\vdots$ \\
$\mathrm{U}_{k_{\mathrm{U}},:}$ & $\mathrm{N}_{k_{\mathrm{U}}, 1}$ & $\mathrm{~N}_{k_{\mathrm{U}}, 2}$ & $\cdots$ & $\mathrm{N}_{k_{\mathrm{U}}, k_{\mathrm{V}}}$ & $\mathrm{N}_{k_{\mathrm{U}},+}$ \\
\hline Somas & $\mathrm{N}_{+, 1}$ & $\mathrm{~N}_{+, 2}$ & $\cdots$ & $\mathrm{N}_{+, k_{\mathrm{V}}}$ & $\mathrm{N}_{+,+}$
\end{tabular}

Albatineh et al. (2006) listaram 22 medidas baseadas em contagem de pares definidas unicamente por meio das varáveis $a, b, c$ e $d$. Por exemplo, o índice J]é definido como

$$
\mathrm{JI}(\mathrm{U}, \mathrm{V}) \triangleq a /(a+b+c)
$$

Alternativamente à matriz de contingência, pode-se calcular as variáveis de pareamento por meio das matrizes de co-associação $\mathrm{J}^{\mathrm{U}}$ e $\mathrm{J}^{\mathrm{V}}$ (Zhang et al., 2012) dos agrupamentos $\mathrm{U} \in M_{\mathrm{e}}$ e $\mathrm{V} \in M_{\mathrm{e}}$, respectivamente:

$$
\mathrm{J}_{i, j}^{\mathrm{U}} \triangleq \begin{cases}1 & \text { se } \exists r \text { tal que } \mathrm{U}_{r, i}=1 \text { e } \mathrm{U}_{r, j}=1 \\ 0 & \text { de outro modo }\end{cases}
$$

A matriz acima pode ser calculada também por $\mathrm{J}^{\mathrm{U}}=\mathrm{U}^{\mathrm{T}} \mathrm{U}$. As variáveis de pareamento podem ser reescritas comd 1

$$
\begin{array}{ll}
a=\sum_{i<j} \mathrm{~J}_{i, j}^{\mathrm{U}} \mathrm{J}_{i, j}^{\mathrm{V}} & b=\sum_{i<j} \mathrm{~J}_{i, j}^{\mathrm{U}}\left(1-\mathrm{J}_{i, j}^{\mathrm{V}}\right) \\
c=\sum_{i<j}\left(1-\mathrm{J}_{i, j}^{\mathrm{U}}\right) \mathrm{J}_{i, j}^{\mathrm{V}} & d=\sum_{i<j}\left(1-\mathrm{J}_{i, j}^{\mathrm{U}}\right)\left(1-\mathrm{J}_{i, j}^{\mathrm{V}}\right)
\end{array}
$$

Como exemplo de uma medida que não é baseada em contagem de pares, considere a medida BCubed (Amigó et al., 2009) (BC). Esta é baseada nos conceitos de precisão e revocação definidas por objeto (Amigó et al. 2009):

$$
\begin{gathered}
\mathrm{BCP}(\mathrm{U}, \mathrm{V}) \triangleq \frac{1}{n} \sum_{i=1}^{n} \frac{\sum_{j=1}^{n} \mathrm{~J}_{i, j}^{\mathrm{U}} \mathrm{J}_{i, j}^{\mathrm{V}}}{\sum_{j=1}^{n} \mathrm{~J}_{i, j}^{\mathrm{U}}} \\
\operatorname{BCR}(\mathrm{U}, \mathrm{V}) \triangleq \frac{1}{n} \sum_{i=1}^{n} \frac{\sum_{j=1}^{n} \mathrm{~J}_{i, j}^{\mathrm{U}} \mathrm{J}_{i, j}^{\mathrm{V}}}{\sum_{j=1}^{n} \mathrm{~J}_{i, j}^{\mathrm{V}}}
\end{gathered}
$$

BC é definida por padrão como:

$$
\mathrm{BC}(\mathrm{U}, \mathrm{V}) \triangleq 2 \cdot \frac{\mathrm{BCP}(\mathrm{U}, \mathrm{V}) \cdot \mathrm{BCR}(\mathrm{U}, \mathrm{V})}{\mathrm{BCP}(\mathrm{U}, \mathrm{V})+\mathrm{BCR}(\mathrm{U}, \mathrm{V})}
$$

Uma versão mais geral dessa medida é apresentada na Seção 4.2.1

\footnotetext{
${ }^{1} \sum_{i<j}$ significa o mesmo que $\sum_{i=1}^{n-1} \sum_{j=i+1}^{n}$.
} 


\subsection{Medidas para agrupamento possibilístico}

Por motivos didáticos serão utilizadas as seguintes abreviações: agrupamento particional exclusivo (APE); agrupamento particional não exclusivo (APNE); agrupamento nebuloso/possibilístico $(\underline{\mathrm{AN}})$; e agrupamento possibilístico $(\underline{\mathrm{AP}})$.

\subsubsection{Revisão da literatura}

Uma visão geral de medidas recentemente propostas voltadas para a comparação dos tipos de agrupamento mais gerais que o APE é dada pela Tabela 4.2.

As medidas 03VI, 03MI e 05MI são baseadas na teoria da informação (Mackay, 2003). Sejam U e V dois ANs com $k_{\mathrm{U}}$ e $k_{\mathrm{V}}$ grupos, respectivamente. A probabilidade conjunta $\mathrm{P}(r, t)$ de um objeto pertencer a ambos o $r$-ésimo grupo de $\mathrm{U}$ e $t$-ésimo grupo de $\mathrm{V}$ é definida dividindo a matriz de contingência $\mathrm{N}$ por $n$, i.e., $\mathrm{P}(r, t) \triangleq \mathrm{N}_{r, t} / n$. A informação mútua entre $\mathrm{U}$ e $\mathrm{V}$ é definida por:

$$
\mathrm{I}(\mathrm{U}, \mathrm{V}) \triangleq \sum_{r, t=1}^{k_{\mathrm{U}}, k_{\mathrm{V}}} \mathrm{P}(r, t) \log \left(\frac{\mathrm{P}(r, t)}{\mathrm{P}(r,+) \mathrm{P}(+, t)}\right)
$$

em que $\mathrm{P}(r,+) \triangleq \sum_{t=1}^{k_{\mathrm{V}}} \mathrm{P}(r, t)$ e $\mathrm{P}(+, t) \triangleq \sum_{r=1}^{k_{\mathrm{U}}} \mathrm{P}(r, t)$ são as somas marginais. A entropia associada a $\mathrm{U}$ é dada por

$$
\mathrm{H}(\mathrm{U}) \triangleq \sum_{r=1}^{k_{\mathrm{U}}} \mathrm{P}(r,+) \log (\mathrm{P}(r,+))
$$

As medidas 03VI, 03MI e 05MI são definidas por:

$$
\begin{aligned}
03 \mathrm{VI}(\mathrm{U}, \mathrm{V}) & \triangleq \mathrm{H}(\mathrm{U})+\mathrm{H}(\mathrm{V})-2 \mathrm{I}(\mathrm{U}, \mathrm{V}) \\
03 \mathrm{MI}(\mathrm{U}, \mathrm{V}) & \triangleq \mathrm{I}(\mathrm{U}, \mathrm{V}) / \sqrt{\mathrm{H}(\mathrm{U}) \mathrm{H}(\mathrm{V})} \\
05 \mathrm{MI}(\mathrm{U}, \mathrm{V}) & \triangleq 2 \mathrm{I}(\mathrm{U}, \mathrm{V}) /(\mathrm{H}(\mathrm{U})+\mathrm{H}(\mathrm{V}))
\end{aligned}
$$

Assume-se a base 2 para a função $\log$ arítmica $\log (\cdot)$ daqui em diante.

07CRI foi desenvolvida por meio de uma reformulação das variáveis de pareamento baseada na teoria dos conjuntos. Sejam U e V dois APEs. Seja $R$ o conjunto de pares não ordenados que pertencem ao mesmo grupo em $U$ e seja $T$ o conjunto de pares não ordenados que pertencem ao mesmo grupo em $\mathrm{V}$. A cardinalidade $|R \cap T|$ leva à variável de pareamento $a$; usando a mesma abordagem, tem-se as variáveis $b, c$ e $d$. Versões nebulosas das variáveis de pareamento foram definidas ao substituir as operações usuais de conjuntos pelas suas contrapartidas da teoria de conjuntos nebulosos (Campello, 2007). Utilizando-se as novas versões de $a, b, c$ e $d$ nas Eqs. 2.35) e 2.36 resultam nas medidas 07CRI e 07CARI, respectivamente, assumindo que U e V são APs.

08BRIp e 08BRIm são generalizações da medida RI foram baseadas nas definições de $a, b$, 
Tabela 4.2: Medidas de similaridade para generalizações do APE.

\begin{tabular}{|c|c|c|c|c|c|}
\hline Medida & APE & AN & APNE & AP & Baseado em \\
\hline $\begin{array}{l}\text { 03VI! }(\text { Meila }, 2003) \\
\text { 03MI! (Strehl e Ghosh, 2003) } \\
\text { 05MI! (I) Fred e Jain, 2005) }\end{array}$ & $*$ & $*$ & & & Teoria da informação \\
\hline $\begin{array}{l}\text { 07CRI! (Campello, 2007) } \\
\text { 07CARI! }\end{array}$ & $*$ & $*$ & $*$ & $*$ & Conjuntos nebulosos $(a, b, c, d)$ \\
\hline $\begin{array}{l}\text { 08BRIp! (Borgelt, 2007) } \\
\text { 08BRIm! }\end{array}$ & $*$ & $*$ & & & $\mathrm{~J}^{\mathrm{U}}(a, b, c, d)$ \\
\hline 09EBC! (Amigó et al., 2009) & $*$ & & $*$ & & Precisão/Revocação \\
\hline $\begin{array}{l}\text { 09CRI! (Ceccarelli e Maratea, 2009) } \\
\text { 09CARI! }\end{array}$ & $*$ & $*$ & $*$ & $*$ & $\dot{\mathrm{N}}(a, b, c, d)^{\dagger}$ \\
\hline 09HI! (Hullermeier e Rifqi, 2009) & $*$ & $*$ & $*$ & $*$ & Distância ( $\mathrm{U}_{:, i}$ e $\mathrm{U}_{:, j}$ ) \\
\hline 09RI! (Rovetta e Masulli, 2009) & $*$ & $*$ & & & $\mathrm{~J}^{\mathrm{U}}(\mathrm{ad}$ hoc $)$ \\
\hline $\begin{array}{l}\text { 09BRI! (Brouwer, 2009) } \\
\text { 09BARI! }\end{array}$ & $*$ & $*$ & $*$ & $*$ & $\mathrm{~J}^{\mathrm{U}}(\mathrm{ad}$ hoc $)$ \\
\hline $\begin{array}{l}\text { 10QRIp! (Quere et al. 2010) } \\
\text { 10QRIm! }\end{array}$ & $*$ & $*$ & $*$ & $*$ & $\mathrm{~J}^{\mathrm{U}}(a, b, c, d)$ \\
\hline $\begin{array}{l}\text { 10ARI! Anderson et al. 2010) } \\
\text { 10AARI! } \\
\text { 10ARIn! } \\
\text { 10AARIn! }\end{array}$ & $*$ & $*$ & $*$ & $*$ & $\mathrm{~N}(a, b, c, d)^{\star}$ \\
\hline 10CSI! (Campello, 2010) & $*$ & & $*$ & & ad hoc \\
\hline $\begin{array}{l}\text { 10CF! Campello, 2010) } \\
\text { 10CFn! }\end{array}$ & $*$ & $*$ & $*$ & $*$ & Distância de edição \\
\hline $\begin{array}{l}\text { 11ARInm! Anderson et al., 2011) } \\
\text { 11AARInm! }\end{array}$ & $*$ & $*$ & $*$ & $*$ & $\mathrm{~N}(a, b, c, d)^{\star}$ \\
\hline 11MD! (Wang, 2010) & $*$ & & $*$ & & $\mathrm{~J}^{\mathrm{U}}(\mathrm{ad}$ hoc $)$ \\
\hline 11D2! (Wang, 2010) & $*$ & & $*$ & & Distância de Hamming \\
\hline 12DB! (Wang, 2012) & $*$ & & $*$ & & Teoria da informação \\
\hline
\end{tabular}

† A matriz de contingência $N$ usada não é a mesma que a original. Ceccarelli e Maratea (2009) definiram como $\dot{\mathrm{N}}_{r, t} \triangleq \sum_{i=1}^{n}\left(\mathrm{U}_{r, i}+\mathrm{V}_{t, i}\right)^{\alpha}$. Adotou-se aqui $\alpha=1$ por simplicidade.

* As medidas 10ARIn, 10AARIn, 11ARInm e 11AARInm usam a matriz de contingência normalizada $\hat{N}$. 
$c$ e $d$ dadas pelas Eqs. (4.4), nas quais uma norma-t arbitrária (da teoria dos conjuntos nebulosos (Klir e Yuan, 1995) substitui o operador de multiplicação usado para calcular $\mathrm{J}^{\mathrm{U}}=\mathrm{U}^{\mathrm{T}} \mathrm{U}$, $\mathrm{J}^{\mathrm{V}}=\mathrm{V}^{\mathrm{T}} \mathrm{V}$ e as variáveis $a, b, c$ e $d$. Adotou-se aqui a norma-t do produto $\left(\top_{\text {prod }}(x, y) \triangleq x y\right) \mathrm{e} \mathrm{a}$ norma-t do mínimo $\left(\top_{\min }(x, y) \triangleq \min \{x, y\}\right)$ para definir 08BRIp e 08BRIm, respectivamente.

A medida 09EBC é baseada na redefinição dos cálculos de precisão e revocação do índice BC (Eqs. (4.5)):

$$
\begin{aligned}
& \operatorname{EBCP}(\mathrm{U}, \mathrm{V}) \triangleq \frac{1}{n} \sum_{i=1}^{n} \frac{\sum_{j=1}^{n} \min \left\{\mathrm{J}_{i, j}^{\mathrm{U}}, \mathrm{J}_{i, j}^{\mathrm{V}}\right\}}{\sum_{j=1}^{n} \mathrm{~J}_{i, j}^{\mathrm{U}}} \\
& \operatorname{EBCR}(\mathrm{U}, \mathrm{V}) \triangleq \frac{1}{n} \sum_{i=1}^{n} \frac{\sum_{j=1}^{n} \min \left\{\mathrm{J}_{i, j}^{\mathrm{U}}, \mathrm{J}_{i, j}^{\mathrm{V}}\right\}}{\sum_{j=1}^{n} \mathrm{~J}_{i, j}^{\mathrm{V}}}
\end{aligned}
$$

As Eqs. 4.5) e 4.6 são equivalentes quando U e V são APEs. 09EBC é definida por padrão como:

$$
09 \operatorname{EBC}(\mathrm{U}, \mathrm{V}) \triangleq 2 \cdot \frac{\operatorname{EBCP}(\mathrm{U}, \mathrm{V}) \cdot \operatorname{EBCR}(\mathrm{U}, \mathrm{V})}{\operatorname{EBCP}(\mathrm{U}, \mathrm{V})+\operatorname{EBCR}(\mathrm{U}, \mathrm{V})},
$$

para matrizes U e V de APNEs.

09CRI e 09CARI são baseadas em uma reformulação da matriz de contingência $\mathrm{N}$, na qual o operador de multiplicação é substituído pelo operador de soma (i.e., $\dot{\mathrm{N}}_{r, t} \triangleq \sum_{i=1}^{n}\left(\mathrm{U}_{r, i}+\mathrm{V}_{t, i}\right)$ ), e o subsequente cálculo das variáveis de pareamento faz uso de uma formulação equivalente (no domínio dos APEs) ao cálculo das Eqs. (4.1) (Eqs. (14), (15), (16) e (21) em (Ceccarelli e Maratea, 2009)). As medidas 09CRI e 09CARI são obtidas ao utilizar-se essas novas variáveis de pareamento nas Eqs. 2.35) e 2.36, respectivamente.

09HI é baseada em cálculos de similaridade entre as colunas de $\mathrm{U}$ e V. Sejam $\mathrm{R}_{i, j}^{\mathrm{U}} \triangleq$ $1-\left\|\mathrm{U}_{:, i}-\mathrm{U}_{:, j}\right\|$ e $\mathrm{R}_{i, j}^{\mathrm{V}} \triangleq 1-\left\|\mathrm{V}_{:, i}-\mathrm{V}_{:, j}\right\|$ para todo $i, j$ as similaridades entre as colunas de $\mathrm{U}$ e $\mathrm{V}$, em que $\|\cdot\|$ é uma norma que fornece valores em $[0,1]$. O grau de concordância entre as distâncias de $\mathrm{U}$ e $\mathrm{V}$ define a medida: $09 \mathrm{HI}(\mathrm{U}, \mathrm{V}) \triangleq 1-\sum_{i<j}\left|\mathrm{R}_{i, j}^{\mathrm{U}}-\mathrm{R}_{i, j}^{\mathrm{V}}\right| /\left(\begin{array}{c}n \\ 2\end{array}\right)$.

A medida 09RI é baseada nas matrizes de co-associação $\mathrm{J}^{\mathrm{U}}$ e $\mathrm{J}^{\mathrm{V}}$. A formulação de 09RI dada pela Eq. (7) de (Rovetta e Masulli, 2009) está incorreta. Rovetta, S. gentilmente forneceu por meio de comunicação pessoal a formulação correta, a qual é apresenta a seguir. Dados $\mathrm{J}^{\mathrm{U}}=$ $\mathrm{U}^{\mathrm{T}} \mathrm{U}$ e $\mathrm{J}^{\mathrm{V}}=\mathrm{V}^{\mathrm{T}} \mathrm{V}$, as seguintes variáveis são calculadas: $\pi \triangleq \sum_{i<j} \mathrm{~J}_{i, j}^{\mathrm{U}} \mathrm{J}_{i, j}^{\mathrm{V}}, \sigma_{\mathrm{U}} \triangleq \sum_{i<j} \mathrm{~J}_{i, j}^{\mathrm{U}} \mathrm{e}$ $\sigma_{\mathrm{V}} \triangleq \sum_{i<j} \mathrm{~J}_{i, j}^{\mathrm{V}}$. A medida 09RI é dada por $1+\left(2 \pi-\sigma_{\mathrm{U}}-\sigma_{\mathrm{V}}\right) /\left(\begin{array}{l}n \\ 2\end{array}\right)$.

09BRI e 09BARI são baseadas nas variáveis de pareamento definidas pelas Eqs. (4.4). Por exemplo, a variável $a$ foi definida como $\left(\sum_{i, j=1}^{n} \dot{\mathrm{j}}_{i, j}^{\mathrm{U}} \dot{\mathrm{j}}_{i, j}^{\mathrm{V}}-n\right) / 2$, cujas matrizes de co-associação são normalizadas: $\dot{\mathrm{J}}_{i, j}^{\mathrm{U}} \triangleq \sum_{r=1}^{k_{\mathrm{U}}}\left(\mathrm{U}_{r, i} \mathrm{U}_{r, j}\right) /\left(\left\|\mathrm{U}_{:, i}\right\|_{\mathrm{e}}\left\|\mathrm{U}_{:, j}\right\|_{\mathrm{e}}\right)^{3}$. O uso dessas novas variáveis nas Eqs. 2.35) e 2.36 fornece 09BRI e 09BARI, respectivamente.

As medidas 10QRIp e 10QRIm são derivadas de 08BRIp e 08BRIm, respectivamente, pela normalização de $\mathrm{J}^{\mathrm{U}}$ e $\mathrm{J}^{\mathrm{V}}$ de forma que todos os termos de suas diagonais sejam iguais a 1,

\footnotetext{
${ }^{2}$ Adotou-se aqui a norma euclidiana para os experimentos do Capítulo 5

${ }^{3}\|\cdot\|_{\mathrm{e}}$ é a norma euclidiana.
} 
sendo U e V APs. A razão por trás dessa normalização é de que um elemento da diagonal $J_{i, i}^{U}$ deve sempre ser o valor máximo da matriz, dado que ele representa o grau com que o objeto $\tilde{o}_{i}$ está no mesmo grupo para o qual o mesmo pertence.

As variáveis de pareamento das medidas 10ARI e 10AARI são definidas por meio da formulação original $\mathrm{N}=\mathrm{UV}^{\mathrm{T}}$ e das Eqs. 4.1). As Eqs. 2.35) e (2.36) são então aplicadas para dar origem a 10ARI e 10AARI, respectivamente. Anderson et al. (2010) notaram que 10ARI não fornece avaliações confinadas no intervalo $[0,1]$ (como é o caso do índice RI) para APs. Eles então propuseram o uso da matriz de contingência normalizada $\hat{\mathrm{N}} \triangleq\left(n / \mathrm{N}_{+,+}\right) \mathrm{N}$ para se ter $\hat{\mathrm{N}}_{+,+}=n$, intencionando contornar o problema acima. Denota-se as versões normalizadas de 10ARI e 10AARI por 10ARIn e 10AARIn, respectivamente.

Foi observado que 10ARIn e 10AARIn não atingem seus valores máximos sempre quando duas soluções equivalentes 4 são comparadas (Anderson et al., 2011). 11ARInm e 11AARInm foram então definidas para solucionar esse problema:

$$
\begin{aligned}
11 \operatorname{ARInm}(\mathrm{U}, \mathrm{V}) & \triangleq 10 \operatorname{ARIn}(\mathrm{U}, \mathrm{V}) / \max \{10 \mathrm{ARIn}(\mathrm{U}, \mathrm{U}), 10 \operatorname{ARIn}(\mathrm{V}, \mathrm{V})\} \\
11 \mathrm{AARInm}(\mathrm{U}, \mathrm{V}) & \triangleq 10 \mathrm{AARIn}(\mathrm{U}, \mathrm{V}) / \max \{10 \mathrm{AARIn}(\mathrm{U}, \mathrm{U}), 10 \operatorname{AARIn}(\mathrm{V}, \mathrm{V})\} .
\end{aligned}
$$

A medida 10CSI foi projetada para lidar com agrupamentos particionais exclusivos e não exclusivos. Sejam $\mathrm{J}^{\mathrm{U}}=\mathrm{U}^{\mathrm{T}} \mathrm{U}$ e $\mathrm{J}^{\mathrm{V}}=\mathrm{V}^{\mathrm{T}} \mathrm{V}$ as matrizes de co-associação. Sejam $\mathrm{U}_{+, i}$ e $\mathrm{V}_{+, i}$ os números de grupos aos quais o objeto $\tilde{o}_{i}$ pertence, de acordo com as suas respectivas soluções. A concordância e a discordância entre $\mathrm{U}$ e $\mathrm{V}$ com relação ao posicionamento relativo dos objetos $\tilde{o}_{i}$ e $\tilde{o}_{j}$ são definidos por 10CSI como:

$$
\begin{aligned}
& a_{i, j}^{g} \triangleq \min \left\{\mathrm{J}_{i, j}^{\mathrm{U}}, \mathrm{J}_{i, j}^{\mathrm{V}}\right\}+\min \left\{\mathrm{U}_{+, i}, \mathrm{~V}_{+, i}\right\}+\min \left\{\mathrm{U}_{+, j}, \mathrm{~V}_{+, j}\right\}-2 \\
& d_{i, j}^{g} \triangleq\left|\mathrm{J}_{i, j}^{\mathrm{U}}-\mathrm{J}_{i, j}^{\mathrm{V}}\right|+\left|\mathrm{U}_{+, i}-\mathrm{V}_{+, i}\right|+\left|\mathrm{U}_{+, j}-\mathrm{V}_{+, j}\right|
\end{aligned}
$$

10CSI é definida por $\sum_{i<j} a_{i, j}^{g} / \sum_{i<j}\left(a_{i, j}^{g}+d_{i, j}^{g}\right)$, a qual se reduz ao índice J] no domínio dos APEs.

As medidas 10CF e 10CFn diferem das outras principalmente porque elas não são baseadas em contagem de pares ou na teoria da informação. 10CF e 10CFn são relacionadas à distância de edição comumente usada para definir a compatibilidade entre duas sequências de letras (Levenshtein, 1966). Campello (2010) definiu a distância de transferência nebulosa $\mathrm{F}_{\mathrm{TD}}(\mathrm{U}, \mathrm{V})$ entre dois APs U e $\mathrm{V}$ como a menor quantidade de pertinência dos objetos que precisa ser transferida entre $\mathrm{U}(\mathrm{V})$ e $\mathrm{V}(\mathrm{U})$ para tornar os agrupamentos equivalentes. Define-se 10CF como $10 \mathrm{CF}(\mathrm{U}, \mathrm{V}) \triangleq 1-\mathrm{F}_{\mathrm{TD}}(\mathrm{U}, \mathrm{V})$ de modo que o resultado pertença ao intervalo $(-\infty, 1] \mathrm{e}$ que atinja 1 se, e somente se, U e V são agrupamentos equivalentes (Campello, 2010). 10CFn é 1 menos a versão normalizada de $\mathrm{F}_{\mathrm{TD}}: 10 \mathrm{CFn}(\mathrm{U}, \mathrm{V}) \triangleq 1-\mathrm{F}_{\mathrm{TD}}(\mathrm{U}, \mathrm{V}) /\left(n \max \left\{k_{\mathrm{U}}, k_{\mathrm{V}}\right\}\right)$. $10 \mathrm{CFn}(\mathrm{U}, \mathrm{V})$ pertence ao intervalo $[0,1]$ (Campello, 2010).

Sejam U e V dois APNEs com $k_{\mathrm{U}}$ e $k_{\mathrm{V}}$ grupos, respectivamente. 11MD e 11D2 são defini-

\footnotetext{
${ }^{4}$ Os agrupamentos U e V são equivalentes se, e somente se, (i) eles possuem o mesmo número de grupos e (ii) $\mathrm{V}$ pode ser transformado em U por permutação de linhas.
} 
das como:

$$
\begin{aligned}
& 11 \mathrm{MD}(\mathrm{U}, \mathrm{V}) \triangleq 1-\frac{1}{n} \sum_{i=1}^{n} \frac{\sum_{j=1}^{n}\left|\mathrm{~J}_{i, j}^{\mathrm{U}}-\mathrm{J}_{i, j}^{\mathrm{V}}\right|}{\sum_{j=1}^{n} \max \left\{\mathrm{J}_{i, j}^{\mathrm{U}}, \mathrm{J}_{i, j}^{\mathrm{V}}\right\}} \\
& 11 \mathrm{D} 2(\mathrm{U}, \mathrm{V}) \triangleq 1-\frac{1}{n^{2}} \sum_{i, j=1}^{n}\left|\mathrm{~J}_{i, j}^{\mathrm{U}}-\mathrm{J}_{i, j}^{\mathrm{V}}\right|
\end{aligned}
$$

em que $\sum_{i, j=1}^{n}\left|\mathrm{~J}_{i, j}^{\mathrm{U}}-\mathrm{J}_{i, j}^{\mathrm{V}}\right|$ é a distância de Hamming quando U e V são APEs.

Seja $\mathrm{A}^{\mathrm{U}}$ a matriz de adjacência de $\mathrm{U}$ definida como:

$$
\mathrm{A}_{i, j}^{\mathrm{U}} \triangleq \begin{cases}1 & \text { se } \exists r: \mathrm{U}_{r, i} \mathrm{U}_{r, j}=1 \\ 0 & \text { se de outra forma }\end{cases}
$$

A disconectividade normalizada de U é dada por padrão como (Wang, 2012):

$$
\operatorname{NDisc}(\mathrm{U}) \triangleq 2\left(1-\frac{1}{n^{2}} \sum_{i, j=1}^{n} \mathrm{~A}_{i, j}^{\mathrm{U}}\right)
$$

Seja Z um agrupamento (possivelmente degenerado) resultante da interseção entre os grupos dos APNEs U e V:

$$
\mathrm{Z}_{\left(r+(t-1) * k_{\mathrm{U}}\right), i} \triangleq \mathrm{U}_{r, i} \mathrm{~V}_{t, i}
$$

A medida 12DB é definida por padrão como:

$$
12 \mathrm{DB}(\mathrm{U}, \mathrm{V}) \triangleq 2 \cdot \mathrm{NDisc}(\mathrm{Z})-\mathrm{NDisc}(\mathrm{U})-\mathrm{NDisc}(\mathrm{V})
$$

\subsubsection{Comentários sobre as medidas}

Alguns autores estenderam medidas baseadas em contagem de pares ao permitir que as matrizes $\mathrm{U}$ e $\mathrm{V}$ representassem outros tipos de agrupamentos (i.e., outros mais gerais que APEs) na definição da matriz de contingência N (e.g., Ceccarelli e Maratea, 2009; Anderson et al., 2010) ou na definição das matrizes de co-associação $\mathrm{J}^{\mathrm{U}}$ e $\mathrm{J}^{\mathrm{V}}$ (e.g., Borgelt e Kruse, 2006; Borgelt, 2007; Quere e Frelicot, 2011), calculando as variáveis $a, b, c$ e $d$ por meio das Eqs. (4.1) ou Eqs. (4.4). Entretanto, as equações das variáveis de pareamento foram deduzidas assumindo que U e V são APEs. Sem uma explicação embasada, não se pode esperar que o uso das mesmas definições em circunstâncias mais gerais resultará em valores de $a, b, c$ e $d$ que façam sentido. Considere, por exemplo, os seguintes APEs:

$$
\mathrm{U} \triangleq \mathrm{V} \triangleq\left(\begin{array}{cc}
1.0 & 0.0 \\
0.0 & 1.0
\end{array}\right)
$$


Tem-se $a=0, b=0, c=0$ e $d=1$, de acordo com as definições dadas pelas Eqs. (4.1) e Eqs. (4.4). Existe apenas um par de objetos e tais objetos estão em grupos diferentes em ambas as soluções. Seja

$$
\dot{\mathrm{V}} \triangleq\left(\begin{array}{cc}
0.9 & 0.0 \\
0.1 & 1.0
\end{array}\right)
$$

um AN muito similar ao representado por V. Comparando U e $\dot{\mathrm{V}}$, tem-se $a=-0.09, b=0.1$, $c=0.09$ e $d=0.9$ de acordo com as Eqs. (4.1), enquanto $a=0, b=0, c=-0.1$ e $d=0.9$ de acordo com as Eqs. (4.4). É difícil atribuir uma interpretação para uma variável de pareamento que fornece um valor negativo. Além disso, os valores obtidos são diferentes dependendo das equações usadas. Esse resultado mostra que a aplicação das Eqs. (4.1) e (4.4) em agrupamentos mais gerais que o APE deve ser acompanhada de uma boa justificativa.

Como será visto na Seção 5.4.1. nenhuma das medidas 03VI, 03MI, 05MI, 07CRI, 07CARI, 08BRIp, 08BRIm, 09CRI, 09CARI, 09RI, 09BRI, 09BARI, 10QRIp, 10QRIm, 10ARI, 10AARI, 10ARIn, 10AARIn, 10CF, 11ARInm e 12DB possui a propriedade desejável de atingir o máximo 1 sempre quando soluções equivalentes forem comparadas. Esse comportamento torna difícil a interpretação dos valores de similaridade produzidos. Além disso, não há razão para esperar que generalizações da medida ARI (i.e., 07CARI, 09CARI, 09BARI, 10AARI, 10AARIn e 11AARInm) sejam, assim como ela, ajustada para aleatoriedade em cenários mais gerais que os de APEs simplesmente porque a medida ARI possui essa propriedade para APEs (essa hipótese é confirmada nos experimentos da Seção 5.4.2). As formulações nas quais essas medidas generalizadas são baseadas foram deduzidas ao assumir que as soluções a serem comparadas são APEs.

\subsection{Avaliação de bi-agrupamento em expressão gênica}

Desde 2000, foram desenvolvidos dezenas de algoritmos de bi-agrupamento para expressão gênica (Madeira e Oliveira, 2004; Tanay et al., 2005; Busygin et al., 2008; Eren et al., 2012). A proposição de um novo algoritmo é usualmente acompanhada de um estudo comparativo incluindo outros algoritmos de bi-agrupamento. Existem quatro abordagens comumente empregadas para avaliar a eficácia dos algoritmos propostos nesses estudos. A primeira depende de análise biológica e interpretação por especialistas da área (Prelić et al., 2006). Os especialistas usam métodos de visualização (e.g., gráficos de coordenadas paralelas e mapas de calor da matriz de dados X) e dependem de um prévio conhecimento sobre os genes e condições biológicas da base de dados (Nepomuceno et al., 2007; Santamaría et al., 2008; Gu e Liu, 2008; Cheng et al. 2008; Dharan e Nair, 2009). Esse método é frequentemente acompanhado de outras abordagens devido à sua natureza subjetiva e é inviável quando vários algoritmos são comparados (Prelić et al., 2006).

Uma abordagem mais objetiva e popular consiste na comparação das soluções pela significância biológica delas (Hanczar e Nadif, 2012; Eren et al., 2012; Gao et al., 2012). Por 
exemplo, pode-se aplicar algoritmos em bases reais cujos genes foram previamente anotados na base Gene Ontology (Consortium, 2012) e, então, realizar a análise de enriquecimento, o que fornece um valor-p indicando a aleatoriedade dos bi-grupos encontrados. Essa análise é muito convincente, mas não considera as condições biológicas e não é aplicável em bases sintéticas.

O terceiro método de comparação consiste na validação interna (Cano et al., 2007; Gremalschi e Altun, 2008; Lee et al., 2011), capaz de avaliar soluções usando apenas informações inerentes aos dados. Cheng e Church (2000) propuseram o resíduo quadrático médio que mede a qualidade do padrão encontrado na matriz de expressão gênica ${ }^{5}$. Índices internos consideram tanto a dimensão dos genes como a das condições biológicas. Portanto, o desempenho do algoritmo é avaliado sob um espectro mais amplo. Entretanto, índices internos fazem suposições quanto aos padrões que um bi-grupo deve possuir, mas os padrões que a expressão gênica de um processo biológico pode apresentar não são totalmente conhecidos.

Uma validação externa pode ser aplicada quando a solução de referência é conhecida (e.g., em experimentos com dados sintéticos). Nesse caso, a medida de similaridade pode ser usada para comparar diretamente o bi-agrupamento encontrado com o de referência (Gao et al., 2012; Hanczar e Nadif, 2012; Eren et al., 2012), e nenhuma suposição quanto aos padrões de expressão gênica precisa ser feito. É apresentada na seção seguinte uma revisão das medidas de similaridade de bi-agrupamentos. Uma análise aprofundada delas, em conjunto com outras duas medidas propostas neste trabalho, é feita no Capítulo 6 .

\subsubsection{Revisão da literatura}

Assume-se aqui que $B \triangleq\left\{B_{i}\right\}_{i=1}^{k}$ e $\dot{B} \triangleq\left\{\dot{B}_{i}\right\}_{i=1}^{q}$ são, respectivamente, os bi-agrupamentos encontrado e de referência. Além disso, medidas de dissimilaridade foram transformadas em medidas de similaridade por motivos de comparação.

Turner et al. (2005) adaptaram a medida-F (Strehl e Ghosh, 2003) para bi-agrupamento, mas usaram um conceito de um modelo específico de bi-agrupamento (modelo de xadrez ou plaid model em inglês) para estabelecer a correspondência entre os bi-grupos encontrados e os de referência, o que severamente estreita a sua aplicabilidade. Por essa razão, essa medida não será mais discutida.

\subsubsection{Medidas $\mathbb{S}_{\text {prel }}$ e $\mathbb{S}_{\text {prec }}$}

Prelić et al. (2006) definiram duas medidas que consideram apenas a dimensão das linhas da matriz de dados, o que fazem delas medidas voltadas para comparação de agrupamentos usuais. As medidas que consideram tanto a dimensão das linhas como a das colunas foram propostas

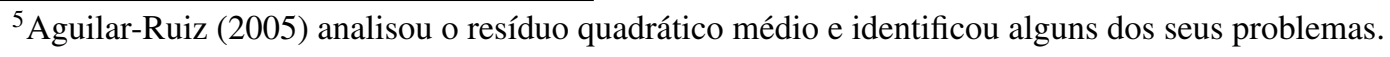


no material suplementar desses autores. Sejam

$$
\begin{aligned}
& S_{\mathrm{r}}(B, \dot{B}) \triangleq \frac{1}{k} \sum_{i=1}^{k} \max _{l \in \mathbb{N}_{1, q}}\left\{\frac{\left|B_{i}^{\mathrm{r}} \cap \dot{B}_{l}^{\mathrm{r}}\right|}{\left|B_{i}^{\mathrm{r}} \cup \dot{B}_{l}^{\mathrm{r}}\right|}\right\} \mathrm{e} \\
& S_{\mathrm{c}}(B, \dot{B}) \triangleq \frac{1}{k} \sum_{i=1}^{k} \max _{l \in \mathbb{N}_{1, q}}\left\{\frac{\left|B_{i}^{\mathrm{c}} \cap \dot{B}_{l}^{\mathrm{c}}\right|}{\left|B_{i}^{\mathrm{c}} \cup \dot{B}_{l}^{\mathrm{c}}\right|}\right\}
\end{aligned}
$$

dois índices de pontuação. As medidas propostas no material suplementar são as seguintes:

$$
\begin{aligned}
& \mathbb{S}_{\text {prel }}(B, \dot{B}) \triangleq \sqrt{S_{\mathrm{r}}(B, \dot{B}) \cdot S_{\mathrm{c}}(B, \dot{B})} \text { e } \\
& \mathbb{S}_{\text {prec }}(B, \dot{B}) \triangleq \mathbb{S}_{\text {prel }}(\dot{B}, B) .
\end{aligned}
$$

A primeira é chamada de medida geral de relevância, enquanto a segunda é chamada de medida geral de revocação.

\subsubsection{Medidas $\mathbb{S}_{\mathrm{rnia}}$ e $\mathbb{S}_{\mathrm{ce}}$}

Patrikainen e Meila (2006) introduziram quatro medidas para comparação de algoritmos de agrupamento em subespaços. As duas medidas que apresentaram resultados teóricos superiores e foram usadas no estudo experimental das autoras são definidas a seguir. Seja $\mathrm{N}_{j_{1}, j_{2}}$ o número de bi-grupos em $B$ para os quais o elemento da matriz correspondente à $j_{1}$-ésima linha e à $j_{2}$ ésima coluna pertence e, analogamente, defina $\dot{\mathrm{N}}_{j_{1}, j_{2}}$ para o bi-agrupamento $\dot{B}$. Os tamanhos dos conjuntos de união e interseção, considerando sobreposição, são definidos por

$$
\begin{gathered}
|U| \triangleq \sum_{j_{1}, j_{2}} \max \left\{\mathrm{N}_{j_{1}, j_{2}}, \dot{\mathrm{N}}_{j_{1}, j_{2}}\right\} \mathrm{e} \\
|I| \triangleq \sum_{j_{1}, j_{2}} \min \left\{\mathrm{N}_{j_{1}, j_{2}}, \dot{\mathrm{N}}_{j_{1}, j_{2}}\right\} .
\end{gathered}
$$

Seja

$$
U_{B} \triangleq \bigcup_{i=1}^{k} B_{i}^{\mathrm{r}} \times B_{i}^{\mathrm{c}}
$$

o conjunto união do bi-agrupamento $B$. Tem-se $|U|=\left|U_{B} \cup U_{\dot{B}}\right|$ e $|I|=\left|U_{B} \cap U_{\dot{B}}\right|$ para biagrupamentos $B$ e $\dot{B}$ sem sobreposição. A medida de área relativa sem interseção Patrikainen e Meila, 2006) é dada por

$$
\mathbb{S}_{\text {rnia }}(B, \dot{B}) \triangleq 1-\frac{|U|-|I|}{|U|}=\frac{|I|}{|U|}
$$


Seja $\left\{\left(t_{i}, y_{i}\right)\right\}_{i=1}^{\min \{k, q\}}$ a relação única $\sqrt{6}$ que maximiza

$$
d_{\max } \triangleq \sum_{i=1}^{\min \{k, q\}}\left|B_{t_{i}}^{\mathrm{r}} \times B_{t_{i}}^{\mathrm{c}} \cap \dot{B}_{y_{i}}^{\mathrm{r}} \times \dot{B}_{y_{i}}^{\mathrm{c}}\right|
$$

O erro de agrupamento (Patrikainen e Meila, 2006) é dado por

$$
\mathbb{S}_{\mathrm{ce}}(B, \dot{B}) \triangleq 1-\frac{|U|-d_{\max }}{|U|}=\frac{d_{\max }}{|U|} .
$$

\subsubsection{Medida $\mathbb{S}_{1 \& w}$}

Liu e Wang (2007) definiram uma medida que se tornou popular (Xiao et al., 2008; Huang et al., 2008; Freitas et al., 2011):

$$
\mathbb{S}_{1 \& \mathrm{w}}(B, \dot{B}) \triangleq \frac{1}{k} \sum_{i=1}^{k} \max _{l \in \mathbb{N}_{1, q}}\left\{\frac{\left|B_{i}^{\mathrm{r}} \cap \dot{B}_{l}^{\mathrm{r}}\right|+\left|B_{i}^{\mathrm{c}} \cap \dot{B}_{l}^{\mathrm{c}}\right|}{\left|B_{i}^{\mathrm{r}} \cup \dot{B}_{l}^{\mathrm{r}}\right|+\left|B_{i}^{\mathrm{c}} \cup \dot{B}_{l}^{\mathrm{c}}\right|}\right\} .
$$

\subsubsection{Medida $\mathbb{S}_{\text {stm }}$}

Seja

$$
\mathbb{D}\left(B_{i}, \dot{B}_{l}\right) \triangleq \frac{2 \cdot\left|B_{i}^{\mathrm{r}} \times B_{i}^{\mathrm{c}} \cap \dot{B}_{l}^{\mathrm{r}} \times \dot{B}_{l}^{\mathrm{c}}\right|}{\left|B_{i}^{\mathrm{r}} \times B_{i}^{\mathrm{c}}\right|+\left|\dot{B}_{l}^{\mathrm{r}} \times \dot{B}_{l}^{\mathrm{c}}\right|}
$$

o índice de Dice (1945) aplicado em $B_{i}$ e $\dot{B}_{l}$. Santamaría et al. (2007) propuseram a medida

$$
\mathbb{S}_{\mathrm{stm}}(B, \dot{B}) \triangleq \frac{1}{k} \sum_{i=1}^{k} \max _{l \in \mathbb{N}_{1, q}}\left\{\mathbb{D}\left(B_{i}, \dot{B}_{l}\right)\right\}
$$

4.3.1.5 Medidas $\mathbb{S}_{\mathrm{wjac}}$ e $\mathbb{S}_{\mathrm{wdic}}$

Seja

$$
\mathbb{J}\left(B_{i}, \dot{B}_{l}\right) \triangleq \frac{\left|B_{i}^{\mathrm{r}} \times B_{i}^{\mathrm{c}} \cap \dot{B}_{l}^{\mathrm{r}} \times \dot{B}_{l}^{\mathrm{c}}\right|}{\left|B_{i}^{\mathrm{r}} \times B_{i}^{\mathrm{c}} \cup \dot{B}_{l}^{\mathrm{r}} \times \dot{B}_{l}^{\mathrm{c}}\right|}
$$

\footnotetext{
6"Relação única" significa "relação binária única à direita e única à esquerda. Por exemplo, $\{(1,3),(4,2)\}$ é uma relação única entre $\mathbb{N}_{1,4}$ e $\mathbb{N}_{1,4}$, mas $\{(1,3),(4,3)\}$ não é.
} 
o índice de Jaccard (1908) aplicado em $B_{i}$ e $\dot{B}_{l}$. As medidas propostas por Lee et al. (2009) consideram os tamanhos dos bi-grupos na avaliação:

$$
\begin{aligned}
& \mathbb{S}_{\mathrm{wjac}}(B, \dot{B}) \triangleq \frac{\sum_{i=1}^{k}\left|B_{i}^{\mathrm{r}} \times B_{i}^{\mathrm{c}}\right| \cdot \max _{l \in \mathbb{N}_{1, q}}\left\{\mathbb{J}\left(B_{i}, \dot{B}_{l}\right)\right\}}{\sum_{i=1}^{k}\left|B_{i}^{\mathrm{r}} \times B_{i}^{\mathrm{c}}\right|} \mathrm{e} \\
& \mathbb{S}_{\mathrm{wdic}}(B, \dot{B}) \triangleq \frac{\sum_{i=1}^{k}\left|B_{i}^{\mathrm{r}} \times B_{i}^{\mathrm{c}}\right| \cdot \max _{l \in \mathbb{N}_{1, q}}\left\{\mathbb{D}\left(B_{i}, \dot{B}_{l}\right)\right\}}{\sum_{i=1}^{k}\left|B_{i}^{\mathrm{r}} \times B_{i}^{\mathrm{c}}\right|}
\end{aligned}
$$

A medida $\mathbb{S}_{\mathrm{wdic}}$ difere da medida $\mathbb{S}_{\mathrm{stm}}$ ao atribuir maior peso na avaliação de bi-grupos maiores.

\subsubsection{Medida $\mathbb{S}_{\mathrm{fabi}}$}

Hochreiter et al. (2010) afirmaram que as medidas anteriores ao trabalho deles não foram projetadas para lidar com a sobreposição entre bi-grupos e nem com a diferença no número de bi-grupos entre a solução encontrada e a de referência. Semelhantemente à medida $\mathbb{S}_{\mathrm{ce}}$, seja $\left\{\left(t_{i}, y_{i}\right)\right\}_{i=1}^{\min \{k, q\}}$ a relação única que maximiza $\sum_{i=1}^{\min \{k, q\}} \mathbb{J}\left(B_{t_{i}}, \dot{B}_{y_{i}}\right)$. A medida fabia é dada por

$$
\mathbb{S}_{\text {fabi }}(B, \dot{B}) \triangleq \frac{\sum_{i=1}^{\min \{k, q\}} \mathbb{J}\left(B_{t_{i}}, \dot{B}_{y_{i}}\right)}{\max \{k, q\}}
$$

\subsubsection{Medidas $\mathbb{S}_{\mathrm{u}}$ e $\mathbb{S}_{\mathrm{e}}$}

Bozdag et al. (2010) definiram as duas medidas seguintes:

$$
\begin{aligned}
& \mathbb{S}_{\mathrm{u}}(B, \dot{B}) \triangleq 1-\frac{\left|U_{\dot{B}}\right|-\left|U_{B} \cap U_{\dot{B}}\right|}{\left|U_{\dot{B}}\right|}=\frac{\left|U_{B} \cap U_{\dot{B}}\right|}{\left|U_{\dot{B}}\right|} \mathrm{e} \\
& \mathbb{S}_{\mathrm{e}}(B, \dot{B}) \triangleq \mathbb{S}_{\mathrm{u}}(\dot{B}, B) .
\end{aligned}
$$

A primeira lida com a porção não descoberta do bi-agrupamento de referência e a segunda lida com a porção extra do bi-agrupamento encontrado.

Ayadi et al. (2012a) usaram as medidas

$$
\begin{aligned}
& \mathbb{S}_{\mathrm{sh}}(B, \dot{B}) \triangleq \frac{\left|U_{B} \cap U_{\dot{B}}\right|}{\left|U_{\dot{B}}\right|} \mathrm{e} \\
& \mathbb{S}_{\mathrm{nsh}}(B, \dot{B}) \triangleq 1-\frac{\left|U_{B}-\left(U_{B} \cap U_{\dot{B}}\right)\right|}{\left|U_{\dot{B}}\right|}
\end{aligned}
$$

baseando-se no trabalho de Cano et al. (2007). Note que $\mathbb{S}_{\mathrm{sh}}(B, \dot{B})=\mathbb{S}_{\mathrm{u}}(B, \dot{B})$ e que $\mathbb{S}_{\text {nsh }}$ pode atribuir avaliações negativas, enquanto $\mathbb{S}_{\mathrm{sh}}$ assume valores em $[0,1]$. Por outro lado, tanto $\mathbb{S}_{\mathrm{u}}$ como $\mathbb{S}_{\mathrm{e}}$ assumem valores em $[0,1]$ e são simétricas em relação à ordem dos parâmetros. Será considerada, então, apenas as medidas $\mathbb{S}_{\mathrm{u}}$ e $\mathbb{S}_{\mathrm{e}}$ no presente estudo. 


\subsubsection{Medida $\mathbb{S}_{\text {ay }}$}

Ayadi et al. (2012b) também propuseram a medida

$$
\mathbb{S}_{\mathrm{ay}}(B, \dot{B}) \triangleq \frac{1}{k} \sum_{i=1}^{k} \max _{l \in \mathbb{N}_{1, q}} \frac{\left|B_{i}^{\mathrm{r}} \cap \dot{B}_{l}^{\mathrm{r}}\right|\left|B_{i}^{\mathrm{c}} \cap \dot{B}_{l}^{\mathrm{c}}\right|}{\left|B_{i}^{\mathrm{r}} \cup \dot{B}_{l}^{\mathrm{r}}\right|\left|B_{i}^{\mathrm{c}} \cup \dot{B}_{l}^{\mathrm{c}}\right|} .
$$

Note que $\left|B_{i}^{\mathrm{r}} \cap \dot{B}_{l}^{\mathrm{r}}\right|\left|B_{i}^{\mathrm{c}} \cap \dot{B}_{l}^{\mathrm{c}}\right|=\left|B_{i}^{\mathrm{r}} \times B_{i}^{\mathrm{c}} \cap \dot{B}_{l}^{\mathrm{r}} \times \dot{B}_{l}^{\mathrm{c}}\right|$, mas $\left|B_{i}^{\mathrm{r}} \cup \dot{B}_{l}^{\mathrm{r}}\right|\left|B_{i}^{\mathrm{c}} \cup \dot{B}_{l}^{\mathrm{c}}\right| \neq\left|B_{i}^{\mathrm{r}} \times B_{i}^{\mathrm{c}} \cup \dot{B}_{l}^{\mathrm{r}} \times \dot{B}_{l}^{\mathrm{c}}\right|$ no geral (o primeiro é sempre maior que ou igual ao segundo), o que torna $\mathbb{S}_{\text {ay }}$ e $\mathbb{S}_{\text {stm }}$ diferentes.

\subsubsection{Medidas $\mathbb{S}_{\text {erel }}$ e $\mathbb{S}_{\text {erec }}$}

Eren et al. (2012) introduziram as medidas

$$
\begin{aligned}
& \mathbb{S}_{\text {erel }}(B, \dot{B}) \triangleq \frac{1}{k} \sum_{i=1}^{k} \max _{l \in \mathbb{N}_{1, q}}\left\{\mathbb{J}\left(B_{i}, \dot{B}_{l}\right)\right\} \mathrm{e} \\
& \mathbb{S}_{\text {erec }}(B, \dot{B}) \triangleq \mathbb{S}_{\text {erel }}(\dot{B}, B) .
\end{aligned}
$$

A medida $\mathbb{S}_{\text {erel }}(B, \dot{B})$ calcula a relevância do bi-agrupamento encontrado, enquanto $\mathbb{S}_{\text {erec }}(B, \dot{B})$ mede a revocação do bi-agrupamento de referência.

\subsubsection{Comentários sobre as medidas}

Dado que o interesse pela área de bi-agrupamento de dados é recente em comparação com o agrupamento tradicional e até com o agrupamento nebuloso de dados (Kaufman e Rousseeuw, 1990; Bezdek, 1981), não existe uma análise teórica das várias medidas de similaridade entre bi-agrupamentos. Por exemplo, nota-se que a maioria das medidas de bi-agrupamento são baseadas em uma associação entre os bi-grupos da solução encontrada com os da solução de referência. Essa abordagem de comparação é análoga à associação de grupos realizada por algumas medidas de comparação para agrupamento de dados. Tal abordagem é fortemente criticada (Amigó et al., 2009; Rosenberg e Hirschberg, 2007; Meila, 2003), principalmente porque as medidas baseadas nela tendem a ignorar partes dos grupos, resultando no problema da insensibilidade quanto à homogeneidade das soluções (a definir no Capítulo 6). Essa questão é tratada mais a fundo no Capítulo 6 para o contexto do bi-agrupamento de dados.

\subsection{Considerações}

A validação externa de agrupamento ainda é uma área de pesquisa bastante ativa na literatura, especialmente devido ao interesse em agrupamentos mais gerais que os particionais exclusivos e em bi-agrupamento. Surgiram dezenas de novas medidas de similaridade para tais cenários nos últimos anos, demandando estudos empíricos e teóricos sobre os seus comportamentos e propriedades. Neste capítulo foram revisadas 28 medidas para agrupamentos mais gerais que o particional exclusivo e 14 medidas para bi-agrupamento. Não é preciso uma 
análise extensa para concluir que grande parte dessas medidas possuem problemas importantes. Por exemplo, na Seção 4.2 .2 foi argumentado que quase todas as medidas de agrupamento apresentadas não possuem a propriedade de atingir o máximo sempre quando duas soluções equivalentes são comparadas. Na Seção 4.3.2 observou-se que o esquema de comparação de bi-agrupamentos usualmente adotado torna as respectivas medidas incapazes de considerar a homogeneidade da solução encontrada. Os fatos acima citados e outros motivaram o desenvolvimento de novas medidas e análises empíricas e teóricas, cujos trabalhos estão relatados no dois capítulos seguintes. 



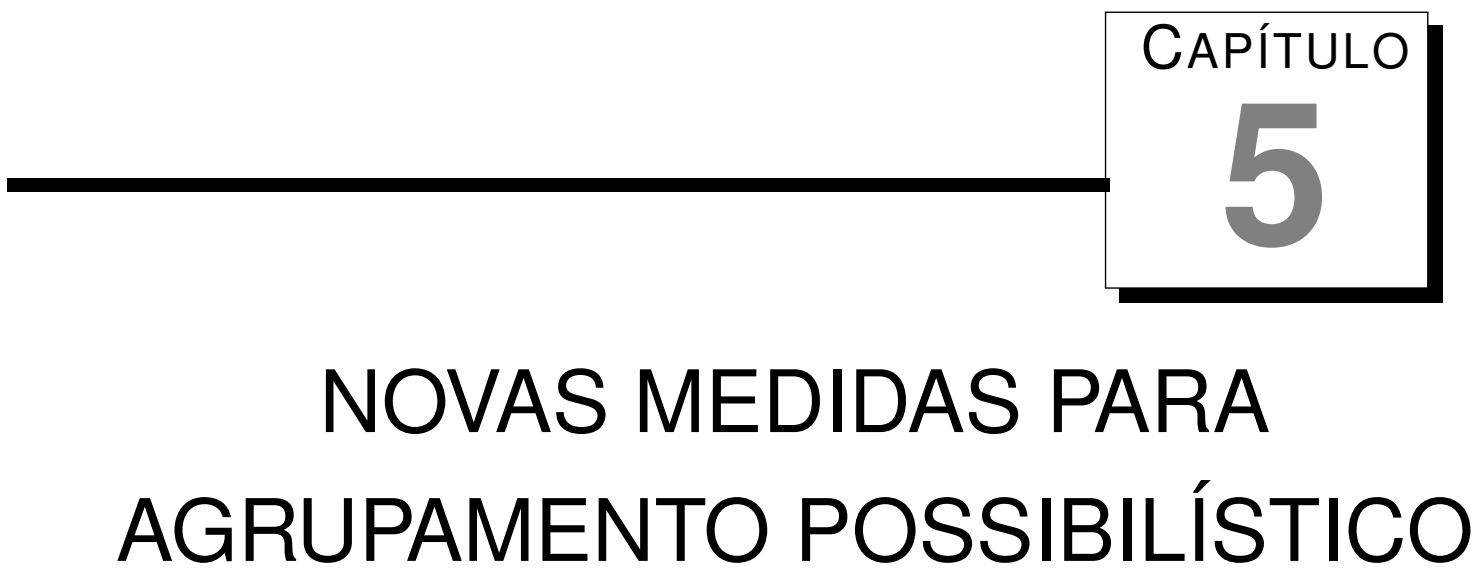

Como discutido no início do Capítulo 4, existem várias aplicações para medidas que avaliam a similaridade entre agrupamentos. No entanto, não existe uma medida, ou um conjunto de medidas, para agrupamentos mais gerais que os particionais exclusivos que se sobressaia na literatura. Houve um crescente interesse por essas medidas nos últimos anos e várias têm sido propostas, o que demanda uma revisão compreensiva delas (já apresentada no Capítulo 4) e uma análise comparativa, apresentada neste capítulo. É também proposto neste capítulo uma medida ajustada para aleatoriedade capaz de lidar com agrupamentos possibilísticos (Seções 5.1 e 5.2), o que engloba a maior parte dos tipos de agrupamentos não hierárquicos encontrados na literatura. Essa medida é comparada com 32 outras em conjuntos de agrupamentos gerados por algoritmos de diferentes naturezas, em dezenas de bases reais e artificiais.

\subsection{Medida 13FRI}

Dado dois ANs U (com $k_{\mathrm{U}}$ grupos) e $\mathrm{V}$ (com $k_{\mathrm{V}}$ grupos) de $n$ objetos, 13FRI transforma cada um em duas matrizes $n$-por- $n$, retendo apenas informações essenciais para a comparação. Seja $I_{k_{\mathrm{U}}}$ a matriz de identidade $k_{\mathrm{U}}$-por- $k_{\mathrm{U}}$ e $\mathbb{1}_{k_{\mathrm{U}}}$ a matriz $k_{\mathrm{U}}$-por- $k_{\mathrm{U}}$ que possui 1 em cada uma de seus elementos. Sejam as matrizes

$$
\begin{aligned}
& \mathrm{J}^{\mathrm{U}} \triangleq \mathrm{U}^{\mathrm{T}} \mathrm{U} \text { e } \\
& \mathrm{S}^{\mathrm{U}} \triangleq \mathrm{U}^{\mathrm{T}}\left(\mathbb{1}_{k_{\mathrm{U}}}-\mathrm{I}_{k_{\mathrm{U}}}\right) \mathrm{U} .
\end{aligned}
$$

As matrizes $\mathrm{J}^{\mathrm{U}}$ e $\mathrm{S}^{\mathrm{U}}$ fornecem toda a informação sobre o pareamento de objetos em $\mathrm{U}$ que a medida 13FRI precisa. Sejam $\mathrm{J}^{\mathrm{V}}$ e $\mathrm{S}^{\mathrm{V}}$ as matrizes correspondentes de V. 13FRI compara $\mathrm{J}^{\mathrm{U}}$ e 
$\mathrm{S}^{\mathrm{U}}$ com $\mathrm{J}^{\mathrm{V}}$ e $\mathrm{S}^{\mathrm{V}}$ para medir o quanto $\mathrm{U}$ e $\mathrm{V}$ concordam com as atribuições de pertinência para cada par de objeto. $\mathrm{O}$ uso dessas matrizes é motivado a seguir.

$\mathrm{J}_{i, j}^{\mathrm{U}}$ e $\mathrm{S}_{i, j}^{\mathrm{U}}$ podem ser interpretadas de várias maneiras. Para APEs, $\mathrm{J}_{i, j}^{\mathrm{U}}=1$ (implicando em $\mathrm{S}_{i, j}^{\mathrm{U}}=0$ ) significa que os objetos $\tilde{o}_{i}$ e $\tilde{o}_{j}$ pertencem ao mesmo grupo da solução $\mathrm{U}$, e $\mathrm{J}_{i, j}^{\mathrm{U}}=0$ (implicando em $\mathrm{S}_{i, j}^{\mathrm{U}}=1$ ) significa que eles pertencem a diferentes grupos em U. No domínio do APE, $\mathrm{J}^{\mathrm{U}}$ representa a mesma matriz definida na Eq. (4.3), e $\mathrm{S}_{i, j}^{\mathrm{U}}=1-\mathrm{J}_{i, j}^{\mathrm{U}}$.

Uma outra interpretação interessante pode ser fornecida para $\mathrm{J}^{\mathrm{U}}$ e $\mathrm{S}^{\mathrm{U}}$ no domínio dos ANs. Considerando que a matriz $\mathrm{U}$ de um AN provê probabilidades dos objetos pertencerem aos grupos (e.g., como nas soluções do algoritmo $(\mathrm{EM})$, i.e., $\mathrm{U}_{r, i}$ é a probabilidade do objeto $\tilde{o}_{i}$ pertencer ao $r$-ésimo grupo, $\mathrm{J}_{i, j}^{\mathrm{U}}$ fornece a probabilidade dos objetos $\tilde{o}_{i}$ e $\tilde{o}_{j}$ pertencerem ao mesmo grupo de acordo com $\mathrm{U}$, e $\mathrm{S}_{i, j}^{\mathrm{U}}=1-\mathrm{J}_{i, j}^{\mathrm{U}}$ fornece a probabilidade que eles pertençam a grupos diferentes de acordo com $\mathrm{U}$, assumindo independência.

Permite-se aqui também que $\mathrm{J}^{\mathrm{U}}$ e $\mathrm{S}^{\mathrm{U}}$ sejam definidas para o problema geral dos APs (Seção 5.2. Portanto, seguem-se mais duas interpretações sobre $\mathrm{J}^{\mathrm{U}}$ e $\mathrm{S}^{\mathrm{U}}$ para o domínio dos APs. Seja $\mathrm{U}$ um APNE, $\mathrm{J}_{i, j}^{\mathrm{U}}$ é o número de vezes que $\tilde{o}_{i}$ e $\tilde{o}_{j}$ pertencem ao mesmo grupo em $\mathrm{U}$, e $\mathrm{S}_{i, j}^{\mathrm{U}}$ é o número de vezes que $\tilde{o}_{i}$ e $\tilde{o}_{j}$ pertencem a diferentes grupos em $\mathrm{U}$. Se U é um agrupamento mais geral, no domínio dos APs ainda, pode-se dizer que $\mathrm{J}_{i, j}^{\mathrm{U}}$ é a possibilidade de $\tilde{o}_{i}$ e $\tilde{o}_{j}$ pertencerem ao mesmo grupo em $\mathrm{U}$, e $\mathrm{S}_{i, j}^{\mathrm{U}}$ é a possibilidade de $\tilde{o}_{i}$ e $\tilde{o}_{j}$ pertencerem a diferentes grupos em $\mathrm{U}$.

A despeito da multitude de interpretações acima mencionadas, entende-se que $\mathrm{J}_{i, j}^{\mathrm{U}}$ representa o grau de veracidade da sentença “ $\tilde{o}_{i}$ e $\tilde{o}_{j}$ pertencem ao mesmo grupo", enquanto $\mathrm{S}_{i, j}^{\mathrm{U}}$ fornece o grau de falsidade da mesma sentença, de acordo com a solução U. Essa linha de raciocínio leva à seguinte redefinição das variáveis de pareamento $a, b, c$ e $d$ :

$$
\begin{aligned}
& \dot{a} \triangleq \sum_{i<j} \min \left\{\mathrm{J}_{i, j}^{\mathrm{U}}, \mathrm{J}_{i, j}^{\mathrm{V}}\right\} \\
& \dot{b} \triangleq \sum_{i<j} \min \left\{\mathrm{J}_{i, j}^{\mathrm{U}}-\min \left\{\mathrm{J}_{i, j}^{\mathrm{U}}, \mathrm{J}_{i, j}^{\mathrm{V}}\right\}, \mathrm{S}_{i, j}^{\mathrm{V}}-\min \left\{\mathrm{S}_{i, j}^{\mathrm{U}}, \mathrm{S}_{i, j}^{\mathrm{V}}\right\}\right\} \\
& \dot{c} \triangleq \sum_{i<j} \min \left\{\mathrm{J}_{i, j}^{\mathrm{V}}-\min \left\{\mathrm{J}_{i, j}^{\mathrm{U}}, \mathrm{J}_{i, j}^{\mathrm{V}}\right\}, \mathrm{S}_{i, j}^{\mathrm{U}}-\min \left\{\mathrm{S}_{i, j}^{\mathrm{U}}, \mathrm{S}_{i, j}^{\mathrm{V}}\right\}\right\} \\
& \dot{d} \triangleq \sum_{i<j} \min \left\{\mathrm{S}_{i, j}^{\mathrm{U}}, \mathrm{S}_{i, j}^{\mathrm{V}}\right\}
\end{aligned}
$$

As variáveis $\dot{a}$ e $\dot{d}$ medem o grau de concordância entre $\mathrm{U}$ e $\mathrm{V}$ com respeito à veracidade e à falsidade da sentença " $\tilde{o}_{i}$ e $\tilde{o}_{j}$ pertencem ao mesmo grupo" para cada par de objetos $\tilde{o}_{i}$ e $\tilde{o}_{j} ; \dot{b}$ e $\dot{c}$ medem o grau de discordância. Para APEs U e V, $\min \left\{\mathrm{J}_{i, j}^{\mathrm{U}}, \mathrm{J}_{i, j}^{\mathrm{V}}\right\}=1$ significa que $\tilde{o}_{i}$ e $\tilde{o}_{j}$ são agrupados conjuntamente em ambos os agrupamentos. De outro modo, $\min \left\{\mathrm{S}_{i, j}^{\mathrm{U}}, \mathrm{S}_{i, j}^{\mathrm{V}}\right\}=1$ significa que $\tilde{o}_{i}$ e $\tilde{o}_{j}$ pertencem a diferentes grupos em ambas as soluções. Em ambos os casos, $\dot{a}+\dot{d}$ é incrementado por $1 . \mathrm{J}_{i, j}^{\mathrm{U}} \neq \mathrm{J}_{i, j}^{\mathrm{V}}$ significa que existe uma discordância entre $\mathrm{U}$ e $\mathrm{V}$ com respeito ao pareamento de $\tilde{o}_{i}$ e $\tilde{o}_{j}$; isso implica que $\min \left\{\mathrm{J}_{i, j}^{\mathrm{U}}, \mathrm{J}_{i, j}^{\mathrm{V}}\right\}=\min \left\{\mathrm{S}_{i, j}^{\mathrm{U}}, \mathrm{S}_{i, j}^{\mathrm{V}}\right\}=0$ e $\dot{b}+\dot{c}$ é incrementado em 1. Esse comportamento lembra a definição descritiva das variáveis $a, b, c \mathrm{e}$ $d$ dada na Seção 2.5. Comparando as definições das Eqs. (4.4) com as das Eqs. (5.2), tem-se $a=\dot{a}, b=\dot{b}, c=\dot{c}$ e $d=\dot{d}$ para a comparação de APEs. Consequentemente, a medida de 
similaridade

$$
13 F R I(U, V) \triangleq \frac{\dot{a}+\dot{d}}{\dot{a}+\dot{b}+\dot{c}+\dot{d}}
$$

se reduz ao índice RI quando U e V são APEs.

Considere o contexto mais geral no qual U e V são ANs. Definiu-se aqui $\dot{a}+\dot{d}(\dot{b}+\dot{c})$ para medir o grau de concordância (ou discordância) entre U e V com relação ao pareamento dos objetos. Por exemplo, o operador min em $\min \left\{\mathrm{S}_{i, j}^{\mathrm{U}}, \mathrm{S}_{i, j}\right\}$ parece prover uma noção razoável sobre a extensão com que as soluções estão em acordo com os objetos $\tilde{o}_{i}$ e $\tilde{o}_{j}$ não serem atribuídos ao mesmo grupo. Quando os elementos de $\mathrm{J}^{\mathrm{U}}$ e $\mathrm{J}^{\mathrm{V}}$ (ou $\mathrm{S}^{\mathrm{U}}$ e $\mathrm{S}^{\mathrm{V}}$ ) apresentam simultaneamente valores alto e baixos, existe uma forte compatibilidade entre $\mathrm{U}$ e $\mathrm{V}$. Isso é refletido em como 13FRI foi definido.

Pode-se perguntar por que $\dot{b}$ (e similarmente o mesmo para $\dot{c}$ ) não foi definido como $\dot{b} \triangleq$ $\sum_{i<j} \min \left\{\mathrm{J}_{i, j}^{\mathrm{U}}, \mathrm{S}_{i, j}^{\mathrm{V}}\right\}$. A razão é que a quantidade $\min \left\{\mathrm{J}_{i, j}^{\mathrm{U}}, \mathrm{J}_{i, j}^{\mathrm{V}}\right\}$ foi usada de $\mathrm{J}_{i, j}^{\mathrm{U}}$ e $\mathrm{J}_{i, j}^{\mathrm{V}}$ para estabelecer a compatibilidade entre $\mathrm{J}_{i, j}^{\mathrm{U}}$ e $\mathrm{J}_{i, j}^{\mathrm{V}}$ em $\dot{a}$. Suponha que $\mathrm{J}_{i, j}^{\mathrm{U}}=\mathrm{S}_{i, j}^{\mathrm{U}}=\mathrm{J}_{i, j}^{\mathrm{V}}=\mathrm{S}_{i, j}^{\mathrm{V}}=x$. Seja $\dot{a}_{i, j} \triangleq \min \left\{\mathrm{J}_{i, j}^{\mathrm{U}}, \mathrm{J}_{i, j}^{\mathrm{V}}\right\}$ e, de maneira análoga, defina $\dot{b}_{i, j}, \dot{c}_{i, j}$ e $\dot{d}_{i, j}$. Sem as subtrações das Eqs. (5.2b) e (5.2c), todas as variáveis $\dot{a}, \dot{b}, \dot{c}$ e $\dot{d}$ seriam acrescidas de $x$ (i.e., $\dot{a}_{i, j}=\dot{b}_{i, j}=$ $\dot{c}_{i, j}=\dot{d}_{i, j}=x$ ), significando que $\mathrm{U}$ e $\mathrm{V}$ teriam apenas $50 \%$ de concordância com relação a $\tilde{o}_{i}$ e $\tilde{o}_{j}$, ao invés de $100 \%$. Isso não acontece com a formulação original porque toda a informação com relação ao posicionamento de $\tilde{o}_{i}$ e $\tilde{o}_{j}$ foi usada na definição de $\dot{a}_{i, j}$ e $\dot{d}_{i, j}$, e então nada foi deixado para a definição de $\dot{b}_{i, j}$ e $\dot{c}_{i, j}$. A Figura 5.1 ilustra os valores $\mathrm{J}_{i, j}^{\mathrm{U}}+\mathrm{S}_{i, j}^{\mathrm{U}}=1 \mathrm{e}$ $\mathrm{J}_{i, j}^{\mathrm{V}}+\mathrm{S}_{i, j}^{\mathrm{V}}=1$ por meio da altura das caixas.
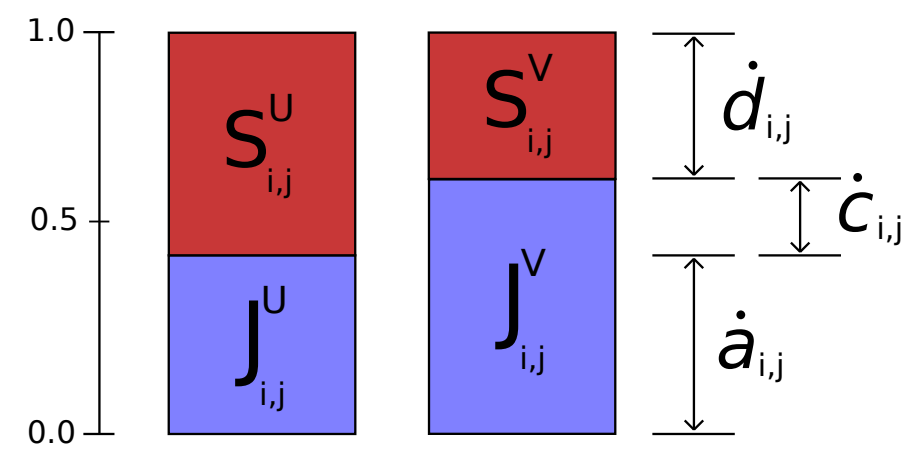

Figura 5.1: Representação ilustrativa de uma avaliação realizada por 13FRI.

A medida 13FRI fornece valores no intervalo [0,1]. Ela atinge o máximo 1 sempre quando soluções equivalentes ${ }^{1}$ são comparadas ${ }^{2}$ e atinge o mínimo 0 apenas quando U e V são APEs

\footnotetext{
${ }^{1}$ Os agrupamentos U e V são equivalentes se, e somente se, possuem o mesmo número de grupos e existe uma permutação de linhas que o fazem serem idênticos.

${ }^{2}$ Note que $\mathrm{J}_{i, j}^{\mathrm{U}}$ e $\mathrm{S}_{i, j}^{\mathrm{U}}$ são independentes de permutação das linhas de U. Se U e V são agrupamentos equivalen-
} 
nos quais um deles tem um grupo e o outro tem $n$ grupos (Proposição 5.1, Seção 5.5). Entretanto, esse último cenário é extremo e tem pouco valor prático (Vinh et al., 2009, 2010), tornando quase impossível a atribuição de valores baixos por 13FRI na prática. É desejável que todo o intervalo $[0,1]$ da medida seja útil, para fins de interpretabilidade. Isso pode ser alcançado por uma medida de similaridade que assume valores próximos de uma constante $\alpha$ ( $\alpha$ pode sempre se tornar zero por uma transformação não linear: subtraindo $\alpha$ da avaliação e multiplicando o resultado por um $\beta$ que faça o valor máximo ser igual a 1) quando comparando soluções aleatórias. Quando tal propriedade está presente, diz-se que a medida apresenta avaliação basal constante. Quando existe uma avaliação basal constante e o usuário conhece o seu valor, aumenta-se a confiança nas conclusões do usuário porque o valor atribuído pela medida pode ser comparada com o valor basal. É apresentada na próxima seção o ajuste do índice 13FRI que o permite assumir valores próximos de zero para soluções geradas aleatoriamente.

\subsubsection{Ajuste para aleatoriedade}

Suponha que a medida atribua $x$ à similaridade entre duas matrizes $\mathrm{U}$ e V de ANs. Como determinar se $x$ não é apenas um valor inerente à flutuação aleatória do índice? Uma abordagem popular para lidar com esse problema consiste em subtrair a esperança da medida e normalizar o valor para assumir 1 como o seu máximo (Hubert e Arabie, 1985; Albatineh et al., 2006; Vinh et al., 2009, 2010):

$$
\operatorname{MSA}(\mathrm{U}, \mathrm{V}) \triangleq \frac{\mathrm{MS}(\mathrm{U}, \mathrm{V})-\mathrm{E}[\mathrm{MS}]_{\mathrm{U}, \mathrm{V}}}{\max \{\mathrm{MS}\}-\mathrm{E}[\mathrm{MS}]_{\mathrm{U}, \mathrm{V}}}
$$

no qual MS é qualquer medida de similaridade, $E[M S]_{U, V}$ é a sua esperança dado $U$ e $V$, $\max \{\mathrm{MS}\}$ é o máximo de MS, e MSA é a sua versão ajustada. MSA assume valores do intervalo $(-\infty, 1]$. Um valor positivo de MSA indica que a similaridade entre $\mathrm{U}$ e $\mathrm{V}$ é maior do que o valor esperado na comparação de soluções aleatórias. Esse ajuste para aleatoriedade também pode fazer com que a medida se torne imparcial com relação ao número de grupos (Vinh et al. 2009, 2010), o que é observado na Seção 5.4.2 para os índices propostos neste capítulo.

A fim de ajustar a medida para aleatoriedade, é necessário especificar um modelo nuld ${ }^{3}$ de acordo com o qual as soluções são geradas (Vinh et al., 2009, 2010). Dado dois ANs U e V, o modelo nulo proposto aqui produz simultaneamente duas soluções de permutações aleatórias e independentes das colunas de $\mathrm{U}$ e $\mathrm{V}$. Sejam $\pi_{1}, \pi_{2}, \ldots, \pi_{n !}$ todas as permutações possíveis dos números do conjunto $\mathbb{N}_{1, n}$, e defina a função $\Gamma_{\pi_{l}}(\mathrm{U}) \triangleq\left[\begin{array}{lll}\mathrm{U}_{:, \pi_{l}(1)} & \mathrm{U}_{:, \pi_{l}(2)} & \ldots\end{array} \mathrm{U}_{:, \pi_{l}(n)}\right]$ que aplica a permutação $\pi_{l}$ na matriz U. Uma permutação particular $\pi_{l}$ de U é escolhida com probabilidade $\mathrm{P}\left(\pi_{l}\right) \triangleq 1 / n$ !, e as permutações de U e V são consideradas eventos independentes. Define-se $\mathrm{P}\left(\pi_{l}, \pi_{q}\right) \triangleq 1 /(n ! n !)$. A esperança de 13FRI assumindo o modelo nulo e as matrizes $\mathrm{U}$ e $\mathrm{V}$ é

tes, tem-se $\mathrm{J}_{i, j}^{\mathrm{U}}=\mathrm{J}_{i, j}^{\mathrm{V}}$ e $\mathrm{S}_{i, j}^{\mathrm{U}}=\mathrm{S}_{i, j}^{\mathrm{V}} \forall i<j$. Isso implica em $\dot{b}=\dot{c}=0$, o que faz $13 \mathrm{FRI}(\mathrm{U}, \mathrm{V})=1$.

${ }^{3}$ Adianta-se que o modelo nulo será violado em todos os experimentos realizados nesta tese. Porém, concluirse-á que o ajuste para aleatoriedade funciona mesmo assim. 
dada por

$$
\mathrm{E}[13 \mathrm{FRI}]_{\mathrm{U}, \mathrm{V}}=\frac{1}{n ! n !} \sum_{l, q=1}^{n !} 13 \mathrm{FRI}\left(\Gamma_{\pi_{l}}(\mathrm{U}), \Gamma_{\pi_{q}}(\mathrm{~V})\right)
$$

Sejam $\dot{a}\left(\mathrm{~J}^{\mathrm{U}}, \mathrm{J}^{\mathrm{V}}\right) \triangleq \sum_{i<j} \min \left\{\mathrm{J}_{i, j}^{\mathrm{U}}, \mathrm{J}_{i, j}^{\mathrm{V}}\right\}$ e $\dot{d}\left(\mathrm{~S}^{\mathrm{U}}, \mathrm{S}^{\mathrm{V}}\right) \triangleq \sum_{i<j} \min \left\{\mathrm{S}_{i, j}^{\mathrm{U}}, \mathrm{S}_{i, j}^{\mathrm{V}}\right\}$. Dado que $\dot{a}+$ $\dot{b}+\dot{c}+\dot{d}$ é uma constante para o modelo nulo proposto (Corolário 5.1, Seção 5.5), considere a esperança reescrita como

$$
\mathrm{E}[13 \mathrm{FRI}]_{\mathrm{U}, \mathrm{V}}=(\dot{a}+\dot{b}+\dot{c}+\dot{d})^{-1}\left(\mathrm{E}[\dot{a}]_{\mathrm{U}, \mathrm{V}}+\mathrm{E}[\dot{d}]_{\mathrm{U}, \mathrm{V}}\right)
$$

na qual

$$
\begin{aligned}
\mathrm{E}[\dot{a}]_{\mathrm{U}, \mathrm{V}} & =\frac{1}{n ! n !} \sum_{l, q=1}^{n !} \dot{a}\left(\mathrm{~J}^{\Gamma_{\pi_{l}}(\mathrm{U})}, \mathrm{J}^{\Gamma_{\pi_{q}}(\mathrm{~V})}\right) \\
& =\frac{1}{n ! n !} \sum_{l, q=1}^{n !} \sum_{i_{1}<j_{1}} \min \left\{\mathrm{J}_{\pi_{l}\left(i_{1}\right), \pi_{l}\left(j_{1}\right)}^{\mathrm{U}}, \mathrm{J}_{\pi_{q}}^{\mathrm{V}}\left(i_{1}\right), \pi_{q}\left(j_{1}\right)\right\} \\
& =\frac{2(n-2) !}{n ! n !} \sum_{q=1}^{n !} \sum_{i_{1}<j_{1}} \sum_{i_{2}<j_{2}} \min \left\{\mathrm{J}_{i_{2}, j_{2}}^{\mathrm{U}}, \mathrm{J}_{\pi_{q}\left(i_{1}\right), \pi_{q}\left(j_{1}\right)}^{\mathrm{V}}\right\} \\
& =\frac{2(n-2) ! 2(n-2) !}{n ! n !} \sum_{i_{1}<j_{1}} \sum_{i_{2}<j_{2}} \sum_{i_{3}<j_{3}} \min \left\{\mathrm{J}_{i_{2}, j_{2}}^{\mathrm{U}}, \mathrm{J}_{i_{3}, j_{3}}^{\mathrm{V}}\right\} \\
& =\frac{4}{n^{2}(n-1)^{2}} \sum_{i_{1}<j_{1}} \sum_{i_{2}<j_{2}} \sum_{i_{3}<j_{3}} \min \left\{\mathrm{J}_{i_{2}, j_{2}}^{\mathrm{U}}, \mathrm{J}_{i_{3}, j_{3}}^{\mathrm{V}}\right\} \\
& =\frac{2}{n(n-1)} \sum_{i_{2}<j_{2}} \sum_{i_{3}<j_{3}} \min \left\{\mathrm{J}_{i_{2}, j_{2}}^{\mathrm{U}}, \mathrm{J}_{i_{3}, j_{3}}^{\mathrm{V}}\right\}
\end{aligned}
$$

e, analogamente,

$$
\mathrm{E}[\dot{d}]_{\mathrm{U}, \mathrm{V}}=\frac{2}{n(n-1)} \sum_{i_{2}<j_{2}} \sum_{i_{3}<j_{3}} \min \left\{\mathrm{S}_{i_{2}, j_{2}}^{\mathrm{U}}, \mathrm{S}_{i_{3}, j_{3}}^{\mathrm{V}}\right\}
$$

De acordo com a transformação dada pela Eq. (5.4), o índice 13FRI ajustado é definido como

$$
13 \operatorname{AFRI}(\mathrm{U}, \mathrm{V}) \triangleq \frac{13 \mathrm{FRI}(\mathrm{U}, \mathrm{V})-\mathrm{E}[13 \mathrm{FRI}]_{\mathrm{U}, \mathrm{V}}}{1-\mathrm{E}[13 \mathrm{FRI}]_{\mathrm{U}, \mathrm{V}}}
$$

Para $\mathrm{E}[13 \mathrm{FRI}]_{\mathrm{U}, \mathrm{V}}=1$, basta definir 13AFRI $(\mathrm{U}, \mathrm{V}) \triangleq 1$ (Proposição 5.8).

13AFRI atinge o máximo 1 da mesma maneira que a medida 13FRI (i.e., sempre quando dois agrupamentos equivalentes são comparados) e fornece 0 quando a medida 13FRI assumiria um valor igual a sua esperança, sob o modelo nulo. 13AFRI pode apresentar avaliações negativas, o que significa que os agrupamentos comparados são mais dissimilares do que o esperado para o caso aleatório. 
Dados dois APEs U e V, tem-se 13AFRI(U, V) = ARI(U, V) (Proposição 5.3, Seção 5.5). Em outras palavras, 13AFRI reduz-se ao índice ARI no domínio dos APEs. Isso indica que o modelo nulo proposto para a medida 13AFRI é apropriado, o qual pode ser estendido para APs (Seção 5.2).

\subsubsection{Considerações}

13FRI pode ser aplicado em APs. Nesse caso, entretanto, 13FRI não forneceria valores de avaliação razoáveis em cenários nos quais a soma de pertinência por objeto (i.e., as somas dos valores de cada coluna da matriz de agrupamento) varia conforme as soluções. Seja U um AN (lembre-se que um AN também é um AP). O resultado da multiplicação de U por um escalar $x \in(0,1)$ é também uma matriz de AP, na qual a soma de pertinência por objeto de cada objeto é decrementada. Note que $13 \mathrm{FRI}(\mathrm{U}, \mathrm{U})=13 \mathrm{FRI}(\mathrm{U}, x \mathrm{U})=13 \mathrm{AFRI}(\mathrm{U}, \mathrm{U})=$ 13AFRI $(\mathrm{U}, x \mathrm{U})=1$ para qualquer $x \in(0,1]$. Isso ocorre porque $\mathrm{J}_{i, j}^{x \mathrm{U}}=\min \left\{\mathrm{J}_{i, j}^{\mathrm{U}}, \mathrm{J}_{i, j}^{x \mathrm{U}}\right\} \mathrm{e}$ $\mathrm{S}_{i, j}^{x \mathrm{U}}=\min \left\{\mathrm{S}_{i, j}^{\mathrm{U}}, \mathrm{S}_{i, j}^{x \mathrm{U}}\right\}$, igualando as variáveis $\dot{b}$ e $\dot{c}$ a zero.

A fim de apresentar um outro cenário problemático, considere as seguintes matrizes:

$$
\mathrm{U} \triangleq\left(\begin{array}{cc}
0.8 & 0.3 \\
0.3 & 0.8
\end{array}\right) \quad \text { e } \quad \mathrm{V} \triangleq\left(\begin{array}{cc}
0.5 & 0.5 \\
0.5 & 0.5
\end{array}\right)
$$

Note que U é um AP mais geral que um AN. Tem-se $\mathrm{J}_{1,2}^{\mathrm{U}}=0.48, \mathrm{~S}_{1,2}^{\mathrm{U}}=0.73, \mathrm{~J}_{1,2}^{\mathrm{V}}=0.5$ e $\mathrm{S}_{1,2}^{\mathrm{V}}=0.5$. As alturas da primeira e da segunda caixa da Figura 5.2 correspondem aos valores $\mathrm{J}_{1,2}^{\mathrm{U}}+\mathrm{S}_{1,2}^{\mathrm{U}}=1.21$ e $\mathrm{J}_{1,2}^{\mathrm{V}}+\mathrm{S}_{1,2}^{\mathrm{V}}=1$, respectivamente. Existe um espaço não utilizado pelas variáveis $a, b, c$ e $d$ de tamanho $\mathrm{J}_{1,2}^{\mathrm{U}}+\mathrm{S}_{1,2}^{\mathrm{U}}-\left(\mathrm{J}_{1,2}^{\mathrm{V}}+\mathrm{S}_{1,2}^{\mathrm{V}}\right)=0.21$ na primeira caixa, o qual 13FRI ignora. Uma medida razoável para APs deveria decrescer a pontuação proporcionalmente à quantidade não utilizada. É proposta uma modificação da medida 13FRI para solucionar o problema encontrado, ao definir uma nova variável de pareamento (variável $e$ ilustrada na Figura 5.2 .

\subsection{Medida 13GRI}

Sejam $\mathrm{T}^{\mathrm{U}} \triangleq \mathrm{J}^{\mathrm{U}}+\mathrm{S}^{\mathrm{U}}$ e $\mathrm{M} \triangleq \max \left\{\mathrm{T}^{\mathrm{U}}, \mathrm{T}^{\mathrm{V}}\right\} \bigsqcup^{4}$. Uma nova variável

$$
\dot{e} \triangleq \max \left\{\sum_{i<j}\left(\mathrm{M}_{i, j}-\mathrm{T}_{i, j}^{\mathrm{U}}\right), \sum_{i<j}\left(\mathrm{M}_{i, j}-\mathrm{T}_{i, j}^{\mathrm{V}}\right)\right\}
$$

é introduzida em 13FRI para dar origem ao índice 13GRI:

$$
13 \mathrm{GRI}(\mathrm{U}, \mathrm{V}) \triangleq \frac{\dot{a}+\dot{d}}{\dot{a}+\dot{b}+\dot{c}+\dot{d}+\dot{e}} .
$$

\footnotetext{
${ }^{4} \mathrm{M}=\max \left\{\mathrm{T}^{\mathrm{U}}, \mathrm{T}^{\mathrm{V}}\right\}$ significa que $\mathrm{M}_{i, j}=\max \left\{\mathrm{T}_{i, j}^{\mathrm{U}}, \mathrm{T}_{i, j}^{\mathrm{V}}\right\}$ para todo $i, j$.
} 


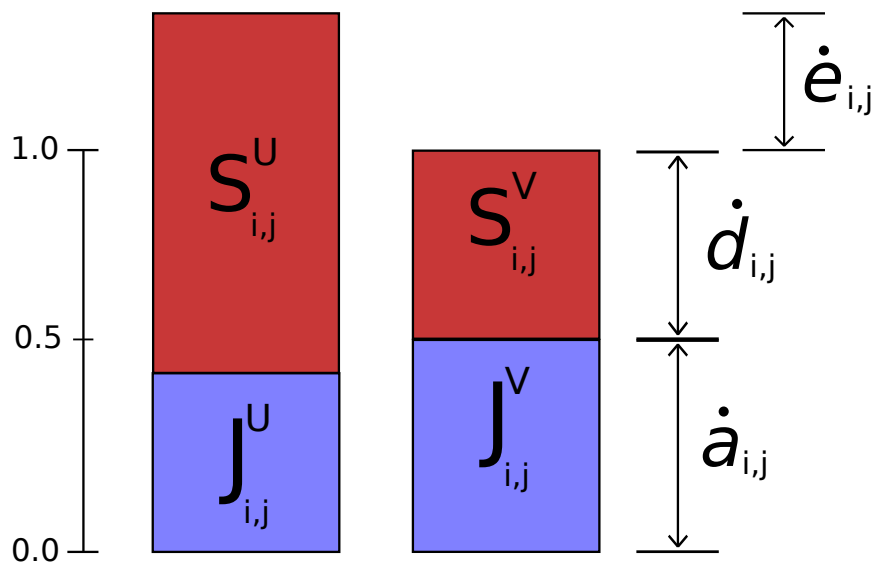

Figura 5.2: Representação gráfica do problema ao usar 13FRI para comparar matrizes que possuem diferentes somas de coluna.

Dados dois objetos $\tilde{o}_{i}$ e $\tilde{o}_{j}, \mathrm{M}_{i, j}-\mathrm{T}_{i, j}^{\mathrm{U}}$ corresponde ao quanto $\mathrm{T}_{i, j}^{\mathrm{V}}$ é maior que $\mathrm{T}_{i, j}^{\mathrm{U}}$. Na Figura 5.2. $\mathrm{M}_{i, j}-\mathrm{T}_{i, j}^{\mathrm{V}}=0.44$, o que é igual à altura do espaço vazio na primeira caixa. A Proposição 5.5 (Seção 5.5) permite que a Eq. (5.11) seja reescrita como

$$
\operatorname{13GRI}(\mathrm{U}, \mathrm{V})=\frac{\sum_{i<j} \min \left\{\mathrm{J}_{i, j}^{\mathrm{U}}, \mathrm{J}_{i, j}^{\mathrm{V}}\right\}+\sum_{i<j} \min \left\{\mathrm{S}_{i, j}^{\mathrm{U}}, \mathrm{S}_{i, j}^{\mathrm{V}}\right\}}{\max \left\{\sum_{i<j} \mathrm{~T}_{i, j}^{\mathrm{U}}, \sum_{i<j} \mathrm{~T}_{i, j}^{\mathrm{V}}\right\}} .
$$

Se U e V são ANs, $\mathrm{T}_{i, j}^{\mathrm{U}}=\mathrm{T}_{i, j}^{\mathrm{V}}=1, \dot{a}+\dot{b}+\dot{c}+\dot{d}+\dot{e}=\max \left\{\sum_{i<j} \mathrm{~T}_{i, j}^{\mathrm{U}}, \sum_{i<j} \mathrm{~T}_{i, j}^{\mathrm{V}}\right\}=n(n-1) / 2$, então 13GRI se reduz a 13FRI. Assim como 13FRI, a medida 13GRI alcança o máximo 1 sempre quando os APs U e V são equivalentes 5

Adotando o mesmo modelo nulo proposto na Seção 5.1.1, e observando que $\dot{a}+\dot{b}+\dot{c}+\dot{d}+\dot{e}$ é constante para ele (Corolário 5.2, Seção 5.5), tem-se E[13GRI $]_{\mathrm{U}, \mathrm{V}}=(\dot{a}+\dot{b}+\dot{c}+\dot{d}+$ $\dot{e})^{-1}\left(\mathrm{E}[\dot{a}]_{\mathrm{U}, \mathrm{V}}+\mathrm{E}[\dot{d}]_{\mathrm{U}, \mathrm{V}}\right)$. A versão ajustada da medida 13GRI é dada por

$$
13 \mathrm{AGRI}(\mathrm{U}, \mathrm{V}) \triangleq \frac{13 \mathrm{GRI}(\mathrm{U}, \mathrm{V})-\mathrm{E}[13 \mathrm{GRI}]_{\mathrm{U}, \mathrm{V}}}{1-\mathrm{E}[13 \mathrm{GRI}]_{\mathrm{U}, \mathrm{V}}}
$$

Para $\mathrm{E}[13 \mathrm{GRI}]_{\mathrm{U}, \mathrm{V}}=1$, basta definir $13 \mathrm{AGRI}(\mathrm{U}, \mathrm{V}) \triangleq 1$ (Proposição 5.9).

Similarmente ao 13AFRI, o índice 13AGRI alcança o máximo 1 da mesma maneira que a medida 13GRI e sua esperança é 0, de acordo com o modelo nulo. É mostrado na Seção 5.4 .2 que 13AGRI de fato exibe uma avaliação basal constante próxima de zero, mesmo quando o modelo nulo é claramente violado.

\footnotetext{
${ }^{5}$ Como para o caso de 13FRI, tem-se $\mathrm{J}_{i, j}^{\mathrm{U}}=\mathrm{J}_{i, j}^{\mathrm{V}}$ e $\mathrm{S}_{i, j}^{\mathrm{U}}=\mathrm{S}_{i, j}^{\mathrm{V}} \forall i<j$ sempre quando U e V são agrupamentos equivalentes. Logo, $\mathrm{T}_{i, j}^{\mathrm{U}}=\mathrm{T}_{i, j}^{\mathrm{V}} \forall i<j$, fazendo com que $\dot{a}$ e $\dot{d}$ sejam os únicos termos não nulos.
} 


\subsection{Complexidade computacional e implementação}

Sejam $I_{k_{\mathrm{U}}}$ a matriz de identidade $k_{\mathrm{U}}$-por- $k_{\mathrm{U}}$ e $\mathbb{1}_{k_{\mathrm{U}}}$ a matriz $k_{\mathrm{U}}$-por- $k_{\mathrm{U}}$ com 1 em cada entrada. Existem $\mathcal{O}\left(n^{2} k_{\mathrm{U}}\right)$ passos computacionais para calcular $\mathrm{J}^{\mathrm{U}}=\mathrm{U}^{\mathrm{T}} \mathrm{U}$ e $\mathrm{S}^{\mathrm{U}}=\mathrm{U}^{\mathrm{T}}\left(\mathbb{1}_{k_{\mathrm{U}}}\right.$ $\left.\mathrm{I}_{k_{\mathrm{U}}}\right) \mathrm{U}$, e $\mathcal{O}\left(n^{2}\right)$ passos para calcular $\mathrm{M}=\max \left\{\mathrm{T}^{\mathrm{U}}, \mathrm{T}^{\mathrm{V}}\right\}$. As variáveis $\dot{a}, \dot{b}, \dot{c}, \dot{d}$ e $\dot{e}$ exigem $\mathcal{O}\left(n^{2}\right)$ passos por causa das somas $\sum_{i<j}$ em suas fórmulas (Eqs. (5.2) e (5.10). 13FRI e 13GRI então requerem $\mathcal{O}\left(n^{2}\left(k_{\mathrm{U}}+k_{\mathrm{V}}\right)\right)$ operações cada. O cálculo de medidas baseadas em contagem de pares para APEs geralmente exigem $\mathcal{O}\left(n k_{\mathrm{U}} k_{\mathrm{V}}\right)$ passos devido ao cálculo da matriz de contingência $\mathrm{N}=\mathrm{UV}^{\mathrm{T}}$. A maior complexidade computacional de 13FRI e 13GRI, em comparação a certos índices, é o ônus decorrente de uma medida de comparação mais geral.

As Eqs. (5.7) e 5.8) podem sugerir que a medida 13AFRI (e 13AGRI) requer $\mathcal{O}\left(n^{4}\right)$ passos computacionais, tornando o seu cômputo inviável para grande parte dos cenários reais. Felizmente, o operador min permite a redução da complexidade computacional das Eqs. (5.7) e (5.8) para apenas $\mathcal{O}\left(n^{2} \log n\right)$ passos. Para entender como isso é possível, suponha que $\mathrm{J}_{1,2}^{\mathrm{U}} \leq \mathrm{J}_{i, j}^{\mathrm{V}}$ para todo $i<j\left(i, j \in \mathbb{N}_{1, n}\right)$ como um caso especial e um exemplo didático. Tem-se $\sum_{i<j} \min \left\{\mathrm{J}_{1,2}^{\mathrm{U}}, \mathrm{J}_{i, j}^{\mathrm{V}}\right\}=\mathrm{J}_{1,2}^{\mathrm{U}} n(n-1) / 2$ computável em tempo constante, reduzindo o custo computacional total. Agora, considere o caso geral para o cálculo de $\mathrm{E}[\dot{a}]_{\mathrm{U}, \mathrm{V}}$ (Eq. (5.7)). Defina

$$
\mathbf{1}_{i_{1}, j_{1}}^{i_{2}, j_{2}} \triangleq \begin{cases}1 & \text { se } \mathrm{J}_{i_{1}, j_{1}}^{\mathrm{U}} \leq \mathrm{J}_{i_{2}, j_{2}}^{\mathrm{V}} \\ 0 & \text { de outro modo }\end{cases}
$$

A Eq. (5.7) pode ser reescrita como

$$
\begin{aligned}
\frac{n(n-1)}{2} \mathrm{E}[\dot{a}]_{\mathrm{U}, \mathrm{V}} & =\sum_{i_{1}<j_{1}} \sum_{i_{2}<j_{2}} \min \left\{\mathrm{J}_{i_{1}, j_{1}}^{\mathrm{U}}, \mathrm{J}_{i_{2}, j_{2}}^{\mathrm{V}}\right\} \mathbf{1}_{i_{1}, j_{1}}^{i_{2}, j_{2}}+\sum_{i_{2}<j_{2}} \sum_{i_{1}<j_{1}} \min \left\{\mathrm{J}_{i_{1}, j_{1}}^{\mathrm{U}}, \mathrm{J}_{i_{2}, j_{2}}^{\mathrm{V}}\right\}\left(1-\mathbf{1}_{i_{1}, j_{1}}^{i_{2}, j_{2}}\right) \\
& =\sum_{i_{1}<j_{1}} \mathrm{~J}_{i_{1}, j_{1}}^{\mathrm{U}} \sum_{i_{2}<j_{2}} \mathbf{1}_{i_{1}, j_{1}}^{i_{2}, j_{2}}+\sum_{i_{2}<j_{2}} \mathrm{~J}_{i_{2}, j_{2}}^{\mathrm{V}} \sum_{i_{1}<j_{1}}\left(1-\mathbf{1}_{i_{1}, j_{1}}^{i_{2}, j_{2}}\right)
\end{aligned}
$$

$\mathrm{O}$ cálculo de $\mathrm{E}[\dot{d}]_{\mathrm{U}, \mathrm{V}}\left(\right.$ Eq. $[5.8)$ ) é análogo; a única diferença está no uso de $\mathrm{S}^{\mathrm{U}}$ e $\mathrm{S}^{\mathrm{V}}$ ao invés de $\mathrm{J}^{\mathrm{U}}$ e $\mathrm{J}^{\mathrm{V}}$.

A estratégia acima pode ser aplicada eficientemente ao rearranjar, primeiramente, as partes triangulares superiores das matrizes $\mathrm{J}^{\mathrm{U}}$ e $\mathrm{J}^{\mathrm{V}}$ nos vetores x e y, respectivamente, e ordenar o vetores resultantes ${ }^{6}$ É mostrado no Algoritmo 5.1 uma implementação da estratégia acima, no qual o primeiro e segundo termos do lado direito da Eq. (5.14) são calculados pelos laços dos Passos 7e 15, respectivamente.

O passo que mais demanda esforço computacional do Algoritmo 5.1 é o Passo 4, o qual ordenada os dois vetores de tamanho $n(n-1) / 2$ em $\mathcal{O}\left(n^{2} \log n\right)$ passos usando, por exemplo, o algoritmo heapsort. As medidas 13AGRI e 13AFRI requerem, portanto, $\mathcal{O}\left(n^{2}\left(k_{\mathrm{U}}+k_{\mathrm{V}}+\log n\right)\right)$ passos computacionais.

\footnotetext{
${ }^{6} \mathrm{~A}$ parte triangular superior de $\mathrm{J}_{i, j}^{\mathrm{U}}$ pode ser rearranjada como segue: $\mathrm{x}_{\pi(i, j)} \triangleq \mathrm{J}_{i, j}^{\mathrm{U}} \forall i<j$, em que $\pi(i, j) \triangleq$ $j-i+\sum_{t=1}^{i-1}(n-t)=j-i(1+i) / 2+n(i-1)$.
} 


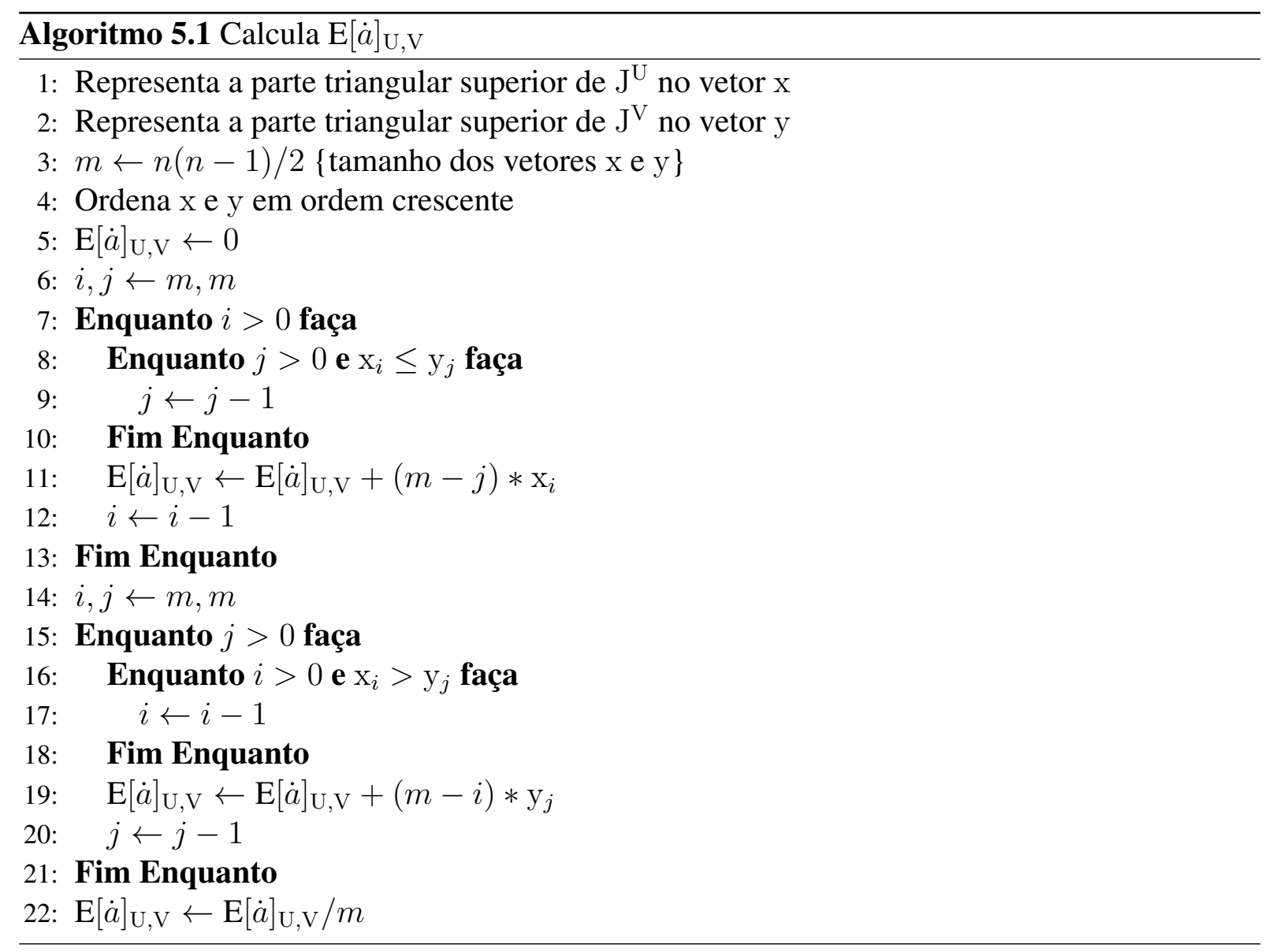

\subsection{Experimentos}

É uma prática comum comparar a acurácia de algoritmos de agrupamento por meio da comparação das similaridades entre as soluções encontradas e a solução de referência. $\mathrm{O}$ algoritmo que gerou os agrupamentos mais similares à solução de referência é considerado o mais acurado. A medida de agrupamento precisa, de alguma forma, avaliar adequadamente a similaridade entre os agrupamentos comparados. Essa característica é avaliada na Seção 5.4.1. onde 34 medidas são aplicadas em soluções com diferentes números de grupos produzidas por diferentes algoritmos. Bases de dados sintéticas foram geradas de acordo com os tipos de agrupamento que tais algoritmos buscam (e.g., é sabido que o k-Means tende a produzir grupos de formato esférico), e a solução de referência para cada base foi definida por meio da aplicação do algoritmo correspondente com uma boa solução inicial. Nesse cenário, é esperado que a dissimilaridade entre o agrupamento gerado e o de referência seja proporcional à diferença do número de grupos.

Em um outro cenário, as medidas são comparadas na Seção 5.4.2 na avaliação de soluções geradas aleatoriamente. Nesse caso, a medida deve apresentar uma avaliação uniforme ao longo dos números de grupos porque qualquer semelhança entre as soluções comparadas é devido apenas à sorte.

A validade das avaliações do índice 13AGRI para ANs é testada na Seção 5.4 .3 para 14 bases reais. A medida 13AGRI é usada na Seção 5.4.4 como uma estatística de estabilidade e 
usada para a estimação do número de grupos em cinco bases reais.

Visto que 13GRI (13AGRI) é mais geral que e se torna equivalente à medida 13FRI (13AFRI) quando aplicada em ANs, só serão apresentados os resultados para o índice 13GRI (13AGRI).

\subsubsection{Similaridade entre agrupamentos}

As medidas foram avaliadas em cinco bases de dados sintéticas (Figs. 5.3), cada qual voltada para um dos seguintes tipos de agrupamento: APE, AN, APNE e AP. A base DAPE (Figura 5.3(a) possui nove grupos bem separados, enquanto a base DAN (Figura 5.3(b)) possui nove grupos com sobreposição. Em ambas as bases, os grupos foram gerados por distribuições gaussianas com iguais variâncias e nenhuma correlação entre os atributos. A base DAPNE (Figura 5.3(c) tem quatro grupos, mas eles se reduzem a dois grupos quando projetados em um único eixd7. A base DAP foi gerada (Figura 5.3(d) de modo que parecesse com uma outra (Zhang e Leung, 2004), mas com ruído adicionado.

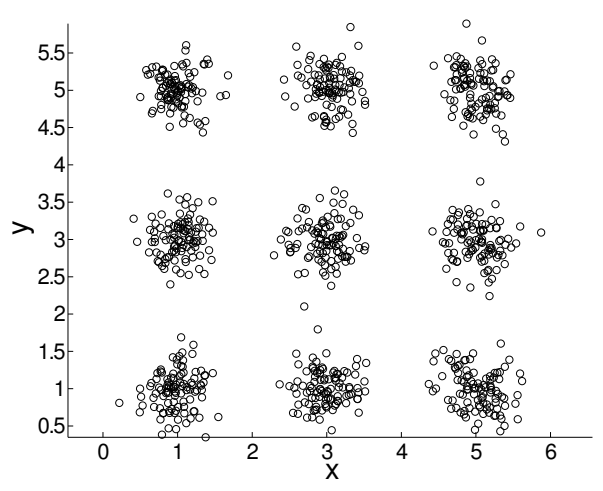

(a) Base para APE(DAPE).

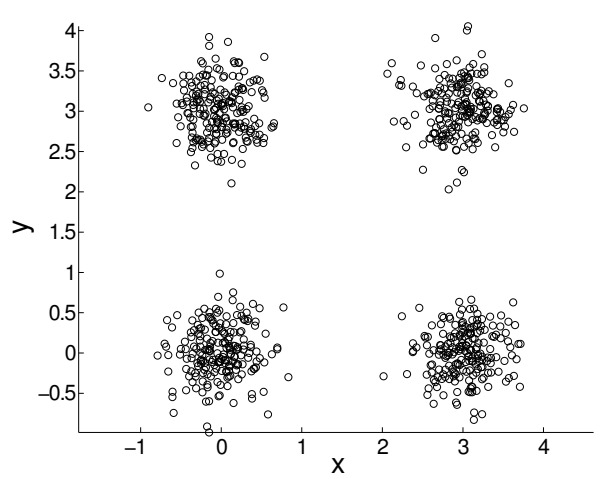

(c) Base para APNE(DAPNE).

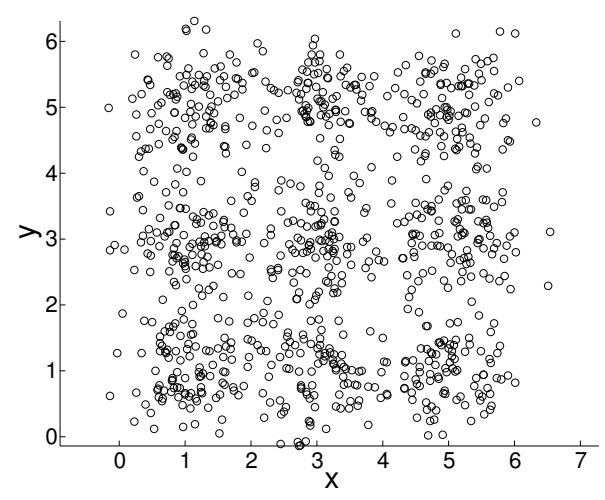

(b) Base para $\mathrm{AN}(\mathrm{DAN})$.

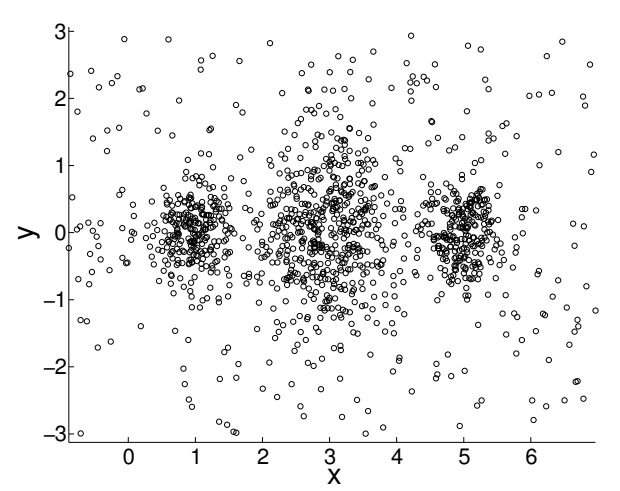

(d) Base para $\mathrm{AP}(\mathrm{DAP})$.

Figura 5.3: Base de dados para cada tipo de agrupamento.

Diferentes algoritmos de agrupamento foram empregados às bases de dados, de acordo com o tipo de agrupamento correspondente: k-Means para DAPE, FCM e Expectation Maximization for Gaussian Mixture (Dempster et al., 1977) (EMGM) para DAN, Density-Connected

\footnotetext{
${ }^{7}$ Pode-se atribuir uma interpretação similar às outras bases. Entretanto, serão considerados apenas subespaços nessa base específica.
} 
Subspace Clustering (Kailing et al., 2004a) (SUBCLU) para DAPNE e Improved Possibilistic $c$-Means 2 (Zhang e Leung, 2004) (IPCM2) para DAP. O expoente $m$ de FCM e IPCM2 foi configurado como 2 (o que é comumente feito na literatura). O parâmetro minpts de SUBCLU foi configurado como 5 e a norma euclidiana foi adotada; a mesma configuração foi usada em todos os experimentos relatados neste capítulo. A solução de referência para a combinação de base e algoritmo (i.e., (DAPE, k-Means), (DAN, ECM), (DAN, EMGM), (DAPNE, [SUBCLU) e (DAP, IPCM2) ) foi produzida ao aplicar o algoritmo de agrupamento com o número correto de grupos (ou um epsilon bem ajustado no caso do algoritmo SUBCLU), e o resultado foi analisado para conferir que de fato a solução poderia ser considerada ideal no espaço de agrupamentos com o qual o correspondente algoritmo lida. Por exemplo, o algoritmo k-Means foi aplicado na base DAPE com $k=9$ grupos, usando as esperanças das distribuições gaussianas (usadas para gerar os grupos) como os centróides iniciais. A solução final possuía praticamente os mesmos centróides iniciais, corroborando com a validade das soluções obtidas.

É importante notar que não se está sugerindo que os algoritmos de agrupamento são inadequados para as bases de dados nas quais não estão sendo aplicados. Por exemplo, o algoritmo FCM consegue encontrar facilmente a estrutura presente na base DAPE, assim como IPCM2 consegue encontrar a estrutura da base DAN. O mais importante é que a base possua a estrutura de dados adequada para o algoritmo sendo aplicado.

Os algoritmos k-Means, FCM, EMGM e IPCM2 foram aplicados 30 vezes para cada número de grupos $k \in\{2,3, \ldots, \sqrt{n}\}$ (é comumente adotado na literatura o limiar superior $\sqrt{n}$ (Pal e Bezdek, 1995; Pakhira et al., 2005)) e o algoritmo SUBCLU foi aplicado 30 vezes para cada epsilon do intervalo $\{0.1,0.2, \ldots, 5.0\}$. As medidas foram aplicadas em cada solução e apenas os maiores valores (significando "os melhores") alcançados para cada $k$ ou epsilon para um dada medida foram retidos para gerar os gráficos das Figs. 5.4, 5.5, 5.6, 5.7 e 5.8, Medidas que apresentaram os mesmos valores foram unidas e representadas por uma única curva, e vários gráficos para os mesmos experimentos foram gerados por motivos de visualização.

Figura 5.4 mostra que a maioria das medidas apresentou os mesmos resultados que RI ou ARI quando avaliando APEs. Isso é esperado porque a maioria das medidas foi definida por meio de generalizações das variáveis usadas nas formulações de RI e ARI. Por exemplo, a medida 07CRI é uma versão nebulosa de $\mathbb{R I}$ na qual as variáveis de pareamento $a, b, c$ e $d$ foram definidas para conjuntos nebulosos. Quando aplicado em APEs, o índice 07CRI se reduz ao RI (Campello, 2007). RI, 09HI, 10CFn, 12DB e as medidas que apresentaram os mesmos resultados que $\mathbb{R I}$ foram fracamente afetadas pela diferença positiva entre o número de grupos obtidos e o número real de grupos. RI é igual a 1 e 0.94 para a soluções com 9 e 30 grupos, respectivamente, o que representa menos que $10 \%$ do intervalo $[0,1]$. Essa fraca sensibilidade ao número de grupos torna difícil decidir se a solução em mãos é realmente boa ou não. O índice 09CRI exibiu uma avaliação crescente ao longo da faixa dos números de grupos e o índice 09CARI produziu apenas pontuações próximas de zero. De fato, 09CARI produziu avaliações próximas de zero em todos os cenários desta seção. Contrariamente, JI, ARI, BC, 09EBC, 10CSI, $11 \mathrm{MD}$ e as medidas que apresentaram os mesmos resultados que ARI (incluindo a 


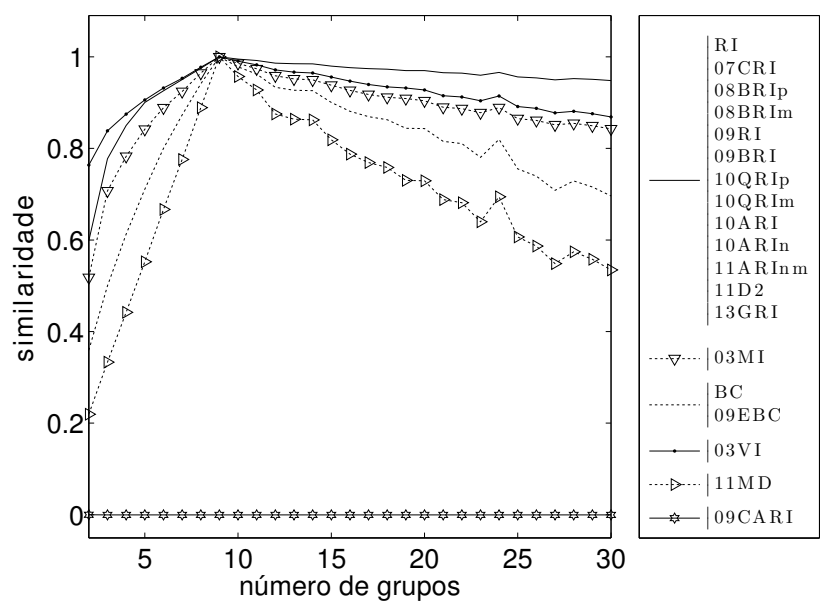

(a)

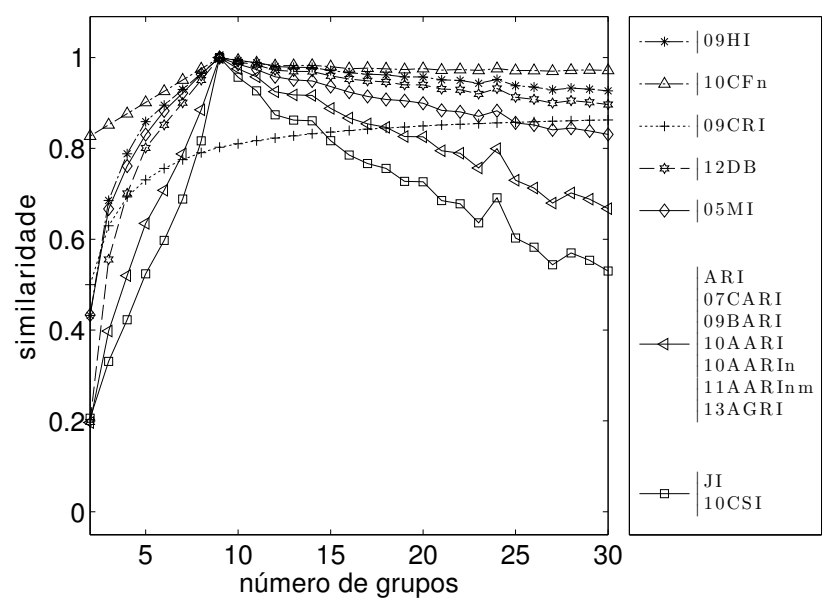

(b)

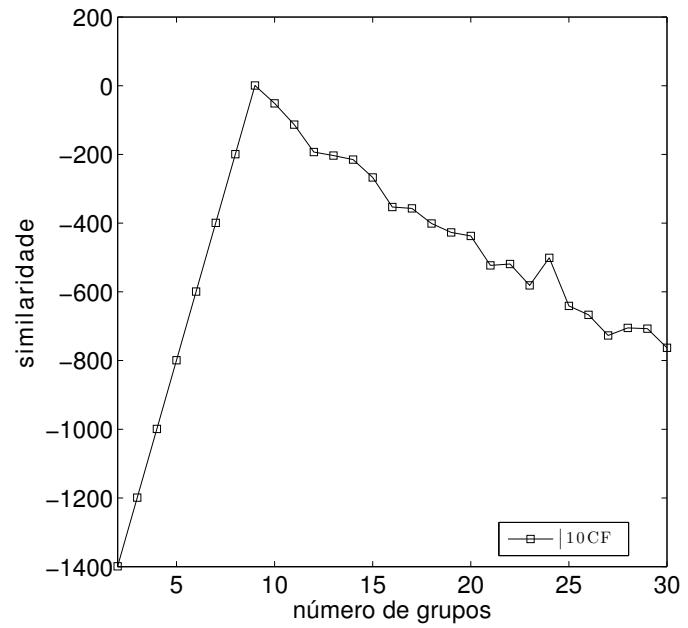

(c)

Figura 5.4: Avaliações das soluções do algoritmo k-Means para a base DAPE. 


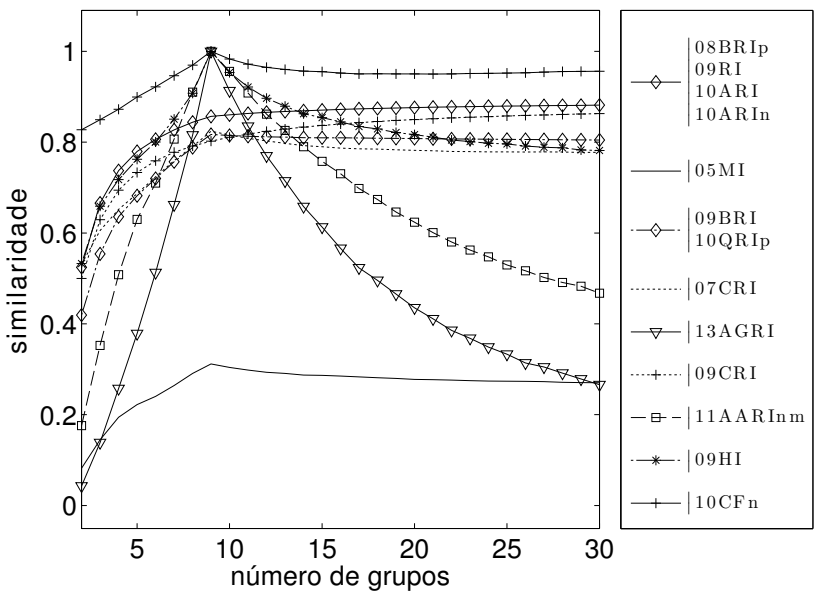

(a)

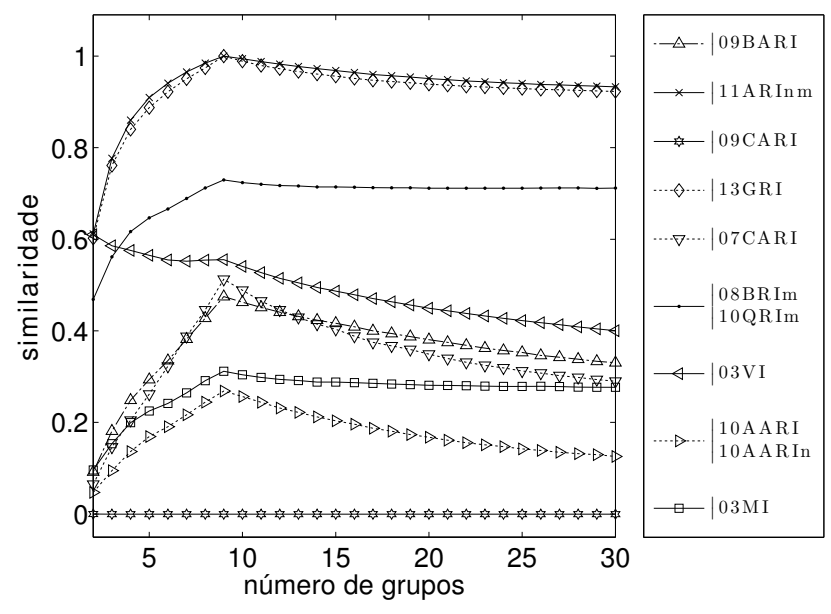

(b)

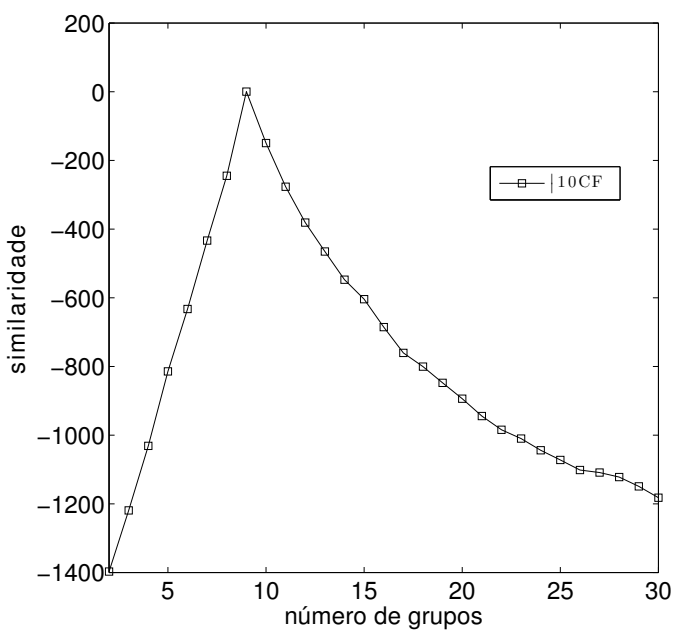

(c)

Figura 5.5: Avaliações das soluções do algoritmo FCM para a base DAN. 
medida 13AGRI proposta aqui) exibiram um decrescimento firme para números altos de grupos. Essa maior responsividade às diferenças nos agrupamentos parece ser mais intuitiva ao usuário. A medida 10CF (Figura 5.4(c)] atingiu o máximo 1 para o número correto de grupos, embora o gráfico não deixe isso claro.

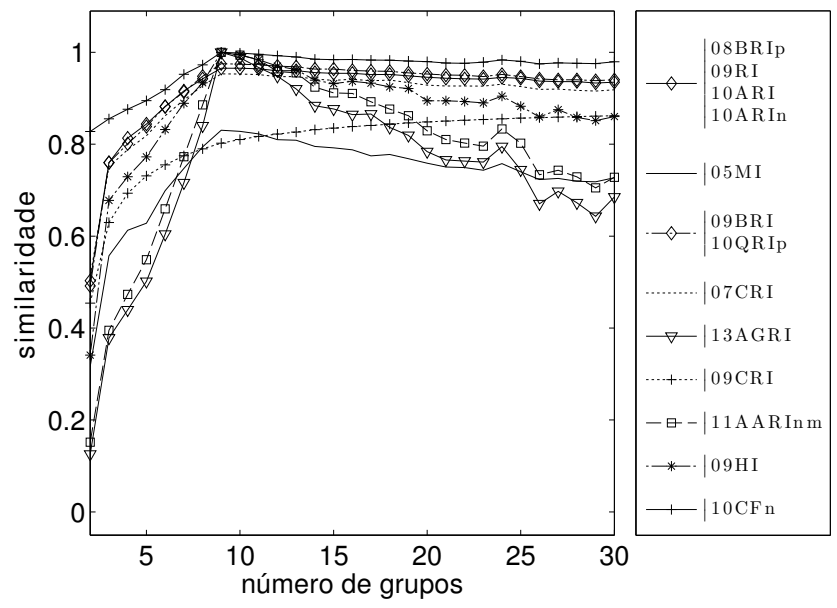

(a)

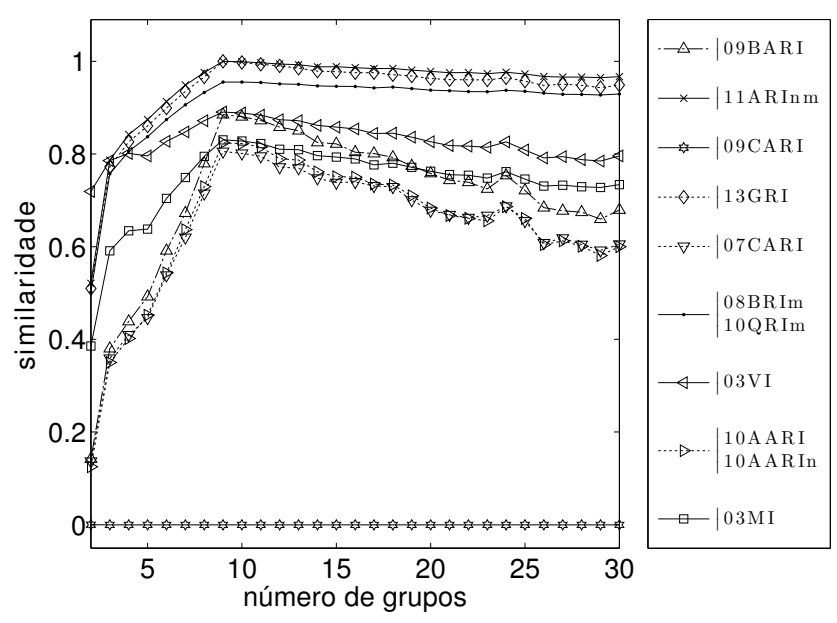

(b)

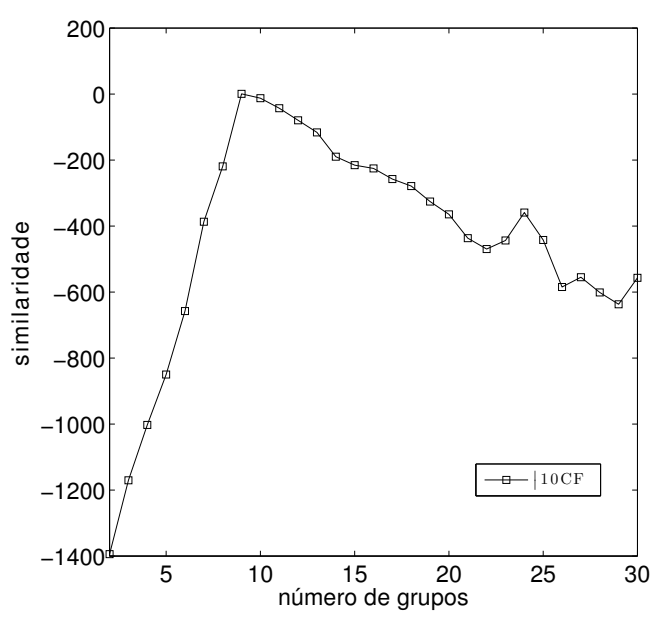

(c)

Figura 5.6: Avaliações das soluções do algoritmo EMGM para a base DAN. 
Figura 5.5 mostra os resultados das medidas para ANs aplicadas nas soluções do algoritmo FCM para a base DAN. Apenas 13AGRI e 11AARInm forneceram o valor máximo para o número correto de grupos e apresentaram um decrescimento firme a medida que a diferença positiva entre o número de grupos obtido e o número correto aumentava. 09HI atribuiu 1 para as soluções com o número correto de grupos, mas apresentou uma curva assintótica para números altos de grupos. 03VI, 08BRIp, 09RI, 09CRI, 09CARI, 10ARI e 10ARIn não conseguiram indicar a solução de referência.

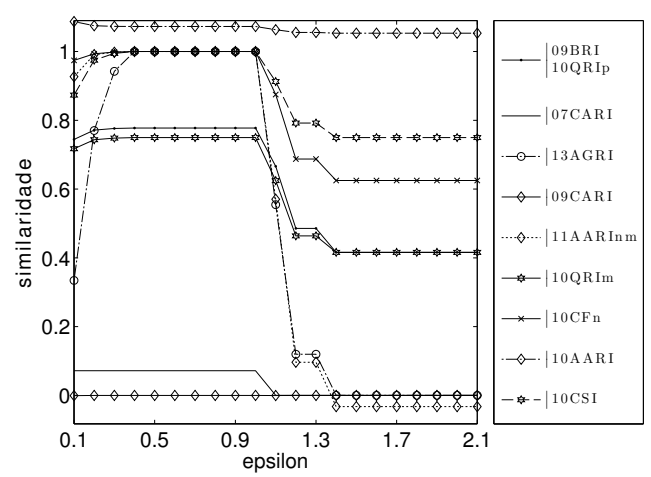

(a)

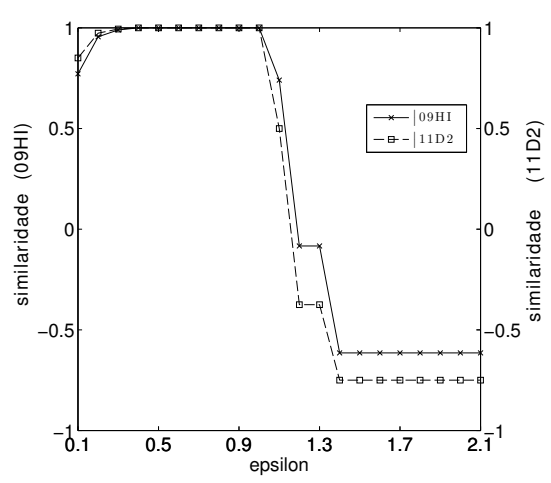

(c)

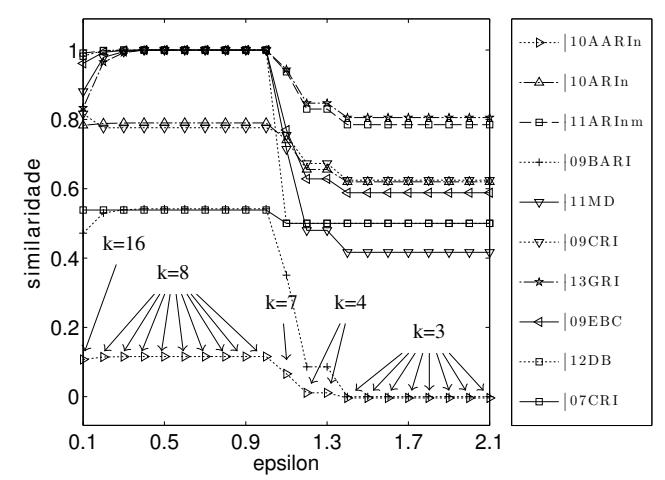

(b)

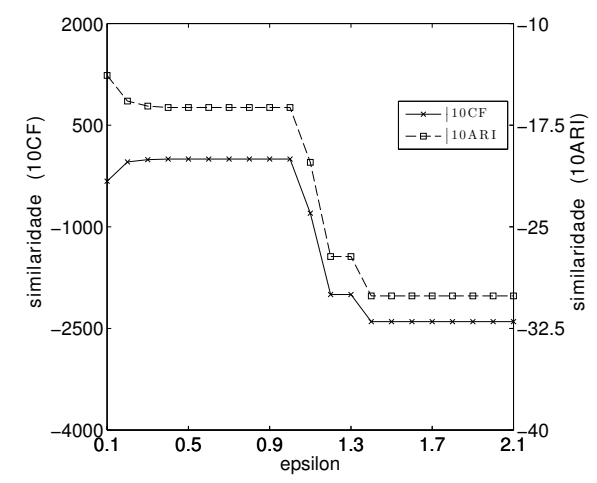

(d)

Figura 5.7: Avaliações das soluções do algoritmo SUBCLU para a base DAPNE.

A Figura 5.6 apresenta os resultados da aplicação das medidas para a soluções do algoritmo EMGM na base DAN. 07CRI, 08BRIp, 08BRIm, 09CRI, 09CARI, 09RI, 09BRI, 10QRIp, 10QRIm, 10ARI, 10ARIn e 11ARInm não conseguiram indicar o número correto de grupos. 09HI, 10CFn, 11AARInm, 13GRI e 13AGRI alcançaram o máximo 1 para o número ideal de grupos. Entretanto, 10CFn e 13GRI quase não apresentaram diferença nas avaliações dos agrupamentos com números de grupos maiores que $k^{*}=9$. 10CF atingiu 0.92 para o número ideal de grupos.

A Figura 5.7, na qual APNEs são avaliados, mostra apenas o intervalo $\{0.1,0.2, \ldots, 2.1\}$ de epsilons, visto que os resultados para os epsilons de 1.4 a 5.0 são idênticos. A solução de referência tem oito grupos: quatro dos dados projetados no plano, dois para os dados projetados no eixo $\vec{x}$ e dois para os dados projetados no eixo $\vec{y}$ (Figura 5.3(c)). Figura 5.7(b) indica o número de grupos encontrados para cada epsilon. SUBCLU]gera a solução de referência apenas para os epsilons de 0.4 a 1.0 (isso foi concluído por inspeção), e a maioria das medidas alcançou 
pontuações altas para esse intervalo. 07CRI, 09CRI, 10ARI e 10AARI julgaram a solução com epsilon igual a 0.1 como a melhor. A maioria das medidas identificaram as soluções corretas, mas apenas 09EBC, 09HI, 10CSI, 10CF, 10CFn, 11AARInm, 11MD, 11D2, 13GRI e 13AGRI atingiram o máximo 1 para elas. 11AARInm e 13AGRI aproximaram rapidamente do zero para epsilons não ótimos.

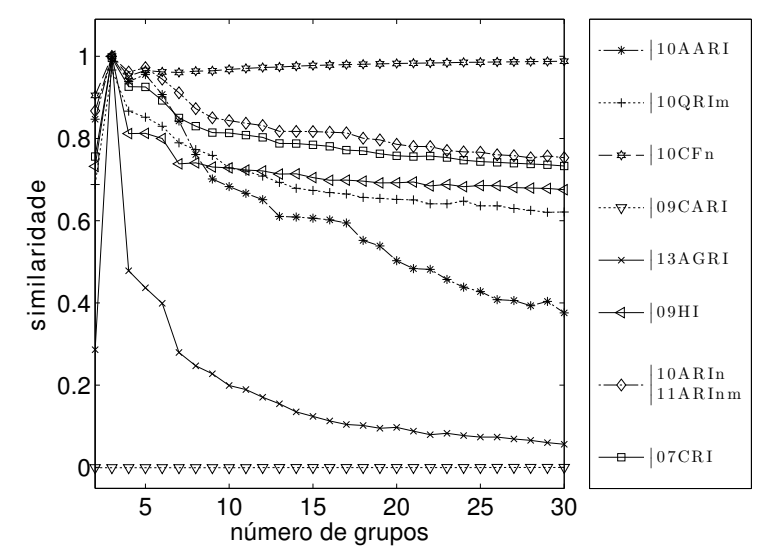

(a)

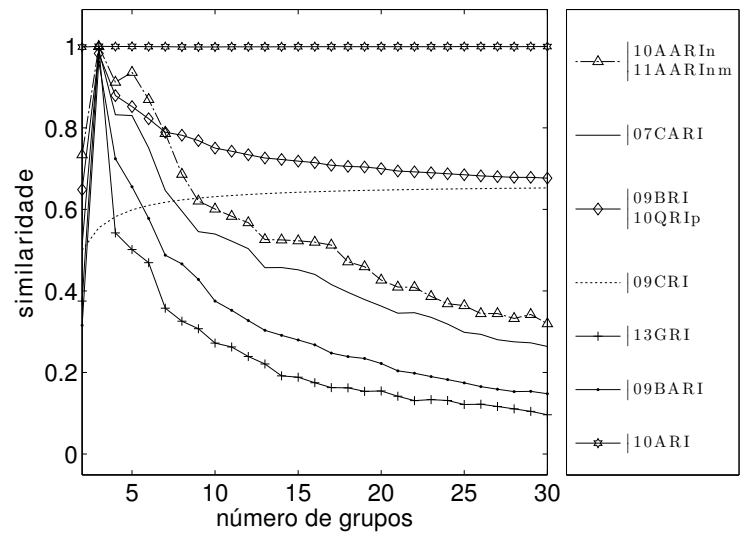

(b)

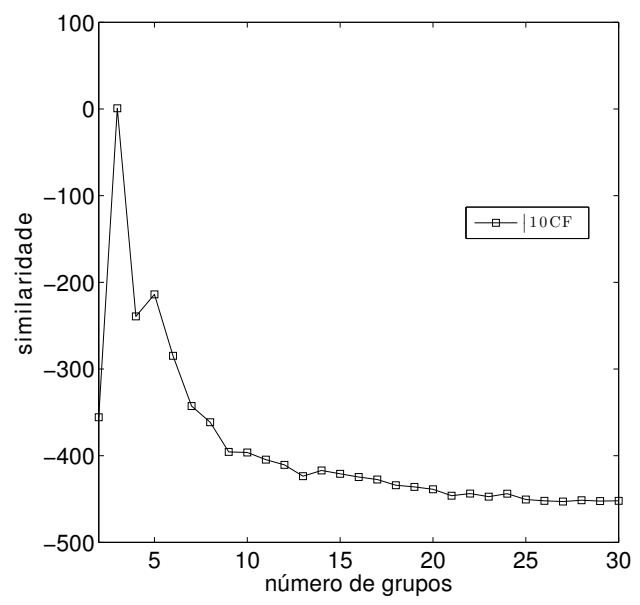

(c)

Figura 5.8: Avaliações das soluções do algoritmo IPCM2 para a base DAP.

Na Figura 5.8, 13GRI e 13AGRI exibiram uma queda brusca em suas avaliações e um pico de 1 para o número ideal de grupos. A base DAP tem apenas três grupos, enquanto os outros têm nove (DAPE e DAN) ou oito (DAPNE) grupos. Uma curva mais acentuada era então esperada. 07CRI, 09HI, 09BRI, 10QRIp, 10QRIm, 10ARI, 10ARIn e 11ARInm atribuíram pontuações altas para uma grande quantidade de números de grupos. As medidas 10ARI e 09CARI não conseguiram discriminar as soluções e a medida 09CRI não conseguiu indicar o número ideal de grupos. 10CFn apresentou uma avaliação crescente para soluções com números de grupos maiores que $k=5$. 10CF indicou o número correto de grupos (Fig 5.8(c)), mas não atribuiu o máximo 1 para as soluções correspondentes (o valor atribuído foi 0.92).

A Tabela 5.1 sumariza os resultados ao indicar com " $k$ "” as medidas que identificaram o agrupamento de referência e com "1" as medidas que atingiram o máximo para, e apenas para, a solução de referência. 09HI, 10CFn, 11AARInm, 13GRI e 13AGRI são as únicas medidas 
Tabela 5.1: Propriedades apresentadas pelas medidas no primeiro experimento.

\begin{tabular}{|c|c|c|c|c|c|}
\hline Medidas & APE & $\mathrm{AN} \sqrt{\mathrm{FCM}}$ & ANEMGM & ANE & AP \\
\hline JI & $k^{*} / 1$ & - & - & - & - \\
\hline $\mathrm{RI}$ & $k^{*} / 1$ & - & - & - & - \\
\hline ARI & $k^{*} / 1$ & - & - & - & - \\
\hline $\mathrm{BC}$ & $k^{*} / 1$ & - & - & - & - \\
\hline 03MI & $k^{*} / 1$ & $k^{*} /$ & $k^{*} /$ & - & - \\
\hline $05 \mathrm{MI}$ & $k^{*} / 1$ & $k^{*} /$ & $k^{*} /$ & - & - \\
\hline 03VI & $k^{*} / 1$ & $\%$ & $k^{*} /$ & - & - \\
\hline 07CRI & $k^{*} / 1$ & $k^{*} /$ &.$/$ & $\%$ & $k^{*} /$ \\
\hline 07CARI & $k^{*} / 1$ & $k^{*} /$ & $k^{*} /$ & $\%$ & $k^{*} /$ \\
\hline 08BRIp & $k^{*} / 1$ & $\%$ &.$/$ & - & - \\
\hline 08BRIm & $k^{*} / 1$ & $k^{*} /$ & $\%$ & - & - \\
\hline 09EBC & $k^{*} / 1$ & - & - & $k^{*} / 1$ & - \\
\hline 09CRI & $\%$ &. & $\%$ & $\%$ & $\%$ \\
\hline 09CARI &.$/$ & $\%$ &.$/$ & $\%$ & $\%$ \\
\hline 09HI & $k^{*} / 1$ & $k^{*} / 1$ & $k^{*} / 1$ & $k^{*} / 1$ & $k^{*} / 1$ \\
\hline 09RI & $k^{*} / 1$ & $\%$ &.$/$ & - & - \\
\hline 09BRI & $k^{*} / 1$ & $k^{*} /$ &.$/$ & $k^{*} /$ & $k^{*} /$ \\
\hline 09BARI & $k^{*} / 1$ & $k^{*} /$ & $k^{*} /$ & $k^{*} /$ & $k^{*} /$ \\
\hline 10QRIp & $k^{*} / 1$ & $k^{*} /$ &.$/$ & $k^{*} /$ & $k^{*} /$ \\
\hline 10QRIm & $k^{*} / 1$ & $k^{*} /$ & $\%$ & $k^{*} /$ & $k^{*} /$ \\
\hline 10ARI & $k^{*} / 1$ & . &.$/$ & $/$ &.$/$ \\
\hline 10AARI & $k^{*} / 1$ & $k^{*} /$ & $k^{*} /$ & $\%$ & $k^{*} / 1$ \\
\hline 10ARIn & $k^{*} / 1$ &.$/$ &.$/$ & $\%$ & $k^{*} / 1$ \\
\hline 10AARIn & $k^{*} / 1$ & $k^{*} /$ & $k^{*} /$ &. & $k^{*} / 1$ \\
\hline 10CSI & $k^{*} / 1$ & - & - & $k^{*} / 1$ & - \\
\hline $10 \mathrm{CF}$ & $k^{*} / 1$ & $k^{*} /$ & $k^{*} /$ & $k^{*} / 1$ & $k^{*} /$ \\
\hline 10CFn & $k^{*} / 1$ & $k^{*} / 1$ & $k^{*} / 1$ & $k^{*} / 1$ & $k^{*} / 1$ \\
\hline 11ARInm & $k^{*} / 1$ & $k^{*} / 1$ & $\%$ & $\%$ & $k^{*} / 1$ \\
\hline 11AARInm & $k^{*} / 1$ & $k^{*} / 1$ & $k^{*} / 1$ & $k^{*} / 1$ & $k^{*} / 1$ \\
\hline $11 \mathrm{MD}$ & $k^{*} / 1$ & - & - & $k^{*} / 1$ & - \\
\hline 11D2 & $k^{*} / 1$ & - & - & $k^{*} / 1$ & - \\
\hline $12 \mathrm{DB}$ & $k^{*} / 1$ & - & - & $\%$ & - \\
\hline 13GRI & $k^{*} / 1$ & $k^{*} / 1$ & $k^{*} / 1$ & $k^{*} / 1$ & $k^{*} / 1$ \\
\hline 13AGRI & $k^{*} / 1$ & $k^{*} / 1$ & $k^{*} / 1$ & $k^{*} / 1$ & $k^{*} / 1$ \\
\hline
\end{tabular}


que apresentaram as propriedades acima para cada cenário. Entretanto, 09HI, 10CFn e 13GRI mostraram uma fraca sensibilidade para variações de solução na maioria dos casos (e.g., Figs. 5.4(a) e 5.4(b) e 10CFn apresentou uma avaliação crescente para soluções progressivamente piores (Figura 5.8(a)). 11AARInm e 13AGRI identificaram a solução de referência, atingindo o máximo 1 para o agrupamento de referência e foram sensíveis à diferença no número de grupos em todos os cenários.

\subsubsection{Agrupamentos gerados aleatoriamente}

O experimento dessa seção é baseado em um publicado anteriormente (Vinh et al., 2009, 2010) que avalia a habilidade das medidas propostas para APEs (baseado em teoria da informação) em fornecer uma avaliação basal constante para soluções geradas aleatoriamente. Para um tipo particular de agrupamento (APE, AN, APNE ou AP), modelo aleatório (uniforme ou beta), $\operatorname{par}\left(n, k^{*}\right)$ e $k \in\left\{2,3, \ldots, 2 k^{*}\right\}$, foram gerados 30 pares de agrupamento com $n$ objetos. Cada par contém um agrupamento com $k$ grupos (representando a solução obtida) e um agrupamento com $k^{*}$ grupos (representando a solução de referência). Usou-se aqui quatro combinações de número de objetos e de grupos: $\left(n=25, k^{*}=5\right),\left(n=100, k^{*}=5\right),\left(n=50, k^{*}=10\right) \mathrm{e}$ $\left(n=200, k^{*}=10\right)$. Os modelos aleatórios usados para gerar os agrupamentos dependem do tipo de agrupamento como se segue:

- Para APE, cada objeto foi atribuído, de maneira uniforme, a um grupo (modelo uniforme);

- Para AN, foram gerados agrupamentos tanto pelo modelo uniforme como pelo modelo beta. Seja $X_{r}^{u}$ uma variável uniforme distribuída de acordo com o modelo uniforme $\mathcal{U}(0,1)$. Para o modelo uniforme, o objeto $\tilde{o}_{i}$ possui um grau de pertinência ao $r$-ésimo grupo distribuído de acordo com $X_{r}^{u} /\left(X_{1}^{u}+X_{2}^{u}+\cdots+X_{k}^{u}\right)$, em que $k$ é o número de grupos. Para o modelo beta, um inteiro é uniformemente escolhido de $r_{i} \in \mathbb{N}_{1, k}$ para cada objeto $\tilde{o}_{i}$ para indicar a qual grupo $\tilde{o}_{i}$ provavelmente possuirá o maior grau de pertinência. Formalmente, sejam $X_{r}^{b}$ e $Y^{b}$ duas variáveis aleatórias distribuídas de acordo com as distribuições beta $\mathrm{Be}(1,5)$ e $\mathrm{Be}(5,1)$, respectivamente. O objeto $\tilde{o}_{i}$ possui um grau de pertinência ao $r$-ésimo grupo $\left(r \neq r_{i}\right)$ distribuído de acordo com $X_{r}^{b} /\left(X_{1}^{b}+\cdots+\right.$ $\left.X_{r_{i}-1}^{b}+Y^{b}+X_{r_{i}+1}^{b}+\cdots+X_{k}^{b}\right)$ e ao $r_{i}$-ésimo grupo de acordo com $Y^{b} /\left(X_{1}^{b}+\cdots+\right.$ $\left.X_{r_{i}-1}^{b}+Y^{b}+X_{r_{i}+1}^{b}+\cdots+X_{k}^{b}\right)$;

- Para APNE, cada objeto $\tilde{o}_{i}$ foi uniformemente atribuído a $k_{i} \in \mathbb{N}_{1, k}$ grupos, em que $k_{i}$ também foi uniformemente selecionado;

- Para AP, foram gerados agrupamentos pelos modelos uniforme e beta. As distribuições usadas por ambos os modelos são similares àquelas usadas para AN. A única diferença é a ausência dos denominadores de normalização nas definições das distribuições.

Uma dada configuração experimental será denotada por uma quadrupla. Por exemplo, $\left(\right.$ APE $, \mathcal{U}, n=25, k^{*}=5$ ) se refere a um conjunto de APEs gerados de acordo com o modelo 


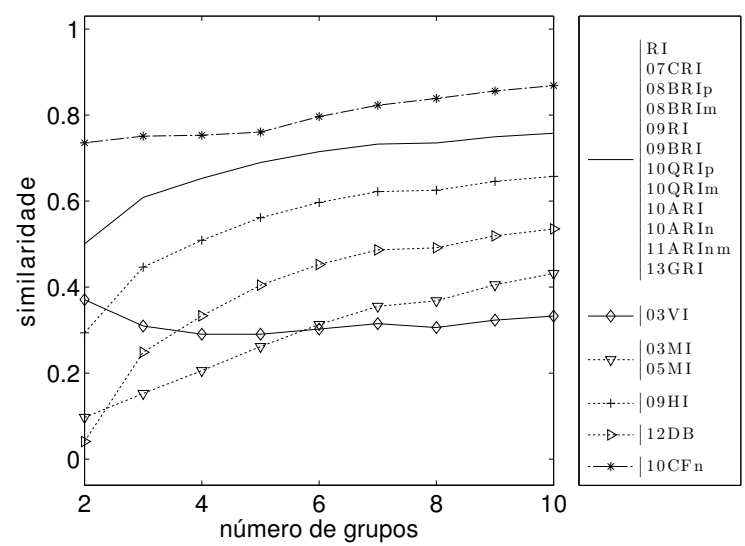

(a)

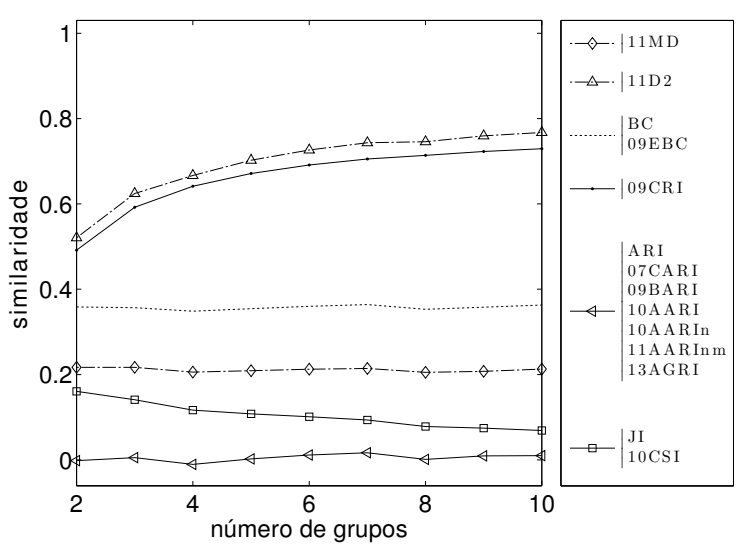

(b)

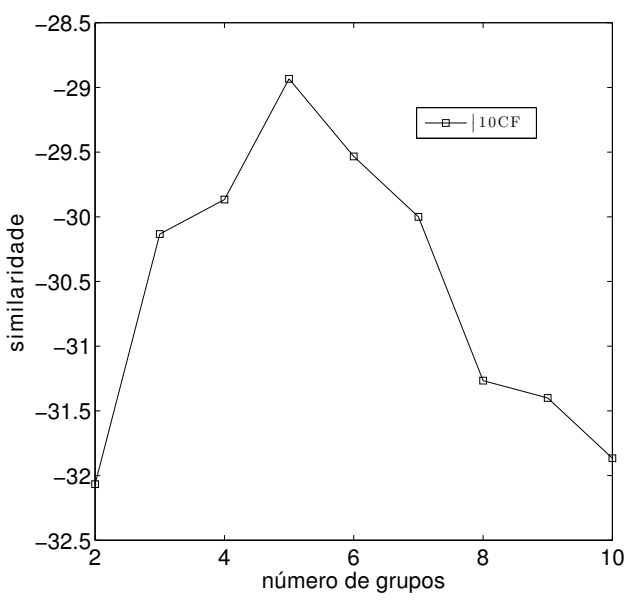

(c)

Figura 5.9: Médias das avaliações para (APE, $\mathcal{U}, n=25, k^{*}=5$ ). 
uniforme, no qual cada agrupamento possui 25 objetos. As soluções de (APE, $\mathcal{U}, n=25, k^{*}=$ 5 ) foram organizadas em 30 pares de APEs para cada $k \in\{2,3, \ldots, 10\}$. Cada par contém um APE com $k$ grupos e um APE com $k^{*}$ grupos. Assim, o conjunto (APE, $\mathcal{U}, n=25, k^{*}=5$ ) tem $30 \cdot 9=270$ pares de agrupamentos. As medidas foram aplicadas para avaliar a similaridade entre os dois agrupamentos de cada par e a avaliação média para cada $k \in\{2,3, \ldots, 10\}$ foi calculada e ilustrada na Figura 5.9. Similarmente, as Figs. 5.10, 5.11 e 5.12 se referem às configurações experimentais $\left(\mathrm{AN}, \mathcal{U}, n=100, k^{*}=5\right),\left(\mathrm{ANE}, \mathcal{U}, n=50, k^{*}=10\right) \mathrm{e}$ (AP, Be, $n=200, k^{*}=10$ ), respectivamente. As figuras remanescentes podem ser encontradas no endereço http://sn.im/25a9h8u.

As Figs. 5.9(a) e 5.9(b) mostram que 11 medidas exibiram as mesmas médias que o índice RI e que seis medidas apresentaram as mesmas médias que o índice ARI, respectivamente. RI e JI (em um menor grau) não possuem a propriedade de avaliação basal constante (Hubert e Arabie, 1985; Albatineh et al., 2006) e esse comportamento foi novamente observado nas Figs. 5.9(a) e 5.9(b), As medidas 13GRI e 13AGRI mostraram as mesmas médias dos índices [RI e ARI, respectivamente, devido à equivalência deles no contexto de APEs (Corolários 5.4 e 5.5 . Seção 5.5). 10CF alcançou o pico para $k=k^{*}$ grupos na Figura 5.9(c) para agrupamentos gerados aleatoriamente. BC, 09EBC, 11MD, ARI e as medidas com valores similares são as únicas que apresentaram uma avaliação basal constante. Os outros índices apresentaram uma tendência a favorecer soluções com números altos ou baixos de grupos.

A Figura 5.10 mostra os resultados da configuração experimental (AN, $\mathcal{U}, n=100, k^{*}=5$ ). 03MI, 05MI, 07CARI, 09BARI e 13AGRI apresentaram uma avaliação basal constante próxima de zero na Figura 5.10(b), 07CRI, 08BRIm e 10QRIm (Figura 5.10(a) também apresentaram avaliações basais constantes, embora não próximas de zero. Essas três medidas não foram formalmente ajustadas para aleatoriedade ou baseada em um índice que tenha sido. Além disso, as mesmas apresentaram uma baixa variância para vários números de grupos na Figura 5.5. Isso leva à suspeita de que o comportamento uniforme apresentado na Figura 5.10(a) seja devido a uma sensibilidade pobre com relação à variação das soluções. 09BRI e 10QRIp exibiram na Figura 5.10(b) uma curva monotonicamente decrescente com baixa variância de valores, assim como as medidas 10AARI e 10AARIn na Figura 5.10(a), 11AARInm produziu valores mais altos que o suposto máximo 1 e apresentou um comportamento contra-intuitivo na Figura 5.10(c) 10CF, 11ARInm e 13GRI apresentaram um pico para o número $k=k^{*}$ de grupos para soluções geradas aleatoriamente.

07CARI, 09BARI e 13AGRI são as únicas medidas que apresentaram uma avaliação basal constante próxima de zero na Figura 5.11, correspondendo aos resultados de (ANE, $\mathcal{U}, n=$ $\left.50, k^{*}=10\right)$. Assim como 10QRIm na Figura 5.10(a), o índice 10QRIp apresentou uma avaliação basal constante na Figura 5.11(a) provavelmente devido a uma baixa sensibilidade na discriminação de soluções, dado que ele não é ajustado para aleatoriedade e é baseado em uma medida $(\underline{\mathbb{R I}})$ conhecida por não ser. O mesmo não pode ser dito sobre 13AGRI, visto que este compara soluções contra um modelo nulo e exibiu uma forte sensibilidade na variação de soluções nos experimentos da Seção 5.4.1. 10AARI apresentou na Figura 5.11(b) valores maiores 


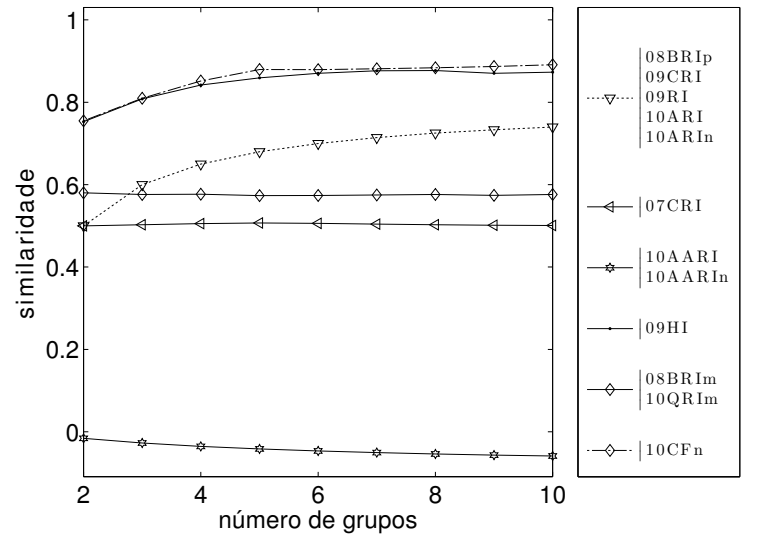

(a)

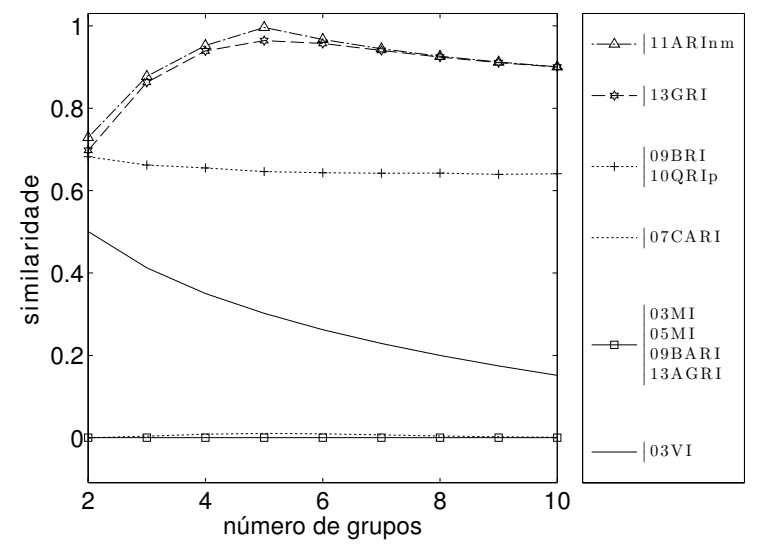

(b)

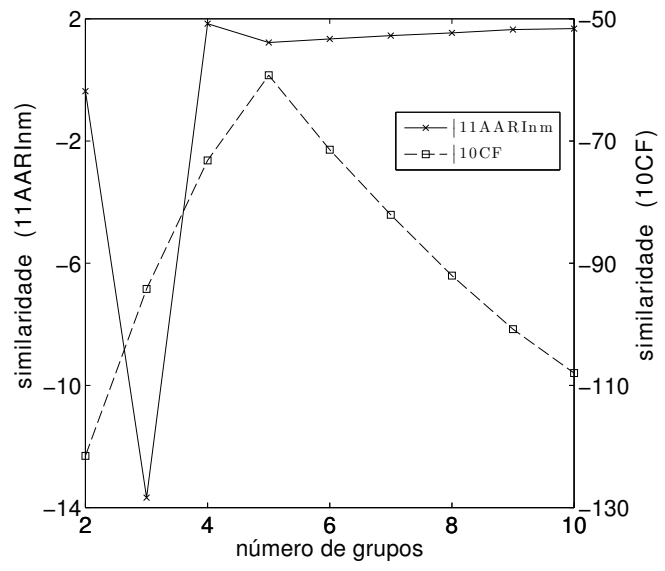

(c)

Figura 5.10: Avaliações médias para $\left(\mathrm{AN}, \mathcal{U}, n=100, k^{*}=5\right)$. 


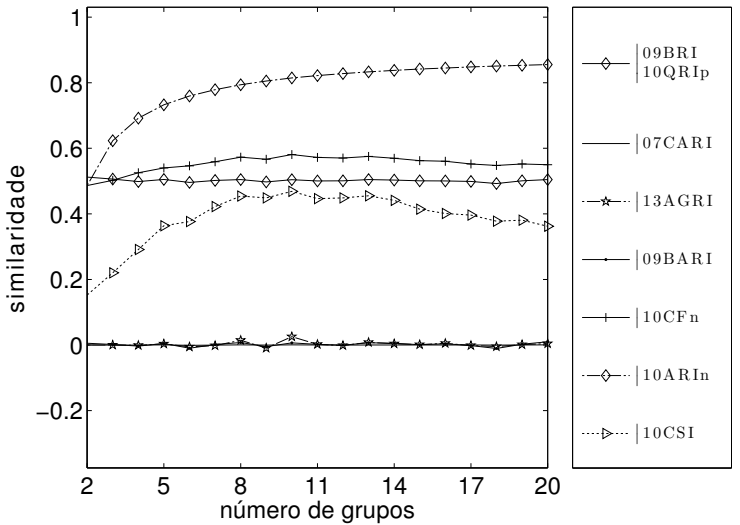

(a)

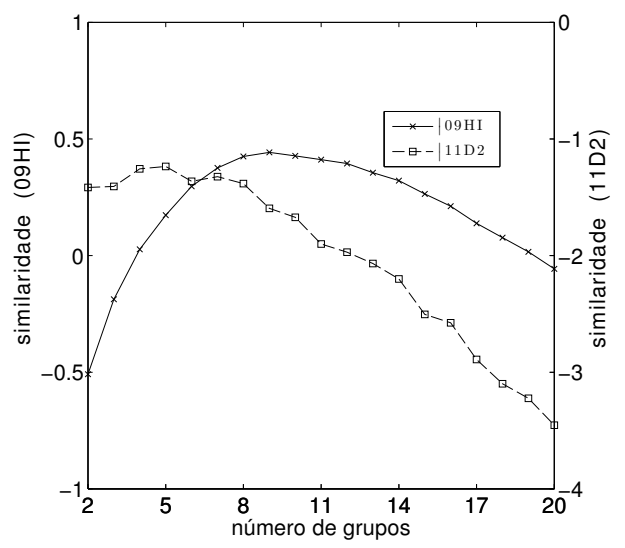

(c)

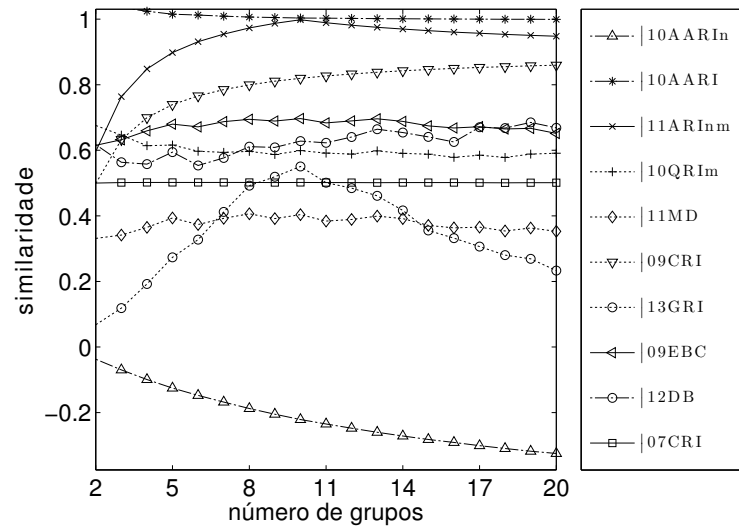

(b)

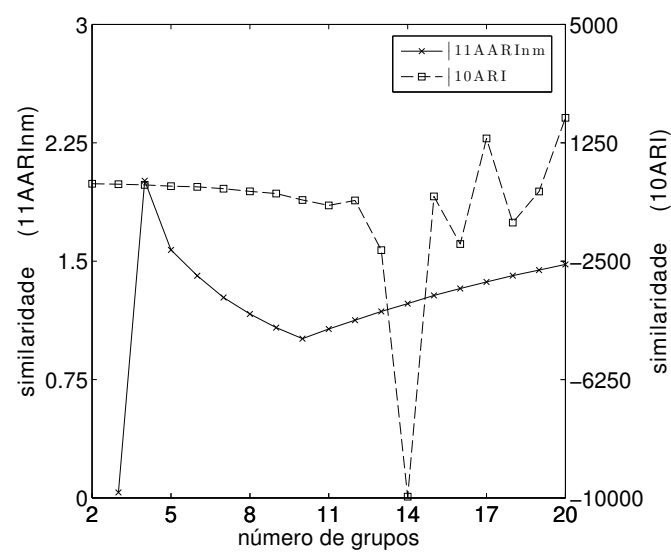

(d)

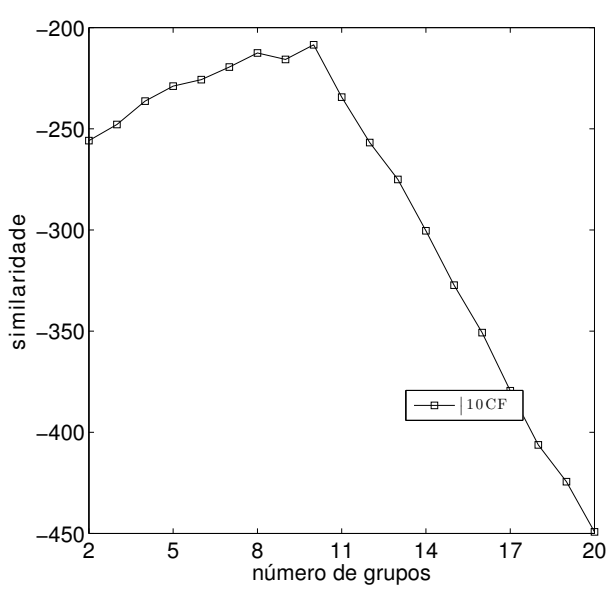

(e)

Figura 5.11: Avaliações médias para (ANE, $\left.\mathcal{U}, n=50, k^{*}=10\right)$. 
que 1 para a maioria das soluções. 10CF (Figura 5.11(e)] e 13GRI (Figura 5.11(b) novamente atingiram o pico para $k=k^{*}$ grupos para soluções geradas aleatoriamente. 10ARI e 11AARInm (Figura 5.11(d) produziram avaliações extremamente irregulares. 11AARInm atribuiu $-\infty$ (transbordamento aritmético) para as soluções com $k=2$ grupos devido a divisões por denominadores próximos de zero.

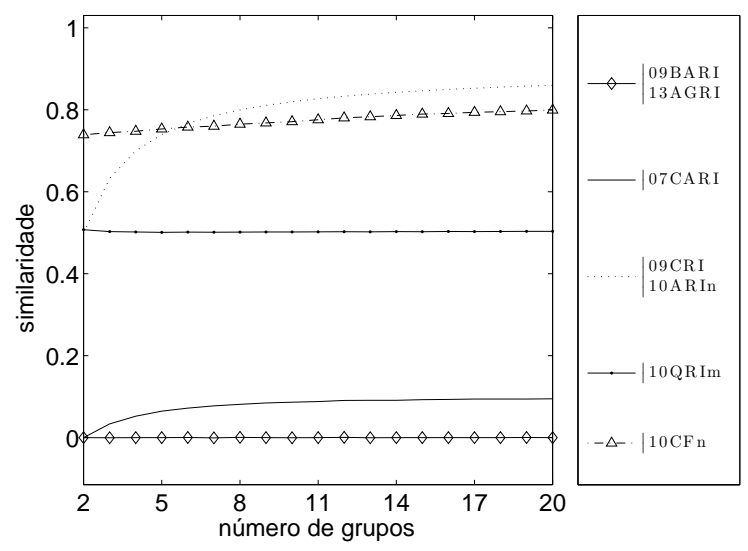

(a)

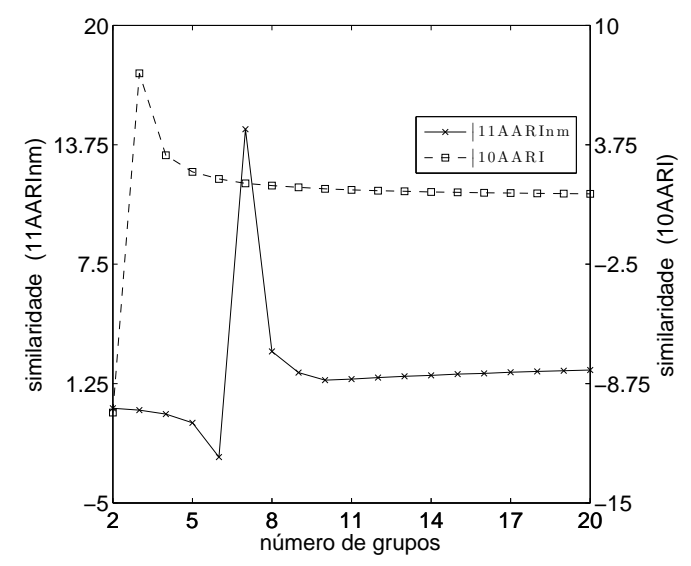

(c)

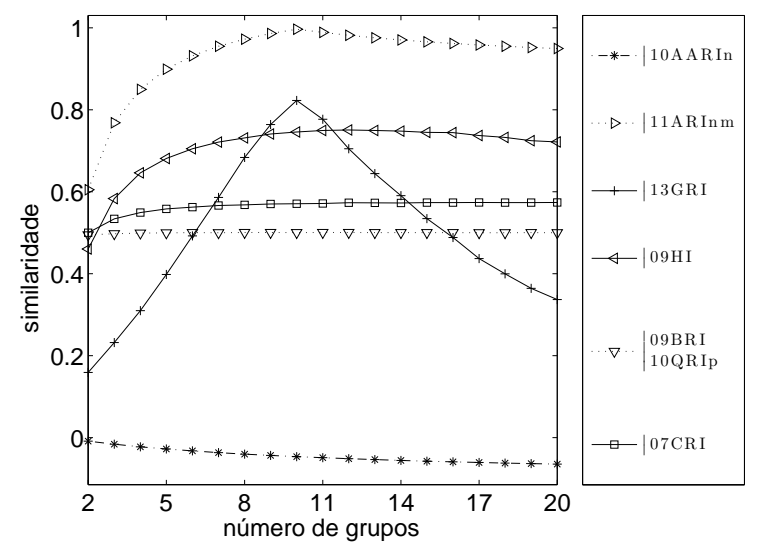

(b)

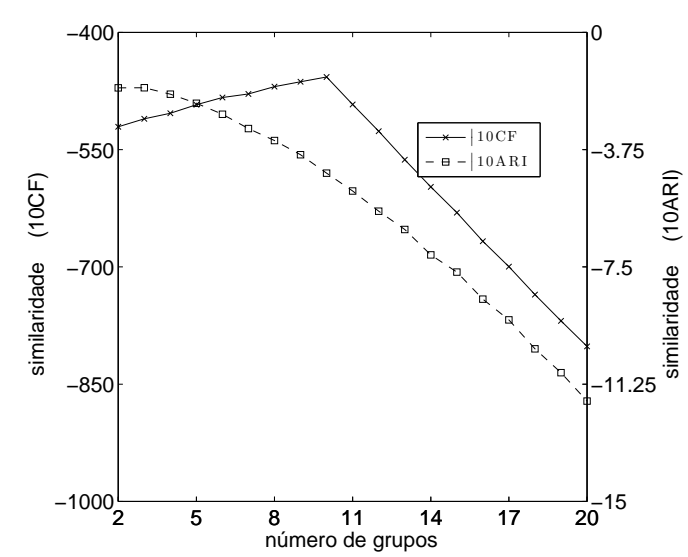

(d)

Figura 5.12: Avaliações médias para (AP, Be, $\left.n=200, k^{*}=10\right)$.

Figura 5.12 ilustra os resultados para (AP, Be, $n=200, k^{*}=10$ ). 09BRI, 09BARI, 10QRIp, 10QRIm e 13AGRI apresentaram avaliações basais constantes e essas avaliações para 13AGRI e 09BARI foram próximas de zero. 10CF (Figura 5.12(d)) e 13GRI (Figura 5.12(b) novamente pontuaram soluções aleatórias com $k=k^{*}$ grupos como sendo as melhores. 10AARI e 11AARInm apresentaram resultados inesperados (Figura 5.12(c)).

Os resultados desta seção são sumarizados na Tabela 5.2. A maioria das medidas não conseguiu prover avaliações imparciais. Essas medidas tendem a favorecer soluções aleatórias com números de grupos altos ou baixos ou apresentam um pico para soluções aleatórias que possuem o mesmo número de grupos que o agrupamento de referência. Esse comportamento não é desejável, uma vez que as soluções comparadas foram geradas de maneira independente. Apenas 09BARI e 13AGRI apresentaram avaliações basais constantes (e próximas de zero) em todos os cenários. O modelo nulo de 13AGRI é claramente violado em cada cenário, o que sugere que 
Tabela 5.2: A medida de similaridade apresentou avaliações basais constantes?

\begin{tabular}{lcccclcccc}
\hline Medidas & APE & AN & APNE & AP & Medidas & APE & AN & APNE & AP \\
\hline \hline JI & $\mathrm{n}$ & - & - & - & 09BARI & $\mathbf{s}$ & $\mathbf{s}$ & $\mathbf{s}$ & $\mathbf{s}$ \\
RI & $\mathrm{n}$ & - & - & - & 10QRIp & $\mathrm{n}$ & $\mathrm{n}$ & $\mathbf{s}$ & $\mathbf{s}$ \\
ARI & $\mathbf{s}$ & - & - & - & 10QRIm & $\mathrm{n}$ & $\mathbf{s}$ & $\mathrm{n}$ & $\mathbf{s}$ \\
BC & $\mathbf{s}$ & - & - & - & 10ARI & $\mathrm{n}$ & $\mathrm{n}$ & $\mathrm{n}$ & $\mathrm{n}$ \\
03MI & $\mathrm{n}$ & $\mathbf{s}$ & - & - & 10AARI & $\mathbf{s}$ & $\mathrm{n}$ & $\mathrm{n}$ & $\mathrm{n}$ \\
05MI & $\mathrm{n}$ & $\mathbf{s}$ & - & - & 10ARIn & $\mathrm{n}$ & $\mathrm{n}$ & $\mathrm{n}$ & $\mathrm{n}$ \\
03VI & $\mathrm{n}$ & $\mathrm{n}$ & - & - & 10AARIn & $\mathbf{s}$ & $\mathrm{n}$ & $\mathrm{n}$ & $\mathrm{n}$ \\
07CRI & $\mathrm{n}$ & $\mathbf{s}$ & $\mathbf{s}$ & $\mathrm{n}$ & 10CSI & $\mathrm{n}$ & - & $\mathrm{n}$ & - \\
07CARI & $\mathbf{s}$ & $\mathbf{s}$ & $\mathbf{s}$ & $\mathrm{n}$ & 10CF & $\mathrm{n}$ & $\mathrm{n}$ & $\mathrm{n}$ & $\mathrm{n}$ \\
08BRIp & $\mathrm{n}$ & $\mathrm{n}$ & - & - & 10CFn & $\mathrm{n}$ & $\mathrm{n}$ & $\mathrm{n}$ & $\mathrm{n}$ \\
08BRIm & $\mathrm{n}$ & $\mathbf{s}$ & - & - & 11ARInm & $\mathrm{n}$ & $\mathrm{n}$ & $\mathrm{n}$ & $\mathrm{n}$ \\
09EBC & $\mathbf{s}$ & - & $\mathrm{n}$ & - & 11AARInm & $\mathbf{s}$ & $\mathrm{n}$ & $\mathrm{n}$ & $\mathrm{n}$ \\
09CRI & $\mathrm{n}$ & $\mathrm{n}$ & $\mathrm{n}$ & $\mathrm{n}$ & 11MD & $\mathbf{s}$ & - & $\mathrm{n}$ & - \\
09CARI & - & - & - & - & 11D2 & $\mathrm{n}$ & - & $\mathrm{n}$ & - \\
09HI & $\mathrm{n}$ & $\mathrm{n}$ & $\mathrm{n}$ & $\mathrm{n}$ & 13GRI & $\mathrm{n}$ & $\mathrm{n}$ & $\mathrm{n}$ & $\mathrm{n}$ \\
09RI & $\mathrm{n}$ & $\mathrm{n}$ & - & - & 13AGRI & $\mathbf{s}$ & $\mathbf{s}$ & $\mathbf{s}$ & $\mathbf{s}$ \\
09BRI & $\mathrm{n}$ & $\mathrm{n}$ & $\mathbf{s}$ & $\mathbf{s}$ & 12DB & $\mathrm{n}$ & - & $\mathrm{n}$ & - \\
\hline
\end{tabular}

o ajuste de 13GRI não é apenas um adorno teórico, mas uma verdadeira correção que torna a comparação de agrupamentos mais justa. Note que, ao contrário do índice 13AGRI, a medida 09BARI não atribuiu a pontuação máxima 1 para a maioria das soluções perfeitas encontradas na seção anterior.

\subsubsection{Medida 13AGRI para agrupamento nebuloso}

Aplicou-se os algoritmos k-Means e FCM 30 vezes para cada número de grupos $k \in$ $\{2,3, \ldots, 20\}$ nas bases de dados da UCI (Frank e Asuncion, 2010) descritas na Tabela 5.3 . A rotulação de referência de cada base de dados foi considerada como sendo o agrupamento de referência. O índice 13AGRI avaliou o melhor agrupamento (melhor de acordo com a respectiva função-custo do algoritmo) para cada número de grupos usando a classificação conhecida como solução de referência; a solução de referência é, portanto, um APE. 13AGRI provê os mesmos resultados que a medida ARI para agrupamentos gerados pelo k-Means dado que este produz APEs (Corolário 5.5. Seção 5.5. FCM é tido como a versão nebulosa do k-Means, no sentido de que ambos buscam por grupos esféricos, e tende ao k-Means quando o expoente $m$ se aproxima de 1 (Yu et al., 2004). Assim, suas soluções são frequentemente similares no sentido de que a conversão de uma solução do FCM em um APE (atribuindo os objetos aos grupos para os quais eles apresentam maior grau de pertinência) resulta em um agrupamento cuja atribuição relativa entre os objetos é similar à atribuição relativa que seria feita pela aplicação do k-Means (i.e., quando os objetos $\tilde{o}_{i}$ e $\tilde{o}_{j}$ são atribuídos ao mesmo grupo em uma solução, eles são frequentemente atribuídos ao mesmo grupo na outra solução). É examinado nesta seção se a medida 13AGRI produz avaliações semelhantes para soluções geradas pelo k-Means e pelo 
Tabela 5.3: Bases de dados da UCI.

\begin{tabular}{|c|c|c|c|}
\hline Nome & \# Objetos & \# Atributos & \# Classes \\
\hline Breast cancer w. d. (bcw-d) & 569 & 30 & 2 \\
\hline Breast cancer w. o. $(\mathrm{bcw}-\mathrm{o})^{\mathrm{a}}$ & 699 & 9 & 2 \\
\hline Synthetic control chart (chart) & 600 & 60 & 6 \\
\hline Ecoli data set (ecoli) & 336 & 7 & 7 \\
\hline Glass identification (glass) & 214 & 9 & 6 \\
\hline Haberman (haberman) & 306 & 3 & 2 \\
\hline Image segmentation (img) & 210 & 19 & 7 \\
\hline Ionosphere (ion) & 351 & 34 & 2 \\
\hline Iris (iris) & 150 & 4 & 3 \\
\hline Pima indians diabetes (pima) & 768 & 8 & 2 \\
\hline Connectionist bench (sonar) & 208 & 60 & 2 \\
\hline SPECT heart (heart) & 267 & 22 & 2 \\
\hline Vehicle silhouettes (vehicle) & 846 & 18 & 4 \\
\hline Wine (wine) & 178 & 13 & 3 \\
\hline
\end{tabular}

a A base de dados original possui 16 objetos com atributos faltantes. Adotou-se o algoritmo dos $k$ vizinhos mais próximos com a distância euclidiana para a imputação (Hastie et al. 1999) e a base resultante foi usada nos experimentos.

FCM. Sendo esse o caso, pode-se adquirir uma confiança maior na validade das avaliações do índice 13AGRI para ANs, visto, também, que 13AGRI e ARI são equivalentes no domínio dos APEs.

Para cada base de dados, a Tabela 5.4 mostra as correlações de Pearson entre avaliações da medida 13AGRI para soluções produzidas pelo k-Means e para soluções produzidas pelo FCM, ao longo da faixa de números de grupos $\{2,3, \ldots, 20\}$. Cinco correlações foram maiores que 0.9 e mais da metade foram maiores que 0.7. As Figs. 5.14(a) e 5.14(b) ilustram as avaliações da medida 13AGRI para as bases de dados correspondentes às três maiores e três menores correlações, respectivamente. As legendas das figuras denotam as bases correspondentes, o tipo de agrupamento e o número de classes da rotulação a priori. Pelo fato das soluções de referência serem APEs, a medida 13AGRI quase sempre apresentou pontuações maiores para APEs do que para ANs. Os baixos valores de correlação parecem ter sido obtidos nas bases de dados para as quais os algoritmos não conseguiram encontrar bons agrupamentos. Para essas bases, as similaridades entre as soluções encontradas e a de referência flutuaram ao longo dos números de grupos porque não existiria um número ideal de grupos para o qual a curva de avaliação apresentaria um pico. Os algoritmos k-Means e FCM produziram soluções pobres para as bases haberman e sonar de acordo com 13AGRI. As avaliações de 13AGRI indicam que o algoritmo k-Means descobriu pelo menos parte da estrutura da base chart porque a pontuação de 0.5 dada pelo índice 13AGRI (a mesma que seria dada pelo ARI) é alta, de acordo com nossa a experiência. Entretanto, não existe uma solução distinta para a faixa de números de grupos. 13AGRI indicou o AN com três grupos como o sendo o mais similar ao agrupamento de referência para a base chart.

Para melhor investigar o comportamento da medida 13AGRI para soluções da base chart, 
Tabela 5.4: Correlação entre as avaliações de 13AGRI para APEs e ANs.

\begin{tabular}{ccccccc} 
bcw-d & iris & wine & bcw-o & img & ecoli & ion \\
\hline 0.99 & 0.99 & 0.98 & 0.98 & 0.91 & 0.89 & 0.83 \\
vehicle & glass & pima & heart & haberman & chart & sonar \\
\hline 0.75 & 0.70 & 0.69 & 0.60 & 0.23 & 0.02 & -0.45
\end{tabular}

reduziu-se por meio da técnica $\mathrm{ACP}$ a dimensionalidade ao projetar os dados (que possuem 60 atributos) nos primeiros nove componentes principais, os quais dizem respeito a $90 \%$ de toda a variância que a base apresenta. Identificou-se dois pares de classes com alto grau de sobreposição (especificamente, as classes decreasing trend com downward shift e increasing trend com upward shift (Alcock, 1999) ) ao projetar os dados em vários planos. Uniu-se então a classe decreasing trend com a classe downward shift e a classe increasing trend com a classe upward shift, resultando em uma classificação de quatro classes. A correlação de Pearson entre as avaliações da medida 13AGRI é agora 0.91, usando a mesma configuração experimental acima. A Figura 5.14 apresenta as avaliações das soluções dos algoritmos k-Means e FCM. $\mathrm{O}$ índice 13AGRI forneceu pontuações altas para as soluções do k-Means com três e quatro grupos, enquanto é sugerido pela medida 13AGRI que a melhor solução do FCMé aquela com três grupos.

Figura 5.13: Avaliações de 13AGRI que exibiram as três maiores (a) e as três menores correlações (b).

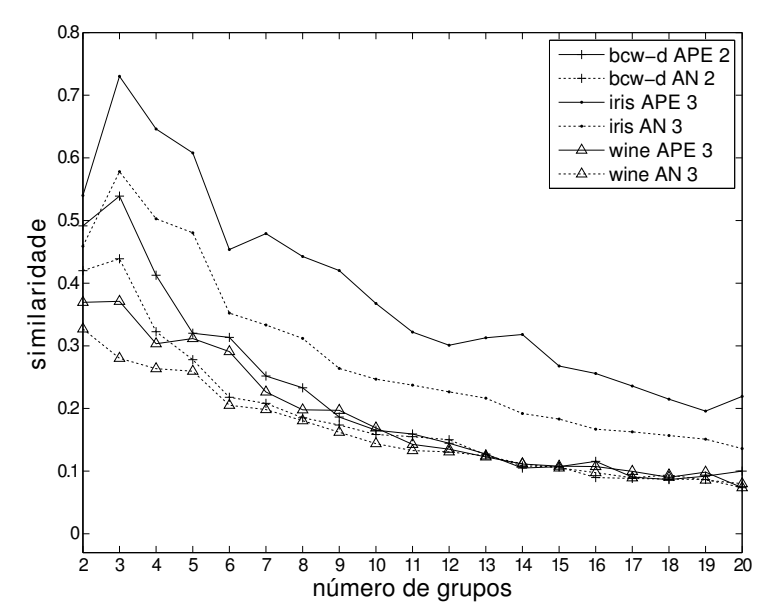

(a)

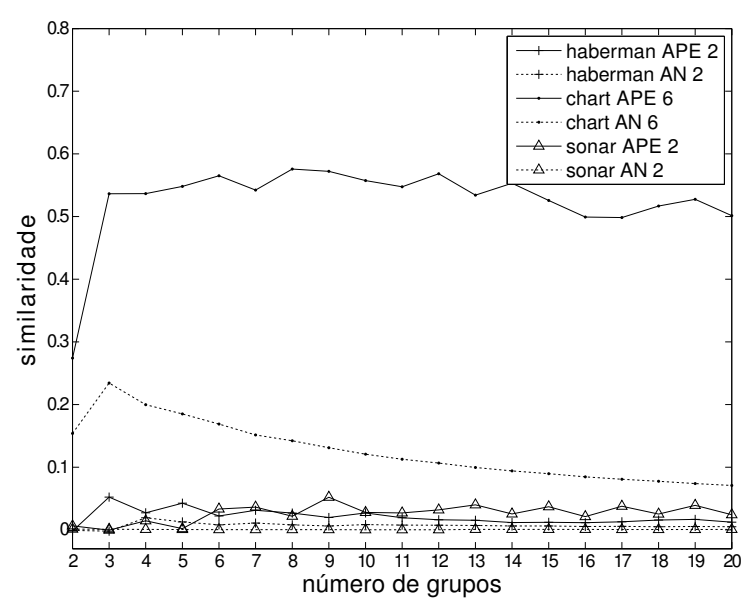

(b)

Os resultados indicam que 13AGRI quando aplicado em ANs se comporta de maneira similar quando o mesmo é aplicado em APEs (lembre-se da equivalência entre 13AGRI e ARI para APEs), particularmente quando as soluções encontram alguma estrutura dos dados. Considerando que ARI é uma das medidas de agrupamento mais confiáveis, os resultados corroboram com a validade da avaliação de 13AGRI para ANs. 
Figura 5.14: Avaliações de 13AGRI para a base chart processada.

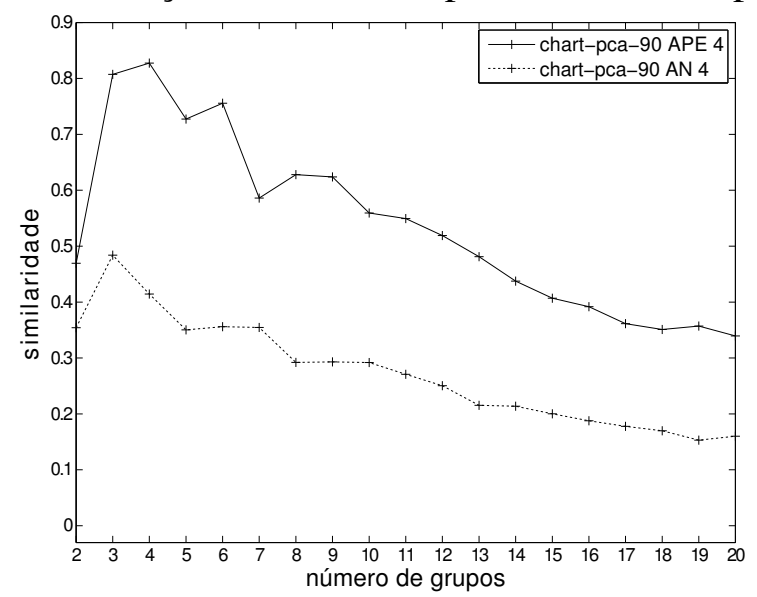

Tabela 5.5: Correlação entre as avaliações de 13AGRI e a estatística de estabilidade.

\begin{tabular}{ccccc} 
bcw-d & bcw-o & wine & iris & img \\
\hline 0.94 & 0.90 & 0.85 & 0.67 & 0.59
\end{tabular}

\subsubsection{Medida 13AGRI como estatística de estabilidade}

O algoritmo EMGM foi aplicado 100 vezes para cada número de grupos $k \in\{2, \ldots, 20\}$ em subamostras das cinco bases da seção anterior que correspondem às cinco maiores correlações (i.e., bcw-d, iris, wine, bcw-o e img), gerando 100 misturas gaussianas para cada número de grupos e base de dados; essas misturas de gaussianas explicam diferentes fenômenos que produziram a base de dados. Para cada mistura de gaussianas encontrada, calculou-se a matriz $\mathrm{U}$ de AN para a base de dados, de forma que $\mathrm{U}_{r, i}$ é a probabilidade de $\tilde{o}_{i}$ pertencer ao $r$-ésimo grupo (i.e., à $r$-ésima componente gaussiana da mistura). 13AGRI compara cada um dos $\left(\begin{array}{c}100 \\ 2\end{array}\right)$ pares de ANs para cada número de grupos e base de dados, e a média das avaliações é interpretada como a estatística de estabilidade (quanto menos diverso for o conjunto de soluções, maior é a estatística de estabilidade) para cada número de grupos e base correspondentes. Subamostras foram geradas ao selecionar aleatoriamente $80 \%$ dos objetos da base, sem reposição, como em (Monti et al. 2003). É descrito pelo Algoritmo 5.2 como a avaliação de estabilidade é feita para estimar o número de grupos e selecionar a solução mais promissora de um conjunto de agrupamentos.

A Tabela 5.5 apresenta as correlações entre valores da estatística de estabilidade (definido pelo Passo 9) e as avaliações de 13AGRI (similaridade entre o protótipo de conjunto de agrupamentos $\mathrm{V}^{k}$, Passo 10 , e a solução de referência) para os diferentes números de grupos. As altas correlações indicam que a estatística de estabilidade, a qual pode ser usada em cenários reais, segue aproximadamente as avaliações de 13AGRI, as quais dependem do agrupamento de referência.

A Figura 5.15 ilustra as avaliações de 13AGRI para cada protótipo de conjunto de agrupamentos (Passo 10 do Algoritmo 5.2) e base de dados. A barra de erro para um dado $k \in\{2, \ldots, 20\}$ e uma dada base foi gerada como segue. Seja $t_{k}$ a estatística de estabili- 


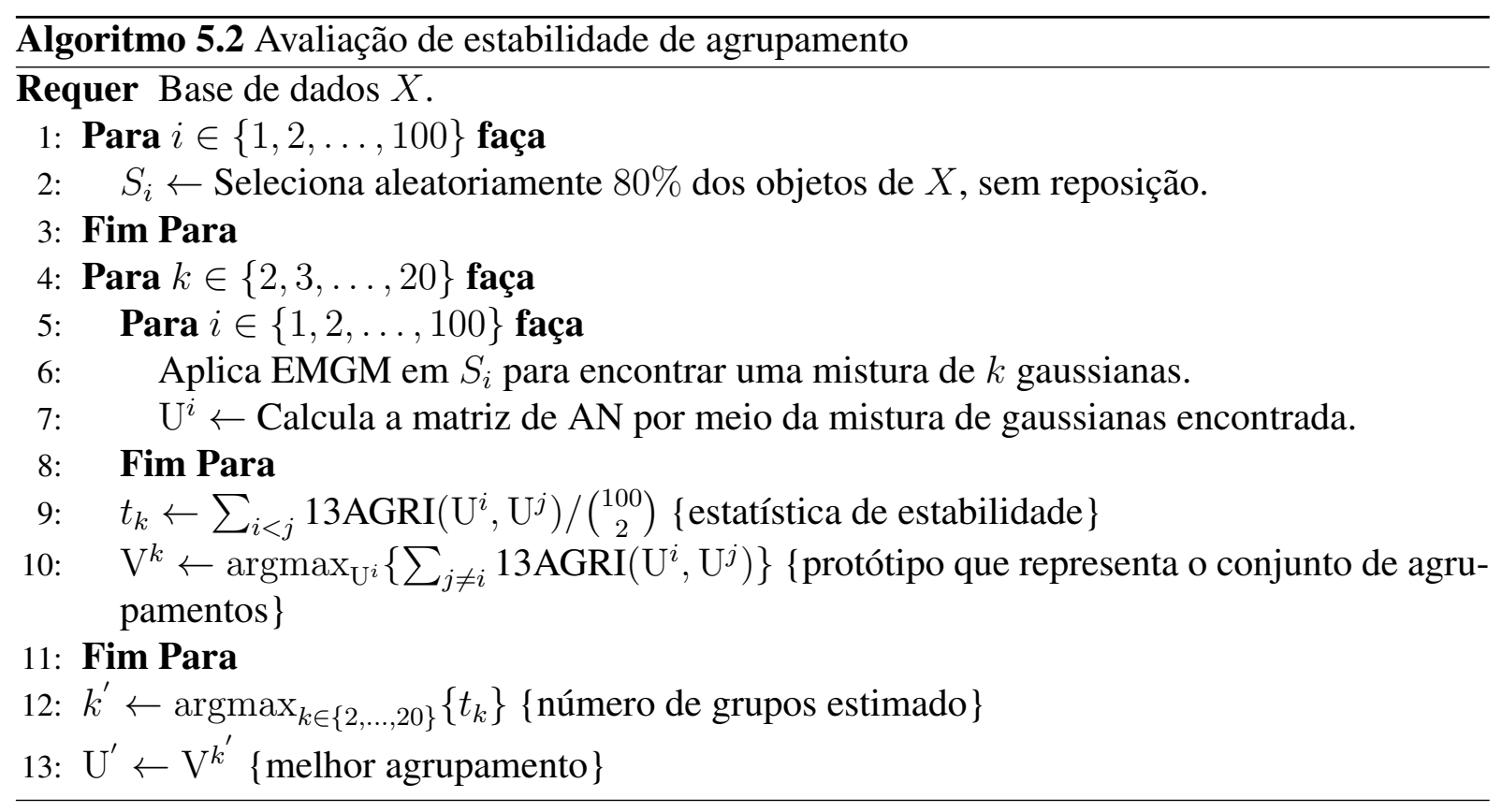

dade para o conjunto de agrupamentos com $k$ grupos cada (Passo99). A barra de erro assume 0 para o conjunto de agrupamentos mais estável (de maior valor de estatística de estabilidade) e 0.1 para o conjunto menos estável, por motivos de visualização. Assim, o valor da barra de erro correspondente ao conjunto de agrupamentos com $k$ grupos é

$$
\frac{t_{k}-\min \left\{t_{2}, t_{3}, \ldots, t_{20}\right\}}{\max \left\{t_{2}, t_{3}, \ldots, t_{20}\right\}-\min \left\{t_{2}, t_{3}, \ldots, t_{20}\right\}} \times 0.1 .
$$

A estatística de estabilidade estimou precisamente o número correto de grupos para as bases bcw-d (Figura 5.15(a)) e bcw-o (Figura 5.15(b)). Os dois conjuntos mais estáveis para a base iris foram aquelas com dois e três grupos. A base iris é classificada em três classes (precisamente, setosa, versicolour e virginica). Entretanto, é conhecido que as classes versicolour e virginica possuem uma alta sobreposição e são frequentemente consideradas um único grupo (Wu e Yang, 2005), o que corrobora com a validade da estatística de estabilidade. Embora não tenha indicado o número exato de grupos, os menores valores de instabilidade para as bases wine e img correspondem aos conjuntos de agrupamentos com números de grupos por volta do número correto. De maneira geral, quanto mais próximo é o número de grupos do conjunto de agrupamentos do número correto, mais estável o conjunto tende a ser. Esses bons resultados preliminares demostram que a medida 13AGRI merece uma investigação futura mais profunda quanto à possibilidade de aplicá-la para estimar o número de grupos no domínio de ANs.

\subsection{Provas teóricas}

Proposição 5.1. Sejam U e V dois ANs tais que 13FRI $(\mathrm{U}, \mathrm{V})=0, n>1$ e $1 \leq k_{\mathrm{U}}, k_{\mathrm{V}} \leq n$. Isso implica que $\mathrm{U}$ e $\mathrm{V}$ são APEs e que $k_{\mathrm{U}}=1$ e $k_{\mathrm{V}}=$ n ou $k_{\mathrm{U}}=$ n e $k_{\mathrm{V}}=1$, o que determina precisamente $\mathrm{U} e \mathrm{~V}$.

Prova. Observe que, pelas Eqs. (5.1), $\sum_{r=1}^{k_{\mathrm{U}}} \mathrm{U}_{r, l}=1 \quad \forall l$ implica em $\mathrm{S}_{i, j}^{\mathrm{U}}=1-\mathrm{J}_{i, j}^{\mathrm{U}}$. Para se 


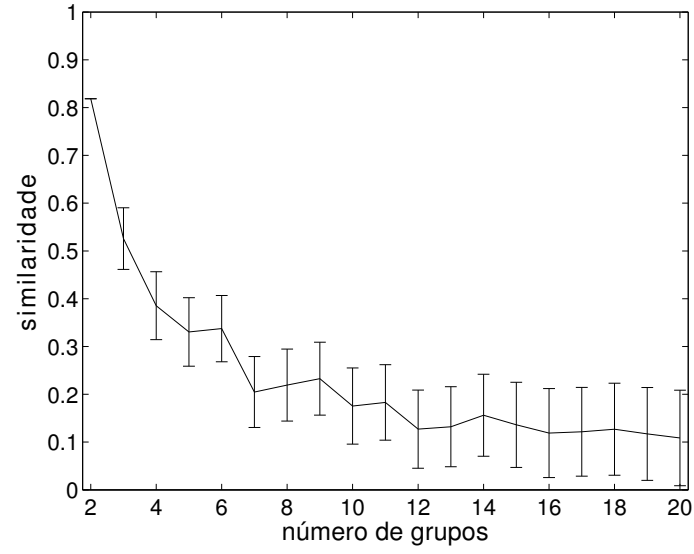

(a) Base: bcw-d $\left(k^{*}=2\right)$.

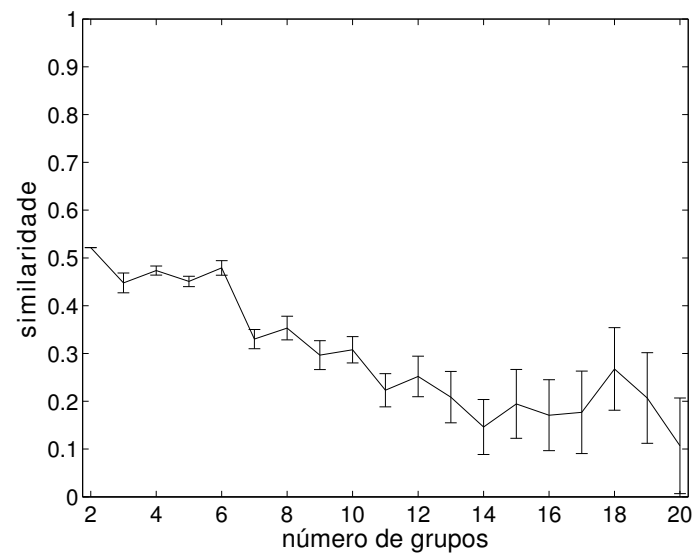

(c) Base: wine $\left(k^{*}=3\right)$.

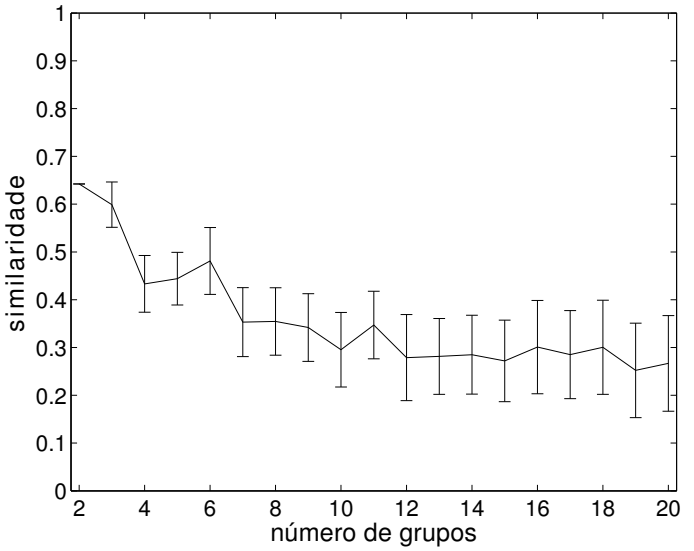

(b) Base: bcw-o $\left(k^{*}=2\right)$.

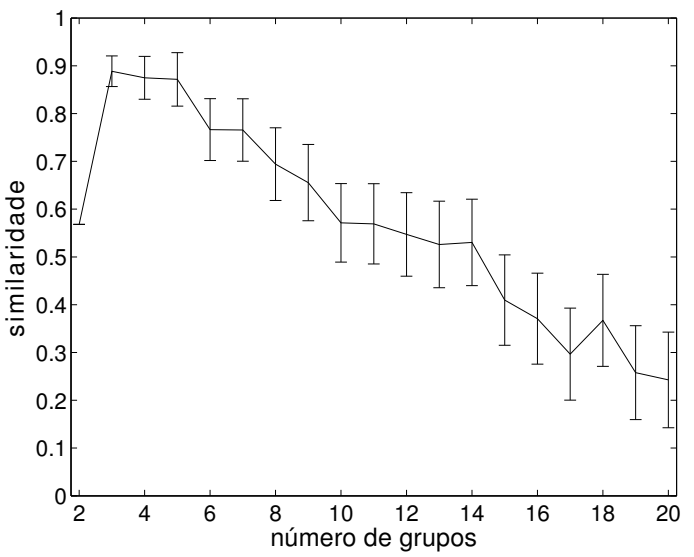

(d) Base: iris $\left(k^{*}=3\right)$.

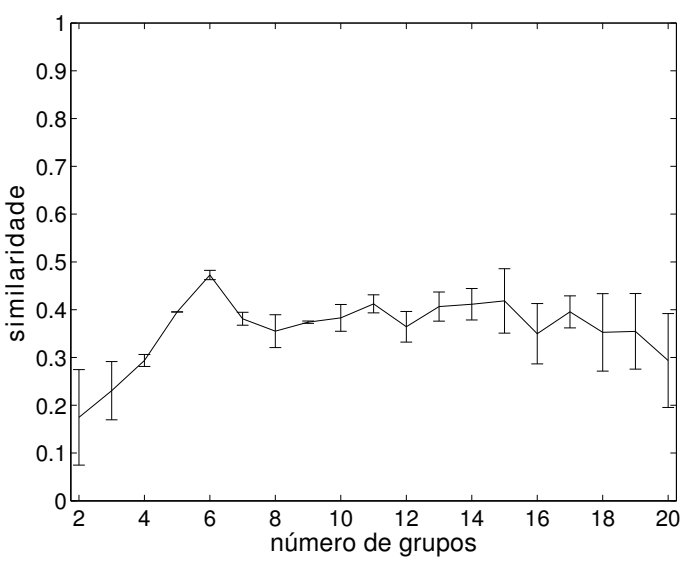

(e) Base: img $\left(k^{*}=7\right)$.

Figura 5.15: Avaliações de 13AGRI com barras de erro indicando a instabilidade dos conjuntos de agrupamentos. 
ter $13 \mathrm{FRI}(\mathrm{U}, \mathrm{V})=0$, é preciso que $\dot{a}=\dot{d}=0$ (Eq. (5.3)), implicando que $\min \left\{\mathrm{J}_{i, j}^{\mathrm{U}}, \mathrm{J}_{i, j}^{\mathrm{V}}\right\}=$ $\min \left\{1-\mathrm{J}_{i, j}^{\mathrm{U}}, 1-\mathrm{J}_{i, j}^{\mathrm{V}}\right\}=0 \forall i<j$ (Eqs. (5.2a) e (5.2d)). Portanto, $\mathrm{J}_{i, j}^{\mathrm{U}}, \mathrm{J}_{i, j}^{\mathrm{V}} \in\{0,1\}$ e $\mathrm{J}_{i, j}^{\mathrm{U}} \neq \mathrm{J}_{i, j}^{\mathrm{V}}$ para todo $i<j$.

Primeiro, prova-se por contradição que U não pode ter uma coluna $i$ e uma linha $r$ para as quais $\mathrm{U}_{r, i} \in(0,1)$ (o mesmo vale para $\mathrm{V}$ ). Assumindo que a $i$-ésima coluna de $\mathrm{U}$ tem $\mathrm{U}_{r, i} \in(0,1)$ para um $r \in \mathbb{N}_{1, k_{\mathrm{U}}}$, tem-se $k_{\mathrm{U}}>1$ e pelo menos dois elementos de $\mathrm{U}_{:, i}$ têm valores no intervalo aberto $(0,1)$ porque $\sum_{t=1}^{k_{\mathrm{U}}} \mathrm{U}_{t, i}=1$. Sem perda de generalidade, assume-se que $i=1$ (as colunas de $\mathrm{U}$ e $\mathrm{V}$ podem ser simultaneamente permutadas sem alteração na avaliação da medida). Sabe-se que $\mathrm{U}_{:, 1}^{\mathrm{T}} \mathrm{U}_{:, j}=\mathrm{J}_{1, j}^{\mathrm{U}}=0 \forall j \in \mathbb{N}_{2, n}$ porque $\mathrm{U}_{:, 1}^{\mathrm{T}} \mathrm{U}_{:, j}$ não pode fornecer 1. Assim, $\mathrm{J}_{1, j}^{\mathrm{V}}=1 \forall j \in \mathbb{N}_{2, n}$. Isso implica que as colunas de $\mathrm{V}$ são todas idênticas e que cada uma tem o elemento 1 , resultando em $k_{\mathrm{V}}=1$ por causa da restrição $\sum_{j=1}^{n} \mathrm{~V}_{t, j}>0 \forall t$. Tem-se, então, $\mathrm{J}_{i_{1}, j_{1}}^{\mathrm{V}}=1 \forall i_{1}<j_{1}$ e $\mathrm{J}_{i_{2}, j_{2}}^{\mathrm{U}}=0 \forall i_{2}<j_{2}$. A última igualdade vale com a restrição $\sum_{j=1}^{n} \mathrm{U}_{t, j}>0 \forall t$ se cada linha de $\mathrm{U}$ tiver exatamente um valor maior que zero. A propriedade $\sum_{t=1}^{k_{\mathrm{U}}} \mathrm{U}_{t, j}=1 \forall j$ para ANs e a suposição $k_{\mathrm{U}} \leq n$ requerem que cada coluna de $\mathrm{U}$ tenha exatamente um valor maior que zero (e tenha $k_{\mathrm{U}}=n$ linhas). Este valor só pode ser o 1 . Isso viola a suposição de que $\mathrm{U}_{r, i} \in(0,1)$, implicando que $\mathrm{U}$ (e $\mathrm{V}$ ) precisa ser uma matriz de apenas zeros e uns.

Suponha que $n=2$. Se as colunas 1 e 2 de U são idênticas, as colunas 1 e 2 de V são diferentes porque foi mostrado que $\mathrm{J}_{i, j}^{\mathrm{U}} \neq \mathrm{J}_{i, j}^{\mathrm{V}}$. Isso pode ocorrer apenas quando $k_{\mathrm{U}}=1 \mathrm{e}$ $k_{\mathrm{V}}=2$ (lembre-se das propriedades de uma matriz de AN). Agora, suponha que $n>2$ e, sem perda de generalidade, que $U_{:, 1}$ e $U_{:, 2}$ são idênticas e que $V_{:, 1}$ e $V_{:, 2}$ são diferentes. Se uma coluna $i>2$ de $\mathrm{U}$ difere das colunas 1 e 2 de $\mathrm{U}$, conclui-se que as colunas 1 e 2 de $\mathrm{V}$ são iguais à coluna $i$ de $\mathrm{V}$. Entretanto, isso implica que as colunas $1 \mathrm{e} 2 \mathrm{de} \mathrm{V}$ são iguais, o que, como sabe-se, não são. Consequentemente, todas as colunas de U precisam ser iguais e todas as colunas de $\mathrm{V}$ precisam ser diferentes. Isso pode ocorrer apenas quando $k_{\mathrm{U}}=1$ e $k_{\mathrm{V}}=n$, o que prova a proposição.

Proposição 5.2. Dadas as matrizes $\mathrm{U} e \mathrm{~V}$ de APEs, tem-se $13 F R I(\mathrm{U}, \mathrm{V})=R I(\mathrm{U}, \mathrm{V})$.

Prova. Note que $\dot{a}, \dot{b}, \dot{c}$ e $\dot{d}$ (Eqs. (5.2) ) são equivalentes a $a, b, c$ e $d$ (Eqs. (4.4)) ao atribuir os valores 0 e 1 às matrizes $\mathrm{J}_{i, j}^{\mathrm{U}}$ e $\mathrm{J}_{i, j}^{\mathrm{V}}$.

Proposição 5.3. Dadas as matrizes $\mathrm{U} e \mathrm{~V}$ de APEs, tem-se $13 A F R I(\mathrm{U}, \mathrm{V})=A R I(\mathrm{U}, \mathrm{V})$.

Prova. ARI e 13AFRI adotam o modelo da Eq. (5.4). A esperança de ARI dado U e V é $\mathrm{E}[\mathrm{ARI}]_{\mathrm{U}, \mathrm{V}}=\left(\mathrm{E}[a]_{\mathrm{U}, \mathrm{V}}+\mathrm{E}[d]_{\mathrm{U}, \mathrm{V}}\right) /(a+b+c+d)($ Hubert e Arabie, 1985). Precisa-se, portanto, mostrar apenas que $\mathrm{E}[a]_{\mathrm{U}, \mathrm{V}}=\mathrm{E}[\dot{a}]_{\mathrm{U}, \mathrm{V}}$ e $\mathrm{E}[d]_{\mathrm{U}, \mathrm{V}}=\mathrm{E}[\dot{d}]_{\mathrm{U}, \mathrm{V}}$, visto que $a=\dot{a}, b=\dot{b}, c=\dot{c}$ e $d=\dot{d}$ pela Proposição 5.2. Sejam $\mathrm{J}^{\mathrm{U}}=\mathrm{U}^{\mathrm{T}} \mathrm{U}, \mathrm{J}^{\mathrm{V}}=\mathrm{V}^{\mathrm{T}} \mathrm{V}$ e $\mathrm{N}=\mathrm{UV}^{\mathrm{T}}$. Dado que $\mathrm{U}$ e $\mathrm{V}$ são APEs, pode-se reescrever $\min \left\{\mathrm{J}_{i, j}^{\mathrm{U}}, \mathrm{J}_{i, j}^{\mathrm{V}}\right\}=\mathrm{J}_{i, j}^{\mathrm{U}} \mathrm{J}_{i, j}^{\mathrm{V}}$. Tanto $\sum_{i<j} \mathrm{~J}_{i, j}^{\mathrm{U}}$ como $\sum_{r=1}^{k_{\mathrm{U}}}\left(\begin{array}{c}\mathrm{N}_{r,+} \\ 2\end{array}\right)$ contam 
o número de pares de objetos não ordenados pertencentes ao mesmo grupo em U. Tem-se então

$$
\begin{aligned}
\mathrm{E}[\dot{a}]_{\mathrm{U}, \mathrm{V}} & =\frac{2}{n(n-1)} \sum_{i_{1}<j_{1}} \mathrm{~J}_{i_{1}, j_{1}}^{\mathrm{U}} \sum_{i_{2}<j_{2}} \mathrm{~J}_{i_{2}, j_{2}}^{\mathrm{V}} \\
& =\sum_{r=1}^{k_{\mathrm{U}}}\left(\begin{array}{c}
\mathrm{N}_{r,+} \\
2
\end{array}\right) \sum_{t=1}^{k_{\mathrm{V}}}\left(\begin{array}{c}
\mathrm{N}_{+, t} \\
2
\end{array}\right) /\left(\begin{array}{l}
n \\
2
\end{array}\right) \\
& \left.=\mathrm{E}[a]_{\mathrm{U}, \mathrm{V}} \text { (Eq. (2) em (Hubert e Arabie, 1985) }\right) .
\end{aligned}
$$

Visto que $\mathrm{J}_{i, j}^{\mathrm{U}}=1-\mathrm{S}_{i, j}^{\mathrm{U}}$ para APEs, tem-se

$$
\begin{aligned}
\mathrm{E}[\dot{d}]_{\mathrm{U}, \mathrm{V}} & =\frac{2}{n(n-1)} \sum_{i_{1}<j_{1}} \sum_{i_{2}<j_{2}}\left(1-\mathrm{J}_{i_{1}, j_{1}}^{\mathrm{U}}\right)\left(1-\mathrm{J}_{i_{2}, j_{2}}^{\mathrm{V}}\right) \\
& =\left(\begin{array}{c}
n \\
2
\end{array}\right)-\sum_{i_{1}<j_{1}} \mathrm{~J}_{i_{1}, j_{1}}^{\mathrm{U}}-\sum_{i_{2}<j_{2}} \mathrm{~J}_{i_{2}, j_{2}}^{\mathrm{V}} \\
& +\sum_{i_{1}<j_{1}} \sum_{i_{2}<j_{2}} \mathrm{~J}_{i_{1}, j_{1}}^{\mathrm{U}} \mathrm{J}_{i_{2}, j_{2}}^{\mathrm{V}} /\left(\begin{array}{l}
n \\
2
\end{array}\right) \\
& =\left(\begin{array}{c}
n \\
2
\end{array}\right)-\sum_{r=1}^{k_{\mathrm{U}}}\left(\begin{array}{c}
\mathrm{N}_{r,+} \\
2
\end{array}\right)-\sum_{t=1}^{k_{\mathrm{V}}}\left(\begin{array}{c}
\mathrm{N}_{+, t} \\
2
\end{array}\right) \\
& +\sum_{r=1}^{k_{\mathrm{U}}}\left(\begin{array}{c}
\mathrm{N}_{r,+} \\
2
\end{array}\right) \sum_{t=1}^{k_{\mathrm{V}}}\left(\begin{array}{c}
\mathrm{N}_{+, t} \\
2
\end{array}\right) /\left(\begin{array}{c}
n \\
2
\end{array}\right) \\
& =\mathrm{E}[d]_{\mathrm{U}, \mathrm{V}} \text { (Eq. (3) em (Hubert e Arabie, 1985) multiplicada por }\left(\begin{array}{c}
n \\
2
\end{array}\right) \mathrm{e} \\
& \text { então subtraída por E } \left.[a]_{\mathrm{U}, \mathrm{V}}\right) .
\end{aligned}
$$

Proposição 5.4. Dadas as matrizes $\mathrm{U} e \mathrm{~V}$ de APs, tem-se $\dot{a}+\dot{b}+\dot{c}+\dot{d}=\sum_{i<j} \min \left\{\mathrm{T}_{i, j}^{\mathrm{U}}, \mathrm{T}_{i, j}^{\mathrm{V}}\right\}$.

Prova. Sejam

$$
\begin{aligned}
& \dot{a}_{i, j} \triangleq \min \left\{\mathrm{J}_{i, j}^{\mathrm{U}}, \mathrm{J}_{i, j}^{\mathrm{V}}\right\}, \\
& \dot{b}_{i, j} \triangleq \min \left\{\mathrm{J}_{i, j}^{\mathrm{U}}-\min \left\{\mathrm{J}_{i, j}^{\mathrm{U}}, \mathrm{J}_{i, j}^{\mathrm{V}}\right\}, \mathrm{S}_{i, j}^{\mathrm{V}}-\min \left\{\mathrm{S}_{i, j}^{\mathrm{U}}, \mathrm{S}_{i, j}^{\mathrm{V}}\right\}\right\}, \\
& \dot{c}_{i, j} \triangleq \min \left\{\mathrm{J}_{i, j}^{\mathrm{V}}-\min \left\{\mathrm{J}_{i, j}^{\mathrm{U}}, \mathrm{J}_{i, j}^{\mathrm{V}}\right\}, \mathrm{S}_{i, j}^{\mathrm{U}}-\min \left\{\mathrm{S}_{i, j}^{\mathrm{U}}, \mathrm{S}_{i, j}^{\mathrm{V}}\right\}\right\} \mathrm{e} \\
& \dot{d}_{i, j} \triangleq \min \left\{\mathrm{S}_{i, j}^{\mathrm{U}}, \mathrm{S}_{i, j}^{\mathrm{V}}\right\} .
\end{aligned}
$$

A proposição será provada ao mostrar que

$$
\dot{a}_{i, j}+\dot{b}_{i, j}+\dot{c}_{i, j}+\dot{d}_{i, j}=\min \left\{\mathrm{T}_{i, j}^{\mathrm{U}}, \mathrm{T}_{i, j}^{\mathrm{V}}\right\}
$$

São mostradas na Tabela 5.6 as seis combinações de ordem entre os valores dos pares $\left(\mathrm{J}_{i, j}^{\mathrm{U}}, \mathrm{J}_{i, j}^{\mathrm{V}}\right)$, $\left(\mathrm{S}_{i, j}^{\mathrm{U}}, \mathrm{S}_{i, j}^{\mathrm{V}}\right)$ e $\left(\mathrm{T}_{i, j}^{\mathrm{U}}, \mathrm{T}_{i, j}^{\mathrm{V}}\right)$, cobrindo todos os cenários possíveis. Eq. (5.15) está correta para cada cenário. Por concisão, serão apresentadas as provas apenas das Combinações 1 e 3. 
Tabela 5.6: Combinações de ordem.

\begin{tabular}{llll}
\hline$\#$ & $\left(\mathrm{~J}_{i, j}^{\mathrm{U}}, \mathrm{J}_{i, j}^{\mathrm{V}}\right)$ & $\left(\mathrm{S}_{i, j}^{\mathrm{U}}, \mathrm{S}_{i, j}^{\mathrm{V}}\right)$ & $\left(\mathrm{T}_{i, j}^{U}, \mathrm{~T}_{i, j}^{\mathrm{V}}\right)$ \\
\hline \hline 1 & $\mathrm{~J}_{i, j}^{\mathrm{U}} \geq \mathrm{J}_{i, j}^{\mathrm{V}}$ & $\mathrm{S}_{i, j}^{\mathrm{U}} \geq \mathrm{S}_{i, j}^{\mathrm{V}}$ & $\mathrm{T}_{i, j}^{U} \geq \mathrm{T}_{i, j}^{V}$ \\
2 & $\mathrm{~J}_{i, j}^{\mathrm{U}} \geq \mathrm{J}_{i, j}^{V}$ & $\mathrm{~S}_{i, j}^{U}<\mathrm{S}_{i, j}^{V}$ & $\mathrm{~T}_{i, j}^{U} \geq \mathrm{T}_{i, j}^{V}$ \\
3 & $\mathrm{~J}_{i, j}^{U} \geq \mathrm{J}_{i, j}^{V}$ & $\mathrm{~S}_{i, j}^{U}<\mathrm{S}_{i, j}^{V}$ & $\mathrm{~T}_{i, j}^{U}<\mathrm{T}_{i, j}^{V}$ \\
4 & $\mathrm{~J}_{i, j}^{U}<\mathrm{J}_{i, j}^{V}$ & $\mathrm{~S}_{i, j}^{U} \geq \mathrm{S}_{i, j}^{V}$ & $\mathrm{~T}_{i, j}^{U} \geq \mathrm{T}_{i, j}^{V}$ \\
5 & $\mathrm{~J}_{i, j}^{U}<\mathrm{J}_{i, j}^{V}$ & $\mathrm{~S}_{i, j}^{U} \geq \mathrm{S}_{i, j}^{V}$ & $\mathrm{~T}_{i, j}^{U}<\mathrm{T}_{i, j}^{V}$ \\
6 & $\mathrm{~J}_{i, j}^{U}<\mathrm{J}_{i, j}^{V}$ & $\mathrm{~S}_{i, j}^{U}<\mathrm{S}_{i, j}^{V}$ & $\mathrm{~T}_{i, j}^{U}<\mathrm{T}_{i, j}^{V}$ \\
\hline
\end{tabular}

Assumindo a Combinação 1, tem-se $\dot{a}_{i, j}=\mathrm{J}_{i, j}^{\mathrm{V}}, \dot{b}_{i, j}=0, \dot{c}_{i, j}=0$ e $\dot{d}_{i, j}=\mathrm{S}_{i, j}^{\mathrm{V}}$, e a Eq. (5.15) está correta. Assumindo a Combinação 3, tem-se $\dot{a}_{i, j}=\mathrm{J}_{i, j}^{\mathrm{V}}, \dot{b}_{i, j}=\min \left\{\mathrm{J}_{i, j}^{\mathrm{U}}-\mathrm{J}_{i, j}^{\mathrm{V}}, \mathrm{S}_{i, j}^{\mathrm{V}}-\mathrm{S}_{i, j}^{\mathrm{U}}\right\}$, $\dot{c}_{i, j}=0$, e $\dot{d}_{i, j}=\mathrm{S}_{i, j}^{\mathrm{U}}$. Note que $\mathrm{T}_{i, j}^{\mathrm{U}}<\mathrm{T}_{i, j}^{\mathrm{V}} \Rightarrow \mathrm{J}_{i, j}^{\mathrm{U}}+\mathrm{S}_{i, j}^{\mathrm{U}}<\mathrm{J}_{i, j}^{\mathrm{V}}+\mathrm{S}_{i, j}^{\mathrm{V}} \Rightarrow \mathrm{J}_{i, j}^{\mathrm{U}}-\mathrm{J}_{i, j}^{\mathrm{V}}<\mathrm{S}_{i, j}^{\mathrm{V}}-\mathrm{S}_{i, j}^{\mathrm{U}}$. Assim, $\dot{b}_{i, j}=\mathrm{J}_{i, j}^{\mathrm{U}}-\mathrm{J}_{i, j}^{\mathrm{V}}$, e a Eq. (5.15) está correta.

Corolário 5.1. Se U e V são duas matrizes de ANs com n colunas cada, tem-se $\mathrm{T}_{i, j}^{\mathrm{U}}=\mathrm{T}_{i, j}^{\mathrm{V}}=1$ e a soma $\dot{a}+\dot{b}+\dot{c}+\dot{d}=n(n-1) / 2$.

Proposição 5.5. Dadas duas matrizes $\mathrm{U}$ e $\mathrm{V}$ de APs, tem-se $\dot{a}+\dot{b}+\dot{c}+\dot{d}+\dot{e}=$ $\max \left\{\sum_{i<j} \mathrm{~T}_{i, j}^{\mathrm{U}}, \sum_{i<j} \mathrm{~T}_{i, j}^{\mathrm{V}}\right\}$.

Prova. Seja $\mathrm{M} \triangleq \max \left\{\mathrm{T}^{\mathrm{U}}, \mathrm{T}^{\mathrm{V}}\right\}$. Se $\mathrm{T}_{i, j}^{\mathrm{U}} \geq \mathrm{T}_{i, j}^{\mathrm{V}}$, então $\min \left\{\mathrm{T}_{i, j}^{\mathrm{U}}, \mathrm{T}_{i, j}^{\mathrm{V}}\right\}+\mathrm{M}_{i, j}-\mathrm{T}_{i, j}^{\mathrm{V}}=\mathrm{T}_{i, j}^{\mathrm{U}}$. Se $\mathrm{T}_{i, j}^{\mathrm{U}}<\mathrm{T}_{i, j}^{\mathrm{V}}$, então $\min \left\{\mathrm{T}_{i, j}^{\mathrm{U}}, \mathrm{T}_{i, j}^{\mathrm{V}}\right\}+\mathrm{M}_{i, j}-\mathrm{T}_{i, j}^{\mathrm{V}}=\mathrm{T}_{i, j}^{\mathrm{U}}$ da mesma forma. Assim, $\mathrm{T}_{i, j}^{\mathrm{U}}=$ $\min \left\{\mathrm{T}_{i, j}^{\mathrm{U}}, \mathrm{T}_{i, j}^{\mathrm{V}}\right\}+\mathrm{M}_{i, j}-\mathrm{T}_{i, j}^{\mathrm{V}}$ e o mesmo raciocínio funciona para $\mathrm{T}_{i, j}^{\mathrm{V}}=\min \left\{\mathrm{T}_{i, j}^{\mathrm{U}}, \mathrm{T}_{i, j}^{\mathrm{V}}\right\}+$ $\mathrm{M}_{i, j}-\mathrm{T}_{i, j}^{\mathrm{U}}$. Sabe-se que $\dot{a}+\dot{b}+\dot{c}+\dot{d}=\sum_{i<j} \min \left\{\mathrm{T}_{i, j}^{\mathrm{U}}, \mathrm{T}_{i, j}^{\mathrm{V}}\right\}$ pela Proposição 5.4. Se $\sum_{i<j} \mathrm{~T}_{i, j}^{\mathrm{U}} \geq \sum_{i<j} \mathrm{~T}_{i, j}^{\mathrm{V}}$, tem-se $\dot{e}=\sum_{i<j}\left(\mathrm{M}_{i, j}-\mathrm{T}_{i, j}^{\mathrm{V}}\right)$ e $\dot{a}+\dot{b}+\dot{c}+\dot{d}+\dot{e}=\sum_{i<j} \mathrm{~T}_{i, j}^{\mathrm{U}} ;$ de outro modo, $\dot{a}+\dot{b}+\dot{c}+\dot{d}+\dot{e}=\sum_{i<j} \mathrm{~T}_{i, j}^{\mathrm{V}}$.

Corolário 5.2. A soma $\dot{a}+\dot{b}+\dot{c}+\dot{d}+\dot{e}$ é constante para qualquer permutação simultânea das colunas de $\mathrm{U} e \mathrm{~V}$ porque elas não alteram as somas $\sum_{i<j} \mathrm{~T}_{i, j}^{\mathrm{U}} e \sum_{i<j} \mathrm{~T}_{i, j}^{\mathrm{V}}$.

Corolário 5.3. 13FRI (13AFRI) e 13GRI (13AGRI) são equivalentes quando aplicadas em ANs porque $\max \left\{\sum_{i<j} \mathrm{~T}_{i, j}^{\mathrm{U}}, \sum_{i<j} \mathrm{~T}_{i, j}^{\mathrm{V}}\right\}=n(n-1) / 2=\dot{a}+\dot{b}+\dot{c}+\dot{d}$.

Corolário 5.4. Dadas as matrizes $\mathrm{U} e \mathrm{~V}$ de APEs, tem-se $13 G R I(\mathrm{U}, \mathrm{V})=R I(\mathrm{U}, \mathrm{V})$ por causa da Proposição 5.2 e Corolário 5.3.

Corolário 5.5. Dadas as matrizes $\mathrm{U} e \mathrm{~V}$, tem-se 13AGRI $(\mathrm{U}, \mathrm{V})=\underline{A R I}(\mathrm{U}, \mathrm{V})$ por causa da Proposição 5.3 e Corolário 5.3

Proposição 5.6. Dados ANs U e V, tem-se $0 \leq \mathrm{E}[\dot{a}]_{\mathrm{U}, \mathrm{V}}+\mathrm{E}[\dot{d}]_{\mathrm{U}, \mathrm{V}} \leq \dot{a}+\dot{b}+\dot{c}+\dot{d}$ (Eqs. (5.7) $e(5.8)$. Equivalentemente, tem-se $0 \leq \mathrm{E}[13 F R I]_{\mathrm{U}, \mathrm{V}} \leq 1$.

Prova. A proposição segue do fato de que $0 \leq 13 \mathrm{FRI}(\mathrm{U}, \mathrm{V}) \leq 1$.

Proposição 5.7. Dados APs, tem-se $0 \leq \mathrm{E}[\dot{a}]_{\mathrm{U}, \mathrm{V}}+\mathrm{E}[\dot{d}]_{\mathrm{U}, \mathrm{V}} \leq \dot{a}+\dot{b}+\dot{c}+\dot{d}+\dot{e}$ (Eqs. 5.7) $e$ (5.8). Equivalentemente, tem-se $0 \leq \mathrm{E}[13 G R I]_{\mathrm{U}, \mathrm{V}} \leq 1$. 
Prova. A proposição segue do fato de que $0 \leq 13 \mathrm{GRI}(\mathrm{U}, \mathrm{V}) \leq 1$.

Proposição 5.8. Dado dois ANs $\mathrm{U} e \mathrm{~V}, \mathrm{E}[\dot{a}]_{\mathrm{U}, \mathrm{V}}+\mathrm{E}[\dot{d}]_{\mathrm{U}, \mathrm{V}}=\dot{a}+\dot{b}+\dot{c}+\dot{d}$ (equivalentemente, $\left.\mathrm{E}[13 F R I]_{\mathrm{U}, \mathrm{V}}=1\right)$ implica em $13 F R I(\mathrm{U}, \mathrm{V})=1$, o que justifica a definição $13 A F R I(\mathrm{U}, \mathrm{V}) \triangleq 1$ para $\mathrm{E}[13 F R I]_{\mathrm{U}, \mathrm{V}}=1$.

Prova. Pela Proposição 5.6, segue que cada uma das permutações do lado direito da Eq. (5.5) resulta no valor 1 . Em particular, $13 \mathrm{FRI}(\mathrm{U}, \mathrm{V})=1$.

Proposição 5.9. Dado dois APs $\mathrm{U} e \mathrm{~V}, \mathrm{E}[\dot{a}]_{\mathrm{U}, \mathrm{V}}+\mathrm{E}[\dot{d}]_{\mathrm{U}, \mathrm{V}}=\dot{a}+\dot{b}+\dot{c}+\dot{d}+\dot{e}$ (equivalentemente, $\left.\mathrm{E}[13 G R I]_{\mathrm{U}, \mathrm{V}}=1\right)$ implica em $13 G R I(\mathrm{U}, \mathrm{V})=1$, o que justifica a definição $13 A G R I(\mathrm{U}, \mathrm{V}) \triangleq 1$ para $\mathrm{E}[13 G R I]_{\mathrm{U}, \mathrm{V}}=1$.

Prova. Vide prova da Proposição 5.8 .

Corolário 5.6. Se 13GRI $(\mathrm{U}, \mathrm{V})<1$, então $\mathrm{E}[\dot{a}]_{\mathrm{U}, \mathrm{V}}+\mathrm{E}[\dot{b}]_{\mathrm{U}, \mathrm{V}}<\dot{a}+\dot{b}+\dot{c}+\dot{d}+\dot{e}$. Equivalentemente, se $13 G R I(\mathrm{U}, \mathrm{V})<1$, então $\mathrm{E}[13 G R I]_{\mathrm{U}, \mathrm{V}}<1$.

Prova. A desigualdade 13GRI $(\mathrm{U}, \mathrm{V})<1$ implica que $\mathrm{E}[\dot{a}]_{\mathrm{U}, \mathrm{V}}+\mathrm{E}[\dot{b}]_{\mathrm{U}, \mathrm{V}}<\dot{a}+\dot{b}+\dot{c}+\dot{d}+\dot{e}$ pelas Proposições $5.7 \mathrm{e} 5.9$.

Corolário 5.7. Se 13GRI(U, V) < 1, então $13 A G R I(U, \mathrm{~V})<1$.

Prova. Pelo Corolário 5.6, o caso especial para o qual 13AGRI(U, V) $=1$ devido a $\mathrm{E}[13 \mathrm{GRI}]_{\mathrm{U}, \mathrm{V}}=1$ não se aplica. Multiplicando por $\dot{a}+\dot{b}+\dot{c}+\dot{d}+\dot{e}$ o numerador e o denominador do lado direito da Eq. (5.13) leva à

$$
13 \operatorname{AGRI}(\mathrm{U}, \mathrm{V})=\frac{\dot{a}+\dot{d}-\mathrm{E}[\dot{a}]_{\mathrm{U}, \mathrm{V}}-\mathrm{E}[\dot{d}]_{\mathrm{U}, \mathrm{V}}}{\dot{a}+\dot{b}+\dot{c}+\dot{d}+\dot{e}-\mathrm{E}[\dot{a}]_{\mathrm{U}, \mathrm{V}}-\mathrm{E}[\dot{d}]_{\mathrm{U}, \mathrm{V}}},
$$

tornando claro que $13 \operatorname{AGRI}(\mathrm{U}, \mathrm{V})<1$ dado que $\dot{a}+\dot{b}+\dot{c}+\dot{d}+\dot{e}>\dot{a}+\dot{d}$.

\subsection{Considerações}

Mostrou-se que várias das medidas voltadas para agrupamentos mais gerais que os agrupamentos particionais exclusivos (APEs) não alcançam o máximo sempre quando duas soluções equivalentes são comparadas e que a maioria das medidas são parciais no que diz respeito ao número de grupos. Além disso, várias medidas são baseadas em equações que foram originalmente desenvolvidas para APEs. Portanto, algumas das medidas exibiram comportamentos inesperados em experimentos envolvendo cenários mais gerais.

A medida 13FRI foi proposta para lidar com agrupamentos nebulosos (ANs) e com agrupamentos particionais não exclusivos, o que inclui os APEs. Foi proposto um modelo nulo para geração aleatória de agrupamentos, servindo de base do ajuste da medida 13FRI para aleatoriedade, dando origem ao índice 13AFRI. Os índices 13FRI e 13AFRI foram então estendidos para lidar com agrupamentos ainda mais gerais, chamados de agrupamentos possibilísticos, o 
que levou à definição das medidas 13GRI e 13AGRI, respectivamente. A análise de complexidade computacional mostrou que as novas medidas são viáveis na prática.

No primeiro experimento, envolvendo cinco algoritmos de agrupamento de naturezas distintas, observou-se que algumas medidas não puderam identificar as melhores soluções, e que vários não proveram uma avaliação fina ao longo da faixa de números de grupos, enquanto 13AGRI sempre alcançou o máximo 1 para o número correto de grupos e apresentou avaliações sensíveis às diferenças das soluções. Foi avaliada no segundo experimento a capacidade das medidas em apresentar uma avaliação imparcial para soluções geradas aleatoriamente com números de grupos diferentes. Uma medida justa deve atribuir uma avaliação uniforme ao longo da faixa de números de grupos, visto que cada solução gerada é independente da solução de referência (Vinh et al., 2010). Esse é o caso do popular índice ARI projetado para APEs. Apenas as medidas 13AGRI e 09BARI (Brouwer, 2009) apresentaram tal avaliação para todos os cenários considerados, o que inclui o contexto dos APEs; entretanto, 09BARI não atingiu o máximo 1 para o número correto de grupos na maioria dos cenários do primeiro experimento. As outras medidas exibiram preferência por certas soluções, fenômeno atribuível unicamente aos seus mecanismos de avaliação. Embora o modelo nulo proposto para 13AGRI incorpore algumas suposições, elas claramente foram violadas pelos experimentos. Mesmo assim, 13AGRI conseguiu fornecer avaliações uniformes próximas de zero nos experimentos com agrupamentos gerados aleatoriamente.

Dois outros experimentos foram realizados envolvendo 14 bases reais e os algoritmos kMeans, FCM e EMGM para determinar a validade das avaliações do índice 13AGRI no domínio de ANs e investigar a sua aplicabilidade na estimação do número de grupos. Argumentou-se que as avaliações das soluções produzidas pelos algoritmos k-Means e FCM para uma mesma base de dados devem ser similares, e que esse comportamento apresentado pela medida 13AGRI é ainda mais importante para a sua validade porque 13AGRI e ARI são equivalentes quando aplicadas em soluções geradas pelo k-Means. A estatística de estabilidade baseado no índice 13AGRI definida no último experimento apresentou bons resultados, indicando que 13AGRI pode também ser aplicado na estimação do número de grupos em ANs.

Foi provado que 13AGRI e ARI são equivalentes no contexto dos APEs. Esse resultado é importante porque ARI é uma das medidas mais confiáveis encontradas na literatura (Steinley, 2004; Albatineh et al., 2006) e porque o modelo nulo de 13AGRI foi desenvolvido para o contexto geral de APs. Como um trabalho futuro, recomenda-se uma investigação das propriedades conceituais que a medida 13AGRI pode apresentar, como sensibilidade à homogeneidade, sensibilidade à completude de grupo e observância dos axiomas métricos (Meila, 2007; Amigó et al., 2009). 
CAPÍTULO

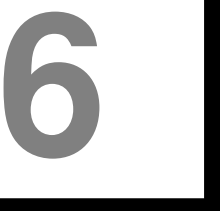

\section{DESENVOLVIMENTO DE MEDIDAS PARA BI-AGRUPAMENTO}

Como mencionado na Seção 4.3.2, não existe ainda uma análise teórica das várias medidas de similaridade entre bi-agrupamentos. Além disso, a maioria das medidas de bi-agrupamento baseia-se em uma abordagem (associação entre grupos/bi-grupos) sabidamente problemática. Portanto, é proposta neste capítulo uma nova abordagem de avaliação de bi-agrupamentos que permite o uso de medidas de agrupamento particionais não exclusivo. São formalizadas oito propriedades de medidas de bi-agrupamento. A importância delas é corroborada por meio de exemplos didáticos e por meio de experimentos empíricos com modelos de bi-grupos e algoritmos de bi-agrupamento extraídos de importantes estudos da literatura de expressão gênica. São comparadas 17 medidas de bi-agrupamento de acordo com as propriedades propostas e por meio de experimentos.

As seguintes definições são utilizadas neste capítulo. Diz-se que os bi-grupos $B_{i}$ e $B_{l}$ são equivalentes, $B_{i} \equiv B_{l}$, se, e somente se, $B_{i}$ e $B_{l}$ têm as mesmas linhas e colunas. Os bi-grupos $B_{i}$ e $B_{l}$ de um mesmo bi-agrupamento são iguais se, e somente se, $i=l$. Dois bi-grupos iguais são, então, equivalentes, mas nem sempre dois bi-grupos equivalentes são iguais. Diz-se que $B \triangleq\left\{B_{i}\right\}_{i=1}^{k}$ e $\dot{B} \triangleq\left\{\dot{B}_{i}\right\}_{i=1}^{q}$ são bi-agrupamentos equivalentes, $B \equiv \dot{B}$, se, e somente se, $k=q$ e existe uma bijeção ${ }^{1}\left\{\left(t_{i}, y_{i}\right)\right\}_{i=1}^{k}$ para a qual $B_{t_{i}} \equiv \dot{B}_{y_{i}}$ para todo $i$. Note que uma solução pode ter bi-grupos consistindo de uma linha e uma coluna. Bi-agrupamentos com tais bi-grupos serão chamados aqui de degenerados por duas razões: (i) esse tipo de solução é dificilmente encontrada na prática e (ii) algumas medidas de bi-agrupamento possuem certas propriedades apenas na presença de soluções não degeneradas. Finalmente, diz-se que dois bi-grupos $B_{i}$ e $B_{l}$ se sobrepõem se, e somente se, $B_{i}^{\mathrm{r}} \times B_{i}^{\mathrm{c}} \cap B_{l}^{\mathrm{r}} \times B_{l}^{\mathrm{c}} \neq \emptyset$.

\footnotetext{
${ }^{1}$ Essa bijeção diz respeito aos conjuntos $\mathbb{N}_{1, k}$ e $\mathbb{N}_{1, q}$. Esse detalhe será daqui em diante omitido em contextos similares.
} 


\subsection{Trabalhos relacionados}

Meila (2003) propôs 12 propriedades de medidas para agrupamento particional exclusivo, discutiu a importância delas, introduziu a medida de Variação de Informação (VI) e analisou esta em conjunto com outras medidas populares. Meila $(2005,2007)$ também mostrou que algumas medidas de agrupamento podem ser completamente caracterizadas por um conjunto de axiomas e usou grafos reticulados para representar e estudar o espaço dos agrupamentos particionais exclusivos. Embora tratem especificamente de medidas para APEs, esses três trabalhos discutem características que serviram de inspiração para a proposição das propriedades de medidas para bi-agrupamento de dados apresentadas na Seção 6.3 .

Prelić et al. (2006) discutiram brevemente os métodos de comparação de bi-agrupamento e introduziram medidas de recuperação e relevância de bi-grupos.

Patrikainen e Meila (2006) propuseram a primeira metodologia de comparação de agrupamento em subespaços. A maior parte do artigo foi dedicada ao caso especial de agrupamento em subespaços alinhados com os eixos (Seção 2.3.1). Embora os algoritmos de bi-agrupamento e de agrupamento em subespaços alinhados com os eixos usualmente procurem por tipos distintos de subestrutura nos dados, ambos produzem o mesmo tipo de solução de agrupamento (i.e., um conjunto de conjuntos consistindo de linhas e colunas da matriz de dados), o que significa que as técnicas discutidas em (Patrikainen e Meila, 2006) também podem ser aplicadas na comparação de bi-agrupamentos. No mesmo artigo foi proposto um conjunto de propriedades desejáveis para a comparação de agrupamentos em subespaços sem sobreposição e analisadas algumas medidas em termos dessas propriedades.

Santamaría et al. (2007) revisaram alguns índices internos, externos e relativos para biagrupamento. Rosenberg e Hirschberg (2007) salientaram a importância de dois aspectos de agrupamento usualmente conflitantes (homogeneidade e completude).

Amigó et al. (2009) propuseram quatro propriedades de medidas de agrupamento particional exclusivo, incluindo os aspectos de homogeneidade e completude usados em (Rosenberg e Hirschberg, 2007). A intuitividade dessas propriedades foi validada em um experimento envolvendo avaliações humanas. Essas propriedades foram comparadas com outras encontradas na literatura. Lee et al. (2009, 2011) revisaram várias medidas de bi-agrupamento e propuseram duas novas.

\subsection{Nova abordagem de comparação}

Patrikainen e Meila (2006) propuseram o uso dos elementos da matriz de dados como o conjunto de elementos a ser agrupado, ao invés dos objetos da base de dados (que seriam as linhas da matriz de dados), para comparar agrupamentos em subespaços alinhados com os eixos sem sobreposição. Elas redefiniram o conceito de tamanho de interseção e de união usado por algumas medidas de similaridade a fim de lidar com agrupamentos que apresentam sobreposição, dando origem às medidas $\mathbb{S}_{\text {rnia }}$ e $\mathbb{S}_{\mathrm{ce}}$ definidas na Seção 4.3.1 e consideradas nas análises 
teórica e experimental relatadas neste capítulo.

É proposta aqui uma abordagem similar para a representação de bi-agrupamentos (com ou sem sobreposição) na qual o conjunto de elementos da matriz de dados é usado como o conjunto de objetos a serem agrupados, mas sem a necessidade da redefinição do tamanho de interseção e de união. Essa abordagem consiste na representação de bi-agrupamento por meio de um agrupamento particional não exclusivo, o que permite o uso de medidas projetadas para lidar com o último tipo de agrupamento.

Considere uma matriz de dados $\mathrm{X} \in \mathbb{R}^{n \cdot p}$. Cada elemento de $\mathrm{X}$ é, agora, um objeto do conjunto $O \triangleq\left\{\tilde{o}_{1}, \tilde{o}_{2}, \ldots, \tilde{o}_{n \cdot p}\right\}$ de pares linha-coluna, cujo mapeamento é definido como

$$
\pi(j, s) \triangleq j+n(s-1) \quad \forall j \in \mathbb{N}_{1, n}, \forall s \in \mathbb{N}_{1, p}
$$

no qual $\tilde{o}_{\pi(j, s)}$ representa o elemento da matriz correspondente à $j$-ésima linha e à $s$-ésima coluna (i.e., o elemento $\left.\mathrm{X}_{j, s}\right)$. Qualquer bi-grupo $B_{i} \triangleq\left(B_{i}^{\mathrm{r}}, B_{i}^{\mathrm{c}}\right) \in B$, em que $B \triangleq$ $\left\{B_{1}, B_{2}, \ldots, B_{k}\right\}$, pode ser convertido em um grupo usual $P_{i}$. Portanto, define-se $P_{i}$ como

$$
P_{i} \triangleq \bigcup_{j \in B_{i}^{\mathrm{r}}, s \in B_{i}^{\mathrm{c}}}\left\{\tilde{o}_{\pi(j, s)}\right\}
$$

Fazendo o mesmo para cada $i \in \mathbb{N}_{1, k}$ e definindo o conjunto $\left\{P_{1}, P_{2}, \ldots, P_{k}\right\}$ produz-se um agrupamento particional não exclusivo de pares linha-coluna pertencentes a pelo menos um bi-grupo. Alguns pares linha-coluna não seriam agrupados ao aplicar a abordagem acima em soluções encontradas na prática. Por exemplo, muitos dos elementos de uma matriz de dados de expressão gênica não exibirão um padrão (Madeira e Oliveira, 2004) e é esperado que não sejam agrupados. Pode-se associar cada um desses elementos chamados aqui de ruidosos a grupos unitários, visto que tais elementos não devem ser agrupados com nenhum outro. Portanto, define-se $P$ como

$$
P \triangleq\left\{P_{1}, P_{2}, \ldots, P_{k}, P_{k+1}, \ldots, P_{k+h}\right\}
$$

em que $P_{i}\left(i \in \mathbb{N}_{1, k}\right)$ é dado pela Eq. (6.2), $P_{i} \triangleq\left\{\tilde{o}_{j_{i}}\right\}\left(i \in \mathbb{N}_{k+1, k+h}\right)$, $\tilde{o}_{j_{i}}\left(\forall i \in \mathbb{N}_{k+1, k+h}\right)$ é um objeto de $O$ que não pertence a nenhum $P_{l}\left(\forall l \in \mathbb{N}_{1, k}\right)$, $h$ é a quantidade desses objetos e os objetos $\tilde{o}_{j_{k+1}}, \tilde{o}_{j_{k+2}}, \ldots, \tilde{o}_{j_{k+h}}$ são diferentes entre si.

Considere o bi-agrupamento representado na Figura 2.5. Tem-se o bi-agrupamento $B=$ $\left\{B_{1}, B_{2}\right\}$, no qual $B_{1}=(\{1,2,3\},\{1,2\})$ e $B_{2}=(\{2\},\{2,3\})$. Usando a representação dada pela Eq. (6.1), tem-se, então, o conjunto $O=\left\{\tilde{o}_{1}, \tilde{o}_{2}, \ldots, \tilde{o}_{12}\right\}$ de pares linha-coluna. Aplicando as Eqs. (6.2) e 6.3), tem-se $P_{1}=\left\{\tilde{o}_{1}, \tilde{o}_{2}, \tilde{o}_{3}, \tilde{o}_{5}, \tilde{o}_{6}, \tilde{o}_{7}\right\}, P_{2}=\left\{\tilde{o}_{6}, \tilde{o}_{10}\right\}, P_{3}=\left\{\tilde{o}_{4}\right\}$, $P_{4}=\left\{\tilde{o}_{8}\right\}, P_{5}=\left\{\tilde{o}_{9}\right\}, P_{6}=\left\{\tilde{o}_{11}\right\}, P_{7}=\left\{\tilde{o}_{12}\right\}$ e $P=\left\{P_{1}, P_{2}, \ldots, P_{7}\right\}$.

Depois de transformar os bi-agrupamentos encontrado e de referência nos agrupamentos particionais não exclusivos $P$ e $\dot{P}$, o passo final da abordagem de avaliação proposta valida $P$ usando $\dot{P}$. Foram selecionadas as medidas 09EBC, 10CSI e 13AGRI de agrupamento particional não exclusivo para as análises teórica e experimental apresentadas mais adiante. As duas 
primeiras medidas foram definidas na Seção 4.2.1 no contexto de representação de agrupamento por meio de matrizes e a medida 13AGRI foi proposta na Seção 5.2. No entanto, a representação de agrupamento particional não exclusivo por meio também de conjuntos facilitaria a análise teórica. Logo, define-se

$$
\begin{aligned}
& \mathbb{S}_{\mathrm{csi}}(B, \dot{B}) \triangleq 10 \mathrm{CSI}(P, \dot{P}), \\
& \mathbb{S}_{\mathrm{ebc}}(B, \dot{B}) \triangleq 09 \operatorname{EBC}(P, \dot{P}) \mathrm{e} \\
& \mathbb{S}_{\mathrm{agri}}(B, \dot{B}) \triangleq 13 \operatorname{AGRI}(\mathrm{U}, \dot{\mathrm{U}}),
\end{aligned}
$$

em que 10CSI e 09EBC são definidas pelas Eqs. (B.7) e (B.2) para o contexto de representação de agrupamento por meio de conjuntos. As matrizes $\mathrm{U}$ e $\dot{\mathrm{U}}$ representam os agrupamentos $P$ e $\dot{P}$, respectivamente.

Similarmente aos bi-grupos, diz-se que $P_{i}$ e $P_{l}$ são equivalentes, $P_{i} \equiv P_{l}$, se, e somente se, $P_{i}$ e $P_{l}$ possuem os mesmos objetos. Os grupos $P_{i}$ e $P_{l}$ em uma mesma solução são iguais se, e somente se, $i=l$. Diz-se que $P \triangleq\left\{P_{i}\right\}_{i=1}^{k}$ e $\dot{P} \triangleq\left\{\dot{P}_{i}\right\}_{i=1}^{q}$ são agrupamentos equivalentes, $P \equiv \dot{P}$, se, e somente se, $k=q$ e existe uma bijeção $\left\{\left(t_{i}, y_{i}\right)\right\}_{i=1}^{k}$ tal que $P_{t_{i}} \equiv \dot{P}_{y_{i}}$ para todo $i$. Note que dois bi-agrupamentos não equivalentes podem ser transformados em um mesmo agrupamento particional não exclusivo (Proposição 6.1. Seção 6.5). Entretanto, isso é possível apenas para soluções degeneradas (Proposição 6.2, Seção 6.5).

\subsection{Análise teórica das medidas}

Nesta seção são comparadas as medidas de bi-agrupamento discutidas na Seção $4.3 .1 \mathrm{em}$ conjunto com $\mathbb{S}_{\mathrm{csi}}$ e $\mathbb{S}_{\mathrm{ebc}}$ em termos de oito propriedades relevantes para a avaliação de biagrupamento. Seja

$$
\mathrm{I}\left(B_{i}, B_{l}\right) \triangleq\left(B_{i}^{\mathrm{r}} \times B_{i}^{\mathrm{c}}\right) \cap\left(B_{l}^{\mathrm{r}} \times B_{l}^{\mathrm{c}}\right)
$$

a interseção entre dois bi-grupos.

Definição 1 (Tamanho de bi-grupos espúrios). Seja $\left\{B_{t_{i}}\right\}_{i=1}^{x}$ o conjunto de bi-grupos em $B$, tal que $\mathrm{I}\left(B_{t_{i}}, \dot{B}_{l}\right)=\emptyset$ para todo $l \in \mathbb{N}_{1, q}$ e $i \in \mathbb{N}_{1, x} .\left\{B_{t_{i}}\right\}_{i=1}^{x}$ é o conjunto de bi-grupos espúrios de B. Seja $\hat{B}$ um bi-agrupamento equivalente a $B$, exceto que um ou mais bi-grupos espúrios foram incrementados de maneira que continuam sendo espúrios. Diz-se que $\mathbb{S}$ é sensível ao tamanho de bi-grupos espúrios se, e somente se, $\mathbb{S}(B, \dot{B})>\mathbb{S}(\hat{B}, \dot{B})$.

Dado que elementos ruidosos não devem ser agrupados, um bi-grupo contendo mais elementos ruidosos deve ser avaliado como pior em termos de qualidade. A Figura 6.1 ilustra esse caso. As estrelas denotam o único bi-grupo do bi-agrupamento de referência $\dot{B}$ e os círculos preenchidos representam elementos ruidosos. As Figs. 6.1(a) e 6.1(b) ilustram dois bi-agrupamentos $B$ e $\hat{B}$ que possuem dois bi-grupos cada. Cada solução contém um bi-grupo composto de elementos ruidosos, mas tais bi-grupos possuem tamanhos distintos. A Tabela 6.1 mostra que a 
metade das medidas ignorou a diferença de tamanho entre os bi-grupos espúrios.

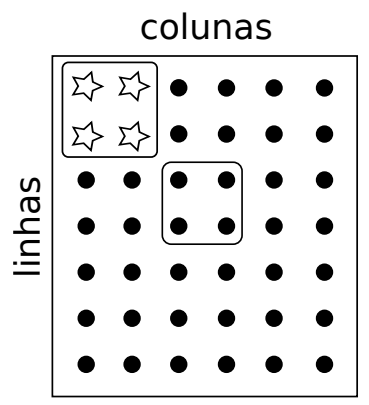

(a) Bi-agrupamento $B$.

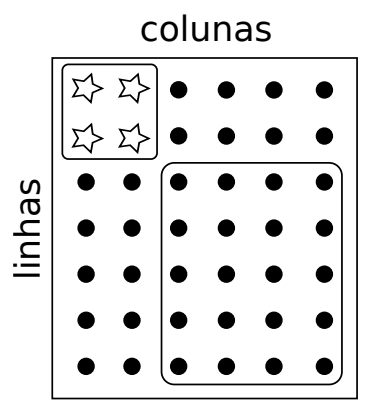

(b) Bi-agrupamento $\hat{B}$.

Figura 6.1: Dois bi-agrupamentos encontrados diferindo apenas no tamanho dos bi-grupos espúrios.

O tamanho de união definido pela Eq. (4.13) aumenta sempre quando um bi-grupo espúrio é incrementado, o que prova que as medidas $\mathbb{S}_{\text {rnia }}$ e $\mathbb{S}_{\text {ce }}$ são sensíveis ao tamanho de bi-grupos espúrios. As medidas $\mathbb{S}_{\text {wjac }}$ e $\mathbb{S}_{\text {wdic }}$ são sensíveis porque os denominadores das Eqs. (4.23) e (4.24) atribuem valores maiores enquanto os nominadores não mudam quando um bi-grupo espúrio é incrementado. A medida $\mathbb{S}_{\mathrm{e}}$ é claramente sensível se o domínio de bi-agrupamentos for restringido aos dos não sobrepostos, mas não é sensível se for considerado o domínio geral dado que bi-grupos espúrios podem ser incrementados sem necessariamente implicar em um aumento do valor de $\left|U_{B}\right|$ (Eq. (4.15)). As medidas $\mathbb{S}_{\mathrm{csi}}$ e $\mathbb{S}_{\mathrm{ebc}}$ são sensíveis, de acordo com as Proposições 6.3 e 6.4. Apesar do resultado exposto na Tabela 6.1. a medida $\mathbb{S}_{\text {agri }}$ não é sensível ao tamanho de bi-grupos espúrios, de acordo com a Proposição 6.5

Tabela 6.1: Avaliação dos bi-agrupamentos da Figura 6.1

\begin{tabular}{lcclcccccc}
\hline Medida & Fig. $6.1(\mathrm{a})$ & Fig. $6.1(\mathrm{~b})$ & Medida & Fig. $6.1(\mathrm{a})$ & Fig. $6.1(\mathrm{~b})$ & Medida & Fig. $6.1(\mathrm{a})$ & Fig. $6.1(\mathrm{~b})$ \\
\hline $\mathbb{S}_{\text {prel }}$ & 0.500 & 0.500 & $\mathbb{S}_{\text {wjac }}$ & $\mathbf{0 . 5 0 0}$ & $\mathbf{0 . 1 6 7}$ & $\mathbb{S}_{\text {erel }}$ & 0.500 & 0.500 \\
$\mathbb{S}_{\text {prec }}$ & 1.000 & 1.000 & $\mathbb{S}_{\text {wdic }}$ & $\mathbf{0 . 5 0 0}$ & $\mathbf{0 . 1 6 7}$ & $\mathbb{S}_{\text {erec }}$ & 1.000 & 1.000 \\
$\mathbb{S}_{\text {rnia }}$ & $\mathbf{0 . 5 0 0}$ & $\mathbf{0 . 1 6 7}$ & $\mathbb{S}_{\text {fabi }}$ & 0.500 & 0.500 & $\mathbb{S}_{\text {csi }}$ & $\mathbf{0 . 5 0 0}$ & $\mathbf{0 . 0 3 1}$ \\
$\mathbb{S}_{\text {ce }}$ & $\mathbf{0 . 5 0 0}$ & $\mathbf{0 . 1 6 7}$ & $\mathbb{S}_{\text {u }}$ & 1.000 & 1.000 & $\mathbb{S}_{\text {ebc }}$ & $\mathbf{0 . 9 6 3}$ & $\mathbf{0 . 7 0 8}$ \\
$\mathbb{S}_{1 \& \text { w }}$ & 0.500 & 0.500 & $\mathbb{S}_{\mathrm{e}}$ & $\mathbf{0 . 5 0 0}$ & $\mathbf{0 . 1 6 7}$ & $\mathbb{S}_{\text {agri }}$ & $\mathbf{0 . 6 6 4}$ & $\mathbf{0 . 0 4 7}$ \\
$\mathbb{S}_{\text {stm }}$ & 0.500 & 0.500 & $\mathbb{S}_{\text {ay }}$ & 0.500 & 0.500 & & & \\
\hline
\end{tabular}

Definição 2 (Cobertura). Assuma que $B$ possua menos bi-grupos que $\dot{B}$ (i.e., $k<q$ ) e que cada bi-grupo de $B$ é equivalente a um bi-grupo de $\dot{B}$. Especificamente, $B$ é dado por um subconjunto próprio de bi-grupos de $\dot{B}$. Assim, $\mathbb{S}$ é uma medida que penaliza soluções por não cobrirem todos os bi-grupos de referência se, e somente se, $\mathbb{S}(B, \dot{B})<1$.

A Figura 6.2 ilustra o caso para o qual o bi-agrupamento encontrado não possui todos os bi-grupos da solução de referência (representada por figuras geométricas não preenchidas), o que é claramente indesejável. Entretanto, a Tabela 6.2 mostra que oito medidas avaliaram $B$ da Figura 6.2 como uma solução perfeita. 
A medida $\mathbb{S}_{\text {fabi }}$ não atinge 1 para soluções que possuem números distintos de grupos, implicando que ela apresenta a propriedade de cobertura. A Proposição 6.6 prova que $\mathbb{S}_{\text {rnia }}$ e $\mathbb{S}_{\text {ce }}$ também possuem a propriedade. Apesar dos seus resultados apresentados na Tabela 6.2 , a Proposição 6.7 prova que $\mathbb{S}_{\text {prec }}, \mathbb{S}_{\mathrm{u}}$ e $\mathbb{S}_{\text {erec }}$ não têm a propriedade. As Proposições 6.8, 6.9 e 6.10 provam que $\mathbb{S}_{\mathrm{csi}}, \mathbb{S}_{\mathrm{ebc}}$ e $\mathbb{S}_{\text {agri }}$ possuem a propriedade para o domínio das soluções não degeneradas.

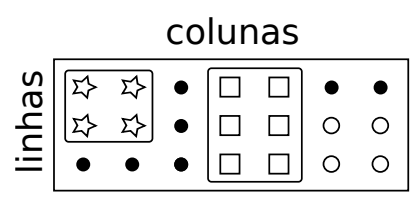

Figura 6.2: Exemplo de bi-agrupamento $B$ que não cobre toda a solução de referência $\dot{B}$.

Tabela 6.2: Avaliação dos bi-agrupamentos da Figura 6.2.

\begin{tabular}{lclcllc}
\hline Medida & Fig. 6.2 & Medida & Fig. 6.2 & Medida & Fig. 6.2 \\
\hline $\mathbb{S}_{\text {prel }}$ & 1.000 & $\mathbb{S}_{\text {wjac }}$ & 1.000 & $\mathbb{S}_{\text {erel }}$ & 1.000 \\
$\mathbb{S}_{\text {prec }}$ & $\mathbf{0 . 7 7 0}$ & $\mathbb{S}_{\text {wdic }}$ & 1.000 & $\mathbb{S}_{\text {erec }}$ & $\mathbf{0 . 6 6 7}$ \\
$\mathbb{S}_{\text {rnia }}$ & $\mathbf{0 . 7 1 4}$ & $\mathbb{S}_{\text {fabi }}$ & $\mathbf{0 . 6 6 7}$ & $\mathbb{S}_{\text {csi }}$ & $\mathbf{0 . 7 7 8}$ \\
$\mathbb{S}_{\text {ce }}$ & $\mathbf{0 . 7 1 4}$ & $\mathbb{S}_{\mathrm{u}}$ & $\mathbf{0 . 7 1 4}$ & $\mathbb{S}_{\text {ebc }}$ & $\mathbf{0 . 9 2 3}$ \\
$\mathbb{S}_{1 \& \text { w }}$ & 1.000 & $\mathbb{S}_{\mathrm{e}}$ & 1.000 & $\mathbb{S}_{\mathrm{agri}}$ & $\mathbf{0 . 8 5 9}$ \\
$\mathbb{S}_{\mathrm{stm}}$ & 1.000 & $\mathbb{S}_{\mathrm{ay}}$ & 1.000 & & \\
\hline
\end{tabular}

Definição 3 (Área sem interseção). Sejam B e $\dot{B}$ dois bi-agrupamentos e seja $S$ o conjunto de elementos da matriz de dados que não estão bi-agrupados em $\dot{B}$. Seja $\hat{B}$ um bi-agrupamento que difere de B apenas pela adição de elementos de $S$ nos bi-grupos de B elou pela criação de outros bi-grupos com apenas elementos de $S$. Uma medida $\mathbb{S}$ penaliza soluções por área sem interseção se, e somente se, $\mathbb{S}(B, \dot{B})>\mathbb{S}(\hat{B}, \dot{B})$.

A propriedade acima é mais geral que a propriedade de mesmo nome definida em (Patrikainen e Meila, 2006) porque $S$ na Def. 3 pode ter elementos bi-agrupados em $B$. A propriedade de área sem interseção traduz a ideia intuitiva de que incrementar a solução $B$ sem o uso de elementos bi-agrupados em $\dot{B}$ deve fazer com que a solução resultante seja menos similar a $\dot{B}$.

Se uma medida penaliza soluções pela área sem interseção com o bi-agrupamento de referência, ela possui a propriedade dada pela Def. 11. Portanto, as únicas medidas que podem satisfazer a Def. 3 são $\mathbb{S}_{\text {rnia }}, \mathbb{S}_{\mathrm{ce}}, \mathbb{S}_{\mathrm{wjac}}, \mathbb{S}_{\mathrm{wdic}}, \mathbb{S}_{\mathrm{csi}}$ e $\mathbb{S}_{\mathrm{ebc}}$.

A medida $\mathbb{S}_{\mathrm{e}}$ possui a propriedade apenas no domínio dos bi-agrupamentos sem sobreposição, o que é evidente devido à Eq. (4.27). As Proposições 6.11 e 6.12 provam que $\mathbb{S}_{\text {rnia }} \mathrm{e}$ $\mathbb{S}_{\mathrm{ce}}$ têm a propriedade em questão. As medidas $\mathbb{S}_{\mathrm{csi}}$ e $\mathbb{S}_{\mathrm{ebc}}$ também possuem a propriedade, mas no domínio das soluções não degeneradas, de acordo com as Proposições 6.15 e 6.17. A Proposição 6.13 mostra um caso no qual $\mathbb{S}_{\mathrm{wjac}}$ e $\mathbb{S}_{\mathrm{wdic}}$ falham no cumprimento da propriedade. 
Definição 4 (Cobertura múltipla). Sejam $B \triangleq\left\{B_{1}\right\}$ e $\dot{B} \triangleq\left\{\dot{B}_{i}\right\}_{i=1}^{q}$ dois bi-agrupamentos, tais que $q>1, B_{1}^{\mathrm{r}} \times B_{1}^{\mathrm{c}}=\bigcup_{i=1}^{q} \dot{B}_{i}^{\mathrm{r}} \times \dot{B}_{i}^{\mathrm{c}}$ e $\dot{B}$ não tenha bi-grupos sobrepostos. Assim, $\mathbb{S}$ é uma medida que penaliza soluções com cobertura múltipla de bi-grupos se, e somente se, $\mathbb{S}(B, \dot{B})<1$.

Deve-se objetivar a geração de bi-agrupamentos que cobrem toda a solução de referência, mas sem misturar elementos da matriz de dados que pertençam a bi-grupos distintos. A propriedade acima formaliza essa ideia e foi proposta em (Patrikainen e Meila, 2006).

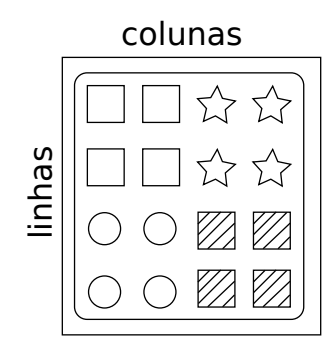

Figura 6.3: Bi-agrupamento encontrado com um bi-grupo e bi-agrupamento de referência com quatro bi-grupos.

A Figura 6.3 mostra um exemplo para o qual uma medida precisa reconhecer a diferença entre as soluções. A Tabela 6.3 mostra que apenas $\mathbb{S}_{\text {rnia }}, \mathbb{S}_{\mathrm{u}}$ e $\mathbb{S}_{\mathrm{e}}$ não identificaram a diferença. A medida $\mathbb{S}_{\text {fabi }}$ não atinge 1 para soluções com diferentes números de bi-grupos, implicando que ela possui a propriedade dada pela Def. 4. Patrikainen e Meila (2006) mostraram que $\mathbb{S}_{\mathrm{ce}}$ tem a propriedade. As Proposições 6.18, 6.19, 6.20, 6.21 e 6.22 provam que as medidas remanescentes também apresentam a propriedade.

Tabela 6.3: Avaliação dos bi-agrupamentos da Figura 6.3

\begin{tabular}{lclcllc}
\hline Medida & Fig. 6.3 & Medida & Fig. 6.3 & Medida & Fig. 6.3 \\
\hline $\mathbb{S}_{\text {prel }}$ & $\mathbf{0 . 5 0 0}$ & $\mathbb{S}_{\text {wjac }}$ & $\mathbf{0 . 2 5 0}$ & $\mathbb{S}_{\text {erel }}$ & $\mathbf{0 . 2 5 0}$ \\
$\mathbb{S}_{\text {prec }}$ & $\mathbf{0 . 5 0 0}$ & $\mathbb{S}_{\text {wdic }}$ & $\mathbf{0 . 4 0 0}$ & $\mathbb{S}_{\text {erec }}$ & $\mathbf{0 . 2 5 0}$ \\
$\mathbb{S}_{\text {rnia }}$ & 1.000 & $\mathbb{S}_{\text {fabi }}$ & $\mathbf{0 . 0 6 2}$ & $\mathbb{S}_{\text {csi }}$ & $\mathbf{0 . 2 0 0}$ \\
$\mathbb{S}_{\text {ce }}$ & $\mathbf{0 . 2 5 0}$ & $\mathbb{S}_{\mathrm{u}}$ & 1.000 & $\mathbb{S}_{\text {ebc }}$ & $\mathbf{0 . 4 0 0}$ \\
$\mathbb{S}_{1 \& \text { w }}$ & $\mathbf{0 . 5 0 0}$ & $\mathbb{S}_{\mathrm{e}}$ & 1.000 & $\mathbb{S}_{\text {agri }}$ & $\mathbf{0 . 0 0 0}$ \\
$\mathbb{S}_{\text {stm }}$ & $\mathbf{0 . 4 0 0}$ & $\mathbb{S}_{\text {ay }}$ & $\mathbf{0 . 2 5 0}$ & & \\
\hline
\end{tabular}

Definição 5 (Bi-grupos repetitivos). Seja $\dot{B}$ uma bi-agrupamento de referência sem sobreposição. Seja B um bi-agrupamento possuindo um ou mais bi-grupos que são equivalentes a bi-grupos de $\dot{B}$. Esses são chamados de bi-grupos ideais. Seja $\hat{B}$ um bi-agrupamento equivalente a $B$, exceto que existem um ou mais bi-grupos ideais em $B$ que foram replicados. Assim, $\mathbb{S}$ é uma medida que penaliza soluções com bi-grupos repetitivos se, e somente se, $\mathbb{S}(B, \dot{B})>\mathbb{S}(\hat{B}, \dot{B})$. 


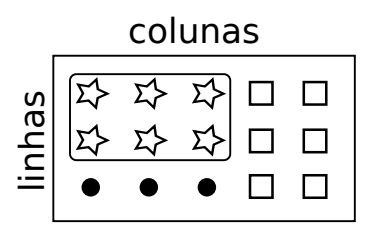

(a) Bi-agrupamento $B$.

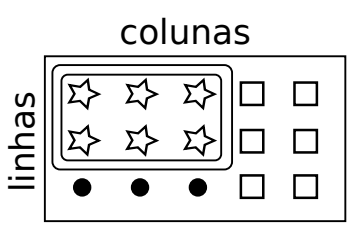

(b) Bi-agrupamento $\hat{B}$.

Figura 6.4: Exemplo de um bi-grupo repetitivo.

A Figura 6.4(a) ilustra um bi-agrupamento $B$ que tem um bi-grupo ideal. Esse bi-grupo foi replicado, dando origem à solução $\hat{B}$ retratada na Figura 6.4(b). O bi-agrupamento $\dot{B}$ é definido por formatos geométricos sem preenchimento. Claramente, $B$ é mais similar a $\dot{B}$ que $\hat{B}$. Entretanto, a Tabela 6.4 mostra que a maioria das medidas não identificou essa diferença. Uma inspeção da Eq. (4.13 leva à conclusão de que $\mathbb{S}_{\text {rnia }}$ e $\mathbb{S}_{\text {ce }}$ seguem a propriedade dada pela Def. 5. As medidas $\mathbb{S}_{\mathrm{csi}}, \mathbb{S}_{\mathrm{ebc}}$ e $\mathbb{S}_{\text {agri }}$ também apresentam a propriedade, de acordo com as Proposições 6.23, 6.24 e 6.25 .

Tabela 6.4: Avaliação dos bi-agrupamentos da Figura 6.4.

\begin{tabular}{|c|c|c|c|c|c|c|c|c|}
\hline Medida & Fig. $6.4(\mathrm{a})$ & Fig. $6.4(\mathrm{~b})$ & Medida & Fig. $6.4(\mathrm{a})$ & Fig. $6.4(\mathrm{~b})$ & Medida & Fig. $6.4(\mathrm{a})$ & Fig. $6.4(\mathrm{~b})$ \\
\hline $\mathbb{S}_{\text {prel }}$ & 1.000 & 1.000 & $\mathbb{S}_{\text {wjac }}$ & 1.000 & 1.000 & $\mathbb{S}_{\text {erel }}$ & 1.000 & 1.000 \\
\hline $\mathbb{S}_{\text {prec }}$ & 0.577 & 0.577 & $\mathbb{S}_{\text {wdic }}$ & 1.000 & 1.000 & $\mathbb{S}_{\text {erec }}$ & 0.500 & 0.500 \\
\hline $\mathbb{S}_{\text {rnia }}$ & 0.600 & 0.375 & $\mathbb{S}_{\mathrm{fabi}}$ & 0.500 & 0.500 & $\mathbb{S}_{\mathrm{csi}}$ & 0.714 & 0.125 \\
\hline $\mathbb{S}_{\mathrm{ce}}$ & 0.600 & 0.375 & $\mathbb{S}_{\mathrm{u}}$ & 0.600 & 0.600 & $\mathbb{S}_{\mathrm{ebc}}$ & 0.889 & 0.800 \\
\hline $\mathbb{S}_{1 \& w}$ & 1.000 & 1.000 & $\mathbb{S}_{\mathrm{e}}$ & 1.000 & 1.000 & $\mathbb{S}_{\text {agri }}$ & 0.800 & 0.103 \\
\hline $\mathbb{S}_{\text {stm }}$ & 1.000 & 1.000 & $\mathbb{S}_{\text {ay }}$ & 1.000 & 1.000 & & & \\
\hline
\end{tabular}

Embora a Def. 5 se aplique ao caso específico de bi-grupos idênticos, são apresentados exemplos na Seção 6.4 gerados por algoritmos de bi-agrupamento para os quais as medidas que não seguem a propriedade em questão têm dificuldade em penalizar soluções com vários bi-grupos similares.

Definição 6 (Simetria). A medida $\mathbb{S}$ é simétrica se, e somente se, $\mathbb{S}(B, \dot{B})=\mathbb{S}(\dot{B}, B)$ para quaisquer $B$ e $\dot{B}$.

A propriedade de simetria é importante porque ela facilita a interpretação da medida (Meila, 2003). Limita-se aqui apontar o leitor para a Tabela 6.6, a qual indica a presença ou ausência da propriedade de simetria para cada medida. As provas são triviais e foram, então, omitidas.

Definição 7 (Homogeneidade). Sejam $B, \hat{B}$ e $\dot{B}$ bi-agrupamentos sem sobreposição. Seja $B_{i_{1}} \in B$ um bi-grupo contendo apenas elementos bi-agrupados em $\dot{B}_{m a\left(i_{1}\right)} \in \dot{B}$ e $\dot{B}_{m i\left(i_{1}\right)} \in \dot{B}$, de modo que $\left|\mathrm{I}\left(B_{i_{1}}, \dot{B}_{m a\left(i_{1}\right)}\right)\right|>\left|\mathrm{I}\left(B_{i_{1}}, \dot{B}_{m i\left(i_{1}\right)}\right)\right|$. Em outras palavras, $\dot{B}_{m a\left(i_{1}\right)}$ é a categoria majoritária de $B_{i_{1}}$ e os elementos restantes pertencem ao bi-grupo $\dot{B}_{m i\left(i_{1}\right)}$ na solução de referência. Sejam $B_{i_{2}}, \dot{B}_{m a\left(i_{2}\right)}$ e $\dot{B}_{m i\left(i_{2}\right)}$ analogamente definidos, de modo que mi $\left(i_{1}\right) \neq m i\left(i_{2}\right)$, $m i\left(i_{1}\right) \neq m a\left(i_{2}\right)$ e mi $\left(i_{2}\right) \neq m a\left(i_{1}\right)$. Seja $\hat{B}$ um bi-agrupamento equivalente a $B$, exceto 
que $x>0$ elementos da categoria minoritária de $B_{i_{1}}$ foram permutados por $x$ elementos da categoria minoritária de $B_{i_{2}}$. Logo, $\mathbb{S}$ é uma medida que penaliza soluções menos homogêneas se, e somente se, $\mathbb{S}(B, \dot{B}) \geq \mathbb{S}(\hat{B}, \dot{B})$, tal que $\mathbb{S}(B, \dot{B})=\mathbb{S}(\hat{B}, \dot{B})$ se, e somente se, $x=\left|\mathrm{I}\left(B_{i_{1}}, \dot{B}_{m i\left(i_{1}\right)}\right)\right|=\left|\mathrm{I}\left(B_{i_{2}}, \dot{B}_{m i\left(i_{2}\right)}\right)\right|$.

A Figura 6.5(a) retrata um bi-agrupamento de referência $\dot{B}$. As Figs. 6.5(b) e 6.5(c) mostram um exemplo de bi-agrupamentos $B$ e $\hat{B}$ que seguem as suposições da Def. 7 . A homogeneidade foi proposta em (Rosenberg e Hirschberg, 2007; Meila, 2007; Amigó et al., 2009) (discutidas em (Meila, 2007) como o "problema de associação") como uma característica desejável de medidas para comparação de agrupamentos, mas sem uma formalização precisa. Uma medida não deve ser sensível apenas à categoria majoritária de um bi-grupo encontrado, mas ela precisa considerar também como o resto do bi-grupo encontrado está organizado.

A solução $B$ (Figura 6.5(b) é claramente mais organizada que $\hat{B}$ (Figura 6.5(c)) e, portanto, deve ser preferida. Entretanto, a Tabela 6.5 mostra que a maioria das medidas não detectou a diferença. A Proposição 6.26 prova que $\mathbb{S}_{\text {prec }}$ e $\mathbb{S}_{\text {erec }}$ não têm a propriedade de homogeneidade, a despeito dos seus resultados apresentados na Tabela 6.5. As Proposições 6.27, 6.28 e 6.29 mostram que $\mathbb{S}_{\mathrm{csi}}, \mathbb{S}_{\mathrm{ebc}}$ e $\mathbb{S}_{\text {agri }}$ têm a propriedade.

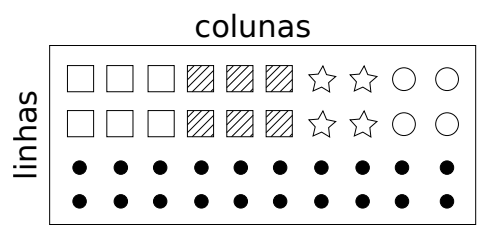

(a) Matriz de dados $\mathrm{X} \in \mathbb{R}^{4 \cdot 10}$.

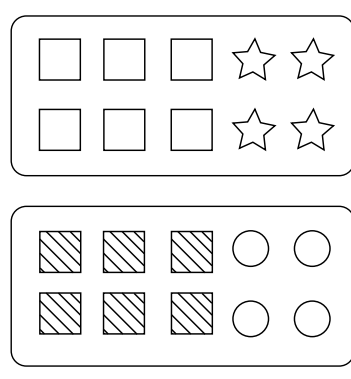

(b) Bi-agrupamento $B$.

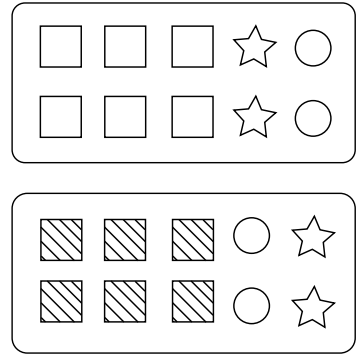

(c) Bi-agrupamento $\hat{B}$.

Figura 6.5: Diferença de homogeneidade.

Tabela 6.5: Avaliação de bi-agrupamentos da Figura 6.5.

\begin{tabular}{lcc|lcc|ccc|}
\hline Medida & Fig. $6.5(\mathrm{~b})$ & Fig. $6.5(\mathrm{c})$ & Medida & Fig. $6.5(\mathrm{~b})$ & Fig. $6.5(\mathrm{c})$ & Medida & Fig. $6.5(\mathrm{~b})$ & Fig. \\
\hline $\mathbb{S}_{\text {prel }}$ & 0.775 & 0.775 & $\mathbb{S}_{\text {wjac }}$ & 0.600 & 0.600 & $\mathbb{S}_{\text {erel }}$ & 0.600 & 0.600 \\
$\mathbb{S}_{\text {prec }}$ & $\mathbf{0 . 7 0 7}$ & $\mathbf{0 . 6 1 9}$ & $\mathbb{S}_{\text {wdic }}$ & 0.750 & 0.750 & $\mathbb{S}_{\text {erec }}$ & $\mathbf{0 . 5 0 0}$ & $\mathbf{0 . 3 8 3}$ \\
$\mathbb{S}_{\text {rnia }}$ & 1.000 & 1.000 & $\mathbb{S}_{\text {fabi }}$ & 0.300 & 0.300 & $\mathbb{S}_{\text {csi }}$ & $\mathbf{0 . 4 6 7}$ & $\mathbf{0 . 3 4 7}$ \\
$\mathbb{S}_{\text {ce }}$ & 0.600 & 0.600 & $\mathbb{S}_{\text {u }}$ & 1.000 & 1.000 & $\mathbb{S}_{\text {ebc }}$ & $\mathbf{0 . 8 6 4}$ & $\mathbf{0 . 8 0 0}$ \\
$\mathbb{S}_{1 \& \text { w }}$ & 0.714 & 0.714 & $\mathbb{S}_{\text {e }}$ & 1.000 & 1.000 & $\mathbb{S}_{\text {agri }}$ & $\mathbf{0 . 6 0 8}$ & $\mathbf{0 . 4 7 7}$ \\
$\mathbb{S}_{\text {stm }}$ & 0.750 & 0.750 & $\mathbb{S}_{\text {ay }}$ & 0.600 & 0.600 & & & \\
\hline
\end{tabular}


Definição 8 (Condições para o máximo). Diz-se que $\mathbb{S}$ obedece as condições necessária e suficiente para o máximo se $\mathbb{S}$ é tal que: $\mathbb{S}(B, \dot{B})=1$ se, e somente se, $B$ e $\dot{B}$ são bi-agrupamentos equivalentes.

A propriedade acima é importante porque garante que não existe uma solução melhor caso a medida atinja o seu máximo. A Proposição 6.30 mostra que $\mathbb{S}_{\mathrm{ce}}(B, \dot{B})=1$ se, e somente se, $B \equiv \dot{B}$, o que não foi provado em (Patrikainen e Meila, 2006) para o caso em que a sobreposição de bi-grupos é permitida. A Proposição6.31 prova que $\mathbb{S}_{\text {fabi }}$ também segue as condições de máximo. As medidas $\mathbb{S}_{\mathrm{csi}}$, $\mathbb{S}_{\mathrm{ebc}}$ e $\mathbb{S}_{\text {agri }}$ não obedecem a condição necessária de máximo (Proposição 6.32 mesmo para soluções não degeneradas (Proposição 6.33). As Tabelas 6.1, 6.4 e 6.5 mostram que as medidas restantes também não seguem a condição necessária de máximo.

Em resumo, a Tabela 6.6 discrimina cada medida de acordo com as propriedades discutidas.

Tabela 6.6: Discriminação de medidas de acordo com as propriedades discutidas.

\begin{tabular}{|c|c|c|c|c|c|c|c|c|c|c|c|c|c|c|c|c|c|}
\hline Propriedades & $\mathbb{S}_{\text {prel }}$ & $\mathbb{S}_{\text {prec }}$ & $\mathbb{S}_{\text {rnia }}$ & $\mathbb{S}_{\mathrm{ce}}$ & $\mathbb{S}_{1 \& w}$ & $\mathbb{S}_{\text {stm }}$ & $\mathbb{S}_{\mathrm{wjac}}$ & $\mathbb{S}_{\text {wdic }}$ & $\mathbb{S}_{\text {fabi }}$ & $\mathbb{S}_{\mathrm{u}}$ & $\mathbb{S}_{\mathrm{e}}$ & $\mathbb{S}_{\text {ay }}$ & $\mathbb{S}_{\text {erel }}$ & $\mathbb{S}_{\text {erec }}$ & $\mathbb{S}_{\mathrm{csi}}$ & $\mathbb{S}_{\text {ebc }}$ & $\mathbb{S}_{\text {agri }}$ \\
\hline Bi. espú. & & & $\checkmark$ & $\checkmark$ & & & $\checkmark$ & $\checkmark$ & & & $\sqrt{ }^{a}$ & & & & $\checkmark$ & $\checkmark$ & \\
\hline Cobert. & & & $\checkmark$ & $\checkmark$ & & & & & $\checkmark$ & & & & & & $\checkmark^{b}$ & $\checkmark^{b}$ & $\checkmark^{b}$ \\
\hline Área sem int. & & & $\checkmark$ & $\checkmark$ & & & & & & & $\checkmark^{\mathrm{a}}$ & & & & $\checkmark^{b}$ & $\checkmark^{b}$ & \\
\hline Cobert. múlt. & $\checkmark$ & $\checkmark$ & & $\checkmark$ & $\checkmark$ & $\checkmark$ & $\checkmark$ & $\checkmark$ & $\checkmark$ & & & $\checkmark$ & $\checkmark$ & $\checkmark$ & $\checkmark$ & $\checkmark$ & $\checkmark$ \\
\hline Bi. rep. & & & $\checkmark$ & $\checkmark$ & & & & & & & & & & & $\checkmark$ & $\checkmark$ & $\checkmark$ \\
\hline Simetria & & & $\checkmark$ & $\checkmark$ & & & & & $\checkmark$ & & & & & & $\checkmark$ & $\checkmark$ & $\checkmark$ \\
\hline Homog. & & & & & & & & & & & & & & & $\checkmark$ & $\checkmark$ & $\checkmark$ \\
\hline Máximo & & & & $\checkmark$ & & & & & $\checkmark$ & & & & & & & & \\
\hline
\end{tabular}

\subsubsection{Considerações}

Visto que $\mathbb{S}_{\text {prel }}, \mathbb{S}_{\text {prec }}, \mathbb{S}_{\text {ce }}, \mathbb{S}_{1 \& w}, \mathbb{S}_{\text {stm }}, \mathbb{S}_{\text {wjac }}, \mathbb{S}_{\text {wdic }}, \mathbb{S}_{\text {fabi }}, \mathbb{S}_{\text {ay }}, \mathbb{S}_{\text {erel }}$ e $\mathbb{S}_{\text {erec }}$ são medidas baseadas em associação entre bi-grupos, elas ignoram o relacionamento entre os elementos da matriz de dados que não pertencem à categoria majoritária do respectivo bi-grupo. Esse tipo de avaliação é análogo ao das medidas baseadas em associação de grupos para a comparação de agrupamentos particionais exclusivos (e.g., critério de Meila e Heckerman (Meila e Heckerman, 2001), critério de Larsen e Aone (Larsen e Aone, 1999), métrica de van Dongen (Dongen, 2000)), os quais apresentam o problema análogo de ignorar o que ocorre com os objetos que não pertencem à categoria majoritária do respectivo grupo (Amigó et al., 2009; Rosenberg e Hirschberg, 2007; Meila, 2003). Não coincidentemente, nenhuma dessas medidas de bi-agrupamento possui a propriedade de homogeneidade dada pela Def. 7( veja a Tabela 6.6). Sabe-se também que a associação de grupos (ou bi-grupos) entre as soluções encontrada e de referência é arbitrária (Steinley, 2003, 2004). Ela pode ser manipulada para gerar avaliações mais ou menos favoráveis, visto que algoritmos de agrupamento (assim como algoritmos de bi-agrupamento) não fornecem tal associação. As medidas $\mathbb{S}_{\text {rnia }}, \mathbb{S}_{\mathrm{u}}$ e $\mathbb{S}_{\mathrm{e}}$ vão além ao ignorarem completamente a relação entre os elementos da matriz de dados ao basear sua avaliação somente na participação ou não dos elementos no bi-agrupamento. Contrariamente, tanto $\mathbb{S}_{\mathrm{csi}}$ como $\mathbb{S}_{\mathrm{ebc}}$ e $\mathbb{S}_{\text {agri }}$ analisam a relação entre cada par de elementos da matriz, de maneira parecida às medidas de similaridade entre agrupamentos particionais exclusivos baseadas em pares (e.g., RI Je ARI). Essa é 
a razão pela qual $\mathbb{S}_{\mathrm{csi}}, \mathbb{S}_{\mathrm{ebc}}$ e $\mathbb{S}_{\text {agri }}$ apresentam a propriedade de homogeneidade.

As medidas $\mathbb{S}_{\mathrm{ce}}$, $\mathbb{S}_{\mathrm{csi}}$ e $\mathbb{S}_{\mathrm{ebc}}$ se destacaram na análise teórica como sendo as melhores. Elas diferem basicamente no que diz respeito às propriedades de homogeneidade e máximo apenas. Embora a medida $\mathbb{S}_{\text {agri }}$ tenha se desempenhado inferiormente às outras na análise teórica apresentada neste capítulo, é importante mencionar que ela, ao contrário (possivelmente) das outras, possui outras propriedades interessantes discutidas no Capítulo 5 . Em particular, a medida $\mathbb{S}_{\text {agri }}$ apresenta uma avaliação basal constante, o que pode torná-la mais atraente em cenários cujas soluções apresentam números distintos de bi-grupos.

\subsection{Experimentos}

São reportados vários experimentos envolvendo modelos de bi-grupos e algoritmos de biagrupamento selecionados de estudos importantes da literatura de expressão gênica. Esses experimentos mostram que as propriedades de medidas de similaridade entre bi-agrupamentos ajudam no entendimento de resultados de estudos empíricos reais.

\subsubsection{Experimento 1}

Esse experimento segue o modelo de regulação-positiva constante para bi-grupo adotado na análise empírica de algoritmos de bi-agrupamento de um estudo publicado recentemente (Eren et al. 2012). Foi gerada uma matriz de dados com 50 linhas e 10 colunas 2 , na qual foi inserido um bi-grupo com um nível de expressão constante 5. Os valores de fundo (i.e., valores dos elementos da matriz de dados que não pertencem a nenhum bi-grupo) foram sorteados de acordo com a distribuição normal padrão $\mathcal{N}(0,1)$. O bi-grupo inserido em $\dot{B}$ é dado por $\dot{B}_{1} \triangleq(\{1,2, \ldots, 30\},\{1,2, \ldots, 8\})$. A Figura 6.6(a) apresenta a base de dados.

Os algoritmos de bi-agrupamento listados na Tabela 6.7 foram aplicados na matriz de dados. A Tabela 6.8 mostra os resultados de dois casos interessantes (correspondentes aos algoritmos pcluster e bibit) e de um bi-agrupamento gerado artificialmente, constituído apenas do primeiro bi-grupo da solução produzida pelo algoritmo pcluster (?). O algoritmo pcluster gerou três bi-grupos muito similares:

$$
\begin{aligned}
& B_{1}=(\{1,2, \ldots, 30\},\{1,2, \ldots, 8,10\}), \\
& B_{2}=(\{1,2, \ldots, 30,46,48\},\{1,2, \ldots, 8\}) \mathrm{e} \\
& B_{3}=(\{1,2, \ldots, 30,46,47,50\},\{1,2, \ldots, 8\}) .
\end{aligned}
$$

Note que as medidas $\mathbb{S}_{\text {rnia }}, \mathbb{S}_{\mathrm{ce}}, \mathbb{S}_{\mathrm{csi}}, \mathbb{S}_{\mathrm{ebc}}$ e $\mathbb{S}_{\text {agri }}$, as quais apresentam a propriedade de detectar bi-grupos repetitivos (Def. 5), penalizaram consideravelmente a solução do algoritmo pcluster. Embora não tenha apresentado essa propriedade, a medida $\mathbb{S}_{\text {fabi }}$ penalizou severamente a solução do algoritmo pcluster. Esse comportamento é explicado pela diferença entre o número de bi-grupos das soluções encontrada e de referência. A terceira coluna mostra os resultados para o

\footnotetext{
${ }^{2}$ Gerou-se um conjunto de dados menor que os usados em (Eren et al. 2012) por motivos didáticos.
} 
Tabela 6.7: Algoritmos de bi-agrupamento usados nos experimentos da Seção6.4.

\begin{tabular}{|c|c|c|c|c|}
\hline Algoritmo & Implementação & Fonte & \multicolumn{2}{|c|}{ Referência } \\
\hline \multirow[t]{2}{*}{$\mathrm{bbc}$} & $\mathrm{C}$ & http://www.people.fas. & Gu e Liu. 2 & 2008) \\
\hline & & harvard.edu/ junliu/BBC/ & & \\
\hline bcca & Matlab & http://sn.im/26fxm0n & \multicolumn{2}{|c|}{ (Bhattacharya e De. 2009) } \\
\hline \multirow[t]{2}{*}{ bibit } & Java & http://www.upo.es/eps/ & \multirow{2}{*}{\multicolumn{2}{|c|}{ (Rodriguez-Baena et al., 2011) }} \\
\hline & & bigs/BiBit_algorithm.html & & \\
\hline \multirow[t]{3}{*}{ bimax } & pacote biclust em R & http://cran.r-project. & \multirow[t]{3}{*}{ (Prelić et al. } & \multirow[t]{3}{*}{$2006)$} \\
\hline & & org/web/packages/biclust/ & & \\
\hline & & index.html & & \\
\hline \multirow[t]{3}{*}{ cc } & pacote biclust em $\mathrm{R}$ & http://cran.r-project. & \multirow{3}{*}{\multicolumn{2}{|c|}{ (Cheng e Church 2000) }} \\
\hline & & org/web/packages/biclust/ & & \\
\hline & & index.html & & \\
\hline \multirow[t]{2}{*}{ las } & Matlab & https://genome.unc.edu/ & \multirow[t]{2}{*}{ Shabalin et a } & \multirow[t]{2}{*}{ 1. (2009) } \\
\hline & & las/ & & \\
\hline \multirow[t]{3}{*}{ msbe } & Java & http://www.cs.cityu.edu. & \multirow[t]{3}{*}{ (Liu e Wang } & \multirow[t]{3}{*}{$2007)$} \\
\hline & & hk/ lwang/software/msbe/ & & \\
\hline & & help.html & & \\
\hline \multirow[t]{2}{*}{ pcluster } & binário do Windows & http://haixun.olidu.com/ & \multirow[t]{2}{*}{ (Wang et al., } & \multirow[t]{2}{*}{ 2002) } \\
\hline & & proj/delta.html & & \\
\hline \multirow[t]{3}{*}{ xmotifs } & pacote biclust em $\mathrm{R}$ & http://cran.r-project. & \multirow[t]{3}{*}{ (Murali e Kasi } & \multirow[t]{3}{*}{ f. 2003$)$} \\
\hline & & org/web/packages/biclust/ & & \\
\hline & & index.html & & \\
\hline
\end{tabular}

bi-agrupamento que possui apenas o bi-grupo $B_{1}$ da solução do algoritmo pcluster. As medidas $\mathbb{S}_{1 \& \mathrm{w}}$ e $\mathbb{S}_{\mathrm{u}}$ apresentaram pouca ou nenhuma diferença de avaliação para a solução completa do algoritmo pcluster, que possui três bi-grupos praticamente idênticos, e o bi-agrupamento $\left\{B_{1}\right\}$ quase perfeito. As medidas $\mathbb{S}_{\text {prel }}, \mathbb{S}_{\text {prec }}, \mathbb{S}_{\text {stm }}, \mathbb{S}_{\text {wjac }}, \mathbb{S}_{\text {wdic }}, \mathbb{S}_{\text {ay }}, \mathbb{S}_{\text {erel }}$ e $\mathbb{S}_{\text {erec }}$ avaliaram $\left\{B_{1}\right\}$ como uma pior solução que a solução completa do algoritmo pcluster, o que não é desejável.

$\mathrm{O}$ algoritmo bibit (Rodriguez-Baena et al., 2011) encontrou 31 bi-grupos. Cada um deles abrange uma grande parte da matriz de dados, o que levou as medidas $\mathbb{S}_{\text {prec }}$, $\mathbb{S}_{\mathrm{u}}$ e $\mathbb{S}_{\text {erec }}$ a atingirem 1. Isso não é ruim por si só porque essas três medidas devem ser consideradas em conjunto com os seus pares $\mathbb{S}_{\text {prel }}, \mathbb{S}_{\mathrm{e}}$ e $\mathbb{S}_{\text {erel }}$. Entretanto, acredita-se que uma boa medida deve avaliar a solução encontrada em questão como muito ruim porque existe uma grande diferença de número de grupos. A Tabela 6.8 mostra que as medidas que possuem a propriedade de detectar bi-grupos repetitivos em conjunto com a medida $\mathbb{S}_{\text {fabi }}$ atingiram avaliações próximas de zero para a solução do algoritmo bibit.

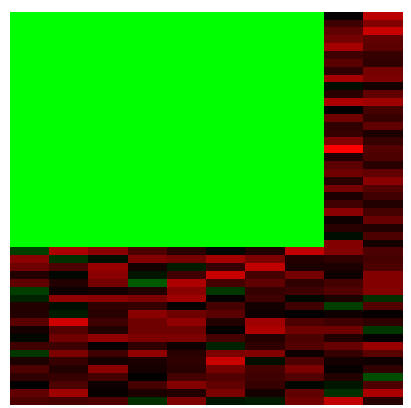

(a)

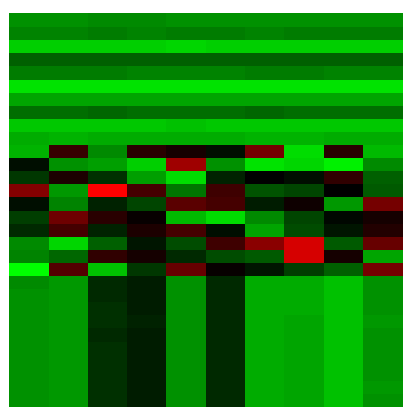

(b)

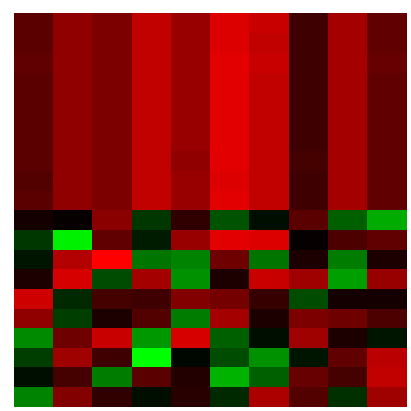

(c)

Figura 6.6: Bases de dados para a análise do comportamento das medidas. 
Tabela 6.8: Avaliações relacionadas à base de dados da Figura 6.6(a).

\begin{tabular}{lccc}
\hline Medida & Pcluster & Pcluster $\left\{B_{1}\right\}$ & Bibit \\
\hline $\mathbb{S}_{\text {prel }}$ & 0.956 & 0.943 & 0.718 \\
$\mathbb{S}_{\text {prec }}$ & 1.000 & 0.943 & 1.000 \\
$\mathbb{S}_{\text {rnia }}$ & 0.304 & 0.889 & 0.024 \\
$\mathbb{S}_{\text {ce }}$ & 0.304 & 0.889 & 0.024 \\
$\mathbb{S}_{1 \& \text { w }}$ & 0.950 & 0.974 & 0.679 \\
$\mathbb{S}_{\text {stm }}$ & 0.954 & 0.941 & 0.688 \\
$\mathbb{S}_{\text {wjac }}$ & 0.911 & 0.889 & 0.531 \\
$\mathbb{S}_{\text {wdic }}$ & 0.954 & 0.941 & 0.677 \\
$\mathbb{S}_{\text {fabi }}$ & 0.312 & 0.889 & 0.032 \\
$\mathbb{S}_{\mathrm{u}}$ & 1.000 & 1.000 & 1.000 \\
$\mathbb{S}_{\mathrm{e}}$ & 0.795 & 0.889 & 0.480 \\
$\mathbb{S}_{\text {ay }}$ & 0.912 & 0.889 & 0.532 \\
$\mathbb{S}_{\text {erel }}$ & 0.912 & 0.889 & 0.549 \\
$\mathbb{S}_{\text {erec }}$ & 0.938 & 0.889 & 1.000 \\
$\mathbb{S}_{\mathrm{csi}}$ & 0.083 & 0.790 & 0.004 \\
$\mathbb{S}_{\text {ebc }}$ & 0.687 & 0.940 & 0.025 \\
$\mathbb{S}_{\text {agri }}$ & 0.048 & 0.842 & 0.000 \\
\hline & & &
\end{tabular}

\subsubsection{Experimento 2}

Madeira e Oliveira (2004) propuseram quatro classes principais de bi-grupos. Uma delas consiste de bi-grupos com valores constantes nas linhas e colunas. Criou-se, aqui, uma base de dados com 30 linhas e 10 colunas possuindo um bi-grupo de colunas constantes e um bi-grupo de linhas constantes, retratada na Figura 6.6(b). A Tabela 6.9 mostra os resultados de quatro algoritmos. O algoritmo pcluster gerou a extrema quantidade de 497 bi-grupos. Apesar disso, várias medidas atribuíram pontuações relativamente altas a essa solução. Como no experimento anterior, apenas as medidas que têm a propriedade de detectar bi-grupos repetitivos em conjunto com a medida $\mathbb{S}_{\text {fabi }}$ atingiram avaliações próximas de zero.

O algoritmo xmotifs (Murali e Kasif, 2003) encontrou apenas o bi-grupo de linhas constantes. As medidas $\mathbb{S}_{\text {rnia }}, \mathbb{S}_{\mathrm{ce}}, \mathbb{S}_{\mathrm{fabi}}, \mathbb{S}_{\mathrm{csi}}, \mathbb{S}_{\mathrm{ebc}}$ e $\mathbb{S}_{\text {agri }}$, que possuem a propriedade de cobertura dada pela Def. 2, detectaram a diferença entre as soluções encontrada e de referência. As medidas $\mathbb{S}_{\text {prec }}, \mathbb{S}_{\mathrm{u}}$ e $\mathbb{S}_{\text {erec }}$, que não possuem a propriedade de cobertura, também detectaram a diferença. A razão para isso é que essas três medidas violam a propriedade de cobertura apenas em situações muito particulares, como na Proposição 6.7. Similarmente, o algoritmo bcca (Bhattacharya e De, 2009) encontrou apenas o bi-grupo de colunas constantes, e os resultados foram os mesmos.

O algoritmo msbe (Liu e Wang, 2007) encontrou seis bi-grupos. Os dois primeiros são equi- 
Tabela 6.9: Avaliações relacionadas à base de dados da Figura 6.6(b).

\begin{tabular}{lcccc}
\hline Medida & Pcluster & Xmotifs & Bcca & Msbe \\
\hline $\mathbb{S}_{\text {prel }}$ & 0.625 & 1.000 & 1.000 & 0.408 \\
$\mathbb{S}_{\text {prec }}$ & 1.000 & 0.707 & 0.707 & 1.000 \\
$\mathbb{S}_{\text {rnia }}$ & 0.004 & 0.500 & 0.500 & 0.855 \\
$\mathbb{S}_{\text {ce }}$ & 0.004 & 0.500 & 0.500 & 0.855 \\
$\mathbb{S}_{\text {\&\&w }}$ & 0.620 & 1.000 & 1.000 & 0.405 \\
$\mathbb{S}_{\text {stm }}$ & 0.607 & 1.000 & 1.000 & 0.333 \\
$\mathbb{S}_{\text {wjac }}$ & 0.439 & 1.000 & 1.000 & 0.855 \\
$\mathbb{S}_{\text {wdic }}$ & 0.603 & 1.000 & 1.000 & 0.855 \\
$\mathbb{S}_{\text {fabi }}$ & 0.004 & 0.500 & 0.500 & 0.333 \\
$\mathbb{S}_{\text {u }}$ & 1.000 & 0.500 & 0.500 & 1.000 \\
$\mathbb{S}_{\mathrm{e}}$ & 0.678 & 1.000 & 1.000 & 0.862 \\
$\mathbb{S}_{\text {ay }}$ & 0.392 & 1.000 & 1.000 & 0.333 \\
$\mathbb{S}_{\text {erel }}$ & 0.443 & 1.000 & 1.000 & 0.333 \\
$\mathbb{S}_{\text {erec }}$ & 0.950 & 0.500 & 0.500 & 1.000 \\
$\mathbb{S}_{\text {csi }}$ & 0.001 & 0.500 & 0.500 & 0.932 \\
$\mathbb{S}_{\text {ebc }}$ & 0.038 & 0.802 & 0.802 & 0.950 \\
$\mathbb{S}_{\text {agri }}$ & 0.000 & 0.609 & 0.609 & 0.957 \\
\hline & & & &
\end{tabular}

valentes aos dois bi-grupos de referência e os outros não possuem sobreposição com nenhum bi-grupo de referência:

$$
\begin{aligned}
& B_{1}=(\{1,2, \ldots, 10\},\{1,2, \ldots, 10\}) \\
& B_{2}=(\{21,22, \ldots, 30\},\{1,2, \ldots, 10\}), \\
& B_{3}=(\{12,15,17\},\{6,8,10\}), \\
& B_{4}=(\{11,19,20\},\{1,4,9\}), \\
& B_{5}=(\{12,15,16,19\},\{3,4\}) \mathrm{e} \\
& B_{6}=(\{11,13,17,20\},\{2,6\}) .
\end{aligned}
$$

Essa solução traz a tona outras características de medidas que não estão diretamente relacionadas às propriedades estudadas. As medidas $\mathbb{S}_{\mathrm{stm}}, \mathbb{S}_{\mathrm{fabi}}, \mathbb{S}_{\text {ay }}$ e $\mathbb{S}_{\text {erel }}$ associaram os bi-grupos $B_{1} \mathrm{e}$ $B_{2}$ com os de referência e somaram as suas contribuições na avaliação. A soma resultante foi dividida pelo número de bi-grupos encontrados, o que explica o porquê delas terem atribuído o valor $2 / 6$. As medidas $\mathbb{S}_{\text {wjac }}$ e $\mathbb{S}_{\text {wdic }}$ alcançaram altos valores porque os tamanhos dos bi-grupos corretos são maiores que os tamanhos dos bi-grupos espúrios. As medidas $\mathbb{S}_{\mathrm{csi}}$, $\mathbb{S}_{\mathrm{ebc}}$ e $\mathbb{S}_{\text {agri }}$ atingiram valores altos devido à abordagem de avaliação baseada em pares. Por exemplo, a submatriz correspondente ao bi-grupo $B_{1}$ tem $\left(\begin{array}{c}100 \\ 2\end{array}\right)=4950$ pares de elementos da matriz de 
dados, os quais são individualmente avaliados por $\mathbb{S}_{\mathrm{csi}}$, $\mathbb{S}_{\text {ebc }}$ e $\mathbb{S}_{\text {agri }}$, enquanto a submatriz correspondente ao bi-grupo espúrio $B_{3}$ tem apenas $\left(\begin{array}{l}9 \\ 2\end{array}\right)=36$ pares. Por outro lado, as outras medidas se baseiam nos elementos da matriz de dados, da maneira individual, para avaliar as soluções, significando que os bi-grupos espúrios encontrados têm uma relevância maior na avaliação. Por exemplo, tanto $\mathbb{S}_{\text {rnia }}$ como $\mathbb{S}_{\text {ce }}$ atribuíram o valor 0.855 .

\subsubsection{Experimento 3}

Criou-se outra base de dados com 20 linhas e 10 colunas, com apenas um bi-grupo de colunas constantes. Ela é ilustrada pela Figura 6.6(c), O algoritmo las (Shabalin et al., 2009) encontrou os bi-grupos

$$
\begin{aligned}
& B_{1}=(\{1,2, \ldots, 10\},\{1,2, \ldots, 10\}) \mathrm{e} \\
& B_{2}=(\{11,13,17,18\},\{1,4,5,6,7,9,10\}),
\end{aligned}
$$

e o algoritmo cc (Cheng e Church, 2000) encontrou

$$
\begin{aligned}
& B_{1}=(\{1,2, \ldots, 10\},\{1,2, \ldots, 10\}) \mathrm{e} \\
& B_{2}=(\{11,15,16\},\{6,10\}) .
\end{aligned}
$$

A solução do algoritmo cc é melhor que a do algoritmo las porque o bi-grupo espúrio é menor. A Tabela 6.10 mostra que as medidas que são insensíveis ao tamanho de bi-grupos espúrios (Def. 1) não detectaram a diferença entre as soluções ou avaliaram a solução do algoritmo las como sendo melhor que a solução do algoritmo cc.

Tabela 6.10: Avaliações relacionadas à base de dados da Figura 6.6(c).

\begin{tabular}{lcclcc}
\hline Medida & Las & Cc & Medida & Las & Cc \\
\hline $\mathbb{S}_{\text {prel }}$ & 0.652 & 0.548 & $\mathbb{S}_{\mathrm{u}}$ & 1.000 & 1.000 \\
$\mathbb{S}_{\text {prec }}$ & 1.000 & 1.000 & $\mathbb{S}_{\mathrm{e}}$ & 0.781 & 0.943 \\
$\mathbb{S}_{\text {rnia }}$ & 0.781 & 0.943 & $\mathbb{S}_{\text {ay }}$ & 0.500 & 0.500 \\
$\mathbb{S}_{\text {ce }}$ & 0.781 & 0.943 & $\mathbb{S}_{\text {erel }}$ & 0.500 & 0.500 \\
$\mathbb{S}_{1 \& \mathrm{w}}$ & 0.646 & 0.543 & $\mathbb{S}_{\text {erec }}$ & 1.000 & 1.000 \\
$\mathbb{S}_{\text {stm }}$ & 0.500 & 0.500 & $\mathbb{S}_{\text {csi }}$ & 0.929 & 0.997 \\
$\mathbb{S}_{\text {wjac }}$ & 0.781 & 0.943 & $\mathbb{S}_{\text {ebc }}$ & 0.928 & 0.987 \\
$\mathbb{S}_{\text {wdic }}$ & 0.781 & 0.943 & $\mathbb{S}_{\text {agri }}$ & 0.950 & 0.998 \\
$\mathbb{S}_{\text {fabi }}$ & 0.500 & 0.500 & & & \\
\hline
\end{tabular}




\subsubsection{Experimentos de Prelić}

Os experimentos do Cenário 1 de (Prelić et al., 2006) envolvem bases de dados com 10 bi-grupos de 10 linhas e 5 colunas cada, posicionados na diagonal da matriz de dados. A Figura 6.7 ilustra uma dessas bases 3 , nas quais foram aplicados os mesmos algoritmos de biagrupamento usados em (Prelić et al., 2006). O algoritmo bimax (Prelić et al., 2006) encontrou apenas um bi-grupo, o qual é equivalente a um dos bi-grupos de referência. De maneira similar aos resultados dos algoritmos xmotifs e bcca da Seção 6.4.2, a Tabela 6.11 mostra que várias medidas avaliaram a solução do algoritmo bimax como sendo perfeita.

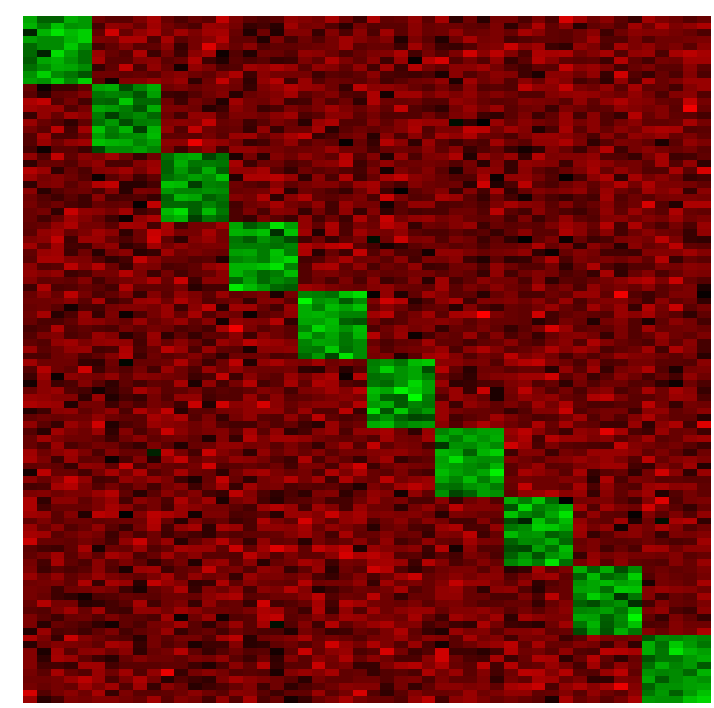

Figura 6.7: Uma base de dados do Cenário 1 de (Prelić et al., 2006).

Tabela 6.11: Avaliação dos bi-agrupamentos da Figura 6.7

\begin{tabular}{lclc}
\hline Medida & Bimax & Medida & Bimax \\
\hline $\mathbb{S}_{\text {prel }}$ & 1.000 & $\mathbb{S}_{\mathrm{u}}$ & 0.100 \\
$\mathbb{S}_{\text {prec }}$ & 0.100 & $\mathbb{S}_{\mathrm{e}}$ & 1.000 \\
$\mathbb{S}_{\text {rnia }}$ & 0.100 & $\mathbb{S}_{\text {ay }}$ & 1.000 \\
$\mathbb{S}_{\mathrm{ce}}$ & 0.100 & $\mathbb{S}_{\text {erel }}$ & 1.000 \\
$\mathbb{S}_{1 \& \mathrm{w}}$ & 1.000 & $\mathbb{S}_{\text {erec }}$ & 0.100 \\
$\mathbb{S}_{\mathrm{stm}}$ & 1.000 & $\mathbb{S}_{\mathrm{csi}}$ & 0.100 \\
$\mathbb{S}_{\text {wjac }}$ & 1.000 & $\mathbb{S}_{\text {ebc }}$ & 0.954 \\
$\mathbb{S}_{\mathrm{wdic}}$ & 1.000 & $\mathbb{S}_{\text {agri }}$ & 0.182 \\
$\mathbb{S}_{\text {fabi }}$ & 0.100 & & \\
\hline
\end{tabular}

A medida $\mathbb{S}_{\mathrm{ebc}}$ atingiu uma valor inesperadamente alto para a solução do algoritmo bimax, o que pode ser explicado da seguinte forma. A precisão dada pela Eq. B.1a é 1, como esperado.

\footnotetext{
${ }^{3}$ Ela se encontra em http://www.tik.ee.ethz.ch/ sop/bimax/SupplementaryMaterial/ Datasets/InSilico/Scenariol/data/em_1,n_0.15.1.txt.h.
} 
A revocação dada pela Eq. (B.1b) é uma média das avaliações dos objetos, o que significa que é uma média sobre as avaliações dos elementos da matriz de dados. Um elemento da matriz de dados que não foi bi-agrupado nas soluções encontrada e de referência recebe o valor 1 de avaliação. Visto que a imensa maioria dos elementos se encaixa nesse caso, a revocação atingiu um valor alto de avaliação (0.91), explicando o valor atribuído por $\mathbb{S}_{\text {ebc }}$. Por outro lado, a medida $\mathbb{S}_{\mathrm{csi}}$ avalia cada par de elementos e os consolida pelas Eqs. (B.5) e (B.6). Os pares de elementos ruidosos (elementos avermelhados da Figura 6.7), os quais são, por uma alta margem de diferença, o tipo de par mais abundante nesse problema e para os quais as soluções encontrada e de referência concordam, não contribuem para a avaliação de $\mathbb{S}_{\text {csi }}$ porque as funções de concordância e discordância (Eqs. (B.3) e (B.4) ) atribuem 0 a eles. Os pares remanescentes consistem em pares de elementos esverdeados e avermelhados, para os quais as soluções encontrada e de referência geralmente discordam. Precisamente, os termos de concordância e discordância (Eqs. (B.5) e (B.6) ) atingiram os valores 1225 e 11025, respectivamente.

\subsubsection{Experimentos de Eren}

Gerou-se uma matriz de dados de 50 linhas e 20 colunas, com três bi-grupos que seguem o modelo de deslocamento usado em (Eren et al., 2012). A Figura 6.8 retrata a matriz de dados. Os bi-grupos de referência são

$$
\begin{aligned}
& \dot{B}_{1} \triangleq(\{1,2, \ldots, 25\},\{1,2, \ldots, 10\}), \\
& \dot{B}_{2} \triangleq(\{1,2, \ldots, 25\},\{11,12, \ldots, 20\}) \mathrm{e} \\
& \dot{B}_{3} \triangleq(\{26,27, \ldots, 50\},\{1,2, \ldots, 10\}) .
\end{aligned}
$$

Foram aplicados os mesmos algoritmos usados em (Eren et al., 2012). O algoritmo bbc (Gu e Liu. 2008) encontrou apenas $\dot{B}_{2}$ e $\dot{B}_{3}$, mas a Tabela 6.12 mostra que várias medidas avaliaram a solução do algoritmo bbc como perfeita, o que pode ser novamente explicado pela ausência da propriedade de cobertura dada pela Def. 2 .

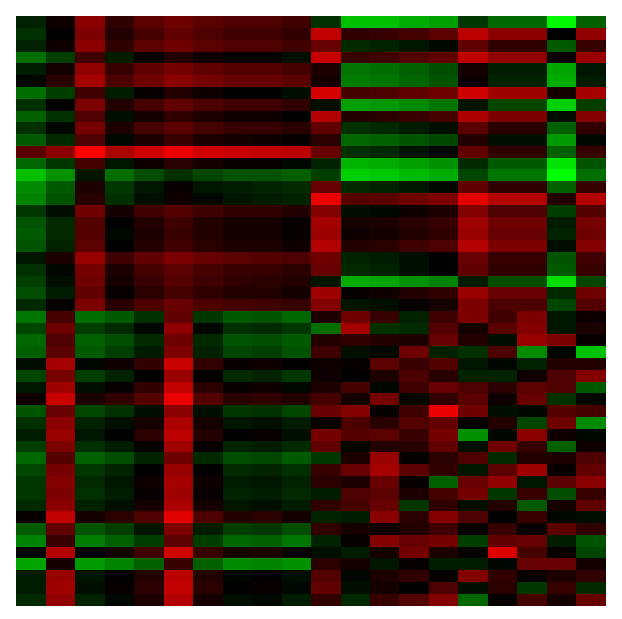

Figura 6.8: Uma base de dados com bi-grupos do modelo de deslocamento (Eren et al., 2012). 
Tabela 6.12: Avaliação dos bi-agrupamentos da Figura 6.8 .

\begin{tabular}{lclc}
\hline Medida & Bbc & Medida & Bbc \\
\hline $\mathbb{S}_{\text {prel }}$ & 1.000 & $\mathbb{S}_{\mathrm{u}}$ & 0.667 \\
$\mathbb{S}_{\text {prec }}$ & 1.000 & $\mathbb{S}_{\mathrm{e}}$ & 1.000 \\
$\mathbb{S}_{\text {rnia }}$ & 0.667 & $\mathbb{S}_{\text {ay }}$ & 1.000 \\
$\mathbb{S}_{\text {ce }}$ & 0.667 & $\mathbb{S}_{\text {erel }}$ & 1.000 \\
$\mathbb{S}_{1 \& w}$ & 1.000 & $\mathbb{S}_{\text {erec }}$ & 0.667 \\
$\mathbb{S}_{\text {stm }}$ & 1.000 & $\mathbb{S}_{\text {csi }}$ & 0.667 \\
$\mathbb{S}_{\text {wjac }}$ & 1.000 & $\mathbb{S}_{\text {ebc }}$ & 0.858 \\
$\mathbb{S}_{\text {wdic }}$ & 1.000 & $\mathbb{S}_{\text {agri }}$ & 0.765 \\
$\mathbb{S}_{\text {fabi }}$ & 0.667 & & \\
\hline
\end{tabular}

As medidas $\mathbb{S}_{\text {rnia }}, \mathbb{S}_{\mathrm{ce}}$ e $\mathbb{S}_{\mathrm{csi}}$ foram as únicas que não apresentaram avaliações claramente contra-intuitivas na análise experimental. A medida $\mathbb{S}_{\text {fabi }}$ falhou no Experimento 3 por não discriminar uma solução claramente superior a outra e a medida $\mathbb{S}_{\text {agri }}$ falhou por avaliar como superior uma solução claramente inferior. A medida $\mathbb{S}_{\text {ebc }}$ atingiu um alto valor para uma solução claramente ruim na Seção 6.4.4, expondo uma falha conceitual da aplicação de $\mathbb{S}_{\text {ebc }}$ na representação de bi-agrupamento por meio de agrupamento particional não exclusivo. A maior parte do comportamento exibido pelas medidas foi explicada com a ajuda das propriedades definidas neste capítulo.

\subsection{Provas teóricas}

Daqui em diante será assumido que $P, \dot{P}$ e $\hat{P}$ são representações baseadas em agrupamento particional não exclusivo de $B, \dot{B}$ e $\hat{B}$, respectivamente, conforme a abordagem de representação de bi-agrupamento proposta na Seção 6.2. Além disso, U, Uं e Û são as representações matriciais de $P, \dot{P}$ e $\hat{P}$, respectivamente.

Proposição 6.1. Existem bi-agrupamentos $B$ e $\dot{B}$ não equivalentes tais que $P \equiv \dot{P}$.

Prova. Seja $B$ um bi-agrupamento tal que alguns elementos da matriz de dados não sejam bi-agrupados e seja $\dot{B}$ o bi-agrupamento $B$ com um novo bi-grupo possuindo apenas um dos elementos que não estão bi-agrupados em $B$. Esse novo bi-grupo é transformado em um grupo unitário de $\dot{P}$ pela Eq. (6.2), enquanto a Eq. (6.3) define um grupo unitário equivalente para $P$. Em outras palavras, o bi-grupo adicionado em $B$ para produzir $\dot{B}$ é supérfluo do ponto de vista da transformação de bi-agrupamento em agrupamento particional não exclusivo.

Proposição 6.2. Se $B$ e $\dot{B}$ são bi-agrupamentos não equivalentes e não degenerados, então $P \not \equiv \dot{P}$. 
Prova. Sejam $k$ e $q$ os números de bi-grupos em $B$ e $\dot{B}$, respectivamente. Suponha que $P \triangleq$ $\left\{P_{i}\right\}_{i=1}^{\bar{k}} \equiv \dot{P} \triangleq\left\{\dot{P}_{i}\right\}_{i=1}^{\bar{q}}$. Assim, $\bar{k}=\bar{q}$ e existe uma bijeção $\left\{\left(t_{i}, y_{i}\right)\right\}_{i=1}^{\bar{k}}$ tal que $P_{t_{i}} \equiv$ $\dot{P}_{y_{i}}$ para todo $i$. Sem perda de generalidade, suponha que $P_{t_{1}}, P_{t_{2}}, \ldots, P_{t_{\underline{k}}}$ (respectivamente, $\left.\dot{P}_{y_{1}}, \dot{P}_{y_{2}}, \ldots, \dot{P}_{y_{q}}\right)$ são grupos não unitários. Claramente, $\underline{k}=\underline{q}=k=q$. A bijeção $\left\{\left(t_{i}, y_{i}\right)\right\}_{i=1}^{\underline{k}}$ implica que existe uma bijeção correspondente entre $B$ e $\dot{B}$ tornando $B \equiv \dot{B}$, o que contradiz a suposição de que $B \not \equiv \dot{B}$.

Adota-se a seguinte notação em várias provas adiante. Sejam $C_{1}$ e $C_{2}$ dois subconjuntos de objetos de $O \triangleq\left\{\tilde{o}_{j}\right\}_{j=1}^{n}$ e seja $f(\cdot, \cdot)$ uma função sobre $O \times O$. Nas provas sobre alguma propriedade da medida $\mathbb{S}_{\mathrm{csi}}, f\left(C_{1}, C_{2}\right)=x$ significa $f\left(\tilde{o}_{j_{1}}, \tilde{o}_{j_{2}}\right)=x$ para todo $\tilde{o}_{j_{1}} \in C_{1}$ e $\tilde{o}_{j_{2}} \in C_{2}$ tal que $j_{1} \neq j_{2}$. Nas provas sobre alguma propriedade da medida $\mathbb{S}_{\mathrm{ebc}}, f\left(C_{1}, C_{2}\right)=x$ significa $f\left(\tilde{o}_{j_{1}}, \tilde{o}_{j_{2}}\right)=x$ para todo $\tilde{o}_{j_{1}} \in C_{1}$ e $\tilde{o}_{j_{2}} \in C_{2}$. Em ambos os casos, para uma dada função $f(\cdot), f\left(C_{1}\right)=x$ significa $f\left(\tilde{o}_{j}\right)=x$ para todo $\tilde{o}_{j} \in C_{1}$.

Proposição 6.3. A medida $\mathbb{S}_{\mathrm{csi}}$ é sensível ao tamanho de bi-grupos espúrios (Def. 1).

Prova. Sejam $B, \hat{B}$ e $\dot{B}$ bi-agrupamentos, como dado na Def. 1 e lembre-se que $O$ é o conjunto de objetos. Seja $\left\{P_{t_{i}}\right\}_{i=1}^{x}$ o conjunto de grupos do agrupamento particional não exclusivo correspondentes aos bi-grupos espúrios $\left\{B_{t_{i}}\right\}_{i=1}^{x}$ em $B$ e defina, similarmente, $\left\{\hat{P}_{t_{i}}\right\}_{i=1}^{x}$ para $\hat{B}$. Defina $C_{\mathrm{s}}^{1} \triangleq \bigcup_{i=1}^{x} P_{t_{i}}$ e $C_{\mathrm{s}}^{2} \triangleq \bigcup_{i=1}^{x} \hat{P}_{t_{i}}$. Note que $C_{\mathrm{s}}^{1} \subset C_{\mathrm{s}}^{2}$. Sabe-se que

$$
\begin{aligned}
\alpha_{\dot{P}}\left(C_{\mathrm{s}}^{2}, O\right) & =\beta_{\dot{P}}\left(C_{\mathrm{s}}^{2}\right)=0, \\
\alpha_{P}\left(O, O-C_{\mathrm{s}}^{2}\right) & =\alpha_{\hat{P}}\left(O, O-C_{\mathrm{s}}^{2}\right), \\
\beta_{P}\left(O-C_{\mathrm{s}}^{2}\right) & =\beta_{\hat{P}}\left(O-C_{\mathrm{s}}^{2}\right), \\
\alpha_{P}\left(C_{\mathrm{s}}^{2}, C_{\mathrm{s}}^{2}\right) & \leq \alpha_{\hat{P}}\left(C_{\mathrm{s}}^{2}, C_{\mathrm{s}}^{2}\right) \mathrm{e} \\
\beta_{P}\left(C_{\mathrm{s}}^{2}\right) & \leq \beta_{\hat{P}}\left(C_{\mathrm{s}}^{2}\right) .
\end{aligned}
$$

Portanto,

$$
\begin{aligned}
a_{G}^{P, \dot{P}}\left(C_{\mathrm{s}}^{2}, O-C_{\mathrm{s}}^{2}\right) & =\min \left\{\beta_{P}\left(O-C_{\mathrm{s}}^{2}\right), \beta_{\dot{P}}\left(O-C_{\mathrm{s}}^{2}\right)\right\} \\
& =\min \left\{\beta_{\hat{P}}\left(O-C_{\mathrm{s}}^{2}\right), \beta_{\dot{P}}\left(O-C_{\mathrm{s}}^{2}\right)\right\} \\
& =a_{G}^{\hat{P}, \dot{P}}\left(C_{\mathrm{s}}^{2}, O-C_{\mathrm{s}}^{2}\right), \\
a_{G}^{P, \dot{P}}\left(C_{\mathrm{s}}^{2}, C_{\mathrm{s}}^{2}\right) & =0=a_{G}^{\hat{P}, \dot{P}}\left(C_{\mathrm{s}}^{2}, C_{\mathrm{s}}^{2}\right) \mathrm{e} \\
a_{G}^{P, \dot{P}}\left(O-C_{\mathrm{s}}^{2}, O-C_{\mathrm{s}}^{2}\right) & =a_{G}^{\hat{P}, \dot{P}}\left(O-C_{\mathrm{s}}^{2}, O-C_{\mathrm{s}}^{2}\right) .
\end{aligned}
$$


Logo, $a_{G}^{P, \dot{P}}(O, O)=a_{G}^{\hat{P}, \dot{P}}(O, O)$. Observe que

$$
\begin{gathered}
d_{G}^{P, \dot{P}}\left(C_{\mathrm{s}}^{2}, O-C_{\mathrm{s}}^{2}\right)=\alpha_{P}\left(C_{\mathrm{s}}^{2}, O-C_{\mathrm{s}}^{2}\right)+\beta_{P}\left(C_{\mathrm{s}}^{2}\right)+\left|\beta_{P}\left(O-C_{\mathrm{s}}^{2}\right)-\beta_{\dot{P}}\left(O-C_{\mathrm{s}}^{2}\right)\right| \\
=\alpha_{\hat{P}}\left(C_{\mathrm{s}}^{2}, O-C_{\mathrm{s}}^{2}\right)+\beta_{P}\left(C_{\mathrm{s}}^{2}\right)+\left|\beta_{\hat{P}}\left(O-C_{\mathrm{s}}^{2}\right)-\beta_{\dot{P}}\left(O-C_{\mathrm{s}}^{2}\right)\right| \\
\leq \alpha_{\hat{P}}\left(C_{\mathrm{s}}^{2}, O-C_{\mathrm{s}}^{2}\right)+\beta_{\hat{P}}\left(C_{\mathrm{s}}^{2}\right)+\left|\beta_{\hat{P}}\left(O-C_{\mathrm{s}}^{2}\right)-\beta_{\dot{P}}\left(O-C_{\mathrm{s}}^{2}\right)\right| \\
=d_{G}^{\hat{P}, \dot{P}}\left(C_{\mathrm{s}}^{2}, O-C_{\mathrm{s}}^{2}\right), \\
d_{G}^{P, \dot{P}}\left(C_{\mathrm{s}}^{2}, C_{\mathrm{s}}^{2}\right) \\
=\alpha_{P}\left(C_{\mathrm{s}}^{2}, C_{\mathrm{s}}^{2}\right)+\beta_{P}\left(C_{\mathrm{s}}^{2}\right)+\beta_{P}\left(C_{\mathrm{s}}^{2}\right) \\
\leq \alpha_{\hat{P}}\left(C_{\mathrm{s}}^{2}, C_{\mathrm{s}}^{2}\right)+\beta_{\hat{P}}\left(C_{\mathrm{s}}^{2}\right)+\beta_{\hat{P}}\left(C_{\mathrm{s}}^{2}\right) \\
=d_{G}^{\hat{P}, \dot{P}}\left(C_{\mathrm{s}}^{2}, C_{\mathrm{s}}^{2}\right)
\end{gathered}
$$

$\mathrm{e}$

$$
d_{G}^{P, \dot{P}}\left(O-C_{\mathrm{s}}^{2}, O-C_{\mathrm{s}}^{2}\right)=d_{G}^{\hat{P}, \dot{P}}\left(O-C_{\mathrm{s}}^{2}, O-C_{\mathrm{s}}^{2}\right)
$$

Logo, $d_{G}^{P, \dot{P}}(O, O) \leq d_{G}^{\hat{P}, \dot{P}}(O, O)$.

Seja $P_{t}$ o grupo do agrupamento particional não exclusivo correspondente ao bi-grupo espúrio $B_{t}$ que foi incrementado, dando origem aos bi-agrupamentos $\hat{B}_{t}$ e $\hat{P}_{t}$. Sejam $\tilde{o}_{j_{1}}$ e $\tilde{o}_{j_{2}}$ dois objetos de $\hat{P}_{t}$ tais que $\tilde{o}_{j_{1}} \in P_{t}$ e $\tilde{o}_{j_{2}} \notin P_{t}$. Logo, $\alpha_{\hat{P}}\left(\tilde{o}_{j_{1}}, \tilde{o}_{j_{2}}\right)>\alpha_{P}\left(\tilde{o}_{j_{1}}, \tilde{o}_{j_{2}}\right)$, $d_{G}^{P, \dot{P}}\left(\tilde{o}_{j_{1}}, \tilde{o}_{j_{2}}\right)<d_{G}^{\hat{P}, \dot{P}}\left(\tilde{o}_{j_{1}}, \tilde{o}_{j_{2}}\right)($ Eq. 6.4 $)$ e $10 \operatorname{CSI}(P, \dot{P})>10 \operatorname{CSI}(\hat{P}, \dot{P})$.

Proposição 6.4. A medida $\mathbb{S}_{\mathrm{ebc}}$ é sensível ao tamanho de bi-grupos espúrios (Def. 1).

Prova. Sejam $B, \hat{B}$ e $\dot{B}$ bi-agrupamentos, como na Def. 1 e lembre-se que $O$ é o conjunto de objetos. Sejam $C_{\mathrm{s}}^{1}$ e $C_{\mathrm{s}}^{2}$ os conjuntos definidos na prova da Proposição 6.3 . Note que

$$
\begin{aligned}
\alpha_{P}\left(C_{s}^{2}, C_{s}^{2}\right) & \geq \alpha_{\dot{P}}\left(C_{s}^{2}, C_{s}^{2}\right), \\
\alpha_{\hat{P}}\left(C_{s}^{2}, C_{s}^{2}\right) & \geq \alpha_{\dot{P}}\left(C_{s}^{2}, C_{s}^{2}\right), \mathrm{e} \\
\alpha_{P}\left(O, O-C_{s}^{2}\right) & =\alpha_{\hat{P}}\left(O, O-C_{s}^{2}\right) .
\end{aligned}
$$

Os nominadores das Eqs. (B.1) são iguais quando se compara $P$ com $\dot{P}$ ou $\hat{P}$ com $\dot{P}$. Assim, $\operatorname{EBCR}(P, \dot{P})=\operatorname{EBCR}(\hat{P}, \dot{P}) \cdot \operatorname{EBCP}(P, \dot{P}) \geq \operatorname{EBCP}(\hat{P}, \dot{P})$ porque $\alpha_{\hat{P}}(O, O) \geq \alpha_{P}(O, O)$.

Seja $P_{t}$ o grupo do agrupamento particional não exclusivo correspondente a um bi-grupo espúrio $B_{t}$ que foi incrementado, dando origem a $\hat{B}_{t}$ e $\hat{P}_{t}$. Sejam $\tilde{o}_{j_{1}}$ e $\tilde{o}_{j_{2}}$ dois objetos de $\hat{P}_{t}$ tais que $\tilde{o}_{j_{1}} \in P_{t}$ e $\tilde{o}_{j_{2}} \notin P_{t}$. Assim, $\alpha_{\hat{P}}\left(\tilde{o}_{j_{1}}, \tilde{o}_{j_{2}}\right)>\alpha_{P}\left(\tilde{o}_{j_{1}}, \tilde{o}_{j_{2}}\right), \operatorname{EBCP}(P, \dot{P})>\operatorname{EBCP}(\hat{P}, \dot{P})$ e $09 \mathrm{EBC}(P, \dot{P})>09 \mathrm{EBC}(\hat{P}, \dot{P})$.

Proposição 6.5. A medida $\mathbb{S}_{\text {agri }}$ não é sensível ao tamanho de bi-grupos espúrios (Def. 1).

Prova. Considere a matriz de dados $\mathrm{X} \in \mathbb{R}^{2 \cdot 3}$. Sejam $B \triangleq\left\{B_{1}, B_{2}\right\}, B_{1} \triangleq(\{2\},\{1,2\}), B_{2} \triangleq$ $(\{2\},\{3,4\}), \dot{B} \triangleq\left\{\dot{B}_{1}, \dot{B}_{2}\right\}, \dot{B}_{1} \triangleq(\{1\},\{1,2,3,4\}), \dot{B}_{2} \triangleq(\{1\},\{1,2,3,4\}), \hat{B} \triangleq\left\{\hat{B}_{1}, \hat{B}_{2}\right\}$, 
$\hat{B}_{1} \triangleq(\{2\},\{1,2\})$ e $\hat{B}_{2} \triangleq(\{2\},\{2,3,4\})$. Tem-se $\mathbb{S}_{\text {agri }}(\mathrm{U}, \dot{\mathrm{U}})=-0.0120$ e $\mathbb{S}_{\text {agri }}(\hat{\mathrm{U}}, \dot{\mathrm{U}})=$ 0.000 .

Proposição 6.6. As medidas $\mathbb{S}_{\text {rnia }} e \mathbb{S}_{\text {ce }}$ penalizam soluções que não abrangem todos os bigrupos de referência (Def. 2).

Prova. Sejam $B$ e $\dot{B}$ como dados pela Def. 2. Tem-se $\dot{\mathrm{N}}_{j_{1}, j_{2}} \geq \mathrm{N}_{j_{1}, j_{2}}$ para todo $j_{1}$ e $j_{2}$ e existem $j_{1}$ e $j_{2}$ tais que $\dot{\mathrm{N}}_{j_{1}, j_{2}}>\mathrm{N}_{j_{1}, j_{2}}$ (Eqs. (4.13) e (4.14)). Logo, $|U|>|I|$ e $\mathbb{S}_{\text {rnia }}$ apresenta a propriedade estabelecida pela Def. 2. Tem-se também $\mathbb{S}_{\text {rnia }}(B, \dot{B}) \geq \mathbb{S}_{\mathrm{ce}}(B, \dot{B})$ pela Proposição 1 de (Patrikainen e Meila, 2006). Assim, $\mathbb{S}_{\mathrm{ce}}$ também possui a propriedade.

Proposição 6.7. As medidas $\mathbb{S}_{\text {prec }}, \mathbb{S}_{\mathrm{u}}$ e $\mathbb{S}_{\mathrm{erec}}$ nem sempre penalizam soluções que não abrangem todos os bi-grupos de referência (Def. 2).

Prova. Sejam $B$ e $\dot{B}$ como dados pela Def. 2, em que $B \triangleq\left\{B_{1}\right\}$ e $\dot{B} \triangleq\left\{\dot{B}_{1}, \dot{B}_{2}\right\}$ tais que $B_{1} \equiv \dot{B}_{1} \equiv \dot{B}_{2}$. Teria-se $\mathbb{S}_{\text {prec }}(B, \dot{B})=\mathbb{S}_{\mathrm{u}}(B, \dot{B})=\mathbb{S}_{\text {erec }}(B, \dot{B})=1$, violando a condição dada pela Def. 2 .

Proposição 6.8. A medida $\mathbb{S}_{\mathrm{csi}}$ penaliza soluções que não abrangem todos os bi-grupos de referência (Def. 2) no domínio dos bi-agrupamentos não degenerados.

Prova. Sejam $B$ e $\dot{B}$ como dados pela Def. 2, com a restrição adicional de não serem degenerados. Tem-se $\alpha_{P}\left(\tilde{o}_{j_{1}}, \tilde{o}_{j_{2}}\right) \leq \alpha_{\dot{P}}\left(\tilde{o}_{j_{1}}, \tilde{o}_{j_{2}}\right)$ para todo $j_{1} \neq j_{2}$ e a desigualdade é atingida para pelo menos um par $\left(j_{1}, j_{2}\right)$. Logo, $d_{G}^{P, \dot{P}}>0,10 \operatorname{CSI}(P, \dot{P})<1$ e $\mathbb{S}_{\mathrm{csi}}(B, \dot{B})<1$.

Proposição 6.9. A medida $\mathbb{S}_{\mathrm{ebc}}$ penaliza soluções que não abrangem todos os bi-grupos de referência (Def. 2) no domínio dos bi-agrupamentos não degenerados.

Prova. Sejam $B$ e $\dot{B}$ bi-agrupamentos não degenerados como dados pela Def. 2, com a restrição adicional de não serem degenerados. Tem-se $\alpha_{P}\left(\tilde{o}_{j_{1}}, \tilde{o}_{j_{2}}\right) \leq \alpha_{\dot{P}}\left(\tilde{o}_{j_{1}}, \tilde{o}_{j_{2}}\right)$ para todos os $j_{1}$ e $j_{2}$. A desigualdade é atingida para pelo menos um par $\left(j_{1}, j_{2}\right)$, implicando que $\operatorname{EBCR}(P, \dot{P})<1$, $09 \mathrm{EBC}(P, \dot{P})<1$ e $\mathbb{S}_{\mathrm{ebc}}(B, \dot{B})<1$.

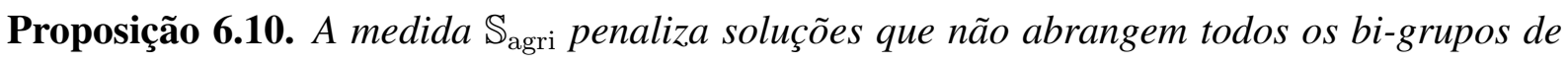
referência (Def. 2) no domínio dos bi-agrupamentos não degenerados.

Prova. Sejam $B$ e $\dot{B}$ como dados pela Def. 2, com a restrição adicional de não serem degenerados. Note que U consiste em U̇ após a remoção de uma ou mais linhas. Logo, tem-se $\mathrm{J}_{j_{1}, j_{2}}^{\mathrm{U}} \leq \mathrm{J}_{j_{1}, j_{2}}^{\dot{U}}$ e $\mathrm{S}_{j_{1}, j_{2}}^{\mathrm{U}} \leq \mathrm{S}_{j_{1}, j_{2}}^{\dot{U}}$ para todos os $j_{1}$ e $j_{2}$. Em particular, existem $j_{1}$ e $j_{2}$ tais que $\mathrm{J}_{j_{1}, j_{2}}^{\mathrm{U}}<\mathrm{J}_{j_{1}, j_{2}}^{\dot{U}}$. Isso implica em $\dot{e}>0$ (Eq. (5.10), 13GRI(U, $\left.\dot{\mathrm{U}}\right)<1$ (Eq. (5.11)), 13AGRI $(\mathrm{U}, \dot{\mathrm{U}})<1$ (Corolário 5.7) e $\mathbb{S}_{\text {agri }}(B, \dot{B})<1$.

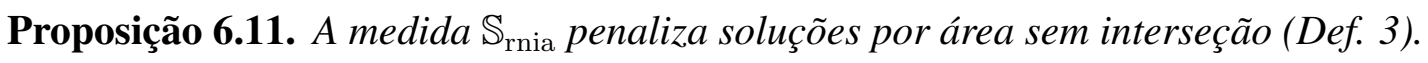

Prova. Sejam $B, \dot{B}$ e $\hat{B}$ três bi-agrupamentos, como na Def. 3 e seja $S$ o conjunto de elementos da matriz de dados, como na Def. 3. Os elementos de $S$ são aqueles correspondentes aos 
índices $j_{1}$ e $j_{2}$ tais que $\dot{\mathrm{N}}_{j_{1}, j_{2}}=0$. Tem-se $\mathrm{N}_{j_{1}, j_{2}}=\hat{\mathrm{N}}_{j_{1}, j_{2}}$ para todos os índices $j_{1}$ e $j_{2}$ tais que $\dot{\mathrm{N}}_{j_{1}, j_{2}}>0$. Assim, $\min \left\{\mathrm{N}_{j_{1}, j_{2}}, \dot{\mathrm{N}}_{j_{1}, j_{2}}\right\}=\min \left\{\hat{\mathrm{N}}_{j_{1}, j_{2}}, \dot{\mathrm{N}}_{j_{1}, j_{2}}\right\}$ para todos os índices $j_{1} \mathrm{e}$ $j_{2}$. Uma vez que $\mathrm{N}_{j_{1}, j_{2}} \leq \hat{\mathrm{N}}_{j_{1}, j_{2}}$ para todos os índices $j_{1}$ e $j_{2}$, tem-se $\max \left\{\mathrm{N}_{j_{1}, j_{2}}, \dot{\mathrm{N}}_{j_{1}, j_{2}}\right\} \leq$ $\max \left\{\hat{\mathrm{N}}_{j_{1}, j_{2}}, \dot{\mathrm{N}}_{j_{1}, j_{2}}\right\}$ para todos os índices $j_{1}$ e $j_{2}$. Portanto, $\mathbb{S}_{\text {rnia }}(B, \dot{B}) \geq \mathbb{S}_{\text {rnia }}(\hat{B}, \dot{B})$. Uma vez que $\mathrm{N}_{j_{1}, j_{2}}<\hat{\mathrm{N}}_{j_{1}, j_{2}}$ para pelo menos um par $\left(j_{1}, j_{2}\right)$ tal que $\dot{\mathrm{N}}_{j_{1}, j_{2}}=0$, tem-se $\max \left\{\mathrm{N}_{j_{1}, j_{2}}, \dot{\mathrm{N}}_{j_{1}, j_{2}}\right\}<\max \left\{\hat{\mathrm{N}}_{j_{1}, j_{2}}, \dot{\mathrm{N}}_{j_{1}, j_{2}}\right\}$ para esse par e $\mathbb{S}_{\text {rnia }}(B, \dot{B})>\mathbb{S}_{\text {rnia }}(\hat{B}, \dot{B})$.

Proposição 6.12. A medida $\mathbb{S}_{\mathrm{ce}}$ penaliza soluções por área sem interseção (Def. 3).

Prova. Sejam $B, \dot{B}$ e $\hat{B}$ três bi-agrupamentos, como na Def. 3 . Sabe-se pela prova da Proposição 6.11 que $|U|$ aumenta ao passar da comparação de $B$ com $\dot{B}$ para a comparação de $\hat{B}$ com $\dot{B}$. Por outro lado, $d_{\max }$ (Eq. (4.17) não muda ao passar da comparação de $B$ com $\dot{B}$ para a comparação de $\hat{B}$ com $\dot{B}$. Logo, $\mathbb{S}_{\mathrm{ce}}(B, \dot{B})>\mathbb{S}_{\mathrm{ce}}(\hat{B}, \dot{B})$.

Proposição 6.13. As medidas $\mathbb{S}_{\mathrm{wjac}}$ e $\mathbb{S}_{\mathrm{wdic}}$ nem sempre penalizam soluções por área sem interseção (Def. 3).

Prova. Considere a matriz de dados $\mathrm{X} \in \mathbb{R}^{4 \cdot 4}$. Sejam $B \triangleq\left\{B_{1}, B_{2}\right\}, B_{1} \triangleq(\{2,3,4\},\{1,2\})$, $B_{2} \triangleq(\{2,3,4\},\{3\}), \dot{B} \triangleq\left\{\dot{B}_{1}\right\}, \dot{B}_{1} \triangleq(\{2,3,4\},\{3,4\}), \hat{B} \triangleq\left\{\hat{B}_{1}, \hat{B}_{2}\right\}, \hat{B}_{1} \triangleq$ $(\{2,3,4\},\{1,2\})$ e $\hat{B}_{2} \triangleq(\{1,2,3,4\},\{3\})$. Note que $B, \dot{B}$ e $\hat{B}$ seguem as definições de bi-agrupamentos dadas pela Def. 3 . Entretanto, $\mathbb{S}_{\mathrm{wjac}}(B, \dot{B})=0.167<0.171=\mathbb{S}_{\mathrm{wjac}}(\hat{B}, \dot{B}) \mathrm{e}$ $\mathbb{S}_{\mathrm{wdic}}(B, \dot{B})=0.22<0.24=\mathbb{S}_{\mathrm{wdic}}(\hat{B}, \dot{B})$.

Proposição 6.14. Sejam $B, \dot{B}$ e $\hat{B}$ três bi-agrupamentos, como na Def. 3. Tem-se $\mathbb{S}_{\mathrm{csi}}(B, \dot{B}) \geq$ $\mathbb{S}_{\mathrm{csi}}(\hat{B}, \dot{B})$.

Prova. Seja $S$ o conjunto de elementos da matriz de dados, como na Def. 3. Defina $C$ como o conjunto de elementos de $O$ correspondentes aos elementos de $S$ e seja $\bar{C} \triangleq O-C$. Tem-se

$$
\begin{aligned}
\alpha_{P}(\bar{C}, \bar{C}) & =\alpha_{\hat{P}}(\bar{C}, \bar{C}), \\
\alpha_{P}(C, \bar{C}) & \leq \alpha_{\hat{P}}(C, \bar{C}), \\
\alpha_{P}(C, C) & \leq \alpha_{\hat{P}}(C, C), \\
\alpha_{\dot{P}}(C, \bar{C}) & =\alpha_{\dot{P}}(C, C)=0, \\
\beta_{P}(\bar{C}) & =\beta_{\hat{P}}(\bar{C}), \\
\beta_{P}(C) & \leq \beta_{\hat{P}}(C) \mathrm{e} \\
\beta_{\dot{P}}(C) & =0 .
\end{aligned}
$$

Assim, $a_{G}^{P, \dot{P}}(\bar{C}, \bar{C})=a_{G}^{\hat{P}, \dot{P}}(\bar{C}, \bar{C})$ e $d_{G}^{P, \dot{P}}(\bar{C}, \bar{C})=d_{G}^{\hat{P}, \dot{P}}(\bar{C}, \bar{C})$. Tem-se

$$
\begin{aligned}
a_{G}^{P, \dot{P}}(C, \bar{C}) & =\min \left\{\beta_{P}(\bar{C}), \beta_{\dot{P}}(\bar{C})\right\} \\
& =\min \left\{\beta_{\hat{P}}(\bar{C}), \beta_{\dot{P}}(\bar{C})\right\} \\
& =a_{G}^{\hat{P}, \dot{P}}(C, \bar{C})
\end{aligned}
$$


$\mathrm{e}$

$$
\begin{aligned}
d_{G}^{P, \dot{P}}(C, \bar{C}) & =\alpha_{P}(C, \bar{C})+\beta_{P}(C)+\left|\beta_{P}(\bar{C})-\beta_{\dot{P}}(\bar{C})\right| \\
& =\alpha_{P}(C, \bar{C})+\beta_{P}(C)+\left|\beta_{\hat{P}}(\bar{C})-\beta_{\dot{P}}(\bar{C})\right| \\
& \leq \alpha_{\hat{P}}(C, \bar{C})+\beta_{\hat{P}}(C)+\left|\beta_{\hat{P}}(\bar{C})-\beta_{\dot{P}}(\bar{C})\right| \\
& =d_{G}^{\hat{P}, \dot{P}}(C, \bar{C}) .
\end{aligned}
$$

Note que

$$
a_{G}^{P, \dot{P}}(C, C)=0=a_{G}^{\hat{P}, \dot{P}}(C, C)
$$

e

$$
\begin{aligned}
d_{G}^{P, \dot{P}}(C, C) & =\alpha_{P}(C, C)+2 \beta_{P}(C) \\
& \leq \alpha_{\hat{P}}(C, C)+2 \beta_{\hat{P}}(C) \\
& =d_{G}^{\hat{P}, \dot{P}}(C, C) .
\end{aligned}
$$

$\operatorname{Logo}, a_{G}^{P, \dot{P}}=a_{G}^{\hat{P}, \dot{P}}, d_{G}^{P, \dot{P}} \leq d_{G}^{\hat{P}, \dot{P}}, 10 \mathrm{CSI}(P, \dot{P}) \geq 10 \mathrm{CSI}(\hat{P}, \dot{P})$ e $\mathbb{S}_{\mathrm{csi}}(B, \dot{B}) \geq \mathbb{S}_{\mathrm{csi}}(\hat{B}, \dot{B})$

Proposição 6.15. A medida $\mathbb{S}_{\mathrm{csi}}$ penaliza soluções por área sem interseção (Def. 3) no domínio dos bi-agrupamentos não degenerados.

Prova. Sejam $B, \dot{B}$ e $\hat{B}$ três bi-agrupamentos, como na Def. 3, com a restrição adicional de não serem degenerados. Seja $S$ o conjunto de elementos da matriz de dados, como na Def. 3 . Defina $C$ como os elementos de $O$ correspondentes aos elementos de $S$. Se existem bi-grupos $\hat{B}$ originados da expansão de bi-grupos de $B$, existe um objeto $\tilde{o}_{j} \in C$ que pertence a mais grupos em $\hat{P}$ do que em $P$, significando que $\beta_{\hat{P}}\left(\tilde{o}_{j}\right)>\beta_{P}\left(\tilde{o}_{j}\right)$. Se existem novos bi-grupos em $\hat{B}$, seja $\hat{B}_{i}$ um desses. Existe um par $\tilde{o}_{j_{1}}, \tilde{o}_{j_{2}} \in C\left(j_{1} \neq j_{2}\right)$ que pertence a mais grupos em $\hat{P}$ do que em $P$, significando que $\alpha_{\hat{P}}\left(\tilde{o}_{j_{1}}, \tilde{o}_{j_{2}}\right)>\alpha_{P}\left(\tilde{o}_{j_{1}}, \tilde{o}_{j_{2}}\right)$. No primeiro caso, $d_{G}^{\hat{P}, \dot{P}}>d_{G}^{P, \dot{P}}$ por causa da Ineq. 6.5). No segundo caso, $d_{G}^{\hat{P}, \dot{P}}>d_{G}^{P, \dot{P}}$ por causa da Ineq. 6.6. Portanto, $10 \mathrm{CSI}(P, \dot{P})>10 \mathrm{CSI}(\hat{P}, \dot{P})$ e $\mathbb{S}_{\mathrm{csi}}(B, \dot{B})>\mathbb{S}_{\mathrm{csi}}(\hat{B}, \dot{B})$.

Proposição 6.16. Sejam $B, \dot{B}$ e $\hat{B}$ três bi-agrupamentos, como na Def. 3 Tem-se $\mathbb{S}_{\mathrm{ebc}}(B, \dot{B}) \geq$ $\mathbb{S}_{\mathrm{ebc}}(\hat{B}, \dot{B})$.

Prova. Seja $S$ o conjunto de elementos da matriz de dados, como na Def. 3 . Defina $C$ como o conjunto de elementos de $O$ correspondentes aos elementos de $S$ e seja $\bar{C} \triangleq O-C$. Tem-se $\alpha_{P}(\bar{C}, \bar{C})=\alpha_{\hat{P}}(\bar{C}, \bar{C}), \alpha_{\dot{P}}(C, \bar{C})=0, \alpha_{\dot{P}}\left(\tilde{o}_{j_{1}}, \tilde{o}_{j_{2}}\right)=0$ para todos os $\tilde{o}_{j_{1}}, \tilde{o}_{j_{2}} \in C$ sujeitos a $j_{1} \neq j_{2}, \alpha_{P}\left(\tilde{o}_{j}, \tilde{o}_{j}\right) \geq \alpha_{\dot{P}}\left(\tilde{o}_{j}, \tilde{o}_{j}\right)$ para todo $\tilde{o}_{j} \in C$ e $\alpha_{\hat{P}}\left(\tilde{o}_{j}, \tilde{o}_{j}\right) \geq \alpha_{\dot{P}}\left(\tilde{o}_{j}, \tilde{o}_{j}\right)$ para todo $\tilde{o}_{j} \in C$. Logo, $\min \left\{\alpha_{P}(O, O), \alpha_{\dot{P}}(O, O)\right\}=\min \left\{\alpha_{\hat{P}}(O, O), \alpha_{\dot{P}}(O, O)\right\}$. Visto que

$$
\begin{aligned}
& \alpha_{\hat{P}}(C, \bar{C}) \geq \alpha_{P}(C, \bar{C}) \mathrm{e} \\
& \alpha_{\hat{P}}(C, C) \geq \alpha_{P}(C, C),
\end{aligned}
$$


tem-se 09EBC $(P, \dot{P}) \geq 09 \mathrm{EBC}(\hat{P}, \dot{P})$ e $\mathbb{S}_{\mathrm{ebc}}(B, \dot{B}) \geq \mathbb{S}_{\mathrm{ebc}}(\hat{B}, \dot{B})$.

Proposição 6.17. A medida $\mathbb{S}_{\mathrm{ebc}}$ penaliza soluções por área sem interseção (Def. 3) no domínio dos bi-agrupamentos sem degeneração.

Prova. Sejam $B, \dot{B}$ e $\hat{B}$ três bi-agrupamentos, como na Def. 3 , com a restrição adicional de serem não degenerados. Seja $S$ o conjunto de elementos da matriz de dados, como na Def. 3. Defina $C$ como o conjunto de elementos de $O$ correspondentes aos elementos de $S$ e seja $\bar{C} \triangleq O-C$. Se existem bi-grupos em $\hat{B}$ originados da expansão de bi-grupos de $B$, existe um par $\tilde{o}_{j_{1}}, \tilde{o}_{j_{2}} \in C$ sujeito a $j_{1} \neq j_{2}$ ou um par $\tilde{o}_{j_{1}} \in C, \tilde{o}_{j_{2}} \in \bar{C}$ que pertence a mais grupos em $\hat{P}$ do que em $P$, significando que $\alpha_{\hat{P}}\left(\tilde{o}_{j_{1}}, \tilde{o}_{j_{2}}\right)>\alpha_{P}\left(\tilde{o}_{j_{1}}, \tilde{o}_{j_{2}}\right)$. Se existem novos bi-grupos em $\hat{B}$, seja $\hat{B}_{i}$ um desses. Existe um par $\tilde{o}_{j_{1}}, \tilde{o}_{j_{2}} \in C$ sujeito a $j_{1} \neq j_{2}$ que pertence a mais grupos em $\hat{P}$ do que em $P$, significando que $\alpha_{\hat{P}}\left(\tilde{o}_{j_{1}}, \tilde{o}_{j_{2}}\right)>\alpha_{P}\left(\tilde{o}_{j_{1}}, \tilde{o}_{j_{2}}\right)$. Em ambos os casos, pode-se concluir das Ineqs. (6.7) e (6.8) que $09 \operatorname{EBC}(P, \dot{P})>09 \operatorname{EBC}(\hat{P}, \dot{P})$ e $\mathbb{S}_{\mathrm{ebc}}(B, \dot{B})>\mathbb{S}_{\mathrm{ebc}}(\hat{B}, \dot{B})$.

Proposição 6.18. A medida $\mathbb{S}_{\mathrm{csi}}$ penaliza soluções por cobertura múltipla de bi-grupos (Def. 4).

Prova. Sejam $B$ e $\dot{B}$ dois bi-agrupamentos, como na Def. 4. Tem-se $\alpha_{P}(O, O) \geq \alpha_{\dot{P}}(O, O)$ e $\beta_{P}(O)=\beta_{\dot{P}}(O)=0$. Existem $\tilde{o}_{j_{1}}$ e $\tilde{o}_{j_{2}}$ sujeitos a $j_{1} \neq j_{2}$ tais que $\alpha_{P}\left(\tilde{o}_{j_{1}}, \tilde{o}_{j_{2}}\right)>\alpha_{\dot{P}}\left(\tilde{o}_{j_{1}}, \tilde{o}_{j_{2}}\right)$, implicando que $d_{G}^{P, \dot{P}}>0,10 \mathrm{CSI}(P, \dot{P})<1$ e $\mathbb{S}_{\mathrm{csi}}(B, \dot{B})<1$.

Proposição 6.19. A medida $\mathbb{S}_{\mathrm{ebc}}$ penaliza soluções por cobertura múltipla de bi-grupos (Def. 4).

Prova. Sejam $B$ e $\dot{B}$ dois bi-agrupamentos, como na Def. 4. Note que $\alpha_{P}(O, O) \geq \alpha_{\dot{P}}(O, O)$. Existem $\tilde{o}_{j_{1}}$ e $\tilde{o}_{j_{2}}$ sujeitos a $j_{1} \neq j_{2}$ tais que $\alpha_{P}\left(\tilde{o}_{j_{1}}, \tilde{o}_{j_{2}}\right)>\alpha_{\dot{P}}\left(\tilde{o}_{j_{1}}, \tilde{o}_{j_{2}}\right)$, implicando que $\operatorname{EBCP}(P, \dot{P})<1,09 \mathrm{EBC}(P, \dot{P})<1$ e $\mathbb{S}_{\mathrm{ebc}}(B, \dot{B})<1$.

Proposição 6.20. As medidas $\mathbb{S}_{\text {prel }}, \mathbb{S}_{\text {prec }} e \mathbb{S}_{1 \& w}$ penalizam soluções por cobertura múltipla de bi-grupos (Def. 4).

Prova. Sejam $B$ e $\dot{B}$ dois bi-agrupamentos, como na Def. 4. Visto que os bi-grupos de $\dot{B}$ não se sobrepõem, tem-se os relacionamentos de subconjunto próprio $\dot{B}_{i}^{\mathrm{r}} \subset B_{1}^{\mathrm{r}}$ para todo $i$ ou $\dot{B}_{i}^{\mathrm{c}} \subset B_{1}^{\mathrm{c}}$ para todo $i$. Logo, $\left|B_{1}^{\mathrm{r}} \cup \dot{B}_{i}^{\mathrm{r}}\right|>\left|B_{1}^{\mathrm{r}} \cap \dot{B}_{i}^{\mathrm{r}}\right|$ para todo $i$ ou $\left|B_{1}^{\mathrm{c}} \cup \dot{B}_{i}^{\mathrm{c}}\right|>\left|B_{1}^{\mathrm{c}} \cap \dot{B}_{i}^{\mathrm{c}}\right|$ para todo $i$. Tem-se $S_{\mathrm{r}}(B, \dot{B})<1$ ou $S_{\mathrm{c}}(B, \dot{B})<1$ (Eqs. (4.9) e (4.10), implicando que $\mathbb{S}_{\text {prel }}(B, \dot{B})<1$. Similarmente, $\mathbb{S}_{\text {prec }}(B, \dot{B})<1$ e $\mathbb{S}_{1 \& \mathrm{w}}(B, \dot{B})<1$.

Proposição 6.21. As medidas $\mathbb{S}_{\mathrm{stm}}, \mathbb{S}_{\mathrm{wjac}}, \mathbb{S}_{\mathrm{wdic}}, \mathbb{S}_{\mathrm{ay}}, \mathbb{S}_{\text {erel }}$ e $\mathbb{S}_{\mathrm{erec}}$ penalizam soluções por cobertura múltipla de bi-grupos (Def. 4).

Prova. Sejam $B$ e $\dot{B}$ dois bi-agrupamentos, como na Def. 4. Visto que os bi-grupos em $\dot{B}$ não se sobrepõem, tem-se relacionamentos de subconjunto próprio $\dot{B}_{i}^{\mathrm{r}} \times \dot{B}_{i}^{\mathrm{c}} \subset B_{1}^{\mathrm{r}} \times B_{1}^{\mathrm{c}}$ para todo $i$. Logo, $\mathbb{D}\left(B_{1}, \dot{B}_{i}\right)<1$ e $\mathbb{J}\left(B_{1}, \dot{B}_{i}\right)<1$ para todo $i$, implicando que $\mathbb{S}_{\text {stm }}(B, \dot{B})<1$, $\mathbb{S}_{\mathrm{wjac}}(B, \dot{B})<1, \mathbb{S}_{\mathrm{wdic}}(B, \dot{B})<1, \mathbb{S}_{\mathrm{erel}}(B, \dot{B})<1$ e $\mathbb{S}_{\mathrm{erec}}(B, \dot{B})<1$. De $\mathbb{S}_{\mathrm{ay}}(B, \dot{B})<$ $\mathbb{S}_{\text {erel }}(B, \dot{B})$, tem-se também $\mathbb{S}_{\text {ay }}(B, \dot{B})<1$. 
Proposição 6.22. A medida $\mathbb{S}_{\text {agri }}$ penaliza soluções por cobertura múltipla de bi-grupos (Def. 4).

Prova. Sejam $B$ e $\dot{B}$ dois bi-agrupamentos, como na Def. 4 . Existem $j_{1}$ e $j_{2}$ (cujos objetos $\tilde{o}_{j_{1}}$ e $\tilde{o}_{j_{2}}$ pertencem a grupos diferentes em $\dot{P}$ ) tais que $\mathrm{J}_{j_{1}, j_{2}}^{\mathrm{U}}=\mathrm{S}_{j_{1}, j_{2}}^{\dot{U}}=1$ and $\mathrm{J}_{j_{1}, j_{2}}^{\dot{U}}=\mathrm{S}_{j_{1}, j_{2}}^{\mathrm{U}}=0$. Logo, $\dot{b}>0$ (Eq. (5.2b)), 13GRI(U, U) $<1$ (Eq. (5.11)), 13AGRI (U, Ü) $<1$ (Corolário 5.7) e $\mathbb{S}_{\text {agri }}(B, \dot{B})<1$.

Proposição 6.23. A medida $\mathbb{S}_{\mathrm{csi}}$ penaliza soluções com bi-grupos repetitivos (Def. 5).

Prova. Sejam $B, \dot{B}$ e $\hat{B}$ bi-agrupamentos, como na Def. 5. Seja $C_{\mathrm{r}}$ o conjunto de objetos de $O$ que correspondem aos elementos dos bi-grupos de $B$ que foram replicados. Tem-se

$$
\begin{aligned}
\alpha_{P}\left(O, O-C_{\mathrm{r}}\right) & =\alpha_{\hat{P}}\left(O, O-C_{\mathrm{r}}\right), \\
\alpha_{\hat{P}}\left(C_{\mathrm{r}}, C_{\mathrm{r}}\right) & >\alpha_{P}\left(C_{\mathrm{r}}, C_{\mathrm{r}}\right) \geq \alpha_{\dot{P}}\left(C_{\mathrm{r}}, C_{\mathrm{r}}\right), \\
\beta_{P}\left(O-C_{\mathrm{r}}\right) & =\beta_{\hat{P}}\left(O-C_{\mathrm{r}}\right) \mathrm{e} \\
\beta_{\hat{P}}\left(C_{\mathrm{r}}\right) & >\beta_{P}\left(C_{\mathrm{r}}\right) \geq \beta_{\dot{P}}\left(C_{\mathrm{r}}\right) .
\end{aligned}
$$

Conclui-se que $a_{G}^{P, \dot{P}}(O, O)=a_{G}^{\hat{P}, \dot{P}}(O, O)$ e $a_{G}^{P, \dot{P}}=a_{G}^{\hat{P}, \dot{P}}$.

Tem-se

$$
\begin{aligned}
d_{G}^{P, \dot{P}}\left(O, O-C_{\mathrm{r}}\right) & =\left|\alpha_{P}\left(O, O-C_{\mathrm{r}}\right)-\alpha_{\dot{P}}\left(O, O-C_{\mathrm{r}}\right)\right| \\
& +\left|\beta_{P}(O)-\beta_{\dot{P}}(O)\right| \\
& +\left|\beta_{P}\left(O-C_{\mathrm{r}}\right)-\beta_{\dot{P}}\left(O-C_{\mathrm{r}}\right)\right| \\
& =\left|\alpha_{\hat{P}}\left(O, O-C_{\mathrm{r}}\right)-\alpha_{\dot{P}}\left(O, O-C_{\mathrm{r}}\right)\right| \\
& +\left|\beta_{P}(O)-\beta_{\dot{P}}(O)\right| \\
& +\left|\beta_{\hat{P}}\left(O-C_{\mathrm{r}}\right)-\beta_{\dot{P}}\left(O-C_{\mathrm{r}}\right)\right| \\
& \leq d_{G}^{\hat{P}, \dot{P}}\left(O, O-C_{\mathrm{r}}\right)
\end{aligned}
$$

e

$$
\begin{aligned}
d_{G}^{P, \dot{P}}\left(C_{\mathrm{r}}, C_{\mathrm{r}}\right) & =\left|\alpha_{P}\left(C_{\mathrm{r}}, C_{\mathrm{r}}\right)-\alpha_{\dot{P}}\left(C_{\mathrm{r}}, C_{\mathrm{r}}\right)\right| \\
& +2\left|\beta_{P}\left(C_{\mathrm{r}}\right)-\beta_{\dot{P}}\left(C_{\mathrm{r}}\right)\right| \\
& <\left|\alpha_{\hat{P}}\left(C_{\mathrm{r}}, C_{\mathrm{r}}\right)-\alpha_{\dot{P}}\left(C_{\mathrm{r}}, C_{\mathrm{r}}\right)\right| \\
& +2\left|\beta_{\hat{P}}\left(C_{\mathrm{r}}\right)-\beta_{\dot{P}}\left(C_{\mathrm{r}}\right)\right| \\
& =d_{G}^{\hat{P}, \dot{P}}\left(C_{\mathrm{r}}, C_{\mathrm{r}}\right) .
\end{aligned}
$$

$\operatorname{Logo}, d_{G}^{\hat{P}, \dot{P}}>d_{G}^{P, \dot{P}}, 10 \operatorname{CSI}(P, \dot{P})>10 \operatorname{CSI}(\hat{P}, \dot{P})$ e $\mathbb{S}_{\mathrm{csi}}(B, \dot{B})>\mathbb{S}_{\mathrm{csi}}(\hat{B}, \dot{B})$

Proposição 6.24. A medida $\mathbb{S}_{\mathrm{ebc}}$ penaliza soluções com bi-grupos repetitivos (Def. 5). 
Prova. Sejam $B, \dot{B}$ e $\hat{B}$ bi-agrupamentos, como na Def. 5. Seja $C_{\mathrm{r}}$ o conjunto de objetos de $O$ que correspondem aos elementos dos bi-grupos de $B$ que foram replicados. Sabe-se das Eqs. 6.9) e 6.10) que os nominadores das Eqs. (B.1) não se alteram ao passar da comparação de $P$ com $\dot{P}$ para a comparação de $\hat{P}$ com $\dot{P}$ e que $\alpha_{\hat{P}}(O, O) \geq \alpha_{P}(O, O)$. Tem-se $\tilde{o}_{j_{1}}$ e $\tilde{o}_{j_{2}}$ (para o qual $j_{1}=j_{2}$ é permitido) tais que $\alpha_{\hat{P}}\left(\tilde{o}_{j_{1}}, \tilde{o}_{j_{2}}\right)>\alpha_{P}\left(\tilde{o}_{j_{1}}, \tilde{o}_{j_{2}}\right)$. Portanto, $\operatorname{EBCP}(P, \dot{P})>$ $\operatorname{EBCP}(\hat{P}, \dot{P}), 09 \operatorname{EBC}(P, \dot{P})>09 \operatorname{EBC}(\hat{P}, \dot{P})$ e $\mathbb{S}_{\mathrm{ebc}}(B, \dot{B})>\mathbb{S}_{\mathrm{ebc}}(\hat{B}, \dot{B})$.

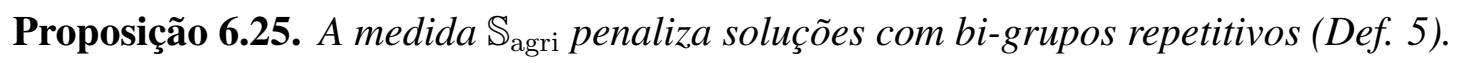

Prova. Sejam $B, \dot{B}$ e $\hat{B}$ bi-agrupamentos, como na Def. 5. Sejam $Q_{\mathrm{dd}}$ o conjunto de tuplas $(i, j)$ cada qual indexando dois objetos pertencentes a um mesmo bi-grupo ideal que foi replicado em $\dot{B}, Q_{\mathrm{df}}$ o conjunto de tuplas $(i, j)$ cada qual indexando um objeto pertencente a um bi-grupo ideal que foi replicado em $\dot{B}$ e outro que não e $Q_{\mathrm{ff}}$ o conjunto das tuplas $(i, j)$ que denotam os pares de objetos remanescentes. Portanto, $Q_{\mathrm{dd}} \cup Q_{\mathrm{df}} \cup Q_{\mathrm{ff}}$ consiste em todas as tuplas $(i, j)$ tais que $i, j \in \mathbb{N}_{1, n}$. Tem-se

$$
\begin{aligned}
& \mathrm{J}_{(i, j) \in Q_{\mathrm{dd}}}^{\hat{\mathrm{U}}}>\mathrm{J}_{(i, j) \in Q_{\mathrm{dd}}}^{\mathrm{U}} \geq \mathrm{J}_{(i, j) \in Q_{\mathrm{dd}}}^{\dot{\mathrm{U}}}=1, \\
& \mathrm{~S}_{(i, j) \in Q_{\mathrm{dd}}}^{\hat{\mathrm{U}}} \geq \mathrm{S}_{(i, j) \in Q_{\mathrm{dd}}}^{\mathrm{U}} \geq \mathrm{S}_{(i, j) \in Q_{\mathrm{dd}}}^{\dot{U}}=0 \text {, } \\
& \mathrm{J}_{(i, j) \in Q_{\mathrm{df}}}^{\hat{\mathrm{U}}}=\mathrm{J}_{(i, j) \in Q_{\mathrm{df}}}^{\mathrm{U}} \geq \mathrm{J}_{(i, j) \in Q_{\mathrm{df}}}^{\dot{\mathrm{U}}}=0, \\
& \mathrm{~S}_{(i, j) \in Q_{\mathrm{df}}}^{\hat{\mathrm{U}}}>\mathrm{S}_{(i, j) \in Q_{\mathrm{df}}}^{\mathrm{U}} \geq \mathrm{S}_{(i, j) \in Q_{\mathrm{df}}}^{\dot{U}}=1, \\
& \mathrm{~J}_{(i, j) \in Q_{\mathrm{ff}}}^{\hat{\mathrm{U}}}=\mathrm{J}_{(i, j) \in Q_{\mathrm{ff}}}^{\mathrm{U}}=\mathrm{J}_{(i, j) \in Q_{\mathrm{ff}}}^{\dot{\mathrm{U}}} \mathrm{e} \\
& \mathrm{S}_{(i, j) \in Q_{\mathrm{ff}}}^{\hat{\mathrm{U}}}=\mathrm{S}_{(i, j) \in Q_{\mathrm{ff}}}^{\mathrm{U}}=\mathrm{S}_{(i, j) \in Q_{\mathrm{ff}}}^{\dot{\mathrm{U}}} .
\end{aligned}
$$

Logo, $\dot{a}^{\mathrm{U}, \dot{\mathrm{U}}}=\dot{a}^{\hat{\mathrm{U}}, \dot{\mathrm{U}}}$ e $\dot{d}^{\mathrm{U}, \dot{\mathrm{U}}}=\dot{d}^{\hat{\mathrm{U}}, \dot{\mathrm{U}}}$. Para provar que $13 \mathrm{GRI}(\mathrm{U}, \dot{\mathrm{U}})>13 \mathrm{GRI}(\hat{\mathrm{U}}, \dot{\mathrm{U}})$, basta provar que o denominador da divisão do lado direito da Eq. (5.12) ao comparar $\hat{U}$ com Uं é maior do que ao comparar U com $\dot{U}$.

Note que $\mathrm{T}_{i, j}^{\hat{\mathrm{U}}} \geq 1, \mathrm{~T}_{i, j}^{\mathrm{U}} \geq 1$ e $\mathrm{T}_{i, j}^{\dot{\mathrm{U}}}=1$. Portanto, $\max \left\{\sum_{i<j} \mathrm{~T}_{i, j}^{\mathrm{U}}, \sum_{i<j} \mathrm{~T}_{i, j}^{\dot{\mathrm{U}}}\right\}=\sum_{i<j} \mathrm{~T}_{i, j}^{\mathrm{U}}$ e $\max \left\{\sum_{i<j} \mathrm{~T}_{i, j}^{\hat{U}}, \sum_{i<j} \mathrm{~T}_{i, j}^{\dot{U}}\right\}=\sum_{i<j} \mathrm{~T}_{i, j}^{\hat{U}}$. Como

$$
\begin{aligned}
\sum_{i<j} \mathrm{~T}_{i, j}^{\mathrm{U}} & =\sum_{\substack{i<j \\
(i, j) \in Q_{\mathrm{dd}}}} \mathrm{T}_{i, j}^{\mathrm{U}}+\sum_{\substack{i<j \\
(i, j) \in Q_{\mathrm{df}}}} \mathrm{T}_{i, j}^{\mathrm{U}}+\sum_{\substack{i<j \\
(i, j) \in Q_{\mathrm{ff}}}} \mathrm{T}_{i, j}^{\mathrm{U}} \\
& =\sum_{\substack{i<j \\
(i, j) \in Q_{\mathrm{dd}}}} \mathrm{T}_{i, j}^{\mathrm{U}}+\sum_{\substack{i<j \\
(i, j) \in Q_{\mathrm{df}}}} \mathrm{T}_{i, j}^{\mathrm{U}}+\sum_{\substack{i<j \\
(i, j) \in Q_{\mathrm{ff}}}} \mathrm{T}_{i, j}^{\hat{\mathrm{U}}} \\
& <\sum_{\substack{i<j \\
(i, j) \in Q_{\mathrm{dd}}}} \mathrm{T}_{i, j}^{\hat{\mathrm{U}}}+\sum_{\substack{i<j \\
(i, j) \in Q_{\mathrm{df}}}} \mathrm{T}_{i, j}^{\hat{\mathrm{U}}}+\sum_{\substack{i<j \\
(i, j) \in Q_{\mathrm{ff}}}} \mathrm{T}_{i, j}^{\hat{\mathrm{U}}}=\sum_{i<j} \mathrm{~T}_{i, j}^{\hat{\mathrm{U}}},
\end{aligned}
$$

tem-se $13 \mathrm{GRI}(\mathrm{U}, \dot{\mathrm{U}})>13 \mathrm{GRI}(\hat{\mathrm{U}}, \dot{\mathrm{U}})$. 
Veja que

$$
\begin{aligned}
\mathrm{E}[\dot{a}]_{\mathrm{U}, \dot{\mathrm{U}}}= & \frac{2}{n(n-1)}\left(\sum_{\left(i_{1}, j_{1}\right) \in Q_{\mathrm{dd}}} \sum_{i_{2}, j_{2}} \min \left\{\mathrm{J}_{i_{1}, j_{1}}^{\mathrm{U}}, \mathrm{J}_{i_{2}, j_{2}}^{\dot{\mathrm{U}}}\right\}+\sum_{\left(i_{1}, j_{1}\right) \in Q_{\mathrm{df}}, \sum_{i_{2}, j_{2}}} \min \left\{\mathrm{J}_{i_{1}, j_{1}}^{\mathrm{U}}, \mathrm{J}_{i_{2}, j_{2}}^{\dot{\mathrm{U}}}\right\}+\right. \\
& \left.\sum_{\left(i_{1}, j_{1}\right) \in Q_{\mathrm{ff}}} \sum_{i_{2}, j_{2}} \min \left\{\mathrm{J}_{i_{1}, j_{1}}^{\mathrm{U}}, \mathrm{J}_{i_{2}, j_{2}}^{\dot{U}}\right\}\right) \\
= & \frac{2}{n(n-1)}\left(\sum_{\left(i_{1}, j_{1}\right) \in Q_{\mathrm{dd}}} \sum_{i_{2}, j_{2}} \min \left\{\mathrm{J}_{i_{1}, j_{1}}^{\mathrm{U}}, \mathrm{J}_{i_{2}, j_{2}}^{\dot{\mathrm{U}}}\right\}+\sum_{\left(i_{1}, j_{1}\right) \in Q_{\mathrm{df}}} \sum_{i_{2}, j_{2}} \min \left\{\mathrm{J}_{i_{1}, j_{1}}^{\mathrm{U}}, \mathrm{J}_{i_{2}, j_{2}}^{\dot{\mathrm{U}}}\right\}+\right. \\
& \left.\sum_{\left(i_{1}, j_{1}\right) \in Q_{\mathrm{ff}}} \sum_{i_{2}, j_{2}} \min \left\{\mathrm{J}_{i_{1}, j_{1}}^{\mathrm{U}}, \mathrm{J}_{i_{2}, j_{2}}^{\dot{\mathrm{U}}}\right\}\right) \\
\leq & \frac{2}{n(n-1)}\left(\sum_{\left(i_{1}, j_{1}\right) \in Q_{\mathrm{dd}}} \sum_{i_{2}, j_{2}} \min \left\{\mathrm{J}_{i_{1}, j_{1}}^{\hat{\mathrm{U}}}, \mathrm{J}_{i_{2}, j_{2}}^{\dot{\mathrm{U}}}\right\}+\sum_{\left(i_{1}, j_{1}\right) \in Q_{\mathrm{df}}} \sum_{i_{2}, j_{2}} \min \left\{\mathrm{J}_{i_{1}, j_{1}}^{\hat{\mathrm{U}}}, \mathrm{J}_{i_{2}, j_{2}}^{\dot{\mathrm{U}}}\right\}+\right. \\
& \left.\sum_{\left(i_{1}, j_{1}\right) \in Q_{\mathrm{ff}}} \sum_{i_{2}, j_{2}} \min \left\{\mathrm{J}_{i_{1}, j_{1}}^{\mathrm{U}}, \mathrm{J}_{i_{2}, j_{2}}^{\mathrm{U}}\right\}\right)=\mathrm{E}[\dot{a}]_{\hat{\mathrm{U}}, \dot{\mathrm{U}}}
\end{aligned}
$$

e, similarmente, $\mathrm{E}[\dot{d}]_{\mathrm{U}, \dot{\mathrm{U}}} \leq \mathrm{E}[\dot{d}]_{\hat{\mathrm{U}}, \dot{\mathrm{U}}} \cdot \operatorname{Logo}, \mathrm{E}[13 \mathrm{GRI}]_{\mathrm{U}, \dot{\mathrm{U}}} \leq \mathrm{E}[13 \mathrm{GRI}]_{\hat{\mathrm{U}}, \dot{\mathrm{U}}} \cdot$

Para $13 \mathrm{GRI}(\mathrm{U}, \dot{\mathrm{U}})=1$, tem-se $13 \mathrm{AGRI}(\mathrm{U}, \dot{\mathrm{U}})=1>13 \mathrm{AGRI}(\hat{\mathrm{U}}, \dot{\mathrm{U}})$, uma vez que $13 \mathrm{GRI}(\hat{\mathrm{U}}, \dot{\mathrm{U}})<1$. Para $13 \mathrm{GRI}(\mathrm{U}, \dot{\mathrm{U}})<1$, tem-se E[13GRI $]_{\mathrm{U}, \dot{\mathrm{U}}}<1$ e E[13GRI $]_{\hat{\mathrm{U}}, \dot{\mathrm{U}}}<1$ e, portanto, pode-se afirmar que

$$
\begin{aligned}
13 \mathrm{AGRI}(\hat{\mathrm{U}}, \dot{\mathrm{U}})\left(1-\mathrm{E}[13 \mathrm{GRI}]_{\hat{\mathrm{U}}, \dot{\mathrm{U}}}\right) & =13 \mathrm{GRI}(\hat{\mathrm{U}}, \dot{\mathrm{U}})-\mathrm{E}[13 \mathrm{GRI}]_{\hat{\mathrm{U}}, \dot{\mathrm{U}}} \\
& <13 \mathrm{GRI}(\mathrm{U}, \dot{\mathrm{U}})-\mathrm{E}[13 \mathrm{GRI}]_{\hat{\mathrm{U}}, \dot{\mathrm{U}}}
\end{aligned}
$$

Seja $\mathrm{E}[13 \mathrm{GRI}]_{\hat{\mathrm{U}}, \dot{\mathrm{U}}} \triangleq \mathrm{E}[13 \mathrm{GRI}]_{\mathrm{U}, \dot{\mathrm{U}}}+x$. Tem-se

$$
\begin{aligned}
13 \operatorname{AGRI}(\hat{\mathrm{U}}, \dot{\mathrm{U}})\left(1-\mathrm{E}[13 \mathrm{GRI}]_{\mathrm{U}, \dot{\mathrm{U}}}\right) & <13 \mathrm{GRI}(\mathrm{U}, \dot{\mathrm{U}})-\mathrm{E}[13 \mathrm{GRI}]_{\mathrm{U}, \dot{\mathrm{U}}}-x(1-13 \mathrm{AGRI}(\hat{\mathrm{U}}, \dot{\mathrm{U}})) \\
& \leq 13 \mathrm{GRI}(\mathrm{U}, \dot{\mathrm{U}})-\mathrm{E}[13 \mathrm{GRI}]_{\mathrm{U}, \dot{\mathrm{U}}} \\
& =13 \mathrm{AGRI}(\mathrm{U}, \dot{\mathrm{U}})\left(1-\mathrm{E}[13 \mathrm{GRI}]_{\mathrm{U}, \dot{U}}\right)
\end{aligned}
$$

implicando em $\mathbb{S}_{\text {agri }}(B, \dot{B})>\mathbb{S}_{\text {agri }}(\hat{B}, \dot{B})$.

Proposição 6.26. As medidas $\mathbb{S}_{\mathrm{prec}}$ e $\mathbb{S}_{\mathrm{erec}}$ não têm a propriedade de homogeneidade (Def. 7 ).

Prova. Considere os bi-agrupamentos sem sobreposição $B$ e $\hat{B}$ representados pelas Figs. 6.9(b) e 6.9(c) e a solução de referência $\dot{B}$ representada pela Figura 6.9(a). Tem-se $\mathbb{S}_{\text {prec }}(B, \dot{B})=$ $\mathbb{S}_{\text {prec }}(\hat{B}, \dot{B})=0.74$ e $\mathbb{S}_{\text {erec }}(B, \dot{B})=\mathbb{S}_{\text {erec }}(\hat{B}, \dot{B})=0.55$.

Proposição 6.27. A medida $\mathbb{S}_{\mathrm{csi}}$ possui a propriedade de homogeneidade (Def. 7).

Prova. Sejam $B, \hat{B}$ e $\dot{B}$ três bi-agrupamentos, como na Def. 7. Sejam $C_{\mathrm{s}}^{1}$ o conjunto de $x$ objetos permutados de $P_{i_{1}}, C_{\mathrm{n}}^{1}$ o número de objetos da categoria minoritária de $P_{i_{1}}$ que não foram permutados e $C_{\mathrm{r}}^{1}$ o número de objetos da categoria majoritária de $P_{i_{1}}$. Analogamente, defina $C_{\mathrm{s}}^{2}, C_{\mathrm{n}}^{2}$ e $C_{\mathrm{r}}^{2}$. Visto que $B, \hat{B}$ e $\dot{B}$ são soluções sem sobreposição, tem-se 
colunas

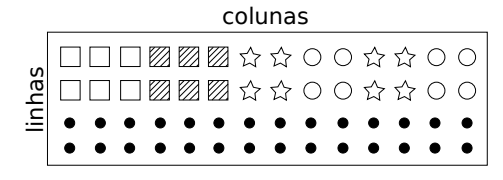

(a) Matriz de dados $\mathrm{X} \in \mathbb{R}^{4 \cdot 14}$.

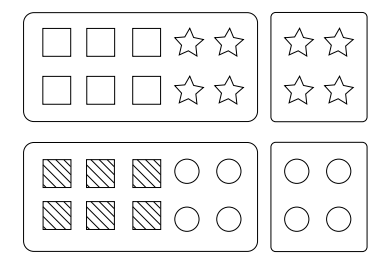

(b) Bi-agrupamento $B$.

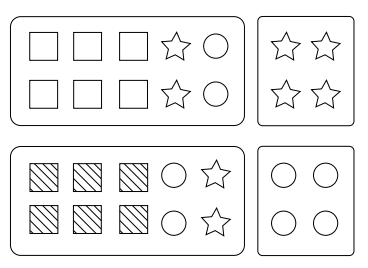

(c) Bi-agrupamento $\hat{B}$.

Figura 6.9: Diferença de homogeneidade.

$\beta_{P}(O)=\beta_{\hat{P}}(O)=\beta_{\dot{P}}(O)=0$. Logo, a eventual diferença entre $a_{G}^{P, \dot{P}}\left(\tilde{o}_{j_{1}}, \tilde{o}_{j_{2}}\right)$ (respectivamente, $\left.d_{G}^{P, \dot{P}}\left(\tilde{o}_{j_{1}}, \tilde{o}_{j_{2}}\right)\right)$ e $a_{G}^{\hat{P}, \dot{P}}\left(\tilde{o}_{j_{1}}, \tilde{o}_{j_{2}}\right)\left(d_{G}^{\hat{P}, \dot{P}}\left(\tilde{o}_{j_{1}}, \tilde{o}_{j_{2}}\right)\right)$ só pode ser devido à eventual diferença entre $\alpha_{P}\left(\tilde{o}_{j_{1}}, \tilde{o}_{j_{2}}\right)$ e $\alpha_{\hat{P}}\left(\tilde{o}_{j_{1}}, \tilde{o}_{j_{2}}\right)$ (veja Eqs. (B.3) e (B.4)).

Tabela 6.13: Diferenças em $a_{G}^{\because \cdot}(\cdot, \cdot)$ ao passar de $P$ para $\hat{P}$.

\begin{tabular}{|l|}
$a_{G}^{P, \dot{P}}\left(C_{\mathrm{s}}^{1}, C_{\mathrm{n}}^{1}\right)=\min \{1,1\}>\min \{0,1\}=a_{G}^{\hat{P}, \dot{P}}\left(C_{\mathrm{s}}^{1}, C_{\mathrm{n}}^{1}\right)$ \\
$a_{G}^{P, \dot{P}}\left(C_{\mathrm{s}}^{2}, C_{\mathrm{n}}^{2}\right)=\min \{1,1\}>\min \{0,1\}=a_{G}^{\hat{P}, \dot{P}}\left(C_{\mathrm{s}}^{2}, C_{\mathrm{n}}^{2}\right)$ \\
\hline$a_{G}^{P, \dot{P}}\left(C_{\mathrm{s}}^{1}, C_{\mathrm{r}}^{1}\right)=\min \{1,0\}=\min \{0,0\}=a_{G}^{\hat{P}, \dot{P}}\left(C_{\mathrm{s}}^{1}, C_{\mathrm{r}}^{1}\right)$ \\
$a_{G}^{P, P}\left(C_{\mathrm{s}}^{2}, C_{\mathrm{r}}^{2}\right)=\min \{1,0\}=\min \{0,0\}=a_{G}^{\hat{P}, \dot{P}}\left(C_{\mathrm{s}}^{2}, C_{\mathrm{r}}^{2}\right)$ \\
\hline$a_{G}^{P, \dot{P}}\left(C_{\mathrm{s}}^{1}, C_{\mathrm{n}}^{2}\right)=\min \{0,0\}=\min \{1,0\}=a_{G}^{\hat{P}, \dot{P}}\left(C_{\mathrm{s}}^{1}, C_{\mathrm{n}}^{2}\right)$ \\
$a_{G}^{P, P}\left(C_{\mathrm{n}}^{1}, C_{\mathrm{s}}^{2}\right)=\min \{0,0\}=\min \{1,0\}=a_{G}^{\hat{P}, \dot{P}}\left(C_{\mathrm{n}}^{1}, C_{\mathrm{s}}^{2}\right)$ \\
\hline$a_{G}^{P, \dot{P}}\left(C_{\mathrm{s}}^{1}, C_{\mathrm{r}}^{2}\right)=\min \{0,0\}=\min \{1,0\}=a_{G}^{\hat{P}, \dot{P}}\left(C_{\mathrm{s}}^{1}, C_{\mathrm{r}}^{2}\right)$ \\
$a_{G}^{P, \dot{P}}\left(C_{\mathrm{r}}^{1}, C_{\mathrm{s}}^{2}\right)=\min \{0,0\}=\min \{1,0\}=a_{G}^{\hat{P}, \dot{P}}\left(C_{\mathrm{r}}^{1}, C_{\mathrm{s}}^{2}\right)$ \\
\hline
\end{tabular}

Conclui-se pela Tabela 6.13 que $a_{G}^{P, \dot{P}}=a_{G}^{\hat{P}, \dot{P}}+\left|C_{\mathrm{s}}^{1}\right|\left|C_{\mathrm{n}}^{1}\right|+\left|C_{\mathrm{s}}^{2}\right|\left|C_{\mathrm{n}}^{2}\right|=a_{G}^{\hat{P}, \dot{P}}+x\left(\left|C_{\mathrm{n}}^{1}\right|+\left|C_{\mathrm{n}}^{2}\right|\right)$, em que $x=\left|C_{\mathrm{s}}^{1}\right|=\left|C_{\mathrm{s}}^{2}\right|$, como na Def. 7. A Tabela 6.14 mostra as diferenças em $d_{G}^{\bullet \cdot}(\cdot, \cdot)$, implicando que $d_{G}^{P, \dot{P}}=d_{G}^{\hat{P}, \dot{P}}-\left|C_{\mathrm{s}}^{1}\right|\left|C_{\mathrm{n}}^{1}\right|-\left|C_{\mathrm{s}}^{2}\right|\left|C_{\mathrm{n}}^{2}\right|-\left|C_{\mathrm{s}}^{1}\right|\left|C_{\mathrm{n}}^{2}\right|-\left|C_{\mathrm{n}}^{1}\right|\left|C_{\mathrm{s}}^{2}\right|-\left|C_{\mathrm{s}}^{1}\right|\left|C_{\mathrm{r}}^{2}\right|-\left|C_{\mathrm{r}}^{1}\right|\left|C_{\mathrm{s}}^{2}\right|+$ $\left|C_{\mathrm{s}}^{1}\right|\left|C_{\mathrm{r}}^{1}\right|+\left|C_{\mathrm{s}}^{2}\right|\left|C_{\mathrm{r}}^{2}\right|=d_{G}^{\hat{P}, \dot{P}}-2 x\left(\left|C_{\mathrm{n}}^{1}\right|+\left|C_{\mathrm{n}}^{2}\right|\right)$. Logo,

$$
10 \operatorname{CSI}(\hat{P}, \dot{P})=\frac{a_{G}^{P, \dot{P}}-x\left(\left|C_{\mathrm{n}}^{1}\right|+\left|C_{\mathrm{n}}^{2}\right|\right)}{a_{G}^{P, \dot{P}}+d_{G}^{P, \dot{P}}+x\left(\left|C_{\mathrm{n}}^{1}\right|+\left|C_{\mathrm{n}}^{2}\right|\right)}
$$

e $\mathbb{S}_{\mathrm{csi}}(B, \dot{B}) \geq \mathbb{S}_{\mathrm{csi}}(\hat{B}, \dot{B})$

Se $x=\left|\mathrm{I}\left(B_{i_{1}}, \dot{B}_{\mathrm{mi}\left(i_{1}\right)}\right)\right|=\left|\mathrm{I}\left(B_{i_{2}}, \dot{B}_{\mathrm{mi}\left(i_{2}\right)}\right)\right|$, então $\left|C_{\mathrm{n}}^{1}\right|=\left|C_{\mathrm{n}}^{2}\right|=0$ e $\mathbb{S}_{\mathrm{csi}}(B, \dot{B})=$ $\mathbb{S}_{\mathrm{csi}}(\hat{B}, \dot{B})$. Se $\mathbb{S}_{\mathrm{csi}}(B, \dot{B})=\mathbb{S}_{\mathrm{csi}}(\hat{B}, \dot{B})$, então $\left|C_{\mathrm{n}}^{1}\right|+\left|C_{\mathrm{n}}^{2}\right|=0$ (porque $x>0$, Def. 7 e $x=\left|\mathrm{I}\left(B_{i_{1}}, \dot{B}_{\operatorname{mi}\left(i_{1}\right)}\right)\right|=\left|\mathrm{I}\left(B_{i_{2}}, \dot{B}_{\operatorname{mi}\left(i_{2}\right)}\right)\right|$.

Proposição 6.28. A medida $\mathbb{S}_{\mathrm{ebc}}$ possui a propriedade de homogeneidade (Def. 7). 
Tabela 6.14: Diferenças em $d_{G} \cdot(\cdot, \cdot)$ ao passar de $P$ para $\hat{P}$.

\begin{tabular}{|l|}
\hline$d_{G}^{P, \dot{P}}\left(C_{\mathrm{s}}^{1}, C_{\mathrm{n}}^{1}\right)=|1-1|<|0-1|=d_{G}^{\hat{P}, \dot{P}}\left(C_{\mathrm{s}}^{1}, C_{\mathrm{n}}^{1}\right)$ \\
$d_{G}^{P, \dot{P}}\left(C_{\mathrm{s}}^{2}, C_{\mathrm{n}}^{2}\right)=|1-1|<|0-1|=d_{G}^{\hat{P}, \dot{P}}\left(C_{\mathrm{s}}^{2}, C_{\mathrm{n}}^{2}\right)$ \\
\hline$d_{G}^{P, \dot{P}}\left(C_{\mathrm{s}}^{1}, C_{\mathrm{r}}^{1}\right)=|1-0|>|0-0|=d_{G}^{\hat{P}, \dot{P}}\left(C_{\mathrm{s}}^{1}, C_{\mathrm{r}}^{1}\right)$ \\
$d_{G}^{P, P}\left(C_{\mathrm{s}}^{2}, C_{\mathrm{r}}^{2}\right)=|1-0|>|0-0|=d_{G}^{\hat{P}, \dot{P}}\left(C_{\mathrm{s}}^{2}, C_{\mathrm{r}}^{2}\right)$ \\
\hline$d_{G}^{P, \dot{P}}\left(C_{\mathrm{s}}^{1}, C_{\mathrm{n}}^{2}\right)=|0-0|<|1-0|=d_{G}^{\hat{P}, \dot{P}}\left(C_{\mathrm{s}}^{1}, C_{\mathrm{n}}^{2}\right)$ \\
$d_{G}^{P, \dot{P}}\left(C_{\mathrm{n}}^{1}, C_{\mathrm{s}}^{2}\right)=|0-0|<|1-0|=d_{G}^{\hat{P}, \dot{P}}\left(C_{\mathrm{n}}^{1}, C_{\mathrm{s}}^{2}\right)$ \\
\hline$d_{G}^{P, \dot{P}}\left(C_{\mathrm{s}}^{1}, C_{\mathrm{r}}^{2}\right)=|0-0|<|1-0|=d_{G}^{\hat{P}, \dot{P}}\left(C_{\mathrm{s}}^{1}, C_{\mathrm{r}}^{2}\right)$ \\
$d_{G}^{P, \dot{P}}\left(C_{\mathrm{r}}^{1}, C_{\mathrm{s}}^{2}\right)=|0-0|<|1-0|=d_{G}^{\hat{P}, \dot{P}}\left(C_{\mathrm{r}}^{1}, C_{\mathrm{s}}^{2}\right)$ \\
\hline
\end{tabular}

Prova. Sejam $B, \hat{B}$ e $\dot{B}$ três bi-agrupamentos, como na Def. 7. Sejam $C_{\mathrm{s}}^{1}$ os $x$ objetos permutados de $P_{i_{1}}, C_{\mathrm{n}}^{1}$ os objetos da categoria minoritária de $P_{i_{1}}$ que não foram permutados e $C_{\mathrm{r}}^{1}$ os objetos da categoria majoritária de $P_{i_{1}}$. Analogamente, defina $C_{\mathrm{s}}^{2}, C_{\mathrm{n}}^{2}$ e $C_{\mathrm{r}}^{2}$. Seja

$$
\delta_{\mathrm{p}}^{P, \dot{P}}\left(\tilde{o}_{j_{1}}\right) \triangleq \frac{\sum_{j_{2}=1}^{n} \min \left\{\alpha_{P}\left(\tilde{o}_{j_{1}}, \tilde{o}_{j_{2}}\right), \alpha_{\dot{P}}\left(\tilde{o}_{j_{1}}, \tilde{o}_{j_{2}}\right)\right\}}{\sum_{j_{2}=1}^{n} \alpha_{P}\left(\tilde{o}_{j_{1}}, \tilde{o}_{j_{2}}\right)}
$$

tal que $\operatorname{EBCP}(P, \dot{P})=(1 / n) \sum_{j_{1}=1}^{n} \delta_{\mathrm{p}}^{P, \dot{P}}\left(\tilde{o}_{j_{1}}\right)$

Tabela 6.15: Diferenças em $\delta_{\mathrm{p}}^{\cdot \bullet}(\cdot)$ ao passar de $P$ para $\hat{P}$.

\begin{tabular}{|c|l|}
\hline$\delta_{\mathrm{p}}^{P, \dot{P}}\left(C_{\mathrm{s}}^{1}\right)=\frac{\left|C_{\mathrm{s}}^{1} \cup C_{\mathrm{n}}^{1}\right|}{\left|P_{i_{1}}\right|}$ & $\delta_{\mathrm{p}}^{\hat{P}, \dot{P}}\left(C_{\mathrm{s}}^{1}\right)=\frac{\left|C_{\mathrm{s}}^{1}\right|}{\left|P_{i_{2}}\right|}$ \\
$\delta_{\mathrm{p}}^{P, \dot{P}}\left(C_{\mathrm{s}}^{2}\right)=\frac{\left|C_{\mathrm{s}}^{2} \cup C_{\mathrm{n}}^{2}\right|}{\left|P_{i_{2}}\right|}$ & $\delta_{\mathrm{p}}^{\hat{P}, \dot{P}}\left(C_{\mathrm{s}}^{2}\right)=\frac{\left|C_{\mathrm{s}}^{2}\right|}{\left|P_{i_{1}}\right|}$ \\
\hline$\delta_{\mathrm{p}}^{P, \dot{P}}\left(C_{\mathrm{n}}^{1}\right)=\frac{\left|C_{\mathrm{s}}^{1} \cup C_{\mathrm{n}}^{1}\right|}{\left|P_{i_{1}}\right|}$ & $\delta_{\mathrm{p}}^{\hat{P}, \dot{P}}\left(C_{\mathrm{n}}^{1}\right)=\frac{\left|C_{\mathrm{n}}^{1}\right|}{\left|P_{i_{1}}\right|}$ \\
$\delta_{\mathrm{p}}^{P, \dot{P}}\left(C_{\mathrm{n}}^{2}\right)=\frac{\left|C_{\mathrm{s}}^{2} \cup C_{\mathrm{n}}^{2}\right|}{\left|P_{i_{2}}\right|}$ & $\delta_{\mathrm{p}}^{\hat{P}, \dot{P}}\left(C_{\mathrm{n}}^{2}\right)=\frac{\left|C_{\mathrm{n}}^{2}\right|}{\left|P_{i_{2}}\right|}$ \\
\hline$\delta_{\mathrm{p}}^{P, \dot{P}}\left(C_{\mathrm{r}}^{1}\right)=\frac{\left|C_{\mathrm{r}}^{1}\right|}{\left|P_{i_{1}}\right|}$ & $\delta_{\mathrm{p}}^{\hat{P}, \dot{P}}\left(C_{\mathrm{r}}^{1}\right)=\frac{\left|C_{\mathrm{r}}^{1}\right|}{\left|P_{i_{1}}\right|}$ \\
$\delta_{\mathrm{p}}^{P, \dot{P}}\left(C_{\mathrm{r}}^{2}\right)=\frac{\left|C_{\mathrm{r}}^{2}\right|}{\left|P_{i_{2}}\right|}$ & $\delta_{\mathrm{p}}^{\hat{P}, \dot{P}}\left(C_{\mathrm{r}}^{2}\right)=\frac{\left|C_{\mathrm{r}}^{2}\right|}{\left|P_{i_{2}}\right|}$ \\
\hline
\end{tabular}

Note que

$$
\sum_{\tilde{o}_{j_{1}} \notin P_{i_{1}} \cup P_{i_{2}}} \delta_{\mathrm{p}}^{P, \dot{P}}\left(\tilde{o}_{j_{1}}\right)=\sum_{\tilde{o}_{j_{1}} \notin P_{i_{1}} \cup P_{i_{2}}} \delta_{\mathrm{p}}^{\hat{P}, \dot{P}}\left(\tilde{o}_{j_{1}}\right) .
$$


Conclui-se pela Tabela 6.15 e depois de algumas manipulações algébricas que

$$
\begin{aligned}
\sum_{\tilde{o}_{j_{1}} \in P_{i_{1}} \cup P_{i_{2}}} \delta_{\mathrm{p}}^{P, \dot{P}}\left(\tilde{o}_{j_{1}}\right) & =\frac{\left(\left|C_{\mathrm{s}}^{1}\right|+\left|C_{\mathrm{n}}^{1}\right|\right)^{2}}{\left|P_{i_{1}}\right|}+\frac{\left|C_{\mathrm{r}}^{1}\right|^{2}}{\left|P_{i_{1}}\right|} \\
& +\frac{\left(\left|C_{\mathrm{s}}^{2}\right|+\left|C_{\mathrm{n}}^{2}\right|\right)^{2}}{\left|P_{i_{2}}\right|}+\frac{\left|C_{\mathrm{r}}^{2}\right|^{2}}{\left|P_{i_{2}}\right|}
\end{aligned}
$$

$\mathrm{e}$

$$
\begin{aligned}
\sum_{\tilde{o}_{j_{1}} \in P_{i_{1}} \cup P_{i_{2}}} \delta_{\mathrm{p}}^{\hat{P}, \dot{P}}\left(\tilde{o}_{j_{1}}\right) & =\frac{\left|C_{\mathrm{s}}^{2}\right|^{2}+\left|C_{\mathrm{n}}^{1}\right|^{2}+\left|C_{\mathrm{r}}^{1}\right|^{2}}{\left|P_{i_{1}}\right|} \\
& +\frac{\left|C_{\mathrm{s}}^{1}\right|^{2}+\left|C_{\mathrm{n}}^{2}\right|^{2}+\left|C_{\mathrm{r}}^{2}\right|^{2}}{\left|P_{i_{2}}\right|} .
\end{aligned}
$$

De $x=\left|C_{\mathrm{s}}^{1}\right|=\left|C_{\mathrm{s}}^{2}\right|$, tem-se

$$
\operatorname{EBCP}(\hat{P}, \dot{P})-\operatorname{EBCP}(P, \dot{P})=\frac{-2 x}{n}\left(\frac{\left|C_{\mathrm{n}}^{1}\right|}{\left|P_{i_{1}}\right|}+\frac{\left|C_{\mathrm{n}}^{2}\right|}{\left|P_{i_{2}}\right|}\right) .
$$

Seja

$$
\delta_{\mathrm{r}}^{P, \dot{P}}\left(\tilde{o}_{j_{1}}\right) \triangleq \frac{\sum_{j_{2}=1}^{n} \min \left\{\alpha_{P}\left(\tilde{o}_{j_{1}}, \tilde{o}_{j_{2}}\right), \alpha_{\dot{P}}\left(\tilde{o}_{j_{1}}, \tilde{o}_{j_{2}}\right)\right\}}{\sum_{j_{2}=1}^{n} \alpha_{\dot{P}}\left(\tilde{o}_{j_{1}}, \tilde{o}_{j_{2}}\right)}
$$

tal que $\operatorname{EBCR}(P, \dot{P})=(1 / n) \sum_{j_{1}=1}^{n} \delta_{\mathrm{r}}^{P, \dot{P}}\left(\tilde{o}_{j_{1}}\right)$. A única diferença entre $\delta_{\mathrm{p}}^{P, \dot{P}}\left(\tilde{o}_{j_{1}}\right)$ e $\delta_{\mathrm{r}}^{P, \dot{P}}\left(\tilde{o}_{j_{1}}\right)$ se deve aos denominadores. Logo,

$$
\begin{aligned}
\sum_{\tilde{o}_{j_{1}} \in P_{i_{1}} \cup P_{i_{2}}} \delta_{\mathrm{r}}^{P, \dot{P}}\left(\tilde{o}_{j_{1}}\right) & =\frac{\left(\left|C_{\mathrm{s}}^{1}\right|+\left|C_{\mathrm{n}}^{1}\right|\right)^{2}}{\left|\dot{P}_{\mathrm{mi}\left(i_{1}\right)}\right|}+\frac{\left(\left|C_{\mathrm{s}}^{2}\right|+\left|C_{\mathrm{n}}^{2}\right|\right)^{2}}{\left|\dot{P}_{\mathrm{mi}\left(i_{2}\right)}\right|} \\
& +\frac{\left|C_{\mathrm{r}}^{1}\right|^{2}}{\left|\dot{P}_{\mathrm{ma}\left(i_{1}\right)}\right|}+\frac{\left|C_{\mathrm{r}}^{2}\right|^{2}}{\left|\dot{P}_{\mathrm{ma}\left(i_{2}\right)}\right|}
\end{aligned}
$$

$\mathrm{e}$

$$
\begin{aligned}
\sum_{\tilde{o}_{j_{1}} \in P_{i_{1}} \cup P_{i_{2}}} \delta_{\mathrm{r}}^{\hat{P}, \dot{P}}\left(\tilde{o}_{j_{1}}\right) & =\frac{\left|C_{\mathrm{s}}^{1}\right|^{2}+\left|C_{\mathrm{n}}^{1}\right|^{2}}{\left|\dot{P}_{\mathrm{mi}\left(i_{1}\right)}\right|}+\frac{\left|C_{\mathrm{s}}^{2}\right|^{2}+\left|C_{\mathrm{n}}^{2}\right|^{2}}{\left|\dot{P}_{\mathrm{mi}\left(i_{2}\right)}\right|} \\
& +\frac{\left|C_{\mathrm{r}}^{1}\right|^{2}}{\left|\dot{P}_{\mathrm{ma}\left(i_{1}\right)}\right|}+\frac{\left|C_{\mathrm{r}}^{2}\right|^{2}}{\left|\dot{P}_{\mathrm{ma}\left(i_{2}\right)}\right|} .
\end{aligned}
$$

Portanto,

$$
\operatorname{EBCR}(\hat{P}, \dot{P})-\operatorname{EBCR}(P, \dot{P})=\frac{-2 x}{n}\left(\frac{\left|C_{\mathrm{n}}^{1}\right|}{\left|\dot{P}_{\mathrm{mi}\left(i_{1}\right)}\right|}+\frac{\left|C_{\mathrm{n}}^{2}\right|}{\left|\dot{P}_{\mathrm{mi}\left(i_{2}\right)}\right|}\right)
$$

Conclui-se das Eqs. 6.11) e 6.12 que $09 \operatorname{EBC}(P, \dot{P}) \geq 09 \mathrm{EBC}(\hat{P}, \dot{P})$ e $\mathbb{S}_{\mathrm{ebc}}(B, \dot{B}) \geq$ $\mathbb{S}_{\mathrm{ebc}}(\hat{B}, \dot{B})$. Se $x=\left|\mathrm{I}\left(B_{i_{1}}, \dot{B}_{\mathrm{mi}\left(i_{1}\right)}\right)\right|=\left|\mathrm{I}\left(B_{i_{2}}, \dot{B}_{\mathrm{mi}\left(i_{2}\right)}\right)\right|$, então $\left|C_{\mathrm{n}}^{1}\right|=\left|C_{\mathrm{n}}^{2}\right|=0$ e $\mathbb{S}_{\mathrm{ebc}}(B, \dot{B})=$ 
$\mathbb{S}_{\mathrm{ebc}}(\hat{B}, \dot{B})$. Se $\mathbb{S}_{\mathrm{ebc}}(B, \dot{B})=\mathbb{S}_{\mathrm{ebc}}(\hat{B}, \dot{B})$, então $\left|C_{\mathrm{n}}^{1}\right|=\left|C_{\mathrm{n}}^{2}\right|=0$ (porque $x>0$, Def. 7) e $x=\left|\mathrm{I}\left(B_{i_{1}}, \dot{B}_{\operatorname{mi}\left(i_{1}\right)}\right)\right|=\left|\mathrm{I}\left(B_{i_{2}}, \dot{B}_{\operatorname{mi}\left(i_{2}\right)}\right)\right|$.

Proposição 6.29. A medida $\mathbb{S}_{\text {agri }}$ possui a propriedade de homogeneidade (Def.7).

Prova. Sejam $B, \hat{B}$ e $\dot{B}$ três bi-agrupamentos, como na Def. 7. Sejam $C_{\mathrm{s}}^{1}$ os $x$ índices dos objetos permutados de $P_{i_{1}}, C_{\mathrm{n}}^{1}$ os índices dos objetos da categoria minoritária de $P_{i_{1}}$ que não foram permutados e $C_{\mathrm{r}}^{1}$ os índices dos objetos da categoria majoritária de $P_{i_{1}}$. Analogamente, defina $C_{\mathrm{s}}^{2}, C_{\mathrm{n}}^{2}$ e $C_{\mathrm{r}}^{2}$.

Como $B, \hat{B}$ e $\dot{B}$ não possuem sobreposição, tem-se $\mathrm{T}_{i, j}^{\mathrm{U}, \dot{\mathrm{U}}}=\mathrm{T}_{i, j}^{\hat{\mathrm{U}}, \dot{\mathrm{U}}}=1$ para todos os $i$ e $j$ e, portanto, o denominador da divisão do lado direito da Eq. (5.12) é igual ao calcularse $13 \mathrm{GRI}(\mathrm{U}, \dot{\mathrm{U}})$ ou $13 \mathrm{GRI}(\hat{\mathrm{U}}, \dot{\mathrm{U}})$. Seja $\dot{a}^{\mathrm{U}}, \dot{\mathrm{U}}\left(C^{1}, C^{2}\right) \triangleq \min \left\{\mathrm{J}_{i, j}^{\mathrm{U}}, \mathrm{J}_{i, j}^{\dot{U}}\right\}$ para algum $i \in C^{1}$ e $j \in C^{2}$ e seja $\dot{d} \mathrm{U}, \dot{\mathrm{U}}\left(C^{1}, C^{2}\right)$ definido de forma análoga. As Tabelas 6.16 e 6.17 apresentam o comportamento das variáveis $\dot{a}$ e $\dot{d}$ para as comparações dos agrupamentos $\mathrm{U}, \dot{\mathrm{U}}$ e $\hat{\mathrm{U}}$. Observe que a soma dos componentes do segundo e do último blocos da Tabela 6.17 resulta em zero. Isto é,

$$
\begin{array}{r}
\sum_{i \in C_{\mathrm{s}}^{1}, j \in C_{\mathrm{r}}^{1}}\left(\min \left\{\mathrm{S}_{i, j}^{\mathrm{U}}, \mathrm{S}_{i, j}^{\dot{\mathrm{U}}}\right\}-\min \left\{\mathrm{S}_{i, j}^{\hat{\mathrm{U}}}, \mathrm{S}_{i, j}^{\dot{\mathrm{U}}}\right\}\right) \\
+\sum_{i \in C_{\mathrm{s}}^{1}, j \in C_{\mathrm{r}}^{2}}\left(\min \left\{\mathrm{S}_{i, j}^{\mathrm{U}}, \mathrm{S}_{i, j}^{\dot{\mathrm{U}}}\right\}-\min \left\{\mathrm{S}_{i, j}^{\hat{\mathrm{U}}}, \mathrm{S}_{i, j}^{\dot{\mathrm{U}}}\right\}\right)=0
\end{array}
$$

$\mathrm{e}$

$$
\begin{array}{r}
\sum_{i \in C_{\mathrm{s}}^{2}, j \in C_{\mathrm{r}}^{2}}\left(\min \left\{\mathrm{S}_{i, j}^{\mathrm{U}}, \mathrm{S}_{i, j}^{\dot{U}}\right\}-\min \left\{\mathrm{S}_{i, j}^{\hat{\mathrm{U}}}, \mathrm{S}_{i, j}^{\dot{U}}\right\}\right) \\
+\sum_{i \in C_{\mathrm{r}}^{1}, j \in C_{\mathrm{s}}^{2}}\left(\min \left\{\mathrm{S}_{i, j}^{\mathrm{U}}, \mathrm{S}_{i, j}^{\dot{\mathrm{U}}}\right\}-\min \left\{\mathrm{S}_{i, j}^{\hat{\mathrm{U}}}, \mathrm{S}_{i, j}^{\dot{\mathrm{U}}}\right\}\right)=0
\end{array}
$$

Pelo primeiro bloco da Tabela 6.16 e pelo terceiro bloco da Tabela 6.17, conclui-se que

\begin{tabular}{|c|}
\hline $\begin{array}{l}\dot{a}^{\mathrm{U}, \dot{\mathrm{U}}}\left(C_{\mathrm{s}}^{1}, C_{\mathrm{n}}^{1}\right)=\min \{1,1\}>\min \{0,1\}=\dot{a}^{\hat{\mathrm{U}}, \dot{\mathrm{U}}}\left(C_{\mathrm{s}}^{1}, C_{\mathrm{n}}^{1}\right) \\
\dot{a}^{\mathrm{U}, \dot{\mathrm{U}}}\left(C_{\mathrm{s}}^{2}, C_{\mathrm{n}}^{2}\right)=\min \{1,1\}>\min \{0,1\}=\dot{a}^{\hat{\mathrm{U}}, \dot{\mathrm{U}}}\left(C_{\mathrm{s}}^{2}, C_{\mathrm{n}}^{2}\right)\end{array}$ \\
\hline $\begin{array}{l}\dot{a}^{\mathrm{U}, \dot{\mathrm{U}}}\left(C_{\mathrm{s}}^{1}, C_{\mathrm{r}}^{1}\right)=\min \{1,0\}=\min \{0,0\}=\dot{a}^{\hat{\mathrm{U}}, \dot{\mathrm{U}}}\left(C_{\mathrm{s}}^{1}, C_{\mathrm{r}}^{1}\right) \\
\dot{a}^{\mathrm{U}, \dot{\mathrm{U}}}\left(C_{\mathrm{s}}^{2}, C_{\mathrm{r}}^{2}\right)=\min \{1,0\}=\min \{0,0\}=\dot{a}^{\hat{\mathrm{U}}, \dot{\mathrm{U}}^{2}}\left(C_{\mathrm{s}}^{2}, C_{\mathrm{r}}^{2}\right)\end{array}$ \\
\hline $\begin{array}{l}\dot{a}^{\mathrm{U}, \mathrm{U}}\left(C_{\mathrm{s}}^{1}, C_{\mathrm{n}}^{2}\right)=\min \{0,0\}=\min \{1,0\}=\dot{a}^{\hat{\mathrm{U}}, \dot{\mathrm{U}}}\left(C_{\mathrm{s}}^{1}, C_{\mathrm{n}}^{2}\right) \\
\dot{a}^{\mathrm{U}, \dot{\mathrm{U}}}\left(C_{\mathrm{n}}^{1}, C_{\mathrm{s}}^{2}\right)=\min \{0,0\}=\min \{1,0\}=\dot{a}^{\hat{\mathrm{U}}, \dot{\mathrm{U}}^{2}}\left(C_{\mathrm{n}}^{1}, C_{\mathrm{s}}^{2}\right)\end{array}$ \\
\hline $\begin{array}{l}\dot{a}^{\mathrm{U}, \mathrm{U}}\left(C_{\mathrm{s}}^{1}, C_{\mathrm{r}}^{2}\right)=\min \{0,0\}=\min \{1,0\}=\dot{a}^{\hat{\mathrm{U}}, \dot{\mathrm{U}}}\left(C_{\mathrm{s}}^{1}, C_{\mathrm{r}}^{2}\right) \\
\dot{a}^{\mathrm{U}, \dot{\mathrm{U}}}\left(C_{\mathrm{r}}^{1}, C_{\mathrm{s}}^{2}\right)=\min \{0,0\}=\min \{1,0\}=\dot{a}^{\hat{\mathrm{U}}, \dot{\mathrm{U}}^{2}}\left(C_{\mathrm{r}}^{1}, C_{\mathrm{s}}^{2}\right)\end{array}$ \\
\hline
\end{tabular}
$13 \mathrm{GRI}(\mathrm{U}, \dot{\mathrm{U}}) \geq 13 \mathrm{GRI}(\hat{\mathrm{U}}, \dot{\mathrm{U}})$ e que a igualdade é atingida se, e somente se, $C_{\mathrm{n}}^{1}=C_{\mathrm{n}}^{2}=\emptyset$ (equivalentemente, $x=\left|\mathrm{I}\left(B_{i_{1}}, \dot{B}_{\operatorname{mi}\left(i_{1}\right)}\right)\right|=\left|\mathrm{I}\left(B_{i_{2}}, \dot{B}_{\operatorname{mi}\left(i_{2}\right)}\right)\right|$ ).

Tabela 6.16: Diferenças em $\dot{a} \cdot \cdot(\cdot, \cdot)$ ao passar de U para $\hat{\mathrm{U}}$. 
Pelo fato dos bi-agrupamentos $B, \hat{B}$ e $\dot{B}$ não possuírem sobreposição, a permutação de elementos descrita pela Def. 7 resulta na mera alteração da ordem das colunas de U para a definição da matriz $\hat{\mathrm{U}}$. Portanto, E[13GRI $]_{\mathrm{U}, \dot{U}}=\mathrm{E}[13 \mathrm{GRI}]_{\hat{\mathrm{U}}, \dot{\mathrm{U}}} \cdot \operatorname{Logo}, 13 \mathrm{AGRI}(\mathrm{U}, \dot{\mathrm{U}}) \geq$ $13 \mathrm{AGRI}(\hat{\mathrm{U}}, \dot{\mathrm{U}})$ e $\mathbb{S}_{\text {agri }}(B, \dot{B}) \geq \mathbb{S}_{\text {agri }}(\hat{B}, \dot{B})$, em que a igualdade ocorre se, e somente se, $x=$ $\left|\mathrm{I}\left(B_{i_{1}}, \dot{B}_{\mathrm{mi}\left(i_{1}\right)}\right)\right|=\left|\mathrm{I}\left(B_{i_{2}}, \dot{B}_{\mathrm{mi}\left(i_{2}\right)}\right)\right|$.

Tabela 6.17: Diferenças em $\dot{d} \cdot \cdot(\cdot, \cdot)$ ao passar de U para $\hat{U}$.

\begin{tabular}{|c|}
\hline $\begin{array}{l}\dot{d}^{\mathrm{U}, \dot{\mathrm{U}}}\left(C_{\mathrm{s}}^{1}, C_{\mathrm{n}}^{1}\right)=\min \{0,0\}=\min \{1,0\}=\dot{d} \hat{\mathrm{U}}, \dot{\mathrm{U}}\left(C_{\mathrm{s}}^{1}, C_{\mathrm{n}}^{1}\right) \\
\dot{d}^{\mathrm{U}, \dot{\mathrm{U}}}\left(C_{\mathrm{s}}^{2}, C_{\mathrm{n}}^{2}\right)=\min \{0,0\}=\min \{1,0\}=\dot{d} \hat{\mathrm{U}}, \dot{\mathrm{U}}\left(C_{\mathrm{s}}^{2}, C_{\mathrm{n}}^{2}\right)\end{array}$ \\
\hline $\begin{array}{l}\dot{d}^{\mathrm{U}, \dot{\mathrm{U}}}\left(C_{\mathrm{s}}^{1}, C_{\mathrm{r}}^{1}\right)=\min \{0,1\}<\min \{1,1\}=\dot{d} \hat{\mathrm{U}}, \dot{\mathrm{U}}\left(C_{\mathrm{s}}^{1}, C_{\mathrm{r}}^{1}\right) \\
\dot{d}^{\mathrm{U}, \dot{\mathrm{U}}}\left(C_{\mathrm{s}}^{2}, C_{\mathrm{r}}^{2}\right)=\min \{0,1\}<\min \{1,1\}=\dot{d}^{\hat{\mathrm{U}}, \dot{\mathrm{U}}}\left(C_{\mathrm{s}}^{2}, C_{\mathrm{r}}^{2}\right)\end{array}$ \\
\hline $\begin{array}{l}\dot{d}^{\mathrm{U}, \dot{\mathrm{U}}}\left(C_{\mathrm{s}}^{1}, C_{\mathrm{n}}^{2}\right)=\min \{1,1\}>\min \{0,1\}=\dot{d}^{\hat{\mathrm{U}}, \mathrm{U}}\left(C_{\mathrm{s}}^{1}, C_{\mathrm{n}}^{2}\right) \\
\dot{d}^{\mathrm{U}, \dot{\mathrm{U}}}\left(C_{\mathrm{n}}^{1}, C_{\mathrm{s}}^{2}\right)=\min \{1,1\}>\min \{0,1\}=\dot{d}^{\hat{\mathrm{U}}, \dot{\mathrm{U}}}\left(C_{\mathrm{n}}^{1}, C_{\mathrm{s}}^{2}\right)\end{array}$ \\
\hline $\begin{array}{l}\dot{d}^{\mathrm{U}, \dot{\mathrm{U}}}\left(C_{\mathrm{s}}^{1}, C_{\mathrm{r}}^{2}\right)=\min \{1,1\}>\min \{0,1\}=\dot{d^{\mathrm{U}}, \dot{\mathrm{U}}}\left(C_{\mathrm{s}}^{1}, C_{\mathrm{r}}^{2}\right) \\
\dot{d}^{\mathrm{U}, \dot{\mathrm{U}}}\left(C_{\mathrm{r}}^{1}, C_{\mathrm{s}}^{2}\right)=\min \{1,1\}>\min \{0,1\}=\dot{d}^{\hat{\mathrm{U}}, \dot{\mathrm{U}}}\left(C_{\mathrm{r}}^{1}, C_{\mathrm{s}}^{2}\right)\end{array}$ \\
\hline
\end{tabular}

Proposição 6.30. Tem-se $\mathbb{S}_{\mathrm{ce}}(B, \dot{B})=1$ se, e somente se, $B \equiv \dot{B}$.

Prova. Claramente, $B \equiv \dot{B}$ implica que $\mathbb{S}_{\mathrm{ce}}=1$. Seja $k$ o número de bi-grupos em $B$ e $q$ o número de bi-grupos em $\dot{B}$. Suponha que $\mathbb{S}_{\mathrm{ce}}(B, \dot{B})=1$ e seja $\left\{\left(t_{i}, y_{i}\right)\right\}_{i=1}^{\min \{k, q\}}$ a relação única que maximiza a Eq. (4.17). Note que $|U| \geq \sum_{j_{1}, j_{2}} \mathrm{~N}_{j_{1}, j_{2}}=\sum_{i=1}^{k}\left|B_{i}^{\mathrm{r}} \times B_{i}^{\mathrm{c}}\right| \geq d_{\max } \mathrm{e}|U| \geq$ $\sum_{j_{1}, j_{2}} \dot{\mathrm{N}}_{j_{1}, j_{2}}=\sum_{i=1}^{q}\left|\dot{B}_{i}^{\mathrm{r}} \times \dot{B}_{i}^{\mathrm{c}}\right| \geq d_{\text {max }}$. Tem-se de $|U|=d_{\max }$ que $\sum_{i=1}^{k}\left|B_{i}^{\mathrm{r}} \times B_{i}^{\mathrm{c}}\right|=d_{\max } \leq$ $\sum_{i=1}^{\min \{k, q\}}\left|B_{t_{i}}^{\mathrm{r}} \times B_{t_{i}}^{\mathrm{c}}\right| \leq \sum_{i=1}^{k}\left|B_{i}^{\mathrm{r}} \times B_{i}^{\mathrm{c}}\right|$, implicando que $\sum_{i=1}^{k}\left|B_{i}^{\mathrm{r}} \times B_{i}^{\mathrm{c}}\right|=\sum_{i=1}^{\min \{k, q\}}\left|B_{t_{i}}^{\mathrm{r}} \times B_{t_{i}}^{\mathrm{c}}\right|$ e $k=\min \{k, q\}$. Similarmente, $\sum_{i=1}^{q}\left|\dot{B}_{i}^{\mathrm{r}} \times \dot{B}_{i}^{\mathrm{c}}\right|=\sum_{i=1}^{\min \{k, q\}}\left|\dot{B}_{y_{i}}^{\mathrm{r}} \times \dot{B}_{y_{i}}^{\mathrm{c}}\right|$ e $q=\min \{k, q\}$. Logo, $k=q$. De $d_{\text {max }}=\sum_{i=1}^{\min \{k, q\}}\left|B_{t_{i}}^{\mathrm{r}} \times B_{t_{i}}^{\mathrm{c}}\right|$ tem-se $B_{t_{i}}^{\mathrm{r}} \times B_{t_{i}}^{\mathrm{c}} \subseteq \dot{B}_{y_{i}}^{\mathrm{r}} \times \dot{B}_{y_{i}}^{\mathrm{c}}$ para todo $i$. Similarmente, $\dot{B}_{y_{i}}^{\mathrm{r}} \times \dot{B}_{y_{i}}^{\mathrm{c}} \subseteq B_{t_{i}}^{\mathrm{r}} \times B_{t_{i}}^{\mathrm{c}}$ para todo $i$ e, então, $B_{t_{i}}^{\mathrm{r}} \times B_{t_{i}}^{\mathrm{c}}=\dot{B}_{y_{i}}^{\mathrm{r}} \times \dot{B}_{y_{i}}^{\mathrm{c}}$ para todo $i$. Logo, $B \equiv \dot{B}$.

Proposição 6.31. Tem-se $\mathbb{S}_{\mathrm{fabi}}(B, \dot{B})=1$ se, e somente $s e, B \equiv \dot{B}$.

Prova. Claramente, $B \equiv \dot{B}$ implica que $\mathbb{S}_{\mathrm{fabi}}=1$. Suponha que $\mathbb{S}_{\mathrm{fabi}}(B, \dot{B})=1$ e seja $\left\{\left(t_{i}, y_{i}\right)\right\}_{i=1}^{\min \{k, q\}}$ a relação única ótima requerida por $\mathbb{S}_{\text {fabi }}$. Os denominadores da Eq. (4.25) mostram que $\mathbb{S}_{\mathrm{fabi}}$ atinge 1 apenas quando comparando soluções com o mesmo número de bigrupos, tornando $\left\{\left(t_{i}, y_{i}\right)\right\}_{i=1}^{\min \{k, q\}}$ uma bijeção. $\mathbb{S}_{\text {fabi }}$ atinge 1 apenas se $\mathbb{J}\left(B_{t_{i}}, \dot{B}_{y_{i}}\right)=1$ para todo $i$ e $\mathbb{J}\left(B_{t_{i}}, \dot{B}_{y_{i}}\right)$ atinge 1 apenas se $B_{t_{i}} \equiv \dot{B}_{y_{i}}$. Logo, $B \equiv \dot{B}$.

Proposição 6.32. Existem dois bi-agrupamentos não equivalentes $B$ e $\dot{B}$ tais que $\mathbb{S}_{\mathrm{csi}}(B, \dot{B})=$ $\mathbb{S}_{\mathrm{ebc}}(B, \dot{B})=\mathbb{S}_{\mathrm{agri}}(B, \dot{B})=1$.

Prova. Seja $B$ um bi-agrupamento no qual um ou mais elementos não foi bi-agrupado. Adicione um bi-grupo em $B$ que possui apenas um dos elementos que não foram bi-agrupados 
e seja $\dot{B}$ a solução resultante. Aplicando a abordagem de avaliação proposta na Seção 6.2 em $B$ e $\dot{B}$, tem-se dois agrupamentos particionais não exclusivos $P$ e $\dot{P}$ equivalentes, implicando que $10 \mathrm{CSI}(P, \dot{P})=09 \mathrm{EBC}(P, \dot{P})=13 \mathrm{AGRI}(\mathrm{U}, \dot{\mathrm{U}})=1$ e $\mathbb{S}_{\mathrm{csi}}(B, \dot{B})=\mathbb{S}_{\mathrm{ebc}}(B, \dot{B})=$ $\mathbb{S}_{\text {agri }}(B, \dot{B})=1$.

Proposição 6.33. Existem dois bi-agrupamentos $B$ e $\dot{B}$ não equivalentes e sem degeneração para os quais $\mathbb{S}_{\mathrm{csi}}(B, \dot{B})=\mathbb{S}_{\mathrm{ebc}}(B, \dot{B})=\mathbb{S}_{\mathrm{agri}}(B, \dot{B})=1$.

Prova. Os bi-agrupamentos da Figura 6.10 são dados por $B \triangleq\left\{B_{i}\right\}_{i=1}^{4}, \dot{B} \triangleq\left\{\dot{B}_{i}\right\}_{i=1}^{3}$, $B_{1} \triangleq(\{1\},\{1,2\}), B_{2} \triangleq(\{2\},\{1,2\}), B_{3} \triangleq(\{3\},\{1,2\}), B_{4} \triangleq(\{1,2,3\},\{1,2\})$, $\dot{B}_{1} \triangleq(\{1,2\},\{1,2\}), \dot{B}_{2} \triangleq(\{2,3\},\{1,2\})$ e $\dot{B}_{3} \triangleq(\{1,3\},\{1,2\})$. Tem-se $P=\left\{P_{i}\right\}_{i=1}^{4}$ e $\dot{P}=\left\{\dot{P}_{i}\right\}_{i=1}^{3}$, para os quais $P_{1}=\left\{\tilde{o}_{1}, \tilde{o}_{4}\right\}, P_{2}=\left\{\tilde{o}_{2}, \tilde{o}_{5}\right\}, P_{3}=\left\{\tilde{o}_{3}, \tilde{o}_{6}\right\}, P_{4}=\left\{\tilde{o}_{j}\right\}_{j=1}^{6}$, $\dot{P}_{1}=\left\{\tilde{o}_{1}, \tilde{o}_{2}, \tilde{o}_{4}, \tilde{o}_{5}\right\}, \dot{P}_{2}=\left\{\tilde{o}_{2}, \tilde{o}_{3}, \tilde{o}_{5}, \tilde{o}_{6}\right\}$ e $\dot{P}_{3}=\left\{\tilde{o}_{1}, \tilde{o}_{3}, \tilde{o}_{4}, \tilde{o}_{6}\right\}$.

Note que $\alpha_{P}\left(\tilde{o}_{j_{1}}, \tilde{o}_{j_{2}}\right)=\alpha_{\dot{P}}\left(\tilde{o}_{j_{1}}, \tilde{o}_{j_{2}}\right)$ para todo $j_{1}$ e $j_{2}$, e tem-se $\beta_{P}\left(\tilde{o}_{j}\right)=\beta_{\dot{P}}\left(\tilde{o}_{j}\right)$ para todo $j$. $\operatorname{Logo}, d_{G}^{P, \dot{P}}=0,10 \operatorname{CSI}(P, \dot{P})=1$ e $\mathbb{S}_{\mathrm{csi}}(B, \dot{B})=1$. Similarmente, $\mathbb{S}_{\mathrm{ebc}}(B, \dot{B})=$ $\mathbb{S}_{\text {agri }}(B, \dot{B})=1$.

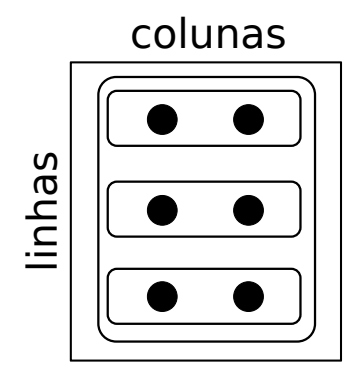

(a) Bi-agrupamento $B$.

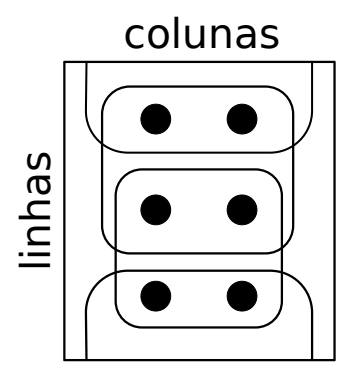

(b) Bi-agrupamento $\dot{B}$.

Figura 6.10: Bi-agrupamentos sem distinção para $\mathbb{S}_{\mathrm{csi}}, \mathbb{S}_{\mathrm{ebc}}$ e $\mathbb{S}_{\text {agri }}$

Visto que $P \not \equiv \dot{P}$, a prova acima contradiz a Proposição 1 de (Campello, 2010), a qual afirma (em termos de matrizes) que $10 \mathrm{CSI}(P, \dot{P})=1$ se, e somente se, $P \equiv \dot{P}$.

\subsection{Considerações}

Neste capítulo foi apresentada uma nova abordagem de avaliação externa de biagrupamentos. Ela consiste na transformação da solução de bi-agrupamento original em um agrupamento particional não exclusivo, permitindo o uso de medidas de similaridade projetadas para esse último tipo de agrupamento na comparação de soluções de bi-agrupamento.

Formalizou-se oito propriedades que uma medida de bi-agrupamento deve possuir, discutiuse sobre a relevância delas e provou-se quais propriedades cada medida apresenta. A relevância prática das propriedades foram aferidas em experimentos baseados em modelos de bi-grupos e algoritmos de bi-agrupamento usados em estudos de grande importância da literatura. 
As medidas $\mathbb{S}_{\mathrm{ce}}$, $\mathbb{S}_{\mathrm{csi}}$ e $\mathbb{S}_{\mathrm{ebc}}$ se destacaram como as melhores na comparação teórica. Entretanto, a medida $\mathbb{S}_{\mathrm{ebc}}$ apresentou um comportamento problemático na análise empírica. A abundância de elementos ruidosos na matriz de dados (o que é comum em dados de expressão gênica) dominou a avaliação realizada por $\mathbb{S}_{\mathrm{ebc}}$, levando-a a atingir um valor muito alto para uma solução claramente ruim. O estudo relatado neste capítulo sugere que as medidas $\mathbb{S}_{\mathrm{ce}}$ e $\mathbb{S}_{\mathrm{csi}}$ devem ser preferidas na comparação de bi-agrupamentos.

Um estudo futuro importante seria o da análise do comportamento das medidas para biagrupamentos gerados aleatoriamente. O Capítulo 5 e outros estudos (Vinh et al., 2009, 2010) mostram a importância de uma medida de similaridade entre agrupamentos apresentar uma avaliação constante para soluções geradas aleatoriamente, como uma estratégia para combater o viés na avaliação. 


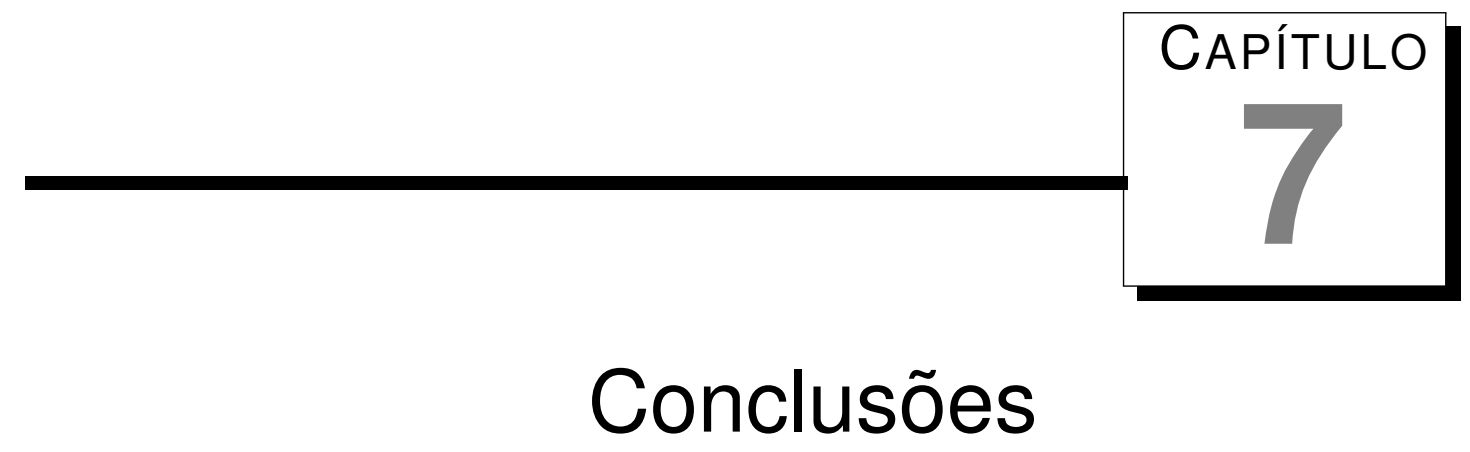

Os algoritmos SCAD e CARD, voltados para bases de dados multi-representadas (i.e., bases descritas por mais de uma representação dos dados), possuem problemas, tais como o não tratamento de pontos críticos de duas de suas equações de otimização, que os impedem de funcionar em determinados cenários. Foi proposta uma nova versão do algoritmo SCAD, referido por ASCAD, imune aos problemas detectados e cujo funcionamento foi provado formalmente. Desenvolveu-se uma versão relacional do algoritmo ASCAD, referida por RASCAD, capaz de lidar com bases de dados descritas por meio apenas das relações de proximidade entre os objetos. Argumentou-se que o algoritmo RASCAD é conceitualmente superior ao algoritmo CARD também relacional, além de não possuir os problemas identificados para esse último. Provou-se a equivalência dos algoritmos ASCAD e RASCAD, sob a suposição de que a matriz relacional é euclidiana. O algoritmo RASCAD foi generalizado para lidar com todos os tipos de matrizes relacionais (i.e., tanto as euclidianas como as não euclidianas) por meio de uma técnica chamada de espalhamento $\beta$.

Os algoritmos propostos foram avaliados experimentalmente quanto à capacidade de ponderar as múltiplas representações dos dados e à robustez a bases com atributos ruidosos, entre outros aspectos. ASCAD e, por consequência da equivalência, RASCAD se desempenharam melhor que as suas contrapartidas voltadas para bases de dados de uma única representação, sugerindo que a agregação ponderada das representações foi responsável pela melhor acurácia dos resultados e corroborando com a Hipótese 1 discutida na Seção 1.

Com relação à Hipótese 2, foi proposto um índice de validação interna e relativa de agrupamento de dados que considera a ponderação das representações na avaliação da solução de agrupamento. Esse índice foi usado em uma abordagem para estimar o melhor número de grupos, apresentando melhores resultados que a sua contrapartida voltada para bases representadas por apenas uma representação dos dados.

Apresentou-se uma revisão de 28 medidas de similaridade entre agrupamentos mais gerais 
que os agrupamentos particionais exclusivos, a saber, agrupamentos particionais não exclusivos, nebulosos/probabilísticos e possibilísticos. Os problemas identificados para as medidas revisadas motivaram a proposição de novas medidas, o que culminou no desenvolvimento da medida 13GRI. Essa medida foi, então, ajustada de forma a atribuir avaliações constantes e próximas de zero para agrupamentos gerados aleatoriamente, dando origem à medida 13AGRI. Esse ajuste é uma estratégia utilizada para evitar que a medida favoreça determinados tipos de agrupamento, a despeito de sua similaridade com o agrupamento com o qual é comparado.

No primeiro conjunto de experimentos, as medidas desenvolvidas e revisadas foram aplicadas na comparação de agrupamentos com diferentes números de grupos gerados por algoritmos de agrupamento de diferentes naturezas. Nesse contexto, uma boa medida deve fornecer uma similaridade entre a solução produzida e a de referência proporcional à diferença do número de grupos. Como os algoritmos eram capazes de encontrar a solução ideal (i.e., a solução de referência), uma boa medida também deve fornecer o valor máximo de similaridade ao comparar a solução ideal (encontrada pelo algoritmo configurado com o número correto de grupos) com a solução de referência. Entretanto, os experimentos mostraram que muitas medidas não atingiram o seu valor máximo para a solução ideal, o que as tornam pouco confiáveis. No segundo conjunto de experimentos, avaliou-se a capacidade das medidas em fornecer avaliações imparciais para soluções geradas aleatoriamente com diferentes números de grupos. Isto é, uma boa medida de similaridade não deve favorecer um determinado tipo de agrupamento quando este é gerado de maneira independente do agrupamento de referência. Apenas as medidas 13AGRI e 09BARI foram imparciais em todos os cenários considerados. É importante mencionar que a medida 09BARI, ao contrário da 13AGRI, não apresentou o comportamento esperado no primeiro conjunto de experimentos; a medida 09BARI não atingiu o seu máximo ao comparar soluções ideais em todos os cenários. Portanto, os experimentos sugerem que a medida 13AGRI deve ser preferida.

Ainda com relação às medidas para agrupamentos gerais, a medida 13AGRI foi avaliada no cenário específico de agrupamentos nebulosos. Esse experimento envolveu a bem conhecida medida ARI, a qual foi aplicada em agrupamentos particionais exclusivos. Argumentou-se que, para o contexto em questão, ambas deviam fornecer valores parecidos, dada à semelhança entre os agrupamentos particionais exclusivos e nebulosos no cenário considerado. Esse comportamento foi observado, corroborando com a validade das avaliações da medida 13AGRI para o contexto nebuloso. Por fim, a medida 13AGRI foi utilizada como uma estatística de estabilidade para conjunto de agrupamentos, com o objetivo de estimar o número correto de grupos em bases de dados reais. Essa abordagem mostrou-se promissora, pois a estatística atribuiu valores baixos de instabilidade para conjuntos de agrupamentos com números de grupos próximos ou iguais ao número correto. Os bons resultados para a medida 13AGRI apontam para a validade da Hipótese 3.

Com relação à Hipótese 4, apresentou-se uma revisão de 14 medidas de similaridade entre bi-agrupamentos. Mencionou-se que a associação entre bi-grupos da solução encontrada com bi-grupos da solução de referência que a maioria das medidas de bi-agrupamento realiza leva 
ao problema da insensibilidade à homogeneidade da solução. Foram propostas e definidas formalmente oito propriedades de medidas de bi-agrupamento. A importância de cada uma foi explicitada e determinada por meio de experimentos envolvendo modelos de bi-grupos e algoritmos de bi-agrupamento propostos em estudos importantes da literatura de expressão gênica. Uma nova abordagem de comparação de bi-agrupamentos foi proposta, a qual permite o uso de medidas de similaridade entre agrupamentos particionais não exclusivos na comparação de bi-agrupamentos. As 14 medidas revisadas em conjunto com três medidas adequadas para agrupamento particional não exclusivo (i.e., $\mathbb{S}_{\mathrm{csi}}, \mathbb{S}_{\mathrm{ebc}}$ e $\mathbb{S}_{\text {agri }}$ ) utilizadas por meio da abordagem proposta foram analisadas teoricamente de acordo com as propriedades definidas. Provou-se quais propriedades cada uma das medidas possui. Por fim, as análises empírica e teórica sugerem que as medidas $\mathbb{S}_{\text {ce }}$ (proposta por Patrikainen e Meila (2006)) e $\mathbb{S}_{\text {csi }}$ (cujo uso foi possibilitado pela abordagem proposta aqui) devem ser preferidas na comparação de bi-agrupamentos, dentre as medidas estudadas.

\subsection{Trabalhos futuros}

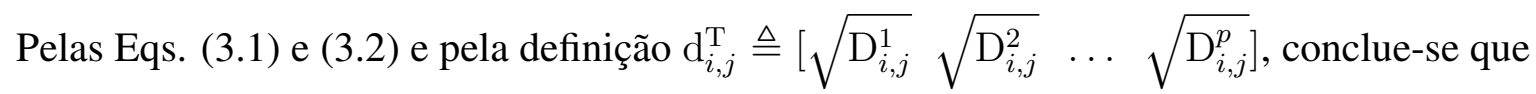
a distância total entre um objeto e um grupo de acordo com o algoritmo ASCAD é dado pelo produto interno definido pela matriz diagonal $\left[\mathrm{Y}_{i}\right]_{s, s} \triangleq \mathrm{Y}_{i, s}$ :

$$
\mathrm{d}_{i, j}^{\mathrm{T}} \mathrm{Y}_{i}^{q} \mathrm{~d}_{i, j}
$$

É portanto interessante investigar o uso de um produto interno mais geral, definido por meio de uma matriz não diagonal, para explorar um relacionamento mais complexo entre as distâncias parciais.

Como mencionado nas conclusões do Capítulo 5 , é interessante uma investigação das propriedades conceituais que a medida 13AGRI pode apresentar, como sensibilidade à homogeneidade, sensibilidade à completude de grupo e observância dos axiomas métricos (Meila, 2007; Amigó et al. 2009). Esse estudo proveria um entendimento mais geral da medida e destacaria eventuais problemas conceituais que poderia motivar o seu melhoramento.

A análise sobre medidas de bi-agrupamento apresentada no Capítulo 6 destacou as medidas $\mathbb{S}_{\mathrm{ce}}$ e $\mathbb{S}_{\mathrm{csi}}$ como sendo as mais confiáveis. No entanto, como não são corrigidas para a aleatoriedade, essas medidas podem apresentar um forte viés para determinados tipos de soluções, como visto no Capítulo 5 para outros tipos de medidas. A medida $\mathbb{S}_{\text {agri }}$ desenvolvida no Capítulo 5 e incluída na análise de medidas de bi-agrupamento no Capítulo 6é corrigida para aleatoriedade. Entretanto, ela não possui três das oito propriedades desejáveis de medidas de bi-agrupamento discutidas (i.e., (i) sensibilidade a bi-grupos espúrios; (ii) sensibilidade a área sem interseção; e (iii) adequação às condições necessária e suficiente para o máximo). Duas importantes frentes de pesquisa seriam, por exemplo, o ajuste das medidas $\mathbb{S}_{\mathrm{ce}}$ e $\mathbb{S}_{\mathrm{csi}}$ para aleatoriedade e a aprimoração da medida $\mathbb{S}_{\text {agri }}$ a fim de satisfazer as três propriedades ausentes. 
Determinadas medidas não apresentaram várias das propriedades analisadas (empírica e teoricamente) nesta tese e outras demonstraram um comportamento claramente inferior às medidas propostas. Com a disseminação desse conhecimento por meio da publicação de artigos e desta tese, espera-se fornecer um maior embasamento para futuras comparações de algoritmos de agrupamento de dados. Em especial, para algoritmos de agrupamentos mais gerais que os particionais exclusivos e para algoritmos de bi-agrupamento de dados. 


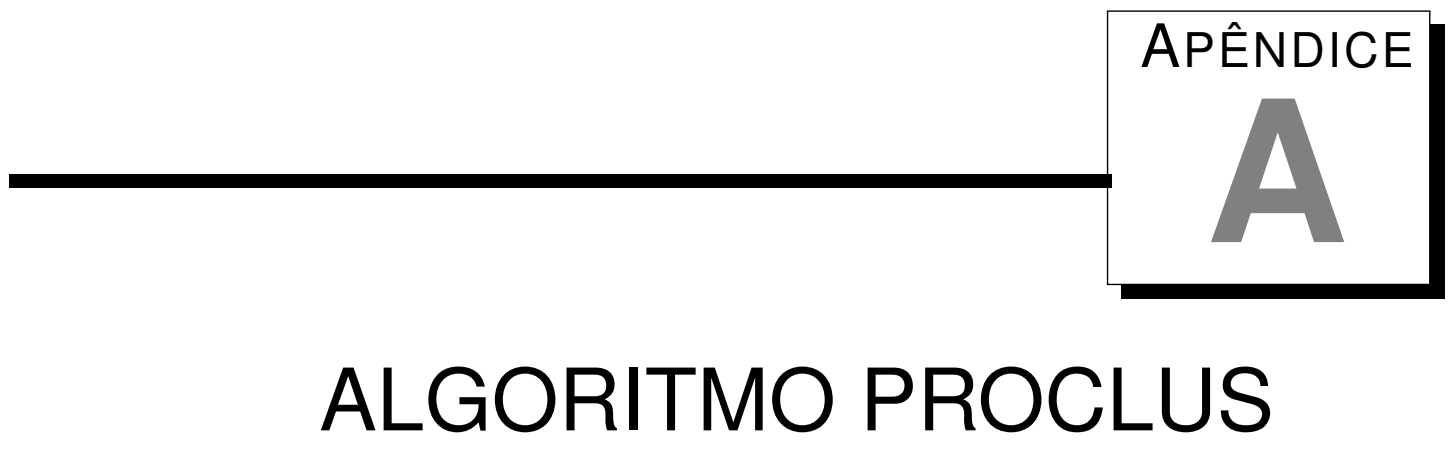

Provavelmente o primeiro algoritmo desenvolvido dessa categoria é o algoritmo PROCLUS (Aggarwal et al., 1999), o qual é descrito para exemplificar a categoria em questão. As soluções produzidas pelo PROCLUS são agrupamentos particionais cujos grupos têm associados a si subconjuntos de atributos sob os quais os objetos estão próximos entre si.

O PROCLUS é baseado nas técnicas que lidam com medóides (Ng e Han, 1994) para representar os grupos. Ele é dividido em três etapas e seu esquema geral pode ser visto pelo Algoritmo A.1. Na primeira etapa, realizada pela função Inicialização (Passo 1), cujo esquema geral se encontra no Algoritmo A.2, é feita uma amostragem $S$ de $a k$ objetos da base, em que $k$ é o número de grupos desejado e $a$ é uma constante escolhida pelo usuário. Em seguida, são realizados $b k$ passos, cuja constante $b$ também é escolhida pelo usuário, para encontrar $b k$ objetos que podem ser representativos. No primeiro passo, um objeto de $S$ é escolhido aleatoriamente e adicionado ao conjunto $\mathcal{M}$. Em cada um dos $b k-1$ passos seguintes, é encontrado o objeto de $S \backslash \mathcal{M}$ cuja menor distância a um dos objetos de $\mathcal{M}$ é a maior dentre os outros objetos de $S \backslash \mathcal{M}$. Esse objeto encontrado é então inserido em $\mathcal{M}$. O objetivo dessa etapa inicial é encontrar pelo menos um objeto de cada um dos $k$ grupos que eventualmente existam na base de dados.

Na segunda etapa, o algoritmo seleciona aleatoriamente $k$ objetos de $\mathcal{M}$ para serem medóides e então define os conjuntos $M_{\text {melhor }}$ e $M_{\text {atual }}$ contendo esses medóides iniciais. Em seguida, o algoritmo define os medóides ruins do conjunto $M_{\text {melhor }}$ O medóide do grupo com o menor número de objetos é considerado um medóide ruim (como os grupos são formados a partir dos medóides é discutido mais adiante). Em adição, os medóides cujos grupos tiverem menos de $(n / k) v$ objetos (em que $v$ é mais uma constante definida pelo usuário, obrigatoriamente menor que 1) também são considerados medóides ruins. Seja $t$ o número de medóides ruins de $M_{\text {melhor }}$. O algoritmo passa então a fazer sorteios aleatórios de $t$ objetos de $\mathcal{M}$, substituindo os medóides ruins de $M_{\text {atual }}$ pelos objetos sorteados. Em cada um desses sorteios, o conjunto de medóides de $M_{\text {atual }}$ é avaliado de acordo com uma função-custo descrita mais adiante. Caso o valor dessa 

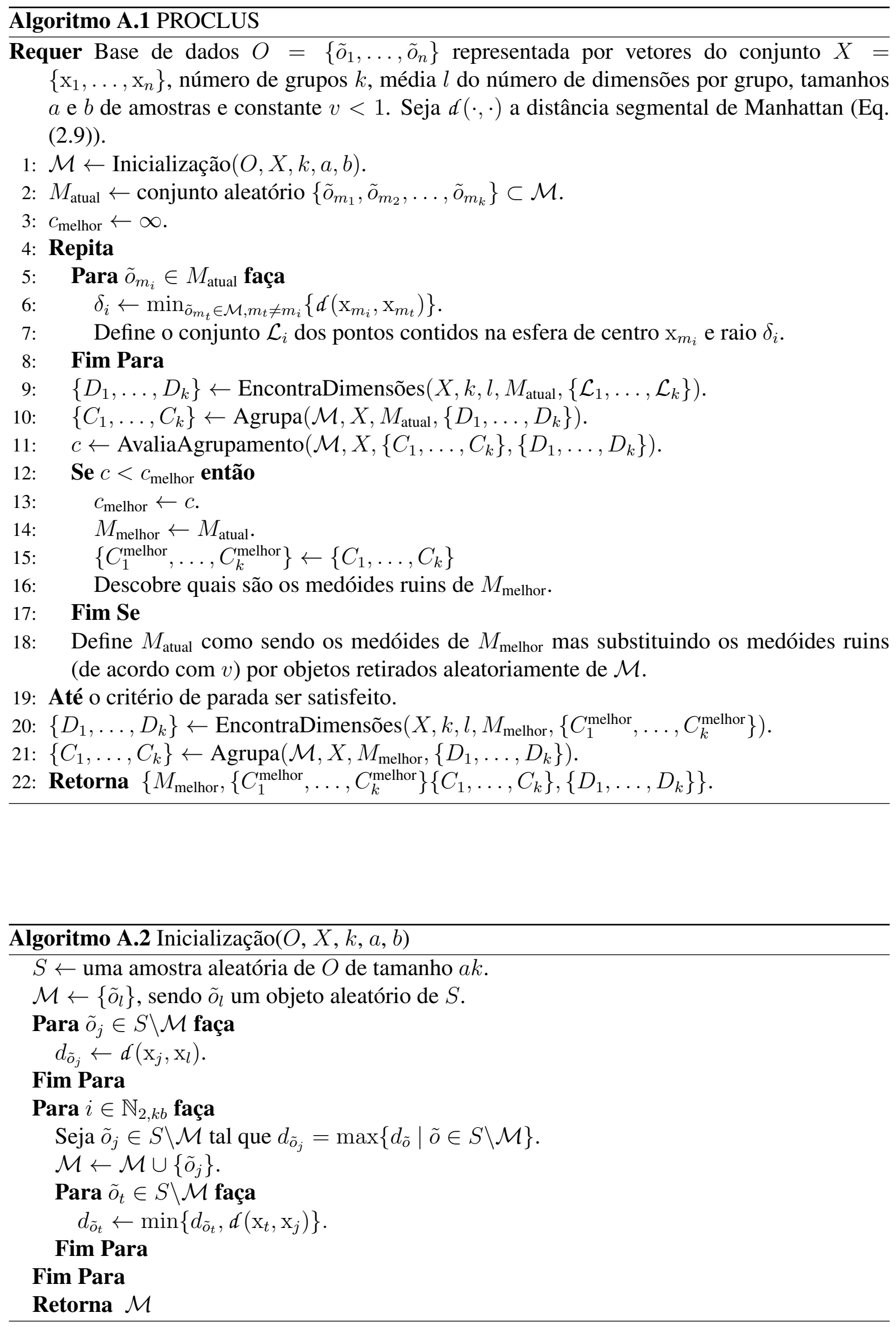
função-custo para os medóides de $M_{\text {atual }}$ seja menor que o valor da função-custo para os medóides de $M_{\text {melhor }}$, substitua todos os elementos de $M_{\text {melhor }}$ pelos de $M_{\text {atual }}$. Repita esse processo até que um critério de parada seja satisfeito; por exemplo, um determinado número de iterações sem encontrar alguma solução que seja superior à encontrada até o momento.
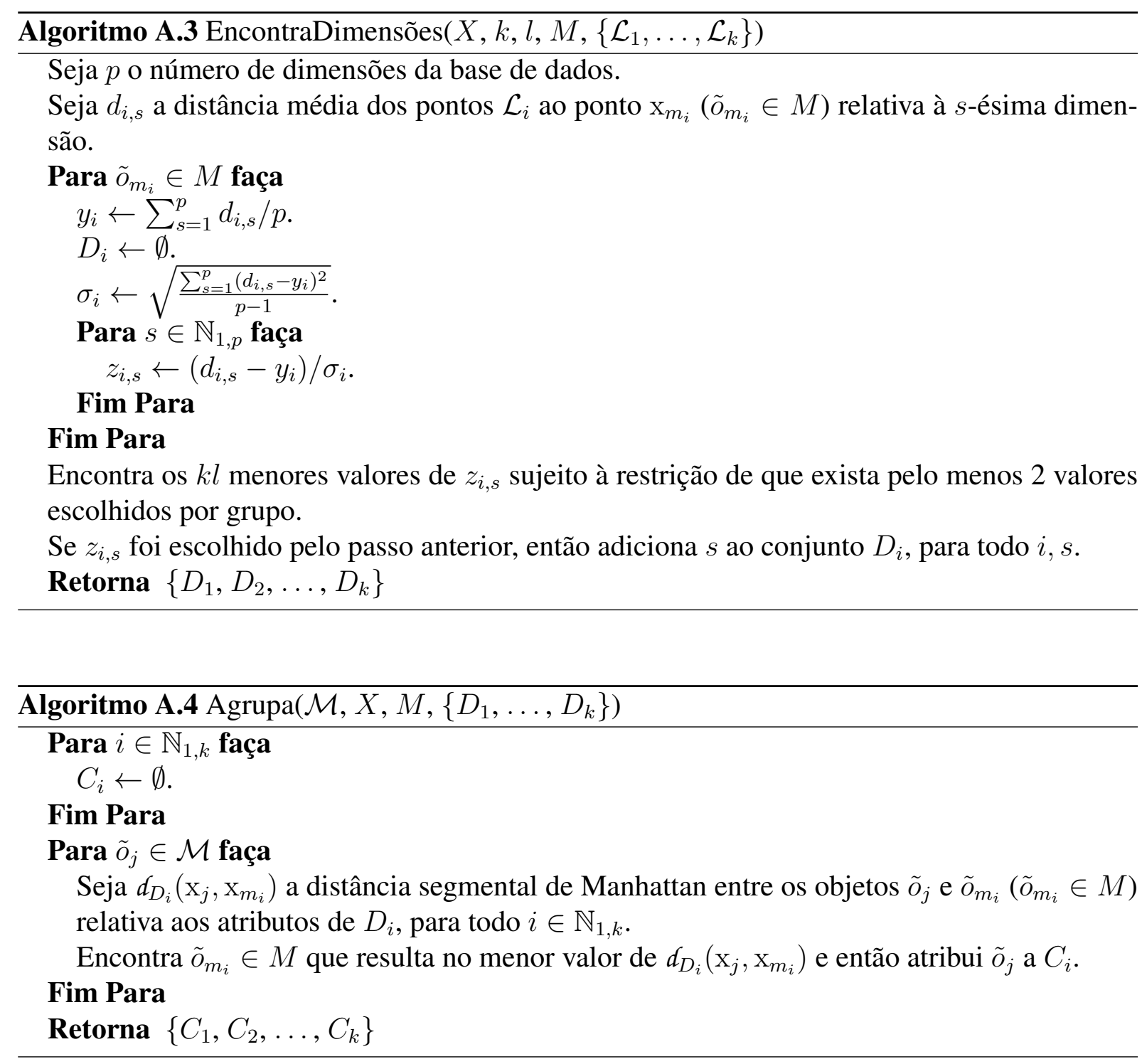

Para avaliar a qualidade dos $k$ medóides selecionados durante as iterações da segunda etapa do algoritmo é necessário resolver dois problemas: (a) encontrar o conjunto apropriado de dimensões para cada um desses medóides; (b) formar os grupos correspondentes aos $k$ medóides. As soluções para esses dois problemas são dadas a seguir.

- Encontra dimensões (Passo 9). Seja $M=\left\{\tilde{o}_{m_{1}}, \tilde{o}_{m_{2}}, \ldots, \tilde{o}_{m_{k}}\right\} \subset \mathcal{M}$ um conjunto de $k$ medóides. Defina os raios $\delta_{i}=\min _{\tilde{o}_{m_{t}} \in \mathcal{M}, m_{t} \neq m_{i}}\left\{d\left(\mathrm{x}_{m_{i}}, \mathrm{x}_{m_{t}}\right)\right\}$ para todo $i \in \mathbb{N}_{1, k}$ e, então, os conjuntos $\mathcal{L}_{1}, \ldots, \mathcal{L}_{k}$ de modo que $\mathcal{L}_{i}$ seja composto pelos pontos que estão dentro de um raio $\delta_{i}$ do ponto $\mathrm{x}_{m_{i}}$. Seja $d_{i, s}$ a média das distâncias dos pontos de $\mathcal{L}_{i}$ ao ponto $\mathrm{x}_{m_{i}}$ considerando apenas o $s$-ésimo atributo. $\mathrm{O}$ objetivo desse passo é associar as dimensões $s \in \mathbb{N}_{1, p}$ ao $i$-ésimo grupo tais que os valores $d_{i, s}$ sejam baixos com relação à esperança estatística (explicada em maiores detalhes mais adiante), sujeita às restrições 
de que o número total de dimensões associadas aos medóides seja igual a $k l$ ( $l$ é mais um parâmetro configurado pelo usuário) e o mínimo de atributos associados por grupo seja 2. Para cada medóide $\tilde{o}_{m_{i}}$, são calculados a esperança estatística $y_{i}=\sum_{s=1}^{p} d_{i, s} / p$ e o desvio-padrão $\sigma_{i}=\sqrt{\frac{\sum_{s=1}^{p}\left(d_{i, s}-y_{i}\right)^{2}}{p-1}}$, em que $p$ é o número de atributos da base de dados. De acordo com os valores $z_{i, s}=\left(d_{i, s}-y_{i}\right) / \sigma_{i}$ calculados para todo $i \in \mathbb{N}_{1, k}$ e $s \in \mathbb{N}_{1, p}, k l$ atributos distintos são associados aos grupos de modo que pelo menos 2 sejam associados a cada grupo e que a soma dos valores $z_{i, s}$ correspondentes aos grupos e aos atributos associados seja mínima;

- Agrupa os dados (Passo 10). Atribua os objetos da base aos grupos dos respectivos medóides mais próximos de acordo com a distância segmental de Manhattan Aggarwal et al., 1999). Naturalmente, no cálculo das distâncias, considere apenas os atributos que foram selecionados para o grupo do medóide em questão.

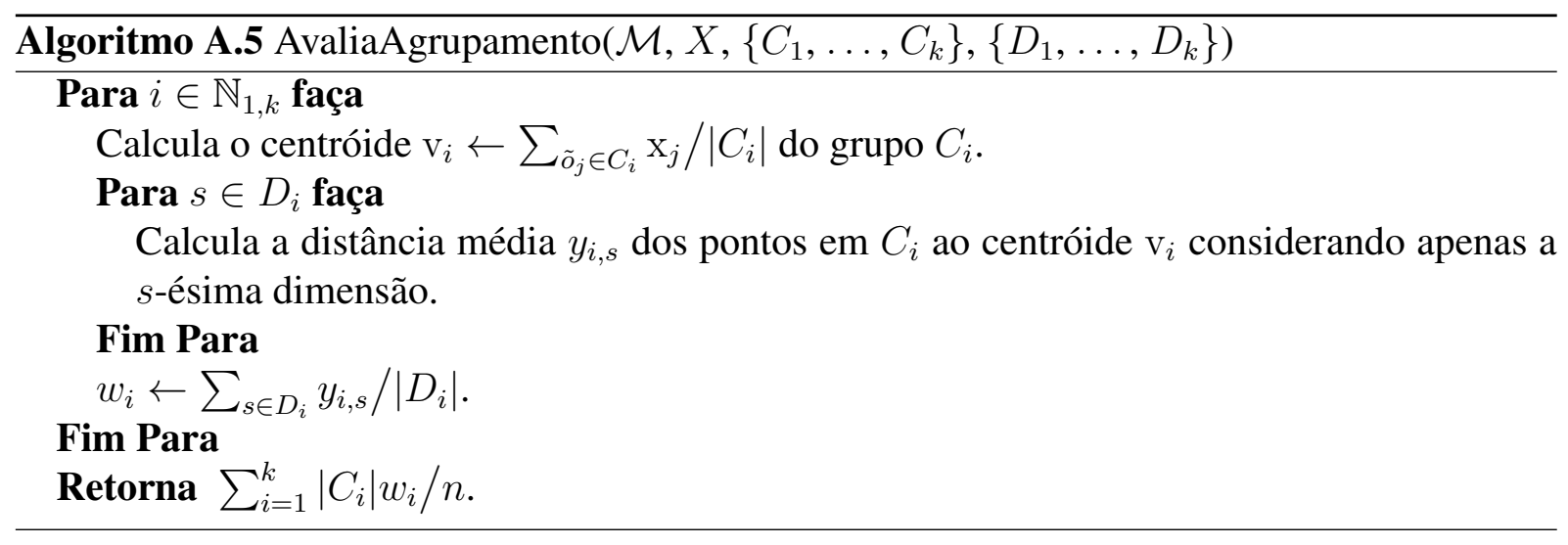

Tendo encontrado o agrupamento e as dimensões correspondentes aos medóides do conjunto $M_{\text {atual, }}$ o algoritmo PROCLUS chama o método descrito no Algoritmo A.5 para avaliar o conjunto $M_{\text {atual }}$ e os seus medóides. Com base nessa avaliação, o algoritmo PROCLUS descarta ou não a solução $M_{\text {atual }}$ e define quais são os medóides ruins.

A terceira e última etapa do algoritmo consiste no refinamento do agrupamento $\left\{C_{1}^{\text {melhor }}, \ldots, C_{k}^{\text {melhor }}\right\}$ encontrado. Para tanto, uma nova associação de dimensões aos grupos é realizada pelo Passo 20. Dessa vez, no entanto, os próprios objetos dos grupos são utilizados para encontrar as dimensões relevantes de cada grupo. Por fim, o melhor conjunto de medóides, o agrupamento e as dimensões associadas aos grupos são retornados como sendo a solução encontrada.

Embora o algoritmo PROCLUS seja de difícil utilização devido ao grande número de parâmetros, ele apresenta uma abordagem bastante utilizada pelos algoritmos baseados em projeção. Isto é, é associado a cada grupo o subconjunto de atributos que minimiza a variância das distâncias dos pontos desse grupo, ao levar em consideração no cálculo das distâncias apenas o subconjunto de atributos correspondente. 


\section{APÊNDICE}

\section{MEDIDAS 09EBC E 10CSI EM TERMOS DE CONJUNTOS}

Seja $O \triangleq\left\{\tilde{o}_{j}\right\}_{j=1}^{n}$ um conjunto de objetos e sejam $P$ e $\dot{P}$ dois agrupamentos particionais não exclusivos. Seja $\alpha_{P}\left(\tilde{o}_{j_{1}}, \tilde{o}_{j_{2}}\right)$ o número de grupos compartilhados pelos objetos $\tilde{o}_{j_{1}}$ e $\tilde{o}_{j_{2}}$ no agrupamento $P$ e seja $\alpha_{\dot{P}}\left(\tilde{o}_{j_{1}}, \tilde{o}_{j_{2}}\right)$ definido de maneira similar. A medida bcubed estendida (09EBC) Amigó et al. (2009) é baseada nas seguintes definições de precisão e revocação:

$$
\begin{array}{r}
\operatorname{EBCP}(P, \dot{P}) \triangleq \frac{1}{n} \sum_{j_{1}=1}^{n} \frac{\sum_{j_{2}=1}^{n} \min \left\{\alpha_{P}\left(\tilde{o}_{j_{1}}, \tilde{o}_{j_{2}}\right), \alpha_{\dot{P}}\left(\tilde{o}_{j_{1}}, \tilde{o}_{j_{2}}\right)\right\}}{\sum_{j_{2}=1}^{n} \alpha_{P}\left(\tilde{o}_{j_{1}}, \tilde{o}_{j_{2}}\right)} \\
\operatorname{EBCR}(P, \dot{P}) \triangleq \frac{1}{n} \sum_{j_{1}=1}^{n} \frac{\sum_{j_{2}=1}^{n} \min \left\{\alpha_{P}\left(\tilde{o}_{j_{1}}, \tilde{o}_{j_{2}}\right), \alpha_{\dot{P}}\left(\tilde{o}_{j_{1}}, \tilde{o}_{j_{2}}\right)\right\}}{\sum_{j_{2}=1}^{n} \alpha_{\dot{P}}\left(\tilde{o}_{j_{1}}, \tilde{o}_{j_{2}}\right)} .
\end{array}
$$

09EBC é definida por padrão como

$$
09 \mathrm{EBC}(P, \dot{P}) \triangleq 2 \cdot \frac{\operatorname{EBCP}(P, \dot{P}) \cdot \operatorname{EBCR}(P, \dot{P})}{\operatorname{EBCP}(P, \dot{P})+\operatorname{EBCR}(P, \dot{P})} .
$$

A medida 10CSI é definida como segue. Seja $\beta_{P}\left(\tilde{o}_{j}\right)$ o número de grupos para os quais o objeto $\tilde{o}_{j}$ pertence em $P$ menos 1 e seja $\beta_{\dot{P}}\left(\tilde{o}_{j}\right)$ definido de maneira similar. as concordâncias e 
discordâncias associadas ao par $\left(j_{1}, j_{2}\right)$ são

$$
\begin{aligned}
a_{G}^{P, \dot{P}}\left(\tilde{o}_{j_{1}}, \tilde{o}_{j_{2}}\right) & =\min \left\{\alpha_{P}\left(\tilde{o}_{j_{1}}, \tilde{o}_{j_{2}}\right), \alpha_{\dot{P}}\left(\tilde{o}_{j_{1}}, \tilde{o}_{j_{2}}\right)\right\} \\
& +\sum_{i=1}^{2} \min \left\{\beta_{P}\left(\tilde{o}_{j_{i}}\right), \beta_{\dot{P}}\left(\tilde{o}_{j_{i}}\right)\right\}
\end{aligned}
$$

$\mathrm{e}$

$$
\begin{aligned}
d_{G}^{P, \dot{P}}\left(\tilde{o}_{j_{1}}, \tilde{o}_{j_{2}}\right) & =\left|\alpha_{P}\left(\tilde{o}_{j_{1}}, \tilde{o}_{j_{2}}\right)-\alpha_{\dot{P}}\left(\tilde{o}_{j_{1}}, \tilde{o}_{j_{2}}\right)\right| \\
& +\sum_{i=1}^{2}\left|\beta_{P}\left(\tilde{o}_{j_{i}}\right)-\beta_{\dot{P}}\left(\tilde{o}_{j_{i}}\right)\right|,
\end{aligned}
$$

respectivamente. Somando $a_{G}^{P, \dot{P}}\left(\tilde{o}_{j_{1}}, \tilde{o}_{j_{2}}\right)$ e $d_{G}^{P, \dot{P}}\left(\tilde{o}_{j_{1}}, \tilde{o}_{j_{2}}\right)$ por todos os pares ordenados $\left(\tilde{o}_{j_{1}}, \tilde{o}_{j_{2}}\right)$ $\left(j_{1}<j_{2}\right)$ resulta na seguintes medidas gerais de concordância $\left(a_{G}^{P, \dot{P}}\right)$ e discordância $\left(d_{G}^{P, \dot{P}}\right)$ entre $P$ e $\dot{P}$ :

$$
\begin{aligned}
& a_{G}^{P, \dot{P}}=\sum_{j_{1}=1}^{n-1} \sum_{j_{2}=j_{1}+1}^{n} a_{G}^{P, \dot{P}}\left(\tilde{o}_{j_{1}}, \tilde{o}_{j_{2}}\right) \mathrm{e} \\
& d_{G}^{P, \dot{P}}=\sum_{j_{1}=1}^{n-1} \sum_{j_{2}=j_{1}+1}^{n} d_{G}^{P, \dot{P}}\left(\tilde{o}_{j_{1}}, \tilde{o}_{j_{2}}\right)
\end{aligned}
$$

A medida 10CSI Campello (2010) é definida como

$$
10 \mathrm{CSI}(P, \dot{P}) \triangleq \frac{a_{G}^{P, \dot{P}}}{a_{G}^{P, \dot{P}}+d_{G}^{P, \dot{P}}} .
$$


APÊNDICE

\section{ALGORITMOS BASEADOS EM MEDÓIDES PARA DADOS MULTI-REPRESENTADOS}

Algoritmos de agrupamento de dados baseados em medóides são aqueles que fazem uso de objetos da própria base de dados para auxiliar na representação dos grupos e, portanto, na realização do agrupamento (Vinod, 1969; Kaufman e Rousseeuw, 1990). Assim como os centróides (Seção 2.4.1), medóides são também chamados de protótipos de grupo, pois são usados na representação de grupos. Como os protótipos nesse caso são objetos da base de dados, o cálculo da distância entre objeto e protótipo se torna trivial. No contexto de base de dados relacionais, basta obter o elemento correspondente da matriz relacional, em contraposição aos algoritmos relacionais propostos nas Seções 3.2 e 3.3 que exigem cálculos complexos. No contexto de base de dados não relacionais, é necessário o cálculo da distância entre o medóide e o objeto em questão, o que pode ser feito antes mesmo da aplicação do algoritmo de agrupamento por meio do cálculo das distâncias entre todos os objetos. Portanto, em ambos os cenários, será assumido que uma matriz relacional $\mathrm{R} \in \mathbb{R}^{n \cdot n}$ é fornecida ao algoritmo baseado em medóides.

Os algoritmos baseados em medóides possuem outra vantagem em relação aos algoritmos relacionais discutidos anteriormente. Esses algoritmos lidam naturalmente com qualquer tipo de matriz relacional, incluindo as não euclidianas (definição na Seção 2.4.2). Para avaliar o potencial de tais algoritmos no contexto de dados multi-representados, são desenvolvidos e analisados neste capítulo dois algoritmos de agrupamento particional exclusivo baseados em medóides. 


\section{C.1 Fundamentação}

Seja $O=\left\{\tilde{o}_{1}, \tilde{o}_{2}, \ldots, \tilde{o}_{n}\right\}$ uma base de dados de $n$ objetos. Os medóides de um agrupamento particional exclusivo de $k$ grupos são denotados por uma $k$-upla $\left(m_{1}, m_{2}, \ldots, m_{k}\right) \in$ $\mathbb{N}_{1, n}^{k}$ de forma que $\tilde{o}_{m_{i}} \in O$ seja o objeto da base que faz o papel de medóide do $i$-ésimo grupo.

Os algoritmos baseados em medóides geralmente minimizam instâncias da Função 2.16, na qual as distâncias entre objetos e protótipos são definidas por

$$
\mathrm{D}_{i, j} \triangleq \mathrm{R}_{j, m_{i}} \quad \forall i \in \mathbb{N}_{1, k}, \forall j \in \mathbb{N}_{1, n}
$$

Por exemplo, a função-custo do algoritmo k-Medoids (T. Hastie, 2003; Bishop, 2006) é uma instância da Função 2.16 na qual $m=1, \mathrm{U} \in M_{\mathrm{e}}$ e as distâncias entre objetos e protótipos são definidas como acima. A função-custo do algoritmo Fuzzy c-Medoids (Krishnapuram et al., 2001) (FCMdd) é também uma instância na qual $m \in(1, \infty), \mathrm{U} \in M_{\mathrm{n}}$ e as distâncias entre objetos e protótipos são definidas da mesma forma.

A fim de atribuir pesos de relevância para as múltiplas representações da base de dados, seja $R \triangleq\left\{\mathrm{R}^{1}, \mathrm{R}^{2}, \ldots, \mathrm{R}^{p}\right\}$ o conjunto de $p$ matrizes relacionais representando as diferentes visões sobre a base $O$. Considere o conjunto $M_{\mathrm{y}}$ de todas as matrizes de ponderação, definido na Seção 2.3.1.2. Seja a função $\mathcal{D}_{\mathrm{mp}}: \mathbb{N}_{1, n}^{k} \mapsto M_{\mathrm{dp}}$, definida como $\mathcal{D}_{\mathrm{mp}}(\tilde{m} ; R) \triangleq\left\{\mathrm{D}^{s}\right\}_{s=1}^{p}, \mathrm{D}^{s} \triangleq\left[\mathrm{D}_{i, j}^{s}\right]$ $\left(\forall s \in \mathbb{N}_{1, p}\right)$ e

$$
\mathrm{D}_{i, j}^{s} \triangleq \mathrm{R}_{j, m_{i}}^{s} \quad \forall i, j, s,
$$

na qual $M_{\mathrm{dp}}$ é a coleção de todos os conjuntos de $p$ matrizes de distância definidas pela Eq. (2.19).

Propõe-se minimizar a função-custo $\mathcal{G}_{m, q}^{\mathrm{mp}}: M_{\mathrm{e}} \times \mathbb{N}_{1, n}^{k} \times M_{\mathrm{y}} \mapsto \mathbb{R}$ definida por

$$
\begin{aligned}
\mathcal{G}_{q}^{\mathrm{mp}}(\mathrm{U}, \tilde{m}, \mathrm{Y} ; R) & =\mathcal{G}_{1, q}\left(\mathrm{U}, \mathcal{D}_{\mathrm{mp}}(\tilde{m} ; R), \mathrm{Y}\right) \\
& =\sum_{i=1}^{k} \sum_{j=1}^{n} \mathrm{U}_{i, j} \cdot \sum_{s=1}^{p}\left(\mathrm{Y}_{i, s}\right)^{q} \cdot \mathrm{R}_{j, m_{i}}^{s}
\end{aligned}
$$

em que $q \in(1, \infty)$ e $\mathcal{G}_{m, q}$ é a Função 2.20). Note que $\sum_{s=1}^{p}\left(\mathrm{Y}_{i, s}\right)^{q} \cdot \mathrm{R}_{j, m_{i}}^{s}$ fornece a distância total (i.e., ao considerar todos as representações da base) e ponderada (pela relevância de cada representação da base para o $i$-ésimo grupo) do objeto $\tilde{o}_{j}$ ao medóide $\tilde{o}_{m_{i}}$.

As distâncias entre os objetos sob uma determinada representação da base podem apresentar valores muito maiores que as distâncias sob uma outra representação da base, tornando a primeira representação muito mais importante para o algoritmo de agrupamento, o que não é necessariamente desejável. Uma opção seria normalizar as matrizes relacionais de forma que as distâncias para diferentes representações da base possuam magnitudes semelhantes. Aqui, será adotada uma abordagem alternativa, na qual a normalização ocorre de forma automática durante o processo de agrupamento dos dados.

Considere a função $\mathcal{D}_{\mathrm{mp}}^{\mathrm{n}}: \mathbb{N}_{1, n}^{k} \mapsto M_{\mathrm{dp}}$, definida como $\mathcal{D}_{\mathrm{mp}}^{\mathrm{n}}(\tilde{m} ; R) \triangleq\left\{\mathrm{D}^{s}\right\}_{s=1}^{p} \in M_{\mathrm{dp}}$, 
$\mathrm{D}^{s} \triangleq\left[\mathrm{D}_{i, j}^{s}\right]\left(\forall s \in \mathbb{N}_{1, p}\right) \mathrm{e}$

$$
\mathrm{D}_{i, j}^{s} \triangleq \mathrm{R}_{j, m_{i}}^{s} / \sum_{l=1}^{n} \mathrm{R}_{l, m_{i}}^{s} \quad \forall i, j, s
$$

Propõe-se, também, minimizar a função-custo $\mathcal{G}_{m, q}^{\mathrm{mpn}}: M_{\mathrm{e}} \times \mathbb{N}_{1, n}^{k} \times M_{\mathrm{y}} \mapsto \mathbb{R}$ definida por

$$
\mathcal{G}_{q}^{\mathrm{mpn}}(\mathrm{U}, \tilde{m}, \mathrm{Y} ; R) \triangleq \mathcal{G}_{1, q}\left(\mathrm{U}, \mathcal{D}_{\mathrm{mp}}^{\mathrm{n}}(\tilde{m} ; R), \mathrm{Y}\right)
$$

A fórmula $\mathrm{R}_{j, m_{i}}^{s} / \sum_{l=1}^{n} \mathrm{R}_{l, m_{i}}^{s}$ representa a fração da distância entre $\tilde{o}_{j}$ e $\tilde{o}_{m_{i}}$ pela soma das distâncias entre o medóide $\tilde{o}_{m_{i}}$ e os objetos da base, sob a $s$-ésima representação.

São apresentados na seção seguinte dois algoritmos de agrupamento baseados em medóides capazes de minimizar as Funções (C.2) e (C.5).

\section{C.2 Algoritmos}

Os algoritmos $k$-Medoids com Ponderação de Visões 1 (KMPV1) e $k$-Medoids com Ponderação de Visões 2 (KMPV2), capazes de minimizar as funções $\mathcal{G}_{q}^{\text {mp }}$ e $\mathcal{G}_{q}^{\text {mpn }}$, respectivamente, são descritos simultaneamente, pois a notação $\mathrm{D}_{i, j}^{s}$ pode ser usada para referir às duas definições de distância: Equações (C.1) e (C.4). Além disso, em várias partes deste texto o termo KMPV é usado para referir a ambos os algoritmos; portanto, com "o algoritmo KMPV" quer-se dizer “os algoritmos KMPV1 e KMPV2]'. Os algoritmos KMPV1 e KMPV2 minimizam as suas funções-custo ao ajustar alternadamente as variáveis $M, \mathrm{Y}, \mathrm{U}$; isto é, em cada etapa dos algoritmos, duas das variáveis são mantidas constantes e a outra variável é alterada de forma a minimizar a função-custo correspondente. Os algoritmos KMPV1 e KMPV2 são subdivididos em três etapas. Na primeira etapa as variáveis Y e U são mantidas constantes. É escolhido para cada grupo um novo medóide de maneira a minimizar as distâncias intragrupo. Formalmente, a tupla $\tilde{m} \triangleq\left(m_{1}, m_{2}, \ldots, m_{k}\right) \in \mathbb{N}_{1, n}^{k}$ de medóides é atualizada por meio da seguinte função. Seja $\mathcal{L}_{q}: M_{\mathrm{e}} \times M_{\mathrm{y}} \mapsto \mathbb{N}_{1, n}^{k}$ uma função definida por $\mathcal{L}_{q}(\mathrm{U}, \mathrm{Y}) \triangleq \tilde{m}^{*} \triangleq\left\{m_{i}^{*}\right\}_{i=1}^{k}$ em que

$$
m_{i}^{*} \leftarrow \underset{l \in\{1, \ldots, n\} \mid \mathrm{U}_{i, l}=1}{\operatorname{argmin}}\left\{\sum_{j=1}^{n} \mathrm{U}_{i, j} \sum_{s=1}^{p}\left(\mathrm{Y}_{i, s}\right)^{q} \cdot \mathrm{D}_{i, j}^{s}\right\} \quad \forall i \in\{1, \ldots, k\}
$$

em que $q \in(1, \infty)$. Quando ocorre um empate, i.e., quando mais de um índice $l \in\{1, \ldots, n\}$ minimiza a soma das distâncias para um mesmo grupo $i$, escolhe-se aleatoriamente um desses índices para representar o medóide. Ao considerar as variáveis $\mathrm{Y}$ e U constantes, a atualização realizada por $\mathcal{L}_{q}$ fornece uma tupla $\tilde{m}^{*}$ que minimiza globalmente as funções-custo $\mathcal{G}_{q}^{\text {mp }}$ e $\mathcal{G}_{q}^{\text {mpn }}$. Para entender o porquê, observe que a escolha do medóide para um determinado $i$-ésimo grupo altera o valor das funções-custo $\mathcal{G}_{q}^{\mathrm{mp}}$ e $\mathcal{G}_{q}^{\mathrm{mpn}}$ de acordo, apenas, com o termo

$$
\sum_{j=1}^{n} \mathrm{U}_{i, j} \sum_{s=1}^{p}\left(\mathrm{Y}_{i, s}\right)^{q} \cdot \mathrm{D}_{i, j}^{s}
$$


Além disso, a escolha do $i$-ésimo medóide não exerce qualquer restrição na escolha dos demais medóides. Portanto, ao manter constantes as variáveis $\mathrm{Y}$ e $\mathrm{U}$, os mínimos de $\mathcal{G}_{q}^{\mathrm{mp}}$ e $\mathcal{G}_{q}^{\mathrm{mpn}}$ podem ser encontrados ao minimizar, independentemente, o custo de cada um dos grupos. Nos algoritmos KMPV1 e KMPV2, essa minimização é feita de maneira exaustiva, testando todos os possíveis medóides para cada grupo.

Na segunda etapa as variáveis $\mathrm{U}$ e $M$ são mantidas constantes e a matriz de ponderação $\mathrm{Y}$ é atualizada por meio da Função $\mathcal{Q}_{1, q}$ (Equações (3.4a), (3.4b) e (3.4c)).

Na terceira etapa as variáveis $\tilde{m}$ e Y são mantidas constantes e o agrupamento é ajustado por meio da atualização da matriz de partição $U$ ao atribuir os objetos aos grupos mais próximos. Isso é feito por meio da função $\mathcal{T}_{q}$ definida na Equação (3.7).

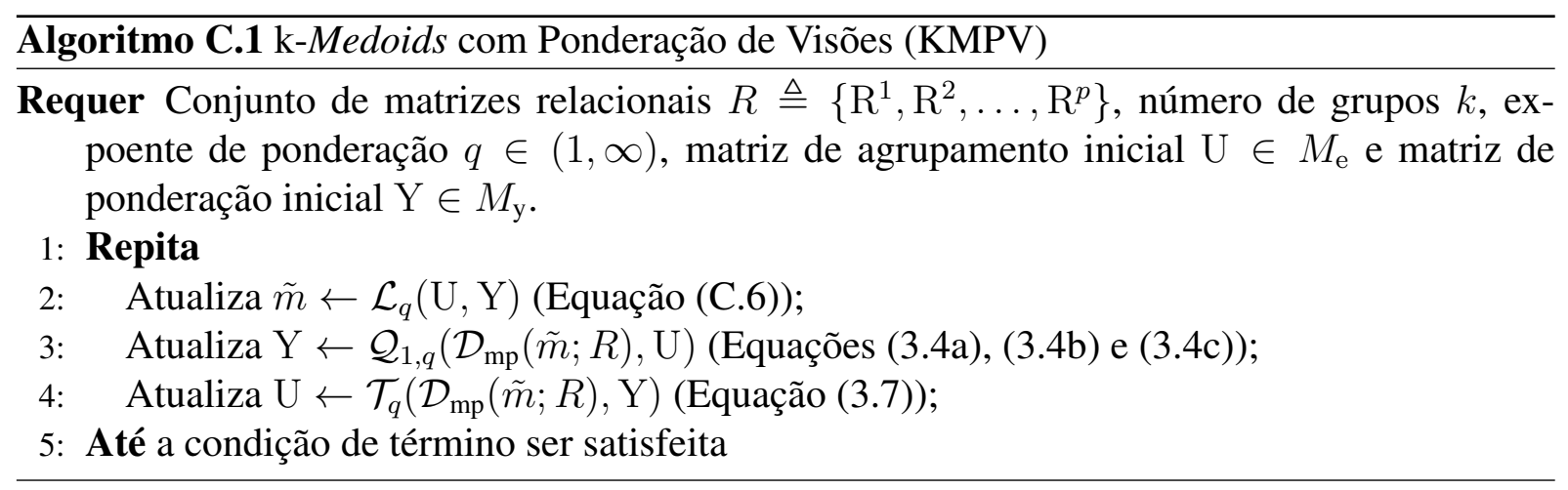

No Algoritmo C.1 são sintetizados os passos dos algoritmos KMPV1 e KMPV2, Além de configurar os parâmetros $k$ e $q$, o usuário precisa fornecer matrizes iniciais de agrupamento e de ponderação. Na Seção C.3 é tratada a questão de inicialização das matrizes U e Y. No entanto, repare que a necessidade de inicialização da matriz de ponderação Y é uma desvantagem em relação aos algoritmos baseados em centróides desenvolvidos no Capítulo 3 .

\section{C.3 Inicialização}

O algoritmo KMPV necessita que o usuário forneça matrizes iniciais U e Y. Para a matriz $\mathrm{Y}$, pode-se adotar a seguinte estratégia:

$$
\mathrm{Y}_{i, s} \leftarrow 1 / p \quad \forall i \in \mathbb{N}_{1, k}, s \in \mathbb{N}_{1, n}
$$

Todas as representações possuem inicialmente a mesma importância.

Quanto à inicialização da matriz $\mathrm{U}$, existem duas estratégias bem simples. A primeira, consiste na atribuição aleatória dos objetos aos grupos, com o cuidado de gerar uma partição de $k$ grupos não vazios. Naturalmente, essa estratégia produz soluções iniciais muito ruins. Além disso, pode-se inferir que as partições iniciais geradas dessa maneira tendem a ser semelhantes. Como os objetos dos grupos produzidos estão espalhados pela base de dados, os objetos mais centrais (i.e., de dissimilaridades menores entre os outros objetos) tendem a ser escolhidos como os medóides da primeira iteração do KMPV. Essa diversidade pobre de partições iniciais pode aumentar as chances do algoritmo cair em mínimos locais. 
Uma segunda abordagem mais adequada consiste em duas etapas. Na primeira, ocorre a escolha aleatória de $k$ medóides distintos. Em seguida, atribui-se os objetos aos grupos de medóides mais próximos (Equação (3.7)), produzindo, assim, uma matriz inicial U de grupos mais compactos.

\section{C.4 Atributos como visões distintas da base}

Nesta seção é investigada a capacidade do algoritmo KMPV1 em ponderar atributos ao serem tratados como representações distintas da base de dados. Para tanto, seja $X \triangleq$ $\left\{\mathrm{x}_{1}, \ldots, \mathrm{x}_{n}\right\} \subset \mathbb{R}^{p}$ o conjunto de vetores que descrevem a base de dados $O$. Considere $p$ matrizes relacionais definidas da seguinte forma:

$$
\mathrm{R}_{j, l}^{s}=\left|\mathrm{x}_{s}^{j}-\mathrm{x}_{s}^{l}\right| \quad \forall s \in \mathbb{N}_{1, p}, \forall j, l \in \mathbb{N}_{1, n}
$$

em que $\mathrm{x}_{s}^{j}$ denota o $s$-ésimo atributo do vetor $\mathrm{x}^{j}$. Seja $\tilde{m}$ um conjunto que representa os medóides. Nesse contexto, a distância total do objeto $\tilde{o}_{j}$ ao medóide $\tilde{o}_{m_{i}}$, dada por $\sum_{s=1}^{p}\left(\mathrm{Y}_{i, s}\right)^{q} \cdot \mathrm{R}_{j, m_{i}}^{s}$, pode ser entendida como a distância de Manhattan de $\mathrm{x}^{j} \mathrm{a} \mathrm{x}^{m_{i}}$ ponderada pelos pesos de relevância. Para entender o efeito disso, na Figura C.1 é apresentado o lugar geométrico dos pontos de $\mathbb{R}^{2}$ que distam 1 da origem, de acordo com a função de distância $f\left(\mathrm{x}^{j}\right) \triangleq \sum_{s=1}^{2}\left(\mathrm{Y}_{1, s}\right)^{2} \cdot\left|\mathrm{x}_{s}^{j}-0\right|$ entre objeto e a origem, para três configurações de $\mathrm{Y}_{1,:}$. Portanto, o algoritmo KMPV1, no espaço $\mathbb{R}^{2}$, atribui dissimilaridades semelhantes a pontos próximos das arestas de um mesmo losango alinhado com os eixos. Considerando que o algoritmo KMPV1 atribui os objetos aos grupos de medóides mais próximos (terceira etapa do algoritmo), é de se esperar que os formatos dos grupos encontrados sejam parecidos com losangos alinhados com os eixos no espaço vetorial $\mathbb{R}^{2}$.

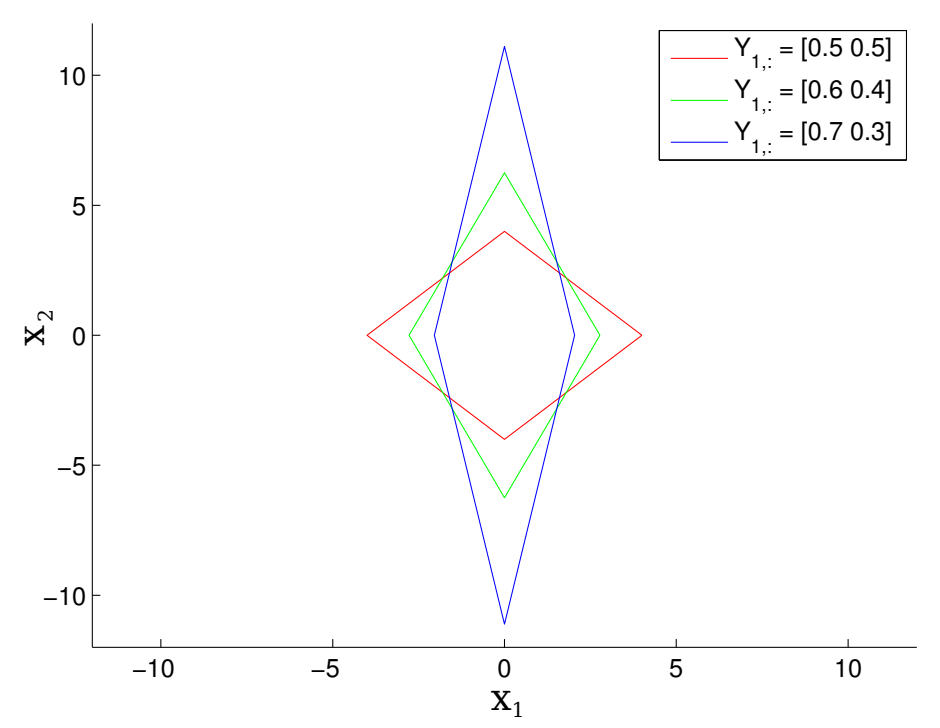

Figura C.1: Lugar geométrico dos pontos de $\mathbb{R}^{2}$ que distam 1 da origem, de acordo com a função de distância $f\left(\mathrm{x}^{j}\right) \triangleq \sum_{s=1}^{2}\left(\mathrm{Y}_{1, s}\right)^{2} \cdot\left|\mathrm{x}_{s}^{j}-0\right|$, para as configurações $\mathrm{Y}_{1,:}=\left[\begin{array}{ll}0.5 & 0.5\end{array}\right]$, $\mathrm{Y}_{1,:}=\left[\begin{array}{ll}0.6 & 0.4\end{array}\right]$ e $\mathrm{Y}_{1,:}=\left[\begin{array}{ll}0.7 & 0.3\end{array}\right]$. (Note que foi utilizado $q=2$.) 
Um outro ponto importante a entender é a influência do expoente de ponderação $q$ no formato dos grupos. Na Figura C.2 está ilustrado o lugar geométrico cujos pontos distam 1 da origem, para cada configuração $q=1.1,1.2,1.3, \ldots, 3.0$, ao utilizar a mesma função de distância entre objeto e a origem e uma matriz $\mathrm{Y}$ tal que $\mathrm{Y}_{1,1}=0.45$ e $\mathrm{Y}_{1,2}=0.55$. Nota-se de imediato que a escolha do valor de $q$ influi no tamanho do losango. De fato, quanto maior o valor de $q$, menores são os valores dos termos $\left(\mathrm{Y}_{i, s}\right)^{q}$ para os quais $\mathrm{Y}_{i, s} \in(0,1)$. Isso implica na diminuição das distâncias $\sum_{s=1}^{p}\left(\mathrm{Y}_{i, s}\right)^{q} \cdot \mathrm{R}_{j, m_{i}}^{s}$ com o aumento de $q$. Além disso, os losangos, para diferentes valores de $q$, parecem se alongar ou contrair. Isto é, pela Figura C.2, a razão $a / b$ (veja Figura C.3(a) parece variar ao tomar valores diferentes de $q$. De fato isso ocorre pelo seguinte motivo. Seja $d>0$ um valor de distância fixo e faça $d=\sum_{s=1}^{2}\left(\mathrm{Y}_{1, s}\right)^{q} \cdot\left|\mathrm{x}_{j, s}-0\right|$. Assim, tem-se $a=d /\left(\mathrm{Y}_{1,2}\right)^{q}$ e $b=d /\left(\mathrm{Y}_{1,1}\right)^{q}$ e, portanto, $a / b=\left(\mathrm{Y}_{1,1} / \mathrm{Y}_{1,2}\right)^{q}$. Desde que $\mathrm{Y}_{1,1} \neq \mathrm{Y}_{1,2}$, a razão $a / b$ tende a 0 ou a 1 quando $q$ tende ao infinito. $\mathrm{O}$ mesmo pode ser observado no caso $p$-dimensional, concluindo-se que grupos alongados são privilegiados, na fase de atribuição dos objetos, na medida que o valor de $q$ é aumentado.

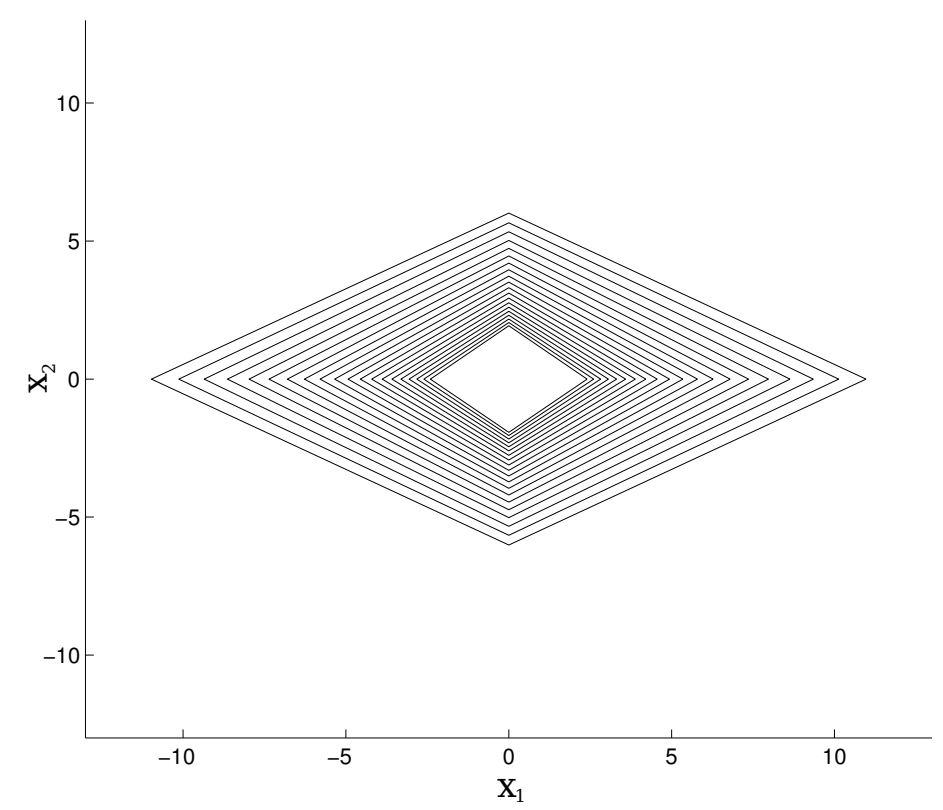

Figura C.2: Lugar geométrico dos pontos de $\mathbb{R}^{2}$ que distam 1 da origem, de acordo com a função de distância $f\left(\mathrm{x}^{j}\right) \triangleq \sum_{s=1}^{2}\left(\mathrm{Y}_{1, s}\right)^{2} \cdot\left|\mathrm{x}_{s}^{j}-0\right|$, em que $\mathrm{Y}_{1,1}=0.45$ e $\mathrm{Y}_{1,2}=0.55$, para as configurações $q=1.1,1.2,1.3, \ldots, 3.0$. $\mathrm{O}$ valor $q=1.1$ corresponde ao losango mais interno e $q=3.0$ ao losango mais externo.

Portanto, embora o aumento do valor de $q$ faça com que a ponderação das representações dos dados (nesse caso, atributos) seja menos drástica (Seção 3.1.3), tornando os pesos de atributos mais ou menos equânimes, a etapa de atribuição dos objetos aos medóides mais próximos do algoritmo KMPV1 acaba por privilegiar formatos de grupos alongados com o aumento de $q$. Logo, no que tange o formato dos grupos encontrados pelo algoritmo KMPV1 e o expoente de ponderação $q$, as etapas de agrupamento (Passo 4 do Algoritmo C.1) e de atualização dos pesos de ponderação (Passo 3 do Algoritmo C.1) são opostas. Em vista disso, não se pode esperar que os grupos encontrados pelo algoritmo KMPV1 se tornem menos alongados com a simples escolha de valores maiores para o expoente $q$. 


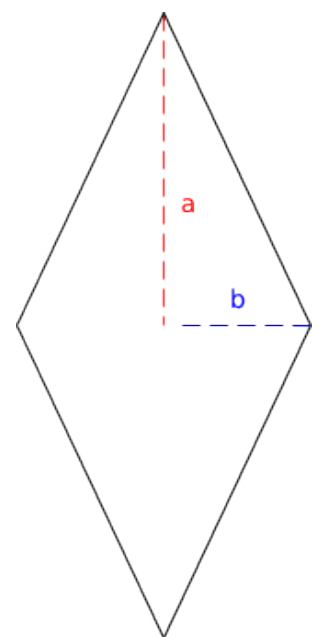

(a) Losango e seus raios $a$ e $b$.

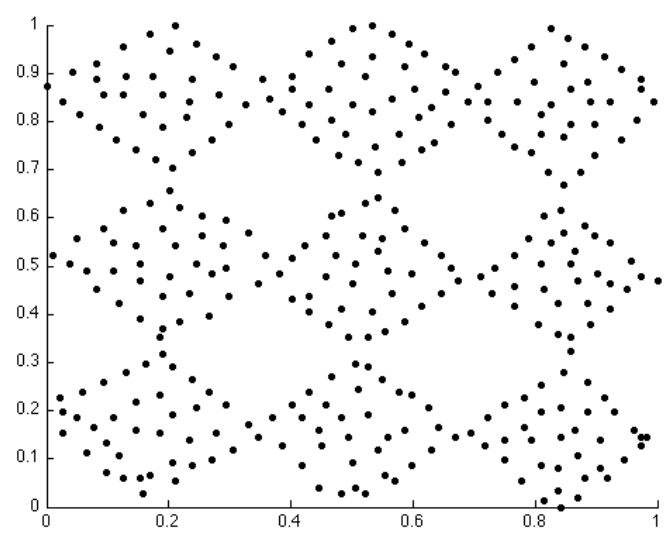

(b) Base de dados 9-Losangos.

Uma maneira de permitir que o usuário de fato controle o quão alongado os grupos tendem a ser consiste na configuração $q=1$ na fase de agrupamento do algoritmo KMPV1, mantendo o valor $q>1$ escolhido inicialmente nas outras duas etapas. Nesse caso, a distância entre $\tilde{o}_{j} \mathrm{e}$ $\tilde{o}_{m_{i}}$, usada na Eq. (3.7), é dada pela combinação convexa $\sum_{s=1}^{p} \mathrm{Y}_{i, s} \cdot \mathrm{R}_{j, m_{i}}^{s} \cdot$ Frigui e Nasraoui (2004) usaram o mesmo artifício para um algoritmo análogo de ponderação de representações. Entretanto, esse artifício não condiz com as equações de minimização deduzidas para o algoritmo KMPV1. Ou seja, é possível que o algoritmo se torne instável em certas execuções. Além disso, não está claro se a oposição entre as etapas de ponderação de representações e de agrupamento na preferência pelo formato dos grupos é uma característica indesejável. Não obstante, essa modificação do algoritmo KMPV1(doravante referida por mKMPV1) é utilizada, por auxiliar a compreensão do comportamento do algoritmo KMPV1 no experimento descrito a seguir.
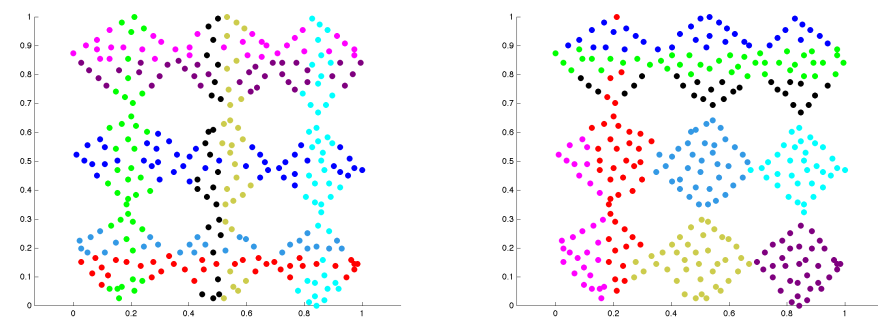

Figura C.3: Melhores partições encontradas pelos algoritmos KMPV1 (figura à esquerda) e mKMPV1 (figura à direita) para $q=2$.

Foi gerada uma base de dados com nove grupos, cada qual possuindo o formato de um losango alinhado com os eixos, denominada 9-Losangos e ilustrada pela Figura C.3(b). Em seguida, os algoritmos KMPV1e mKMPV1 foram aplicados nela 30 vezes para cada configuração de $q=2,4,6,8,10$. Nas Figuras C.3, C.4, C.5, C.6 e C.7 são apresentadas as melhores partições de cada uma das 30 aplicações para os diferentes valores de $q$, de acordo com as suas respectivas funções-custo. 

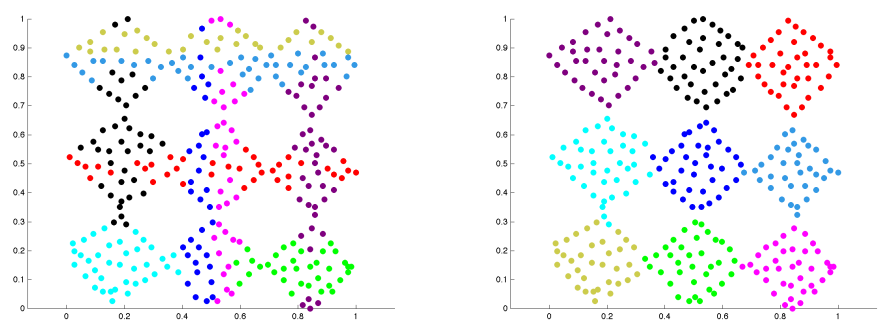

Figura C.4: Melhores partições encontradas pelos algoritmos KMPV1 (figura à esquerda) e mKMPV1 (figura à direita) para $q=4$.
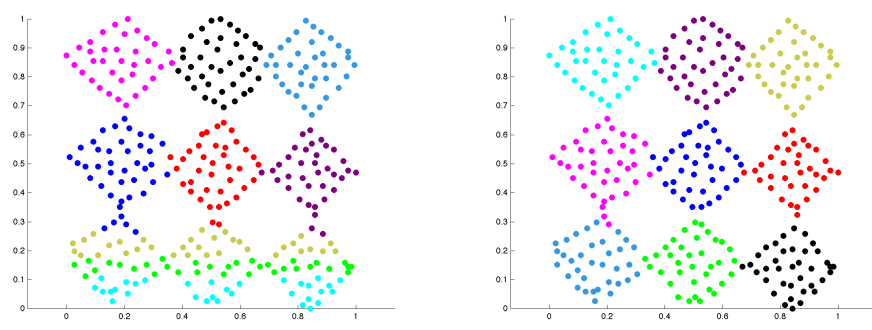

Figura C.5: Melhores partições encontradas pelos algoritmos KMPV1 (figura à esquerda) e mKMPV1 (figura à direita) para $q=6$.
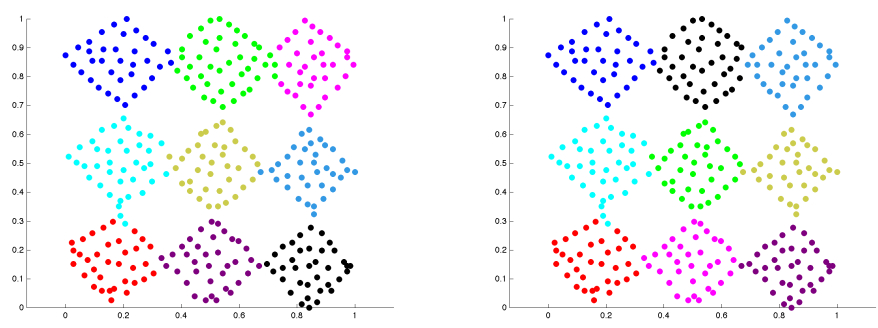

Figura C.6: Melhores partições encontradas pelos algoritmos KMPV1 (figura à esquerda) e mKMPV1 (figura à direita) para $q=8$.
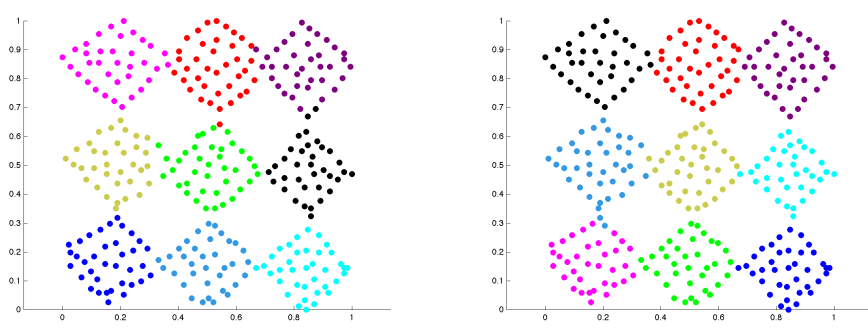

Figura C.7: Melhores partições encontradas pelos algoritmos KMPV1 (figura à esquerda) e mKMPV1 (figura à direita) para $q=10$. 
Para valores baixos de $q$, o algoritmo KMPV1 privilegiou grupos alongados. Somente a partir do valor $q=6$ o algoritmo KMPV1 passou a encontrar os grupos corretos. Por outro lado, o algoritmo mKMPV1, cuja etapa de agrupamento não possui a tendência de alongar os grupos devido à modificação no cálculo das distâncias, passou a encontrar os grupos corretos já a partir de $q=4$. Esse resultado sugere que a preferência por formato de grupo do algoritmo KMPV1é ditada preponderantemente pela etapa de ponderação de representações, o que indica que o formato dos grupos pode ser controlado pelo usuário por meio do expoente $q$.

\section{C.5 Considerações}

A Seção C.2 apresentou dois algoritmos baseados em medóides KMPV1 e KMPV2 que realizam a ponderação de múltiplas representações da base de dados. Suas funções-custo são muito parecidas, sendo basicamente a soma dos erros correspondentes às atribuições dos objetos aos grupos. A Seção C.3 forneceu estratégias para a inicialização das matrizes U e Y, exigida pelos algoritmos KMPV1 e KMPV2. Na Seção C.4 foi apresentado um estudo teórico sobre o formato dos grupos quando o algoritmo KMPV1 é usado em bases de dados nas quais as representações são os próprios atributos. Por fim, os resultados experimentais apresentados na Seção C.4 sugerem que valores baixos de $q$ não são adequados quando o algoritmo KMPV1é aplicado em bases de dados cujos atributos são tratados como representações. 



\section{APÊNDICE} D

\section{Medida-F para bi-agrupamento de Turner et al.}

Turner et al. (2005) propuseram um índice para comparação de bi-agrupamentos baseada na medida-F (Strehl e Ghosh, 2003) para bi-agrupamento e desenvolvido especificamente para o modelo de bi-agrupamento para expressão gênica chamado modelo de xadrez. Esse modelo de bi-agrupamento foi proposto por Lazzeroni e Owen (2000) e se baseia na representação da matriz dos dados $\mathrm{X}$ por meio da soma de camadas, em que cada camada (excetuando a primeira) corresponde a um bi-grupo e a primeira camada corresponde aos efeitos globais dos dados. Cada camada é descrita por uma matriz de números reais $\Theta^{i}$ e de dimensão $n$-por- $p$, em que $n$ é o número de genes e $p$ é o número de condições experimentais. Assim, a expressão do $i$-ésimo gene com relação à $j$-ésima condição experimental e ao $s$-ésimo bi-grupo é definida por $\Theta_{j, l}^{i}$. A matriz $\Theta_{j, l}^{0}$ define a camada dos efeitos globais dos dados. Por fim, a matriz de dados é definida por

$$
\mathrm{X}_{j, l}=\Theta_{j, l}^{0}+\sum_{i=1}^{k} \Theta_{j, l}^{i} \mathbf{1}_{B_{s}^{\mathrm{r}}}(j) \mathbf{1}_{B_{s}^{\mathrm{c}}}(l)+\epsilon_{j, l}
$$

em que $\epsilon_{j, l}$ representa o erro residual e $\mathbf{1}_{A}$ é a função indicadora:

$$
\mathbf{1}_{A}(x) \triangleq \begin{cases}1 & \text { se } x \in A \\ 0 & \text { se } x \notin A\end{cases}
$$

As matrizes $\Theta_{j, l}^{s}$ correspondentes aos bi-grupos são usadas na definição do índice de Turner et al. Mas antes de usá-las, considere as seguintes definições de revocação e precisão para 
bi-grupos:

$$
\begin{aligned}
& \mathbb{S}_{\mathrm{tre}}\left(B_{i}, \dot{B}_{l}\right) \triangleq \frac{\left|B_{i}^{\mathrm{r}} \cap \dot{B}_{l}^{\mathrm{r}}\right|}{\left|\dot{B}_{l}^{\mathrm{r}}\right|} \frac{\left|B_{i}^{\mathrm{c}} \cap \dot{B}_{l}^{\mathrm{c}}\right|}{\left|\dot{B}_{l}^{\mathrm{c}}\right|} \mathrm{e} \\
& \mathbb{S}_{\mathrm{tpr}}\left(B_{i}, \dot{B}_{l}\right) \triangleq \mathbb{S}_{\mathrm{tre}}\left(\dot{B}_{l}, B_{i}\right) .
\end{aligned}
$$

A medida-F entre os bi-grupos $B_{i}$ e $\dot{B}_{l}$ é definida por

$$
\mathbb{S}_{\mathrm{tu}}\left(B_{i}, \dot{B}_{l}\right) \triangleq \frac{2 \cdot \mathbb{S}_{\mathrm{tre}}\left(B_{i}, \dot{B}_{l}\right) \cdot \mathbb{S}_{\mathrm{tpr}}\left(B_{i}, \dot{B}_{l}\right)}{\left|B_{i}^{\mathrm{r}} \times B_{i}^{\mathrm{c}}\right|+\left|\dot{B}_{l}^{\mathrm{r}} \times \dot{B}_{l}^{\mathrm{c}}\right|}
$$

É atribuído a cada bi-grupo de referência um peso definido por

$$
w_{i} \triangleq \sum_{j \in \dot{B}_{i}^{\mathrm{r}}} \sum_{l \in \dot{B}_{i}^{\mathrm{c}}}\left(\Theta_{j, l}^{i}\right)^{2} \text {. }
$$

Esses pesos representam os graus de importância dos bi-grupos e são utilizados tanto na associação entre os bi-grupos encontrados e os de referência como no cálculo final do índice. O índice em questão assume que o número de bi-grupos da solução encontrada é igual ao número de bi-grupos da solução de referência. Portanto, é feita uma associação de um-para-um entre os bi-grupos das soluções. A associação é determinada da seguinte forma. Ao bi-grupo $\dot{B}_{l_{1}}$ de maior peso $w_{l_{1}}$ é associado o bi-grupo $B_{i_{1}}$ da solução encontrada que maximiza $\mathbb{S}_{\mathrm{tu}}\left(B_{i_{1}}, \dot{B}_{l_{1}}\right)$. Em seguida, ao bi-grupo $\dot{B}_{l_{2}}$ que possui o segundo maior peso $w_{l_{2}}$ é associado o bi-grupo $B_{i_{2}}$ da solução encontrada que maximiza $\mathbb{S}_{\mathrm{tu}}\left(B_{i_{2}}, \dot{B}_{l_{2}}\right)$, e assim por diante. No evento de todos os valores da medida-F entre os bi-grupos forem zero, a associação do bi-grupo de referência correspondente é postergada para o final.

Por fim, o índice de Turner et al. é definido por

$$
\mathbb{S}_{\mathrm{tur}}(B, \dot{B}) \triangleq \frac{\sum_{s=1}^{k} w_{l_{s}} \mathbb{S}_{\mathrm{tu}}\left(B_{i_{s}}, \dot{B}_{l_{s}}\right)}{\sum_{s=1}^{k} w_{l_{s}}} .
$$




\section{- \\ COMENTÁRIOS SOBRE TESTE DE HIPÓTESE}

Neste capítulo alguns conceitos de teste de hipótese são abordados com o objetivo de orientar o leitor na escolha do tipo de hipótese alternativa mais adequada para o problema em mãos e em como obter conclusões a respeito das hipóteses do teste. Além disso, são discutidos alguns detalhes e vantagens do teste de hipótese da soma dos postos de Wilcoxon (denotado por Wilcoxon rank-sum test ou pela sigla WRST neste texto) (Hollander e Wolfe, 1999). Embora alguns conceitos de teste de hipótese sejam introduzidos, é pressuposto que o leitor possui uma certa familiaridade com o assunto.

\section{E.1 Hipótese estatística}

Teste de hipótese é o processo de inferência, a partir de amostragens, sobre a veracidade de uma dada afirmação sobre a(s) população(ões) (Conover, 1998). Para tal fim, os testes de hipótese (também chamados de testes estatísticos) empregam uma hipótese nula $\left(H_{0}\right)$ e uma hipótese alternativa $\left(H_{1}\right)$. Tanto uma quanto a outra, podem ser classificadas em hipótese não direcional ou hipótese direcional. Por exemplo, sejam $X$ e $Y$ duas variáveis aleatórias cujas esperanças (numericamente desconhecidas) são $E(X)$ e $E(Y)$. Uma hipótese nula passível de relevância é a da igualdade das esperanças: $H_{0}: E(X)=E(Y)$ (hipótese não direcional). Nesse caso, a hipótese alternativa pode ser uma das seguintes: $H_{1}: E(X)<E(Y)$ (hipótese direcional), $H_{1}: E(X) \neq E(Y)$ (hipótese não direcional) ou $H_{1}: E(X)>E(Y)$ (hipótese direcional). Note que a hipótese alternativa não direcional só afirma que as médias são diferentes, ou seja, não faz afirmação sobre a direção das médias.

Para formalizar a direcionalidade de uma hipótese é preciso introduzir o conceito de estatística de teste. Grosso modo, estatística refere-se a uma característica (representada numerica- 
mente) de amostragem (e.g., a média de amostragem) (Sheskin, 2004). Quando uma estatística é usada em um teste de hipótese, ela é chamada de estatística de teste. Com base nela, o teste de hipótese avalia a veracidade da hipótese nula. Portanto, todo teste de hipótese tem sua estatística de teste. Por exemplo, o Single-sample z test (Sheskin, 2004), que avalia a hipótese nula $H_{0}: E(X)=c$ ( $c$ é uma constante qualquer), tem como estatística de teste a média amostral normalizada $z=\frac{\bar{X}-c}{\sigma_{\bar{X}}}$, em que $\bar{X}$ é a média da amostra e $\sigma_{\bar{X}}$ é o desvio padrão da amostra. Assim, a medida que $z$ se distancia de zero, o que significa que a média amostral $\bar{X}$ se distancia de $c$, a veracidade da hipótese nula se torna menos provável. De modo mais formal, sejam $\alpha$ o nível de significância estatística e $H_{1}: E(X)>c$ a hipótese alternativa. Nesse caso, o teste de hipótese: (i) rejeita a hipótese nula caso $z \geq z_{\alpha}$; (ii) não rejeita a hipótese nula caso $z<z_{\alpha}\left(z_{\alpha}\right.$ define a região crítica na qual a hipótese nula pode ser rejeitada com nível de significância estatística $\alpha$ ). De modo análogo, seja $H_{1}: E(X)<c$ a hipótese alternativa. Nesse caso, o teste de hipótese: (i) rejeita a hipótese nula caso $z \leq-z_{\alpha}$; (ii) não rejeita a hipótese nula caso $z>-z_{\alpha}$. Por fim, seja $H_{1}: E(X) \neq c$ a hipótese alternativa. Nesse caso, o teste de hipótese: (i) rejeita a hipótese nula caso $z \leq-z_{\alpha / 2}$ ou $z \geq z_{\alpha / 2}$; (ii) não rejeita a hipótese nula caso $z>-z_{\alpha / 2} \mathrm{e}$ $z<z_{\alpha / 2}$.

Para todos os testes estatísticos, a estatística de teste é comparada de modo análogo como foi feito com o valor $z$ do Single-sample z test. Note que, quando a(s) amostra(s) apresenta(m) uma estatística de teste que tende para um lado (e.g., $z$ é positivo), é mais fácil rejeitar a hipótese nula quando se usa a hipótese alternativa direcional que indica o mesmo lado (e.g., $H_{1}: E(X)>c$ ) em comparação com a hipótese alternativa não direcional. Isso ocorre porque, no caso não direcional, o nível de significância é divido pela metade e cada metade é usada para definir as duas regiões críticas que correspondem aos valores baixos (e.g., $z \leq-z_{\alpha / 2}$ ) e altos (e.g., $z \geq z_{\alpha / 2}$ ) da estatística de teste. Em face disso, o pesquisador que utiliza o teste de hipótese para avaliar os seus experimentos pode se sentir tentado a escolher, de acordo com o valor resultante da estatística de teste, a hipótese alternativa direcional que facilita a rejeição da hipótese nula. Por exemplo, suponha que se esteja usando o Single-sample z test e que o valor da estatística de teste tenha dado positivo $(z>0)$. Nesse caso, o pesquisador mal informado (ou mal intencionado) usaria $H_{1}: E(X)>c$ e rejeitaria a hipótese nula se $z \geq z_{\alpha}$. Caso o valor da estatística de teste tenha dado não positivo $(z \leq 0)$, o pesquisador usaria $H_{1}: E(X)<c$ e rejeitaria a hipótese nula caso $z \leq-z_{\alpha}$. Ocorre que esse procedimento é equivalente a usar a hipótese alternativa não direcional com nível de significância $2 \alpha$ mas reportar o uso da hipótese alternativa direcional com nível de significância $\alpha$. Sob esse ângulo, está claro que esse procedimento não é apropriado.

Supondo que a hipótese nula seja não direcional, uma questão que surge com frequência é "Qual hipótese alternativa devo usar?". Segundo Motulsky (1995), a hipótese alternativa direcional é apropriada quando o pesquisador tem certeza, antes de coletar qualquer dado, que a hipótese nula é a correta ou a respectiva hipótese direcional é a correta; i.e., a outra hipótese direcional (contrária à hipótese direcional usada) não pode ser verdadeira. Se o pesquisador não consegue especificar essa direção da hipótese alternativa direcional de antemão, antes de realizar os experimentos, então a hipótese alternativa não direcional deve ser usada. Em caso 
de dúvida, use a não direcional.

Porém, segundo Sheskin (2004), não há um consenso sobre quais condições cada hipótese alternativa deve ser usada. Alguns pesquisadores acreditam que a hipótese alternativa não direcional deva ser sempre usada, não importando as expectativas quanto ao resultado dos experimentos. Outros pesquisadores acreditam que a hipótese alternativa não direcional deva ser usada apenas quando não se tem expectativas quanto ao resultado dos experimentos. Entretanto, uma abordagem prudente é sempre usar a hipótese alternativa não direcional. Caso não haja expectativa quanto ao resultado dos experimentos, essa decisão não entra em conflito com nenhuma das duas visões de uso das hipóteses. Quando se tem expectativa sobre a direção da estatística de teste, a escolha da alternativa não direcional é mais conservadora, pois, nesse caso, a probabilidade de rejeição da hipótese nula é sempre menor ou igual (caso a expectativa se confirme) à probabilidade de rejeição ao usar a hipótese alternativa direcional correspondente.

Por fim, vale mencionar que, ao rejeitar a hipótese nula e aceitar a hipótese alternativa não direcional, é sempre possível conhecer a direção da estatística de teste que levou à rejeição da hipótese nula e reportá-la. Por exemplo, na página 278 de (Conover, 1998) é fornecido um exemplo em que a hipótese nula

$$
H_{0} \text { : O sílex (uma rocha específica) das regiões A e B são de igual rigidez }
$$

é rejeitada em favor da hipótese alternativa não direcional

$$
H_{1} \text { : O sílex das regiões A e B não são de igual rigidez. }
$$

Além disso, com base na direção da estatística de teste resultante, é concluído que o sílex da região B é mais rígido que o da região A. Na próxima seção é descrito um exemplo em que o Wilcoxon rank-sum test é usado em uma situação análoga.

\section{E.2 Wilcoxon rank-sum test}

O Wilcoxon rank-sum test (WRST) foi inventado em 1945 por Wilcoxon. De maneira independente, outro teste, mas equivalente ao WRST, foi desenvolvido por Mann e Whitney em 1947 (Sprent e Smeeton, 2007). Em decorrência disso, esses dois testes são referidos de diversas maneiras na literatura: Wilcoxon rank-sum test, Wilcoxon test, Mann-Whitney test, WilcoxonMann-Whitney test, Mann-Whitney-Wilcoxon test, Mann-Whitney U test (Conover, 1998; Sheskin, 2004; Sprent e Smeeton, 2007). O WRST é um teste de hipótese não paramétrico, o que significa que não é exigida uma determinada função de distribuição para as variáveis aleatórias de interesse. Ele é aplicável em dados pelo menos ordinais e em situações que envolvam hipótese sobre duas amostras independentes. Essa sua flexibilidade em aceitar mesmo dados não numéricos advém da transformação dos valores originais dos dados em postos (ranks em inglês). Embora essa transformação implique em perda de informação, o WRST é apenas ligeiramente menos efetivo que a sua contrapartida paramétrica (t-test (Sheskin, 2004)) quando a 
suposição, correspondente ao t-test, de normalidade dos dados é satisfeita (Hollander e Wolfe, 1999). Além disso, o WRST é relativamente insensível a outliers (Hollander e Wolfe, 1999).

Sejam $X$ e $Y$ duas variáveis aleatórias. A eficiência relativa assintótica (ERA) do WRST, quando $X$ e $Y$ possuem distribuições de mesmo formato (diferem possivelmente nas médias/medianas), em comparação com o t-test quando a suposição da normalidade é satisfeita ( $X$ e $Y$ possuem distribuições normais) é 0.955 (Conover, 1998). Grosso modo, isso significa que, quando as amostras fornecidas ao WRST são de tamanho $n_{1}$, o $t$-test precisa de amostras de tamanho $0.955 \times n_{1}$ para obter o mesmo nível de significância $\alpha$ e probabilidade $\beta$ de cometer erro do tipo II que o WRST. Se $X$ e $Y$ são uniformes, então a ERA é 1.0. Se $X$ e $Y$ são variáveis de distribuições exponenciais duplas, então a ERA é 1.5. Além disso, a ERA do WRST em comparação com o t-test nunca pode ser menor que 0.864 (Conover, 1998). Em certos casos, essa ERA pode tender ao infinito (Hollander e Wolfe, 1999). Portanto, quando a natureza das distribuições de $X$ e $Y$ não é conhecida, é mais seguro usar o WRST ao invés do t-test.

Baseando-se no livro (Hollander e Wolfe, 1999), sejam $X_{1}, \ldots, X_{m}$ observações da variável aleatória $X$ e $Y_{1}, \ldots, Y_{n}$ observações da variável aleatória $Y$. As suposições do WRST são:

1. $X_{1}, \ldots, X_{m}$ são mutuamente independentes e identicamente distribuídas. O mesmo vale para as observações $Y_{1}, \ldots, Y_{n}$;

2. As observações $X_{1}, \ldots, X_{m}$ e $Y_{1}, \ldots, Y_{n}$ são mutuamente independentes;

3. $X$ e $Y$ são variáveis aleatórias contínuas (entretanto, em (Conover, 1998) é mencionado que existem pesquisas mostrando que essa pressuposição é desnecessária).

Sejam $f_{x}$ e $f_{y}$ as funções de distribuição de $X$ e $Y$, respectivamente. Segundo Hollander e Wolfe (1999), as hipóteses não direcionais do WRST podem ser:

$$
\begin{gathered}
H_{0}: f_{x}(t)=f_{y}(t), \text { para todo } t \\
H_{1}: f_{x}(t) \neq f_{y}(t), \text { para algum } t
\end{gathered}
$$

Para casos práticos, a Hipótese Alternativa (E.1) não costuma ser interessante. No entanto, segundo Conover (1998), qualquer diferença entre as distribuições, na prática, implica que $P(X>Y)$ não é mais igual a $1 / 2$. Portanto, as seguintes hipóteses não direcionais podem ser usadas:

$$
\begin{aligned}
& H_{0}: f_{x}(t)=f_{y}(t), \text { para todo } t \\
& H_{1}: P(X>Y) \neq P(X<Y) .
\end{aligned}
$$

Por exemplo, a hipótese alternativa pode ser que algum dos dois algoritmos é computacionalmente mais rápido que o outro, quando as variáveis aleatórias $X$ e $Y$ são tempos computacionais. Ao assumir mais a seguinte pressuposição:

4. As distribuições de $X$ e $Y$ possuem o mesmo formato, a não ser possivelmente pelo deslocamento. Formalmente, é assumido que $f_{x}(t)=f_{y}(t+\Delta)$ para todo $t$ e algum $\Delta$. 
Nesse caso, as hipóteses não direcionais podem ser

$$
\begin{aligned}
& H_{0}: \Delta=0 ; \\
& H_{1}: \Delta \neq 0 .
\end{aligned}
$$

De maneira equivalente, tem-se

$$
\begin{aligned}
& H_{0}: M(X)=M(Y) \\
& H_{1}: M(X) \neq M(Y)
\end{aligned}
$$

em que $M(\cdot)$ fornece a mediana da variável aleatória. Quando as esperanças das variáveis $X \mathrm{e}$ $Y$ existem, as seguintes hipóteses são equivalentes às Hipóteses (E.3) e (E.4):

$$
\begin{aligned}
& H_{0}: E(X)=E(Y) ; \\
& H_{1}: E(X) \neq E(Y) .
\end{aligned}
$$





\section{APÊNDICE}

\section{政}

\section{COMMENTS ON "RELATIONAL GENERALIZATIONS OF CLUSTER VALIDITY INDICES"}

\section{Abstract}

In the above paper (Sledge et al., 2010), the authors proposed an ingenious framework to generalize many clustering validity indices to a relational context in which the data to be clustered are described solely by pairwise dissimilarities. This letter is intended to complement that framework in such a way that some particular distances can now be exactly calculated.

\section{F.1 Introduction}

Consider a data set $O=\left\{o_{1}, \ldots, o_{n}\right\}$ of $n$ objects to be clustered. To get started, one has to objectively describe the data objects in a suitable way so that a computational procedure can be applied. Usually, the objects are described by a set $X=\left\{\vec{x}_{1}, \ldots, \vec{x}_{n}\right\} \subset \mathbb{R}^{z}$ of attribute vectors ( $z$-dimensional points), where $\vec{x}_{i}$ represents the $i$ th object, $o_{i}$. In a number of application scenarios, however, only the parwise dissimilarities between objects are available. These dissimilarities can be conveniently disposed in a relational matrix $R=\left[r_{i, j}\right]_{n \times n}$, in which $r_{i, j}$ is a measure of distance between objects $o_{i}$ and $o_{j}$. As in (Sledge et al., 2010), it is assumed here that $r_{i, j} \in \mathbb{R}_{+}$(non-negative reals), $r_{i, i}=0$, and $r_{i, j}=r_{j, i}$, for all $i, j \in \mathbb{N}_{1, n}$.

In an interesting paper, Sledge et al. (Sledge et al., 2010) generalized three well-known clustering validity indices to the context of relational data, thus making it possible the use of such indices to evaluate results obtained by relational clustering algorithms. In doing so, these 
authors also developed a framework to convert many other validity indices to a relational form. A complication that they encountered (please, see the 3rd paragraph, 2nd column, page 775 of Sledge et al. (2010) ) regards the calculation of the distances between cluster centroids. Such distances are needed so that some validity indices can be computed, but the geometric centroids of clusters are not accessible in a relational domain. Sledge et al. proposed an approximate method to estimate these distances by first searching for the most representative object of each cluster and, then, taking the distances between these objects (from the relational matrix $R$ ) as an estimate of the distances between the centroids of the corresponding clusters. In the next section, we show how the distances between cluster centroids can be exactly calculated in a relational domain, given the same assumptions made in Sledge et al. (2010).

\section{F.2 Distances between Centroids - Relational Case}

One common way of representing a partition of a given data set $O$ into $c$ clusters is by means of a matrix $U=\left[u_{i, j}\right]_{c \times n}$ in which $u_{i, j}$ represents the belongingness of object $o_{j}$ to the $i$ th cluster. A general form of $U$ is the so-called possibilistic partition matrix, which comprises hard and probabilistic partitions as particular cases. The set of all those matrices relative to $c$-partitions of $O$ is defined as:

$$
M_{\mathrm{pcm}}=\left\{U \in \mathbb{R}^{c \times n} \mid u_{i, j} \in[0,1] \forall i, j ; \quad 0<\sum_{j=1}^{n} u_{i, j}<n \forall i ; \quad \max _{1 \leq i \leq c} u_{i, j}>0 \forall j\right\} .
$$

Sledge et al. (Sledge et al. 2010) supported their work on the following equality:

$$
\left\|\vec{x}_{j}-{ }_{o} \vec{v}_{l}\right\|_{A}^{2}=\left(R \cdot{ }_{r} \vec{v}_{l}\right)_{j}-\frac{{ }_{r} \vec{v}_{l}^{T} \cdot R \cdot{ }_{r} \vec{v}_{l}}{2}
$$

which was proven in (Hathaway et al., 1989) for a data partition $U \in M_{\text {pcm }}$ and an Euclidean relational matrix $R$ such that $r_{i, s}=\left\|\vec{x}_{i}-\vec{x}_{s}\right\|_{A}^{2} \quad \forall i, s \in \mathbb{N}_{1, n}$. Vectors ${ }_{o} \vec{v}_{l}$ and ${ }_{r} \vec{v}_{l}$ are the $z \times 1$ geometric centroid and the $n \times 1$ relational centroid of the $l$ th cluster, respectively, i.e.:

$$
\begin{aligned}
{ }_{o} \vec{v}_{l}=\frac{\sum_{j=1}^{n} u_{l, j}^{m} \cdot \vec{x}_{j}}{\sum_{j=1}^{n} u_{l, j}^{m}} & l \in \mathbb{N}_{1, c}, \\
{ }_{r} \vec{v}_{l}=\frac{\left(u_{l, 1}^{m}, u_{l, 2}^{m}, \ldots, u_{l, n}^{m}\right)^{T}}{\sum_{j=1}^{n} u_{l, j}^{m}} & l \in \mathbb{N}_{1, c} .
\end{aligned}
$$

These centroids are the cluster prototypes used by the widespread hard $(m=1)$ and fuzzy / possibilistic $(m \in(1, \infty)) c$-means algorithms in their original (non-relational) (Bezdek, 1981; Krishnapuram e Keller, 1993; Bezdek et al., 1999) and relational Hathaway et al. (1989); Davenport e Hathaway (1995) forms.

In the following theorem, we generalize (F.2) to the case in which both points $\vec{w}$ and $\vec{y}$ of $\|\vec{w}-\vec{y}\|_{A}^{2}$ are geometric centroids of clusters. 
Teorema F.1. Let $X=\left\{\vec{x}_{1}, \ldots, \vec{x}_{n}\right\} \subset \mathbb{R}^{z}$ be any set of data objects and let $R$ be an associated $n \times n$ relational data matrix such that, for each $1 \leq i, j \leq n$, the relational elements are given by $r_{i, j}=\left\|\vec{x}_{i}-\vec{x}_{j}\right\|_{A}^{2}$, where $\|\cdot\|_{A}$ is any inner-product-induced norm of $\mathbb{R}^{z}$ and $A$ is the symmetric positive-definite matrix that defines this inner-product. In addition, let $U \in M_{p c m}$ be a $c \times n$ partition matrix according to $(F .1)$. Then, the following equality holds:

$$
\begin{aligned}
\left\|\vec{v}_{i}-{ }_{o} \vec{v}_{l}\right\|_{A}^{2} & =-\frac{{ }_{r} \vec{v}_{i}^{T} \cdot R \cdot{ }_{r} \vec{v}_{i}}{2}+{ }_{r} \vec{v}_{i}^{T} \cdot R \cdot{ }_{r} \vec{v}_{l}-\frac{{ }_{r} \vec{v}_{l}^{T} \cdot R \cdot{ }_{r} \vec{v}_{l}}{2} \\
& =\left({ }_{r} \vec{v}_{i}-{ }_{r} \vec{v}_{l}\right)^{T} \cdot \Phi \cdot\left({ }_{r} \vec{v}_{i}-{ }_{r} \vec{v}_{l}\right)
\end{aligned} \quad \forall i, l \in \mathbb{N}_{1, c},
$$

where ${ }_{o} \vec{v}_{j}$ and ${ }_{r} \vec{v}_{j}$ (for all $j \in \mathbb{N}_{1, c}$ ) are defined by equations $($ F.3 $)$ and $\left(\right.$ F.4 with $m \in[1, \infty){ }^{1}$ respectively, and $\Phi=-\frac{1}{2} R$.

Prova. For the sake of compactness, let us define $\left|C_{i}\right|=\sum_{j=1}^{n} u_{i, j}^{m} \quad \forall i \in \mathbb{N}_{1, c}$ and let $\langle\cdot, \cdot\rangle_{A}$ be the inner-product induced by the symmetric positive-definite matrix $A$. Then, it follows that:

$$
\begin{aligned}
\left\|_{o} \vec{v}_{i}-{ }_{o} \vec{v}_{l}\right\|_{A}^{2} & =\left\langle\frac{\sum_{j=1}^{n} u_{i, j}^{m} \cdot \vec{x}_{j}}{\sum_{j=1}^{n} u_{i, j}^{m}}-\frac{\sum_{j=1}^{n} u_{l, j}^{m} \cdot \vec{x}_{j}}{\sum_{j=1}^{n} u_{l, j}^{m}}, \text { idem }\right\rangle_{A} \\
& =\alpha\left\langle\sum_{s=1}^{n} u_{l, s}^{m} \sum_{j=1}^{n} u_{i, j}^{m} \cdot \vec{x}_{j}-\sum_{s=1}^{n} u_{i, s}^{m} \sum_{j=1}^{n} u_{l, j}^{m} \cdot \vec{x}_{j}, \text { idem }\right\rangle_{A} \\
& \left(\text { where } \alpha=\left(\left|C_{i}\right|\left|C_{l}\right|\right)^{-2}\right) \\
& =\alpha\left(\left\langle\sum_{s=1}^{n} u_{l, s}^{m} \sum_{j=1}^{n} u_{i, j}^{m} \cdot \vec{x}_{j}, \beta\right\rangle_{A}-\left\langle\sum_{s=1}^{n} u_{i, s}^{m} \sum_{j=1}^{n} u_{l, j}^{m} \cdot \vec{x}_{j}, \beta\right\rangle_{A}\right) \\
& \left(\text { where } \beta=\sum_{s=1}^{n} u_{l, s}^{m} \sum_{j=1}^{n} u_{i, j}^{m} \cdot \vec{x}_{j}-\sum_{s=1}^{n} u_{i, s}^{m} \sum_{j=1}^{n} u_{l, j}^{m} \cdot \vec{x}_{j}\right)
\end{aligned}
$$

\footnotetext{
${ }^{1}$ The particular case in which $m=1$ was demonstrated in (Horta 2010), pages 71-73.
} 


$$
\begin{aligned}
& =\alpha \sum_{s=1}^{n} \sum_{j=1}^{n} u_{l, s}^{m} \cdot u_{i, j}^{m}\left(\left\langle\vec{x}_{j}, \beta\right\rangle_{A}-\left\langle\vec{x}_{s}, \beta\right\rangle_{A}\right) \\
& =\alpha \sum_{s=1}^{n} \sum_{j=1}^{n} u_{l, s}^{m} \cdot u_{i, j}^{m}\left\{\sum_{a=1}^{n} \sum_{b=1}^{n} u_{l, a}^{m} \cdot u_{i, b}^{m}\left(\left\langle\vec{x}_{j}, \vec{x}_{b}\right\rangle_{A}-\left\langle\vec{x}_{j}, \vec{x}_{a}\right\rangle_{A}\right)\right. \\
& \left.-\sum_{a=1}^{n} \sum_{b=1}^{n} u_{l, a}^{m} \cdot u_{i, b}^{m}\left(\left\langle\vec{x}_{s}, \vec{x}_{b}\right\rangle_{A}-\left\langle\vec{x}_{s}, \vec{x}_{a}\right\rangle_{A}\right)\right\} \\
& =-\frac{\alpha}{2} \sum_{s=1}^{n} \sum_{j=1}^{n} u_{l, s}^{m} \cdot u_{i, j}^{m} \sum_{a=1}^{n} \sum_{b=1}^{n} u_{l, a}^{m} \cdot u_{i, b}^{m}\left(\left\langle\vec{x}_{j}, \vec{x}_{j}\right\rangle_{A}-\left\langle\vec{x}_{j}, \vec{x}_{j}\right\rangle_{A}\right. \\
& +\left\langle\vec{x}_{s}, \vec{x}_{s}\right\rangle_{A}-\left\langle\vec{x}_{s}, \vec{x}_{s}\right\rangle_{A}+\left\langle\vec{x}_{b}, \vec{x}_{b}\right\rangle_{A}-\left\langle\vec{x}_{b}, \vec{x}_{b}\right\rangle_{A}+\left\langle\vec{x}_{a}, \vec{x}_{a}\right\rangle_{A}-\left\langle\vec{x}_{a}, \vec{x}_{a}\right\rangle_{A} \\
& \left.-2\left\langle\vec{x}_{j}, \vec{x}_{b}\right\rangle_{A}+2\left\langle\vec{x}_{j}, \vec{x}_{a}\right\rangle_{A}+2\left\langle\vec{x}_{s}, \vec{x}_{b}\right\rangle_{A}-2\left\langle\vec{x}_{s}, \vec{x}_{a}\right\rangle_{A}\right) \\
& =-\frac{\alpha}{2} \sum_{s=1}^{n} \sum_{j=1}^{n} u_{l, s}^{m} \cdot u_{i, j}^{m} \sum_{a=1}^{n} \sum_{b=1}^{n} u_{l, a}^{m} \cdot u_{i, b}^{m}\left\{\left(\left\langle\vec{x}_{j}, \vec{x}_{j}\right\rangle_{A}-2\left\langle\vec{x}_{j}, \vec{x}_{b}\right\rangle_{A}+\left\langle\vec{x}_{b}, \vec{x}_{b}\right\rangle_{A}\right)\right. \\
& -\left(\left\langle\vec{x}_{j}, \vec{x}_{j}\right\rangle_{A}-2\left\langle\vec{x}_{j}, \vec{x}_{a}\right\rangle_{A}+\left\langle\vec{x}_{a}, \vec{x}_{a}\right\rangle_{A}\right)-\left(\left\langle\vec{x}_{s}, \vec{x}_{s}\right\rangle_{A}-2\left\langle\vec{x}_{s}, \vec{x}_{b}\right\rangle_{A}+\left\langle\vec{x}_{b}, \vec{x}_{b}\right\rangle_{A}\right) \\
& \left.+\left(\left\langle\vec{x}_{s}, \vec{x}_{s}\right\rangle_{A}-2\left\langle\vec{x}_{s}, \vec{x}_{a}\right\rangle_{A}+\left\langle\vec{x}_{a}, \vec{x}_{a}\right\rangle_{A}\right)\right\} \\
& =-\frac{\alpha}{2} \sum_{s=1}^{n} \sum_{j=1}^{n} u_{l, s}^{m} \cdot u_{i, j}^{m} \sum_{a=1}^{n} \sum_{b=1}^{n} u_{l, a}^{m} \cdot u_{i, b}^{m}\left(\left\|\vec{x}_{j}-\vec{x}_{b}\right\|_{A}^{2}-\left\|\vec{x}_{j}-\vec{x}_{a}\right\|_{A}^{2}\right. \\
& \left.-\left\|\vec{x}_{s}-\vec{x}_{b}\right\|_{A}^{2}+\left\|\vec{x}_{s}-\vec{x}_{a}\right\|_{A}^{2}\right) \\
& =-\frac{\alpha}{2} \sum_{s=1}^{n} u_{l, s}^{m} \sum_{j=1}^{n} u_{i, j}^{m} \sum_{a=1}^{n} u_{l, a}^{m} \sum_{b=1}^{n} u_{i, b}^{m}\left(r_{j, b}-r_{j, a}-r_{s, b}+r_{s, a}\right) . \\
& =
\end{aligned}
$$

Accordingly, the relational matrix provides all the information needed to calculate distances between centroids, as long as $R$ corresponds to pairwise distances in any real inner-product space. The following development shows how to describe $\left\|_{o} \vec{v}_{i}-{ }_{o} \vec{v}_{l}\right\|_{A}^{2}$ in matricial form:

$$
\begin{aligned}
\left\|_{o} \vec{v}_{i}-{ }_{o} \vec{v}_{l}\right\|_{A}^{2} & =-\frac{\alpha}{2} \sum_{s=1}^{n} \sum_{j=1}^{n} u_{l, s}^{m} \cdot u_{i, j}^{m} \sum_{a=1}^{n} u_{l, a}^{m}\left\{\sum_{b=1}^{n} u_{i, b}^{m}\left(r_{j, b}-r_{s, b}\right)+\left|C_{i}\right|\left(r_{s, a}-r_{j, a}\right)\right\} \\
& =-\frac{\alpha}{2} \sum_{s=1}^{n} \sum_{j=1}^{n} u_{l, s}^{m} \cdot u_{i, j}^{m}\left\{\left|C_{l}\right| \sum_{b=1}^{n} u_{i, b}^{m}\left(r_{j, b}-r_{s, b}\right)+\left|C_{i}\right| \sum_{a=1}^{n} u_{l, a}^{m}\left(r_{s, a}-r_{j, a}\right)\right\}
\end{aligned}
$$




$$
\begin{aligned}
& =-\frac{\alpha}{2} \sum_{s=1}^{n} u_{l, s}^{m}\left\{\left|C_{l}\right| \sum_{b=1}^{n} u_{i, b}^{m}\left(\sum_{j=1}^{n} u_{i, j}^{m} \cdot r_{j, b}-\left|C_{i}\right| r_{s, b}\right)\right. \\
& \left.+\left|C_{i}\right| \sum_{a=1}^{n} u_{l, a}^{m}\left(\left|C_{i}\right| r_{s, a}-\sum_{j=1}^{n} u_{i, j}^{m} \cdot r_{j, a}\right)\right\} \\
& =-\frac{\alpha}{2}\left\{\left|C_{l}\right|^{2} \sum_{b=1}^{n} u_{i, b}^{m} \sum_{j=1}^{n} u_{i, j}^{m} \cdot r_{j, b}-\left|C_{l}\right|\left|C_{i}\right| \sum_{b=1}^{n} u_{i, b}^{m} \sum_{s=1}^{n} u_{l, s}^{m} \cdot r_{s, b}\right. \\
& \left.+\left|C_{i}\right|^{2} \sum_{a=1}^{n} u_{l, a}^{m} \sum_{s=1}^{n} u_{l, s}^{m} \cdot r_{s, a}-\left|C_{i}\right|\left|C_{l}\right| \sum_{a=1}^{n} u_{l, a}^{m} \sum_{j=1}^{n} u_{i, j}^{m} \cdot r_{j, a}\right\} .
\end{aligned}
$$

Let the $i$ th row of $U$, with its elements raised to the $m$ th power, be represented as the $1 \times n$ row vector $u_{i}^{m}=\left(u_{i, 1}^{m}, u_{i, 2}^{m}, \cdots, u_{i, n}^{m}\right)$ for all $i \in \mathbb{N}_{1, c}$. Then, from $\sum_{s=1}^{n} u_{l, s}^{m} \sum_{j=1}^{n} u_{i, j}^{m} \cdot r_{s, j}=$ $u_{l}^{m} R\left(u_{i}^{m}\right)^{T}$ it follows that:

$$
\begin{aligned}
\left\|_{o} \vec{v}_{i}-{ }_{o} \vec{v}_{l}\right\|_{A}^{2} & =-\frac{\alpha}{2}\left(\left|C_{l}\right|^{2} u_{i}^{m} R\left(u_{i}^{m}\right)^{T}-2\left|C_{l}\right|\left|C_{i}\right| u_{i}^{m} R\left(u_{l}^{m}\right)^{T}+\left|C_{i}\right|^{2} u_{l}^{m} R\left(u_{l}^{m}\right)^{T}\right) \\
& =-\frac{{ }_{r} \vec{v}_{i}^{T} \cdot R \cdot{ }_{r} \vec{v}_{i}}{2}+{ }_{r} \vec{v}_{i}^{T} \cdot R \cdot{ }_{r} \vec{v}_{l}-\frac{{ }_{r} \vec{v}_{l}^{T} \cdot R \cdot{ }_{r} \vec{v}_{l}}{2} .
\end{aligned}
$$

Note that Theorem F.1 can also be used to compute distances between data objects and cluster centroids. To that end, suppose the $i$ th cluster is a singleton, i.e., $\left(u_{i, 1}, \ldots, u_{i, j-1}, u_{i, j}, u_{i, j+1}, \ldots, u_{i, n}\right)=(0, \ldots, 0,1,0, \ldots, 0)$. This implies ${ }_{o} \vec{v}_{i}=\vec{x}_{j}$ and ${ }_{r} \vec{v}_{i}=(0, \ldots, 0,1,0, \ldots, 0)$. Accordingly, equation (F.5) reduces to:

$$
\begin{aligned}
\left\|\vec{x}_{j}-{ }_{o} \vec{v}_{l}\right\|_{A}^{2} & =-\frac{R_{i, i}}{2}+\left(R \cdot{ }_{r} \vec{v}_{l}\right)_{j}-\frac{{ }_{r} \vec{v}_{l}^{T} \cdot R \cdot{ }_{r} \vec{v}_{l}}{2} \\
& =\left(R \cdot{ }_{r} \vec{v}_{l}\right)_{j}-\frac{{ }_{r} \vec{v}_{l}^{T} \cdot R \cdot{ }_{r} \vec{v}_{l}}{2},
\end{aligned}
$$

which is precisely equation (F.2), proven in (Hathaway et al., 1989). Hence, (F.2) is a special case of (F.5) when the $i$ th centroid represents a single object.

Finally, it is worth noticing that Theorem F.1 can even be used to compute distances between cluster centroids and the data centroid (grand mean). The Variance Ratio Criterion (VRC so-called Calinski-Harabasz index) (Calinski e Harabasz, 1974), for instance, is a well-known validity index that requires such distances. This index ranked among the best ones in comprehensive comparative studies by Milligan \& Cooper Milligan e Cooper (1985) (involving 30 indices) and Vendramin et al. Vendramin et al. (2010) (involving 40 indices). Computing the distances between cluster centroids and the centroid of the entire data set can be accomplished by simply defining an additional, artificial cluster (the $(c+1)$ th cluster) as one that unequivocally contains all data objects? 2 i.e.: $\left(u_{c+1,1}, u_{c+1,2}, \ldots, u_{c+1, n}\right)=(1,1, \ldots, 1)$. Then, distances

\footnotetext{
${ }^{2}$ Although definition F.1. requires that a cluster neither be empty nor fully contain all the data objects, equation
} 
$\left\|\vec{v}_{i}-{ }_{o} \vec{v}_{c+1}\right\|_{A}^{2}$ for $i \leq c$ can be readily computed by using equation $(\overrightarrow{F .5})$.

\section{F.3 Conclusions}

A theorem has been shown that allows the exact computation (in $\mathcal{O}\left(n^{2}\right)$ time) of distances between geometric centroids of data clusters in a relational domain in which the data are described by an Euclidean relational matrix, i.e., a distance matrix $R$ that corresponds to a configuration of points in some Euclidean space. When $R$ is not an Euclidean matrix, the $\beta$ spread transformation described in (Hathaway e Bezdek, 1994; Sledge et al., 2010) can be employed to turn $R$ into an Euclidean one. This letter is intended to be a complement to those theoretical findings in (Sledge et al., 2010). 


\section{Bibliografia}

Achtert, E., Bohm, C., Kriegel, H.-P., Kroger, P., e Zimek, A. (2007). On exploring complex relationships of correlation clusters. In Scientific and Statistical Database Management, 2007. SSBDM '07. 19th Intl. Conference on, page 7.

Achtert, E., Bohm, C., Kroger, P., e Zimek, A. (2006a). Mining hierarchies of correlation clusters. In Proc. of the 18th Intl. Conference on Scientific and Statistical Database Management, pages 119-128, Washington, DC, USA. IEEE Computer Society.

Achtert, E., Kriegel, H.-P., Pryakhin, A., e Schubert, M. (2006b). Clustering multi-represented objects using combination trees. In Proceedings of the 10th Pacific-Asia conference on Advances in Knowledge Discovery and Data Mining, PAKDD'06, pages 174-178, Berlin, Heidelberg. Springer-Verlag.

Aggarwal, C., Hinneburg, A., e Keim, D. (2001). On the surprising behavior of distance metrics in high dimensional space. In Van den Bussche, J. e Vianu, V., editors, Database Theory, ICDT 2001, volume 1973 of Lecture Notes in Computer Science, pages 420-434. Springer Berlin / Heidelberg.

Aggarwal, C., Wolf, J., Yu, P., Procopiuc, C., e Park, J. (1999). Fast algorithms for projected clustering. SIGMOD Record (ACM Special Interest Group on Management of Data), 28(2):61-72.

Aggarwal, C. e Yu, P. (2000). Finding generalized projected clusters in high dimensional spaces. SIGMOD Record (ACM Special Interest Group on Management of Data), 29(2):70-81.

Agrawal, R., Gehrke, J., Gunopulos, D., e Raghavan, P. (1998). Automatic subspace clustering of high dimensional data for data mining applications. SIGMOD Record, 27(2):94-105.

Agrawal, R. e Srikant, R. (1994). Fast algorithms for mining association rules in large databases. In Bocca, J. B., Jarke, M., e Zaniolo, C., editors, VLDB'94, Proc. of 20th Intl. Conference on Very Large Data Bases, September 12-15, 1994, Santiago de Chile, Chile, pages 487-499. Morgan Kaufmann. 
Aguilar-Ruiz, J. S. (2005). Shifting and scaling patterns from gene expression data. Bioinformatics, 21(20):3840-3845.

Albatineh, A. N., Niewiadomska-Bugaj, M., e Mihalko, D. (2006). On similarity indices and correction for chance agreement. Journal of Classification, 23:301-313. 10.1007/s00357006-0017-z.

Alcock, R. (1999). Synthetic control chart time series data set.

Almuallim, H. e Dietterich, T. G. (1991). Learning with many irrelevant features. In Proc. of the Ninth National Conference on Artificial Intelligence (AAAI-91), volume 2, pages 547-552, Anaheim, California. AAAI Press.

Amigó, E., Gonzalo, J., Artiles, J., e Verdejo, F. (2009). A comparison of extrinsic clustering evaluation metrics based on formal constraints. Inf. Retr., 12(4):461-486.

Anderson, D. T., Bezdek, J. C., Popescu, M., e Keller, J. M. (2010). Comparing fuzzy, probabilistic, and possibilistic partitions. IEEE Trans. Fuzzy Syst., 18(5):906-918.

Anderson, D. T., Keller, J. M., Sjahputera, O., Bezdek, J. C., e Popescu, M. (2011). Comparing soft clusters and partitions. In Fuzzy Systems (FUZZ), 2011 IEEE Intl. Conference on, pages $924-931$.

Ayadi, W., Elloumi, M., e Hao, J.-K. (2012a). Bicfinder: a biclustering algorithm for microarray data analysis. Knowl. Inf. Syst., 30(2):341-358.

Ayadi, W., Maatouk, O., e Bouziri, H. (2012b). Evolutionary biclustering algorithm of gene expression data. In Database and Expert Systems Applications (DEXA), 2012 23rd Intl. Workshop on, pages 206-210.

Beringer, J. e Hüllermeier, E. (2007). Fuzzy Clustering of Parallel Data Streams, pages 333352. John Wiley \& Sons, Ltd.

Bezdek, J. C. (1981). Pattern Recognition with Fuzzy Objective Function Algorithms. Kluwer Academic Publishers, Norwell, MA, USA.

Bezdek, J. C., Pal, M. R., Keller, J., e Krisnapuram, R. (1999). Fuzzy Models and Algorithms for Pattern Recognition and Image Processing. Kluwer Academic Publishers, Norwell, MA, USA.

Bhattacharya, A. e De, R. K. (2009). Bi-correlation clustering algorithm for determining a set of co-regulated genes. Bioinformatics, 25(21):2795-2801.

Bickel, S. e Scheffer, T. (2004). Multi-view clustering. In Proceedings of the Fourth IEEE International Conference on Data Mining, ICDM '04, pages 19-26, Washington, DC, USA. IEEE Computer Society. 
Bishop, C. M. (2006). Pattern Recognition and Machine Learning. Springer.

Blum, A. e Langley, P. (1997). Selection of relevant features and examples in machine learning. Artificial Intelligence, 97(1-2):245-271.

Blum, A. e Mitchell, T. (1998). Combining labeled and unlabeled data with co-training. In Proceedings of the eleventh annual conference on Computational learning theory, COLT' 98, pages 92-100, New York, NY, USA. ACM.

Böhm, C., Kailing, K., Kröger, P., e Zimek, A. (2004). Computing clusters of correlation connected objects. In Proc. of the 2004 ACM SIGMOD international conference on Management of data, SIGMOD '04, pages 455-466, New York, NY, USA. ACM.

Borgelt, C. (2007). Resampling for fuzzy clustering. Intl. Journal of Uncertainty, Fuzziness and Knowledge-Based Systems, pages 595-614.

Borgelt, C. e Kruse, R. (2006). Finding the number of fuzzy clusters by resampling. In Fuzzy Systems, 2006 IEEE Intl. Conference on, pages 48-54.

Bozdag, D., Kumar, A. S., e Çatalyürek, U. V. (2010). Comparative analysis of biclustering algorithms. In 2010 ACM Intl. Conf. on Bioinformatics and Computational Biology, pages 265-274.

Brouwer, R. (2009). Extending the rand, adjusted rand and jaccard indices to fuzzy partitions. Journal of Intelligent Information Systems, 32:213-235. 10.1007/s10844-008-0054-7.

Busygin, S., Prokopyev, O., e Pardalos, P. M. (2008). Biclustering in data mining. Computers \& Operations Research, 35(9):2964 - 2987.

Califano, A., Stolovitzky, G., e Tu, Y. (2000). Analysis of gene expression microarrays for phenotype classification. Proc. of the Intl. Conference on Intelligent Systems for Molecular Biology, 8:75-85.

Calinski, T. e Harabasz, J. (1974). A dendrite method for cluster analysis. Communications in statistics, 3:1-27.

Campello, R. J. G. B. (2007). A fuzzy extension of the rand index and other related indexes for clustering and classification assessment. Pattern Recognition Letters, 28(7):833 - 841.

Campello, R. J. G. B. (2010). Generalized external indexes for comparing data partitions with overlapping categories. Pattern Recogn. Lett., 31(9):966-975.

Campello, R. J. G. B. e Hruschka, E. R. (2006). A fuzzy extension of the silhouette width criterion for cluster analysis. Fuzzy Sets and Systems, 157:2858-2875.

Cano, C., Adarve, L., López, J., e Blanco, A. (2007). Possibilistic approach for biclustering microarray data. Computers in Biology and Medicine, 37(10):1426 - 1436. 
Ceccarelli, M. e Maratea, A. (2009). Concordance indices for comparing fuzzy, possibilistic, rough and grey partitions. Int. J. Knowl. Eng. Soft Data Paradigm., 1(4):331-344.

Cheng, C.-H., Fu, A. W., e Zhang, Y. (1999). Entropy-based subspace clustering for mining numerical data. In KDD '99: Proc. of the fifth ACM SIGKDD international conference on Knowledge discovery and data mining, pages 84-93, New York, NY, USA. ACM.

Cheng, K.-O., Law, N.-F., Siu, W.-C., e Liew, A. (2008). Identification of coherent patterns in gene expression data using an efficient biclustering algorithm and parallel coordinate visualization. BMC Bioinformatics, 9(1):210.

Cheng, Y. e Church, G. M. (2000). Biclustering of expression data. In Proc. 8th Intl. Conf. on Intelligent Systems for Molecular Biology, pages 93-103. AAAI Press.

Conover, W. J. (1998). Practical Nonparametric Statistics. John Wiley \& Sons.

Consortium, G. O. (2012). Gene ontology website. Page http://www • geneontology • org/.

Dave, R. e Sen, S. (2002). Robust fuzzy clustering of relational data. IEEE Trans. Fuzzy Syst., 10(6):713-727.

Davenport, J. W. e Hathaway, R. J. (1995). Possibilistic c-means clustering for relational data. In Proc. of the First Intl. Conference on Neural, Parallel and Scientific Computations, volume 1, pages 139-142, USA. Dynamic Publishers Inc.

Davies, D. e Bouldin, D. (1979). A cluster separation measure. IEEE Trans. Pattern Recognition Machine Intell, PAMI-1(2):224-227.

De França, F., Coelho, G., e Von Zuben, F. (2013). Predicting missing values with biclustering: A coherence-based approach. Pattern Recognition, 46(5):1255-1266.

Dempster, A. P., Laird, N. M., e Rubin, D. B. (1977). Maximum likelihood from incomplete data via the em algorithm. Journal of the Royal Statistical Society. Series B (Methodological), 39(1):1-38.

Dharan, S. e Nair, A. (2009). Biclustering of gene expression data using reactive greedy randomized adaptive search procedure. BMC Bioinformatics, 10(Suppl 1):S27.

Dhillon, I. S., Mallela, S., e Modha, D. S. (2003). Information-theoretic co-clustering. In Proc. 9th ACM SIGKDD Intl. Conf. on Knowledge, Discovery, and Data Mining, KDD '03, pages 89-98, New York. ACM.

Dice, L. R. (1945). Measures of the amount of ecologic association between species. Ecology, 26(3):pp. 297-302. 
Divina, F. e Aguilar-Ruiz, J. S. (2006). Biclustering of expression data with evolutionary computation. IEEE Trans. on Knowl. and Data Eng., 18(5):590-602.

Domeniconi, C., Papadopoulos, D., Gunopulos, D., e Ma, S. (2004). Subspace clustering of high dimensional data. Proc. Fourth SIAM Int'l Conf. Data Mining.

Dongen, S. (2000). Performance criteria for graph clustering and markov cluster experiments. Technical report, National Research Institute for Mathematics and Computer Science, Amsterdam, The Netherlands, The Netherlands.

Dunn, J. C. (1973). A fuzzy relative of the isodata process and its use in detecting compact well-separated clusters. Cybernetics and Systems, 3(3):32-57.

Dunn, J. C. (1974). Well separated clusters and optimal fuzzy-partitions. Journal of Cybernetics, 4:95-104.

Eren, K., Deveci, M., Küçüktunç, O., e Çatalyürek, U. V. (2012). A comparative analysis of biclustering algorithms for gene expression data. Briefings in Bioinformatics.

Ester, M., peter Kriegel, H., S, J., e Xu, X. (1996). A density-based algorithm for discovering clusters in large spatial databases with noise. pages 226-231. AAAI Press.

Everitt, B. S., Landau, S., e Leese, M. (2001). Cluster Analysis. Arnold Publishers.

Falkenauer, E. (1998). Genetic Algorithms and Grouping Problems. John Wiley \& Sons, Inc., New York City, NY, USA.

Ferris, M. C. e Mangasarian, O. L. (1995). Breast cancer diagnosis via linear programming. Computational Science Engineering, IEEE, 2(3):70-71.

Frank, A. e Asuncion, A. (2010). UCI machine learning repository.

Fred, A. L. N. e Jain, A. K. (2005). Combining multiple clusterings using evidence accumulation. IEEE Trans. Pattern Anal. Mach. Intell., 27(6):835-850.

Freitas, A., Afreixo, V., Pinheiro, M., Oliveira, J., Moura, G., e Santos, M. (2011). Improving the performance of the iterative signature algorithm for the identification of relevant patterns. Statistical Analysis and Data Mining, 4(1):71-83.

Friedman, J. H. e Meulman, J. J. (2004). Clustering objects on subsets of attributes. Journal of the Royal Statistical Society. Series B: Statistical Methodology, 66(4):815-839.

Frigui, H., Hwang, C., e Rhee, F. C.-H. (2007). Clustering and aggregation of relational data with applications to image database categorization. Pattern Recognition, 40(11):3053 - 3068.

Frigui, H. e Mahdi, R. (2007). Semi-supervised clustering and feature discrimination with instance-level constraints. IEEE Intl. Conference on Fuzzy Systems, pages 1-6. 
Frigui, H. e Nasraoui, O. (2000). Simultaneous clustering and attribute discrimination. IEEE Intl. Conference on Fuzzy Systems, 1:158-163.

Frigui, H. e Nasraoui, O. (2004). Unsupervised learning of prototypes and attribute weights. Pattern Recognition, 37(3):567 - 581.

Frigui, H. e Salema, S. (2003). Fuzzy clustering and subset feature weighting. IEEE Intl. Conference on Fuzzy Systems, 2:857-862.

Gao, B., Griffith, O., Ester, M., Xiong, H., Zhao, Q., e Jones, S. (2012). On the deep orderpreserving submatrix problem: A best effort approach. IEEE Trans. Knowl. and Data Eng., 24(2):309-325.

Getz, G., Levine, E., e Domany, E. (2000). Coupled two-way clustering analysis of gene microarray data. Proc. of the National Academy of Sciences of the United States of America, 97(22):12079-12084.

Geusebroek, J.-M., Burghouts, G. J., e Smeulders, A. W. M. (2005). The amsterdam library of object images. Int. J. Comput. Vision, 61:103-112.

Gremalschi, S. e Altun, G. (2008). Mean squared residue based biclustering algorithms. In Proc. 4th Intl. Conf. on Bioinformatics Research and Applications, ISBRA'08, pages 232243, Berlin, Heidelberg. Springer-Verlag.

Gu, J. e Liu, J. (2008). Bayesian biclustering of gene expression data. BMC Genomics, 9(Suppl 1):S4.

Halkidi, M., Batistakis, Y., e Vazirgiannis, M. (2001). On clustering validation techniques. Journal of Intelligent Information Systems, 17:107-145.

Halkidi, M., Batistakis, Y., e Vazirgiannis, M. (2002a). Cluster validity methods: part i. SIGMOD Rec., 31(2):40-45.

Halkidi, M., Batistakis, Y., e Vazirgiannis, M. (2002b). Clustering validity checking methods: part ii. SIGMOD Rec., 31:19-27.

Hanczar, B. e Nadif, M. (2012). Ensemble methods for biclustering tasks. Pattern Recognition, 45(11):3938 - 3949.

Hartigan, J. A. (1972). Direct clustering of a data matrix. Journal of the American Statistical Association, 67(337):123-129.

Hastie, T., Tibshirani, R., Sherlock, G., Eisen, M., Brown, P., e Botstein, D. (1999). Imputing missing data for gene expression arrays. Technical report, Stanford University.

Hathaway, R. J. e Bezdek, J. C. (March 1994). Nerf c-means: Non-euclidean relational fuzzy clustering. Pattern Recognition, 27(3):429-437. 
Hathaway, R. J., Davenport, J. W., e Bezdek, J. C. (1989). Relational duals of the c-means clustering algorithms. Pattern Recognition, 22(2):205-212.

Hochreiter, S., Bodenhofer, U., Heusel, M., Mayr, A., Mitterecker, A., Kasim, A., Khamiakova, T., Van Sanden, S., Lin, D., Talloen, W., Bijnens, L., Guhlmann, H. W. H., Shkedy, Z., e Clevert, D.-A. (2010). FABIA: factor analysis for bicluster acquisition. Bioinformatics, 26(12):1520-1527.

Hollander, M. e Wolfe, D. A. (1999). Nonparametric statistical methods.

Horta, D. (2010). Evolutionary algorithms for relational data clustering (in portuguese). Dissertação de Mestrado, University of São Paulo, São Carlos - SP, Brazil.

Horta, D. e Campello, R. J. G. B. (2012). Automatic aspect discrimination in data clustering. Pattern Recognition, 45(12):4370-4388.

Hruschka, E. R., Campello, R. J. G. B., e de Castro, L. N. (2006). Evolving clusters in geneexpression data. Information Sciences, 176:1898-1927.

Hruschka, E. R., de Castro, L. N., e Campello, R. J. G. B. (2004). Evolutionary algorithms for clustering gene-expression data. In Data Mining, 2004. ICDM '04. Fourth IEEE Intl. Conference on, pages 403-406.

Huang, Q., Lu, M., e Yan, H. (2008). An evolutionary algorithm for discovering biclusters in gene expression data of breast cancer. In IEEE Congress on Evolutionary Computation, pages $829-834$.

Hubert, L. e Arabie, P. (1985). Comparing partitions. Journal of Classification, 2(1):193-218.

Hullermeier, E. e Rifqi, M. (2009). A fuzzy variant of the rand index for comparing clustering structures. In Proc. IFSA, page 1-6, Lisbon, Portugal.

Jaccard, P. (1908). Nouvelles recherches sur la distribution florale. Bulletin de la Société Vaudoise de Sciences Naturelles, 44:223-370.

Jain, A. K. e Dubes, R. C. (1988). Algorithms for clustering data. Prentice-Hall, Inc., Upper Saddle River, NJ, USA.

Jain, A. K., Murty, M. N., e Flynn, P. J. (1999). Data clustering: A review. ACM Computing Surveys, 31:264-323.

Jiang, D., Tang, C., e Zhang, A. (2004). Cluster analysis for gene expression data: A survey. IEEE Trans. on Knowl. and Data Eng., 16(11):1370-1386.

Jing, L., Ng, M. K., e Huang, J. Z. (2007). An entropy weighting k-means algorithm for subspace clustering of high-dimensional sparse data. IEEE Trans. on Knowl. and Data Eng., 19(8):1026-1041. 
Jolliffe, I. T. (2002). Principal Component Analysis. Springer, second edition.

Kailing, K., Kriegel, H.-P., e Kröger, P. (2004a). Density-connected subspace clustering for high-dimensional data. In IN: PROC. SDM. (2004), pages 246-257.

Kailing, K., Kriegel, H.-P., Pryakhin, A., e Schubert, M. (2004b). Clustering multi-represented objects with noise. In Proceedings of the Pacific-Asia Conference on Knowledge Discovery and Data Mining, pages 394-403.

Kaufman, L. e Rousseeuw, P. J. (1990). Finding Groups in Data: an introduction to cluster analysis. Wiley.

Kira, K. e Rendell, L. A. (1992a). A practical approach to feature selection. In Proc. of the Ninth Intl. Workshop on Machine Learning, ML '92, pages 249-256, San Francisco, CA, USA. Morgan Kaufmann Publishers Inc.

Kira, K. e Rendell, L. A. (1992b). The Feature Selection Problem: Traditional Methods and a New Algorithm. In AAAI, pages 129-134, Cambridge, MA, USA. AAAI Press and MIT Press.

Klir, G. J. e Yuan, B. (1995). Fuzzy sets and fuzzy logic: theory and applications. Prentice-Hall, Inc., Upper Saddle River, NJ, USA.

Kluger, Y., Basri, R., Chang, J., e Gerstein, M. (2003). Spectral biclustering of microarray data: Coclustering genes and conditions. Genome Research, 13(4):703-716.

Kolen, J. e Hutcheson, T. (2002). Reducing the time complexity of the fuzzy c-means algorithm. IEEE Trans. on Fuzzy Systems, 10(2):263-267.

Kriegel, H.-P., Kröger, P., e Zimek, A. (2009). Clustering high-dimensional data: A survey on subspace clustering, pattern-based clustering, and correlation clustering. ACM Trans. on Knowledge Discovery from Data, 3(1).

Krishnapuram, R., Joshi, A., Nasraoui, O., e Yi, L. (2001). Low-complexity fuzzy relational clustering algorithms for web mining. IEEE Trans. on Fuzzy Systems, 9(4):595-607.

Krishnapuram, R. e Keller, J. (1993). A possibilistic approach to clustering. IEEE Trans. on Fuzzy Systems, 1(2):98-110.

Kuncheva, L. I. e Vetrov, D. P. (2006). Evaluation of stability of k-means cluster ensembles with respect to random initialization. IEEE Trans. Pattern Analysis and Machine Intelligence, 28(11): $1798-1808$.

Larsen, B. e Aone, C. (1999). Fast and effective text mining using linear-time document clustering. In Proc. of the fifth ACM SIGKDD international conference on Knowledge discovery and data mining, KDD '99, pages 16-22, New York, NY, USA. ACM. 
Lazzeroni, L. e Owen, A. (2000). Plaid models for gene expression data. Statistica Sinica, 12:61-86.

Lee, Y., Lee, J., e Jun, C.-H. (2011). Stability-based validation of bicluster solutions. Pattern Recognition, 44(2):252 - 264.

Lee, Y., Lee, J.-H., e Jun, C.-H. (2009). Validation measures of bicluster solutions. Industrial Engineering \& Management Systems, 8(2):101-108.

Levenshtein, V. I. (1966). Binary codes capable of correcting deletions, insertions, and reversals. Soviet Physics Doklady, 10(8):707-710.

Liu, G., Li, J., Sim, K., e Wong, L. (2007). Distance based subspace clustering with flexible dimension partitioning. In Data Engineering, 2007. ICDE 2007. IEEE 23rd Intl. Conference on, pages $1250-1254$.

Liu, H. e Yu, L. (2005). Toward integrating feature selection algorithms for classification and clustering. IEEE Trans. on Knowl. and Data Eng., 17(4):491-502.

Liu, X. e Wang, L. (2007). Computing the maximum similarity bi-clusters of gene expression data. Bioinformatics, 23(1):50-56.

Mackay, D. J. C. (2003). Information Theory, Inference and Learning Algorithms. Cambridge University Press, 1 edition.

MacQueen, J. B. (1967). Some methods for classification and analysis of multivariate observations. In Cam, L. M. L. e Neyman, J., editors, Proc. of the fifth Berkeley Symposium on Mathematical Statistics and Probability, volume 1, pages 281-297. University of California Press.

Madeira, S. C. e Oliveira, A. L. (2004). Biclustering algorithms for biological data analysis: A survey. IEEE/ACM Trans. Comput. Biol. Bioinformatics, 1(1):24-45.

Mardia, K. V., Bibby, J. M., e Kent, J. T. (1979). Multivariate analysis. Academic Press, London; New York.

Meila, M. (2003). Comparing clusterings by the variation of information. In Schölkopf, B. e Warmuth, M., editors, Learning Theory and Kernel Machines, volume 2777 of Lecture Notes in Computer Science, pages 173-187. Springer Berlin / Heidelberg.

Meila, M. (2005). Comparing clusterings: an axiomatic view. In Proc. of the 22nd international conference on Machine learning, ICML '05, pages 577-584, New York, NY, USA. ACM.

Meila, M. (2007). Comparing clusterings-an information based distance. J. Multivar. Anal., 98:873-895. 
Meila, M. e Heckerman, D. (2001). An experimental comparison of model-based clustering methods. Machine Learning, 42:9-29.

Milligan, G. (1981). A monte carlo study of thirty internal criterion measures for cluster analysis. Psychometrika, 46(2):187-199.

Milligan, G. W. e Cooper, M. C. (1985). An examination of procedures for determining the number of clusters in a data set. Psychometrika, 50:159-179.

Mirkin, B. (1996). Mathematical Classification and Clustering. Springer.

Mitra, P., Murthy, C., e Pal, S. (2002). Unsupervised feature selection using feature similarity. IEEE Trans. on Pattern Analysis and Machine Intelligence, 24(3):301-312.

Monti, S., Tamayo, P., Mesirov, J., e Golub, T. (2003). Consensus clustering: A resamplingbased method for class discovery and visualization of gene expression microarray data. Mach. Learn., 52:91-118.

Motulsky, H. (1995). Intuitive Biostatistics. Oxford University Press.

Murali, T. M. e Kasif, S. (2003). Extracting conserved gene expression motifs from gene expression data. In Pacific Symposium on Biocomputing, pages 77-88.

Nagesh, H., Goil, S., e Choudhary, A. (2001). Adaptive grids for clustering massive data sets. In In 1st SIAM Intl. Conference Proc. on Data Mining.

Nepomuceno, J. A., Lora, A. T., Aguilar-Ruiz, J. S., e García-Gutiérrez, J. (2007). Biclusters evaluation based on shifting and scaling patterns. In Proc. 8th Intl. Conf. on Intelligent Data Engineering and Automated Learning, IDEAL'07, pages 840-849, Berlin, Heidelberg. Springer-Verlag.

$\mathrm{Ng}$, R. T. e Han, J. (1994). Efficient and effective clustering methods for spatial data mining. In Proc. of the 20th Intl. Conference on Very Large Data Bases, pages 144-155. Morgan Kaufmann Publishers Inc.

Pakhira, M. K., Bandyopadhyay, S., e Maulik, U. (2005). A study of some fuzzy cluster validity indices, genetic clustering and application to pixel classification. Fuzzy Sets and Systems, 155(2):191 - 214.

Pal, N., Pal, K., Keller, J., e Bezdek, J. (2005). A possibilistic fuzzy c-means clustering algorithm. IEEE Trans. on Fuzzy Systems, 13(4):517-530.

Pal, N. R. e Bezdek, J. C. (1995). On cluster validity for the fuzzy c-means model. IEEE Trans. on Fuzzy Systems, 3(3):370-379.

Patrikainen, A. e Meila, M. (2006). Comparing subspace clusterings. IEEE Trans. on Knowl. and Data Eng., 18(7):902-916. 
Prelić, A., Bleuler, S., Zimmermann, P., Wille, A., Buhlmann, P., Gruissem, W., Hennig, L., Thiele, L., e Zitzler, E. (2006). A systematic comparison and evaluation of biclustering methods for gene expression data. Bioinformatics, 22(9):1122-1129.

Quere, R. e Frelicot, C. (2011). A normalized soft window-based similarity measure to extend the rand index. In Fuzzy Systems (FUZZ), 2011 IEEE Intl. Conference on, pages 2513 -2520.

Quere, R., Le Capitaine, H., Fraisseix, N., e Frelicot, C. (2010). On normalizing fuzzy coincidence matrices to compare fuzzy and/or possibilistic partitions with the rand index. In Proc. of the 2010 IEEE Intl. Conference on Data Mining, pages 977-982, Washington, DC, USA. IEEE Computer Society.

Rand, W. M. (1971). Objective criteria for the evaluation of clustering methods. Journal of the American Statistical Association, 66(336):846-850.

Rodriguez-Baena, D. S., Perez-Pulido, A. J., e Aguilar-Ruiz, J. S. (2011). A biclustering algorithm for extracting bit-patterns from binary datasets. Bioinformatics, 27(19):2738-2745.

Rosenberg, A. e Hirschberg, J. (2007). V-measure: A conditional entropy-based external cluster evaluation measure. In Proc. of the 2007 Joint Conference on Empirical Methods in Natural Language Processing and Computational Natural Language Learning, pages 410-420.

Rousseeuw, P. J. (1987). Silhouettes: A graphical aid to the interpretation and validation of cluster analysis. Journal of Computational and Applied Mathematics, 20:53-65.

Rovetta, S. e Masulli, F. (2009). An experimental validation of some indexes of fuzzy clustering similarity. In Proc. of the 8th Intl. Workshop on Fuzzy Logic and Applications, WILF '09, pages 132-139, Berlin, Heidelberg. Springer-Verlag.

Santamaría, R., Quintales, L., e Therón, R. (2007). Methods to bicluster validation and comparison in microarray data. In Yin, H., Tino, P., Corchado, E., Byrne, W., e Yao, X., editors, Intelligent Data Engineering and Automated Learning - IDEAL 2007, volume 4881 of Lecture Notes in Computer Science, pages 780-789. Springer Berlin / Heidelberg.

Santamaría, R., Therón, R., e Quintales, L. (2008). A visual analytics approach for understanding biclustering results from microarray data. BMC Bioinformatics, 9(1):247.

Saporta, G. e Youness, G. (2002). Comparing two partitions: Some proposals and experiments. In Härdle, W., editor, Proc. in Computational Statistics. Physica-Verlag.

Shabalin, A., Weigman, V., Perou, C., e Nobel, A. (2009). Finding large average submatrices in high dimensional data. Annals of Applied Statistics, 3(3):985-1012.

Sharan, R. e Shamir, R. (2000). Click: A clustering algorithm with applications to gene expression analysis. In ISMB'00, pages 307-316. AAAI Press, Menlo Park, CA. 
Sheng, Q., Moreau, Y., e De Moor, B. (2003). Biclustering microarray data by gibbs sampling. Bioinformatics, 19(suppl 2):ii196-ii205.

Sheskin, D. J. (2004). Parametric and Nonparametric Statistical Procedures. Chapman \& Hall/CRC.

Sledge, I., Bezdek, J., Havens, T., e Keller, J. (2010). Relational generalizations of cluster validity indices. IEEE Trans. on Fuzzy Systems, 18(4):771 -786.

Sprent, P. e Smeeton, N. C. (2007). Applied Nonparametric Statistical Methods. Chapman \& Hall/CRC, fourth edition.

Steinbach, M., Ertöz, L., e Kumar, V. (2003). The challenges of clustering high-dimensional data. In In New Vistas in Statistical Physics: Applications in Econophysics, Bioinformatics, and Pattern Recognition. Springer-Verlag.

Steinley, D. (2003). Local optima in k-means clustering: what you don't know may hurt you. Psychol Methods, 8(3):294-304.

Steinley, D. (2004). Properties of the hubert-arabie adjusted rand index. Psychological Methods, 9(3):386-396.

Strehl, A. e Ghosh, J. (2003). Cluster ensembles — a knowledge reuse framework for combining multiple partitions. Journal of Machine Learning Research, 3:583-617.

T. Hastie, R. Tibshirani, J. H. F. (2003). The Elements of Statistical Learning. Springer.

Tan, P.-N., Steinbach, M., e Kumar, V. (2005). Introduction to Data Mining. Addison Wesley.

Tanay, A., Sharan, R., e Shamir, R. (2005). Biclustering algorithms: A survey. In In Handbook of Computational Molecular Biology Edited by: Aluru S. Chapman \& Hall/CRC Computer and Information Science Series, pages 26-1.

Theodoridis, S. e Koutroumbas, K. (2006). Pattern Recognition, Third Edition. Academic Press.

Tucker, W. T. (1987). Counterexamples to the convergence theorem for fuzzy isodata clustering algorithms, volume 3, chapter 7, pages 109-121. CRC Press, Boca Raton, FL.

Turner, H., Bailey, T., e Krzanowski, W. (2005). Improved biclustering of microarray data demonstrated through systematic performance tests. Computational Statistics \& Data Analysis, $48(2): 235-254$.

Vendramin, L., Campello, R. J. G. B., e Hruschka, E. R. (2008). A robust methodology for comparing performances of clustering validity criteria. In SBIA, pages 237-247, Salvador. 
Vendramin, L., Campello, R. J. G. B., e Hruschka, E. R. (2009). On the comparison of relative clustering validity criteria. In Proc. of the SIAM Intl. Conference on Data Mining, pages 733-744. SIAM.

Vendramin, L., Campello, R. J. G. B., e Hruschka, E. R. (2010). Relative clustering validity criteria: A comparative overview. Statistical Analysis and Data Mining, 3(4):209-235.

Vinh, N. X. e Epps, J. (2009). A novel approach for automatic number of clusters detection in microarray data based on consensus clustering. In Proc. of the 2009 Ninth IEEE Intl. Conference on Bioinformatics and Bioengineering, BIBE '09, pages 84-91, Washington, DC, USA. IEEE Computer Society.

Vinh, N. X., Epps, J., e Bailey, J. (2009). Information theoretic measures for clusterings comparison: is a correction for chance necessary? In Proc. of the 26th Annual Intl. Conference on Machine Learning, ICML '09, pages 1073-1080, New York, NY, USA. ACM.

Vinh, N. X., Epps, J., e Bailey, J. (2010). Information theoretic measures for clusterings comparison: Variants, properties, normalization and correction for chance. Journal of Machine Learning Research, 11:2837-2854.

Vinod, H. D. (1969). Integer programming and the theory of grouping. Journal of the American Statistical Association, 40:506-519.

Wang, H., Wang, W., Yang, J., e Yu, P. S. (2002). Clustering by pattern similarity in large data sets. In Proc. of the 2002 ACM SIGMOD international conference on Management of data, SIGMOD '02, pages 394-405, New York, NY, USA. ACM.

Wang, L. e Fu, X. (2005). Data Mining with Computational Intelligence (Advanced Information and Knowledge Processing). Springer-Verlag New York, Inc., Secaucus, NJ, USA.

Wang, Z. (2010). Metrics for overlapping clustering comparison. Page http:// etaxonomy.org/mw/File:Sigs.pdf.

Wang, Z. (2012). Entropy on covers. Data Mining and Knowledge Discovery, 24:288-309. 10.1007/s10618-011-0230-1.

Wettschereck, D., Aha, D. W., e Mohri, T. (1997). A review and empirical evaluation of feature weighting methods for a class of lazy learning algorithms. Artificial Intelligence Review, $11: 273-314$.

Wu, K.-L. e Yang, M.-S. (2005). A cluster validity index for fuzzy clustering. Pattern Recogn. Lett., 26:1275-1291.

Xia, T., Tao, D., Mei, T., e Zhang, Y. (2010). Multiview spectral embedding. Trans. Sys. Man Cyber. Part B, 40(6):1438-1446. 
Xiao, J., Wang, L., Liu, X., e Jiang, T. (2008). Finding additive biclusters with random background. Lecture Notes in Computer Science (including subseries Lecture Notes in Artificial Intelligence and Lecture Notes in Bioinformatics), 5029 LNCS:263-276.

$\mathrm{Xu}, \mathrm{R}$. e Wunsch, D. (2005). Survey of clustering algorithms. IEEE Trans. on Neural Networks, 16(3):645-678.

Yang, W.-H., Dai, D.-Q., e Yan, H. (2011). Finding correlated biclusters from gene expression data. IEEE Trans. on Knowl. and Data Eng., 23(4):568-584.

Yu, J., Cheng, Q., e Huang, H. (2004). Analysis of the weighting exponent in the fcm. Systems, Man, and Cybernetics, Part B: Cybernetics, IEEE Transactions on, 34(1):634 - 639.

Yu, Z., Wong, H.-S., e Wang, H. (2007). Graph-based consensus clustering for class discovery from gene expression data. Bioinformatics, 23(21):2888-2896.

Zhang, J.-S. e Leung, Y.-W. (2004). Improved possibilistic c-means clustering algorithms. Fuzzy Systems, IEEE Transactions on, 12(2):209-217.

Zhang, S., Wong, H.-S., e Shen, Y. (2012). Generalized adjusted rand indices for cluster ensembles. Pattern Recognition, 45(6):2214 - 2226. 